DANIEL ALEXANDER FLÓREZ-ORREGO

PROCESS SYNTHESIS AND OPTIMIZATION OF SYNGAS AND AMMONIA PRODUCTION IN NITROGEN FERTILIZERS COMPLEXES:

EXERGY, ENERGY INTEGRATION AND $\mathrm{CO}_{2}$ EMISSIONS ASSESSMENT 
PROCESS SYNTHESIS AND OPTIMIZATION OF SYNGAS AND AMMONIA PRODUCTION IN NITROGEN FERTILIZERS COMPLEXES:

EXERGY, ENERGY INTEGRATION AND CO2 EMISSIONS ASSESSMENT

Thesis submitted in partial fulfillment of the requirements of the Degree of Doctor in Science of Mechanical Engineering (Energy and Fluids) at the Polytechnic School of the University of São Paulo 


\section{PROCESS SYNTHESIS AND OPTIMIZATION OF SYNGAS AND AMMONIA PRODUCTION IN NITROGEN FERTILIZERS COMPLEXES: \\ EXERGY, ENERGY INTEGRATION AND CO2 EMISSIONS ASSESSMENT}

Thesis submitted in partial fulfillment of the requirements of the Degree of Doctor in Science of Mechanical Engineering (Energy and Fluids) at the Polytechnic School of the University of São Paulo

Area: Mechanical Engineering - Energy and Fluids.

Supervisor: Prof. Dr. Silvio de Oliveira Júnior 
I hereby authorize the partial or total reproduction, publication and distribution of this text by whatsoever manner, conventional or electronic, for study and research purposes, provided that the original source is properly cited.

The author and his supervisor are the only responsible for the revisited and corrected version from the original manuscript,

São Paulo, November 30 ${ }^{\text {th }}, 2018$.

Candidate's Signature

Supervisor's Signature

\section{CATALOGRAPHIC CARD}

Flórez-Orrego, Daniel Alexander

Process Synthesis and Optimization of Syngas and Ammonia Production in Nitrogen Fertilizers Complexes: Exergy, Energy Integration and $\mathrm{CO} 2$ Emissions Assessment /

D. A. Flórez-Orrego. São Paulo, 2018.

$247 \mathrm{p}$.

Thesis (Ph.D.) - Polytechnic School of the University of São Paulo. Department of Mechanical Engineering.

1. Ammonia 2.Energy Integration 3.Fertilizers 4.Optimization 5.CO2 Emissions

I. University of São Paulo. Polytechnic School. Department of Mechanical Engineering II.t. 
É preciso sair da ilha para ver a ilha. Não nos vemos se não saímos de nós.

José Saramago (1922-2010)

49

Où sont les hommes?' reprit enfin le petit prince. 'On est un peu seul dans le désert.'

'On est seul aussi chez les hommes', dit le serpent.

Antoine de Saint-Exupéry (1943)

G

Trabajo y rectitud, (...) Quienes no llenéis todas estas condiciones, haríais mejor en volver a vuestras casas, porque llegaríais con el tiempo a ser la deshonra de la Escuela y solo contribuirías a la ruina de nuestra querida patria.

Tulio Ospina (1857)

Qui sait d'ailleurs si la raison de l'existence de l'homme, ne serait pas dans son existence même? Peut-être a-t-il été jeté au hasard sur un point de la surface de la Terre, sans qu'on puisse savoir ni comment, ni pourquoi; mais seulement qu'il doit vivre et mourir .(...) Quelle folie de tant se tourmenter pour ce qu'il est impossible de connaître, et ce qui ne nous rendrait pas plus heureux, quand nous en viendrions à bout. 
In honor of those who many times sacrificed their joy so that I could pursue my own joy. To my family, the unconditional support of all my endeavors. 


\section{ACKNOWLEDGEMENTS}

Firstly, I would like to express my sincere gratitude to my advisor Prof. Silvio de Oliveira Junior for the continuous support of my doctoral course and the guidance through the publication and presentation of the results. For his patience, motivation and genuine sense of humor. I am glad I could find not only a mentor but also a great friend for supervising my doctoral thesis. I am also grateful to the Examining Committee for their valuable suggestions and observations that contributed to the improvement of the final version of this thesis.

I have to acknowledge my mother Gloria Orrego Velez, my father Jaime Flórez Palacio and my sisters Sandra and Paula, for all the earnest attention and comprehension. Those relentless people whose exemplarity always encouraged me to do my best against whatsoever the difficulty had to be faced. This thesis would not have been possible without their support.

I also would like to thanks Prof. Dr. Emílio C. Nelli Silva, Prof. Dr. Flávio A. Sanzovo Fiorelli, Prof. Dr. Jorge A. Wilhelms Gut, Prof. Dr. Thiago de Castro Martins, Prof. Dr. Galo A. Carillo Le Roux and Prof. Dr. Ardson S. Vianna Junior for the precious instruction received in their classes and Prof. Dr. Hector Velásquez from Universidad Nacional de Colombia, Medellin, for the invitation to collaborate with his projects. My sincere thanks also goes to Prof. Dr. François Maréchal, who provided me an opportunity to join the IPESE laboratory at the École Polytechnique Fédérale de Lausanne, Switzerland, in the frame of the Swiss Government Excellence Scholarship. His support for publishing and presenting the partial results in San Diego, CA, and for interacting with a highly qualified team of scientists is sincerely appreciated. Special thanks to Shivom, Maziar, Nils, Ivan, Katy, Ligang, Hur, Raluca, Jean-Loup, Dilan, Theodoros, Alessio, Sylvie, as well as Aude Rapin and Co. and the Gomez family in Valais.

I also would like to thank to my fellow labmates Yamid Carranza, Pablo Silva, Izabela Batista, Cadu Mady, Tuong-van Nguyen, Sofia Russo, Ronaldo Freire, Amauri Almeida, Bruno Teixeira de Carvalho and Ali Allahyarzadeh, for the stimulating (and the bizarre!) discussions. Special thanks to Fernanda N. Silva and Rafael N. Nakashima with whom I had the great experience of working and publishing together in topics that directly contributed to the development of this thesis. Be strong!. I also would like to thank Bruno Hannud and Julio A. M. Silva for the technical discussions, Beethoven, Luis and Juan, and all the colleagues of the various departments of the University of Sao Paulo.

I also would like to acknowledge the Brazilian National Agency of Petroleum, Natural Gas and Biofuel (ANP) and the Colombian Administrative Department of Science, Technology and Innovation (COLCIENCIAS), for the financial support. Special thanks to Ms. Vilma Guerra and Prof. Dr. Marcelo R. Martins for their great job in the management of the scholarship and the PRH19/ANP. Finally, I would like to acknowledge the valuable job of all the personnel and secretaries of the PPGEM and COSEAS.

In short, thanks to the countless people from all over the world I met during the last 4 years. I irreversibly got permeated by the richness of the cultures of the earth globe. On peut dire sans aucune doute que cela a été uma lição impagável sobre diversidade e humildade. Gracias. 


\begin{abstract}
The primary aim of this thesis is to propose a method to develop the process synthesis and optimization of the production of syngas and ammonia, as well as other byproducts such as marketable $\mathrm{CO}_{2}$, in synthetic nitrogen fertilizers (SNF) complexes. The baseline analysis relies on a typical $1000 \mathrm{t} /$ day ammonia production plant composed of syngas production, purification $\left(\mathrm{CO}_{2}\right.$ capture) and compression systems, along with an industrial ammonia synthesis unit. Initially, exergy and exergoeconomy analyses are used to identify the most important sources of energy consumption and irreversibility associated to the operation units of the integrated plant.
\end{abstract}

From these results, a variety of alternatives for the improvement of the performance at the plantwide and component level are thoroughly examined along the different chapters. Due to the large amount of possible configurations and interrelations, the optimization process may become a formidable engineering task to be solved by using merely trial and error approaches. Accordingly, a systematic approach, based on the combination of heuristics, thermodynamic principles and mathematical programming is used to identify, evaluate, and determine the best configurations in terms of exergy consumption, degree of energy integration, process irreversibility, atmospheric $\mathrm{CO}_{2}$ emissions and operating costs. In this way, the process synthesis and optimization include not only the modification of the main process parameters but also the arrangement of the chemical plant components, suitably integrated to the waste heat recovery and cogeneration systems. Several aspects concerning: (i) the choice of the syngas purification system, (ii) the nature of the energy resources consumed, (iii) the exploitation of the thermodynamic potential at higher temperatures, (iv) the increase of the pre-combustion carbon capture by introducing chemically recuperated concepts, (v) the gradual variation of operating conditions by applying Le Châtelier and Counteraction principles, as well as (vi) the environmental benefits of using alternative energy sources to decarbonize the SNF sector are analyzed in the light of the reduced room of improvement found in modern conventional ammonia production facilities.

The results show significant potentials for decreasing the exergy intensity and environmental impact of those facilities. This allows issuing relevant recommendations for revamping the existing plants or embracing new approaches that attempt to minimize the economic costs, the process inefficiencies and mitigate the environmental impact produced. An efficiency increase of about $8-10 \%$ can be achieved by using more efficient combined cycle cogeneration systems with an affordable marginal investment cost. Alternative configurations with enhanced precombustion carbon capture, using either a CRGT system or upgraded biomass residues, may help cutting down the overall $\mathrm{CO}_{2}$ emissions in the syngas production in $20-28 \%$ or even promoting the decarbonization of the $\mathrm{SNF}$ sector at net rates of atmospheric $\mathrm{CO}_{2}$ depletion close to $-2.3 \mathrm{t}_{\mathrm{CO} 2} / \mathrm{t}_{\mathrm{NH} 3}$, respectively. The reduction of the process irreversibility has been also reduced by $10-13 \%$ in an industrial ammonia synthesis unit through the application of dual pressure systems and the introduction of a purge gas treatment process.

Keywords: Ammonia; Energy Integration; Fertilizers; Exergy; Cost; $\mathrm{CO}_{2}$ Emissions. 


\section{FIGURES}

Fig. 3.1. Evolution of the energy consumption in fertilizers and ammonia production.............6

Fig. 3.2. Effect the utilization of the Haber Bosch process on the population growth..............7

Fig.3.3. Classification of the synthetic nitrogen fertilizers and producers in Brazil...............8

Fig. 4.1. Plantwide layout of an integrated syngas and ammonia production plant.................10

Fig. 4.2. Syngas production via SMR and WGS process .....................................13

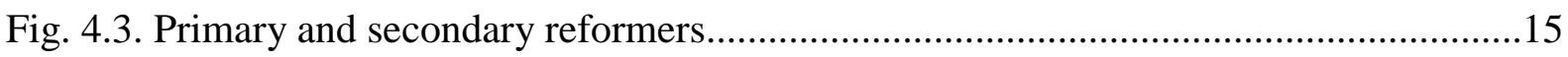

Fig. 4.4. Syngas purification unit based on a chemical absorption system ...........................20

Fig. 4.5. Syngas purification unit based on a physical absorption system ........................21

Fig. 4.6. Flow diagrams of various ammonia loop arrangements ..................................22

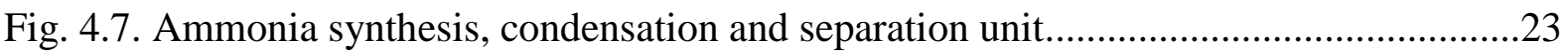

Fig. 4.8. Equilibrium concentration of ammonia at different temperature and pressures.........24

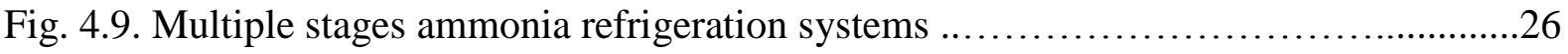

Fig. 4.10. Cryogenic recovery system for hydrogen-rich purge gas............................27

Fig. 4.11. Simplified steam network and utility systems of the conventional syngas and

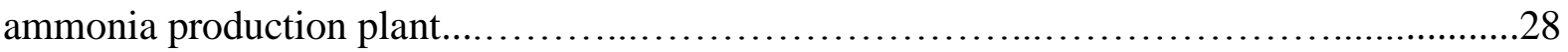

Fig. 5.1. Comparison of the reaction rates for the catalyst data given in Table 5.2.................38

Fig. 5.2. Variation of equilibrium conversion with temperature for (a) an exothermic reaction

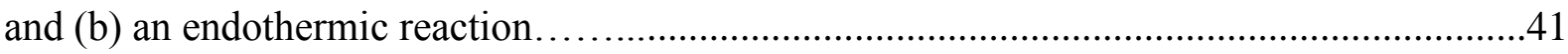

Fig. 5.3. Lines of constant reaction rate in a conversion vs. temperature plot. Montecatini

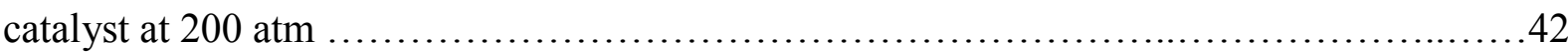

Fig. 5.4. Adiabatic operation lines and maximum adiabatic temperature attainable at

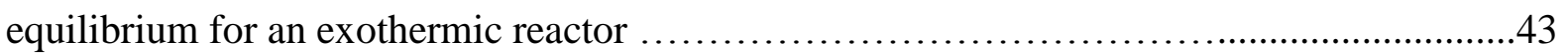

Fig. 5.5. Comparison between direct or indirect cooling of the ammonia reactor...............44

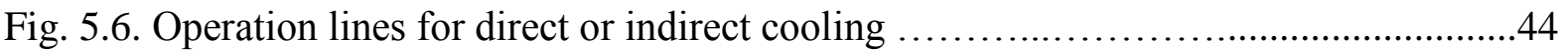

Fig. 5.7. Locus of maximum conversion for a each reaction rate for a Montecatini Catalyst at

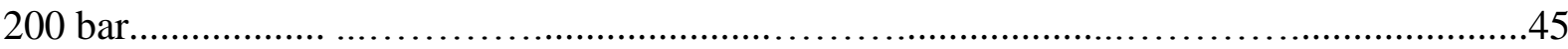

Fig. 5.8. Construction of the cold and hot composite curves.............................................52

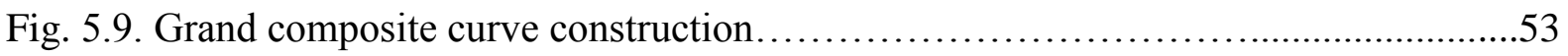

Fig.5.10. Mechanical vapor recompression systems, closed and open configurations..........54

Fig. 5.11. Thermodynamic decomposition of chemical reactors for energy integration

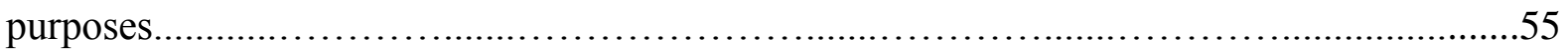

Fig. 5.12. Determination of the T-H profile for an endothermic reactor............................56

Fig. 5.13. Coarse and detailed representation of an endothermic reactor: $\mathrm{T}-\mathrm{H}$ profile..............56

Fig. 5.14. Determination of the T-H profile for an exothermic reactor...............................57

Fig. 5.15. Integrated system used for calculation of cumulative exergy consumption and $\mathrm{CO} 2$ emission of fuels, chemicals and transportation services, including the synthetic nitrogen fertilizers.

Fig. 5.16. Control volumes used for the (a) Exergy flows and (b) Exergy and $\mathrm{CO} 2$ emission

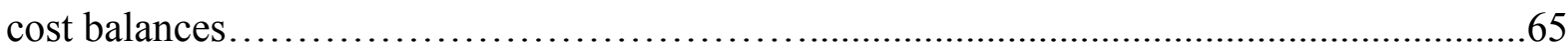

Fig. 5.17. Representation of the HRCT used for the unit exergy cost allocation....................66 
Fig. 5.18. Representation of the heat recovery convection train (HRCT) used for the allocation of the $\mathrm{CO}_{2}$ emissions.

Fig. 5.19. Cost allocation in dissipative components.

Fig. 5.20. Graphical representation of the exergy destruction in the HEN, Grand Composite Curve GCC, Carnot Grand Composite Curves, CGCC.

Fig. 5.21. Graphical summary of the systematic framework used in the chemical process synthesis.

Fig. 5.22. Chemical process overview: Extended, Plantwide and Detailed Layout

Fig. 6.1. Detailed layout of the baseline syngas and ammonia production plant

Fig. 6.2. Heat recovery convection train (HRCT) of the steam methane reformer

Fig. 6.3. Heat recovery convection train of the primary reformer: T-H diagram. Carnot factorH diagram.

Fig. 6.4. Distribution of the cooling requirement between major users by process

Fig. 6.5. Distribution of the power generation and utilization amongst the major users by process.

Fig. 6.6. Multistage ammonia refrigeration systems......................................... 84

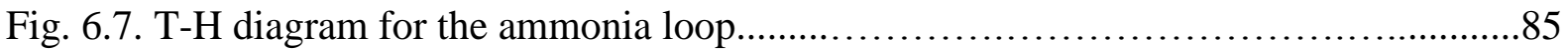

Fig. 6.8. Carnot factor-H diagram for the ammonia loop $(\mathrm{T}$ in $\mathrm{K}$, and $\mathrm{To}=298.15 \mathrm{~K}$ )..........86

Fig. 6.9. Unit exergy costs and $\mathrm{CO}_{2}$ emissions of representative input and output streams of

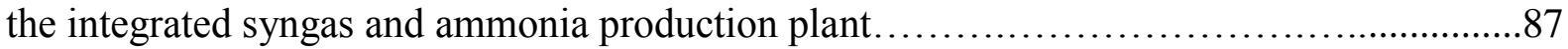

Fig. 6.10. Exergy efficiency for representative components and units.....................91

Fig. 6.11. Breakdown of the exergy destruction in the syngas and ammonia production

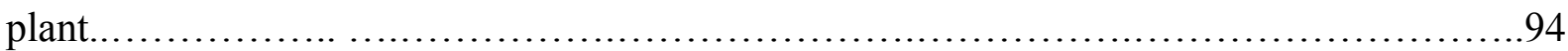

Fig. 6.12. Exergy destruction rates for selected units................................94

Fig. 7.1. Superstructure of the chemical processes, utility units and resources network in the ammonia production plant...................................................... 97

Fig. 7.2. Definition of the layer concept used in the optimization of the components of the utility system

Fig. 7.3. Composite (CC, left) and grand composite (GCC, right) curves for the simulated ammonia plant with MDEA-based, DEA-based and DEPG-based carbon capture unit........104 Fig. 7.4. T-H profile modifications by means of the incorporation of other energy technologies in the chemical system................................................ 105

Fig. 7.5. Integrated Composite curves........................................................... 107

Fig. 7.6. Plantwide and extended exergy consumption and $\mathrm{CO}_{2}$ emission figures for the various configurations studied........................................................... 110

Fig. 7.7. Operating revenues for the various configurations studied..................... 112

Fig. 7.8. Breakdown of power generation and consumption in each plant configuration......114

Fig. 7.9. Plantwide and extended exergy consumption figures for the various configurations studied..... 116

Fig. 7.10. Exergy destruction breakdown for each plant configuration.................................118

Fig. 7.11. Carnot integrated curves for the AUTO and AUTO GT scenarios........................120 
Fig.8.1. Conventional configuration. Steam network-based utility system

Fig. 8.2. Single Pressure, Chemically Recuperated Gas Turbine-based configuration (Single

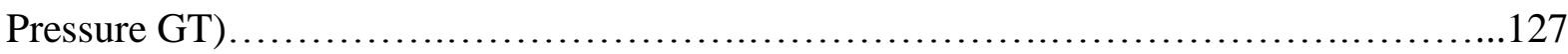

Fig. 8.3. Dual Pressure, Chemically Recuperated Gas Turbine-based configuration (Dual Pressure GT).

Fig. 8.4. Single Pressure, Chemically Recuperated Gas Turbine-based configuration with Steam Network (Single CC)

Fig. 8.5. Dual Pressure, Chemically Recuperated Gas Turbine-based configuration with Steam Network (Dual CC) ......................................................... 129

Fig. 8.6. Comparison between the specific exergy consumption (left) and specific $\mathrm{CO}_{2}$ emissions (right) of the syngas production configurations

Fig. 8.7. Distribution of the power supply and demand (in $\mathrm{kW}$ ) for the (a) conventional, (b) single and (c) dual pressure GT, and (d) single and (e) dual pressure CC configurations......136 Fig. 8.8. Carnot Grand Composite curves (CGCC) for the conventional, single and dual pressure GT configurations and for single and dual pressure $\mathrm{CC}$ configurations....

Fig. 8.9. Plantwide exergy efficiencies for the syngas production plants studied.................138 Fig. 8.10. Exergy efficiency of the integrated cogeneration systems in the syngas production plants

Fig. 8.11. Specific exergy destruction for the studied syngas production plants

Fig. 8.12. Exergy destruction breakdown for representative components of the studied syngas production plants.

Fig. 9.1. Flowsheet of the industrial ammonia production unit

Fig. 9.2. Conversion vs. Temperature. (a) Base-case 150 bar, (b) Optimal case 150 bar, (c) Base-case 200 bar, (d) Optimal case 200 bar...................................... 152 Fig. 9.3. Exergy destruction of representative components of the ammonia loop.................153 Fig. 9.4. Exergy destruction breakdown for representative components of the ammonia loop 154

Fig. 9.5. Plantwide exergy efficiencies of the base-case and optimal scenarios. 154

Fig. 9.6. Exergy efficiencies for representative components of the ammonia loop (150 bar) 155

Fig. 9.7. Exergy efficiencies for representative components of the ammonia loop (200 bar) 156

Fig. 9.8. Exergy destruction in the ammonia loop as a function of the catalytic bed inlet temperatures $\mathrm{T}_{1}$ and $\mathrm{T}_{3}$ (Loop pressure: 150 bar) 160 Fig. 9.9. Exergy destruction in the ammonia loop as a function of the catalytic bed inlet temperatures $\mathrm{T}_{1}$ and $\mathrm{T}_{3}$ (Loop pressure: 200 bar). 161

Fig. 10.1. Single pressure ammonia synthesis flowsheet. ... 164

Fig. 10.2. Dual Pressure Ammonia Synthesis flowsheet.

Fig. 10.3. Conversion vs. Temperature plots. (a) Single pressure 150 bar, (b) Single pressure 200 bar, (c) Dual configuration, once-through pressure 83 bar, (d) Dual configuration, loop pressure 200 bar.

Fig. 10.4. Plantwide exergy efficiencies for the various ammonia configurations... 
Fig. 10.5. Consumed-produced exergy efficiency of representative components of the ammonia production units

Fig. 10.6. Exothermic reactor represented as a chemical exergy-driven heat pump and a set of van't Hoff equilibrium boxes.

Fig. 10.7. Consumed-produced (CP) efficiency of the reactor beds and its relation with the reactor conversion and the autothermal heat exchanger (ATHE) performance. 180

Fig. 10.8. Exergy destruction breakdown for representative components of the ammonia production units.

Fig. 10.9. Exergy destruction of representative components of the ammonia production units.

Fig. 10.10. Carnot composite curves (CCC; left) and Carnot grand composite curves (CGCC; right) for the ammonia production units

Fig.11.1. Conventional natural gas-fueled ammonia production plant

Fig. 11.2. Superstructure used in the process synthesis and optimization of the utility systems of the biomass-based ammonia production plant

Fig. 11.3. Modeling and simulation steps of the twin circulated bed BCL bagasse gasifier..193

Fig. 11.4. Concept of layer used in the optimization of the utility systems

Fig. 11.5. Plantwide and extended exergy consumption figures for the various configurations studied

Fig. 11.6. Overall and detailed (biogenic and fossil, directly and indirectly emitted, and avoided) $\mathrm{CO}_{2}$ emissions for the various configurations studied.........................................201

Fig. 11.7.Power consumption breakdown of the selected scenarios.................................204

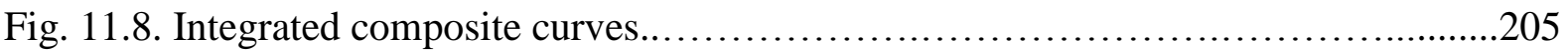

Fig. 11.9. Exergy destruction breakdown for the selected scenarios......................206

Fig. 11.10. Comparison between the relative and extended relative exergy efficiency of the various conventional and alternative ammonia production plant configurations

Fig. 12.1. Detailed superstructure of the various production routes of upgraded feedstock..210

Fig. 12.2. SNG and hydrogen production routes

Fig. 12.3. Overall and utility system exergy efficiency in the various upgraded SNG and hydrogen production routes.

Fig. 12.4. Exergy destruction breakdown in the various upgraded SNG and hydrogen production routes

Fig. 12.5. Integrated curves of the various production routes of $\mathrm{SNG}$ and $\mathrm{H}_{2}$ without and with the integration of a combined cycle.

Fig. A1.1. Graphical representation of the Nelder Mead method...................................252

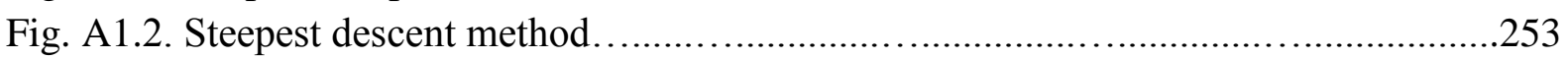

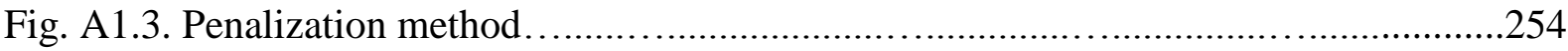

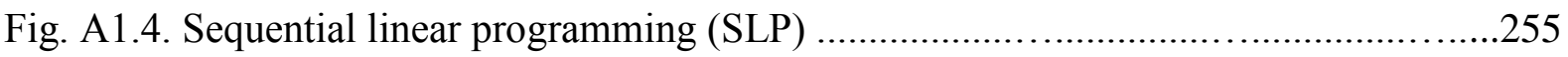

Fig. A1.5. Breadth first (left) and depth first (right) searches used in the solution of MILP and MINLP problems with the Branch and Bound method.............................................256

Fig. A2.1. Influence of the investment cost share on the overall production revenues..........262 


\section{TABLES}

Table 4.1. Relative plant investment and relative energy consumption for various feedstock used in the ammonia production.

Table 5.1. Coefficient of the equilibrium constant $\mathrm{K}_{\mathrm{P}}$

Table 5.2. Pre-exponential factor and activation energy for forward and backward reaction rates constant....

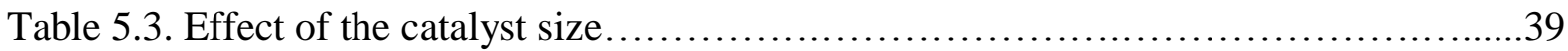

Table 5.4 Variation of the equilibrium constant with temperature ..........................41

Table 5.5. Exergy efficiency definitions for representative equipment and units

Table 6.1. Process data of the heat recovery convection train of the primary reformer.

Table 6.2. Selected process data calculated for the baseline scenario for the integrated syngas and ammonia production plant $\left(1,000\right.$ metric $\mathrm{t}_{\mathrm{NH} 3} /$ day $)$

Table 7.1. Plantwide exergy efficiency definitions of the ammonia production plants. 99

Table 7.2. Optimal process variables of the studied ammonia production facilities.

Table 7.3. Exergy destruction and exergy efficiencies for the chemical and physical absorption-based ammonia production plant configurations

Table 8.1. Plantwide exergy efficiency definitions of the syngas production systems 130

Table 8.2. Optimization problem definition.

Table 8.3. Main process variables of the syngas production facilities studied

Table 9.1. Comparison between the exergy efficiency definitions of the overall ammonia synthesis unit.

Table 9.2. Comparison of the exergy efficiency definitions for representative equipment....146

Table 9.3. Optimization problem definition. 148

Table 9.4. Main process variables of the base-case and calculated optimal unit operating conditions for a $1,000 \mathrm{t}_{\mathrm{NH} 3} /$ day industrial ammonia unit.

Table 10.1. Selected process data of the ammonia synthesis unit operating at 150 bar........166

Table 10.2. Selected process data of the ammonia synthesis unit operating at 200 bar.

Table 10.3. Selected process data of the ammonia synthesis unit operating at dual pressure $83 / 200$ bar 168

Table 10.4. Main process parameters of the various configurations studied.

Table 10.5. Cooling duty and total exergy associated at selected heat transfer temperature..185

Table 11.1. Plantwide exergy efficiency definitions of the ammonia production plants........195

Table 11.2. Optimal process variables of the studied ammonia production facilities............202

Table 11.3. Exergy destruction and exergy efficiencies for the studied configurations.........207

Table 12.1. Exergy consumption and production remarks for each production route...........214 
ABBREVIATIONS

ANAD

ANADH2

ANDA

ASC

ATE

ATHE

AUTO

AUTO GT

ATR

BA

BAT

BCL

BFW

CAPEX

$\mathrm{CC}$

CGCC

CHP

CH

CL

Comb.

Compr.

Cond.

CO

CHP

COP

CPO

CRGT

CryoPG, CRY

CW

DEA

DEPG

Desorb.

DP

EE

EES

EI

ENRTL

EOS

ES

EVA

Evap.

FAFEN

FOSFERTIL
Anaerobic digestion process

Hydrogen production via anaerobic digestion process

National Association for the Fertilizer Dissemination

Ammonia Synthesis and Condensation

Approach to Equilibrium temperature difference

Autothermal Heat Exchanger

Autonomous operation mode

Autonomous operation mode with combined cycle

Autothermal Reforming

Bahia State

Best Available Technology

Battelle Columbus Laboratory

Boiler feedwater

Investment or Capital Cost

Combined Cycle, Composite Curve

Carnot Grand Composite Curve

Combined Heat and Power Production

Chemical

Chemical Looping reactor

Combustion

Compressor

Condensate, Condenser

Carbon Dioxide

Combined Heat and Power production

Coefficient of performance

Catalytic Partial Oxidation

Chemically Recuperated Gas Turbine

Cryogenic Purge Gas Unit

Cooling Water

Di Ethanol Amine

Di Ethyl Propylene Glycol

Desorber

Dual Pressure configuration

Electricity

Engineering Equation Solver

Energy Integration

Electrolytic Non-Random Two-Liquid

Equation of State

Espirito Santo State

Nuclear powered SMR reactors

Evaporador

Nitrogen Fertilizers Factory

Phosphorous Fertilizers Factory 
GA

GASIF

GASIFH2

GASIFCH4

GCC

GHG

GHR

GJ

GPU

GRID

GT

H/C

HE

HEN

$\mathrm{H}_{2} / \mathrm{N}_{2}$

HP

HRCT

HRSG

HTS

IFA

IO

INCO

InterCW

IPESE

KAAP

KKT

LETE

LHV

Liq.

LP

L/R

LT

LTS

Max.

MDEA

MEA

MER

MG

MILP

Min.

MINLP

MIXED

MP

MS
Genetic Algorithm

Gasification process

Hydrogen production via gasification process

Synthetic Natural gas production via gasification process

Grand Composite Curve

Green House Gas emission

Gas Heated Reformer

GigaJoule

Gas Purification Unit

Grid operation mode

Gas Turbine

Hydrogen to Carbon molar ratio

Heat Exchanger

Heat Exchanger Network

Hydrogen to Nitrogen molar ratio

High Pressure

Heat Recovery Convection Train

Heat Recovery Steam Generator

High Temperature Shift

International Fertilizers Association

Input/output efficiency

Net Economic Incomes

Intercooling Cooling Water

Industrial Process and Energy Systems Engineering group

Kellogg Advanced Ammonia Process

Karush-Kuhn-Tucker stationary condition

Laboratory of Environmental and Thermal Engineering

Lower heating Value

Liquid

Low Pressure, Linear programming

Lean to Rich Amine Heat Exchanger

Low Temperature

Low Temperature Shift

Maximum

Methyl Di Ethanol Amine

Mono Ethanol Amine

Minimum Energy Requirement

Minas Gerais State

Mixed Integer Linear Programming

Minimum

Mixed Integer Non-Linear Programming

Mixed operation mode

Medium Pressure

Mato Grosso do Sul State 


\begin{tabular}{|c|c|}
\hline MVR & Mechanical Vapor Recompression \\
\hline MW & Megawatt \\
\hline NG & Natural gas \\
\hline OCDE & Organization for Economic Co-operation and Development \\
\hline OGTC & Open Gas Turbine Cycle \\
\hline OPEX & Operating Cost \\
\hline PBR & Packed Bed Reactor \\
\hline PFHE & Plate Fin Heat Exchanger \\
\hline PFR & Plug Flow Reactor \\
\hline PH & Physical \\
\hline POGT & Partial Oxidation Gas Turbine \\
\hline POX & Partial Oxidation \\
\hline Proc. & Process \\
\hline Prod. & Product \\
\hline PR & Paraná State, Peng Robinson EOS \\
\hline PSA & Pressure Swing Adsorption \\
\hline $\mathbf{R C}$ & Rankine Cycle \\
\hline Ref. & Reformer \\
\hline REV & Economic Revenues \\
\hline $\mathbf{S} / \mathbf{C}$ & Steam to Carbon ratio \\
\hline SE & Sergipe State \\
\hline SG & Syngas fuel \\
\hline SHR & Shift Reaction processes \\
\hline SMR & Steam Methane Reforming \\
\hline SNF & Synthetic Nitrogen Fertilizers \\
\hline SNG & Synthetic Natural Gas \\
\hline SP & Sao Paulo State, Single pressure configuration \\
\hline SRK & Soave-Redlich-Kwong EOS \\
\hline SLP & Sequential Linear Programming \\
\hline SQP & Sequential Quadratic Programming \\
\hline Refrig & Refrigeration \\
\hline TOEX & Total Cost \\
\hline UMR & Unmixed Reforming \\
\hline UT & Utilities Plant \\
\hline Vap. & Vapor \\
\hline VBA & Visual Basic for Applications \\
\hline WF & Without Fuel \\
\hline WGS & Water Gas Shift \\
\hline
\end{tabular}




\section{SYMBOLS}

\section{Latin symbols}

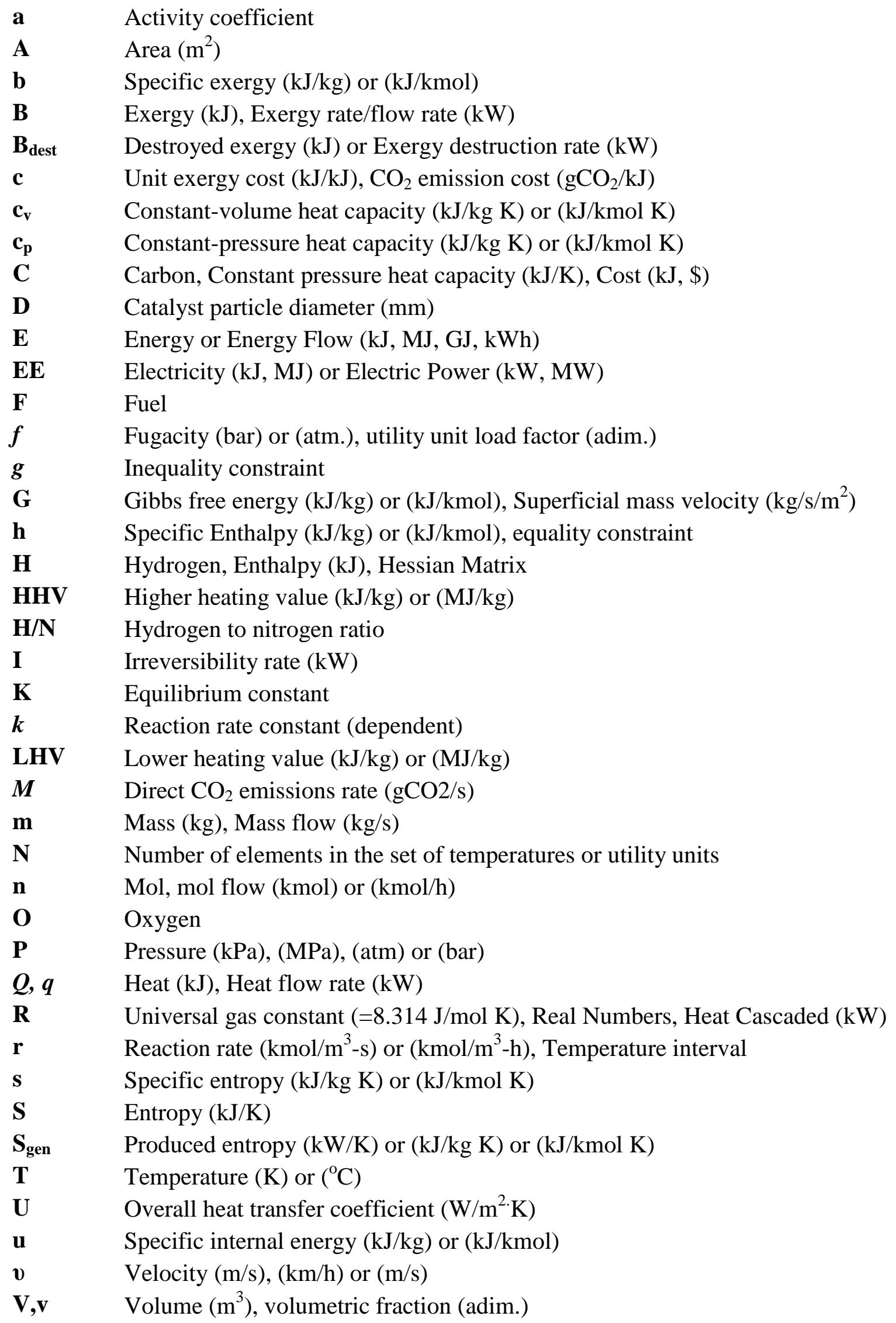


w Discrete optimization variable sizing and nominal capacities

W Work $(\mathrm{kJ})$, Power $(\mathrm{kW})$

y Mass fraction, integer optimization variable

$\mathbf{x} \quad$ Molar fraction, continuous optimization variable

$\mathbf{Z} \quad$ elevation (m), Annualized equipment cost (\$/s), Compressibility (Ideal Gas Dev.)

$\mathbf{z} \quad$ Binary variable

\section{Greek symbols}

$\boldsymbol{\mu} \quad$ Chemical potential $(\mathrm{kJ} / \mathrm{kmol})$, Dynamic viscosity $(\mathrm{kg} / \mathrm{s} . \mathrm{m})$

$\boldsymbol{\alpha} \quad$ Temkin-Phyzev exponential factor

$\varphi \quad$ Chemical exergy to Lower heating value ratio

$\phi \quad$ Void fraction, Fugacity coefficient of species

$\xi \quad$ Nitrogen reaction conversion

$\boldsymbol{\eta} \quad$ Efficiency

$\Delta \quad$ Difference

$\rho \quad$ Density $\left(\mathrm{kg} / \mathrm{m}^{3}\right)$

$\boldsymbol{\delta} \quad$ Infinitesimal flow

$\boldsymbol{\psi} \quad$ Bagasse moisture (\% mass)

$\gamma \quad$ Activity coefficient

$\Theta \quad$ Molar inlet flow ratio for species i-th to nitrogen

$\boldsymbol{\sigma} \quad$ Volumetric entropy generation rate $\left(\mathrm{kJ} /\left(\mathrm{kmol} . \mathrm{K} . \mathrm{m}^{3} . \mathrm{h}\right)\right)$

$\boldsymbol{\Omega}$ Reaction quotient, region of feasible solutions

$\boldsymbol{\omega} \quad$ Utility unit index for optimization

\section{Subscript}

$\begin{array}{ll}\mathbf{1} & \text { State } 1,1^{\text {st }} \text { reformer } \\ \mathbf{2} & \text { State } 2,2^{\text {nd }} \text { reformer } \\ \mathbf{a} & \text { Activation energy } \\ \mathbf{b} & \text { Exergetic efficiency, backward reaction } \\ \text { BFW } & \text { Boiler feedwater } \\ \text { Carnot } & \text { Carnot Ideal thermal cycle } \\ \text { CO } & \text { Carbon monoxide } \\ \text { CO } 2 & \text { Carbon dioxide } \\ \text { Comp. } & \text { Compressor } \\ \text { cond. } & \text { Condenser } \\ \text { CP } & \text { Consumed-Produced } \\ \text { CV } & \text { Control Volume } \\ \text { DEA } & \text { Di ethanol amine } \\ \text { Dest. } & \text { Destroyed } \\ \text { EE } & \text { Electric energy (kJ, MJ, kWh) } \\ \text { F } & \text { Fuel } \\ \text { gen } & \text { Generated } \\ \text { eq. } & \text { True Equilibrium Constant }\end{array}$




\begin{tabular}{|c|c|}
\hline $\mathbf{H}$ & High temperature \\
\hline $\mathbf{I}$ & First law / Energetic \\
\hline In & Inlet stream \\
\hline $\mathbf{F}$ & Fuel \\
\hline $\mathbf{f}$ & Forward reaction \\
\hline $\mathbf{F}$ & Fuel \\
\hline$\circ$ & Reference state, Standard Value \\
\hline HP & High pressure \\
\hline$i$ & i-th flow, i-th reaction rate \\
\hline ise & Isentropic \\
\hline $\mathbf{L P}$ & Low pressure \\
\hline $\mathbf{L}$ & Low temperature \\
\hline $\mathbf{L M}$ & Logarithmic mean temperature \\
\hline MP & Medium pressure \\
\hline NR & Non Renewable \\
\hline $\mathbf{N}_{2}$ & Nitrogen \\
\hline $\mathbf{0}$ & Environmental conditions $25^{\circ} \mathrm{C}$ and $1 \mathrm{~atm}$. \\
\hline $\mathbf{O}_{2}$ & Oxygen \\
\hline Out & Outlet stream \\
\hline $\mathbf{P}$ & Physical exergy pressure component, Product \\
\hline $\mathbf{p}$ & Ideal Gas pressure based equilibrium constant \\
\hline $\mathbf{Q}$ & Heat, Reaction Quotient \\
\hline $\mathbf{R}$ & Renewable, Reaction, Reference for Enthalpy of Reaction \\
\hline Ref & Reformer, reference temperature \\
\hline Rxn & Reaction derived $\mathrm{CO}_{2}$ emissions \\
\hline rev & Reversible \\
\hline $\mathbf{T}$ & Physical exergy thermal component \\
\hline Tot & Total \\
\hline UT & Utilities plant \\
\hline
\end{tabular}

\section{Superscript}

$\begin{array}{ll}\mathbf{C H} & \text { Chemical exergy } \\ \mathbf{i} & \text { i-th flow } \\ \mathbf{j} & \text { j-th input } \\ \mathbf{K} & \text { Kinetic exergy } \\ \mathbf{k} & \text { k-th HRCT module } \\ \mathbf{M} & \text { Mass flow rate exergy } \\ \mathbf{n} & \text { n-th (last) HRCT module } \\ \mathbf{0} & \text { 0-th (first) HRCT module } \\ \mathbf{P} & \text { Potential exergy } \\ \mathbf{P H} & \text { Physical exergy } \\ \mathbf{Q} & \text { Heat Exergy } \\ \mathbf{T} & \text { Total Exergy } \\ \mathbf{W} & \text { Work }\end{array}$


Acknowledgements..............................................................................................................................................vii

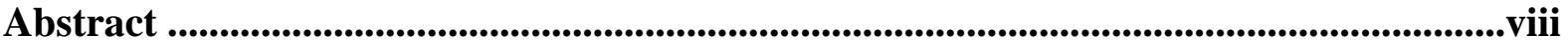

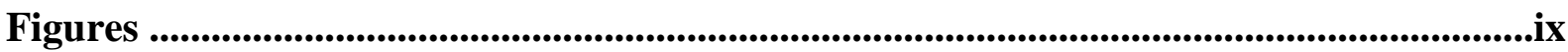

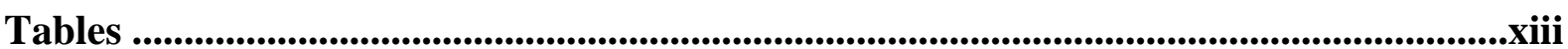

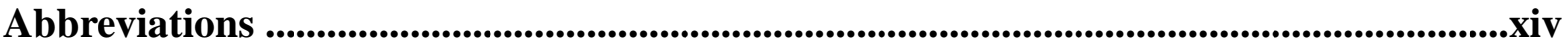

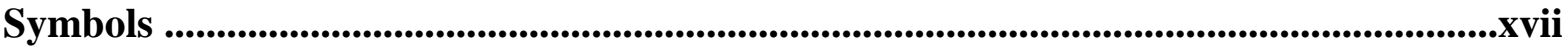

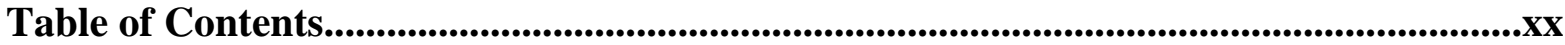

CHAPTER 1. INTRODUCTION......................................................................................................1

CHAPTER 2. OBJECTIVES AND RELEVANCE ....................................................4

CHAPTER 3. SYNTHETIC NITROGEN FERTILIZERS: AN OVERVIEW..................5

3.1. The Brazilian synthetic nitrogen fertilizers scenario: An overview ...............................

CHAPTER 4. SYNGAS AND AMMONIA PRODUCTION TECHNOLOGIES.............10

4.1. Syngas and hydrogen production technologies........................................................... 11

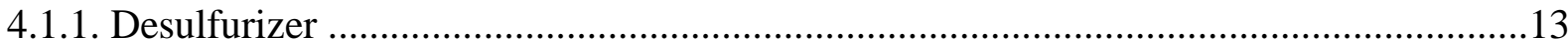

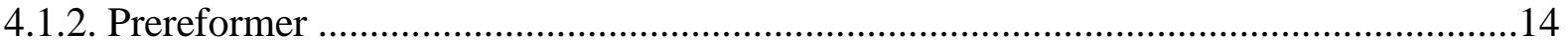

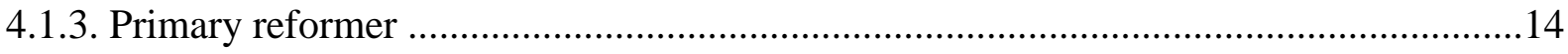

4.1.4. Autothermal and Secondary reformer ............................................... 17

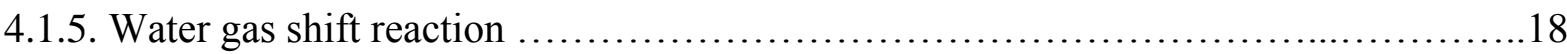

4.1.6. Syngas Purification: Carbon capture and methanation.............................. 18

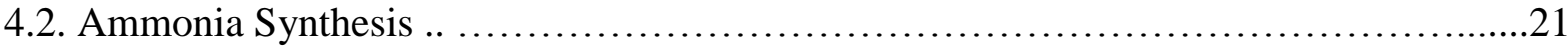

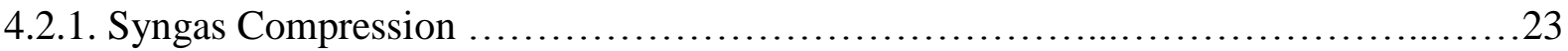




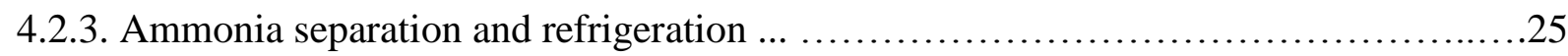

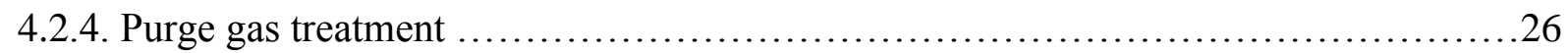

4.3. Combined heat and power production........................................................28

\section{CHAPTER 5. PROCESS SYNTHESIS: MODELING, SIMULATION AND}

OPTIMIZATION

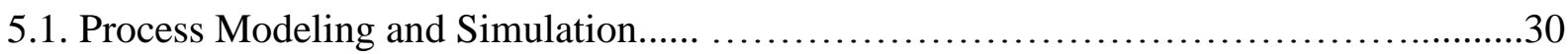

5.2. Process Synthesis and Optimization... .......................................47

5.2.1. Heuristic methods.......................................................49

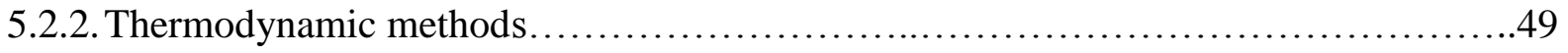

5.2.2.1. Energy integration and Minimum energy requirements.........................51

5.2.2.2. Exergy and Exergoeconomy Analysis.........................................58

5.2.3. Algorithmic methods.................................................. 71

5.2.3.1. General definition of an optimization problem............................. 72

5.3. Summary of the systematic framework used for the chemical process synthesis.............73

\section{CHAPTER 6. DIAGNOSTIC OF THE BASELINE SYNGAS AND AMMONIA}

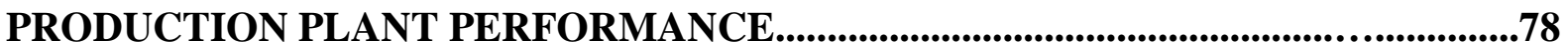

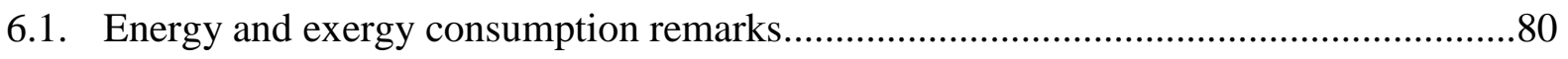

6.2. Cumulative unit exergy cost and specific $\mathrm{CO}_{2}$ emissions........................................86

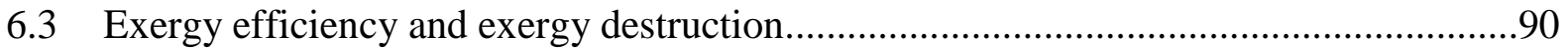

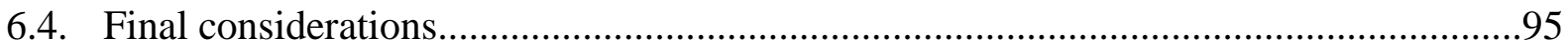


7.1. Superstructure of the chemical process and the utility system. .96

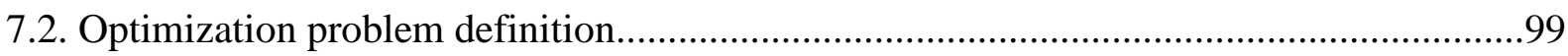

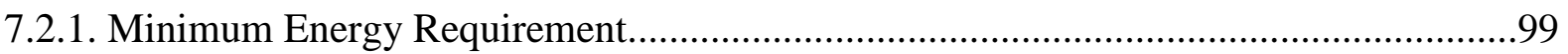

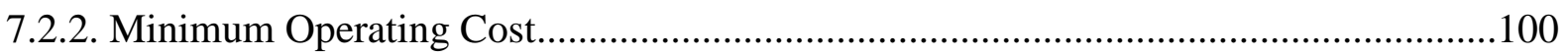

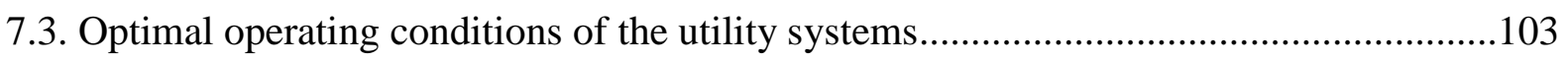

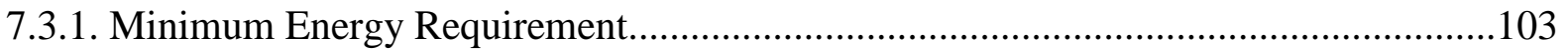

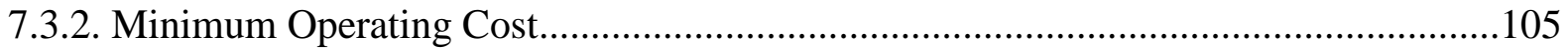

7.4. Exergy analysis of the integrated ammonia production plants.....................................116

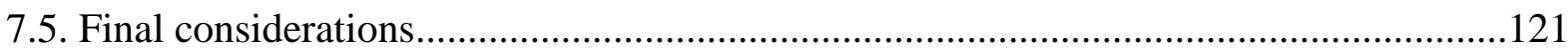

CHAPTER 8. IMPROVED SYNGAS PRODUCTION AND CARBON CAPTURE BY USING A NOVEL CRGT APPROACH....................................................................122

8.1. An overview on the conventional and novel approaches for syngas production.............122

8.2. Syngas Production with Thermo-Chemically Recuperated Gas Turbine Systems..........125

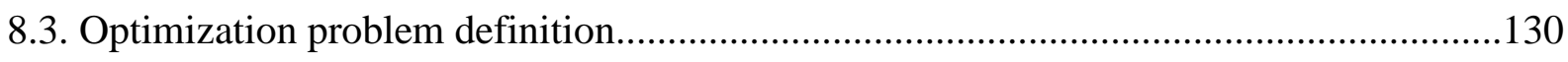

8.4. Optimal operating conditions of the conventional and novel configurations..................131

8.5. Energy Integration and Carnot Grand Composite Curves...........................................137

8.6. Exergy analysis of the conventional and proposed syngas production units...................138

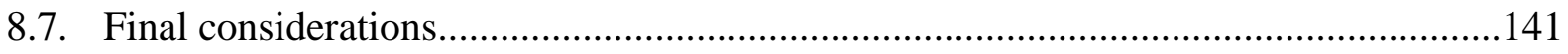

CHAPTER 9. MODELING AND OPTIMIZATION OF AN INDUSTRIAL AMMONIA

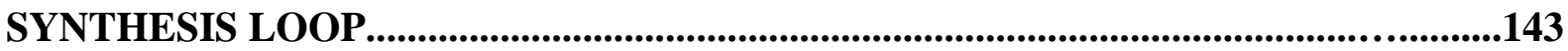

9.1. Performance indicators proposed for the industrial ammonia synthesis loop................143

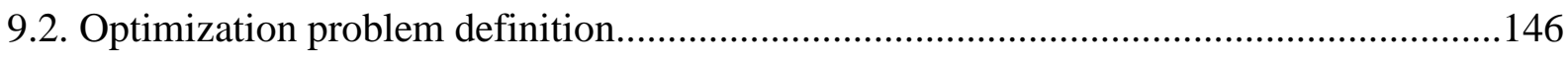

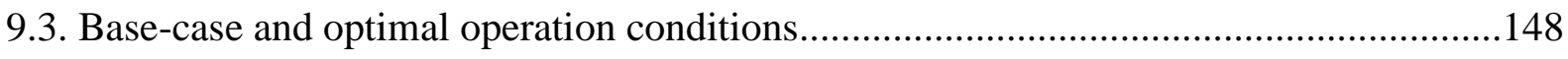

9.4. Reaction kinetics in the intercooled, multiple bed catalytic reactor............................150 
CHAPTER 10. SINGLE AND DUAL PRESSURE INDUSTRIAL AMMONIA UNITS: THE COUNTERACTION PRINCIPLE . .163

10.1. Description of the single and dual pressure ammonia synthesis unit.... 164

10.2. Exergy efficiency definition of the industrial ammonia units. 169

10.3. Counteraction principle and the application to the ammonia synthesis..... 169

10.4. Operation conditions of the single and dual pressure ammonia synthesis units. 170

10.5. Reaction kinetics in the intercooled, multiple bed catalytic reactor 172

10.6. Exergy analysis of the single and dual pressure ammonia synthesis units 174

10.7. Combined exergy and energy integration analysis 183

10.8. The role of the purge gas treatment unit .185

10.9. Final considerations 185

CHAPTER 11. SYNGAS AND AMMONIA PRODUCTION FROM BIOMASS 187

11.1. Biomass as a source of hydrogen for ammonia production 187

11.2. Natural gas and Biomass-based Ammonia Production Plants 189

11.3. Optimization problem definition.

11.4. Optimal scenarios of the conventional and alternative routes of ammonia production. 199

11.5. Combined exergy and energy integration analysis 205

11.6. Final considerations .208

CHAPTER 12. UPGRADING BIOMASS RESIDUES TO HYDROGEN AND SNG 
12.2. Optimization problem definition

12.3. Optimal operating conditions of the upgrading processes of the biorefinery residues

12.4. Final considerations

CHAPTER 13. CONCLUSIONS AND SUGGESTIONS FOR FUTURE WORKS......218

CITED REFERENCES

APPENDIX 1. ALGORITHMIC METHODS USED IN PROCESS SYNTHESIS AND OPTIMIZATION OF CHEMICAL PROCESSES .252

APPENDIX 2. AN OVERVIEW OF THE INVESTMENT AND OVERALL COSTS OF THE AMMONIA PRODUCTION. .259 



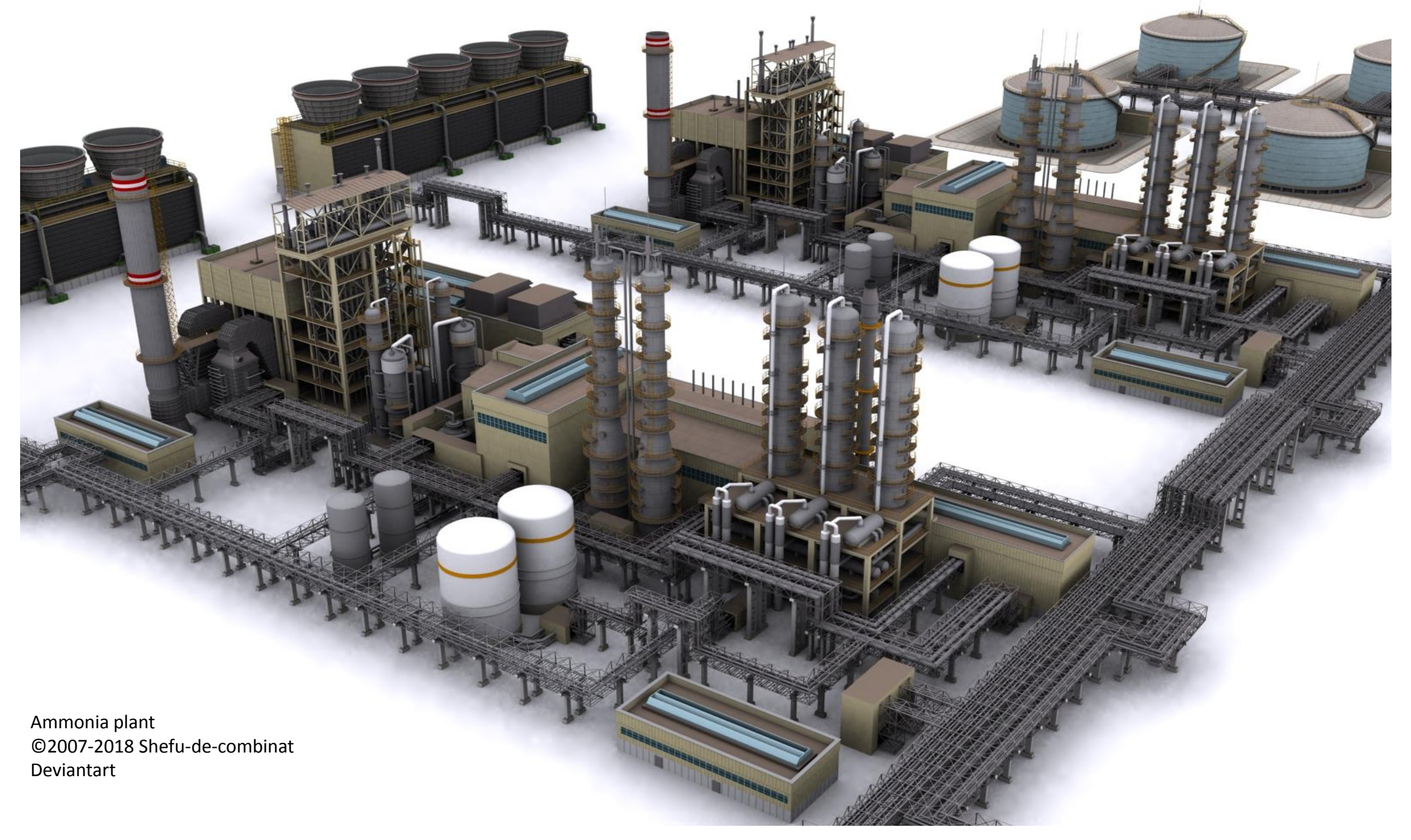




\section{CHAPTER 1}

\section{INTRODUCTION}

Some fundamental mineral nutrients limiting the vegetable growing, e.g. carbon and oxygen, can be easily obtained by the plants through the soil and the surrounding air. Meanwhile, other components, such as the atmospheric nitrogen, must be fixed into more accessible forms to the plant before they can be effectively absorbed. Currently, nitrogen fixation is possible at an industrial scale thanks to the production of synthetic nitrogen fertilizers (SNF) [1], assumed to be responsible for at least fifty percent of the global yield of food crops [2]. However, the manufacture of SNF is an energy intensive industry that fundamentally depends on the consumption of large amounts of non-renewable resources, contributing further to the environmental strain already associated to extensive farming. Consequently, in the last years, SNF plants have deserved an increased legal surveillance, having to comply with more stringent controls of atmospheric emissions and wastes production [3, 4]. In order to fulfill the new regulations, SNF technology has undergone radical developments in terms of both design and equipment, most of them focused on the reduction of the power and feedstock demands [5-9], the improvement of the waste heat recovery network [10-16], the cutdown in the energy consumption of the $\mathrm{CO}_{2}$ removal process [17-19] and the design of better and more active catalysts [5, 20-23].

In spite of the comprehensive research on energy integration and optimization achieved so far, the technological lag in the sector is still associated to the use of old existent plants relying on less efficient practices and conversion technologies [24]. Actually, it is estimated that more than $45 \%$ of the total existing plants in the world are older than 30 years [25]. It is also worthy to notice that the minimum theoretical consumption in modern ammonia plants (18$\left.21 \mathrm{GJ} / \mathrm{t}_{\mathrm{NH} 3}\right)$ is still much lower [25] compared to the best figures reported in literature (28-31 GJ/t $\left.\mathrm{t}_{\mathrm{NH} 3}\right)$, which may vary widely depending on project-specific requirements $[5,9]$. Thus, according to the European Roadmap of Process Intensification (PI - PETCHEM), the potential benefits in the ammonia production sector are significant. In the short to mid-term (10-20 years), an increase of 5 percent in the overall energy efficiency is expected, whereas 20 percent higher energy efficiency is envisaged in the long term (30-40 years) [26]. Other studies [27] are less optimistic and estimate more moderate improvement rates in the near future in comparison with those experienced over the 1991-2003 period. For instance, the improvement in the fuel consumption is predicted to be $35 \%$ lower than during the previous decade. Anyhow, all those studies admit the potential benefits of the enhanced process integration during the design of new ammonia and urea plants, with lower or minor capital costs increments and with a reduced ratio of carbon emission-to-ammonia throughput.

Despite those promising figures, it must be born in mind that any additional room for an efficiency increase or a reduced environmental impact might not come about but through breakthrough approaches [28, 29], including a radical diversification of the energy resources 
in the fertilizers sector [30-32], whereas some other mitigation options may become rather impracticable (e.g. post-combustion carbon capture) [33]. Anyway, practitioners still count on increased efficiencies as the first step towards the reduction of the large energy consumption associated to the SNF industry [34]. In addition, the mitigation approaches for the massive atmospheric $\mathrm{CO}_{2}$ emissions point towards an increase of the pre-combustion carbon capture [33]. In this way, the captured $\mathrm{CO}_{2}$ can be used in the production of other chemical processes including urea, methanol, polymers, synthetic fuels and food industry, decreasing both the energy consumption and wastes, which certainly should be accounted for in an overall environmental analysis.

In the light of these circumstances, an extended assessment encompassing the feedstock production, supply chain and conversion stages in the integrated syngas and ammonia production plant, along with the mitigation and commercialization of the emissions generated, is still necessary. Thus, the primary goal of this thesis is to develop a systematic methodolody based on the application of thermodynamic, economic and environmental methods that assists in identifying, evaluating and optimizing existing and proposed configurations of syngas, hydrogen, carbon dioxide and ammonia production in SNF plants. A comprehensive comparison between the various setups designed for accomplishing the energy integration of the chemical processes and the utility systems is performed. Additionally, a comparative assessment between the conventional and alternative routes for the production of chemicals and the decarbonization of the fertilizers sector is also presented. The text is structured as follows:

In the second chapter, the objectives and relevance of this thesis are briefly presented,

The third chapter shows an overview of the history of the SNF production and their relevance, as well as the the challenges that SNF industry faces in the Brazilian economy.

Chapter four presents a thorough revision on the main energy conversion technologies used in the conventional syngas and ammonia production plants. The unconventional designs analyzed in subsequent chapters basically rely on the same operating principles shown in Chapter 4, although more detailed descriptions are provided in each chapter when necessary.

The fifth chapter presents an overview of the systematic framework used in the process synthesis. The methodologies and tools used, including heuristics, thermodynamic and algorithmic methods, are briefly discussed. This chapter also summarizes the challenges and research question along with the strategies used to address the decision-making and optimization problems that will come about along the thesis.

Chapter 6 presents a diagnostic of the performance of the most relevant units and components of the baseline syngas and ammonia production plant. The obtained results are used to prioritize the efforts towards the reduction of the energy consumption and $\mathrm{CO}_{2}$ emissions of the energy conversion processes that present the highest rates of exergy destruction and the most critical environmental impact. In this way, the benefits of revamping or substituting the outdated technologies can be highlighted. 
In the seventh chapter, the mathematical programming problem consisting of the selection of the most suitable components for the utility systems from a resourceful superstructure, that includes all the relevant energy technologies, is presented. The formulated problem aims to minimize the energy requirements of the overall ammonia production plant while reducing the operating costs. An special attention is aimed to the role of the syngas purification unit adopted, as it drastically modifies the way in which the chemical plant and the utility system are optimally integrated.

Chapter 8 describes a novel approach based on the chemically recuperated gas turbine concept, proposed for the syngas production section at the frontend of the ammonia plant. This new setup aims to improve the energy integration capabilities at higher temperatures and to reduce the amount of atmospheric emissions, by directly intensifying the rates of precombustion carbon capture.

In Chapter 9, some critical operation parameters of the backend ammonia loop such as the loop pressure, feed temperature, $\mathrm{H}_{2}$ to $\mathrm{N}_{2}$ ratio, inert fraction are further analyzed through the use of a series of case studies in order to reduce the process irreversibility in comparison with the base-case scenario. Chapter 10 expands the analysis on the performance of the backend ammonia synthesis unit by introducing a dual pressure loop that reduces the power consumption and the exergy destroyed by introducing an additional reactive component.

The last two Chapters 11 and 12) deal with the study of the utilization of biomass as an alternative source of energy that may help decarbonizing the SNF sector, while taking advantage of the readily available wastes of Brazilian sugar cane mills. The opportunities related to the potential depletion of the atmospheric carbon dioxide through the production of chemicals via biomass-based routes are highlighted.

In the closure chapters of this thesis, the main conclusions and the suggestions for future works are presented, whereas the annexes show an overview of some of the algorithmic methods used in the optimization of chemical processes and some commentaries on the investment and overall production costs of an ammonia production plant. Finally, some published papers, conferences and seminars attended along the time frame of the graduate course, including the research fellowship performed at the École Polytechnique Fédérale de Lausanne, Switzerland, are summarized. Along these pages, the author attempts to contribute towards the knowledge about:

(i) how to better exploit the thermodynamic potential of costly, high temperature utility streams and exothermic reactor effluents,

(ii) how to reduce the energy consumption and increase the pre-combustion $\mathrm{CO}_{2}$ capture, or even decarbonize the fertilizer sector by using alternative fuels,

(iii) which are the most adequate operating modes considering the project specific conditions,

(iv) how to univocally define the performance indicators (e.g. exergy efficiency) so that they can be reasonably attainable, and

(v) whether the counteraction and Le Châtelier principles trigger irreconcilable trade-offs in terms of process irreversibility and reactions rates. 


\section{CHAPTER 2}

\section{OBJECTIVES AND RELEVANCE}

\section{GENERAL OBJECTIVE}

The main objective of this thesis is the development and application of a systematic methodology, based on the use of a variety of methods and optimization tools, for proposing optimum and sustainable configurations of syngas, hydrogen, carbon dioxide and ammonia production in SNF plants.

\section{SPECIFIC OBJECTIVES}

- To conceive and develop a methodology for modeling and analyzing SNF plants, or major parts thereof, with respect to multiple criteria (e.g. themodynamic, environmental, economic), in order to determine the exergy and economic expenditure of the production processes.

- To integrate and capitalize on various computational tools for synthesizing and optimizing such facilities;

- To suggest improvement opportunities for each scenario based on suitable performance indicators, so that the exergy consumption, the environmental impact and the operating costs can be minimized in SNF plants.

\section{RELEVANCE}

The fact that the demand for synthetic nitrogen fertilizers has significantly surpassed Brazilian production capacity makes the country vulnerable to variations in prices in the international markets, shipping costs and logistical problems at Brazilian ports. Additionaly, in spite of the intensive energy integration already undergone by the modern ammonia production plants, the consideration of unconventional approaches represents an opportunity to issue relevant recommendations for the future developments of the synthetic fertilizers sector. Morever, few research works have deal with the analysis of the production process in the SNF plants accounting for the various manufacturing aspects (thermodynamic, environmental, economic).

Accordingly, the process synthesis and optimization of the SNF facilities in the light of the Second Law of the Thermodynamics by using heuristic, themodynamic and algorithmic methods might allow for a more rational and comprehensive point of comparison between the SNF production routes and even with other chemical sectors. Actually, this topic has not been thoroughly discussed, often based on heuristic approaches, focused on production aspects or concerned with standalone equipment improvement, rarely based on environmental aspects. Certainly, although this issues are expectedly subject to economic and geopolitical aspects, increasing the efficiency of the SNF domestic production could be the first step towards the reduction of the large non-renewable exergy consumption and environmental impact that SNF industry is responsible for. 


\section{CHAPTER 3}

\section{SYNTHETIC NITROGEN FERTILIZERS: AN OVERVIEW}

Nitrogen is an essential constituent of the molecules contained in all the amino acids that, in turn, make up the proteins required for the functioning of all the living beings. Unlike the plants, the animals are unable to utilize more simple forms of nitrogen and, therefore, rely on the intake of food sources for protein, which can then be digested to amino acids and used for the protein synthesis in their bodies [1]. But, even the plants cannot directly profit from the atmospheric nitrogen found in about $79 \%$ of the volume of the surrounding air, since its molecules are strongly joined through a covalent triple bond, and the nutrients can only be absorbed if they are present in easily dissolved chemical compounds. Thus, nitrogen must be first decomposed and combined into more accessible forms to the plants by means of a process called fixation. This process is accomplished by means of either natural phenomena or anthropogenic activities, which have been traditionally used to supply the main plant nutrients to the soil, namely nitrogen, phosphorus and potassium [35].

Since early years, humans have thought out several ways to restitute the essential nutrients taken from the soil, from using leguminous plants rotation, rotten stalks, silage and other organic material such as manure and Peruvian guano, or even the nitrogen-rich natural deposits of saltpeter (sodium nitrate) from the nineteen century Chilean mines [36]. However, in spite of its natural origin, organic fertilizers had much lower concentrations of plant nutrients and presented the economic problems of the collection, treatment, transportation and distribution. Therefore, it was not until the twentieth century (specifically from 1902 to 1913), that, in order to meet the increasing demand for the nitrogen fertilizers, a variety of methods were invented to convert the atmospheric nitrogen into commercial fertilizers [21]. First, it was the electric arc process, ca. 1905, which produced nitric oxide via the reaction of nitrogen with oxygen at high temperatures and, thus, consuming high amounts of energy (50-80 kWh of electricity per $\mathrm{kg}$ of nitrogen) [22]. Afterwards, the cyanamide process, introduced few years later (c. 1910) and based on the reaction of nitrogen with calcium carbide to produce calcium cyanamide, reduced the energy consumption to about $180 \mathrm{GJ} / \mathrm{t}$ [21]. Calcium cyanamide could be used directly or as the base to produce nitrogen-rich organic compounds. However, the era of the artificial fertilizers was born when the catalytic ammonia synthesis was invented by Fritz Haber, who devised a catalytic reactor to convert the nitrogen in the air into liquid ammonia $\left(\mathrm{NH}_{3}\right)$, and then Carl Bosch applied this process to the industrial production. According to the International Fertilizer Industry Association [37], more than 95\% of the nitrogen fertilizers globally produced are manufactured by means of the Haber Bosch process.

Ammonia is a concentrated source of nitrogen (85\% mass) and the basic feedstock for all upgraded nitrogen fertilizers, so it can be either directly applied to the soil or further processed to produce urea or nitrates [38]. The first ammonia plant built in 1913 had a 
production of 30 tons of ammonia per day [39], and by 1940, the original version of the current Haber Bosch process consumed $100 \mathrm{GJ} / \mathrm{t}$ by using coal and coke [22]. Subsequently, by 1960, natural gas replaced coal as feedstock, and between 1955 and 1966, the energy requirements of the best plants dropped from $55 \mathrm{GJ} / \mathrm{t}$ to $40 \mathrm{GJ} / \mathrm{t}$. The decline in the exergy expenditure since 1950 to the present days has been more gradual [40]. A major advance in the technology of ammonia production occurred in the early 60's, when the M.W. Kellogg Company introduced the jumbo-size single train ammonia plants equipped with centrifugal compressors, where ammonia could be produced at half of the costs of smaller plants equipped with the conventional reciprocal compressors [36]. Figure 3.1 shows the evolution of the energy consumption of various production technologies for some fertilizers including ammonia.

Fig. 3.1. Evolution of the energy consumption in fertilizers and ammonia production.

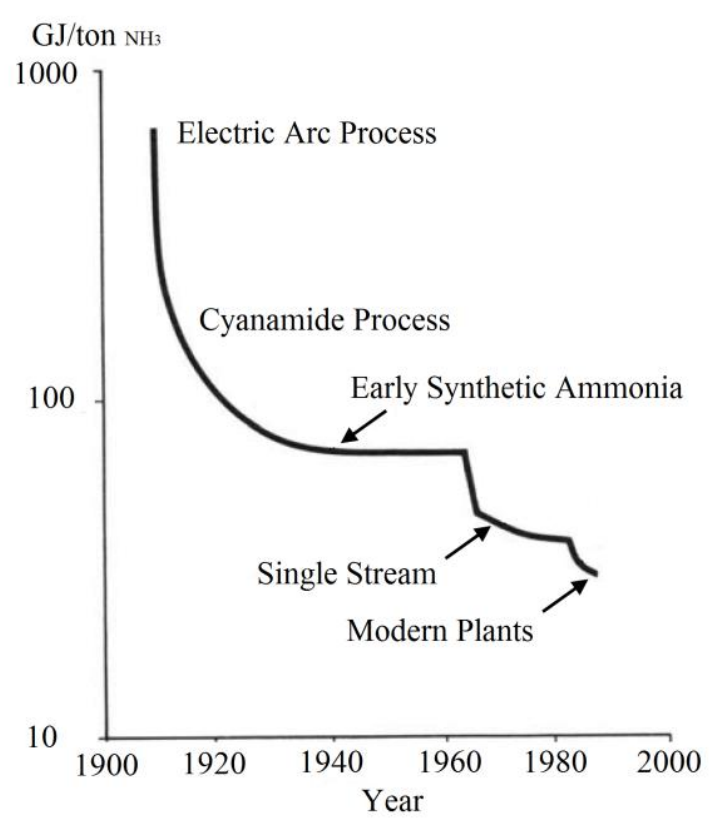

Source: [41].

As expected, the effect in the agriculture was immediate up to the point that almost half of the yield of the global crops is nowadays attributed to commercial fertilizers (Fig. 3.2), with the remaining share coming from organic sources, native soil reserves and biological nitrogen fixation cycles [2]. Currently, the fertilizers production represents the second largest share in energy consumption in agriculture (24\%), only surpassed by fuels and followed far by pesticides (1.6\%) [35], accounting for about 2-3\% of the total world energy demand [42]. In the last years, the use of commercial fertilizers has steadily increased and, in the case of the nitrogen fertilizers, the demand has risen at almost a rate of 2 million of tons $(\mathrm{N})$ per year [43, 44]. Accordingly, alongside the global population growth and food consumption, the demand of SNF is expected to increase from 105.3 million of tons in 2011 up to more than 119.4 million of tons in 2018. The total increase in demand is more likely to be distributed among Asia 58\%, the Americas 22\%, Europe 11\%, Africa 8\% and Oceania 1\%; whereas in Latin America, Brazil, Argentina, Colombia and Mexico are expected to dominate the increasing trend [45]. 
Currently, the nitrogen fertilizers production is a highly fragmented and regionalized business, preferably installed close to widely available natural gas sources. The ten largest nitrogen producers account for approximately 19 percent of global ammonia capacity [46] and, currently, the largest global producer of nitrogen fertilizers is the Norwegian company Yara International [38].

Fig. 3.2. Effect of the utilization of the Haber-Bosch process on the population growth.

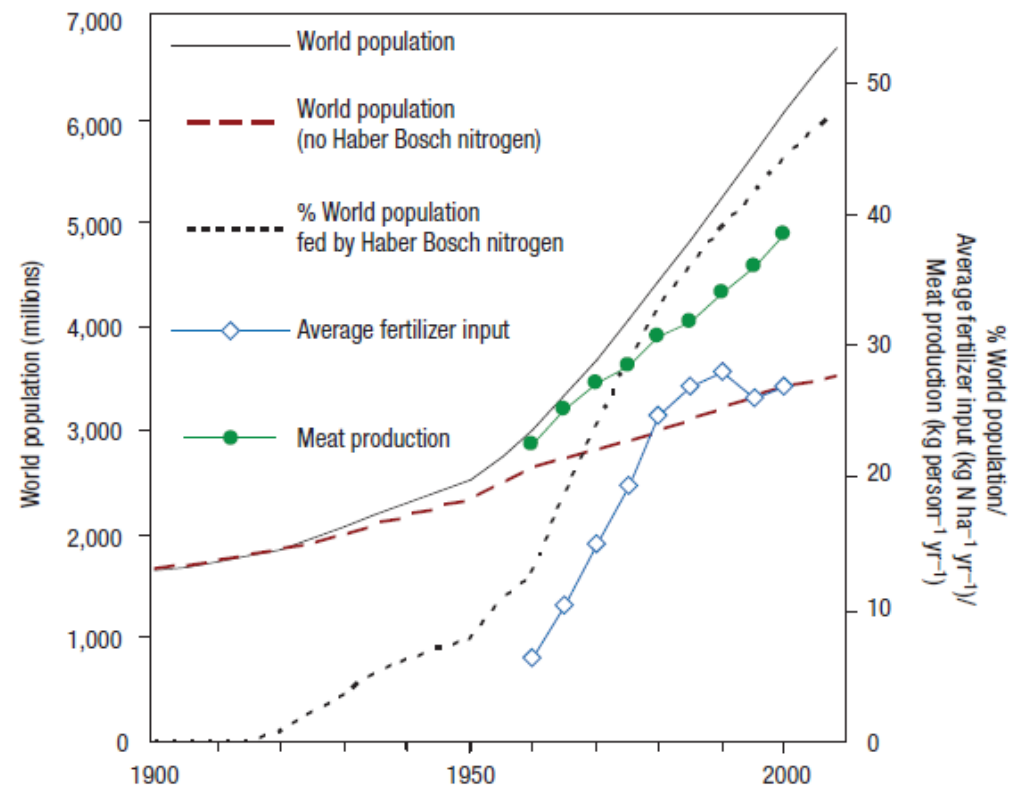

Source: [2].

\subsection{The Brazilian synthetic fertilizers scenario: An overview}

It dates back to 1970 in Cubatao, when Petrobras first decided to participate in the production of raw materials used in the manufacture of fertilizers [47]. Nowadays, according to ANDA (National Association for the Fertilizer Dissemination), in Brazil are produced ammonium nitrate (FOSFERTIL), urea (FOSFERTIL and Petrobras FAFEN), ammonium phosphate and di-ammonium phosphate (COPEBRAS and FOSFERTIL), and ammonium sulfate (BUNGE, BRASKEM and UNIGEL) [48].

Figure 3.3 shows the products classified in the category of SNF, namely ammonia, urea, nitric acid and ammonium nitrate. As a common fundamental stage, ammonia $\left(\mathrm{NH}_{3}\right)$ is the main feedstock used for the production of other SNF and represents the most energy intensive step [42]. Two main technologies are currently employed in the ammonia production in Brazil. The first type consists of the catalytic steam reforming of light hydrocarbons, used in the Petrobras FAFEN plants in Camacari (BA), with a yield of 474000 t/year of ammonia, and in Laranjeiras (SE), with a production of $456000 \mathrm{t} / \mathrm{year}$ of ammonia, both consuming natural gas. The FOSFERTIL plant in Cubatao (SP), producing $191000 \mathrm{t} /$ year of ammonia, also uses the catalytic steam reforming technology, but consumes refinery gas. As a second type of technology, the FOSFERTIL ammonia plant in Araucaria (PR) uses the partial oxidation of asphaltic residue to produce 438000 t/year of ammonia $[48,49]$. In order to guarantee the 
supply of about $87 \%$ of the national ammonia demand by 2020 , an ambitious expansion plan to project and build three new fertilizer plants in Três Lagoas (MS), Uberaba (MG, with 519000 t/year of ammonia) and Linhares (ES) has been lately announced [47, 49].

Fig. 3.3. Classification of the synthetic nitrogen fertilizers and producers in Brazil.

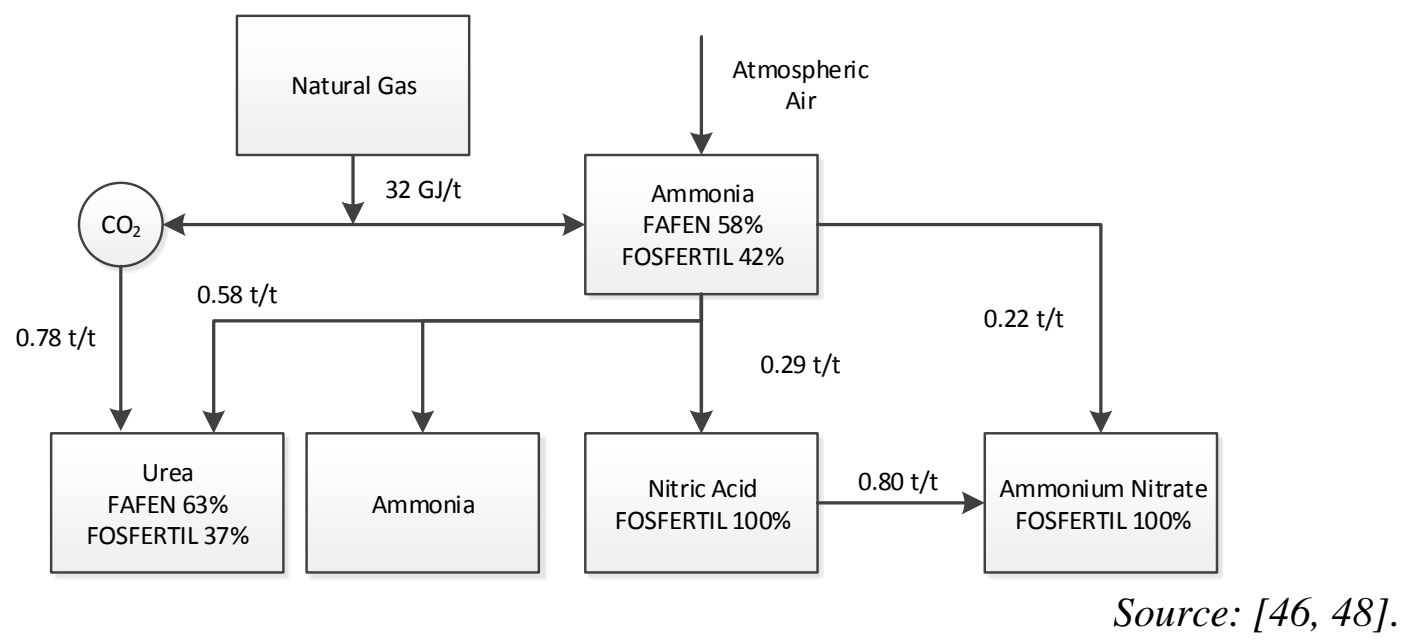

Brazil ranks fourth among the world largest consumers of minerals for the production of synthetic fertilizers, just behind China, India and the United States [50]. In Latin America, Brazil is by far the main producer of phosphate (90\%), the second producer of potash (44\%), and the largest producer of nitrogen (34\%), sharing also about $57 \%$ of the total consumption of fertilizers [51]. The major users of fertilizers in Brazil are the West-Central (30\%) and South-Central (29\%) regions.

However, despite the increasing domestic production $[48,50]$, more than $60 \%$ of the nitrogen fertilizers consumed in Brazil must still be imported [49]. This circumstance leaves the country vulnerable to variations of prices in the international markets, including oil and natural gas prices, shipping costs and logistical problems at ports [51]. It explains the fact that the fertilizers industry has been the segment that contributed the most (25\%) towards the total deficit in the Brazilian chemical sector [4]. This concern should not be taken lightly, if it is considered that Brazil is a major player in global agricultural trade, accounting for $7.3 \%$ of the global agricultural exports and is the world's third-largest exporter of agricultural products, only behind the European Union and the United States [52].

Furthermore, several economic and governance bottlenecks must be addressed to allow the Brazilian fertilizers sector truly expand including the high initial capital costs, the long-term return over investment, the high taxation burdens, the high domestic natural gas price, the unsatisfactory environment of the agriculture business (prioritizing the imports over the domestic production), and the existing difficulties to obtain the environmental licenses [48]. As a result, the domestic fertilizers consumption only increased 30\% (22.8 to 29.6 million of tons) between 2003-2012, while the food production in the country is expected to increase above $40 \%$ between 2010-2020 according to the OCDE [47]. Accordingly, aiming to reduce the foreign dependence of the Brazilian fertilizers sector to only 13\% by 2020 [47], as well as to meet more stringent controls on environmental emissions, the governmental agencies have 
contemplated further investments in the construction of new fertilizer plants or revamping the old ones [4, 47, 49, 53]. Moreover, the exploitation of the natural gas reserves in the Pre-salt region may be a worthwhile strategy to stabilize the gas price fluctuations and the dependence on the imported natural gas, although the technological and logistical adaptations for the extraction and distribution of that resource still remain inadequate [54]. 


\section{CHAPTER 4}

\section{SYNGAS AND AMMONIA PRODUCTION TECHNOLOGIES}

The conventional syngas and ammonia production plant can be generally divided into four main sub-process (see Fig. 4.1), namely the syngas production unit (reforming and shift reactions), the syngas purification unit $\left(\mathrm{CO}_{2}\right.$ removal and methanation), the ammonia synthesis loop (conversion, refrigeration, separation, purge), and utility system (steam and power production, heat exchanger network) [55]. The operation condition of one subprocesses is strongly related to each other, thus any variation in one system simultaneously affects the different sections of the plant concept. In practice, several configurations of processes and technologies may be licensed depending on site-specific conditions. More than 20 commercial ammonia synthesis processes are described in the literature, and commercial vendors include Imperial Chemical Industries (until 2007), Kellogg, Brown \& Root (KBR), Linde, Haldor Topsoe, Ammonia Casale and ThyssenKrupp-Uhde [32].

Fig. 4.1. Plantwide layout of an integrated syngas and ammonia production plant.

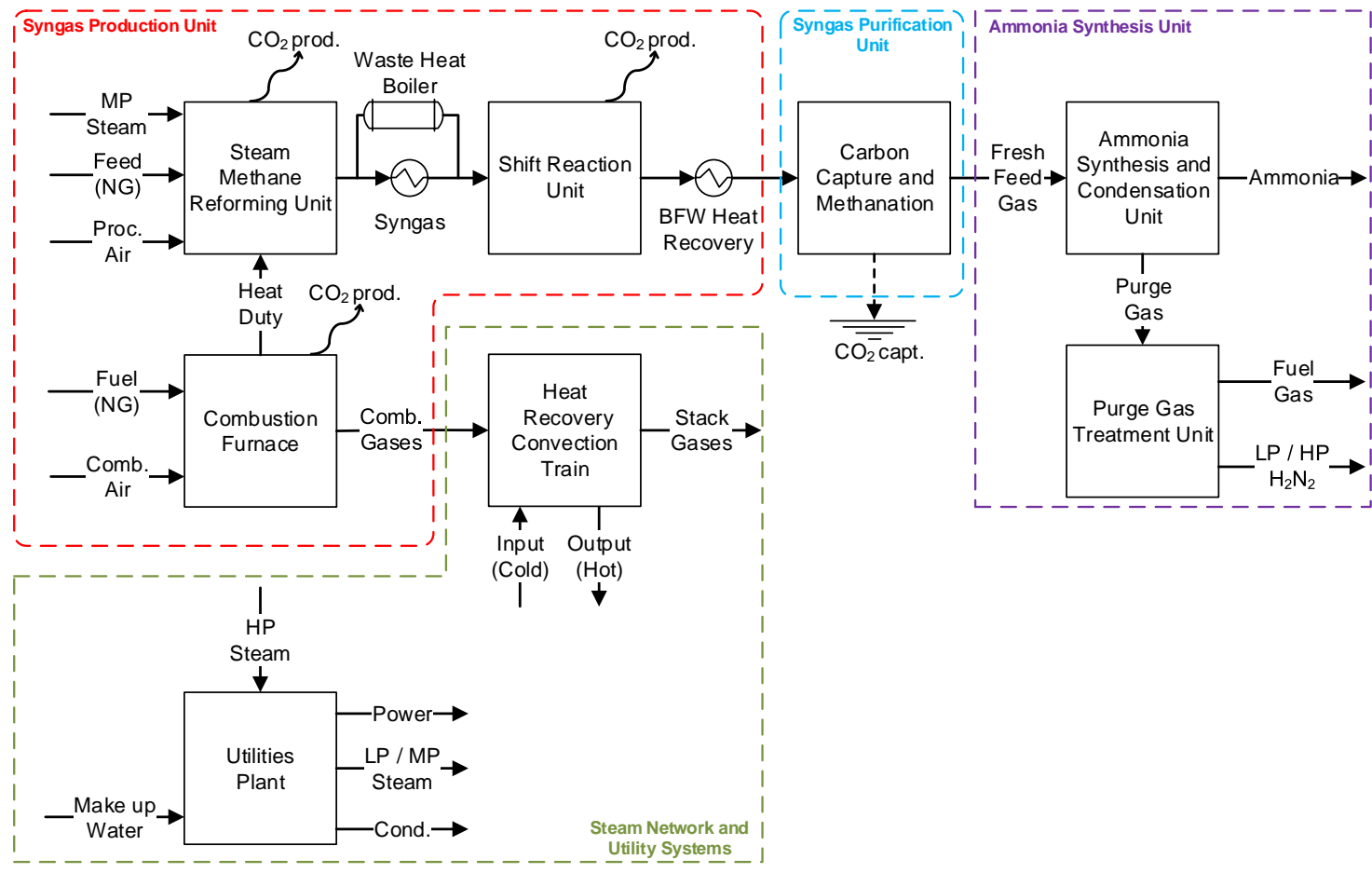

Source: Author.

The objective of the integrated plant shown in Fig. 4.1 is to produce ammonia from a mixture of nitrogen and hydrogen in a stoichiometric proportion of 3:1 from water, natural gas and air. The overall reaction of syngas and ammonia production based, for example, on the reforming of methane is given by (R.4.1) [56]:

$\mathrm{CH}_{4}+0.303 \mathrm{O}_{2}+1.131 \mathrm{~N}_{2}+1.395 \mathrm{H}_{2} \mathrm{O} \rightarrow \mathrm{CO}_{2}+1.131 \mathrm{~N}_{2}+3.395 \mathrm{H}_{2} \rightarrow \mathrm{CO}_{2}+2.262 \mathrm{NH}_{3}$ (R.4.1) 
In this chapter, the energy conversion technologies for syngas production, carbon capture and ammonia synthesis, existing in the conventional ammonia production plants, are briefly presented.

\subsection{Syngas and hydrogen production technologies}

Among the various sources of industrial hydrogen production are (i) the reaction of steam with a metal, (ii) the electrolysis of an electrolyte aqueous solution, (iii) the gasification of carbonaceous materials, (iv) the partial oxidation and (iv) the reforming process [57]. The first production process consists of a cyclic oxidation $\left(\mathrm{H}_{2} \mathrm{O}+\right.$ metal $\rightarrow$ metal oxide $\left.+\mathrm{H}_{2}\right)$ and reduction (metal oxide $+\mathrm{CH}_{4} \rightarrow$ metal $+\mathrm{CO}_{2}+\mathrm{H}_{2} \mathrm{O}$ ) process in which a metal $(\mathrm{Cu}$, Fe, etc.) is reacted with steam to produce $\mathrm{H}_{2}$ and $\mathrm{CO}_{2}$.

In the electrolysis process, the feedstock consists of purified water to which potassium hydroxide is added in order to increase its conductivity, without taking part in the reaction. The cost of the hydrogen production via electrolysis is not as dependent on the plant size as on the cost of electricity. Thus, its competitiveness will depend largely on the plant localization, typically installed where cheaper electricity from hydro or other renewable power plants is available [22]. Typical power consumption figures in electrolysis achieve 4.3 $\mathrm{kWh}$ per $\mathrm{m}^{3}$ of $\mathrm{H}_{2}$ produced (approx. $190 \mathrm{GJ} / \mathrm{t}_{\mathrm{H} 2}$ ) [22, 25]. Considering that, for ammonia synthesis, additional energy is required to separate the nitrogen from the air and to compress the reactants, the total energy consumption may achieve $10,200 \mathrm{kWh} / \mathrm{t}_{\mathrm{NH} 3}$ (or $37 \mathrm{GJ} / \mathrm{t}_{\mathrm{NH} 3}$ ). This value is comparable to those of the reforming processes, provided that an electricity mix with a very efficient generation process is considered $[22,58]$. Otherwise, the energy consumption may sharply increase, depending on the technology used for the electricity generation. Moreover, since the water electrolysis process does not produce $\mathrm{CO}_{2}$, it cannot be associated to an urea facility, unless $\mathrm{CO}_{2}$ is available from another source. A major advantage of the water electrolysis is the production of a gas containing only $0.1-0.2 \%$ of oxygen, compared to the syngas produced from hydrocarbons [22]. This is a key issue, since a small part of the hydrogen produced has to be spent to remove $\mathrm{O}_{2}$ in downstream processes as it represents a poison to the ammonia catalyst. Despite the fact that this technology is currently used in plants of up to $500 \mathrm{t}_{\mathrm{NH} 3} /$ day, the process is generally not considered economically sustainable, except for some specific scenarios [22].

For many years, the predominant feedstock for ammonia synthesis was coke, but currently it is only used under special economic and geographical circumstances, e.g. China, where more than tree fifths of the production relies on coke [25]. At this moment, less than $10 \%$ of the ammonia production is based on coal, coke or lignite. In general the process is similar to the original Haber Process, but involving coal gasification and a gas purification process [22]. In fact, it is more likely other feedstock such as light and heavy hydrocarbons to dominate the ammonia production scenario in the middle term. However, in the very long term it would be expected coal to substitute them [59].

Mainly three technologies are typically employed to produce hydrogen from hydrocarbon fuels, namely steam methane reforming (SMR), auto thermal reforming (ATR) and partial 
oxidation (POX), and each one produces different $\mathrm{H}_{2}$ to $\mathrm{CO}$ ratios (SMR, 3.0 - 5.0; ATR, 1.5 2.5; POX, 1.5 - 1.8) [60,61]. Moreover, all of them present specific operating advantages but also technological challenges [62]. For instance, SMR does not requires oxygen, operates at the lowest process temperature and yields the major $\mathrm{H}_{2}$ to $\mathrm{CO}$ ratios, notwithstanding it produces the largest atmospheric emissions. The ATR process runs on a lower temperature than POX as well as presents a lower methane slip (i.e. unconverted), but its commercial experience is more limited. In turn, POX does not need any catalyst and also presents low methane slip, however it produces the lowest $\mathrm{H}_{2}$ to $\mathrm{CO}$ ratios and operates at very high temperatures, which increases the production of soot, adding purification complexities to the process.

Accordingly, it would be expected that the most economic hydrocarbon feedstock for ammonia production were the one having the highest $\mathrm{H}_{2}$ to $\mathrm{CO}$ ratio and requiring the lowest energy consumption and capital cost. It explains the fact that the steam reforming of natural gas accounts for about $80 \%$ of the feedstock for the ammonia synthesis [54] and it is nowadays considered as the most cost-efficient technology for this application [63]. Table 4.1 compares the relative plant investment and the relative energy consumption for various feedstock used in the ammonia production. The overall energy consumption in the best available technologies (BAT) for steam reforming ranges between 28.4-30.2 $\mathrm{GJ} / \mathrm{t}_{\mathrm{NH} 3}$ [59].

According to Table 4.1, about $30-40 \%$ of the natural gas used in ammonia plants is consumed as fuel and the balance as feedstock. Thus, sometimes it may be advantageous to use less expensive fuels for heating as well as for steam and power generation purposes. For instance, when natural gas cannot be affordable as both fuel and feedstock, a different fuel e.g. light fuel oil may be used [22].

Table 4.1. Relative plant investment and relative energy consumption for various feedstock used in the ammonia production [25, 57, 59].

\begin{tabular}{lccccc}
\hline & $\begin{array}{c}\text { SMR } \\
\text { Natural Gas }\end{array}$ & $\begin{array}{c}\text { ATR } \\
\text { Natural Gas }\end{array}$ & $\begin{array}{c}\text { POX } \\
\text { Fuel Oil }\end{array}$ & $\begin{array}{c}\text { Gasification } \\
\text { Coal }\end{array}$ & $\begin{array}{c}\text { Electrolysis } \\
\text { Water }\end{array}$ \\
\hline $\begin{array}{l}\text { Relative } \\
\text { Investment }\end{array}$ & 1.0 & 1.2 & 1.5 & $1.8-2.1$ & $2.2-2.5$ \\
\hline $\begin{array}{l}\text { Feedstock } \\
\left(\mathrm{GJ} / \mathrm{t}_{\mathrm{NH} 3}\right)\end{array}$ & 22.1 & 24.8 & 28.8 & - & - \\
\hline Fuel $\left(\mathrm{GJ} / \mathrm{t}_{\mathrm{NH} 3}\right)$ & $7.9-9.0$ & $3.6-7.2$ & $5.4-9.0$ & - & - \\
\hline $\begin{array}{l}\text { Total } \\
\left(\mathrm{GJ} / \mathrm{t}_{\mathrm{NH} 3}\right)\end{array}$ & $30.0-31.1$ & $28.4-32.0$ & $34.2-37.8$ & $49.0-59.0$ & 37.0 \\
\hline
\end{tabular}

Partial oxidations offers the advantage of a broader choice of feedstock and a better tolerance to fuel impurities. The most common feedstock in POX is heavy residual oil from petroleum refining process, which is also consumed for steam and electricity generation [59]. The partial oxidation is a non-catalytic process taking place at high pressures and temperatures (> 50bar and $>1400^{\circ} \mathrm{C}$ ) [22]. Furthermore, heavy oil partial oxidation may be attractive as long as it offers an alternative for the utilization of viscous hydrocarbons residues and plastic wastes [59]. 
Even though in an early research stage, some hydrogen production methods use the solar energy and special organisms to produce hydrogen instead of oxygen during photosynthesis. They include the direct and indirect biophotolysis, biological water gas shift reaction, photo fermentation and dark fermentation. [64]. Some studies reported the direct solar ammonia production occurs naturally via exposure of nitrogen and water in presence of catalytic surfaces of the desert sand [65], which has already been experimentally confirmed in laboratory. Other sources, such as ethanol steam reforming represent experimental prospects $[7,66]$, even if for the near term, steam reforming process of light hydrocarbons is forecasted to dominate the syngas production for ammonia synthesis.

In the following sections, the various unit operations used to produce hydrogen via the steam methane reforming (SMR) and water gas shift (WGS) processes are briefly described (see Fig. 4.2), with special attention to the conventional operating conditions.

Fig. 4.2. Syngas production via SMR and WGS process

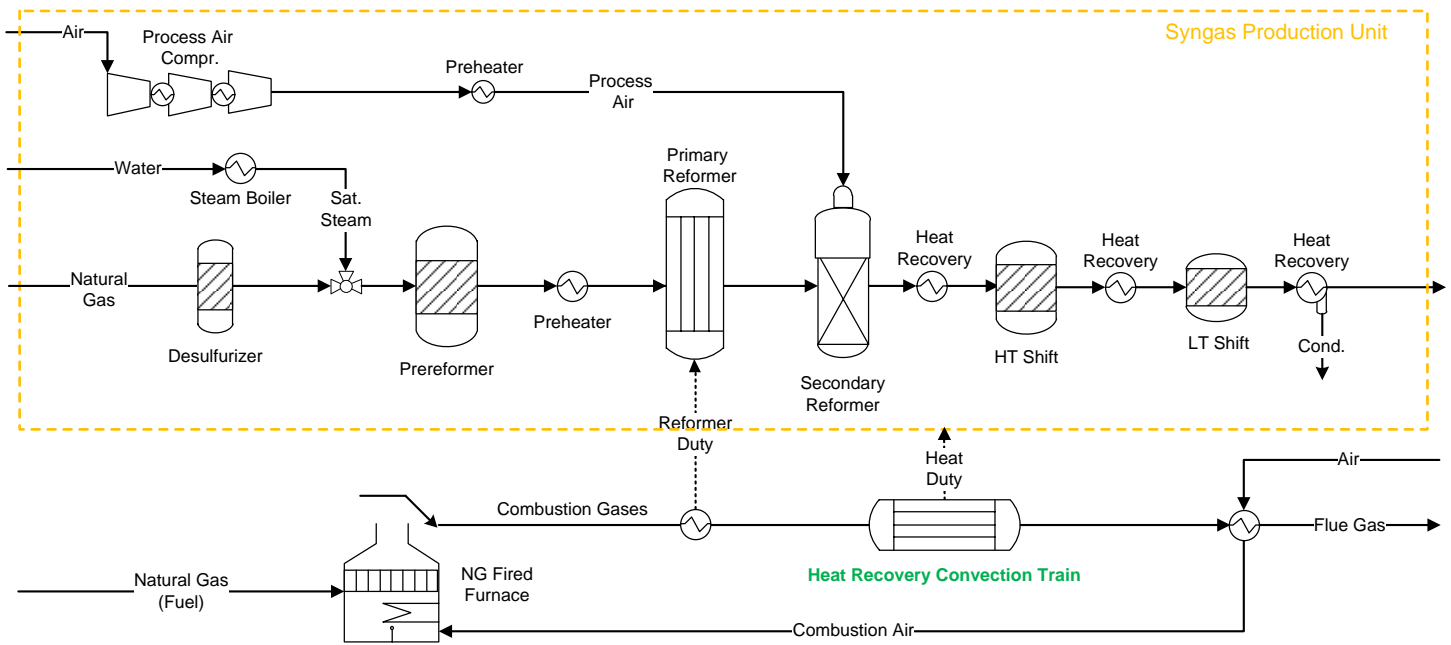

Source: Author.

\subsubsection{Desulfurizer}

In order to avoid the poisoning of catalysts in the downstream processes, the sulfurous compounds in the natural gas must be removed by using a two-step hydrogenation-absorption process called hydrodesulphurization [42]. First, a small amount of hydrogen is added to the feedstock, which is heated up to $400^{\circ} \mathrm{C}$ and passed through a cobalt molybdenum or nickel molybdenum catalyst. There, sulfur is converted into hydrogen sulfide $\left(\mathrm{H}_{2} \mathrm{~S}\right)$, according to (R.4.2 and R.4.3) [67]:

$$
\begin{gathered}
\mathrm{CH}_{3} \mathrm{SH}+\mathrm{H}_{2} \rightarrow \mathrm{CH}_{4}+\mathrm{H}_{2} \mathrm{~S} \\
\mathrm{C}_{2} \mathrm{H}_{5} \mathrm{SH}+\mathrm{H}_{2} \rightarrow \mathrm{C}_{2} \mathrm{H}_{6}+\mathrm{H}_{2} \mathrm{~S}
\end{gathered}
$$

Next, hydrogen sulfide is removed from the feedstock gas through an absorption process producing zinc sulfide (R.4.4) by involving zinc oxide pellets $(\mathrm{ZnO})$ that can be later regenerated [41].

$$
\mathrm{ZnO}+\mathrm{H}_{2} \mathrm{~S} \rightarrow \mathrm{ZnS}+\mathrm{H}_{2} \mathrm{O}
$$


The traces of other compounds found in the feedstock can be removed by using methanation or other methods [21].

\subsubsection{Prereformer}

The prereformer is an adiabatic reactor filled with a high activity catalyst that operates at lower reforming temperatures $\left(<700^{\circ} \mathrm{C}\right)$ than in the conventional primary reformer, located downstream [25]. Although it follows the same sequence of reactions than in the primary reformer (see Section 4.1.3), the equilibrium is established at far lower temperatures. In the prereformer, a preheated mixture of methane, higher hydrocarbons and steam $\left(530^{\circ} \mathrm{C}\right)$ is partially converted into another composed of carbon oxides, hydrogen and methane [68].

A main feature is the irreversible, complete conversion of the heavier hydrocarbons that natural gas may contain, which allows reducing the steam consumption and decreasing the thermal and cracking loads in the tubes of the primary reformer [69]. The use of a prereformer can increase the capacity of the syngas plant inasmuch as it alleviates the primary reformer duty by partially reforming the feed gas. Some authors have reported a reduction of natural gas consumption up to $10 \%$ when a prereformer is introduced [68-70]. On the other hand, since the overall reaction is endothermic, the outlet temperature drops approx. $60-80^{\circ} \mathrm{C}$, requiring to reheat the mixture that is going to be fed to the primary reformer up to a suitable temperature below $650^{\circ} \mathrm{C}$, in order to avoid the thermal cracking of the reactants [25].

\subsubsection{Primary reformer}

The primary reformer is a multi-tubular reactor concept, externally heated by a natural gas fired radiant furnace (see Fig. 4.3). Therein, the reforming reactions (R.4.5-R.4.9) take place across a packed nickel catalyst in which a prereformed mixture of natural gas, preheated in the furnace convection train $\left(580^{\circ} \mathrm{C}\right)$, reacts with the process steam (7-35 bar) supplied by an extraction steam turbine [70]. 
Fig. 4.3. Primary and secondary reformers: (1) prereformed mixture inlet, (2) burner, (3) catalytic reformer tubes, (4) syngas outlet, (5) process air inlet, (6) secondary reformer catalyst and (7) gas outlet.

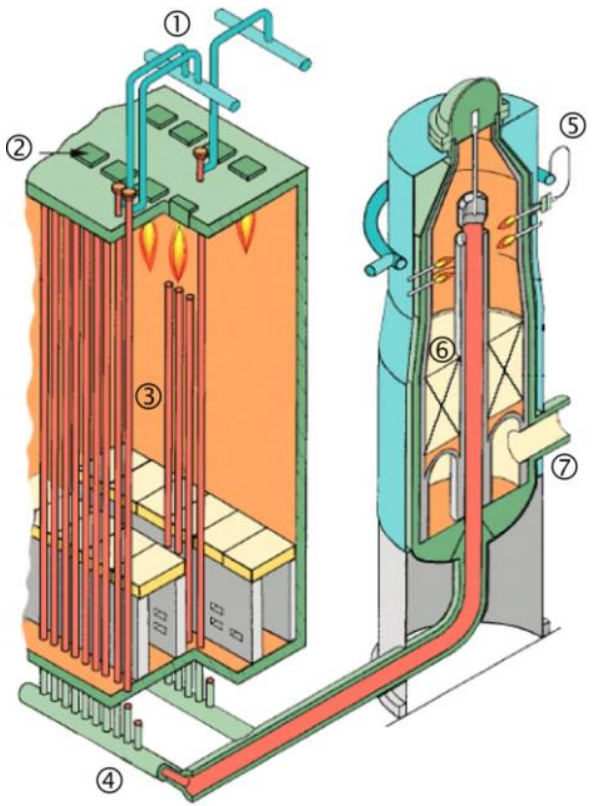

Source: [42].

The primary reformer is the largest and most expensive unit operation, as well as the most energy intensive [71], with a thermal duty of 54.6 MW for a typical $1000 \mathrm{t}_{\mathrm{NH} 3} /$ day plant [55].

Steam reforming reactions [72]

$$
\mathrm{CH}_{4}+\mathrm{H}_{2} \mathrm{O} \rightarrow \mathrm{CO}+3 \mathrm{H}_{2} \quad\left(\Delta H_{298 K}^{0}=206 \mathrm{~kJ} / \mathrm{mol}\right)
$$

Dry reforming reaction [73]

$$
\mathrm{CH}_{4}+\mathrm{CO}_{2} \rightarrow 2 \mathrm{CO}+2 \mathrm{H}_{2} \quad\left(\Delta H_{298 K}^{0}=165 \mathrm{~kJ} / \mathrm{mol}\right)
$$

Homogeneous water reaction or gas shift reaction [70]

$$
\mathrm{CO}+\mathrm{H}_{2} \mathrm{O} \rightarrow \mathrm{CO}_{2}+\mathrm{H}_{2} \quad\left(\Delta H_{298 K}^{0}=-41 \mathrm{~kJ} / \mathrm{mol}\right)
$$

Boudouard reaction [70]

$$
2 \mathrm{CO} \rightarrow \mathrm{C}+\mathrm{CO}_{2} \quad\left(\Delta H_{298 K}^{0}=-173 \mathrm{~kJ} / \mathrm{mol}\right)
$$

Methane catalytic decomposition [70]

$$
\mathrm{CH}_{4} \rightarrow \mathrm{C}+2 \mathrm{H}_{2} \quad\left(\Delta \mathrm{H}_{298 \mathrm{~K}}^{0}=75 \mathrm{~kJ} / \mathrm{mol}\right)
$$

Although the overall stoichiometric steam to carbon ratio $(\mathrm{S} / \mathrm{C})$ ratio is about 1.4 (see R.4.5), most processes operate with higher $\mathrm{S} / \mathrm{C}$ values, typical varying from 2.5 to 4.5 . This selection depends on the type and quality of the feedstock, the existence of purge gas recovery, the reformer capacity, the operation conditions of the shift reactors as well as the steam balance of the plant [59]. A relatively higher S/C ratio has many advantages. First, it shifts the equilibrium of reaction (R.4.5) towards $\mathrm{H}_{2}$ production, thus lowering the $\mathrm{CH}_{4}$ slip. It also provides the necessary steam for shift conversion reactions (R.4.7), increasing the $\mathrm{H}_{2}$ production. Moreover, a high S/C ratio inhibit the occurrence of local overheating and carbon deposition side reactions (R.4.8-4.9), which could lead to deactivation of the catalyst [41, 70, 
72]. In spite of this, some authors have reported substantial energy savings through the reduction of the $\mathrm{S} / \mathrm{C}$ ratio $[70,74]$. Since the excess steam must be condensed by cooling the syngas produced, the larger the amount of excess steam produced, the larger the low-grade waste heat released. Moreover, since the surplus condensed steam contains fractions of carbon dioxide, amines or ammonia, among other components, it may require additional treatment processes (such as steam stripping). Accordingly, the use of excess steam consumes an extra amount of energy both in its generation and in the disposal of its condensate [74]. Thus, a typical S/C ratio 3.0 is normally selected as trade-off between the energy required for the excess steam generation and the reduction of the methane slip [41].

As concerns the operating temperature of the catalytic tubes, higher values are expected to favor both the rate of reaction and the equilibrium conversion of the highly endothermic reforming process. However, the mechanical and metallurgical restrictions following the severe hydrogen-rich operation conditions set a maximum to the allowable temperature and heat flux. Furthermore, since the reacting mixture increases the volume as the reaction evolves, according to Le Châtelier principle, the lower the reactor pressure, the lower the methane slip [59]. On the other hand, higher levels of pressures at the frontend syngas production unit reduce the equipment size and the compression power necessary to further compress the purified syngas up to the backend ammonia loop pressure. Thus, there must be a compromise between the lower methane slip and a higher syngas production pressure [70]. The negative effect of the increased pressure is often offset by increasing the temperature and $\mathrm{S} / \mathrm{C}$ ratio [74], whereas the operating conditions remain limited to $700-830^{\circ} \mathrm{C}$ and $15-40$ bar $[25,75]$. Some performance indicators can be used to quantify the actual methane slip in the primary reformer. The approach to equilibrium (ATE) calculates the deviation of the actual outlet temperature from the expected equilibrium temperature (given the outlet composition) [76]. An increased ATE also results in higher purge rates from the ammonia synthesis loop and, thus, increased energy consumption for the plant. Conservative design values of ATE vary typically between $15-28^{\circ} \mathrm{C}$ [77].

Currently, the syngas production process is a mature technology, although it has been subject to various improvements in the last decades. For instance, some approaches have proposed shifting of a fraction of the primary reformer duty to the secondary reformer, thus reducing the size of the former and avoiding excess firing [69]. However, this approach requires a higher amount of air to the secondary reformer to fully reform the natural gas into hydrogen. This results in a higher nitrogen content which must be removed by different methods [71]. Other variations of the conventional steam reforming process include carbon dioxide reforming (or dry reforming), which produces a mixture with a higher $\mathrm{CO}$ content $(\mathrm{H} / \mathrm{C}<$ 1:1). Since this process entails a greater risk of carbon deposits, it requires suitable catalysts

[78]. Even though dry reforming has not significant commercial application by itself, a number of major advantages related to the thermochemical energy storage has renewed the interest in producing fuels with low $\mathrm{H}_{2} / \mathrm{CO}$ ratio $[73,78]$. Other systems, such as the nuclearbased methane reforming consists of a circuit of helium heated to $950^{\circ} \mathrm{C}$ and cooled by indirect heat exchange with a mixture of methane and steam going through the reformer tubes [22]. Since 1971, these so-called EVA reactors have been used for commercial hydrogen 
production. Other sources of hydrogen production, based on the sunlight concentration in a central receiver that supplies the reforming duty, have been more recently proposed [25].

Finally, previous studies [69] have shown that the main exergy losses in the externally fired primary reformer are not only the result of the highly irreversible fuel combustion in the reformer furnace. Indeed, some avoidable exergy losses are due to the large temperature difference between the flame and the reactants inside the tubes, as well as due to the fumes leaving the furnace stack. According to some authors, the colder gases of a gas turbine system could be used instead of hot combustion gases in order to supply the reformer duty and reduce the process irreversibility $[79,80]$. Since this arrangement provides the heat load to the reformer while generates the electricity and process steam for the plant, the exergy losses due to the excess steam production could be avoided [42].

\subsubsection{Autothermal and Secondary reformer}

The syngas that leaves the primary reformer is reacted with a compressed (35 bar) and preheated air stream $\left(540^{\circ} \mathrm{C}\right)$ inside of a non-tubular, refractory lined adiabatic reactor, called secondary reformer. In a first reaction zone, an homogeneous partial combustion of the syngas increases the reactants temperature $\left(970^{\circ} \mathrm{C}\right)$, meeting both the required $\mathrm{H}_{2}-$ to- $\mathrm{N}_{2}$ ratio and the reactor heat balance $[41,70,73]$. Then, the mixture goes through a heterogeneous reaction zone filled with high temperature resistant nickel catalyst, where the same set of reactions (R.4.5-R.4.9) occur. The intercooled air compression process represents about 20-30\% of the total power consumption in a typical ammonia plant [55]. Despite the fact that the most common applications rely on the air combustion to separate the atmospheric nitrogen, other sources may include liquid air fractioning plants [57]. At the exit of the secondary reformer, almost all of the methane has been converted, although a residual $0.45 \%$ mol content (dry basis) can be still present in the syngas. The reactor effluent must be cooled down from $970^{\circ} \mathrm{C}$ to about $350^{\circ} \mathrm{C}$, a feed temperature suitable for the high temperature shift (HTS) reactor [56]. The waste heat recovery is typically performed by using a watertube boiler with natural circulation in order to produce saturated high pressure steam (100 bar).

The secondary reformer is a type of autothermal reformer (ATR), i.e. a standalone process in which the feedstock conversion is performed by means of the combination of a set of initial homogeneous reactions followed by heterogeneous reactions in presence of a suitable catalyst [81]. The terms secondary reformer applies when it is fed with gases coming from a primary reformer [25]. Despite the fact that the secondary reformer is the most frequent application of commercial ATRs, other types of autothermal reformers used in the ammonia production industry include the partial oxidation (POX) and the catalytic partial oxidation (CPO) reformers. In the POX reactor, a partial combustion of the hydrocarbons or other carbonaceous materials (e.g. biomass gasification) produces higher amounts of carbon monoxide due to a lean oxygen atmosphere [81]. Meanwhile, the CPO process spares the homogenous combustion zone, as the catalytic reforming reactions are performed at milder temperatures. The ATR technology is more flexible than tubular primary reformers as more severe operation conditions and higher throughputs can be attained. Furthermore, since the pressure difference across the catalytic tube wall is avoided, the need for high pressure alloys 
in the internal components is overcome [25]. Some authors also reported that the ATR leads to reduced consumption of resources, lower capital costs and less environmental burdens [69].

On the other hand, the recovery of the high-grade waste heat available from the cooling process of the secondary reformer effluent $\left(>1000^{\circ} \mathrm{C}\right)$ by merely using a watertube steam boiler $\left(300-500^{\circ} \mathrm{C}\right)$ is recognized as an important source of exergy destruction. Thus, various configurations that eliminate the natural gas-fired primary reformer and its associate flue stack $\mathrm{CO}_{2}$ emissions have been proposed [56, 57]. For instance, if the cooling process of the autothermal reformer effluent is used to heat the catalytic tubular reformer, the system is called tandem process or combined ATR with gas heated reformer (GHR) [59]. Nevertheless, the available waste heat is often not enough to completely supply the heating requirement of the primary reformer [69]. Furthermore, since the ATR effluent is not anymore used to raise the process steam that drive all the power consumers [59], the alteration of the waste heat balance implies further modifications in the operation conditions of the combined heat and power production (CHP).

\subsubsection{Water gas shift reaction}

According to the reaction (R.4.7), an additional amount of hydrogen can be produced at the expense of the $\mathrm{CO}$ and water still available in the syngas $\left(\sim 10 \% \mathrm{CO}, 33 \% \mathrm{H}_{2} \mathrm{O}\right.$ molar) by using a high temperature shift reactor (HTS) filled with iron chromium catalyst [55]. After the HTS reactor, the $\mathrm{CO}$ content is reduced to $2.2 \%$ whereas temperature rises up to $350^{\circ} \mathrm{C}-430^{\circ} \mathrm{C}$ [55]. The exothermic water gas shift (WGS) reaction is equilibrium-limited but kinetically favored at higher temperatures which results in an incomplete conversion of carbon monoxide (3\% mol CO at the outlet). In order to reduce the CO content to less than $1 \%$ mol, the WGS reaction is preferably achieved in two high (HTS) and low temperature shift (LTS) reactors [59]. Accordingly, after the mixture is partially converted in the HTS, the syngas is once again cooled down to $220^{\circ} \mathrm{C}$ and fed to a LTS (copper-zinc catalyst) reactor, wherein the concentration of $\mathrm{CO}$ is reduced up to $0.3 \%$ and the temperature rises to $232^{\circ} \mathrm{C}$ [55]. The intercooling of the HTS and LTS reactors as well as the separation of the excess steam is provided by preheating the boiler feedwater (BFW) and by using a large amount of cooling water $(\mathrm{CW})$. The cold syngas produced $\left(35^{\circ} \mathrm{C}\right)$ is sent to the purification unit.

\subsubsection{Syngas Purification: Carbon capture and Methanation}

The cold syngas leaving the shift conversion $\left(35^{\circ} \mathrm{C}\right)$ consist of a mixture of nitrogen $(20.3 \%$ molar), hydrogen $(60.9 \%)$, carbon dioxide $(17.7 \%)$ and non-condensate process steam $(0.2 \%)$, apart from traces of argon and methane (0.66\%) [55]. In order to avoid poisoning the catalyst in the ammonia synthesis and keep $\mathrm{CO}_{2}$ from forming carbamate and ammonium carbonate, all the oxygen compounds $\left(\mathrm{CO}, \mathrm{CO}_{2}, \mathrm{O}_{2}\right.$ and $\left.\mathrm{H}_{2} \mathrm{O}\right)$ must be removed before the syngas enters the ammonia synthesis loop [59, 70]. Catalyst poisoning may reduce nitrogen absorption, increase solution viscosity and foaming, and cause corrosion [82].

Among some commercial acid gas removal technologies are the water scrubbing and the physical and chemical absorption processes. Despite its wide use in older syngas production plants, the main disadvantages of water scrubbing are the low capture rate and the hydrogen 
drift $[22,25]$. Therefore, the aqueous solutions of amines and potassium carbonate are more suitable for the operation conditions in the modern reforming plants [22, 25]. Some advantages of the potassium carbonate system (e.g. Benfield Process) are the low $\mathrm{CO}_{2}$ desorption duty and relatively large gas throughput that can be handled [25]. Furthermore, since the stripper runs cooler than the absorber, a rich-to-lean solvent heat exchanger is spared, thus reducing the investment costs [56].

Figure 4.4 shows the typical configuration of a carbon capture system that operates with a $35 \%$ wt. aqueous solution of di-ethanolamine (DEA), in order to chemically absorb the $\mathrm{CO}_{2}$ present in the syngas produced at relatively high concentrations (> $18 \%$ molar) and partial $\mathrm{CO}_{2}$ pressures (> 6 bar) $[82,83]$. The concentration of the amine solution determines the capacity of the solvent, however, the maximum concentration is limited with regard to its corrosivity and degradation susceptibleness, which requires a solution temperature lower than $120^{\circ} \mathrm{C}$ [25]. Different additives can be used to increase the absorption rates, whereas some inhibitors could be used to increase the amine stability and reduce the corrosion as the amine concentration and acid gas load are increased [70]. The maximum rich DEA and minimum lean DEA $\mathrm{CO}_{2}$ loadings are typically set around 0.45 and $0.04 \mathrm{kmol}_{\mathrm{CO} 2} / \mathrm{kmol}_{\mathrm{DEA}}$, respectively [84].

According to Fig.4.4, in the first column (i.e. the absorber), a major part of the $\mathrm{CO}_{2}$ gas (99.7\%) chemically reacts with the solvent producing a $\mathrm{CO}_{2}$-rich bottoms effluent. Next, the rich solution is expanded and preheated up to 2 bar and $93^{\circ} \mathrm{C}$, respectively [55]. The solution enters a desorption column, where the reaction is reversed and the $\mathrm{CO}_{2}$ is stripped out at higher temperatures and lower pressures. The desorption process demands a substantial amount of heat at the reboiler, close to $48 \mathrm{MW}$ for a typical $1000 \mathrm{t}_{\mathrm{NH} 3} / \mathrm{day}$ plant, which is supplied by low pressure steam ( 7 bar, $0.1 \mathrm{~kg}_{\text {Steam }} / \mathrm{L}_{\text {DEA Solution }}$ ) $[75,85,86]$. Accordingly, the reboiler duty must supply: (i) the energy to raise the temperature of the rich solution up to that of the reboiler, (ii) the enthalpy of reaction to break the chemical bond between the $\mathrm{CO}_{2}$ gas and the amine, and (iii) the enthalpy of vaporization of the water to produce the steam that acts as the stripping source $[84,85]$.

For the simulated conventional case an specific steam consumption of $3.41 \mathrm{MJ} / \mathrm{kg}_{\mathrm{CO} 2}$ is calculated, slightly lower than those reported in literature $[87,88]$, mostly above $4.0 \mathrm{MJ} / \mathrm{kg}$ $\mathrm{CO}_{2}$. This is explained by the reduced enthalpy of reaction and, thus, energy requirement of aqueous DEA solutions compared with MEA [25, 89]. DEA solvent also allows a higher solvent capacity, reducing the solvent circulation rates compared to MEA [25]. 
Fig. 4.4. Syngas purification unit based on a chemical absorption system.

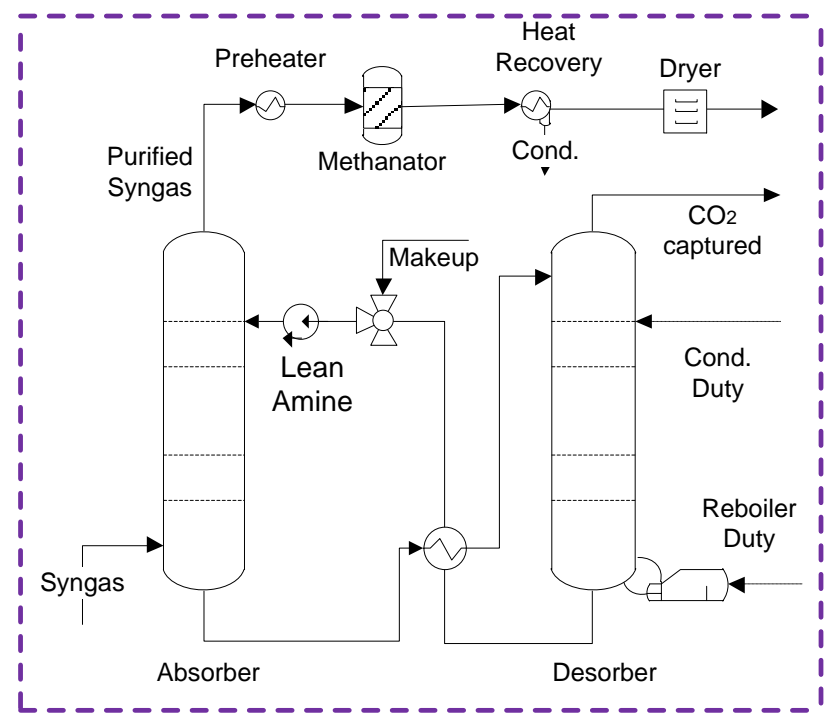

Source: Author

The overhead $\mathrm{CO}_{2}$-enriched gas leaving the desorber is condensate at the top of the desorber column to provide the reflux of water and small amounts of amine vaporized. The captured $\mathrm{CO}_{2}$ gas is cooled, purified and sent to compression [85]. Meanwhile, the partially stripped lean amine solution (92.3\% $\mathrm{CO}_{2}$ removed) is withdrawn through the desorber bottoms and cooled by using a lean-rich (L/R) amine heat exchanger. Since some amount of water is carried over with the overhead streams in the absorber and the desorber, makeup water is required at a rate of $15.5 \mathrm{~m}^{3} / \mathrm{h}$ to keep the desired concentration of the DEA solution. Differently from MEA absorption systems, reclaiming of DEA solution is in most of the cases not necessary or not economically feasible [85]. Thus, the lean DEA stream is finally pumped and recycled back to the absorber.

Physical absorption systems are generally favored over chemical solvents in processes with very high partial pressures and $\mathrm{CO}_{2}$ concentrations [22]. Figure 4.5 shows a typical syngas purification unit based on the physical absorption principle. In the high pressure absorber (30 bar) a mixture of dimethyl ethers of polyethylene glycol (DEPG) contacts the syngas, producing a $\mathrm{CO}_{2}$-rich bottoms solution, analogously to the chemical absorber unit. However, unlike the heat-consuming chemical desorption process, the rich DEPG solution is fairly regenerated by means of a gradual pressure letdown that can recover the expansion energy [90]. The most notorious advantage of using DEPG is the lower energy requirement for the solvent regeneration due to the lack of a reboiler component. Furthermore, DEPG performs a partial gas dehydration during the absorption processes and the $\mathrm{CO}_{2}$ separation temperatures are close to the ambient conditions. Last but not least, DEPG presents as a more stable and non-corrosive operating condition, so that special metallurgy is not required, reducing relative capital and operating costs [91]. 
Fig. 4.5. Syngas purification unit based on a physical absorption system.

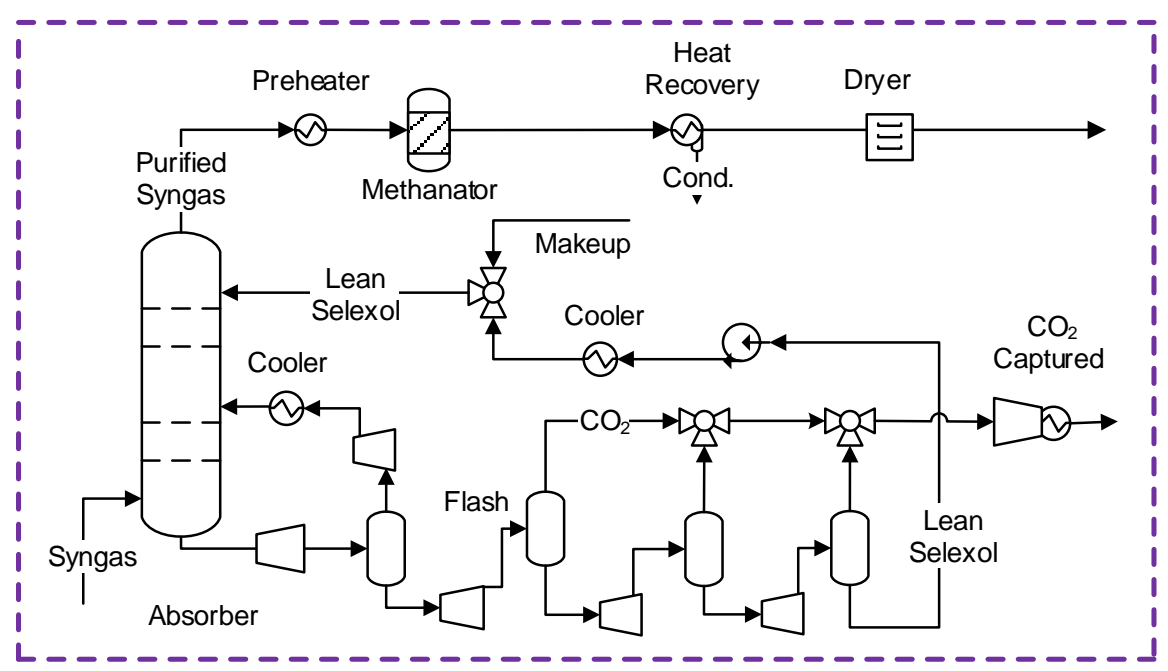

Source: Author

After the $\mathrm{CO}_{2}$ removal, the syngas may still contain up to $0.32 \% \mathrm{~mol} \mathrm{CO}$ and $600 \mathrm{ppm}$ of $\mathrm{CO}_{2}$ [55]. Thus, the methanation system removes the residual oxygen compounds at expense of the consumption of a fraction of the hydrogen produced. Poisonous components are converted into inert methane over a nickel catalyst [25, 70]. Three highly exothermic reactions are involved in methanation unit corresponding to (R.4.7) and reverse reforming reactions (R.4.5R.4.6), all of them carried out at temperatures about $290-317^{\circ} \mathrm{C}[72,92]$. In other to reduce the energy requirements, the methanator outlet stream can be used to preheat the feed stream. Downstream the methanator, the purified syngas is cooled and the moisture is removed [93]. It is claimed that by using an additional nitrogen washing step (especially in the POX process), the ammonia synthesis (see Section 4.2) could be operated without gas purge and, consequently, higher efficiencies and gas purities could be obtained [22].

\subsection{Ammonia Synthesis}

At the end of the purification process, the syngas produced has the desired $\mathrm{N}_{2}-t_{0}-\mathrm{H}_{2}$ ratio and contains a small percentage of inerts. Then, the mixture is further compressed and introduced into the ammonia synthesis loop. According to Figs. 4.6a-e, the quality of the syngas (e.g. level of impurity) along with other technical and economic considerations (e.g. the costly compression) clearly affect the choice about the most suitable point at which the makeup gas should be fed to the synthesis loop $[41,94]$. These arrangements also differ with respect to the points at which the refrigeration of the partially converted gas mixture, as well as the ammonia and purge withdrawal, must be accomplished [95]. As expected, the best point for the purge gas withdrawal corresponds to the point where the concentration of inerts is higher, i.e. after the ammonia bulk is removed and right before the makeup syngas is added. 
Fig. 4.6. Flow diagrams of various ammonia loop arrangements.

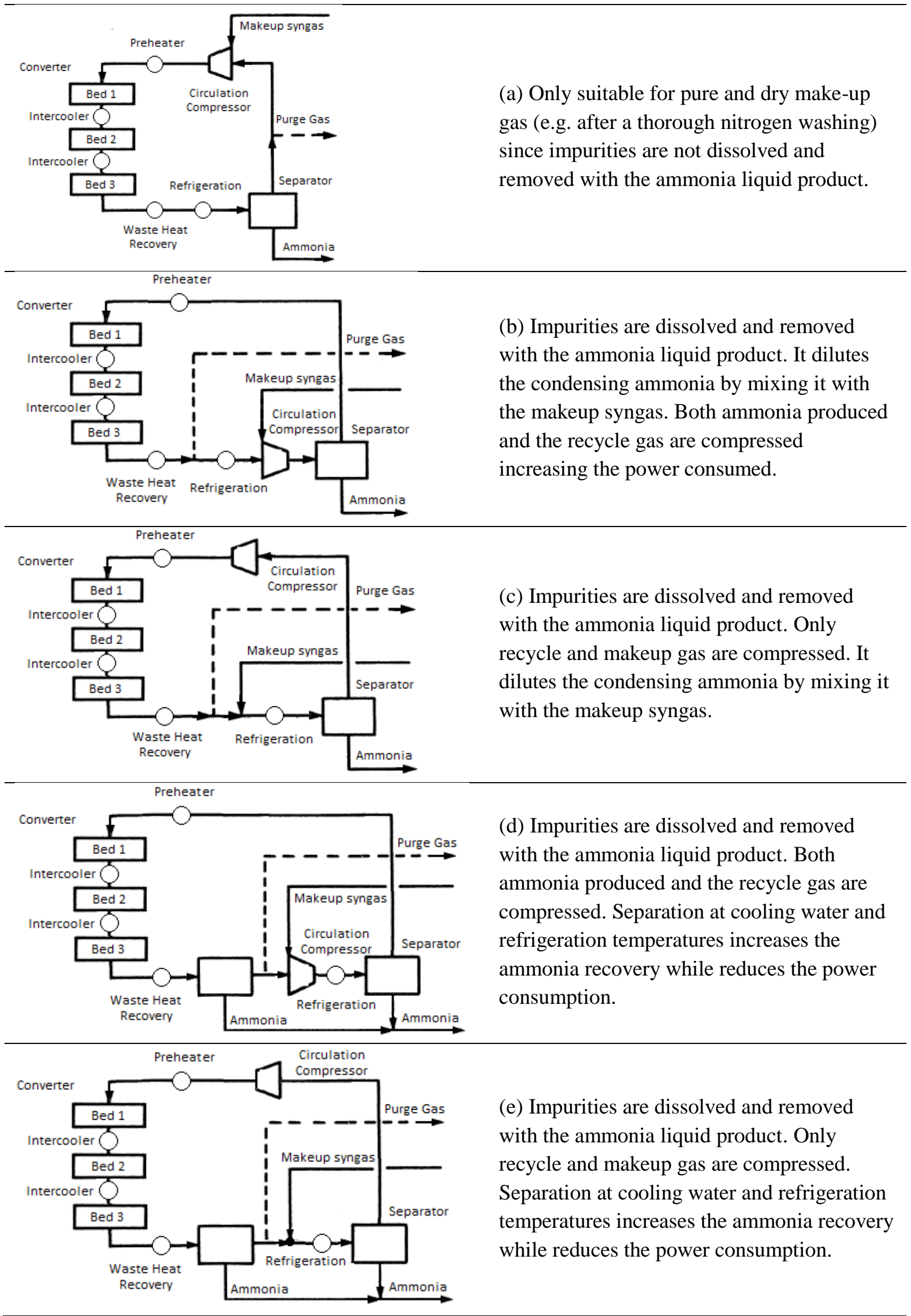


Figure 4.6e presents the configuration that offers the best characteristics in terms of ammonia separation, control of impurity to the converter beds, as well as reduced power compression and purge losses. Figure 4.7 depicts the basic components of the Haber-Bosch ammonia synthesis loop, namely the fresh syngas compressors, the catalytic converter beds, the waste heat recovery and condensation system, the refrigeration and the purge gas recovery unit (not shown) [19]. All these process are further described in the following sections.

Fig. 4.7. Ammonia synthesis, condensation and separation unit.

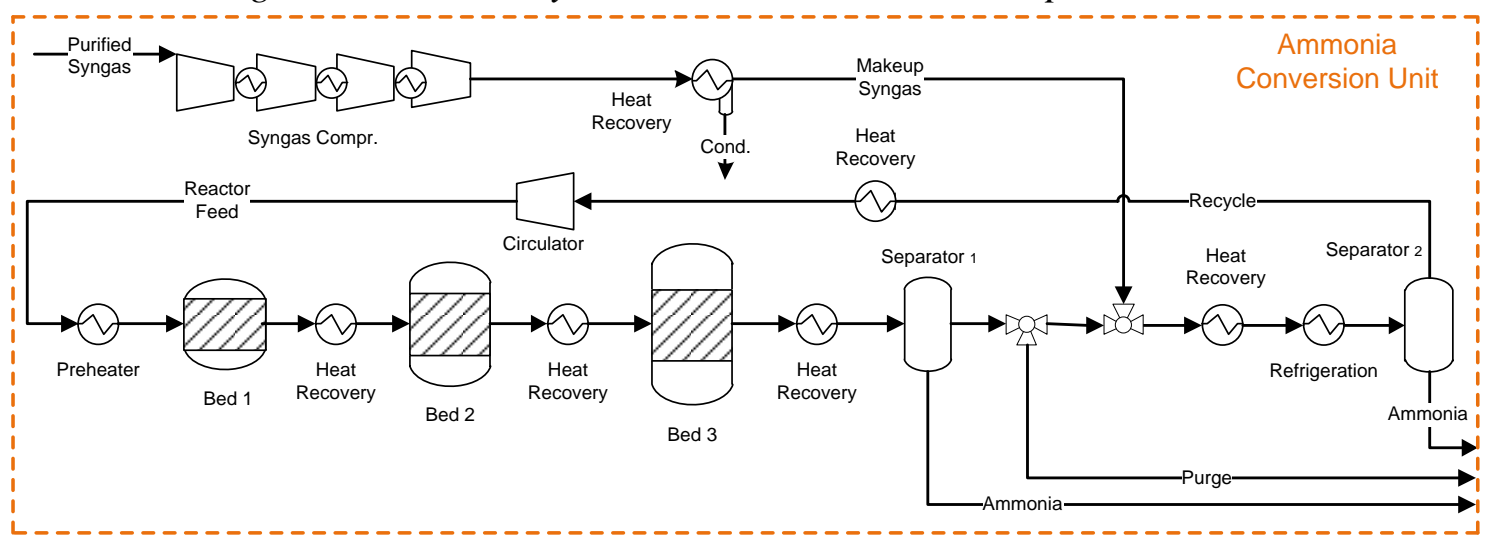

Source: [55].

\subsubsection{Syngas Compression}

Due to the favorable conversion and separation conditions, the ammonia synthesis units typically operate at pressures above 150 bar [48]. Thus, the syngas leaving the purification unit is further compressed in an intercooled, multi-stage compression system (Fig. 4.7), usually driven by high pressure extraction steam turbines [41]. Moreover, since the reactants are not totally converted, the unreacted mixture must be recompressed along with the makeup syngas, and recycled as feed to the ammonia converter [96]. This is performed by means of a circulator that increases the gas pressure by 10-15bar to overcome the pressure drop around the loop [41]. The syngas compressor and circulator reach a combined power consumption of approx. $43 \%$ of the total power consumption in the ammonia plant. Centrifugal compressors driven by steam turbines, instead of reciprocating compressors, allow for higher production rates and achieves further economics [65]. Moreover, although circulator flow rates can be as much as five times higher than in the makeup syngas compression train, the circulator power consumption is quite lower than in the latter, due to the lower pressure difference around the synthesis loop [41].

\subsubsection{Syngas Conversion}

The mixture of the fresh and the recycled syngas gas at $200 \mathrm{bar}$ and $35^{\circ} \mathrm{C}$ is preheated and introduced to the converter, where the synthesis reaction (R.4.10) takes place in the presence of an iron based catalyst. Therein, typical fractional conversions between 10-30\% can be achieved [41].

$$
\mathrm{N}_{2}+3 \mathrm{H}_{2} \rightarrow 2 \mathrm{NH}_{3} \quad\left(\Delta H_{298 K}^{o}=92 \mathrm{~kJ} / \mathrm{kmol}\right)
$$


Contrary to the reforming process, the ammonia synthesis is highly exothermic. Hence, according to the Le Châtelier principle, theoretical equilibrium conversions close to the unity could be achieved by reducing enough the temperature and increasing reactor pressure [23] (Fig. 4.8). However, the reaction rate at lower temperatures is extremely low, whereas, at higher temperatures, it initially speeds up and then decreases on approaching to equilibrium $[23,59]$. This fact entails a set of maximum conversion rates which, in turn, implies lower catalyst volumes requirements. Technically, higher temperatures accelerate the loss of the catalyst activity, whereas abnormally low temperatures threaten the self-sustainability of the exothermic reactor. In practice, moderate temperatures $\left(350-550^{\circ} \mathrm{C}\right)$ are used as a compromise between suitable equilibrium conversions and acceptable reaction rates.

As the synthesis reaction proceeds, the temperature rises sharply, roughly $14-18^{\circ} \mathrm{C}$ per each percent of nitrogen converted [23, 25]. Thus, in order to control the catalyst temperature and increase the per-pass conversion in comparison with a single adiabatic reactor, three or more sequential catalytic beds with a suitable intercooling system are often desirable [25]. In this way, an optimum profile of reaction temperatures can be obtained by regulating the inlet conditions of each bed by means of either a direct (cold shot or quenching) or indirect (e.g. steam generation) cooling process.

Fig. 4.8. Equilibrium concentration of ammonia at different temperatures and pressures. The effect of the inert content in the reactor feed is not shown. For an increment on $10 \%$ of inerts, the ammonia equilibrium composition drops down between 14-17\%.

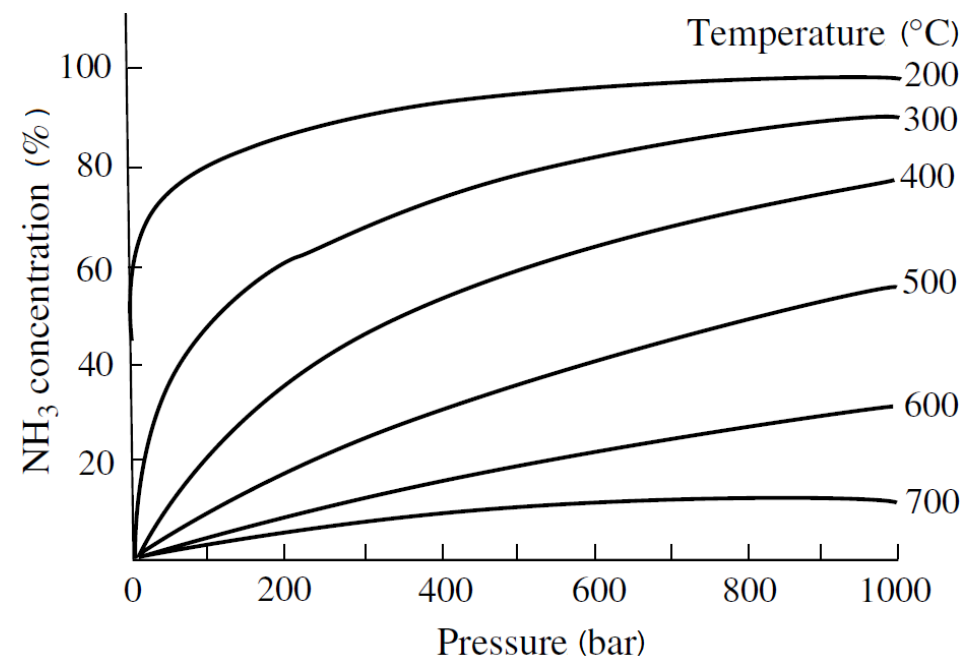

Source: [25].

Moreover, despite the fact that lower pressures at the ammonia loop may bring about lower compression power and reduced equipment costs, several drawbacks arise from the reduced extent of reaction (Fig. 4.8). A lower synthesis loop pressure also hinders the operation of the refrigeration and separation system, affecting the overall performance of the unit. This problem has motivated recent research works on advanced catalysts, more active at lower pressures and temperatures [5, 20]. For instance, the ruthenium catalyst used in the Kellogg Advanced Ammonia Process (KAAP) is claimed to have between tenfold to twentyfold the activity of conventional iron-based catalyst at lower temperatures. Currently, it finds 
application in the Haldor Topsoe S300 ammonia reactor, wherein it is used in conjunction with conventional magnetite catalyst. Energy savings about $1.17 \mathrm{GJ} / \mathrm{t}_{\mathrm{NH} 3}$ have been reported $[22,41]$, showing how the performance of the reactor may be enhanced by means of a set of catalysts sorted along the beds.

Finally, it is worthy to notice that the enthalpy of reaction of the ammonia synthesis is equivalent to about $8.8 \%\left(2.72 \mathrm{GJ} / \mathrm{t}_{\mathrm{NH} 3}\right)$ of the overall fuel consumption in the conventional ammonia plants. Accordingly, there is a strong incentive in recovering as much as possible of this waste heat [41]. However, by carrying out the ammonia synthesis at lower temperatures (e.g. $<300^{\circ} \mathrm{C}$ ), the low-grade waste heat is not further attractive for generating high pressure steam, which drastically modifies the overall energy integration of the ammonia plant [74]. As a conclusion, the performance of the ammonia converter and, consequently, the overall efficiency of the ammonia loop are strongly affected by the reactor pressure, the amount of recycled inerts and non-condensable ammonia, the preheat temperature of the feed syngas, the design of the heat removal system and the characteristics of the catalyst $[19,42,97]$

\subsubsection{Ammonia Separation and Refrigeration}

The bulk of the ammonia produced is preliminarily condensed by using a cheaper cooling water method and then removed in a first vapor-liquid separation vessel. Notwithstanding, the ammonia condensation is not completely satisfactory if only water is used as the cooling medium [59]. For this reason, the recycled stream joining the makeup syngas is refrigerated to approx. $-20^{\circ} \mathrm{C}$ by means of a two-stage R717 vapor compression refrigeration system (Fig. 4.9) [42]. Vapor compression refrigeration is reportedly being more economical than absorption refrigeration for higher loop pressures ( $>150$ bar) [70]. Additionally, R717 refrigerant outperforms other substances in terms of heat transfer and power consumption [98, 99].

However, as it is also exhibited by other industrial refrigerants, R717 experiences a dramatic increase in the compressor discharge temperature $\left(>120^{\circ} \mathrm{C}\right)$ if a single stage of compression is used. This feature increases the breakdown rate of the lubricating oil and the likelihood of material fatigue [100]. Fortnately, the discharge temperature can be controlled by using the two-stage vapor compression refrigeration system with intercooling, according to Fig. 4.9 [98]. Therein, the gas discharged from the low pressure compressor is desuperheated by means of the direct contact with the liquid or the vapor returning from the condenser. This procedure not only reduces the discharge temperature, but also the amount of vapor handled by the high pressure compressor, the compression ratios and the throttling loses $[55,101]$.

Moreover, it is worthy to notice that, since the R717 vapor from the throttling process at high pressure is not passed to the low pressure stage, the quality of the refrigerant entering the evaporator reduces, improving the refrigeration effect. However, case (b) results in a slightly superheated inlet condition at high pressure compressor, apart from an increased cooling requirement and power consumptions. 
Fig. 4.9. Multiple stages ammonia refrigeration systems with vapor-liquid flash (a) for intercooling and (b) for vapor separation only
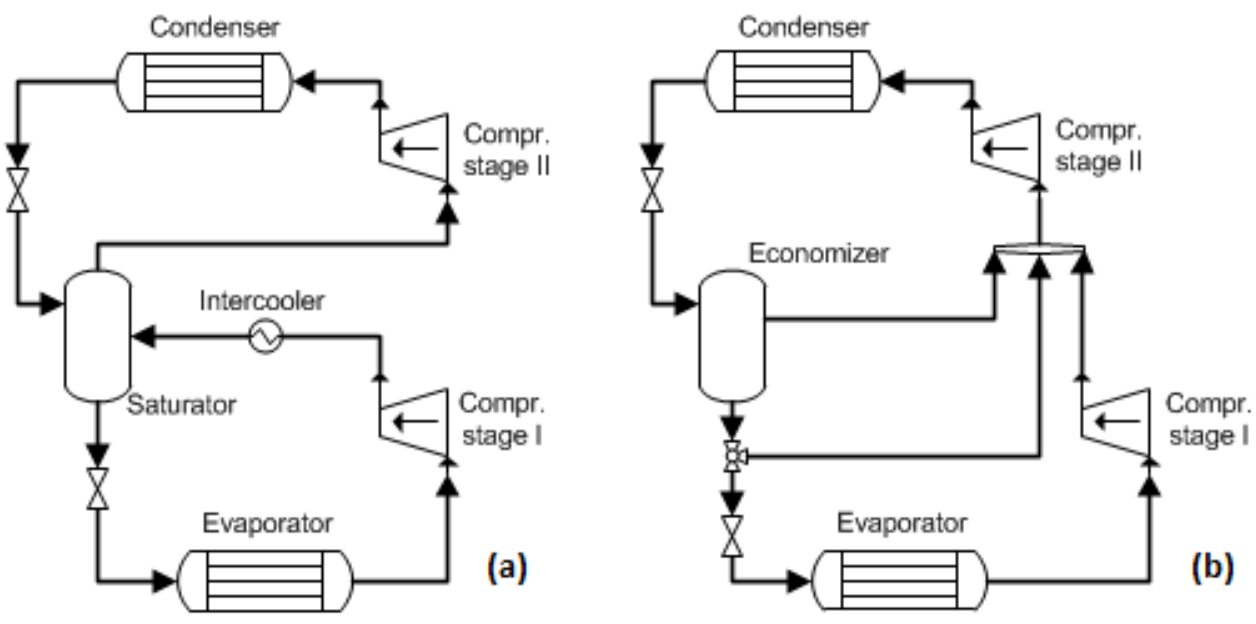

Source: [100].

Finally, the condenser pressure (11-14 bar) is determined by the temperature of the available cooling water, whose utilization is only justified in large industrial refrigeration systems [101]. By considering that the lower the evaporator pressures, the higher the compression power, an evaporator slightly above ambient pressure is preferable $(115 \mathrm{kPa})$. Both evaporator and condenser operate at a minimum temperature approach about $5^{\circ} \mathrm{C}$.

\subsubsection{Purge gas treatment}

Albeit the small fractions of methane and argon slipped into the synthesis loop behave as inerts, the excessive built-up of such components must be controlled since they reduce the reactants conversion and increases the circulation rate [69, 104, 105]. This is achieved by means of a continuous purge of a portion of the hydrogen-rich recycled gas to maintain the overall concentration of inerts down to an acceptable level $(<8 \% \mathrm{~mol})$. As an heuristic approach, a purge fraction of $7 \%$ of the total makeup synthesis gas is recommended [25].

The purge gas contains about $54 \% \mathrm{H}_{2}, 23 \% \mathrm{~N}_{2}$, and other gases $\left(\mathrm{NH}_{3}, \mathrm{Ar}\right.$ and $\left.\mathrm{CH}_{4}\right)$ of which $\mathrm{H}_{2}$ is the most valuable since it can be either returned to the synthesis loop or used as fuel [97, 102]. Due to the considerable amount of hydrogen lost with the purge, its partial recovery and recycle may lead to the reduction of the amount of ammonia produced per unit of feedstock, which may increase the overall efficiency of the loop. Notwithstanding, the decision whether hydrogen recovery is or not beneficial depends on a trade-off between the wastage of the valuable hydrogen and the additional compression power required to operate the recovery unit and recompress the recycled hydrogen-rich gas [102].

Pressure swing adsorption (PSA), membrane systems and cryogenic condensation units are among the purge gas recovery processes available [22, 99, 103]. In the PSA, the purge gas passes through a sort of molecular sieves at elevated pressure, where the non-adsorbed hydrogen is separated from the gaseous mixture. The set of parallel beds operate such that as one ensures the continuous $\mathrm{H}_{2}$ recovery, the other is regenerated by depressurization [25]. PSA is suggested for low pressures (20-30 bar) and the recovery efficiency (70-85\%) is lower 
than that of membrane systems and cryogenic units [22, 104]. Membrane gas separation systems are based on the differences in permeation coefficients of gaseous components, which entails a considerable pressure drop [56, 105]. The hydrogen recovery efficiency of the membranes may vary between $85-95 \%$ [106]. On the other hand, cryogenic recovery can achieve a high recovery efficiency of hydrogen (> 99.5\%), operating at high pressures and large molar flows $[104,105]$. Hence, since this process progresses at very low temperatures, the adiabaticity of the equipment is a decisive factor in the efficiency of the unit [94].

In the cryogenic $\mathrm{H}_{2}$ recovery unit (Fig. 4.10), the ammonia-rich purge gas is expanded and flashed due to the pressure limitation on the brazed aluminum heat exchangers (50-80 bar) [97, 102]. Later, the mixture is water scrubbed in order to separate the substances that, otherwise, would solidify downstream. Meanwhile, scrubbed ammonia is distilled out of the aqueous mixture as aqua-ammonia (used as fertilizer) [107] and the moisture in the ammoniafree purge gas is removed by means of molecular sieves [97].

In the cold box, the ammonia-free purge gas is cooled down to about $-190^{\circ} \mathrm{C}$ in order to separate the lower boiling point components from argon and methane. In this way, a hydrogen recovery of $94.96 \%$ and a nitrogen recovery of $18.54 \%$ can be achieved [97, 102]. The cooling effect is supplied by the expansion of the cryogenic liquid produced along with an auxiliary R717 refrigeration system. Finally, the major part of the hydrogen-rich vapor phase out from the cryogenic separator is recompressed up to essentially the same pressure of the makeup syngas ( 200 bar). A compression stage mechanically coupled to the purge expander is used to this purpose [94].

Fig. 4.10. Cryogenic recovery system for hydrogen-rich purge gas.

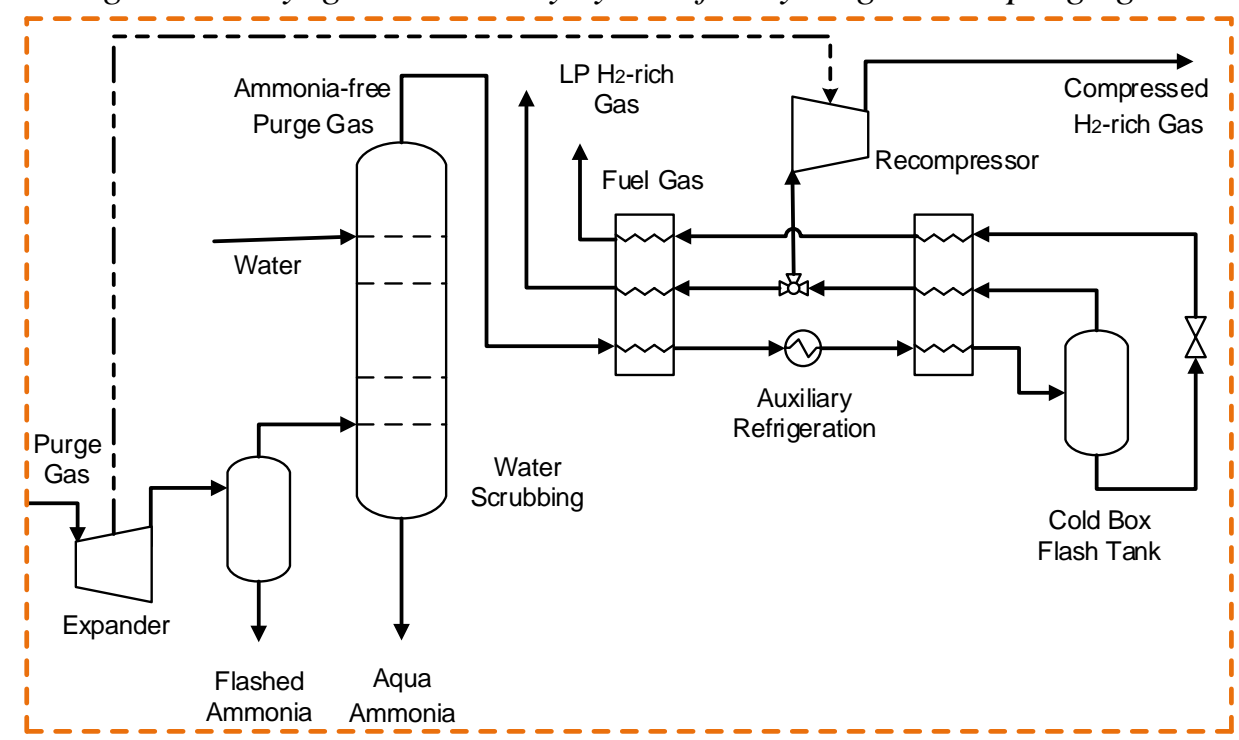

Source: Author.

Meanwhile, the methane-rich liquid phase at lower pressure can be returned to the plant fuel system [94]. Additionally, the uncompressed fraction of the hydrogen-rich gas (71 bar) could be either externally recompressed and recycled to ammonia loop [104] or used as fuel in the ammonia plant to reduce the energy consumption [103]. 


\subsection{Combined Heat and Power production}

Since no additional energy input is needed other than the natural gas fed to the fired furnace of the primary reformer, the modern conventional ammonia plants can be fairly considered as self-sufficient in terms of both steam and power requirements [70]. This is possible thanks to an extensive waste heat recovery system that produces steam at various levels of pressure (0.12-100 bar). Steam is consumed (i) in the extraction turbines that drive pumps and gas compressors, (ii) in the reboiler of the chemical absorption-based purification system, as well (iii) as feedstock in the primary reformer. A simplified representation of the combined heat and power production in the utility system is shown in Fig. 4.11. The industrial cooling tower considered has typical approach temperatures (i.e. the difference between the temperature of the water leaving the cooling tower and the web bulb temperature) ranging between $5-8^{\circ} \mathrm{C}$ [108]. Cooling water inlet and outlet temperatures are set as $40^{\circ} \mathrm{C}$ and $25^{\circ} \mathrm{C}$, respectively, for a 50\% relative air humidity. The water cooling process is very sensitive to these temperatures, and even some studies reported an increment of $0.96 \mathrm{GJ} / \mathrm{t}_{\mathrm{NH} 3}$ in the energy consumption when the cooling water temperature increased from $20^{\circ} \mathrm{C}$ to $35^{\circ} \mathrm{C}$ [22]. Total volumetric water circulation rate is estimated about $7636 \mathrm{~m}^{3} / \mathrm{h}$ whereas the make-up water due to drift, evaporation and blown down is estimated as $2.74 \%$ of the circulating water flow.

Fig. 4.11. Simplified steam network and utility systems of the conventional syngas and ammonia production plant.

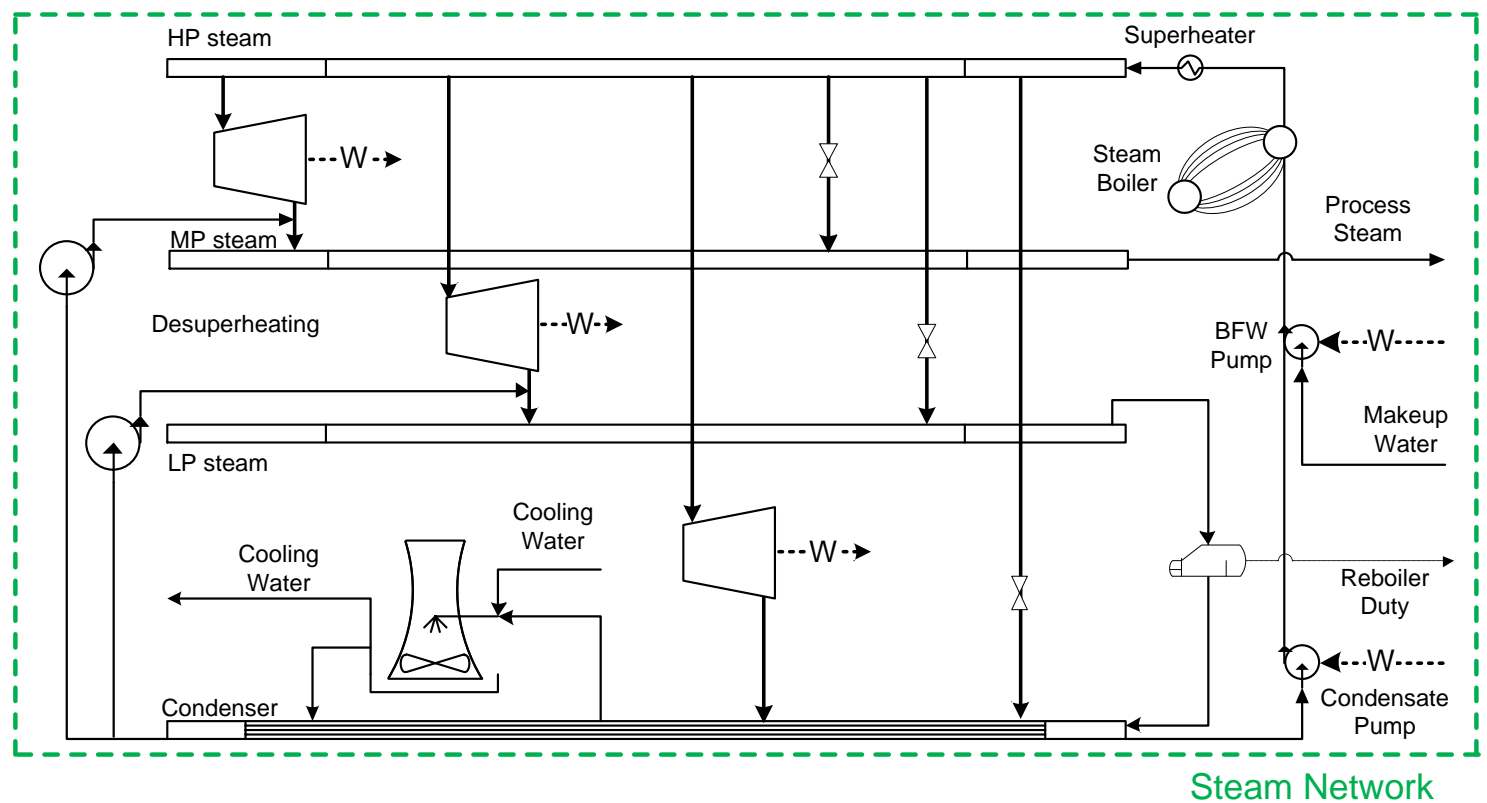

Source: Author.

In old ammonia plants, surplus steam was considered as an undesirable byproduct. Indeed, its excessive generation could be interpreted, to some extent, as an indicator of the degradation of the chemical energy of the feedstock [74, 109]. Nevertheless, even in more advanced plants, the mass of steam produced from waste heat is still as high as three or fourfold the ammonia produced [56]. Correspondingly, a large amount of avoidable irreversibility arises from the overutilization of the waste heat steam boilers $[9,110]$. 
According to this, the goal would rather consist of optimizing the recovery of the enthalpy of reactions along the chemical plant and minimizing the energy export (or import), depending on the local conditions of the facilities. For instance, as concerns the self-sufficiency of the ammonia production plant, some authors [9] reported lower exergy efficiencies for the standalone combined heat and power production (CHP) when compared with the energy integration of the utility systems and the chemical plant. This still holds even if an additional amount of fuel may be needed.

Thus, as it will be discussed in more detail later, some approaches may alleviate the load on the steam production system by shifting the power generation to more efficient cogeneration systems (combined cycle and chemically recuperated gas turbine systems), by partially or totally outsourcing the electricity consumed or by using cheaper and readily available, alternative fuels to supply the heat and power demands. 


\section{CHAPTER 5}

\section{PROCESS SYNTHESIS: MODELING, SIMULATION AND OPTIMIZATION}

The process synthesis consists of the systematic proposition, evaluation, selection, feedback and improvement of the various designed setups or flowsheets that meet the economic, thermodynamic and environmental objectives of any industrial process [99]. Nonetheless, since the thorough fulfillment of all the potential competing targets can be a priori a cumbersome task, partial objectives such as operating and capital costs, production capacity, energy consumption, environmental impact and irreversibility rate are often considered one at time. Therefore, the process synthesis may deal with a variety of tasks, from the design of a new plant up to the revamp of a part of an existing one.

The complete process synthesis of a chemical plant includes the development of a feasible and reliable representation of the physical problem in terms of an adequate mathematical language (modeling), a means to evaluate the alternatives proposed (simulation), and a strategy that allows searching for the best configuration among the vast amount of options that satisfy the predefined tasks and restrictions of the plant (optimization).

Accordingly, apart from selecting the most suitable components (e.g. single unit operations or complete groups of them) out from a resourceful superstructure, the process synthesis must figure out the best way in which those components would operate, how they could be interconnected and should interact in terms of transfer of mass, energy and information. Indeed, even if no breakthrough technologies were to be considered yet, the process synthesis is already combinatorial in nature, and the number of alternatives may substantially increase if new approaches come on scene [111]. Thus, were it not for an appropriate methodology to systematically deal with the process synthesis and optimization, the determination of the best configuration(s) may become an overwhelming, not to say impossible task in a reasonable time frame.

In the following, the description of the mathematical modeling and the simulation processes, as well as the tools used to calculate the thermodynamic properties of the substances involved, are presented. Moreover, the proposed exergy, economy and environmental performance indicators, used to compare the candidate to optimal flowsheets, are discussed. Finally, a brief presentation of the general definition of an optimization problem and the methodology used to optimize the operating conditions and the structure of the various setups proposed for the syngas and ammonia production plant, are outlined.

\subsection{Process modeling and simulation}

The very first step of the synthesis problem is the generation of an abstract representation of the real system, namely a model. A mathematical model is the collection of equations and 
inequalities that define the relationships between the system variables and the acceptable values that those variables can assume. In general, the model will be composed of the material and energy balances, engineering design and constitutive equations, and the correlations that are used to calculate the physical properties of the substances involved [112]. These equations are normally complemented by inequalities that define allowable operating ranges, maximum and minimum bounds for the required performance and for the resources availability. Of course, the level of complexity of the model will depend on the scope of the study and must be consistent with the quality of the information available. Moreover, the assumptions imposed by the model must be clearly formulated and the results have to be interpreted by recognizing its limitations [113]. This explains why the construction of the model is generally the most expensive and time consuming part of the process synthesis, and at some extent, the most important. Actually, the simulation outcome and the possibility to find a meaningful solution of the optimization problem will depend on the ability to develop an appropriate model that closely represents the physical phenomena studied [112].

The simulation process consists of working out one or more operational solutions of the mathematical model. This is achieved by conducting a series of virtual experiments that aim to understand the behavior of the system, evaluate alternative strategies, and tune the model on experimental data [114]. In other words, in the simulation process, the independent variables of the mathematical model are set to specified values so that the system performance indicators can be evaluated. From those results, further modifications to the model or the current operating conditions can be envisaged. The graphical representation of the simulated components and their interconnections is called flowsheet. Correspondingly, flowsheeting is referred as the use of computational and graphical user interface tools that aid setting the selected values of the independent variables in order to perform mass and energy balances, equipment sizing and cost calculations in steady state or dynamic simulations. The main merit of the simulation is thus to be able to elucidate operational configurations well ahead the detailed design and optimization. Next, the base-case can be selected from these sub-optimal configurations and submitted to further improvement [99]. Henceforth, an optimization procedure can be used to find out the effect of the variation of the independent variables on the value of the specified objective, requiring so the simulation to be repeated until certain convergence criterion is achieved.

Three main modeling and simulation approaches used in the process synthesis that deserve a brief description are the response surface, the sequential modular and equation oriented approaches [112]. In the response surface approach, the entire plant or some components are represented by surface fitting formulas with cross-correlation terms, written as a function of the independent variables and other process parameters of the system analyzed. The fitting parameters are adjusted by using directly measured or indirectly collected response data, even derived from virtual experiments. This approach can be advantageous when the detailed calculations take longer or the experimental setups would increase the costs prohibitively. For instance, the simulation of complex energy systems that involve recycling and reactive distillation, such as the syngas purification unit, would have not to be run each time if the system is only a small part of a broader flowsheet optimization [115]. In those cases, the 
output data such as the energy consumption and the $\mathrm{CO}_{2}$ capture rate can be rather correlated to the flow rate, the $\mathrm{CO}_{2}$ feed concentration and other input variables of the purification system. On the other hand, although this approach is useful in identifying the important variables and their interrelation, the developed formulas are only valid within the interpolation limits [116].

In the sequential modular (MS) approach, the mathematical models representing individual operation units (e.g. valve, reactor, compressor) or groups of components (e.g. distillation column with reboiler and condenser) are coded separately at subroutines. These subroutines are called in succession, with the output of one unit serving as the input of the following. For this reason, when there are recycles in the process, the loops must be torn at suitable points and estimated values assigned to those streams for initialization purposes. Recycle loops are sequentially solved until assumed values of the tear streams match the computed information of stream. Consequently, since the recycles must be decomposed into several calculation sequences, this approach may require a large number of iterations for convergence when multiple tear streams are present. However, due to an easier initialization and more intuitive representation, the sequential modular based simulators have been the most widely used type in the industry [117].

Finally, in the equation oriented (EO) approach, all the equations related to the model are assembled in a sparse system of non-linear algebraic equations. Quadrature formulas can be also considered when integral equations are involved. Indeed, the architecture of the EO approach resembles that of common engineering equation solvers (e.g. EES, Excel, MATLAB, among others). Moreover, since the governing equations of each process unit are solved simultaneously, the need for nested iteration loops as in the case of the MS approach is not anymore a concern. Despite the enhanced treatment of the recycle calculations, EO approach still requires a more demanding programing effort, a good initial estimate (so that all the variables can achieve convergence), and a robust general-purpose, non-linear equation solver.

Accordingly, the systematic methodology used in this thesis relies mostly in SM and EO approaches for modeling and simulating the interconnection of the technological components and streams involved in the syngas and ammonia plant, and includes:

- Mass, energy, exergy and cost balances of each process under interest. These balances are established by the use of various process simulation software widely used in the chemical industry, such as Aspen Hysys ${ }^{\circledR}[118]$ and Aspen Plus ${ }^{\circledR}$ [119]. These are powerful software used for modeling systems involving complex electrolyte chemistry, such as carbon dioxide capture units, and for predicting the energy demand of the chemical processes. Other software that serves this purpose is EO Engineering Equation Solver (EES) [120], which includes libraries of thermodynamic properties of several substances as well as an optimization toolbox. Zero Brain Studio $\odot$ IDE and Lua $\odot$ programming language are also used in the definition of the mathematical model of the utility systems in the OSMOSE Lua platform [121]. 
- Semi-empirical correlations used to determine the thermodynamic properties of each flow present in multi-component and multi-phase systems, as well as the vapor liquid equilibrium (VLE) of the mixtures. They include the equations of state (EoS) of Peng-Robinson-BostonMathias (PR-BM), Soave-Redlich-Kwong (SRK) and Perturbed Chain Statistical Associating Fluid Theory (PC-SAFT) [122]. The latter is particularly useful to determine the chemical properties of the substances involved in the physical absorption-based syngas purification. Activity coefficient models such as the Electrolytic Non-Random Two-Liquid (ENRTL-RK) and proprietary Acid Gas ${ }^{\circledR}$ fluid packages [122] are used for taking into account the strongly non-ideal liquid properties, Henry components and the dissociation chemistry present in the reactive absorption-desorption systems. Refprop ${ }^{\circledR}[123]$ and Coolprop® [124] software with the IAPWS Formulation 1995 are also used to calculate the properties of water in the steam network. These correlations are evaluated to calculate other properties such as the physical and chemical exergy of the substances.

- Visual Basic for Applications (VBA) ® scripts defined as user defined functions [125] for automated physical and chemical exergy calculations. Chemical and total exergy calculations are not straightforward in Aspen Hysys or Plus [126-128], and they are used to propose suitable performance indicators for each configuration of the plants analyzed, which allows accomplishing systematic comparisons between different designed setups.

- Aspen Plus ${ }^{\circledR}$-embedded Fortran subroutines used to force certain design specifications to be accomplished.

- Bare module costs of equipment for the ammonia loop are calculated by using the methodology proposed by Turton et al. [129] and correlations from Hamelinck et al. [130].

By way of illustration, the mathematical modeling of the equilibrium-limited ammonia reactor is described next. The special interest put on this system follows the complex interactions brought into play when the reaction section is embedded in a reaction - separation - purge recycle flowsheet. As it will be demonstrated in next chapters, the reaction systems is the key component that determines the performance of the entire loop.

In some cases, an approach-to-equilibrium (ATE) estimate is accurate enough for preliminary calculations of a chemical reactor [77]. However, since the equilibrium is rarely attained in the ammonia synthesis (e.g. the reactor volume is too small or the space velocity is too large), the composition of the reactor effluent is rather kinetics-driven, rendering the modeling and simulation particularly more complicated [25]. Certainly, the knowledge of kinetics is important for solving the industrial problem of reactor sizing, for determining the internal profile of optimal operation conditions as well as in applications involving control of dynamic systems [131].

In the following, the relations between the equilibrium constant, the reaction rates, the fugacities, the activities and activity coefficients, as well as the definition of the equilibrium conversion and maximum conversion lines for an ammonia catalytic packed bed reactor are described. The general expression for the molar balance of a catalytic chemical reactor can be written in terms of the catalyst volume $V$ and the reaction rate $r$, according to the Eq.(5.1) [131]: 


$$
n_{i, \text { inlet }}-n_{i, \text { outlet }}+\int_{V} r_{i} d V=\left.\frac{d n_{i}}{d t}\right|_{C . V .}
$$

Moreover, the fractional conversion of nitrogen into ammonia (R.5.1) is defined as Eq.(5.2):

$$
\begin{aligned}
\xi= & \frac{n_{N_{2}, \text { inlet }}-n_{N_{2}, \text { outlet }}}{n_{N_{2}, \text { inlet }}} \\
& \mathrm{N}_{2}+3 \mathrm{H}_{2} \rightarrow 2 \mathrm{NH}_{3}
\end{aligned}
$$

The reaction extent, $d n_{i} / v_{i}=\varsigma$, relates the stoichiometric coefficients $v_{i}(>0$ for products and $<0$ for reactants) and the variation of the moles of the component $i, d n_{i}$. Meanwhile, the mol fractions of the individual components $i$ at the reactor outlet are calculated from Eqs.(5.35.7):

$$
\begin{aligned}
& x_{N_{2}}=\frac{n_{N_{2}, \text { inlet }}(1-\xi)}{n_{\text {Total }, \text { inlet }}-2 n_{N_{2}, \text { inlet }} \xi} \\
& x_{H_{2}}=\frac{n_{H_{2}, \text { inlet }}-3 n_{N_{2}, \text { inlet }} \xi}{n_{\text {Total, } \text {,inlet }}-2 n_{N_{2}, \text { inlet }} \xi} \\
& x_{N H_{3}}=\frac{n_{N H_{3}, \text { inlet }}+2 n_{N_{2}, \text { inlet }} \xi}{n_{\text {Total, } \text {,inlet }}-2 n_{N_{2}, \text { inlet }} \xi} \\
& x_{A r}=\frac{n_{A r, \text { inlet }}}{n_{\text {Total, inlet }}-2 n_{N_{2}, \text { inlet }} \xi} \\
& x_{C H_{4}}=\frac{n_{C H_{4}, \text { inlet }}}{n_{\text {Total, inlet }}-2 n_{N_{2}, \text { inlet }} \xi}
\end{aligned}
$$

where $n_{\text {Total,inlet }}=\sum_{i} n_{i, \text { inlet }}$ is the total number of moles entering the ammonia reactor. It is also worthy to notice the simpler layout of Eqs.(5.6-5.7) as methane and argon are considered inerts while going through the catalytic bed.

For a packed bed reactor operating in steady state, the molar balance for each species given by Eq.(5.1) can be simplified to Eq.(5.8), [131]:

$$
\frac{d n_{i}}{d V}=r_{i} \rightarrow \frac{d\left(n_{i, \text { inlet }}-n_{i, \text { outlet }}\right)}{d V}=r_{i}
$$

where the reaction rates for the different components (reactants and products) are related by the expression $r_{i} / v_{i}=$ constant. Thus, by determining the reaction rate of the nitrogen consumption, the reaction rate of the remaining species can be easily calculated as $r_{\mathrm{N}_{2}} / 1=r_{\mathrm{H}_{2}} / 3=-r_{\mathrm{NH}_{3}} / 2$. For instance, the nitrogen mole balance in terms of the reactor conversion can be written as:

$$
\frac{d\left(n_{N_{2}, \text { inlet }}-n_{N_{2}, \text { inlet }} \xi\right)}{d V}=r_{N_{2}} \rightarrow n_{N_{2}, \text { inlet }} \frac{d \xi}{d V}=-r_{N_{2}}
$$


From experimental data, Temkin and Phyzev [132] initially published a general expression for the reaction rate of ammonia synthesis over a magnetite catalyst. The expression is consistent with the equilibrium constant and given by the Eq. (5.10):

$$
-r_{N_{2}}=\frac{r_{N H 3}}{2}=k_{f} P_{N_{2}}\left(\frac{P_{H_{2}}^{3}}{P_{N H_{3}}^{2}}\right)^{\alpha}-k_{b}\left(\frac{P_{N H_{3}}^{2}}{P_{H_{2}}^{3}}\right)^{1-\alpha}
$$

Temkin kinetics is based on the hypothesis that the dissociative adsorption of nitrogen is a fundamental step in the ammonia synthesis, assuming that all surface is dominated by $\mathrm{N}^{*}$ species [132]. Equation 5.10 is claimed to predict the mole fraction of the ammonia produced with a maximum deviation of 10 to $20 \%$ in the pressure range of commercial interest (150$300 \mathrm{~atm})$. It is worthy to notice that Equation 5.10 fails to predict the ammonia production rate when there is not any ammonia fraction in the feed, since the first right hand side term diverges. Despite this limitation, in commercial applications the ammonia content of the recycle feed gas is always greater than zero. The forward and backward reaction rate 'constants', $k$, are calculated from the Arrhenius relation:

$$
\frac{d \ln k}{d(1 / T)}=-\frac{E_{a}}{R} \rightarrow k=k_{0} e^{-\frac{E_{a}}{R T}}
$$

where $R$ denotes the universal gas constant $(8.314 \mathrm{~kJ} / \mathrm{kmol} \mathrm{K}), T$ is the reaction temperature $(\mathrm{K}), E_{\mathrm{a}}$ is the activation energy $(\mathrm{kJ} / \mathrm{kmol})$ and $k_{0}$ is the pre-exponential factor. It is important to mention that pioneering experimental research works focused in the reaction of the ammonia decomposition (R.5.2), instead of its synthesis (R.5.1). Thus, the forward preexponential factor, $k_{f}$, is often indirectly calculated by using the reverse reaction data and the true equilibrium constant $K_{\mathrm{eq}}$ of the reaction, according to Eq.(5.12): [133]:

$$
\begin{gathered}
\frac{1}{2} N_{2}+\frac{3}{2} H_{2} \leftarrow N H_{3} \\
\frac{k_{f}}{k_{b}}=K_{e q}^{2}
\end{gathered}
$$

In order to calculate the true equilibrium constant $K_{e q}$, the activity of the each component $i$ must be calculated by using Eq.(5.13):

$$
a_{i}=\frac{f_{i}}{\hat{f}_{i}}
$$

where $\hat{f}_{i}$ is the fugacity of the component $i$ at an arbitrary chosen standard state, e.g. the fugacity of the pure component $i$ at a pressure of $1 \mathrm{~atm}$ and at a temperature equal to the temperature of the system. On the other hand, $f_{i}$ is the fugacity of the component $i$ at the partial pressure of the component in the system. The following approximation given by the Lewis Randall rule is adopted to calculate the value of $f_{i}[134]$ :

$$
f_{i}=x_{i} \cdot f_{i}^{\circ} \rightarrow a_{i}=x_{i} \cdot f_{i}^{\circ}
$$

where $f_{i}^{\circ}$ is the pure component fugacity at the temperature and pressure of the system. In other words, the fugacity of the component in the mixture is proportional to the mol fraction 
of the component at the same temperature of the system. It is worthy to notice that it was assumed $\hat{f}_{i}=1 \mathrm{~atm}$. Finally, the value of $f_{i}^{\circ}$ is estimated by using Eq.(5.15):

$$
f_{i}^{\circ}=\gamma_{i} \cdot P
$$

where $\gamma_{i}$ and $P$ are the activity coefficient of the component $i$ and the total pressure of the system respectively. The activity coefficients are calculated by using Eqs.(5.16-5.18) [132]:

$$
\begin{gathered}
\gamma_{H_{2}}=\exp \left(e^{-3.8402 T^{0.125}+0.541} \cdot P-e^{-0.1263 T^{0.5}-15.980} \cdot P^{2}+300 \cdot e^{-0.011901 T-5.941}\left(e^{-P / 300}-1\right)\right) \\
\gamma_{N_{2}}=0.93431737+0.3101804 E-3 \cdot T+0.295896 E-3 \cdot P \\
-0.2707279 E-5 \cdot T^{2}+0.4775207 E-6 \cdot P^{2} \\
\gamma_{N H_{3}}=0.1438996+0.2028538 E-2 \cdot T-0.4487672 E-3 \cdot P \\
-0.1142945 E-5 \cdot T^{2}+0.2761216 E-6 \cdot P^{2}
\end{gathered}
$$

where the pressure is given in atm and the temperature in $\mathrm{K}$. To grasp the practical meaning of fugacities, it can thought as an effective pressure of the real substances when interacting with other substances of a mixture. Thus, for ideal gases, fugacities are equal to the respective partial pressures. However, in the case of non-ideal gases, fugacity equals the fugacity coefficient times the partial pressure of the substance [96]. Accordingly, a gas with a fugacity coefficient less than 1 has an effective pressure lower than its partial pressure, and vice versa [131].

Now, by considering Eqs.(5.12-5.18), the true equilibrium constant $K_{e q}$ can be calculated by using Eq.(5.19):

$$
K_{e q}=\frac{a_{N H_{3}}}{a_{H_{2}}^{3 / 2} a_{N_{2}}^{1 / 2}}=\frac{\left(\gamma_{N H_{3}} \cdot x_{N_{3}} \cdot P\right)}{\left(\gamma_{H_{2}} \cdot x_{H_{2}} \cdot P\right)^{3 / 2}\left(\gamma_{N_{2}} \cdot x_{N_{2}} \cdot P\right)^{1 / 2}}=K_{P} \cdot K_{\gamma}
$$

It is noteworthy that the equilibrium constant $K_{\mathrm{P}}$ is the ideal gas limit for $K_{e q}$ (i.e. at high temperature and low pressure) [133]. Actually, at increased pressures (> 10 bar) and close to the saturated vapor states, activities coefficients should be used instead of partial pressures, since they account for the real behavior of individual gas components [25].

Meanwhile, $K_{\mathrm{P}}$ can be determined as a function only of the temperature for a given chemical reaction, regardless of the reactant or product concentrations, total pressure or the presence of catalyst. The value of $K_{\mathrm{P}}$ is calculated by using the Gillespie and Beattie correlation and the constants reported in Table 5.1 [133]:

$$
\log _{10} K_{P}=A \cdot \log _{10} T+B \cdot T+C \cdot T^{2}+\frac{D}{T}+E \quad \text { with } T \text { in } \mathrm{K}
$$

Table 5.1. Coefficient of the equilibrium constant $K_{\mathrm{P}}$ [133].

\begin{tabular}{cccccc}
\hline Coefficient & A & B & C & D & E \\
\hline Value & -2.691122 & $-5.519265 \mathrm{E}-5$ & $1.848863 \mathrm{E}-7$ & 2001.6 & 2.6899
\end{tabular}


Since original Temkin-Phyzev equation, Eq.(5.10) has been proposed six decades ago, it has gone through several modifications to adapt the performance of different catalysts and increase the accuracy of the results. Some alternative forms based on the original TemkinPhyzev equation revisited include:

(i) Elementary reactions rates based on component partial pressures (in $\mathrm{kmol} / \mathrm{m}^{3}$ cat $\cdot \mathrm{s}, T$ in $\mathrm{K}$ and $P$ in atm) [135]:

$$
-r_{N_{2}}=k_{f} P_{H_{2}}^{1.5} P_{N H_{3}}^{0.5}-k_{b} P_{N H_{3}}^{1} \quad \text { for } \quad \frac{1}{2} N_{2}+\frac{3}{2} H_{2} \rightarrow N H_{3}
$$

(ii) Reactions rate expressions based on the original TP equation form, with $\alpha=0.5$ but including a correction factor $f=4.75$ to account for the lower activity of the old catalyst [136]:

$$
-r_{N_{2}}=f \cdot\left[k_{f} P_{N_{2}}\left(\frac{P_{H_{2}}^{1.5}}{P_{N H_{3}}}\right)-k_{b}\left(\frac{P_{N H_{3}}}{P_{H_{2}}^{1.5}}\right)\right]
$$

(iii) Reactions rate expressions in terms of fugacities (or activities) and the corresponding equilibrium constant $K_{P}$ [132]:

$$
-r_{N_{2}}=k_{b}\left[K_{P}^{2} f_{N_{2}}\left(\frac{f_{H_{2}}^{3}}{f_{N H_{3}}^{2}}\right)^{\alpha}-\left(\frac{f_{N H_{3}}^{2}}{f_{H_{2}}^{3}}\right)^{1-\alpha}\right]
$$

Additionally, some proprietary catalyst data reported in literature can be used to calculate both the forward $(f)$ and the backward $(b)$ reactions rates constants of the ammonia synthesis. These data is compared in Table 5.2.

Table 5.2. Pre-exponential factor and activation energy for forward $(f)$ and backward $(b)$ reaction rate constants.

\begin{tabular}{ccccccc}
\hline Equation & $\mathbf{k}_{\mathbf{0 f}}$ & $\begin{array}{c}\mathbf{E a}_{\mathbf{f}} \\
\mathrm{kJ} / \mathrm{kmol}\end{array}$ & $\mathbf{k}_{\mathbf{0 b}}$ & $\begin{array}{c}\mathbf{E a}_{\mathbf{b}} \\
\mathrm{kJ} / \mathrm{kmol}\end{array}$ & $\boldsymbol{\alpha}$ & Observations [Reference] \\
\hline$(5.24)$ & -- & -- & $2.57 \mathrm{E}+14$ & 163,500 & 0.55 & $\begin{array}{c}\text { Montecatini catalyst, } \\
\text { fugacities in atm. [137] }\end{array}$ \\
\hline$(5.25)$ & $1.79 \mathrm{E}+4$ & 87,090 & $2.57 \mathrm{E}+16$ & 198,464 & 0.5 & $\begin{array}{c}\text { Partial pressures in bar } \\
\text { Araujo et. al [136] }\end{array}$ \\
\hline$(5.26)$ & -- & -- & $8.03 \mathrm{E}+14$ & 179,592 & 0.692 & $\begin{array}{c}\text { Haldor Topsoe catalyst, } \\
\text { fugacities in atm. [137] }\end{array}$ \\
\hline$(5.27)$ & -- & -- & $8.85 \mathrm{E}+14$ & 170,683 & 0.5 & $\begin{array}{c}\mathrm{k}_{f}=K_{\mathrm{P}} \mathrm{k}_{b} \\
\text { fugacities in atm } \\
\text { Dyson et al. [132] }\end{array}$ \\
\hline$(5.28)$ & $1.0 \mathrm{E}+4$ & $9.1 \mathrm{E}+4$ & $1.3 \mathrm{E}+10^{*}$ & $1.4 \mathrm{E}+5$ & -- & Partial press. in atm. [135] \\
\hline
\end{tabular}

$*$ The reaction rate is given en unit of $\mathrm{kmol} /\left(\mathrm{m}_{\text {gas }}^{3} \mathrm{~s}\right)$.

The units of the reaction rate constants $\mathrm{k}_{f}$ and $\mathrm{k}_{b}$ will depend on the basis used (partial pressures, fugacities or activities). Thus, except for expression (i) and Eq.(5.28), in all the above expressions, the reaction rate has units of $\mathrm{kmol} /\left(\mathrm{m}^{3}\right.$ cat $\left.\mathrm{h}\right)$. 
For the sake of comparison, Fig. 5.1 shows different profiles of the reaction rates obtained from the catalyst data given in Table 5.2, for an arbitrary reactor length of $10 \mathrm{~m}$, diameter 2 $\mathrm{m}$, and feed conditions of $350^{\circ} \mathrm{C}$ and 200 bar. As it can be seen, despite the observable variation between the curves, there certainly exists a monotonous decreasing trend of the reaction rate along the catalyst length, except for one catalyst curve. As expected, the utilization of catalyst data openly reported in the literature carries with an intrinsic uncertainty. However, since liable data is not provided by commercial manufacturers, in order to perform engineering calculations, the Montecatini catalyst data as reported by [137] has been selected.

Fig. 5.1. Comparison of the reaction rates for the catalyst data given in Table 5.2. (200 bar, reactor length $10 \mathrm{~m}$, reactor feed at $350^{\circ} \mathrm{C}$, reactor diameter $2 \mathrm{~m}$ )

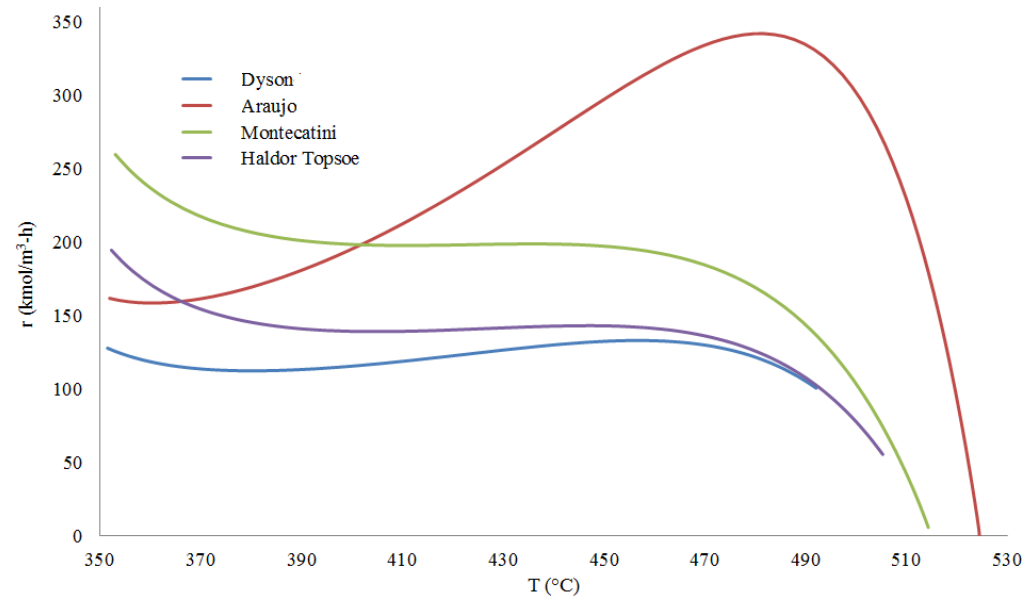

Source: Author.

Alternatively, the reaction rate can be written in units of $\mathrm{kmol} . \mathrm{m}^{-3}$ void. $\mathrm{h}^{-1}$, i.e. per unit volume of reacting gas (void) per hour, instead of per unit volume of catalyst. This is done by means of the definition of the void fraction, i.e. the portion of the reactor that is not filled with catalyst. This is the actual way in which the reaction rate data in Aspen HYSYS® simulator is input:

$$
r_{\text {Void }}=r_{\text {Catalyst }} \cdot \frac{(1-\phi)}{\phi}
$$

where $\phi$ is called the void fraction, defined by Eq.(5.30):

$$
\phi=\frac{V_{\text {Void }}}{V_{\text {Bed }}} \quad \text { and } \quad(1-\phi)=\frac{V_{\text {Catalyst }}}{V_{\text {Bed }}}
$$

Typical void fraction used in the commercial ammonia reactors ranges from 0.33 to 0.5 [138]. Notably, larger $\phi$ values may require larger reactor volumes, and lower values lead to larger pressure drop. Therefore, the value of $\phi$ is also useful to roughly estimate the pressure drop along the reactor bed by using the Ergun correlation [131], Eq.(5.31):

$$
\frac{d P}{d z}=-\frac{G}{\rho D_{p}} \cdot \frac{(1-\phi)}{\phi^{3}} \cdot\left[\frac{150(1-\phi) \mu}{D_{p}}+1.75 G\right]
$$


where $\rho=\rho_{0} \cdot \frac{T_{0}}{T} \cdot \frac{P}{P_{0}} \cdot \frac{\dot{n}_{\text {Total }, 0}}{\dot{n}_{\text {Total }}} \frac{Z_{0}}{Z}$ is the variable ideal gas density calculated from the initial and final conditions in the non-isothermal reactor, $G=\dot{m} / A$ is the superficial mass velocity of the reacting gases, and $D_{\mathrm{P}}$ is the catalyst diameter. It is worthy to notice that the size of the catalyst particle not only affect the pressure drop along the reactor. Since the rate of reaction is limited by diffusion, some measurements performed with a variety of particle sizes show a marked influence at high temperatures. The effect of the catalyst size has been summarized in Table 5.3.

Table 5.3. Effect of the catalyst size [138].

\begin{tabular}{cc}
\hline Particle Size & Reaction rate $\left(\mathbf{k m o l}_{\mathbf{N} 2} / \mathbf{s}-\mathbf{m}_{\text {cat }}^{\mathbf{3}}\right)$ \\
\hline Crushed $(0.6-1.21 \mathrm{~mm})$ & 0.0833 \\
\hline $3 \mathrm{~mm}-4.8 \mathrm{~mm}$ & 0.0311 \\
\hline $6.4 \mathrm{~mm}-9.5 \mathrm{~mm}$ & 0.0169 \\
\hline
\end{tabular}

Moreover, it is assumed that the effectiveness factor of the small catalyst particles used (3 mm, bulk density $2300 \mathrm{~kg} / \mathrm{m}^{3}$, sphericity 1) is close to the unity, thus the reactants concentration inside the particles is closer to the bulk concentration.

Hitherto, the influence of the operation parameters such as pressure, temperature and composition on the kinetics of the chemical mixture in the ammonia reactor has been analyzed. In the following, the equilibrium thermodynamics is described in more detail so that the impact of selected operating conditions on the reactor performance can be better elucidated. Thus, the effect of the inlet temperature and the number of catalytic beds on the maximum per-pass conversion attainable, as well as on the energy balance in the reactor, is discussed.

The so-called equilibrium curve can be plotted in a conversion vs temperature plane by setting the rate of reaction to zero $\left(\mathrm{r}_{\mathrm{N} 2}=0\right)$ with $\alpha=0.5$, according to Eq.(5.32):

$$
k_{2}\left[K_{e q}^{2} a_{N_{2}}\left(\frac{a_{H_{2}}^{3}}{a_{N H_{3}}^{2}}\right)^{0.5}-\left(\frac{a_{N H_{3}}^{2}}{a_{H_{2}}^{3}}\right)^{0.5}\right]=0 \rightarrow K_{e q}=\frac{a_{N H_{3}}}{a_{H_{2}}^{3 / 2} a_{N_{2}}^{1 / 2}}=\frac{\left(\gamma_{N H_{3}} \cdot x_{N H_{3}} \cdot P\right)}{\left(\gamma_{H_{2}} \cdot x_{H_{2}} \cdot P\right)^{3 / 2}\left(\gamma_{N_{2}} \cdot x_{N_{2}} \cdot P\right)^{1 / 2}}
$$

where $a_{i}=\gamma_{i} \cdot x_{i} \cdot P$ is the activity coefficient as described above. Moreover, by considering that the molar flow of the reactor bed outlet stream can be written in terms of conversion:

$$
\begin{gathered}
n_{N_{2}, \text { outlet }}=n_{N_{2}, \text { inlet }}-n_{N_{2}, \text { inlet }} \xi \\
n_{H_{2}, \text { outlet }}=n_{H_{2}, \text { inlet }}-3 n_{N_{2}, \text { inlet }} \xi \\
n_{\mathrm{NH}_{3}, \text { outlet }}=n_{\mathrm{NH}_{3}, \text { inlet }}+2 n_{N_{2}, \text { inlet }} \xi \\
n_{A r, \text { outlet }}=n_{A r, \text { inlet }} \\
n_{\mathrm{CH}_{4}, \text { outlet }}=n_{\mathrm{CH}_{4}, \text { inlet }}
\end{gathered}
$$

and by adding Eqs.(5.33-5.37), the Eq.(5.38) is obtained:

$$
n_{\text {Total, outlet }}=n_{\text {Total, inlet }}-2 n_{N_{2}, \text { inlet }} \xi
$$


Accordingly, the mol fraction of the component $i$ can be expressed as:

$$
x_{i, \text { outlet }}=\frac{n_{i, \text { outlet }}}{n_{\text {Total, outlet }}}
$$

On the other hand, $\Theta_{i}$ is defined as the ratio between the inlet molar flow of each species $i$ and the inlet molar flow of nitrogen, according to Eq.(5.40):

$$
\Theta_{i}=\frac{n_{i, \text { inlet }}}{n_{N_{2}, \text { inlet }}}
$$

Thus, the true equilibrium constant can be written in terms of the conversion and other process parameters, such as the reactor pressure, the inlet composition and the fugacities of the substances, as in Eq.(5.41):

$$
K_{e q}=\frac{\Theta_{N_{3}}+2 \xi}{(1-\xi)^{1 / 2}\left(\Theta_{H_{2}}-3 \xi\right)^{3 / 2}} \cdot \frac{\left(n_{\text {Total } \text {,inlet }}-2 n_{N_{2}, \text { inlet }} \xi\right)}{n_{N_{2}, \text { inlet }}} \cdot \frac{\gamma_{N H_{3}}}{\gamma_{H_{2}}^{3 / 2} \cdot \gamma_{N_{2}}^{1 / 2}} \cdot \frac{1}{P}
$$

Consequently, if the true equilibrium constant were expressed in terms of the temperature, the equilibrium conversion for ammonia production could be also written as a function of temperature. This is done by means of the van't Hoff equation, which relates the variation of the equilibrium constant with the temperature. In effect, since for a reversible chemical reaction [139]:

$$
K_{e q}=k_{1} / k_{2} \rightarrow d \ln K_{e q}=d \ln k_{1}-d \ln k_{2}
$$

And according to the Arrhenius equation:

$$
d \ln k / d(1 / T)=-E_{a} / R \rightarrow\left\{\begin{array}{l}
d \ln k_{f} / d(1 / T)=-E_{a, f} / R \\
d \ln k_{b} / d(1 / T)=-E_{a, b} / R
\end{array}\right.
$$

By subtracting both equations and letting be $E_{a, f}-E_{a, b}=\Delta H_{R}$, as well as by neglecting the difference between specific heat capacity of the products and reactants, i.e. $\Delta C_{p} \approx 0[131]$, Eq.(5.43) can be written as:

$$
\frac{d \ln K_{e q}}{d(1 / T)}=-\frac{\Delta H_{R}(T)}{R} \rightarrow \ln \left[\frac{K_{e q}\left(T_{1}\right)}{K_{e q}\left(T_{2}\right)}\right]=\frac{\Delta H_{R}}{R} \cdot\left[\frac{1}{T_{1}}-\frac{1}{T_{2}}\right]
$$

It is important to point out that, for the reaction of hydrogen and nitrogen at $400^{\circ} \mathrm{C}$, the enthalpy of reaction increases by only $6 \%$ if the pressure is raised from 1atm to $200 \mathrm{~atm}$ [131] and, as such, the effect of the pressure variation can be neglected. Therefore, if it is adopted that $T_{2}>T_{1}$, the following conclusions can be drawn from Eq.(5.44):

(i) For endothermic reaction $\left(\Delta H_{R}>0\right)$, as the temperature increases, the equilibrium constant also increases, whereas

(ii) For exothermic reaction $\left(\Delta H_{R}<0\right)$, as the temperature rises, the equilibrium constant decreases. Those results are summarizes in Table 5.4. 
Table 5.4. Variation of the equilibrium constant with temperature [139].

\begin{tabular}{cccc}
\hline Reaction & Enthalpy of reaction & If temperature & Equilibrium \\
\hline Endothermic & $\Delta H_{R}>0$ & $T \uparrow$ & $K_{e q}=k_{1} / k_{2} \uparrow$ \\
\hline Exothermic & $\Delta H_{R}<0$ & $T \downarrow$ & $K_{e q}=k_{1} / k_{2} \downarrow$ \\
\hline
\end{tabular}

Bearing in mind that the generalized Gibbs free energy change, $\Delta G$, for a system with reaction coefficient, $\Omega$, is given by:

$$
\Delta G=\Delta G^{0}+R T \ln \Omega
$$

And since as the systems strives to reach an equilibrium state, $\Omega \rightarrow K_{e q}$ and thus $\Delta G=0$, the true equilibrium constant is calculated as a function of $\mathrm{T}$ according to Eq.(5.46):

$$
\ln K_{e q}=\frac{\Delta G^{0}}{-R T}=f(T, P)
$$

Finally, by solving the Eqs.(5.41) and (5.46) for the conversion $\xi$ (e.g. use Excel® Solver or EES( ) at a given reaction temperature and pressure, the plot of equilibrium conversion vs temperature for either an endothermic or exothermic reaction, can be represented by Fig. 5.2(a-b).

Fig. 5.2. Variation of equilibrium conversion with temperature for (a) an exothermic reaction and $(b)$ an endothermic reaction

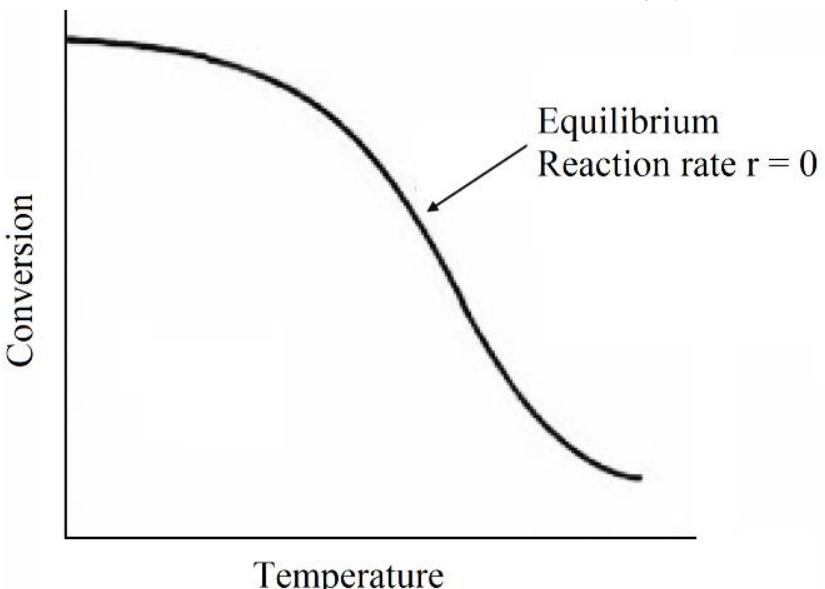

(a)

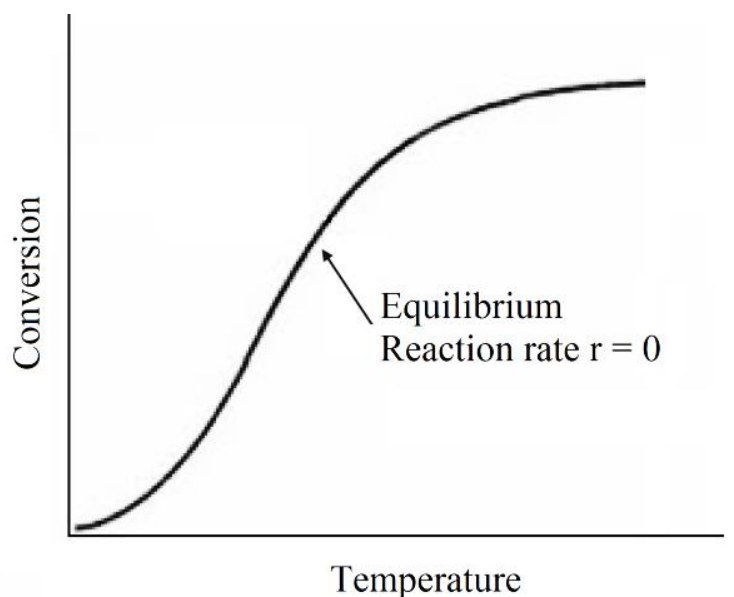

(b)

Source: [139].

These plots prove to be extremely useful for determining the optimal reaction temperature profile that increases the reaction conversion, while guaranteeing near maximum reaction rates.

For instance, Fig. 5.3 shows the distribution of the lines of constant reaction rate and the locus of the maximum conversion for each reaction rate corresponding to the Montecatini catalyst operating at $200 \mathrm{~atm}$. This figure can be obtained by imposing a value of constant reaction rate different from zero in Eq.(5.32) and solving for the conversion $\xi$ (e.g. use Excel ${ }^{\circledR}$ Solver or $\mathrm{EES}\left({ }^{\circ}\right)$ at a given reaction temperature and pressure, analogously to the determination of the equilibrium line in Fig. 5.2. 
Fig. 5.3. Lines of constant reaction rate in a conversion vs. temperature plot. Montecatini catalyst at $200 \mathrm{~atm}$.

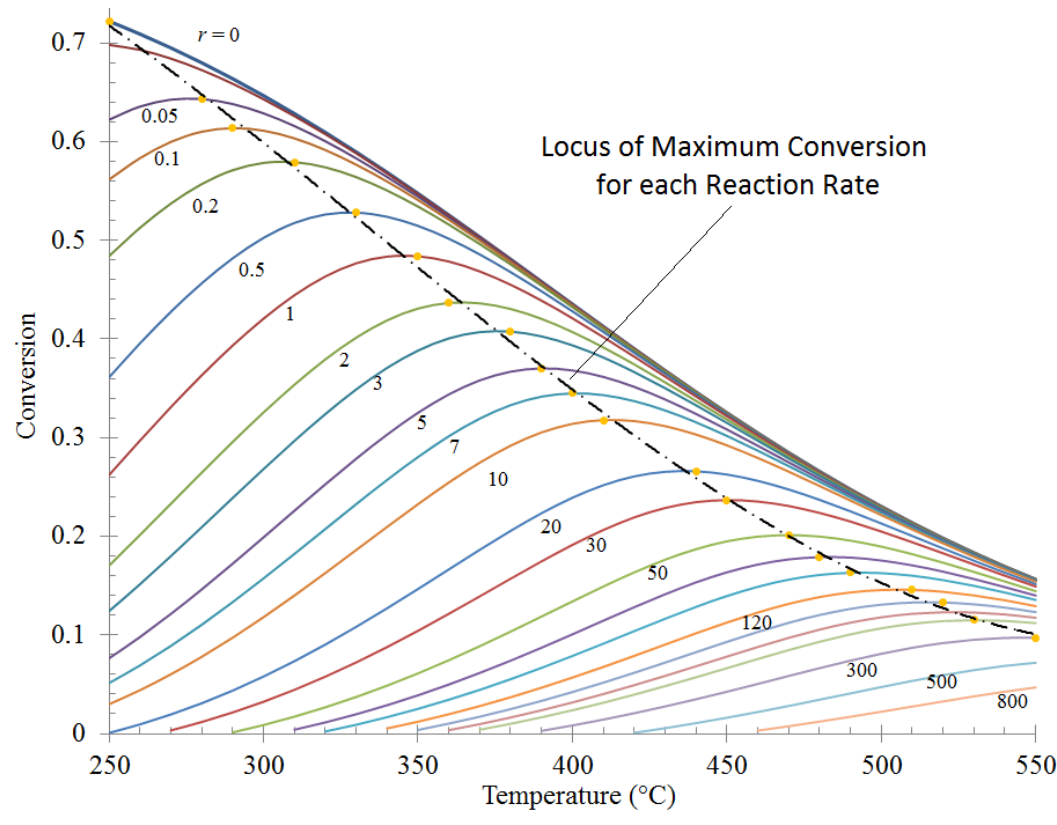

Source: Author.

In the following, the mathematical formulation of the concept of the adiabatic operation line and its relationship with the reactor conversion are described. For a non-isothermal reactor in steady state, the general energy balance can be written as [131]:

$$
Q-n_{N_{2}, \text { inlet }} \sum \Theta_{i} C_{p, i}\left(T-T_{1}\right)-n_{N_{2}, \text { inlet }}\left[\Delta H_{R}^{\circ}\left(T_{R}\right)+\Delta C_{p}\left(T-T_{R}\right)\right] \cdot \underset{\substack{\text { Energy } \\ \text { Balance }}}{\xi}=0
$$

where $Q$ is the heat transfer from/to the reactor; $C_{p, i}$ is the specific heat capacity of the components; $T-T_{1}$ is the temperature difference between reactants and products; $\Delta H_{R x}^{\circ}\left(T_{R}\right)$ $+\Delta C_{p}\left(T-T_{R}\right)$ is the enthalpy of reaction at the reactor temperature $T ; T_{R}$ is the reference temperature to calculate the enthalpy of reaction at the reference state (e.g. $298 \mathrm{~K}$ and $1 \mathrm{~atm}$ ) and $\xi_{\text {Energy }}$ is the nitrogen conversion calculated independently from the energy balance. Since each one of the reactor beds can be considered as adiabatic, the conversion achieved on each of them is given by Eq.(5.48) [131]:

$$
\xi_{\text {Energy }}^{\text {Balance }}=\frac{\sum \Theta_{i} C_{p, i}\left(T-T_{1}\right)}{-\left[\Delta H_{R}^{\circ}\left(T_{R}\right)+\Delta C_{p}\left(T-T_{R}\right)\right]}
$$

Since, the second term in the denominator is often negligible compared with $\Delta H_{R}^{\circ}\left(T_{R}\right)$, the operation curve of the adiabatic bed can be approximated by a linear function with a slope $\beta \approx \sum \Theta_{i} C_{p, i} /-\left[\Delta H_{R}^{\circ}\left(T_{R}\right)\right]$ in a $\xi v s . T$ plot. Furthermore, it is noteworthy that, although the conversion $\xi$ in Eq.(5.48) is calculated through the energy balance, in lieu of the molar balance, Eq.(5.9), both values must match for a given operating condition. For instance, in the ideal case in which the adiabatic reactor bed outlet attains the equilibrium, the solution of the 
system of Eqs.(5.9) and (5.48) corresponds to the intersection point of the equilibrium and the adiabatic operation line, as shown in Fig. 5.4. As a result, the highest per-pass conversion that can be achieved in a single reactor bed for an inlet temperature $T_{1}$ is the equilibrium conversion at $\mathrm{T}$.

Referring again to Fig. 5.4, it is observed that, even for an ideal infinite, single-bed reactor, the nature of the equilibrium curve prevents the total reactants conversion. Accordingly, in order to shift the mixture away from the state of thermodynamic equilibrium and thus increase the fractional conversion, a directly or indirectly intercooled, multiple bed ammonia reactor may be necessary [25].

Fig. 5.4. Adiabatic operation lines and maximum adiabatic temperature attainable at equilibrium for an exothermic reactor.

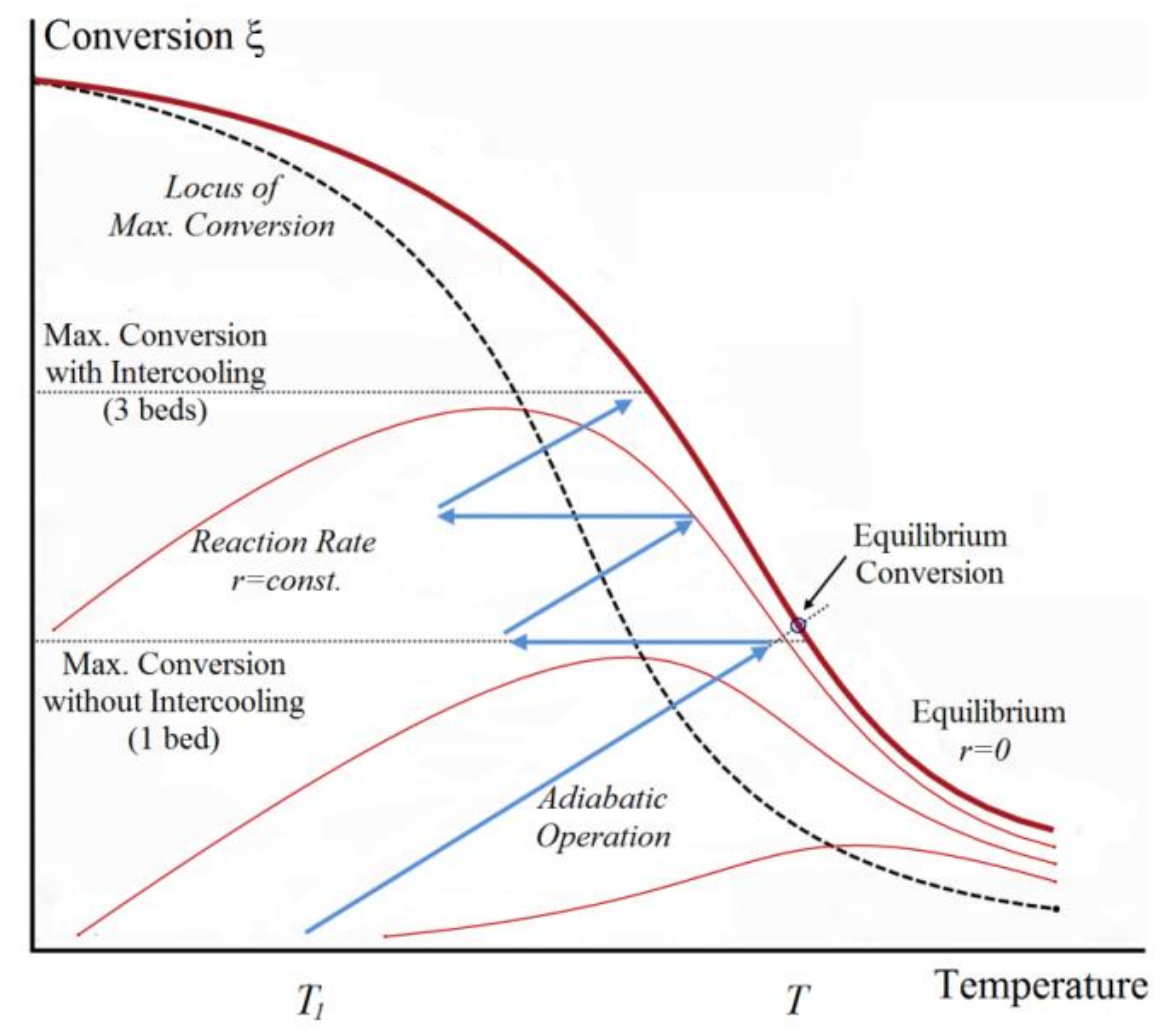

Source: Author.

Figure 5.5 compares the most popular cooling methods, which vary in the way the heat recovery is achieved. In early plants, the quenching (or 'cold shot') process (Fig. 5.5 a) was more popular due to its simpler design. In this process, only a fraction of the reactor feed stream passes through the first catalytic bed, leaving it at about $500^{\circ} \mathrm{C}$. Thereafter, the outlet gas temperature is reduced to approx. $430^{\circ} \mathrm{C}$ by mixing it with a fraction of the bypassed cold feed gas at $150-200^{\circ} \mathrm{C}$, before entering the next bed [22]. This procedure is repeated along all of the remaining beds. On the other hand, the indirectly cooled converter (Fig. 5.5 b) is equipped with a waste heat recovery system that cools down the outlet gas from the converter beds. This is accomplished by either preheating the reactor feed stream or producing steam. In this way, not only a higher concentration of ammonia is obtained at the converter outlet, but also the steam system is directly integrated to the chemical plant [70]. In the case of 
quenching application, the required catalyst volume is reportedly higher (10-15\%) than in the indirectly cooled version $[41,140]$.

Fig.5.5. Comparison between direct (a) and indirect $(b)$ cooling of the ammonia reactor.

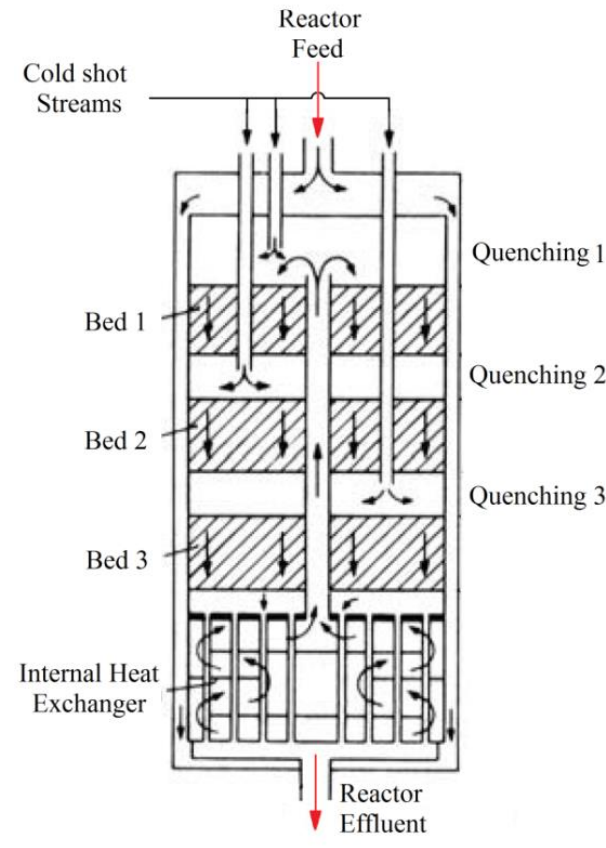

(a)

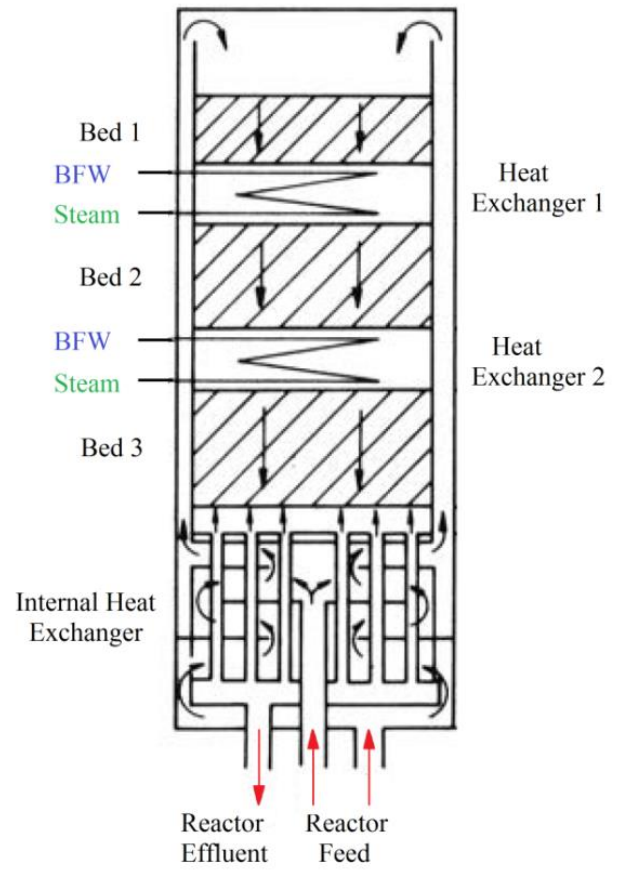

(b)

Source: [25].

Figure 5.6 illustrates the adiabatic lines of the reactor beds corresponding to the indirect and direct intercooling, respectively. As expected, since the reactive mixture has been repeatedly shifted away from equilibrium, the overall equilibrium conversion has been considerably increased.

Fig. 5.6. Operation lines for direct (a) or indirect (b) cooling of the adiabatic, exothermic reactor beds.

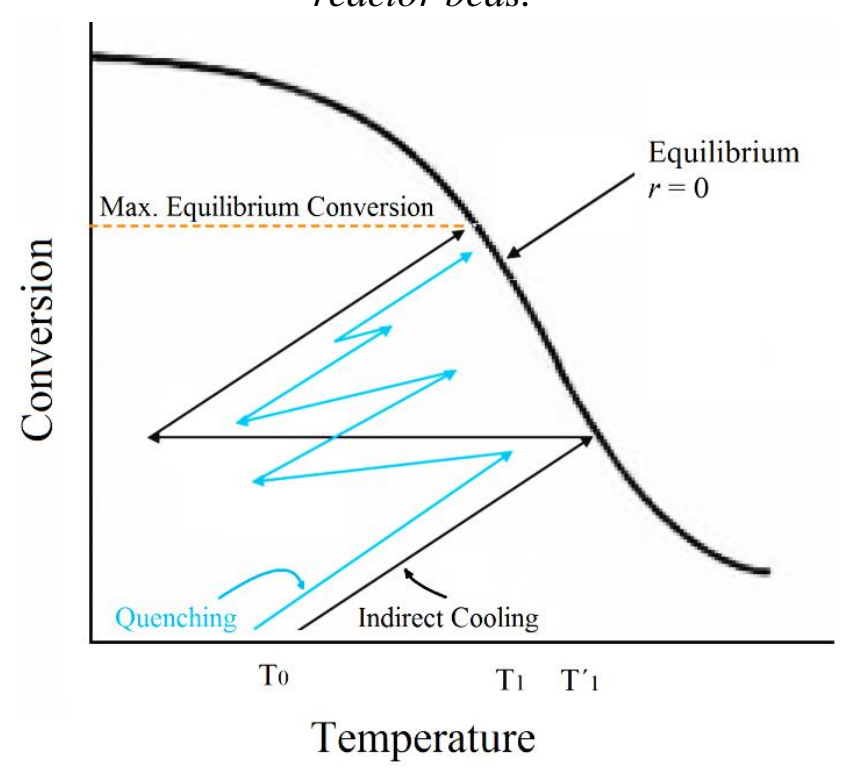

Source: [25]. 
However, the design of the intercooled ammonia reactor shown in Fig. 5.6 must cope with two conflicting effects [141]. According to Figs 5.3 and 5.4, as the reaction starts far away from the equilibrium, the kinetics is favored by an increase of the reaction temperature. In this case, both forward and backward reaction rate constants increase and, thus, the conversion increases. However, since the maximum attainable conversion (at $r=0$ ) diminishes with the temperature, the reaction rate eventually starts to fall when approaching to equilibrium. In the latter case, the rate of the ammonia decomposition outweighs the reactant conversion at higher temperatures [23, 139]. Consequently, there should exist a set of maximum conversion for a given reaction rate that could be attained if the optimum reactor temperatures are suitably manipulated [41]. The curve that contains all the points of maxima is called the locus of maximum conversion for a given reaction rate [139], mathematically represented by Eq.(5.49) and depicted in Fig. 5.7:

$$
\left.\frac{d r_{N_{2}}}{d T}\right|_{\xi=\text { constant }}=0
$$

The existence of the locus of maximum conversion for a given reaction rate entails the existence of a design of minimum reactor volume. For this reason, the concept of optimal operation lines is used in many exothermal processes such as methanol synthesis and sulfur dioxide oxidation. In the case of ammonia reaction, the locus of maximum conversion for a given reaction rate runs almost parallel to the equilibrium curve, but shifted $30-70^{\circ} \mathrm{C}$ toward the lower temperature range [23, 141].

Fig. 5.7. Locus of maximum conversion for a each reaction rate for a Montecatini Catalyst at

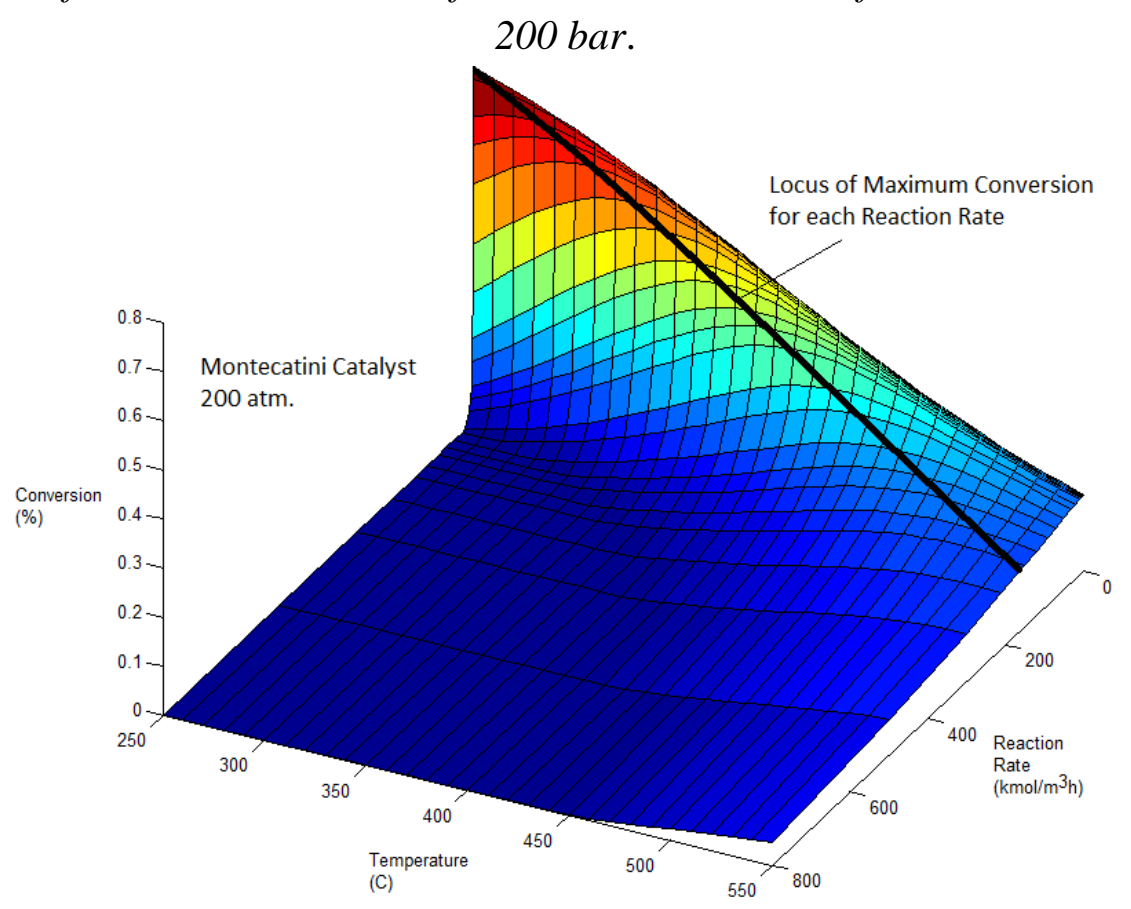

Source: Author.

Certainly, a more detailed reactor simulation may include the coupling of the modeling of the non-linear reaction kinetics to the computational fluid dynamics. However, this would 
considerably increase the computational effort at expense of a small gain in precision for the preliminary conceptual design. Accordingly, in the simulation stage, the effective volume of the reactor is calculated by considering a cylindrical packed bed reactor, in contrast to commercial, non-circular cross-sectioned configurations. It is also assumed that the axial diffusion effect diminishes with the increase of the flow rate. In other words, the ammonia synthesis bed is simplified to a packed bed reactor (PBR) in Aspen Hysys ${ }^{\circledR}$ with a very fast radial mass transport as well as a large reactor diameter compared to pellet diameter. The flow is assumed turbulent so that the rapid mixing of reactants is guaranteed. In this way, a compromise between the effect of the reactor performance and reasonable computational time frames is obtained. Additionally, the effect of the catalyst aging in its activity variation remains out of the scope of this thesis.

Similar analyses, such as the previously described for the ammonia synthesis reactor, could be carried out for the reformers, water gas shift reactors, and alike. Those analyzes are accomplished by taking into account the kinetics of the reforming reaction $\left(\mathrm{T}>586^{\circ} \mathrm{C}\right)$ and the water gas shift reaction, shown in Eqs.(5.50-5.51) in $\mathrm{kmol} / \mathrm{m}^{3}-\mathrm{s}$ ( $\mathrm{T}$ in $\mathrm{K}, \mathrm{P}$ in $\mathrm{atm}$.), respectively [142]:

$$
-r_{\mathrm{CH}_{4}}=k_{f}\left[P_{\mathrm{CH}_{4}} P_{\mathrm{H}_{2} \mathrm{O}}-\frac{P_{\mathrm{CO}_{2}} P_{\mathrm{H}_{2}}^{3}}{K_{e q, R E F}}\right]
$$

where $K_{e q, R E F}=\exp \left(-\frac{27464}{T}+30.707\right)$,

$k_{f}=k_{0, f} \cdot e^{-\frac{E_{a, f}}{R T}}$,

$k_{0, \mathrm{f}}=200 \mathrm{kmol} / \mathrm{m}^{3} . \mathrm{s}$,

$E_{\mathrm{a}, \mathrm{f}}=16,000 \mathrm{~kJ} / \mathrm{kmol}$,

It is worthy to mention that, in comparison with the SMR reaction, Eq.(5.50), the equilibrium composition of the WGS reaction in not affected by the total pressure [25], due to the conservation of the quantity of moles associated to the latter reaction.

$$
-r_{\mathrm{CO}}=k_{2}\left[P_{\mathrm{CO}} P_{\mathrm{H}_{2} \mathrm{O}}-\frac{P_{\mathrm{CO}} P_{\mathrm{H}_{2}}}{K_{e q, 2}}\right]
$$

where $K_{e q, S H}=\exp \left(-\frac{4048}{T}+3.765\right)$,

$k_{2}=k_{0,2} \cdot e^{-\frac{E_{a, 2}}{R T}}$,

$k_{0,2}=100 \mathrm{kmol} / \mathrm{m}^{3} . \mathrm{s}$,

$E_{\mathrm{a}, 2}=16,000 \mathrm{~kJ} / \mathrm{kmol}$.

Finally, due the complex vapor-liquid equilibrium (VLE) in the high pressure ammonia separation, the Raoult's law cannot be applied anymore to model and simulate this process. For higher pressures and for species above their critical temperatures, the fugacity of the species $i$ in the non-ideal liquid phase is rather calculated in terms of the system pressure $f_{\mathrm{i}}=$ $x_{\mathrm{i}} \cdot \phi_{\mathrm{i}} \cdot P$ (i.e. instead of in terms of their vapor pressure $f_{\mathrm{i}}=x_{\mathrm{i}} \cdot \gamma_{\mathrm{i}} \cdot p_{\mathrm{i}}$ ). This is preferable, 
especially for substances whose vapor pressure $p_{i}$ may not be defined above their critical temperatures (e.g. dissolved hydrogen). The fugacity coefficient of species $i, \phi^{\prime}{ }_{i}$, in both liquid $\left(x_{\mathrm{i}}\right)$ and vapor $\left(y_{\mathrm{i}}\right)$ phases is thus computed from the same equation of state $(\mathrm{EoS})$, as well as the other thermodynamic properties such as densities, enthalpies and heat capacities [143].

\subsection{Process Synthesis and Optimization}

Following the formulation of an operational base-case design, the next step in the synthesis of the chemical process deals with finding out the causes of the suboptimal functioning. To this end, the performance of each component or unit is hierarchized via either thermodynamic, environmental or economic indicators. Thereafter, the goal consists of seeking for an improved scheme that achieves a predefined target while complies the restrictions imposed to the model. However, as one goes from the process modeling and simulation to the process improvement, the problem becomes much more difficult not only from a computational but also from a methodological point of view. Accordingly, a systematic optimization framework is necessary in order to smartly search within the space of independent variables while keeping the number of simulations at a minimum [116].

Notwithstanding, even when systematic procedures are used, the number of challenges posed by the retrofitting, revamping or grass-root chemical plant optimization is not less formidable. For instance, in the case of the integrated syngas and ammonia production plants, the following thermodynamics and economic challenges are envisaged:

- Numerous old plants cope with the challenge of remaining competitive in front of the new technology plants. On the other hand, even though new plants can give an advantage in terms of production performance, this is only at expense of a much higher capital cost. Indeed, according to some authors [70], on condition that the projected increase of the steam reforming capacity is between $10-50 \%$, the payoff of the revamp is often more cost-efficient than acquiring a new plant. However, revamps address further parametrical or structural modifications to existing equipment that already operates close to the limit capacity (identified as the bottleneck), rendering the revamp more challenging to be planned than grassroots plants.

- Fertilizers plants are generally designed in sophisticated ways that involves the recycling and integration of the mass and energy streams of the chemical units. This intrinsic characteristic entails the likelihood of facing a variety of convergence problems when using the commercial sequential modular simulators. One of the most common drawbacks is called the snowball effect [135], which refers to a situation in which a small disturbance, for example, in the composition of the reactor feed, causes a disproportionate change in the flow rate of the recycle stream. In such scenario, the reactor, the separator, or both systems, may not be able to handle the increased throughput. Whether the snowball effect occurs or not will depend on the formulation of the optimization constraints and an efficient control system [99]. 
- The process of optimization is both theoretical and practical as it is based on linear and non-linear mathematical models that use data obtained from actual plants found in the literature. Once formulated, the system of equations needs to be solved by analytical or numerical methods which often requires of powerful computer hardware as well as sophisticated, efficient and robust solvers [144]. However, since those solvers may be not included in the simulation software, advanced integration frameworks, that orchestrate the interaction and the data entry/retrieval between the client software and the self-devised subroutines, will be necessary.

- As it will be discussed in the next section, the energy integration method, also known as pinch method, is based on the univocal determination of the initial and final temperatures as well as the change of the enthalpy flow rate of each stream involved in the heat exchanger network (HEN). However, once the solver sets new values to the independent variables of the optimization problem, the simulator calculates updated values for the physical properties of the integrated streams. Consequently, a totally different HEN will be generated which, in turn, will have an important impact in the choice of the new values of the independent variables $[11,145]$. Therefore, the temperature and exchanged heat values defined univocally for each stream become a set of variables that will depend on the optimization problem [3].

- The isolated optimization of a major plant component is facilitated when this is strategically located at the frontend of the chemical process. However, for other major components located downstream in the production process, it cannot be automatically expected that, when optimized in isolation, the remaining components also operate in optimal conditions [13, 146, 147].

- One of the main difficulties found when performing comprehensive optimization analyses of complex chemical systems is the lack of reliable data of the operation parameters and costs. According to some authors [147], the way in which manufacture engineers calculate costs is not always compatible with the way these data are needed for optimization purposes, e.g. in terms of physical and thermodynamic variables. Catalyst properties, reactor sizes and other industrial variables make often part of the intellectual property of licensors, with an almost inexistent transference of knowledge between the industry and the academic centers.

- The simultaneous pursuit of conflicting physical, commercial and environmental targets triggers off a set of relatively suboptimal operation conditions with regards to one objective, while attempting to improve the remaining ones. The graphical representation of the compromise between these relative suboptimal solutions is called Pareto front. Since at some extent all the solutions in the Pareto front are optimal (non-dominated), the expertise of the designer will be fundamental when ruling out certain solutions.

In order to tackle these problems, a number of powerful systematic techniques have emerged to support the integrated process synthesis. A preliminary classification includes three broad classes of methods, namely (i) heuristic, (ii) thermodynamic and (iii) algorithmic [10, 114]. The suitability of each approach for a particular application depends on (a) the amount of 
available information about the performance and cost of each system component, (b) the complexity of the total system, (c) the objectives of the optimization and (d) the required accuracy [148]. These methods have been shown to be successful at least for particular classes of problems, even when the number of degrees of freedom is large and the models involved are highly non-linear and complex [149].

\subsubsection{Heuristic methods}

Various preliminary screening guidelines known as heuristic rules or rules of thumb, based on experience and engineering judgment, can be used to figure out functional chemical plant configurations more quickly, thus radically reducing the optimization search space. However, since the applicability of the heuristics developed depends on specific operating scenarios, they have to be constantly updated [117]. Of course, heuristic method does not provide a guarantee of optimality and, even worse, it may create an opposite effect when extrapolated to breakthrough approaches [111]. The use of heuristics in the design of syngas and ammonia production plants involves the selection of steam to carbon or steam to biomass ratios; boundary operation conditions and approach to equilibrium temperatures in some reactors; range and approach temperature differences in cooling towers; minimum temperature approaches of heat exchangers; strength and load of chemical solvents in the syngas purification unit (utterly important to attain convergence of the model simulation); refrigerants operating conditions; maximum compression ratios in centrifugal compressors (surge prevention); metallurgical and mechanical safety issues; characteristic heat transfer coefficients (boiling, condensation, single phase, gas, liquids); selection of the suitable correlations for thermodynamic properties calculation; initialization values of tear stream (smart guesses), among others.

\subsubsection{Thermodynamic methods}

Since the Principles of the Thermodynamics rely on observed physical laws, they can be used to predict upper and lower bounds of the attainable optimal solution, regardless of the energy technology selected (top-notch, conventional) or the energy conversion process performed (reversible, irreversible, adiabatic, etc.). In this way, many configurations can be excluded from further investigation in early stages of design, largely reducing the extent of the search space. The energy integration (or pinch method) [150] and the exergy method [151] are among the most common thermodynamic methods used in the chemical plant diagnosis. In the former, the minimum consumption of the utility resources is pursued through the integration of the chemical plant with the heat recovery utility system. In the second, the aim is to identify the causes of irreversibility (proportional to the entropy generation) in the design, so that the operation conditions can be adjusted in order to reduce the degradation of those resources.

After the causes of the suboptimal functioning of the system are pinpointed, various principles and methodologies, all based on the Second Law of the Thermodynamics, can be used to either maximize the energy savings or minimize the avoidable large driving forces. Some of them are the Le Châtelier Principle, the Counteraction principle, the Driving Force analysis and the Quasi-Static analysis [69]. In the last two methodologies, the analysis of a real system 
operating under finite driving forces is compared to an equivalent theoretical limiting, quasistatic process that runs with zero driving forces. Thus, the goal is to devise the engineering means to reduce the avoidable driving forces, so that the real process approaches to quasistatic operating conditions. In this thesis, the first two principles, i.e. Le Châtelier Principle and the Counteraction principle, are used to counterbalance the clashing optimization outcomes set off by the simultaneous reduction of the exergy losses (i.e. by reducing the driving force), whereas attempting to enhance the chemical process throughput (i.e. by increasing the driving force).

According to Le Châtelier Principle "Any system in chemical equilibrium that is subjected to a variation in its concentration, temperature, volume, or pressure, will try to adjust itself in order to partly reestablish the stable equilibrium conditions temporarily lost due to the change applied" [152]. In other words, if a finite driving force is set to either a chemical reactor in equilibrium, a heat exchanger network, or a $\mathrm{CO}_{2}$ desorption network, etc. the system will attempt to attain the equilibrium by increasing the rates of conversion, mass and heat transfer. Apart from the more evident heat transfer example (e.g. the higher the temperature difference, the faster the heat transfer rates), in chemical reactors, the increase of the reactive driving force, $-\Delta \mathrm{G}$, may be triggered by:

- A decrease of the temperature of an exothermic reactor or an increase of the temperature of an endothermic one;

- An increase of the pressure or a decreasing-volume reaction or a decrease of the pressure of an increasing-volume one.

On the other hand, the Counteraction principle is used in conjunction with the exergy analysis and the Le Châtelier principle to work out means of reducing the excessive driving forces so that an improved exergetic performance can be achieved [153]. However, this is often accomplished through practices contrary to those advised by the Le Châtelier principle. As it will be explained later, this reasoning follows the minimization of the large driving forces, $-\Delta \mathrm{G}$, in the reactor, in order to increase the recovery of the exergy embodied in the reactants. Actually, this has been one of the major challenges found along the development of this thesis, since "to maximize the recovery of the useful work (or obtain similar positive results), it is necessary to use great ingenuity" [69]. Not to mention that "the decrease in the driving forces in some stages of an industrial process should not lead to negative results at succeeding stages of the process".

Despite their powerfulness, the standalone application of the thermodynamic methods may require of a considerable amount of trial and error attempts to find solutions that are close to the predicted bounds [146]. Moreover, although the design featuring the highest energy efficiencies may sometimes entail also close-to-optimum economic solutions, in the practice, this not necessarily true [154]. Thus, aside from the thermodynamic aspects already discussed, other features related to the capital investment, operation, maintenance, decommissioning, and environmental impact should be also be accounted for.

For instance, the thermodynamic methods have proven to be useful to effectively perform cost allocation analyses as well as to quantify the environmental impact [155]. In the next sections, 
a brief description of the energy integration method is presented. Afterwards, the exergoeconomy methodology used to allocate the exergy cost and $\mathrm{CO}_{2}$ emissions among the various products of an integrated ammonia plant is introduced. To this end, a novel set of auxiliary equations for cost allocation is proposed to overcome the inconveniences presented by the conventional costing theory [156].

\subsubsection{Energy integration and Minimum Energy Requirements}

A large portion of the energy consumed as feedstock in an industrial ammonia plant is eventually transformed and released in the form of low-grade heat, which leads to a reduction of the amount of ammonia produced per unit of feedstock consumed. Therefore, it would be desirable to reutilize most the energy released in order to preheat the cold streams of the same production process. The excess heat comes mostly from the exothermic reactions involved in the partial oxidation, shift and ammonia synthesis, as well as from the intercooled air and syngas compression. However, due to the reduced levels of temperature associated to the lowgrade waste heat, only a fraction of it can be effectively recovered. Accordingly, an additional amount of fuel input would still be necessary in order to supply the demands of the primary reformer and the syngas purification system.

Thus, the main goal of the energy integration (EI) method consists of determining the maximum amount of waste heat that can be recovered (and, thus, the minimum amount of fuel required), well ahead of a detailed design of the heat exchanger network (HEN) be performed [3]. The EI method, also known as Pinch method, is reportedly formalized by Bodo Linnhoff in 1972 and, since then, it has been comprehensively revisited in his research work and by coworkers [157]. By upgrading the waste heat that otherwise would be irremediably dissipated by the cooling system to the environment, the EI method not only reduces the consumption of the costly external resources used in the utility systems (e.g. fossil fuels) but also the wastes and emissions associated. Next, a graphical description of the concepts used in the Pinch method is briefly presented.

The EI method requires (i) the knowledge of the number of cold and hot streams considered in the integration problem, (ii) the corresponding supply and target temperatures, and (iii) the value of either the heat capacity $\left(\mathrm{m}^{\prime} \mathrm{c}_{\mathrm{p}}\right.$ in $\left.\mathrm{kJ} / \mathrm{K}\right)$ or the change in the enthalpy flow rate of each stream $(\Delta \mathrm{H}$ in $\mathrm{kW})$. The calculation of the minimum heating and cooling requirements starts from the representation of the single cold and hot streams in a Temperature vs. Enthalpy flow rate ( $T$ vs. $H$ ) plot, as shown in Fig. 5.8a. It is worthy to notice that, in this diagram, the scale of the abscissa is only a relative difference, thus the arrows can be moved side to side [111].

By merging the representations of the single cold or hot process streams, the cold and hot composite curves (CC), shown in Fig. 5.8b, can be constructed. In this diagram, the vertical distances between the cold and hot composite curves correspond to the temperature difference along the overall heat exchange process. Moreover, the amount of the overlapping of the hot and cold composite curves along the horizontal axis indicates the extent of heat transfer match between the cold and hot process streams pertaining to the interval of temperature concerned. At last, a physical and economics restriction is typically imposed on the minimum overall temperature difference allowed $\left(\Delta \mathrm{T}_{\mathrm{min}}\right)$. This ensures finding a HEN with the minimum utility 
consumption that also achieves practical heat transfer rates in the industrial applications. The value of $\Delta \mathrm{T}_{\min }$ clearly depends on the nature of the fluid involved. Thus, an approach that separately assigns a contribution of each stream to the overall $\Delta \mathrm{T}_{\min }$ is recommendable [158]. Typically, values of $\Delta \mathrm{T}_{\min }$ ranging between $10-100^{\circ} \mathrm{C}$ or more can be assumed, depending on the various characteristics of the substances studied (pressure, phase, etc.) [159].

Fig. 5.8. Construction of the cold and hot composite curves. Actual temperatures shown in ${ }^{\circ} \mathrm{C}$.(a) Single hot and cold streams, (b) Cold and hot composite curves (CC)

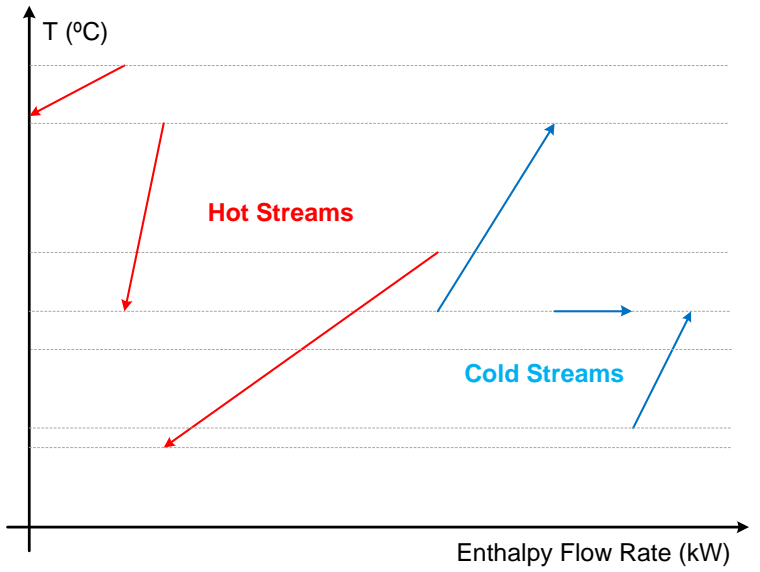

(a)

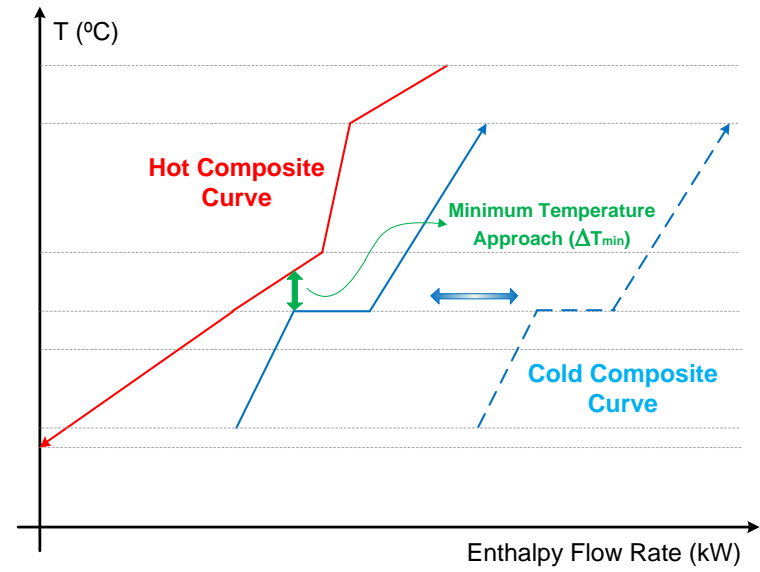

(b)

Source: Author.

Figure 5.9b illustrates the construction of the Grand Composite Curve (GCC) by means of the calculation of the cascaded heat data and based on the shifted- $T$ vs. $H$ diagram shown in Fig. 5.9a. It is worthy to mention that the shifted temperatures are not intended to replace the actual temperatures of the initial simulation, but only serve to ensure the $\Delta \mathrm{T}_{\min }$ restriction in the actual HEN. The GCC shown in Fig. 5.9b proves to be a useful representation of the waste heat recovery potential. In effect, it aids in the choice and integration of one or more energy conversion technologies of the utility systems, looking into an increase of the overall process performance. Moreover, the minimum energy requirements (MER) can be determined from Fig. 5.9b, represented by the horizontal distance from the Y-axis and the GCC of the chemical process at the highest and lowest temperatures. The point at which the GCC of the chemical process touches the $\mathrm{Y}$-axis is the pinch point temperature. In the next sections of this thesis, the linear programming optimization problem that minimizes the MER will be mathematically defined. 
Fig. 5.9.Grand composite curve construction. Shifted temperatures shown in ${ }^{\circ}$ C.(a) Cold and hot composite curves (SCC), (b) Grand composite curve (GCC).

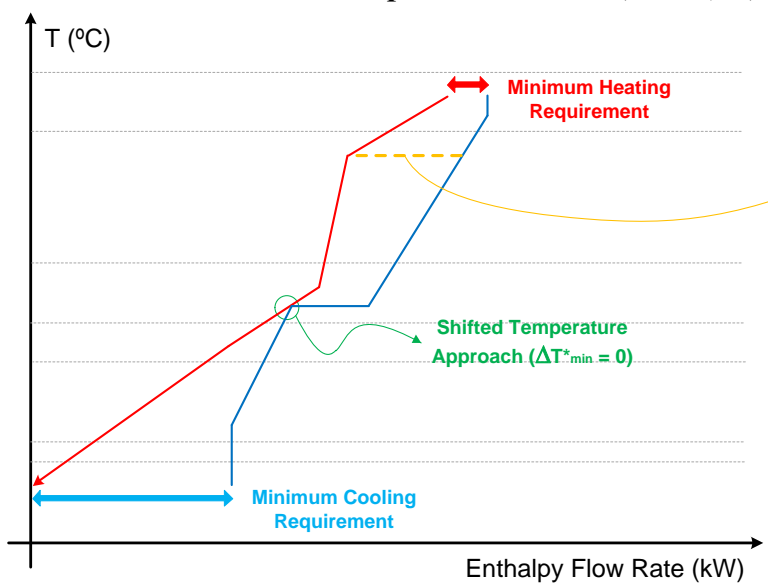

(a)

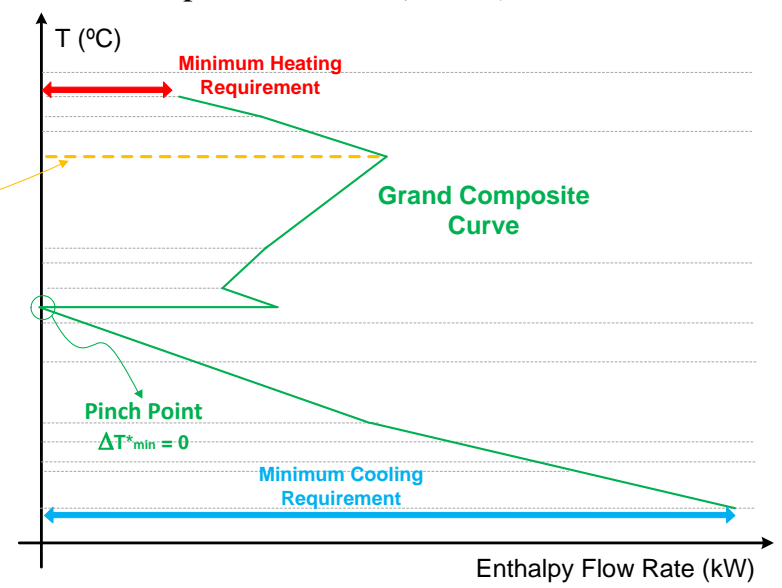

(b)

Source: Author.

As it can be seen from Fig.5.9b, the cascaded heat is null at the pinch point temperature. Thus, the localization of this point serves to determine the extent of the penalty due to the transference of heat across the pinch from a hot to a cold stream. Actually, if an operating condition with the minimum energy requirement (MER) is aimed, the transference of heat across the pinch point should be in principle avoided. Furthermore, the utilization of hot utility streams to heat below - and cold utility streams to cool above - the pinch will only bring about an increase of MER. A special case in which the heat transfer across the pinch could be considered thermodynamically advantageous would be the upgrade of the low-grade waste heat available below the pinch to increase the amount of energy available above the pinch. The preheating of the reactants or the use of a mechanical vapor recompression systems [160] are examples of process that invert the transference of waste heat across the pinch.

In the mechanical vapor recompression systems (MVR) shown in Fig. (5.10), the enthalpy of condensation of a recompressed stream is used to boil up the column bottoms, instead of the consumption of a high temperature utility stream (e.g. steam). According to some authors, the power consumed by the MVR can be as small as 10-15\% of the boiling or condensation duty (energy basis) [161]. Two configurations, namely, an open and closed MVR system can be devised, differing in the type of fluid that is being recompressed. In the closed system a suitable substance, such as R717, can be used as the heat transfer media, similarly to the operation of a heat pump, with the evaporator providing the condensation effect of the column. In the open cycle configuration, the substance leaving the top of the desorber can be used as the heat transfer fluid after recompression. 
Fig. 5.10.Mechanical vapor recompression systems (a) closed and (b) open configuration.

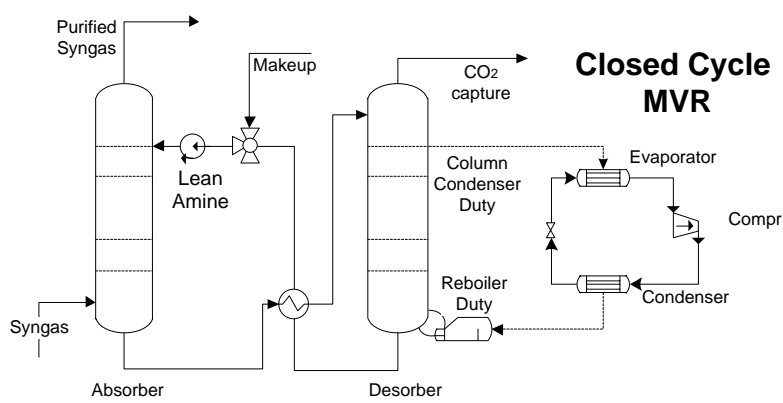

(a)

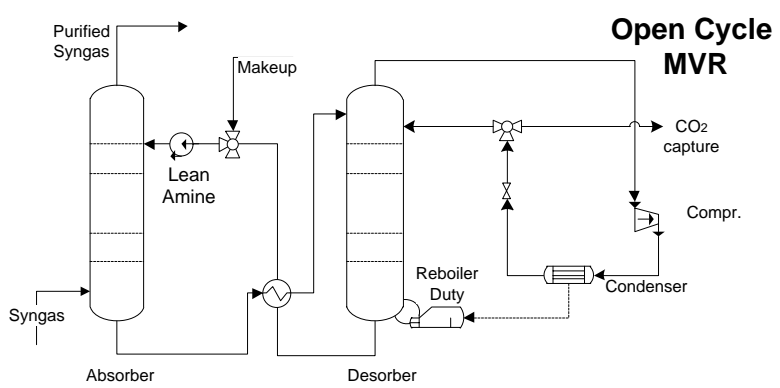

(b)

Source: [161].

Alternatively, the waste heat available below the pinch may become available above the pinch through the preheating of the reactants, which results in an increase of temperature at the reactor outlet (e.g. gas turbine combustors, ATRs, furnaces, etc.). Reaction-driven components are the most important energy conversion systems in the chemical processes as they transform the raw materials into value-added products. Indeed, reactors fundamentally determine the design and performance of the remaining unit operations [162, 163], and the chemical reactions rates and equilibrium conversions are interrelated with the heat recovery and transport rates. Thus, the reactor performance is directly related to the balance of steam and power generation, which directly affects the energy consumption profile. For the sake of example, in a typical 1000 t/day ammonia plant, up to $120 \mathrm{MW}$ of heat must be dissipated through cooling water, either for enhancing the reactor yield or due to safety and reliability issues.

For this reason, an adequate representation of the $T v s$. $H$ profile within any chemical reactor susceptible to energy integration is necessary [164]. This becomes especially true when trying to identify the opportunities for energy recovery and combined heat and power production. However, the reactor profiles are often neglected or misinterpreted, as discussed by Glavic et al. [140]. Most of the energy integration analyses only consider the reactor feed and effluents as simple cold or hot process streams that need to be heated up to (or cooled from) a representative reactor temperature. For instance, some studies arbitrarily assume that the temperature and duty in the radiant furnace is constant, thus simplifying the integration problem of an endothermic reactor into a threshold problem that presents only cooling requirements [165]. This clearly ignores the existence of a reaction-driven chemical utility that should be adequately integrated along with other hot and cold utility streams, so that the overall energy consumption in the system can be minimized [99]. Previously, some authors [166] suggested decoupling the contribution of the reaction enthalpy and the heat effectively transferred. The endothermic reactor was virtually coupled with a fictitious heat exchange unit that allowed calculating the feed preheating and the reaction enthalpy separately. However, depending on the extent of the endothermic duty required, that approach may lead to the calculation of an infeasible fictitious temperature profile that fail to represent the reactor performance in an energy integration process. Other studies [6,7], which decompose the reformer unit into its representative components (i.e. fuel-steam mixing, reforming, heat 
transfer and combustion), have found that, in order to reduce the irreversibility, the temperatures of the hot utility (combustion gases) and the reformed mixture must be better matched. It can be achieved by using more or less excess air, which eventually creates a trade-off between the exergy destruction in the combustion process or in the waste heat recovery system [7].

According to Fig. 5.11, the reactor unit can be thermodynamically decomposed into its functions in order to better approximate the detailed $T-H$ profile on the inside thereof. The reactor functions can be enumerated as the (i) development of the reaction enthalpy (release or absorption of chemical energy); (ii) transfer of the reaction enthalpy to the products; (iii) heat transfer from (to) the hot (cold) products to (from) the fresh reactants; and (iv) exchange between the reactor and the surroundings [140]. In this way, by successfully integrating the reactor profiles to the remaining energy conversion systems, neither the heat recovery opportunities nor the alternatives for the reduction of the irreversibility will remain hidden or missing.

Fig. 5.11. Thermodynamic decomposition of chemical reactors for energy integration purposes: (a) endothermic, $(b)$ exothermic reactor.

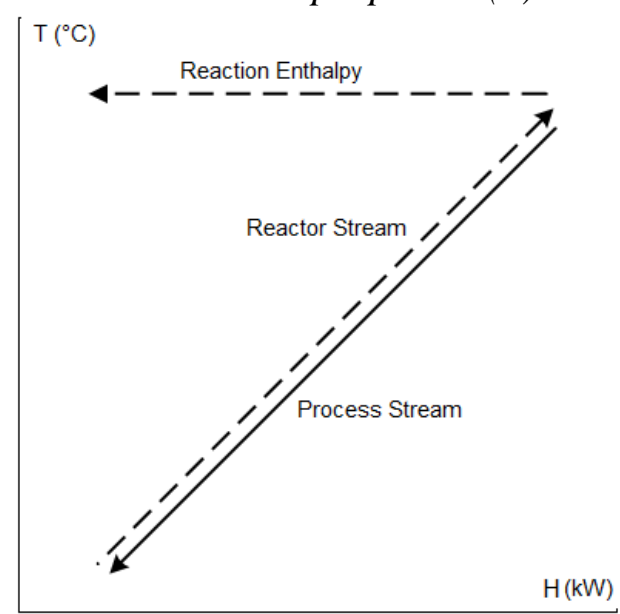

(a)

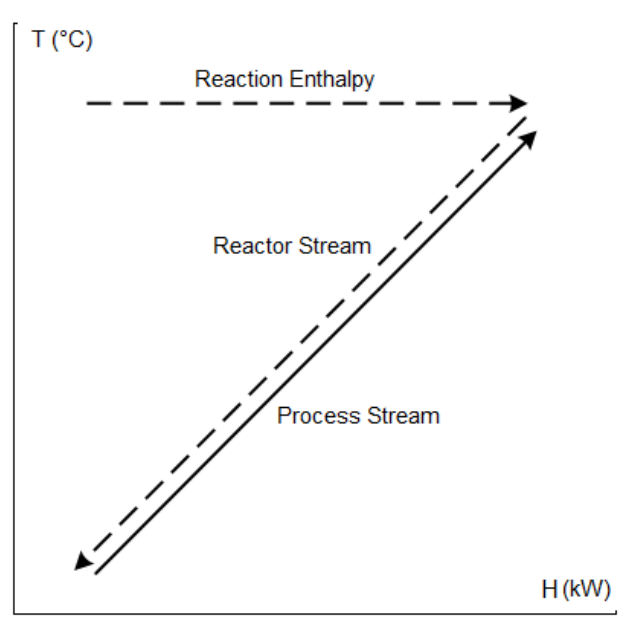

(b)

Adapted from Source: [140].

Figure 5.12 depicts the strategy used for the determination of the endothermic reactor profile in which the arrows indicate the direction of the heat transfer. The reactor feed is initially heated up to the reactor inlet temperature $\left(\mathrm{T}_{\mathrm{in}}\right)$. Then, the mixture is assumed to virtually attain the overall reactor outlet condition $\left(\mathrm{T}_{\text {out,global }}\right)$. However, due to the endothermic nature of the system, the temperature falls down to an intermediate temperature $\left(T_{r x n, b e d, i}\right)$ standing for the reaction temperature that the mixture would attain if no additional heat would be supplied to continue the endothermic reaction. Only then, the reactive mixture is allowed to perform an isothermal endothermic reaction that increases the enthalpy of the reactants proportionately to the reaction enthalpy at the operating temperature $\left(\mathrm{T}_{\text {rxn,bed, }}\right)$. After this partial conversion process has been achieved, the remaining reactive mixture repeats the aforementioned procedure until the global reactor outlet temperature and composition is attained. 
The described approach allows decoupling the reaction and heat transfer processes and can be extended to calculate the optimal temperature profile of the reactor that reduces the irreversibility along thereof. It is worthy to notice that, by decomposing the reformer in its thermodynamic functions, the whole assembly resembles a series of prereformer units with reheating. This scheme helps identifying the potential energy savings in the reformer duty. For instance, this approach may be helpful in deciding whether using hot effluents from the secondary reformer to heat the primary reformer tubes (e.g. Gas Heated Reformer concept, GHR), thus reducing the consumption of natural gas [81, 167].

Fig. 5.12. Determination of the T-H profile for an endothermic reactor.

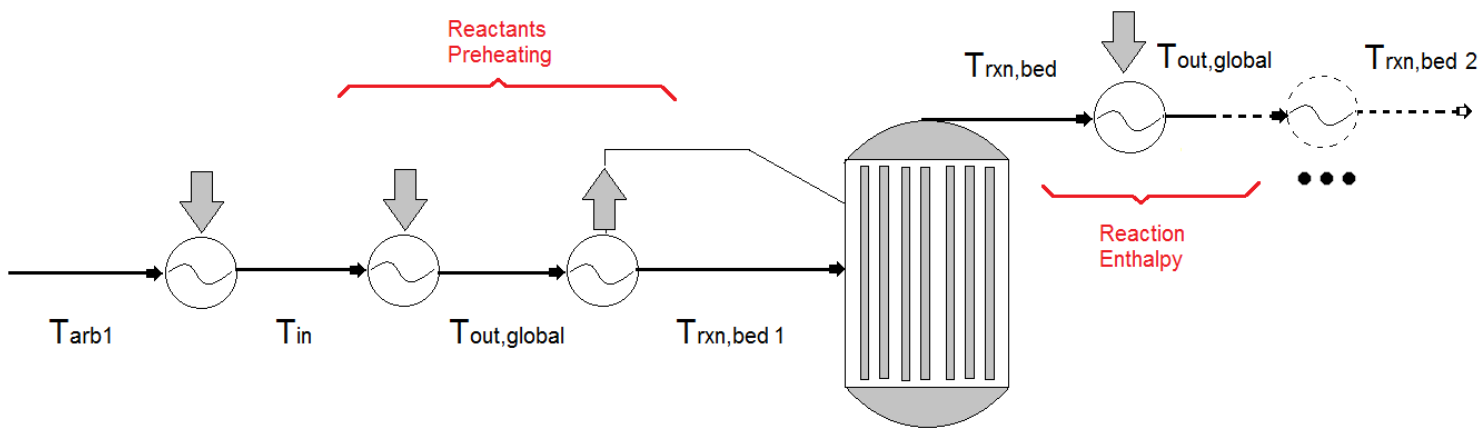

Source: Author.

Figure 5.13 compares the traditional (coarse) and the proposed (detailed) representation of the endothermic reformer [168]. The coarse reactor profile considers the reactor feed stream as a cold stream $\left(580^{\circ} \mathrm{C}\right)$ required to be preheated up to the reactor operating (outlet) temperature (approx. $790^{\circ} \mathrm{C}$ ). Next, the reaction is assumed to evolve isothermally while consuming the reaction enthalpy required by the reforming process. The total enthalpy of reaction is the same that the actual reformer and, thus, the energy requirement of the process is satisfied. However, many integration shortcomings may arise when the reactor profile is aimed to be integrated by using, for instance, a low-grade temperature source (e.g. the waste heat from the plant).

Fig. 5.13. Coarse and detailed representation of an endothermic reactor: $T$-H profile

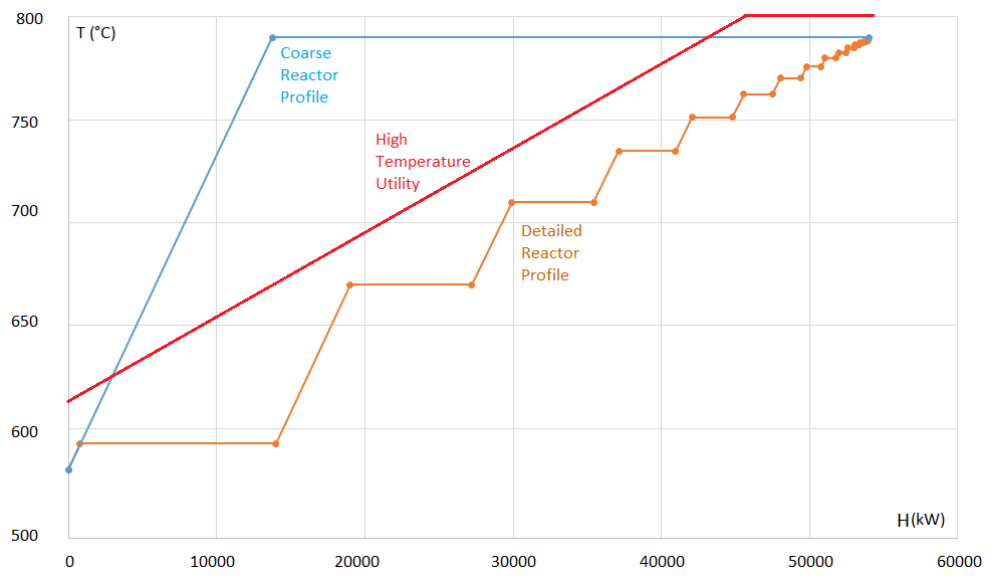

Source: Author. 
In contrast, when a detailed profile is represented, it allows for a better approximation of the operating conditions throughout the reactor. It also helps to analyze the effect of the main reactor parameters (e.g. temperature, composition, conversion, etc.) on the whole process integration, since the reaction temperature can be considered as a decision variable [162]. Interestingly, an endothermic reactor can be compared with a heat engine in which a portion of the high temperature exergy heat provided by the fuel is chemically converted into 'shaft' work (or embodied exergy into the reformed mixture), while a fraction is irremediably destroyed [140].

Analogously, the exothermic adiabatic reactor (Fig. 5.14) can be considered as a heat pump wherein the mechanical work input is equivalent to the chemical work (i.e. the variation of the chemical exergy of the reactants), which increases the physical exergy of the reactor product $[140,169]$. In this way, the exothermic reactor differs in the representation of the endothermic reactor, since now the chemical utility provides a 'free-ride' or internal preheating to the process stream.

Fig. 5.14. Determination of the T-H profile for an exothermic reactor.

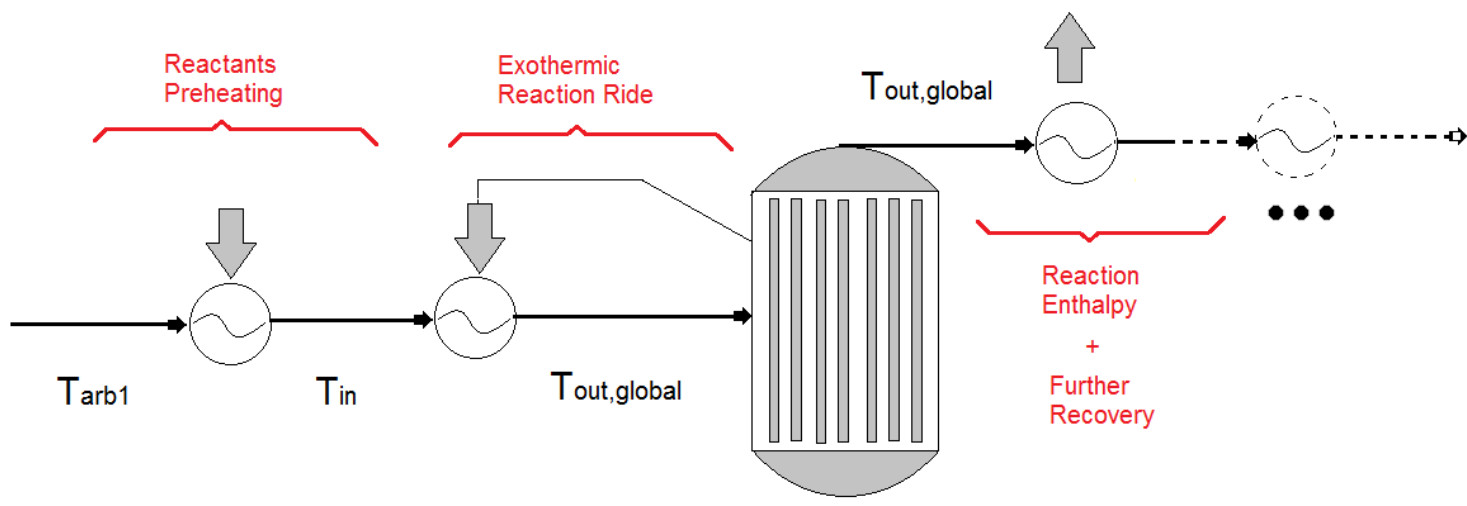

Source: Author.

Finally, the last step of the integration of the reactor profile consists of the determination of the best alternative to recover as much as possible the enthalpy of reaction embodied in the reactor effluent. For the sake of comparison, the reaction enthalpy of the ammonia synthesis is about $8.8 \%\left(2.718 \mathrm{MJ} / \mathrm{t}_{\mathrm{NH} 3}\right)$ of the total consumption of the integrated ammonia production plant [41], which clearly renders mandatory the recovery of this excess heat.

As a final remark, it should be observed that a detailed calculation of the heat exchanger network in the early stages of conceptual design may only add more computational effort without ostensibly increasing the accuracy of the solution [158]. Accordingly, an approximate calculation of the area of the HEN can be performed by considering the heat transfer coefficient, $\bar{U}\left(\mathrm{~W} / \mathrm{m}^{2} \mathrm{~K}\right)$ [159] and the logarithmic mean temperature difference, $\Delta T_{L M}$, calculated as Eq.(5.52):

$$
A=\frac{\Delta H}{U \cdot \Delta T_{L M}} \quad \text { with } \quad \Delta T_{L M}=\frac{\Delta T_{\text {in }}-\Delta T_{\text {out }}}{\ln \left(\Delta T_{\text {in }} / \Delta T_{\text {out }}\right)} \quad \text { and } \quad \frac{1}{\bar{U}}=\frac{1}{U_{\text {cold }}}+\frac{1}{U_{\text {hot }}}
$$


where $A$ is the heat exchanger area $\left(\mathrm{m}^{2}\right), \Delta H$ is the heat exchange rate $(\mathrm{kW}), \Delta T_{\text {in }}$ and $\Delta T_{\text {out }}$ are the inlet and outlet temperature differences in a countercurrent heat exchanger $(\mathrm{K})$, and $U_{\text {cold }}$ and $U_{\text {hot }}$ are the representative heat transfer coefficients of the cold and hot stream $\left(\mathrm{W} / \mathrm{m}^{2} \cdot \mathrm{K}\right)$, widely reported in literature [170].

\subsubsection{Exergy and Exergoeconomy Analysis}

In the last decades, several methods based on the First and Second Laws of Thermodynamics have been developed for defining indicators to assess the performance of chemical and industrial processes [171]. The combination of these two laws led to the concept of exergy. Exergy is defined as the maximum available work that can be obtained from a thermodynamic system when it interacts with the environment by means of reversible processes until the equilibrium state (mechanical, thermal and chemical) with the environment components is attained [151]. Total exergy accounts for the potential $(\mathrm{P})$, kinetic $(\mathrm{K})$, thermo-mechanical or physical $(\mathrm{PH})$ and chemical $(\mathrm{CH})$ exergy components, calculated by using Eqs. (5.53-5.56), respectively:

$$
\begin{gathered}
B^{K}=0.5 \cdot m \cdot|\vec{v}|^{2} \\
B^{P}=m g z \\
B^{P H}=\int m c_{p} d T+\int\left[V-T\left(\frac{\partial V}{\partial T}\right)_{P}\right] d P-T_{O}\left[\int m c_{p} \frac{d T}{T}-\int \frac{V}{T} d P\right] \\
B^{C H}=n_{m i x} \bar{b}^{C H}=n_{m i x}\left[\sum_{i} x_{i} b_{i}^{C H}+R_{u} T_{O} \sum_{i} x_{i} \ln \gamma_{i} x_{i}\right]
\end{gathered}
$$

where $\vec{v}$ and $z$ are the velocity and elevation of the system, respect to a stationary reference; $x_{i}$ and $\gamma_{i}$ are the mol fraction and the activity coefficient of the component $i$ in the mixture; $b_{i}^{C H}$ is the standard chemical exergy of component $i ; R_{u}$ is the universal constant of gases, and $n_{\text {mix }}$ is the total molar flow of the mixture. Equation (5.56) is especially useful when calculating the chemical exergy of gaseous fuels, since the chemical composition of gases can be readily determined and thermochemical data for those components are thoroughly reported [151].

However, solid and liquid industrial fuels and other substances are often mixtures of numerous chemical compounds, usually of unknown nature. Therefore, by assuming that the ratio of the chemical exergy to the lower heating value $\left(\varphi=b^{C H} / L H V\right)$ is invariant for pure chemical substances having the same ratios of chemicals constituents (H/C, O/C, N/C), Szargut and Styrylska derived correlations that express the dependence of $\varphi$ on those atomic ratios [151]. In this way, the chemical exergy of complex substances can be estimated from an equivalent chemical formula (e.g. DEA solvent: $\mathrm{C}_{4} \mathrm{H}_{11} \mathrm{NO}_{2}$ ) along with the thermodynamic data and other correlations for lower (LHV) and higher (HHV) heating values, as those available in Refs. [172-174]. For biomass and other solid and dry organic substances composed of $\mathrm{C}, \mathrm{H}, \mathrm{O}$ and $\mathrm{N}$, and $0.5<\mathrm{O} / \mathrm{C}<2$, the value of $\varphi$ can be calculated as: 


$$
\varphi_{\text {seco }}=\frac{1.044+0.0160(H / C)-0.3493(O / C)(1+0.0531(H / C))+0.0493(N / C)}{1-0.4124(O / C)}
$$

Furthermore, if the fuel contains moisture and sulfur, the chemical exergy is calculated according to Eq.(5.58):

$$
\begin{aligned}
\bar{b}_{F}^{C H}=\left(P C I_{F}+\left[W \cdot h_{l v}\right]_{\text {agua }}\right) \cdot \varphi_{\text {seco }}+\left[\bar{b}^{C H}-P C I\right]_{\text {azufre }} & \cdot S \\
& =\left(P C I_{F}+[W \cdot 2442]_{\text {agua }}\right) \cdot \varphi_{\text {seco }}+9417 \cdot S
\end{aligned}
$$

where $W$ and $S$ are the mass fractions of water and sulfur, respectively; $P C I_{\mathrm{F}}$ is the lower heating value of the fuel $(\mathrm{kJ} / \mathrm{kg})$, and $h_{\mathrm{iv}}$ is the enthalpy of vaporization of water at $\mathrm{T}_{\mathrm{o}}$ in $\mathrm{kJ} / \mathrm{kg}$.

Meanwhile, it is worthy to notice that, since real processes are based on finite-driving forces, they are irremediably irreversible, and a portion of the exergy is always destroyed as the system evolves towards equilibrium. The exergy destruction or irreversibility accounts for the system inefficiencies and gives a useful measure of the way in which resources are consumed and degraded. The exergy balance formulation applied to a control volume encompassing a component or set of them operating in steady state allows to calculate the overall amount of irreversibility generated in the system, $B_{\text {dest }}$, according to Eq.(5.59):

$$
B_{\text {dest }}=\sum_{e} m \cdot b-\sum_{s} m \cdot b+\int\left(1-\frac{T_{O}}{T}\right) \delta Q-W_{\text {useful }}
$$

where $b$ is the specific exergy of the mass flows going through the control volume, Eq.(5.60):

$$
b=\left(h-h_{O}\right)-T_{O}\left(s-s_{O}\right)+1 / 2\left(m v^{2}\right)+m g z+b^{C H}
$$

The terms related to the kinetic and potential exergy in Eq.(5.60) can be often neglected in comparison with the chemical and physical exergy of the mass flow. Furthermore, $Q$ and $W_{\text {useful }}$ are the rate of heat transfer and useful power, respectively, used to calculate the exergy interactions related to the heat and work that enter or exit the control volume.

Fortunately, exergy losses are not always inevitable and, in some cases, a portion of them may be avoided. To this end, optimal operation conditions can be implemented, so that the actual process efficiency can approach the limit value set by the ideal conditions. In other words, the exergy analysis is a valuable tool that allows comparing the actual and the ideal performances, limiting the design of technological developments to feasible thermodynamic solutions. For this reason, the exergy method is generally used to define suitable indicators to evaluate the performance of the processes present in industrial and chemical plants. However, the proposition of a general exergy efficiency definition for the various equipment and units of the integrated syngas and ammonia production plant is not straightforward.

On the one hand, due to the variety of chemical species, equipment, operation conditions and product specifications, each operation unit needs a conscientious definition of the most suitable exergy efficiency, one that truly outlines the useful product and the actual exergy consumption. On the other hand, several issues come about, especially when large mass exergy flows of feedstock, process syngas, chemical and physical solvents, and inert streams overwhelm the exergy terms related to the transference of power and heat exergy (Eq. 5.59). 
From an overview of several works on exergy efficiency definitions reported in the literature $[147,151,175,176]$, it is evident that any formulation of exergy performance is open to interpretation and, sometimes, it remains undefined for specific units and operating conditions. This leads to confusions when various processes are compared in a quantitative manner under different metrics.

Most of the proposed exergy efficiencies can be broadly classified into two types: (i) inputoutput, and (ii) consumed-produced efficiencies. Terms such as 'exergy input', 'exergy output', 'exergy loss', 'fuel', 'exergy expenditure', 'useful product', 'non-exergetic raw feed' or 'transit exergy' are inherent to them. The input-output definitions consider the ratio of all the exergy rate or flow rate leaving the system to the exergy rate or flow rate fed to it. Meanwhile, the consumed-produced definition attempts to differentiate the exergy effectively consumed (or produced) by the system from the transit exergy by calculating the exergy change of specific streams on the way to product. Despite its simple formulation, the inputoutput exergy efficiency may provide misleading results, as it deceptively assume values close to the unity, even for operations which, from an engineering point of view, have a poor performance [177]. In fact, its sensitivity is reduced as the amount of untransformed components increases. Thus, as suggested by some authors [147], this approach may be preferably applied when the system consists of a large number of unit operations.

Table 5.5, Eqs.(5.61-5.72), summarizes the consumed-produced exergy efficiency definitions used in this work for representative components of the ammonia plant. The comparison with the respective simpler input-output efficiencies is also provided. Differently from the inputoutput approaches, the consumed-produced formulation considers an increase (or decrease) of the chemical exergy of specific streams as a contribution to the useful product (or to the consumption) of the respective unit. Similarly, an increase (or decrease) of the physical exergy of the product, compared to that of the feed stream, can be regarded as a useful output (or an expenditure) of the system. Other contributions such as power and heat interactions are also accounted for as produced or consumed exergy rates, whichever it is the case. It is also worthy to notice that, aside from the internal irreversibility inherent to the real processes (e.g. finite driving forces, dissipative processes), external losses (e.g. heat loss, exhausted gases, cooling water) are owed to exergy flows deliberately rejected to the environment, and as such, they should not be considered as useful exergy exiting the control volume [151].

Table 5.5. Exergy efficiency definitions for representative equipment and units. T: Total exergy calculated as the sum of the CH: chemical and PH: physical exergy. cf. Figs. 4.1-4.11.

\begin{tabular}{lcc} 
& \multicolumn{3}{c}{ Exergy efficiency } \\
\hline Unit (Eq.) & $\begin{array}{c}\text { Input-Output } \\
\text { (a) }\end{array}$ & $\begin{array}{c}\text { Consumed-Produced } \\
\text { (b) }\end{array}$ \\
\hline $\begin{array}{c}\text { Combustion } \\
\text { Furnace } \\
(5.61)\end{array}$ & $\eta_{\text {Furnace }}=1-\frac{B_{\text {Dest, Furnace }}}{B_{\text {Fuel }}^{C H}}$ & $\eta_{\text {Furnace }}=\frac{B_{\text {Combustion }}^{\text {GH }}-B_{\text {Combustion }}^{\text {PH }}}{B_{\text {Fuel }}^{C H}}$ \\
\hline
\end{tabular}




\section{Exergy efficiency}

Unit (Eq.)

\section{Input-Output}

(a)

\section{Consumed-Produced}

(b)

\begin{tabular}{|c|c|c|}
\hline $\begin{array}{l}\text { Primary } \\
\text { reformer } \\
(5.62)\end{array}$ & $\eta_{1^{\circ} \text { Reformer }}=1-\frac{B_{\text {Dest }, 1^{\circ} \text { Reformer }}}{\left(\begin{array}{c}B_{1^{\circ} \text { Reformer }}^{T}+B_{\text {Feed }}^{T} \\
\text { Gases }\end{array}\right)}$ & 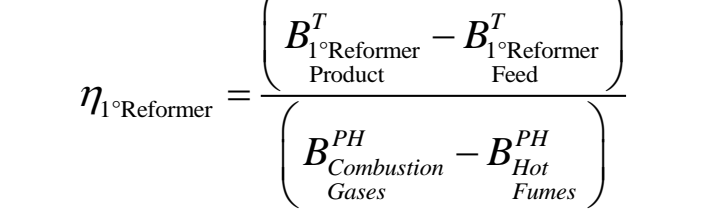 \\
\hline $\begin{array}{l}\text { Secondary } \\
\text { reformer } \\
(5.63)\end{array}$ & $\eta_{2^{\circ} \text { Reformer }}=1-\frac{B_{\text {Dest }, 2^{\circ} \text { Reformer }}}{\left(\begin{array}{c}B_{2^{\circ} \text { Reformer }}^{T}+B_{\text {Proed }}^{P H} \\
\text { Air }\end{array}\right)}$ & 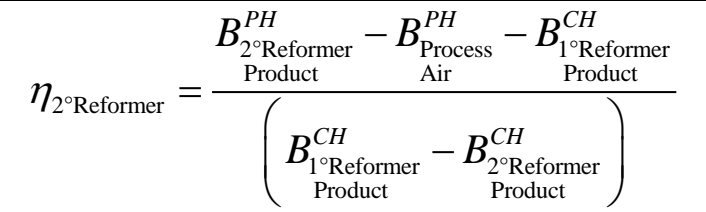 \\
\hline $\begin{array}{l}\text { High T Shift } \\
\qquad(5.64)\end{array}$ & $-\frac{B_{\text {Dest }, \text { HTS }}}{\left(B_{\text {HTS Feed }}^{T}\right)}$ & $=\frac{B_{\text {HT Shift }}^{P H}-B_{\text {HT Shift }}^{P H}}{\left(\begin{array}{c}\text { Feed } \\
B_{\text {HT Shift }}^{C H}-B_{\text {Feed }}^{C H} \\
\text { Product }\end{array}\right)}$ \\
\hline $\begin{array}{l}\text { Low T Shift } \\
\qquad(5.65)\end{array}$ & $\frac{B_{\text {Dest }, \text { LTS }}}{\left(B_{\text {LTS Feed }}^{T}\right)}$ & $=\frac{B_{\text {LT Shift }}^{P H}-B_{\text {LT Shift }}^{P H}}{\left(\begin{array}{c}\text { Feed } \\
\text { Product }\end{array}\right.}$ \\
\hline $\begin{array}{c}\text { Steam Boiler } \\
(5.66)\end{array}$ & $\eta_{\text {Boiler }}=1-\frac{B_{\text {Dest }, \text { Steam Boiler }}}{\left(\begin{array}{c}B_{2^{\circ} \text { Reformer }}^{T}+B_{B F W}^{T o t} \\
\text { Product }\end{array}\right)}$ & $\eta_{\text {Boiler }}=\frac{\left(B_{\text {Steam }}^{P H}-B_{B F W}^{P H}\right)}{\left(\begin{array}{c}B_{2^{\circ} \text { Reformer }}^{P H}-B_{\text {Product }}^{P H} \\
\text { Feed }\end{array}\right)}$ \\
\hline $\begin{array}{c}\text { Absorber } \\
(5.67)\end{array}$ & $\eta_{\text {Absorb }}=1-\frac{B_{\text {Dest, Absorber }}}{W_{\substack{\text { Solvent } \\
\text { Pump }}}+B_{\substack{\text { Raw } \\
\text { Syngas }}}^{T}+B_{\text {Lean }}^{T}}$ & 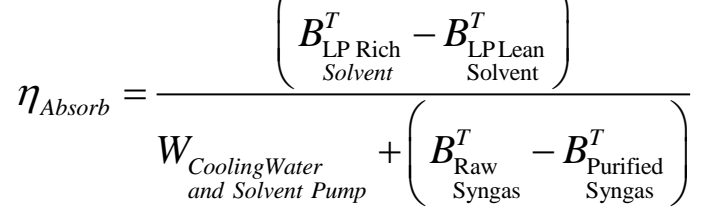 \\
\hline $\begin{array}{c}\text { Desorber } \\
(5.68)\end{array}$ & $\eta_{\text {Desorb }}=1-\frac{B_{\text {Dest, Desorber }}}{B_{\substack{\text { Desorber } \\
\text { Feed }}}+W_{\substack{\text { Cooling } \\
\text { Water }}}+B_{\substack{\mathrm{LP} \\
\text { Steam }}}^{P H}}$ & 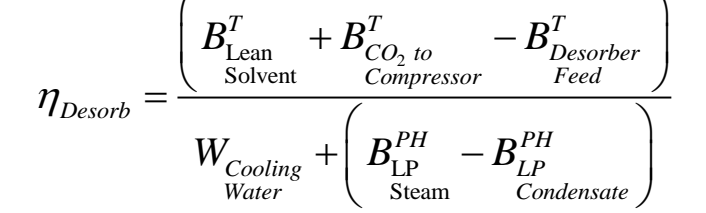 \\
\hline $\begin{array}{l}\text { Ammonia } \\
\text { converter } \\
(5.69)\end{array}$ & $\eta_{\substack{\text { Ammonia } \\
\text { Reactor }}}=1-\frac{B_{\text {Dest }, \text { Converter }}}{B_{\text {Reactor Feed }}^{T}}$ & $\eta_{\substack{\text { Reactor } \\
\text { Renmia }}}=\frac{B_{\text {Reactor Product }}^{P H}-B_{\text {Reactor Feed }}^{P H}}{\left(B_{\text {Reactor Feed }}^{C H}-B_{\text {Reactor Product }}^{C H}\right)}$ \\
\hline $\begin{array}{l}\text { Utility plant } \\
\qquad(5.70)\end{array}$ & $\eta_{\text {Utility }}=1-\frac{B_{\text {Dest }, \text { Utility }}}{B_{\text {HPSteam }}^{P H}+W_{\substack{\text { BFW } \\
\text { Pump }}}+W_{\substack{\text { Cooling } \\
\text { Water }}}}$ & 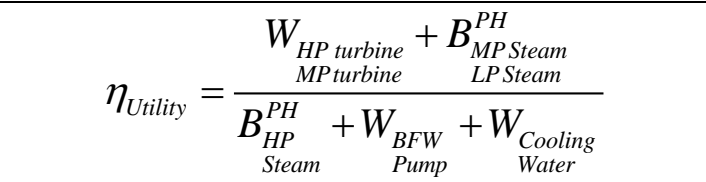 \\
\hline $\begin{array}{l}\text { Refrigeration } \\
\text { cycle } \\
(5.71)\end{array}$ & $\eta_{\text {Refrig }}=1-\frac{B_{\text {Dest }, \text { Refrigeration }}}{W_{\text {Compressor }}+W_{\substack{\text { Cooling } \\
\text { Water }}}+B_{\text {Evaporator }}^{Q}}$ & $\eta_{\text {Refrig }}=\frac{Q_{\text {Evaporator }}\left(T_{0} / T_{\text {Evaporator }}-1\right)}{W_{\text {Compressor }}+W_{\text {Cooling }}}=\frac{C O P_{\text {actual }}}{C O P_{\text {Carnot }}}$ \\
\hline
\end{tabular}




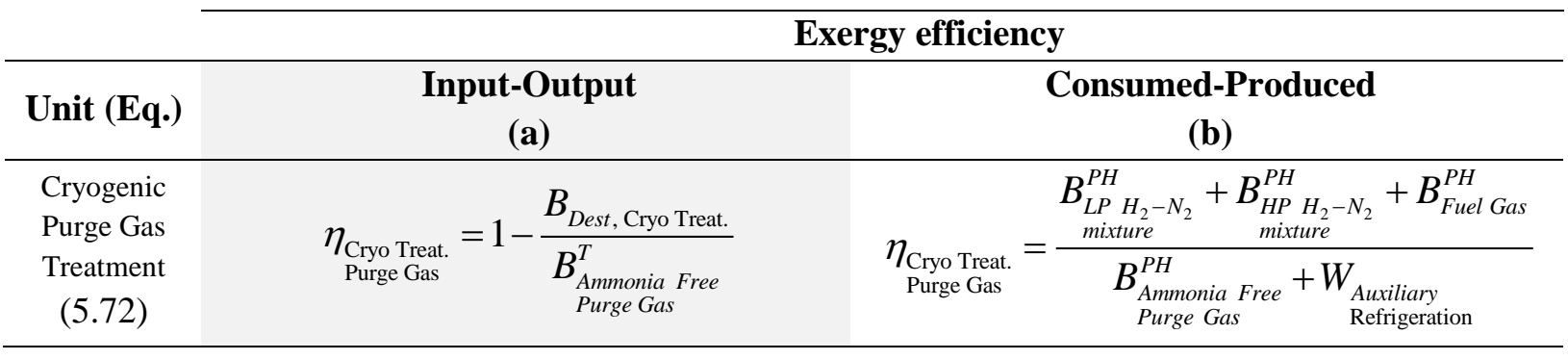

According to Eq.(5.61b), the combustion chamber aims to increase the physical exergy of the products at the expense of the consumption of a fraction of the chemical exergy of the fuel. This goal is also considered for other exothermic chemical reactors such as the secondary reformer, the high and low temperature shift reactors and the ammonia converter Eq. (5.63b5.65b, 5.69b) [69]. Actually, in the secondary reformer, the reactants increase their physical exergy by consuming a part of the chemical exergy in a partial oxidation process. Meanwhile, in the HTS and LTS reactors, the remaining carbon monoxide is consumed, increasing the physical exergy of the syngas, which is then used to preheat the boiler feedwater. For the sake of clarity, it must be warned about the impact of the terms subtracted in the numerators of Eqs. (5.61b), (5.63b-5.65b) and (5.69b), namely the physical exergy of the input streams. If those terms were considered as exergy inputs and added in the denominator, the calculated value of the exergy efficiency would be drastically modified, owed to the large transiting values of the input physical exergy compared with the difference of chemical exergy between the input and output streams.

On the other hand, according to Eq.(5.62), the endothermic reactions in the primary reformer aims to increase of the total exergy of the reactants at expense of the exergy decrease of the hot combustion gases. The combustion gases go through the externally fired reformer and release the excess exergy in the convection train. The exergy efficiency of the primary reforming obtained in this way is in agreement with the exergy efficiency calculated by other authors using various methodologies [7, 177].

Regarding the $\mathrm{CO}_{2}$ absorption unit, it is considered that the exergy of the rich solvent increases at the expense of the total exergy decrease of the purified syngas. Equation (5.67b) also includes the exergy losses due to the throttling of the solvent and the pumping required by the absorption process. Meanwhile, the desorption column is responsible for the separation of the chemical species at the expense of (i) the heat exergy supplied to the reboiler, and (ii) the utilities used in the reflux condenser and the cooling process of the $\mathrm{CO}_{2}$ captured. Therefore, the desorber product is the total exergy difference between the product and feed streams.

The efficiency of the refrigeration cycle, Eq. (5.71b), is calculated by considering the exergy of the heat removed at the evaporator temperature. Finally, due to the absence of chemical reactions in the purge gas treatment process along with an additional refrigeration demand, the exergy efficiency of the cryogenic unit, Eq.(5.72), is approximated as the ratio between the physical exergy of the separated products and the exergy fed to the system (ammonia free purge gas and power). Strinctly speaking, since a separation process is performed in the 
cryogenic purge gas treatment, chemical exergy is slightly affected. However, due the large values of transit chemical exergy involved, this variation can be considered as negligible, compared with the variation of physical exergy.

But, apart from its advantages for defining conversion efficiencies, the exergy concept can be also applied to perform the exergy costing and the partitioning of $\mathrm{CO}_{2}$ emissions. In fact, as long as the exergy stands for the useful energy required for an economic activity to be accomplished, it seems reasonable to evaluate the cost of the energy on the basis of the exergy content [178]. Furthermore, since exergy can be considered as a measure of the departure of the environmental conditions, it also serves as an indicator of environmental impact. Actually, exergy analysis can take into account both the performance of the supply chain (i.e. from primary exergy inputs up to the plant's gate) and the production process itself (e.g. an entire syngas and ammonia production plant) [179]. The discipline that combines the economy and exergy analyses is called exergoeconomy and can be used to rationally distribute the exergy costs and $\mathrm{CO}_{2}$ emissions among the several products and by-products of the chemical plant (hot water, steam, $\mathrm{CO}_{2}$ gas, fuel gas, electricity, etc.) [180].

Based on the theory of the thermoeconomy [147, 148, 181-185], also by considering the contribution of the exergy costs and $\mathrm{CO}_{2}$ emissions of the petroleum derivatives to the national energy matrix, a series of works including this thesis [186-190] have extended the analysis of the cumulative unit exergy cost to a variety of industrial facilities. The integrated system presented in Fig. 5.15 is proposed to calculate the cumulative exergy consumption of several number of products encompassing fuels, chemicals and transportation services [191] in petrochemical refineries, biorefineries (combined ethanol, sugar and electricity production mills as well as biodiesel plants) [190], fertilizers complexes (e.g. syngas and ammonia production chemical plant) [55] and last but not least, the Brazilian electricity mix [192]. In this thesis, the utilization of alternative energy resources, other than natural gas (e.g. bagasse and electricity from the grid), has been also considered through the process synthesis and optimization analyses, so that the optimal operation conditions in terms of exergy consumption and efficiency, operating cost and $\mathrm{CO}_{2}$ emissions can be determined. 
Fig. 5.15. Integrated system used for calculation of the cumulative exergy consumption and $\mathrm{CO}_{2}$ emission in the production of fuels, chemicals and transportation services, including synthetic nitrogen fertilizers (SNF).

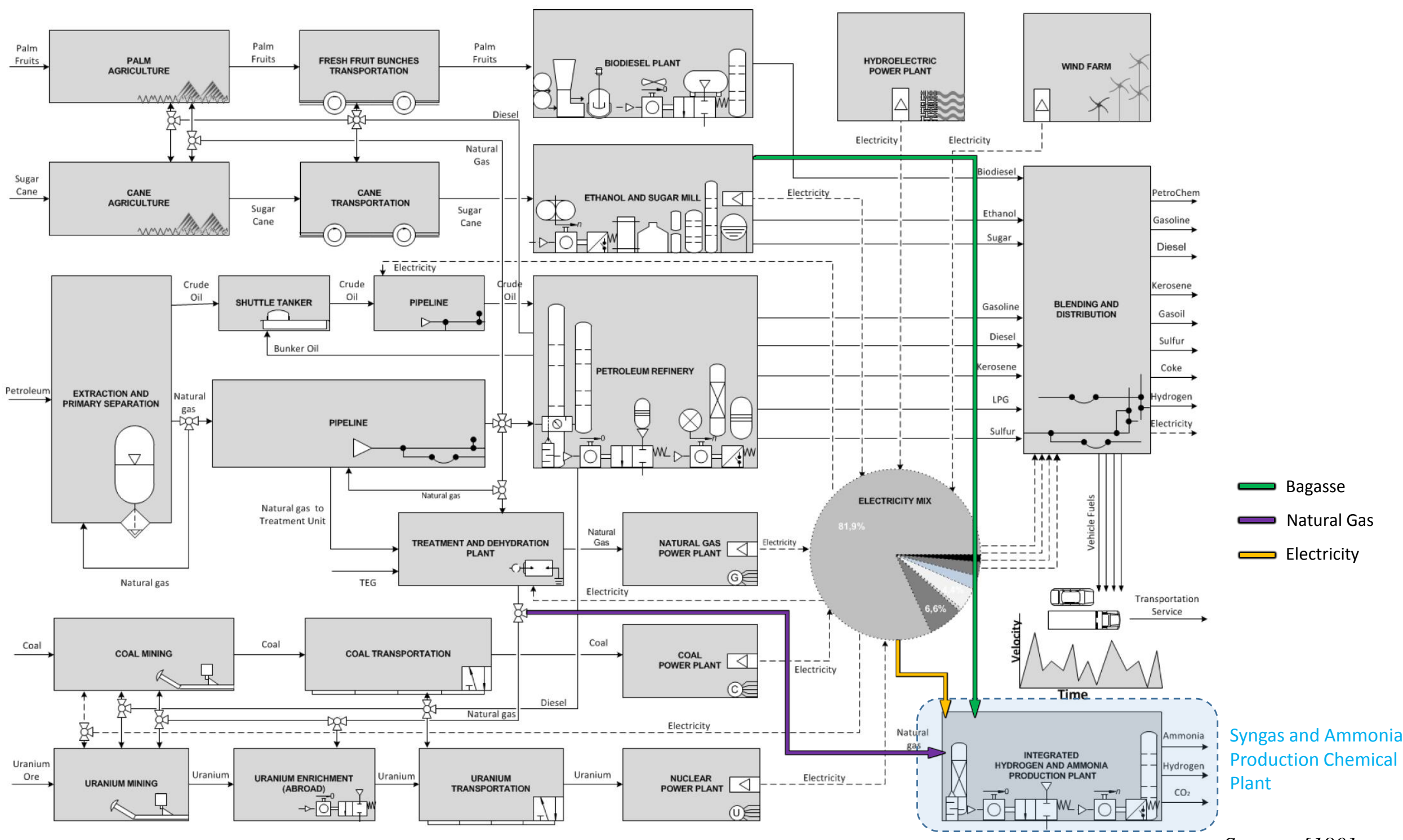

Source: [180]. 
Thus, motivated by the heterogeneity of the energy resources involved in the production of the SNF (see Fig. 5.15), a well-established approach [186-190] has been used to breakdown the total unit exergy cost into its renewable and non-renewable components. It also helps in the allocation of the internally and externally produced greenhouse gas emissions along the supply chains of fuels, chemicals and electricity, as explained next.

The Non-Renewable Unit Exergy Cost $\left(\mathrm{c}_{\mathrm{NR}}\right)[\mathrm{kJ} / \mathrm{kJ}]$ is defined as the rate of non-renewable exergy necessary to produce one unit of exergy rate (or flow rate) of a substance, electricity fuel, work or heat, whereas the Total Unit Exergy Cost $\left(\mathrm{c}_{\mathrm{T}}\right)$ comprises both the Renewable $\left(\mathrm{c}_{\mathrm{R}}\right)$ and the Non-Renewable Unit Exergy Costs. Analogously, the $\mathrm{CO}_{2}$ emission cost $\left(\mathrm{c}_{\mathrm{CO} 2}\right)$ $\left[\mathrm{g}_{\mathrm{CO} 2} / \mathrm{MJ}\right]$ is defined as the rate of $\mathrm{CO}_{2}$ emitted to obtain one unit of exergy rate (or flow rate). By considering a control volume enclosing each process unit (Fig. 5.16), the exergoeconomy balances of the total and non-renewable exergy costs can be written as in Eqs. (5.73-5.74):

$$
\begin{gathered}
\sum_{j} c_{T, P}^{j} B_{T, P}^{j}=\sum_{i} c_{T, F}^{i} B_{T, F}^{i} \\
\sum_{j} c_{N R, P}^{j} B_{N R, P}^{j}=\sum_{i} c_{N R, F}^{i} B_{N R, F}^{i}
\end{gathered}
$$

where $B$ stands for the exergy rate (or flow rate) of the inputs $(F)$ and products $(P)$ of the respective control volume. Similarly, the $\mathrm{CO}_{2}$ emission cost balances can be written as in Eq.(5.75), where the direct $\mathrm{CO}_{2}$ emissions, either produced by burning the fuel $i$ or arisen from the chemical reactions of the supplied fuels (e.g. steam reforming, gasification, shift), are accounted for in the $M_{C O 2, F}^{i}$ and $M_{C O 2, R x n}$ terms $\left[\mathrm{gCO}_{2} / \mathrm{s}\right]$, respectively:

$$
\sum_{j} c_{\mathrm{CO}_{2}, P}^{j} B_{T, P}^{j}=\sum_{i}\left(c_{\mathrm{CO}_{2}, F}^{i} B_{T, F}^{i}+M_{\mathrm{CO}_{2}, F}^{i}\right)+M_{\mathrm{CO}_{2}, \mathrm{Rxn}}
$$

It is worthy to notice that, in the case of the allocation of $\mathrm{CO}_{2}$ emissions, the initial input values for the specific $\mathrm{CO}_{2}$ emission must be considered as equal to zero (or a known value). This fact differs from the typical assumption of adopting the unity (or a known value from previous analyses) for the unit exergy cost of an external input entering the control volume.

Fig. 5.16. Control volumes used for the (a) Exergy flows and (b) Exergy and $\mathrm{CO}_{2}$ emission cost balances. Fuel inputs $(F)$, Product outputs $(P)$

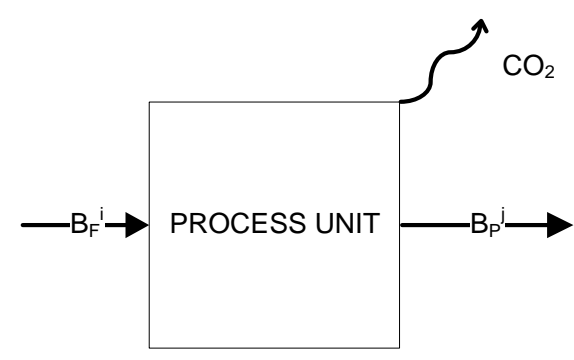

(a)

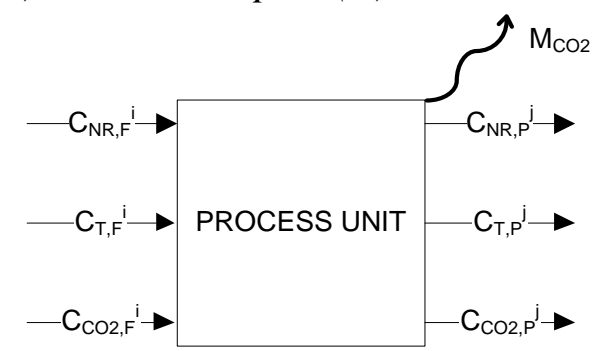

(b)

Source: Author.

However, whenever more than one product is produced in a single plant component or a set of them (e.g. steam and power in the utility system, or severa preheated streams in the heat recovery convection train of the primary reformer, HRCT), complementary equations to those 
given in Eqs.(5.73-5.75) are necessary. Accordingly, the following auxiliary equations are used to allocate the unit exergy costs and $\mathrm{CO}_{2}$ emissions among the different products.

- Auxiliary equations for the allocation of the unit exergy cost in the HRCT.

According to Fig. 5.17, natural gas is consumed as both fuel in the furnace and feedstock in the catalyst tubes of the primary reformer. The main purpose of the furnace is to supply the heat exergy necessary for the endothermic reactions in the primary reformer to proceed [148]. However, since the hot gases leaving the radiant section of the reformer $\left(B^{0}\right)$ still contain a huge amount of exergy, a modular heat recovery convection train (HRCT) becomes necessary in order to minimize the exergy losses associated to the stack emissions.

Fig. 5.17. Representation of the HRCT used for the unit exergy cost allocation.

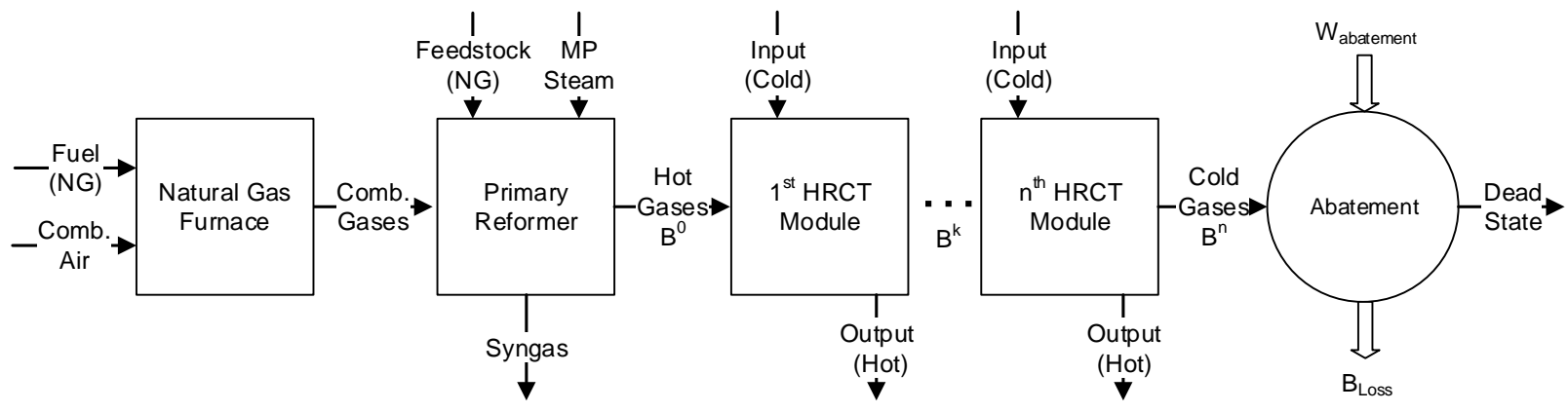

Source: Author.

On the one hand, since the flue stack gases released to the environment are no longer either thermodynamically or economically useful, the unit exergy cost and $\mathrm{CO}_{2}$ emissions cost attributed to them must be set to zero [182]. On the other hand, according to extraction criterion [182], if the whole or a fraction of the exergy input to a given module of the HRCT is equal to the exergy decrease of the hot gases that go through the respective module, then the unit exergy cost of those gases should remain constant along the entire module (i.e. $\left.c^{0}=\ldots=c^{k}=\ldots=c^{n}=\dot{C}^{n} / B^{n}\right)$. However, by carelessly applying this criterion, it results in cold rejected flue gas stream having a unit exergy cost greater than the unity (or equivalently, some exergy costs would remain unallocated to the HRCT products). Consequently, an incautious application of the extraction criterion may produce an artificially lowered cost for the products of the HRCT [193].

Meanwhile, if extraction criterion is still applied but now a zero-value cost is assigned only to the exhaust stream of the HRCT (i.e. $\dot{C}^{n}=0$ ), all the costs would be improperly discharged on the product of the last component of the HRCT (namely, the hot combustion air), penalizing so the combustion air preheating in a non-uniform way [194, 195]. To overcome this problem, some authors [196] proposed a monetary analysis $(\$ / \mathrm{kJ})$ in which the unit exergy cost of the hot combustion gases is allocated along the coils of a heat recovery steam generator (HRSG), linearly proportional to the exergy reduction in each coil. They have also imposed the condition that flue gases exiting the stack of the HRSG have a zero economic cost. However, differently from the monetary approach in which a unit exergy cost less than the unity is still physically meaningful, an stream with a unit exergy cost lower than the unity 
is physically senseless. The reason is that the exergy required to produce the stream cannot be lower than the exergy embodied in the stream itself.

Thus, aiming to deal with these shortcomings, an abatement fictitious unit (cf. Fig. 5.17) is proposed to calculate the cost of the exergy loss $\left(\dot{C}_{\text {Loss }}\right)$ when releasing the stack gases to the atmosphere, according to the exergoeconomy balance given in Eq. (5.76):

$$
\dot{C}_{\text {Loss }}+\dot{\ell}_{\text {Dead }}^{0}=c^{n} \cdot B^{n}+\dot{C}_{\text {abatement }}
$$

where the outlet cost flows of such fictitious unit consist of the cost of flue stack gases at absolute dead state $\dot{C}_{\text {Dead }}$ (thus, zero cost) and the cost of exergy loss. The term $\dot{C}_{\text {abatement }}$ considers the cost of the additional power required for the abatement of the conditions of the released gases, for instance, the power consumed in a post-combustion carbon capture and storage (CCS) unit (if applicable).

Moreover, in other to apportion the total cost of the exergy loss among the product of the $k$-th module of the HRCT ( $\dot{C}_{\text {Loss }}^{k}$ ), weighting criteria based on the exergy decrease of the hot gases along each module $k$, Eq.(5.77), can be adopted:

$$
\dot{C}_{\text {Loss }}^{k}=\frac{B^{k-1}-B^{k}}{B^{\text {Combustion Gases }}-B^{n}} \dot{C}_{\text {Loss }}
$$

In this way, neither the last module of the HRCT is overcharged with the total cost of the exergy loss, nor exergy costs are left unallocated to the final products of the HRCT.

- Auxiliary equations for the allocation of the $\mathrm{CO}_{2}$ emission cost in the HRCT.

Both natural gas burning and primary reforming process lead to a massive production of direct $\mathrm{CO}_{2}$ emissions, whose costs must be rationally distributed among the various products of the HRCT and the reforming unit. The shift reaction process also produces a large amount of $\mathrm{CO}_{2}$ emissions, but its allocation is more straightforward than in the two former cases. Moreover, none of the remaining units of the integrated syngas and ammonia production plant has associated direct $\mathrm{CO}_{2}$ emissions.

Although various approaches have been proposed to deal with allocation and taxation of emissions on the combined heat and power production (CHP) systems [197-199], they yield different results and, thus far, are not universally accepted or sometimes are inconsistent with thermoeconomy theories. Another particular mistake is the allocation of zero $\mathrm{CO}_{2}$ emissions to the so-called 'zero carbon fuels' (i.e. biomass), which clearly ignores the contribution of the fossil inputs at the upstream stages of their supply chains [188, 189]. A further drawback of the methods proposed so far is that they ignore the quality of energy and focus only on the quantities involved, overestimating the share of the emissions allocated to the electricity. For instance, some studies [199] have assumed that the amount of fuel required to generate each unit of electricity is as much as twice the required to generate each unit of heat by wrongly considering average First Law efficiencies of both the electricity generation (25-50\%) and the steam production in fired boilers (50-90\%). Consequently, the carbon intensity of the 
electricity is fixed at twice that of the steam, which misleads purchasers of steam, electricity (or even $\mathrm{CO}_{2}$ ) to wrongly believe they are trading with totally different carbon taxes. Other methods, although showing a sound mathematical reasoning, seem overly complex in notation, avoiding potential policy makers to be aware of their benefits. On the other hand, some works have focused on the distribution of the $\mathrm{CO}_{2}$ emissions among the various unit operations of the plant, instead of performing the cost allocation on its streams [200].

Accordingly, in this thesis, a proposed procedure is used for the allocation of the $\mathrm{CO}_{2}$ emissions, successfully applied to biomass and fossil fuels production processes [186, 187, 189], highly integrated electricity mixes [188] and comparative assessments of the end-use of vehicle fuels [201]. Figure 5.18 shows the representation of the heat recovery convection train (HRCT) used for the allocation of the $\mathrm{CO}_{2}$ emissions.

Fig. 5.18. Representation of the heat recovery convection train (HRCT) used for the allocation of the $\mathrm{CO}_{2}$ emissions.

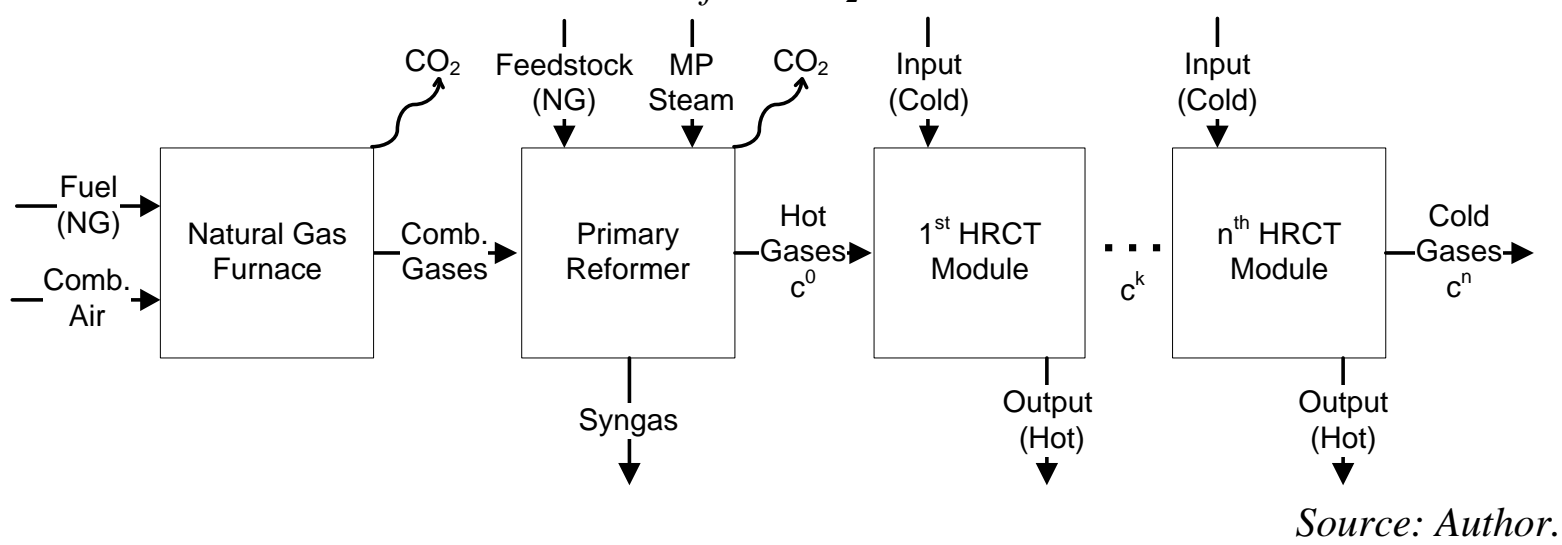

Since the specific $\mathrm{CO}_{2}$ emissions are given in a mass basis $\left(\mathrm{g}_{\mathrm{CO} 2} / \mathrm{kJ}\right)$, there are not restrictions regarding the minimum positive values that they can assume. Accordingly, a procedure analogous to that suggested by Borelli et al [196] for the allocation of the economic cost can be performed. Starting from the $\mathrm{CO}_{2}$ emission cost data of both natural gas (fuel) [189] and preheated combustion air, the $\mathrm{CO}_{2}$ emission cost of the gases leaving the combustion furnace ('Comb. Gases' in Fig. 5.18) can be calculated by using Eq.(5.75). Next, the previously calculated value along with Eq.(5.78) are used to calculate the specific $\mathrm{CO}_{2}$ emissions of the hot gases leaving the primary reformer $\left(c^{0}\right)$.

$$
c_{\mathrm{CO}_{2}}^{k}=\left(1-\frac{B^{k-1}-B^{k}}{B^{\text {Combustion Gas }}-B^{n}}\right) c_{C O_{2}}^{k-1}+\left(\frac{B^{n-1}-B^{n}}{B^{\text {Combustion Gas }}-B^{n}}-1\right) c_{C O_{2}}^{n-1}
$$

In Eq. (5.78), $k$ stands for the "following" hot gas stream (i.e. after the $k$-th module of the HRCT) and $k-1$ stands for the "preceding" one ( $k=1$ to $\mathrm{n}$ ). This procedure is iteratively done until the cost of the stack gases of the last component of the HRCT is determined $\left(c^{n}\right)$. The second term in the right hand side of Eq.(5.78) imposes the restriction that the $\mathrm{CO}_{2}$ emission cost of the exiting cold stack gases is null. Thus, the set of equations given by Eq.(5.75) together with the auxiliary Eqs.(5.78) allow calculating the specific $\mathrm{CO}_{2}$ emission associated to the products of the HRCT. 
Finally, it can be argued that the $\mathrm{CO}_{2}$ embodied in the rejected gases can be taken down to a minimal pollution potential in the environment, in analogy to the abatement unit of Fig. 5.17. Such mitigation process could be performed whilst consuming an additional amount of exergy, provided that it is technically and economically viable (capture, compression, injection). In that case, the additional consumptions and its related $\mathrm{CO}_{2}$ emissions will have to be accounted for too.

- Auxiliary equation for cost allocation in dissipative devices.

In the case of the dissipative components (Fig. 5.19), such as the vacuum condenser and other cooling water $(\mathrm{CW})$ exchangers, all the costs associated with their internal irreversibility and exergy consumption must be directly charged to the component(s) served by them [148, 202]. Since the unit exergy cost of the cooled stream remains constant through the dissipative components (due to the extraction criterion), the cost of the exergy loss is calculated as in Eq.(5.79):

$$
\dot{C}_{\text {Loss }}=c\left(B_{\text {Condensate }}^{\text {Hot }}-B_{\text {Condensate }}^{\text {Cold }}\right)+\dot{C}_{\text {abatement }}
$$

where $\dot{C}_{\text {abatement }}$ is the cost of the auxiliary exergy consumption required to operate the dissipative component (e.g., the pump and fan of the cooling tower), and $B_{\text {Condensate }}^{\text {Hot }}, B_{\text {Condensate }}^{\text {Cold }}$ are the exergy of the hot inlet and cold outlet condensate, respectively (Fig. 5.19):

Fig. 5.19. Cost allocation in dissipative components.

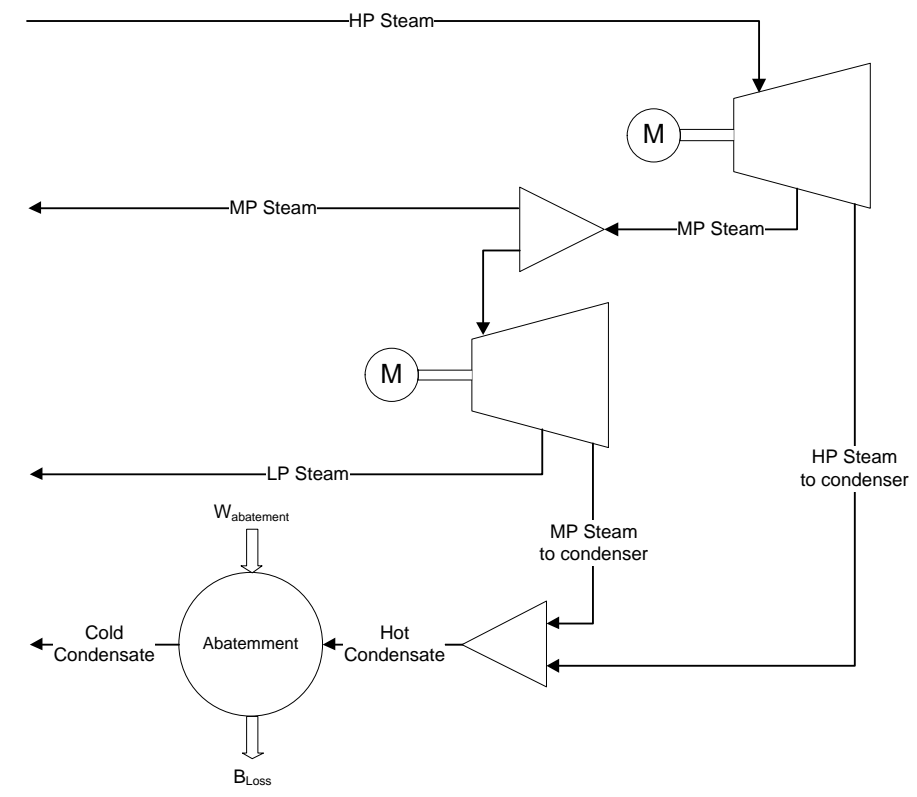

Source: Author.

Generally, the steam network condenser is regarded as a means to lower the output pressure of the steam turbine [203]. Thus, the cost of the exergy loss within this dissipative component is allocated to the products of the extraction high pressure (HP) and medium pressure (MP) steam turbines. The apportioning of the cost of the exergy loss among the products of both steam turbines is based on the amount of exergy dissipated in the condenser by each effluent 
of the HP and MP condensation steam turbines ( $B_{\text {to Condenser }}^{\text {From } H P}$ and $B_{\text {to Condenser }}^{\text {From } M P}$ ), as shown in Eqs.(5.80-5.81). These values are then considered as input costs in the cost balances of the respective steam turbine.

$$
\begin{gathered}
\dot{C}_{\text {Loss }}^{H P}=\frac{B_{\text {to Condenser }}^{\text {From } H P}}{\left(B_{\text {to Condenser }}^{\text {From } H P}+B_{\text {to Condenser }}^{\text {From MP }}\right)} \cdot \dot{C}_{\text {Loss }} \\
\dot{C}_{\text {Loss }}^{M P}=\frac{B_{\text {to Condensser }}^{\text {From MP }}}{\left(B_{\text {to Condenser }}^{\text {From } H P}+B_{\text {to Condenser }}^{\text {From }}\right)} \cdot \dot{C}_{\text {Loss }}
\end{gathered}
$$

Finally, the concept of energy integration introduced in the previous section can be extended to represent the exergy destruction in the heat exchanger network (HEN). In fact, when the vertical axis of the Grand Composite Curve GCC shown in Fig. 5.20a is substituted for the Carnot factor $\left(\theta=1-\mathrm{T}_{\mathrm{o}} / \mathrm{T}\right)$, the new representation obtained, shown in Fig. 5.20b, is called the Carnot Grand Composite curve (CGCC).

Fig. 5.20. Graphical representation of the exergy destruction in the HEN, (a) Grand Composite Curve GCC, (b-c) Carnot Grand Composite Curve, CGCC.

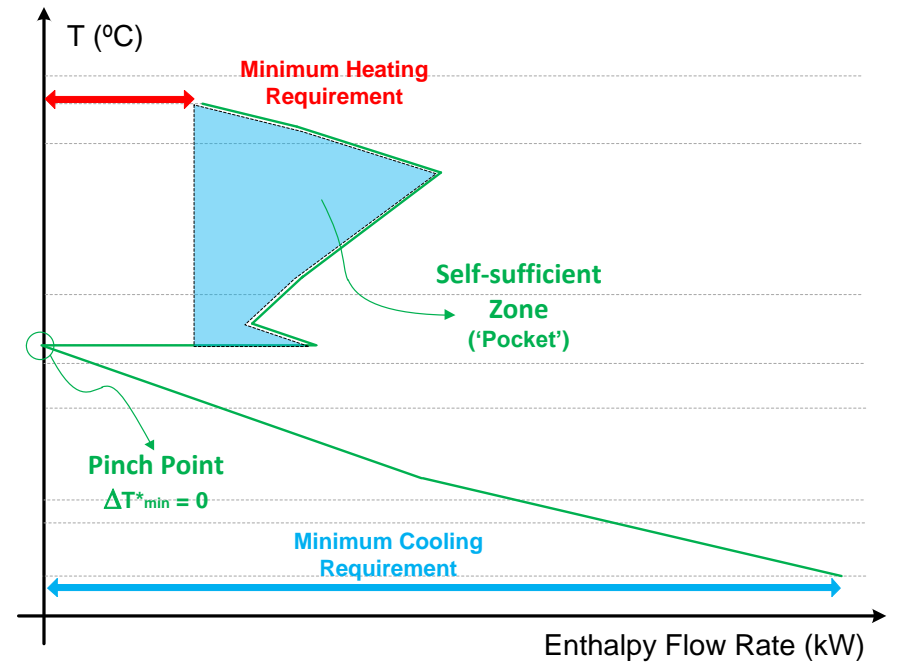

(a)

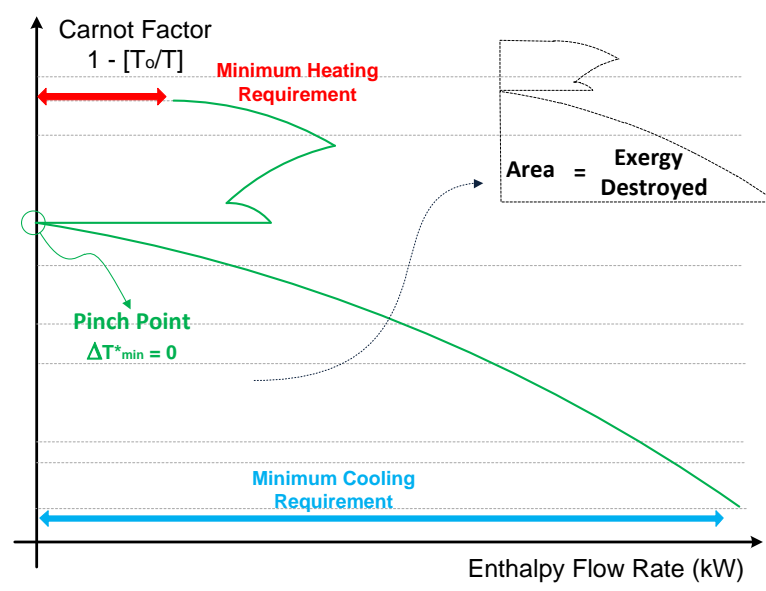

(b)

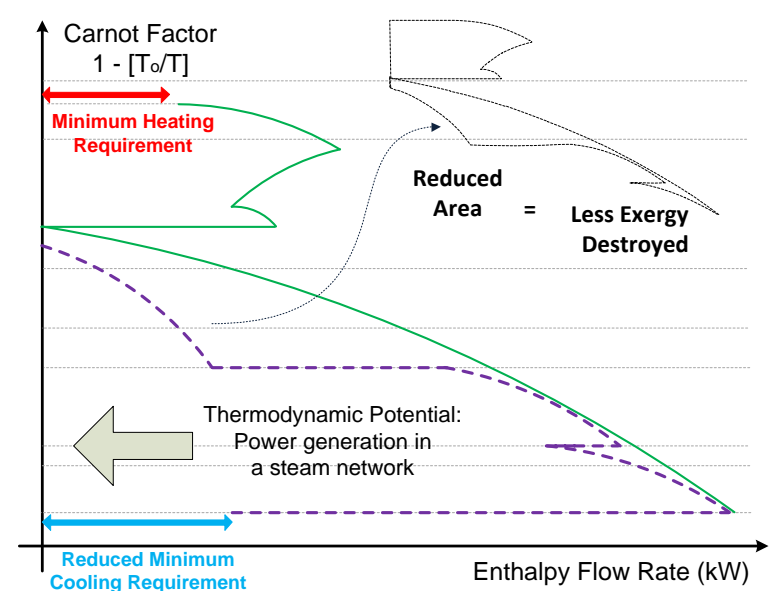

(c)

Source: Author. 
According to Fig. 5.20b, the area enclosed between the CGCC of the chemical process and the vertical axis can be interpreted as a metrics of the exergy destroyed in the HEN. It can be inferred by acknowledging that, for a given interval of temperature in the CGCC, a finite amount of heat cascaded is transferred from a higher temperature (source) to a lower temperature (sink). Thus, the exergy destroyed due to the heat transfer with finite difference of temperature can be estimated by the integral given in Eq.(5.82):

$$
B_{\text {Dest, HEN }}=\int_{C G C C}\left(1-\frac{T_{o}}{T}\right) d H
$$

The CGCC can be used to determine the thermodynamic potential for exploiting the selfsufficient zones (pockets) or profit from the low-grade heat available below the pinch point. For this reason, it proves to be useful to propose the integration of a series of energy technologies that allow to modify the area enclosed in the Fig. 5.20b, in order to reduce the irreversibility. For instance, the integration of an steam network to the chemical system may result in the (i) maximization the mechanical power generation, while (ii) reducing the exergy destroyed as well as the cooling requirements (Fig. 5.20c).

\subsubsection{Algorithmic methods}

In the previous sections, engineering heuristics and physical principles used to build feasible configurations of chemical plants were discussed. Despite the relevance of those methods, the configurations designed may still operate anywhere amidst the upper and lower bounds of the feasible space of the independent variables. Thus, it is highly likely that they at most represent local optimum operating conditions in terms of one or more performance criteria adopted.

In order to overcome this issue, other methods, based on algorithmic routines, aim to provide a more systematic framework that handles the synthesis of the process flowsheet as a mathematical programming problem [146]. Clearly, since those routines rely on multiple evaluations of the model parameters, constrains and performance indicators, a higher computational effort may be required. Additionally, there is still no full guarantee that the converged solutions correspond to global optima. Indeed, the optimality of the solution will be subject to the alternatives initially considered for building the search space. In the worst scenario, even unfeasible or unpractical solutions can be obtained [13]. Fortunately, finding a 'better' local optimal solution can be considered sufficient in many cases.

Anyway, the interest in the algorithmic methods has rapidly increased due to the mixed integer nonlinear modeling inherent to the chemical and industrial processes, also encouraged by the widespread utilization of commercial process simulators [204]. Reliable algorithmic methods must be (i) robust, i.e. their performance must be satisfactory for all reasonable choices of the initial variables; (ii) efficient, i.e. minimum computational time or storage is desired; and (iii) accurate, i.e. it should be able to identify a solution without being overly sensitive to truncation or round off errors [205]. 


\subsubsection{General definition of an optimization problem}

After the development and simulation of the model that represents as satisfactorily as possible the chemical process, various mathematical programming methods can be applied for optimizing the base-case configuration(s) obtained hitherto. The adopted method(s) will have to deal with both the choice of the operating parameters of the base-case design as well as the proposal of alternative setups and interconnections that optimize one or more targets of the chemical plant. Maximum profit, product yield, surplus electricity, heat recovery, return over investment and minimum exergy destruction, wastes and byproducts, energy consumption, GHG emissions, among others, are examples of targets that can be used to build the objective function, $f$, of the optimization problem given in Eq.(5.83), expressed in terms of the selected decision variables [149]:

$$
\text { Minimize or Maximize } f(x, y, z) \quad f: \mathbb{R}^{p} \rightarrow \mathbb{R}
$$

Subject to

$$
h_{i}(x, y, z)=0
$$

and

$$
g_{j}(x, y, z) \leq 0
$$

$h_{i}: \mathbb{R}^{p} \rightarrow \mathbb{R}$ with $i=1,2, \ldots, n$

$$
g_{j}: \mathbb{R}^{p} \rightarrow \mathbb{R}, \text { with } j=1,2, \ldots, m,
$$

in the region of feasible solutions,

$\Omega=\left\{(x, y, z) \in \mathbb{R}^{p}: h_{i}(x, y, z)=0, i=1,2, \ldots, n ; g_{j}(x, y, z) \leq 0, i=1,2, \ldots, m\right\}$

where:

$x$ is the set of the continuous real independent variables corresponding to design and operating parameters of the components of the chemical plant (mass flow, pressure, temperature, reaction rates, etc.)

$y$ is the set of the discrete independent variables used in the design optimization (nominal capacities, geometric standardized dimensions, etc.). The sizing and design correlations for the different components and equipment can be given by simple algebraic equations (explicit or implicit functions in terms of the parameters of the system) or in the form of complex partial differential equations [112]. In the latter case, quadrature methods such as orthogonal collocation could be required.

$z$ represents the set of the integer independent variables used to determine the structure of the optimal flowsheet (i.e. existence of selected components, interconnections, etc.) starting from a proposed superstructure [11-13]. Thus, only one variable of this kind is assigned to a component or stream, indicating whether the component exists in the optimal configuration or not. It may be binary $(0,1)$ or integer, with a 0 value indicating the lack of the component in the final flowsheet [111]. In this way, by using some techniques (e.g. Big-M, propositional logic, etc.), it is possible to control continuous or integer variables by using binary ones [99]. 
Furthermore, the search through the space of alternatives often requires to be subject to a set of equality or inequality constraints, imposed by the reliability, availability, maintenance, operability and environmental impact of the energy systems. Therefore,

$h_{i}(x)$ is the set of equality restrictions, which contains the equations derived from the conservation laws (mass, energy, momentum, cost) and the constitutive equations of the model, variable connections, correlations for physical and chemical property, and

$g_{j}(x)$ is the set of inequality restrictions, which contains upper and lower bounds for mass and heat transfer rates, temperatures, pressures, concentrations as well as limits for the environmental and safety-related parameters [147].

Appendix 1 briefly describes some of the algorithmic methods used in the process synthesis and optimization of the industrial production plants. It also discusses some of the advantages of the stochastic methods when compared to the deterministic search methods, especially when it comes to the optimization of highly non-linear chemical processes. In any case, the definition of the specific optimization problem and the algorithmic methods utilized are opportunely described for each scenario studied along the various chapters of this thesis.

\subsection{Summary of the systematic framework used for the chemical process synthesis.}

The systematic framework adopted for conducting the process synthesis of the existing and retrofitted ammonia production plants is graphically summarized in Fig. 5.21. Although the proposed methodology formerly proceeds through sequential steps of modeling, simulation and optimization of the chemical system, the adjustment of the model developed and the recursive evaluation of the objective function actually entail feedback interactions between those steps.

The methodology relies on the utilization of either heuristics, thermodynamics or algorithmic methods for identifying, hierarchizing and mitigating the main causes of the unsatisfactory performance, the latter being determined from the simulated baseline scenario. The combined application of these research methods capitalizes on both open source and licensed Computer Aided Process Engineering (CAPE) tools. These tools may include robust and efficient solvers for systems of algebraic and differential equations, optimization routines, together with user interfacing and visualization tools, thermophysical properties databases and software integration environments.

The proposed framework aims to reduce the chemical process inefficiencies and mitigate the environmental impact, while reducing the operating costs of complex chemical and industrial production routes, such as that illustrated in Fig. 5.22. To this end, the detailed optimization analyses focus on the main four plant sections, namely (A) the syngas purification unit, (B) the syngas production unit, $(C)$ the ammonia synthesis loop and (D) the cogeneration system. These analyses also consider the thermodynamic and environmental performance of the upstream supply chains of the process feedstock and examine the effect of the consumption of 
various energy resources on the thermodynamic, economic, environmental performance of the chemical plant.

Fig. 5.21. Graphical summary of the systematic framework used in the chemical process synthesis.

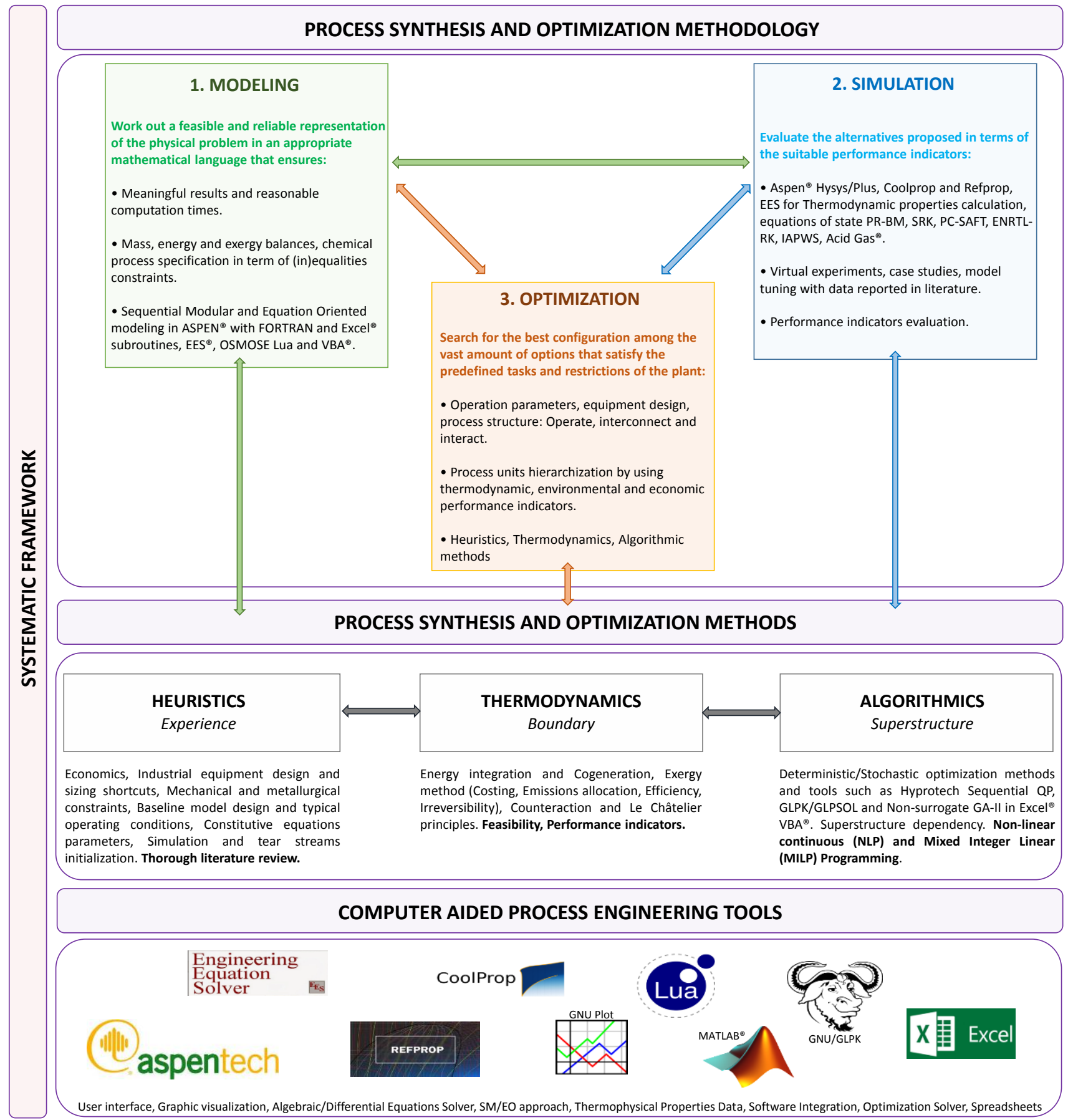

Source: Author 
Fig. 5.22. Chemical process overview: Extended, Plantwide and Detailed Layout.
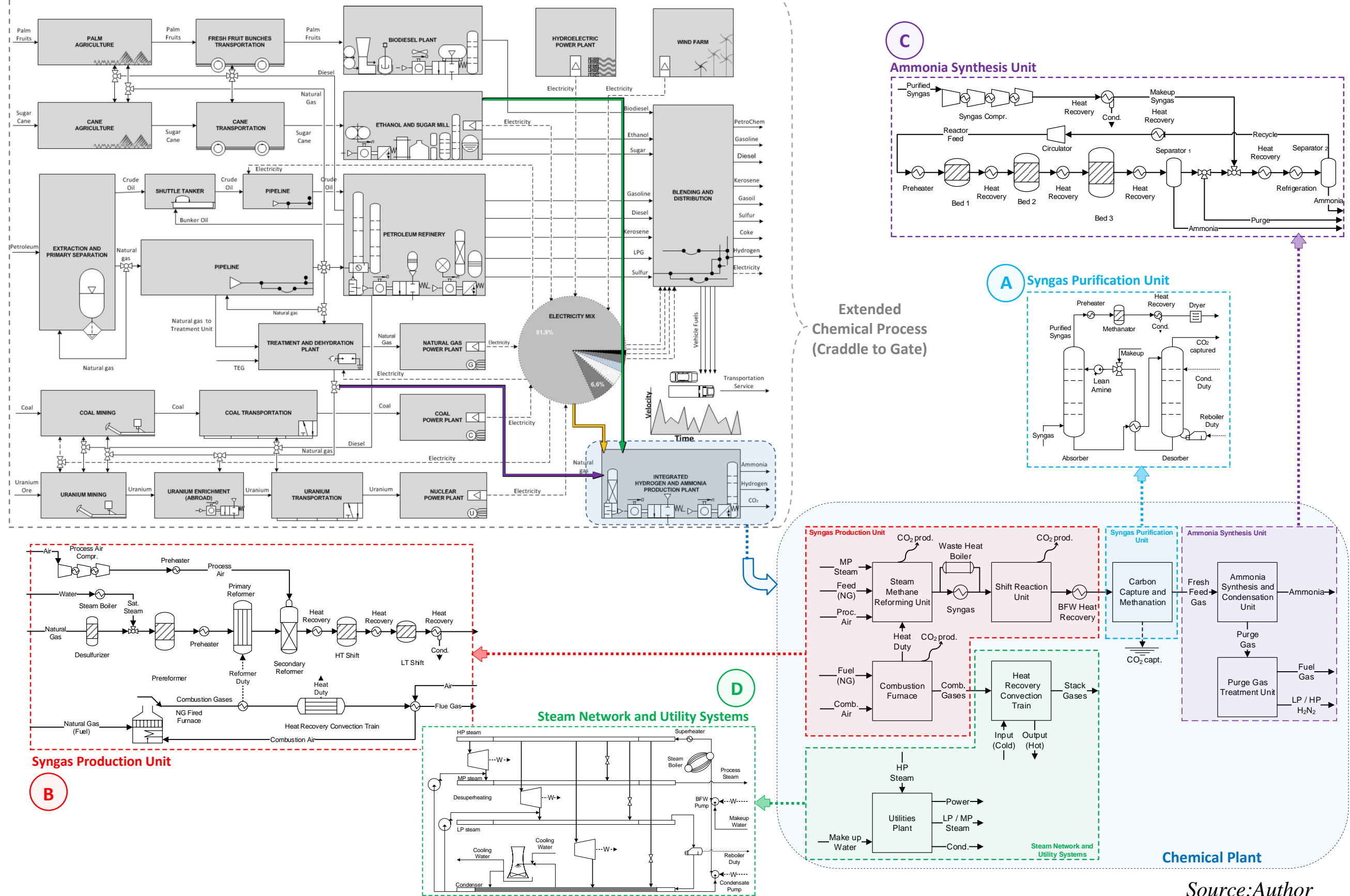

Source:Author 
The research questions and the suggested potential improvements in each section of the plant (cf. Fig. 5.22) that will be addressed in more detail in the following chapters of this thesis can be summarized as follows:

Section A. How does the selection of the syngas purification unit and the cogeneration system influence the overall energy integration and the plantwide performance (power and steam balance, overall $\mathrm{CO}_{2}$ emissions, operating costs)?

The syngas purification section is an energy intensive process that must treat the total amount of syngas produced in the plant frontend. Thus, it is expected to be a critical flow-through operation unit that largely influences the overall plant performance. For this reason, the effect of the selected syngas purification process in the choice and integration of the most suitable energy conversion technologies, as well as in the exergy consumption and the operating revenues of the chemical plant is initially studied. As a result, the utilization of physical solvents, electricity import and enhanced cogeneration cycles proves to be a useful pathway to improve the overall performance of the baseline, autonomous ammonia production plant.

Section B. How can the thermodynamic potential of the waste heat produced at higher temperatures be used to readily produce power and provide the required energy to the endothermic reactions, whereas increasing the pre-combustion carbon capture rate and the overall process efficiency of the frontend syngas production section?

The integration of breakthrough energy conversion systems may help thermally coupling and chemically recuperating the exothermic and endothermic enthalpy of reactions of the syngas production and treatment processes, thus reducing the fossil fuel consumption. Furthermore, these systems may be designed to satisfy the power demand by introducing concepts of more advanced cogeneration systems (e.g. steam injection, air enrichment, gas reheating and chemical recuperation). Moreover, since the proposed systems aim also to lessen the relatively large reactive and heat transfer driving forces, the amount of energy degradation that the bulky, externally fired primary reformers and furnaces are responsible for can be reduced or even avoided. This approach also poses an advantage in terms of environmental impact, as the flue stack gases originally released to the atmosphere can be appropriately embodied into the pressurized process gas, increasing the pre-combustion carbon capture and cutting down the energy consumption in the purification system.

Section C. How to deal with the clashing objectives of a reduced process irreversibility and increased reaction rates in reaction-separation-purge-recycle systems that present relatively low conversions? At which extent do the ammonia reactor performance and the operating pressure affect the overall loop performance? Does the introduction of an additional source of irreversibility such as a separate once-through converter help alleviating the intensive power demand and the overall process irreversibility in the backend of the ammonia plant?

Differently from the case of the the standalone optimization of the loop converter, the overall performance of the ammonia loop is rather a complicated function of the individual performance of the various loop components and the interrelation thereof. Fortunately, the integration of dual pressure reactive systems and cryogenic purge gas treatment processes is 
an interesting operational configuration that may lead to a considerable reduction of the energy consumption and the exergy destruction, especially as concerns the compression and refrigeration power demands in the high pressure ammonia loop, whereas bringing about increased process revenues.

Section D. Can the fossil energy consumption (and its associated $\mathrm{CO}_{2}$ emissions) in the utility systems and the chemical process be sharply reduced by totally or partially substituting the non-renewable energy resources required by the fertilizers plants? Could there be any operating cost benefits, or are the associated advantages limited to environmentally friendly outcomes?

In the last part of this thesis, it is demonstrated that better energy integration technologies that exploit the thermodynamic potential at higher temperatures and maximizes the waste heat recovery along the chemical plant, together with integrated biomass gasification processes and partial utilization of the largely renewable electricity mix may help coping with the large amount of atmospheric emissions and the dependence of costly non-renewable resources. This can be accomplished by profiting from the inexpensive biomass potential in tropical countries. However, due to the currently immature and low scale developments on the biomass energy conversion systems, the investment cost and risk as well as the economy of scale still pose important challenges to the massification of the use of alternative energy resources in the SNF industry for totally replacing the natural gas consumption. 


\section{CHAPTER 6}

\section{DIAGNOSTIC OF THE BASELINE SYNGAS AND AMMONIA PRODUCTION PLANT PERFORMANCE}

The detailed layout of the combined syngas and ammonia production plant shown in Fig. 6.1 is built on the process components described in more detail in the previous chapters. In this chapter, the typical operating figures pertaining to a base-case configuration with a throughput of $1000 \mathrm{t}_{\mathrm{NH}} 3 /$ day are briefly presented.

According to Fig. 6.1, the integrated syngas and ammonia production plant is composed of a frontend syngas production unit and an intermediate syngas purification system that supply the required feed to the backend ammonia synthesis, condensation and separation loop. The comprehensive steam network, whose connections are not shown, recovers the waste heat produced along the chemical plant, allowing for the reduction of the fuel consumption in the utility systems. The heat recovery convection train (HRCT), shown in Fig. 6.2, also plays a fundamental role in the improvement of the overall plant performance as it reduces the exergy losses to the environment from the stack. Thus, a high degree of energy integration between different thermochemical energy conversion processes of the complex ammonia production units come into sight, with pressures ranging from less than atmospheric (12 kPa) up to about $200 \mathrm{bar}$, as well as temperatures varying between $-198^{\circ} \mathrm{C}$ and $1100^{\circ} \mathrm{C}$. This clearly entails an interesting field of study for energy integration and exergy assessments, but also a challenging task, since the large chemical and physical driving forces mean higher rates of energy degradation along the industrial process.

Accordingly, a thorough description of the main energy and exergy consumption remarks of the baseline ammonia plant, namely the heating and cooling requirements and the overall power consumption, are discussed in the first part of this chapter. Next, the renewable and non-renewable unit exergy costs and the $\mathrm{CO}_{2}$ emissions are allocated among all the products of the plant by using the methodology proposed in Chapter 5. These emissions mainly arise from the combustion of natural gas in the furnace, as well as from the reforming and shift reactions in the frontend syngas production units. Withal, in light of the intricate interplay of the various process streams via the heat recovery network and the utility system, a major attention is given to the allocation of the unit exergy cost and $\mathrm{CO}_{2}$ emissions in the heat recovery convection train (HRCT) of the primary reformer. Last but not least, the analysis of the exergy efficiency and the exergy destruction at both single component and plantwide levels is used to spotlight the most critical components in terms of exergy destruction. 
Fig. 6.1. Detailed layout of the baseline syngas and ammonia production plant. cf. Table 6.2 for stream properties.

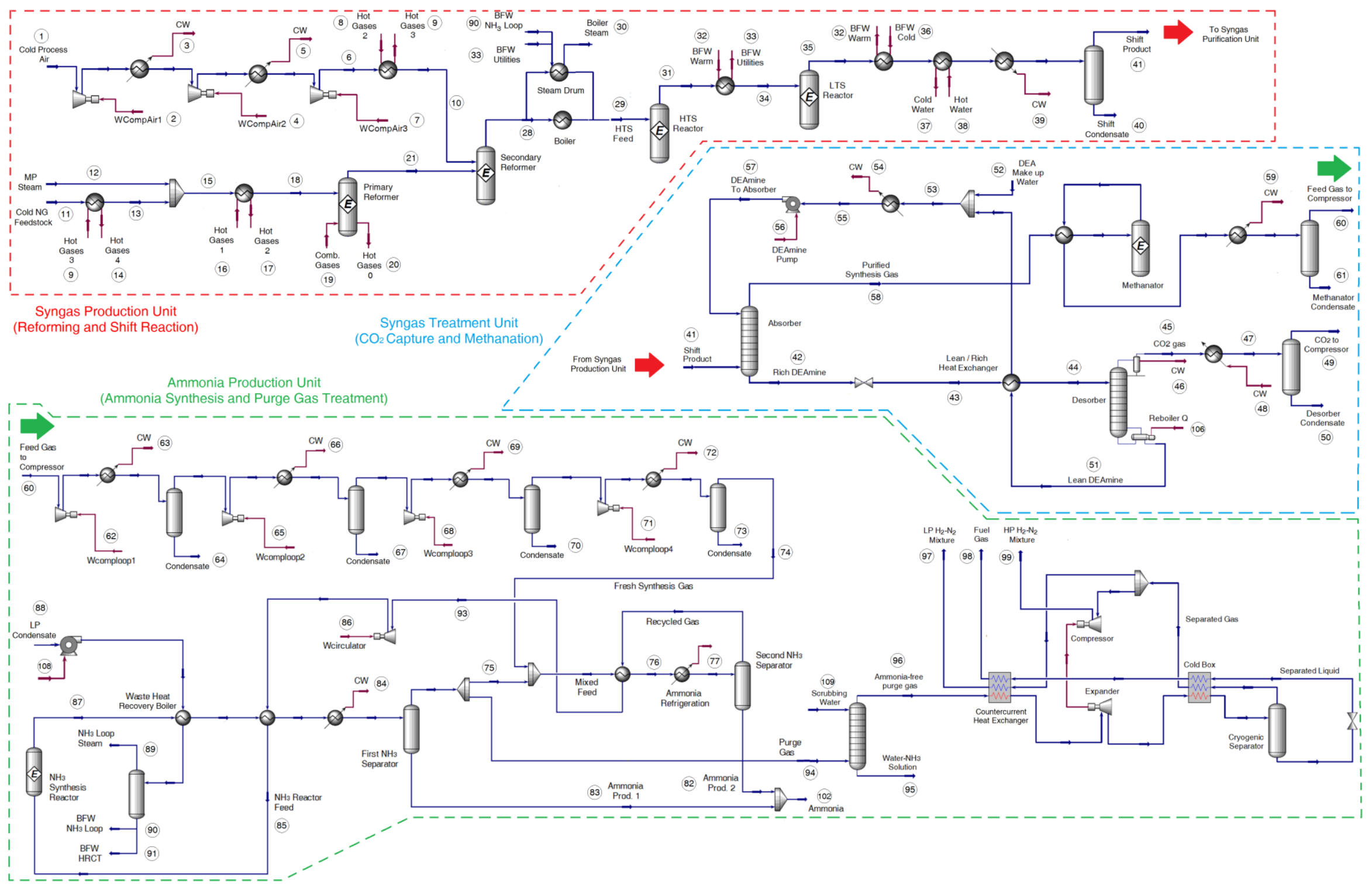




\subsection{Energy and exergy consumption remarks}

In the conventional SMR process, about $20-30 \%$ of the total supply of natural gas is burnt as fuel in the fired furnace, whereas the balance is consumed as feedstock in both primary and secondary reformers [22]. Indeed, the primary reformer is the most exergy-intensive processes of the plant, requiring a heat exergy duty of $54,630 \mathrm{~kW}$ at about $800^{\circ} \mathrm{C}$, provided by a highgrade hot utility in the radiant box [55]. Additionally, since the flue gases leaving the furnace still have as much as half of their total exergy, it must be further recovered in the heat recovery convection train (HRCT) shown in Fig. 6.2 [55].

Figure 6.2. Heat recovery convection train (HRCT) of the steam methane reformer. See Table

\section{1 for streams properties}

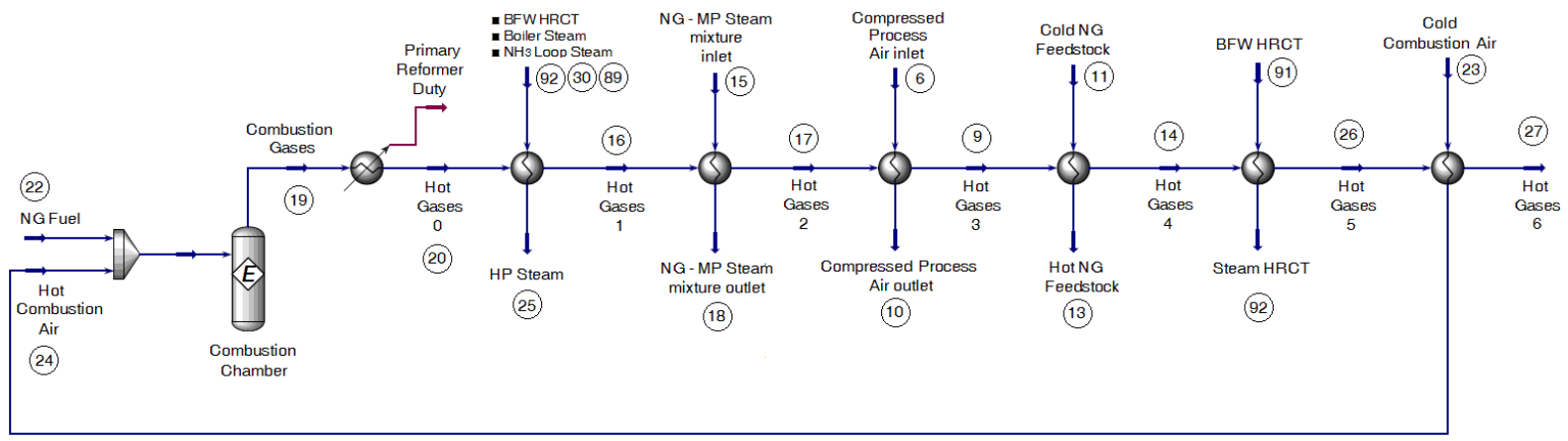

Source: Author.

Table 6.1 summarizes the process streams data obtained for the heat recovery convection train (HRCT) of the primary reformer, shown in Figure 6.2 by using the cost balances, Eqs.(7.37.5) and the auxiliary equations for cost allocation given by Eqs.(7.7-7.8).

Table 6.1. Process data of the heat recovery convection train of the primary reformer; cf. Fig. 6.2 for stream number

\begin{tabular}{|c|c|c|c|c|c|c|c|c|c|}
\hline $\mathbf{N}^{\mathbf{o}}$ & Module & & $\begin{array}{c}\mathbf{n} \\
(\mathbf{k m o l} / \mathbf{h})\end{array}$ & $\begin{array}{c}\mathbf{T} \\
\left({ }^{\circ} \mathbf{C}\right)\end{array}$ & $\begin{array}{c}\mathbf{P} \\
\text { (bar) }\end{array}$ & $\begin{array}{c}\mathbf{B} \\
(\mathbf{k W})\end{array}$ & $\begin{array}{c}\mathbf{c}_{\mathbf{T}} \\
(\mathbf{k J} / \mathbf{k} \mathbf{J})\end{array}$ & $\begin{array}{c}\mathbf{c}_{\mathrm{NR}} \\
(\mathbf{k J} / \mathbf{k J})\end{array}$ & $\begin{array}{c}\mathrm{c}_{\mathrm{CO} 2} \\
\left(\mathrm{~kg}_{\mathrm{CO} 2} / \mathrm{MJ}\right)\end{array}$ \\
\hline $92,30,89$ & \multirow{2}{*}{$\begin{array}{c}\text { HP steam } \\
\text { superheater }\end{array}$} & inlet & \multirow{2}{*}{10,390} & 310 & 100 & 60,451 & 1.5940 & 1.5260 & 0.0518 \\
\hline 25 & & outlet & & 460 & 100 & 72,505 & 1.7170 & 1.6450 & 0.0524 \\
\hline 15 & \multirow{2}{*}{$\begin{array}{l}\text { Primary reactor } \\
\text { feed preheater }\end{array}$} & inlet & \multirow{2}{*}{4,800} & 361 & 35 & 299,844 & 1.2530 & 1.2030 & 0.0109 \\
\hline 18 & & outlet & & 580 & 35 & 307,714 & 1.2720 & 1.2210 & 0.0115 \\
\hline 6 & \multirow{2}{*}{$\begin{array}{c}\text { Process air } \\
\text { preheater }\end{array}$} & inlet & \multirow{2}{*}{1,690} & 197 & 35 & 4,673 & 3.0980 & 2.9580 & 0.0943 \\
\hline 10 & & outlet & & 540 & 35 & 7,332 & 2.7390 & 2.6190 & 0.0738 \\
\hline 11 & \multirow{2}{*}{$\begin{array}{l}\text { NG feedstock } \\
\text { preheater }\end{array}$} & inlet & \multirow{2}{*}{1,200} & 25 & 35 & 280,084 & 1.1780 & 1.1310 & 0.0071 \\
\hline 13 & & outlet & & 400 & 35 & 282,258 & 1.1910 & 1.1430 & 0.0073 \\
\hline 91 & \multirow{2}{*}{$\begin{array}{l}\text { BFW heat } \\
\text { recovery }\end{array}$} & inlet & \multirow{2}{*}{1,646} & 310 & 100 & 4,123 & 1.8090 & 1.7240 & 0.0888 \\
\hline 92 & & outlet & & 310 & 100 & 9,577 & 1.8550 & 1.7740 & 0.0446 \\
\hline 23 & \multirow{2}{*}{$\begin{array}{c}\text { Combustion air } \\
\text { preheater }\end{array}$} & inlet & \multirow{2}{*}{5,403} & 25 & 1 & 0 & 1.0000 & 0 & 0 \\
\hline 24 & & outlet & & 310 & 1 & 4,054 & 1.8940 & 1.7800 & 0.0047 \\
\hline
\end{tabular}


Table 6.1 (cont'd.). Process data of the heat recovery convection train of the primary reformer

\begin{tabular}{ccccccccc}
\hline $\mathbf{N}^{\mathbf{0}}$ & Stream & $\begin{array}{c}\mathbf{n} \\
(\mathbf{k m o l} / \mathbf{h})\end{array}$ & $\begin{array}{c}\mathbf{T} \\
\left({ }^{\circ} \mathbf{C}\right)\end{array}$ & $\begin{array}{c}\mathbf{P} \\
(\mathbf{b a r})\end{array}$ & $\begin{array}{c}\mathbf{B} \\
(\mathbf{k W})\end{array}$ & $\begin{array}{c}\mathbf{c}_{\mathbf{T}} \\
(\mathbf{k J} / \mathbf{k J})\end{array}$ & $\begin{array}{c}\mathbf{c}_{\mathbf{N R}} \\
(\mathbf{k J J} / \mathbf{k J})\end{array}$ & $\begin{array}{c}\mathbf{c}_{\mathbf{C O 2}} \\
(\mathbf{k g} \mathbf{C O 2} / \mathbf{M J})\end{array}$ \\
\hline 20 & Hot Gases 0 & 5,919 & 1287 & 1 & 46,953 & 1.5890 & 1.5250 & 0.0254 \\
\hline 16 & Hot Gases 1 & 5,919 & 933 & 1 & 29,540 & 1.5890 & 1.5250 & 0.0177 \\
\hline 17 & Hot Gases 2 & 5,919 & 714 & 1 & 19,953 & 1.5890 & 1.5250 & 0.0130 \\
\hline 9 & Hot Gases 3 & 5,919 & 628 & 1 & 16,487 & 1.5890 & 1.5250 & 0.0096 \\
\hline 14 & Hot Gases 4 & 5,919 & 525 & 1 & 12,667 & 1.5890 & 1.5250 & 0.0063 \\
\hline 26 & Hot Gases 5 & 5,919 & 325 & 1 & 6,300 & 1.5890 & 1.5250 & 0.0030 \\
\hline 27 & Hot Gases 6 & 5,919 & 82 & 1 & 1,651 & 1.5890 & 1.5250 & 0 \\
\hline
\end{tabular}

Figures 6.3a-b illustrate the variation of the temperature and enthalpy flow rate along the HRCT in the $T$ vs. $H$ and the Carnot factor vs. $H$ diagrams. The heat recovery process includes the preheating of the combustion air (balloon 7, Fig. 6.3), the saturated (balloon 8) and superheated (balloon 12) steam generation, the preheating of the reactants (balloons 9,11) and the process air (balloon 10) fed to the primary and secondary reformer, respectively.

Fig. 6.3. Heat recovery convection train of the primary reformer of a $1000 \mathrm{t} /$ day ammonia plant. Balloon: 1-6. Flue gases, 7. Combustion air preheating, 8. Steam saturation, 9. Natural gas preheating, 10. Process air preheating, 11. Reformer feed preheating, 12. Steam superheating. (Cont'd page 82)

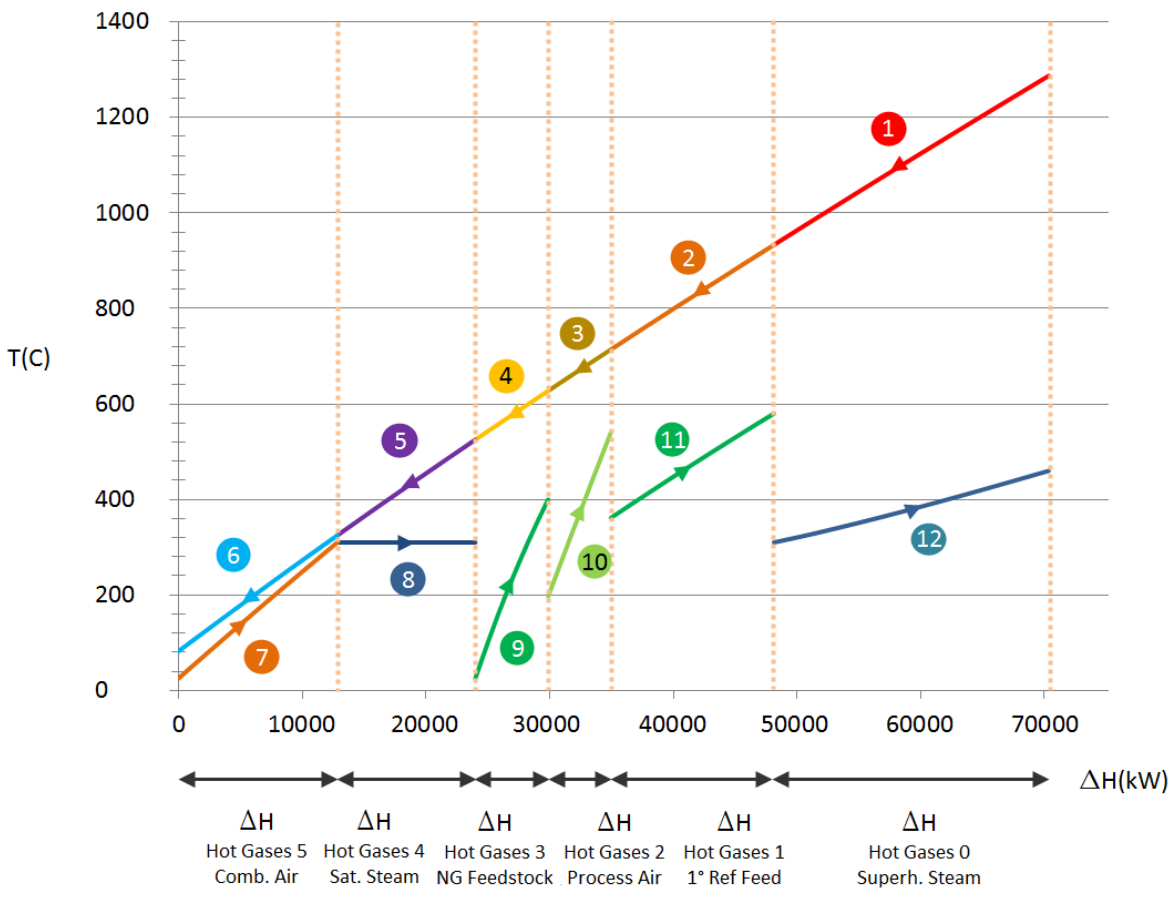

(a) T-H diagram. Minimum temperature approach $=14^{\circ} \mathrm{C}$ 


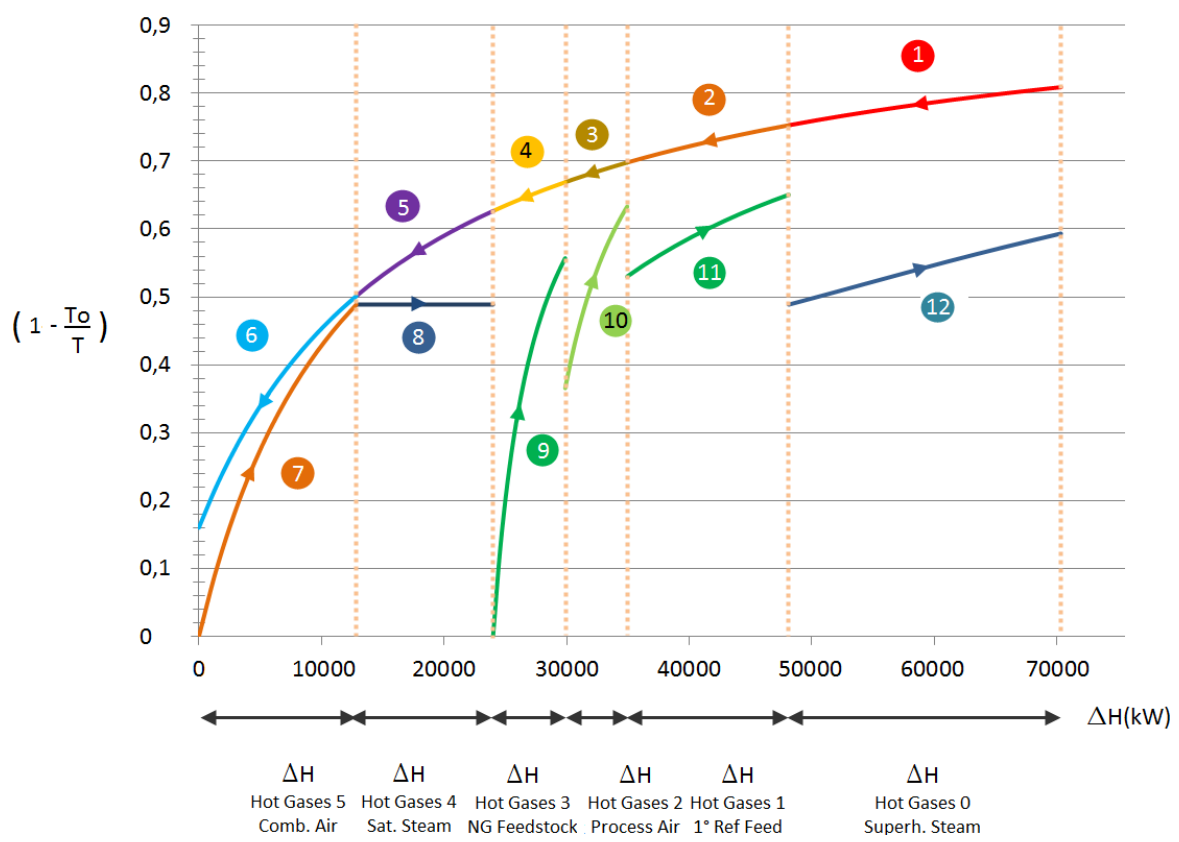

(b) Carnot factor-H diagram ( $T$ in $K$, and $T_{o}=298.15 K$ ).

Source: Author.

According to Fig. 6.3, the highest driving forces triggered by the large temperature difference in the HRCT come mainly from the steam superheating and the reactants preheating. This is explained by the metallurgical and mechanical restrictions that limit the increase of the pressure of the superheated steam. Furthermore, depending on the presence of a pre-reformer, the maximum allowable preheating temperature of the reformer reactants is limited to 550$650^{\circ} \mathrm{C}$. The reason is the increased risk of cracking of the hydrocarbons when natural gassteam mixtures are heated above $650^{\circ} \mathrm{C}$. Thus, cracking also limits the temperature at which the exergy heat is recovered [25]. According to some authors, the use of a gas heated prereformer in combination with an autothermal reformer would allow sparing the use of an externally fired tubular reformer. Hence, it may represent a potential strategy to reduce the natural gas fuel consumption and debottleneck the size of the reforming section [81].

As concerns the syngas purification unit, the major part of the $\mathrm{CO}_{2}$ gas is chemically absorbed into the DEA solvent (99.7\%). The desorption of $\mathrm{CO}_{2}$ requires a large amount of heat $(47,870$ $\mathrm{kW})$ supplied to the reboiler by means of low pressure steam ( $7 \mathrm{bar}, 0.1 \mathrm{~kg}_{\text {Steam }} / \mathrm{L}_{\mathrm{DEA}}$ Solution $)$. Together, the $\mathrm{CO}_{2}$ emissions of the desorber vent (69\%) and the reformer stack (21\%) achieve $1,757.5 \mathrm{t}_{\mathrm{CO} 2} /$ day (or $1.76 \mathrm{t}_{\mathrm{CO} 2} / \mathrm{t}_{\mathrm{NH} 3}$ ). This is close to $1.87 \mathrm{t}_{\mathrm{CO} 2} / \mathrm{t}_{\mathrm{NH} 3}$ reported in [33], with $\mathrm{CO}_{2}$ typically compressed and dehydrated for urea production. However, these figures will be later revisited in light of the exergoeconomy theory and considering ammonia byproducts (e.g. $\mathrm{CO}_{2}$ gas) as also carrying with associated $\mathrm{CO}_{2}$ emissions. Moreover, it is worthy to notice that the calculated reboiler duty by using DEA $\left(3.41 \mathrm{MJ} / \mathrm{kgCO}_{2}\right)$ is slightly lower compared with MEA (typically $>4 \mathrm{MJ} / \mathrm{kgCO}_{2}$ ) partly explained by the lower enthalpy of reaction and higher solvent loads attainable with the former $[25,89]$.

Despite of the improved exergy consumption of the DEA system, the syngas purification unit still presents the largest share of consumption of cooling utility, specifically in the lean 
ammonia cooler and the desorber condenser. According to Fig. 6.4, these two processes together achieve $41 \%$ of the total cooling duty of the plant. That value is comparable to the amount of waste heat released to the environment through the condenser of the CHP system. For the sake of comparison, only one fourth of the overall cooling duty is used in the ammonia synthesis loop. Yet, although a large amount of waste heat is rejected for ammonia condensation purposes, a considerable portion of the exergy heat has been already recovered in the form of steam, reducing so the avoidable losses.

A mechanical draft cooling tower supplies the cooling water to the steam network condenser as well as to the ancillary coolers in a closed circulating system. Cooling tower power consumption including pumps and fans is estimated as $833 \mathrm{~kW}$ [207], whereas total heat rejection (energy basis) is calculated as $117,165 \mathrm{~kW}$ [207]. Total volumetric water circulation rate achieves approx. $7,636 \mathrm{~m}^{3} / \mathrm{h}$. Meanwhile, the water make up due to drift, evaporation and blown down is estimated as $2.74 \%$ of the overall rate of circulating water. The process condensates are treated and recovered to the water system $\left(15,162 \mathrm{~kg} / \mathrm{h}\right.$ at $\left.35^{\circ} \mathrm{C}\right)$.

Fig. 6.4. Distribution of the cooling requirement between major users by process. Total cooling duty: 117,165 kW (energy basis), for a 1000 t/day ammonia plant.

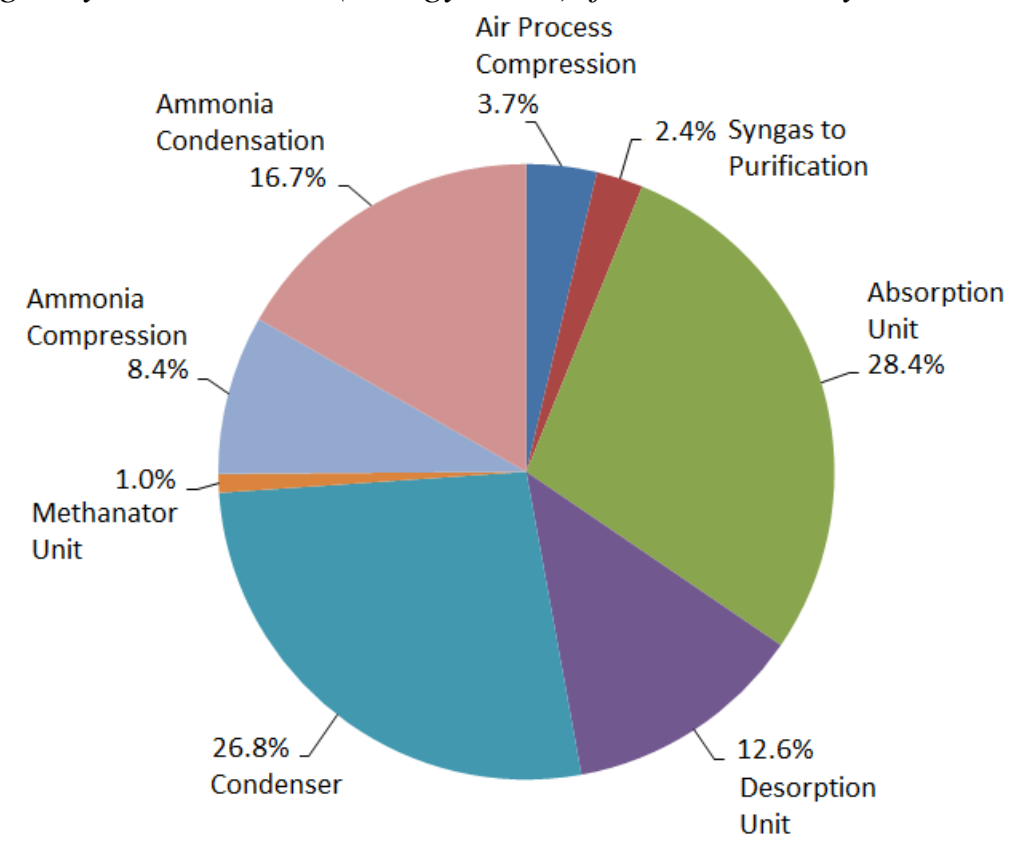

Source: Author.

Figure 6.5 shows the distribution of the main power consumers and producers of the plant. For a typical $1000 t_{N H 3} /$ day plant, the total power consumption achieves $22,617 \mathrm{~kW}$ out of which $42 \%$ is consumed by the energy-intensive syngas compression, followed by the process air compression $(28 \%)$ and the ammonia refrigeration cycle (12\%). The remaining power is used throughout the plant to drive various pumps and compressors. A small surplus power $(4.2 \%)$ can be sold to the grid or used in associated urea plants. 
Fig. 6.5. Distribution of the power generation and utilization amongst the major users by process. Total power production: $23,603 \mathrm{~kW}$.

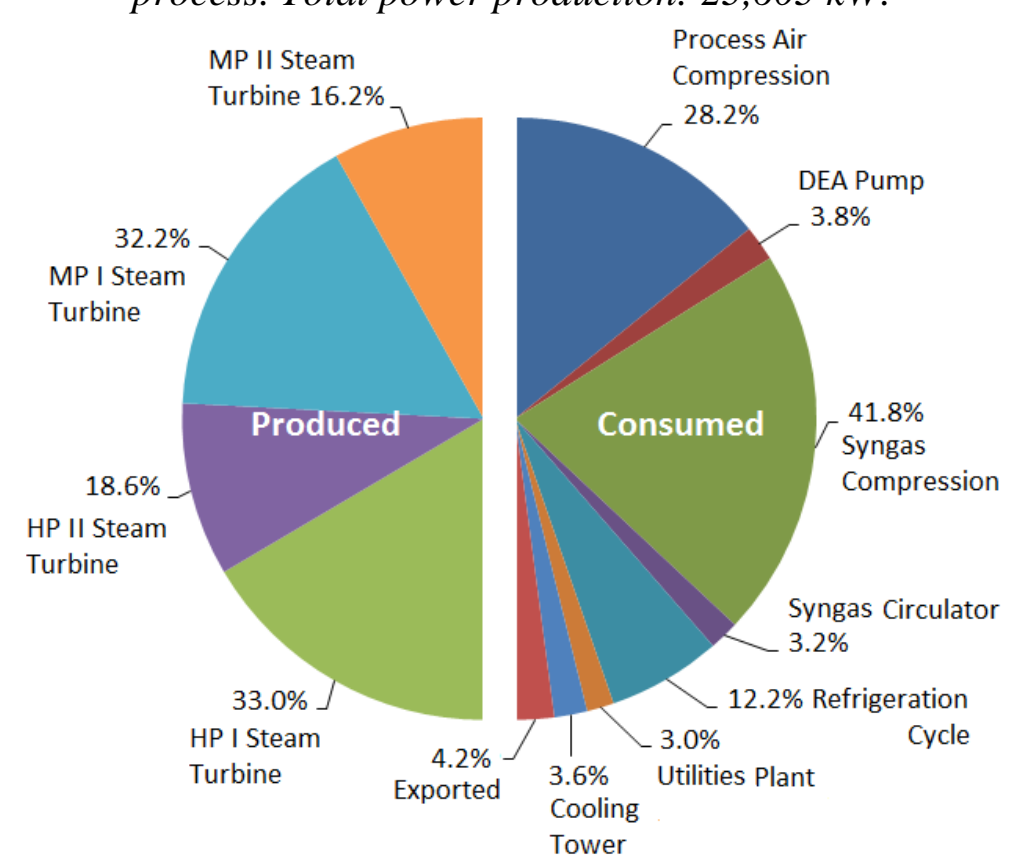

Source: Author.

Figure 6.6 compares two refrigeration systems proposed in which the discharged gas from the medium pressure stage compressor is desuperheated by direct contact with vapor or liquid refrigerant. In case (b), medium pressure discharge vapor is mixed with the flashed vapor, instead of passing through the flash tank, which results in a slightly superheated inlet condition at the high pressure discharge. Since cooling water duty and power consumption are higher in case (b), thus, case (a) has been adopted.

Fig. 6.6. Multistage ammonia refrigeration systems with a flash tank operating (a) as an intercooler and (b) as a vapor separator only.

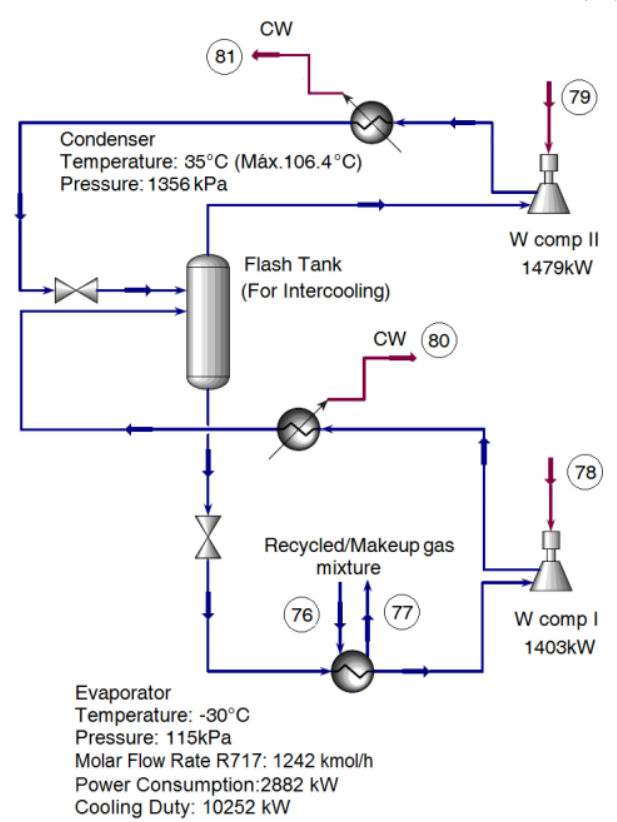

(a)

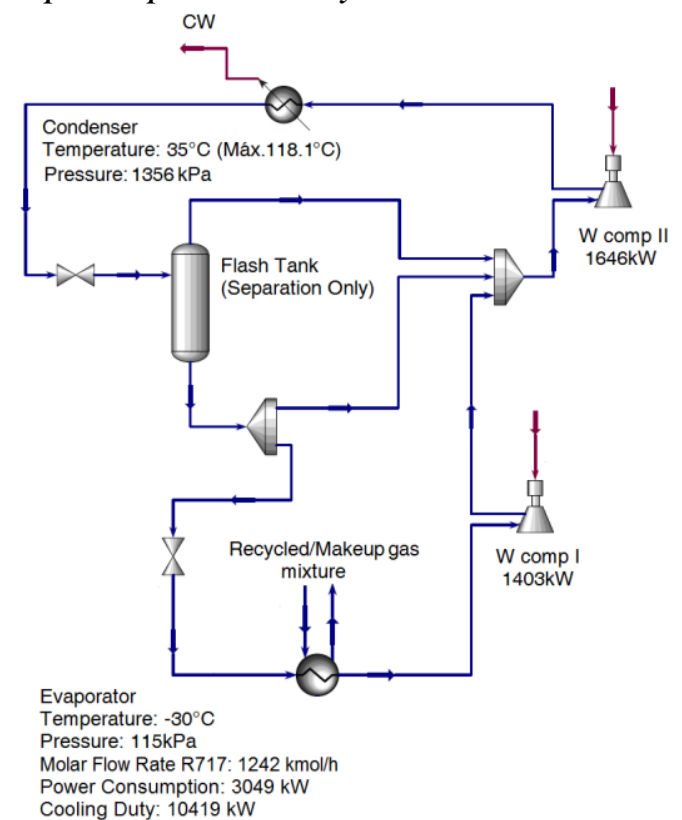

(b)

Source: [100]. 
Figure 6.7 and 6.8 show the $T$ vs. $H$ and Carnot factor- $H$ diagrams for the heat recovery and condensation system of the simulated ammonia loop with a reactor with degree of conversion [19] of $18.97 \%$.

Fig. 6.7. T-H diagram for the ammonia loop, minimum temperature approach: $10^{\circ} \mathrm{C}$. $\Delta H_{\text {reaction }}=32,000 \mathrm{~kW}\left(47 \mathrm{~kJ} / \mathrm{mol}_{\mathrm{NH} 3}\right)$. Balloon 1,12.Synthesis reactor, 2-6. Unconverted syngas and ammonia mixture cooling, 7. Refrigeration, 8,10,12. Recycled gas preheating,

9. Cooling water, 11. Steam boiler.

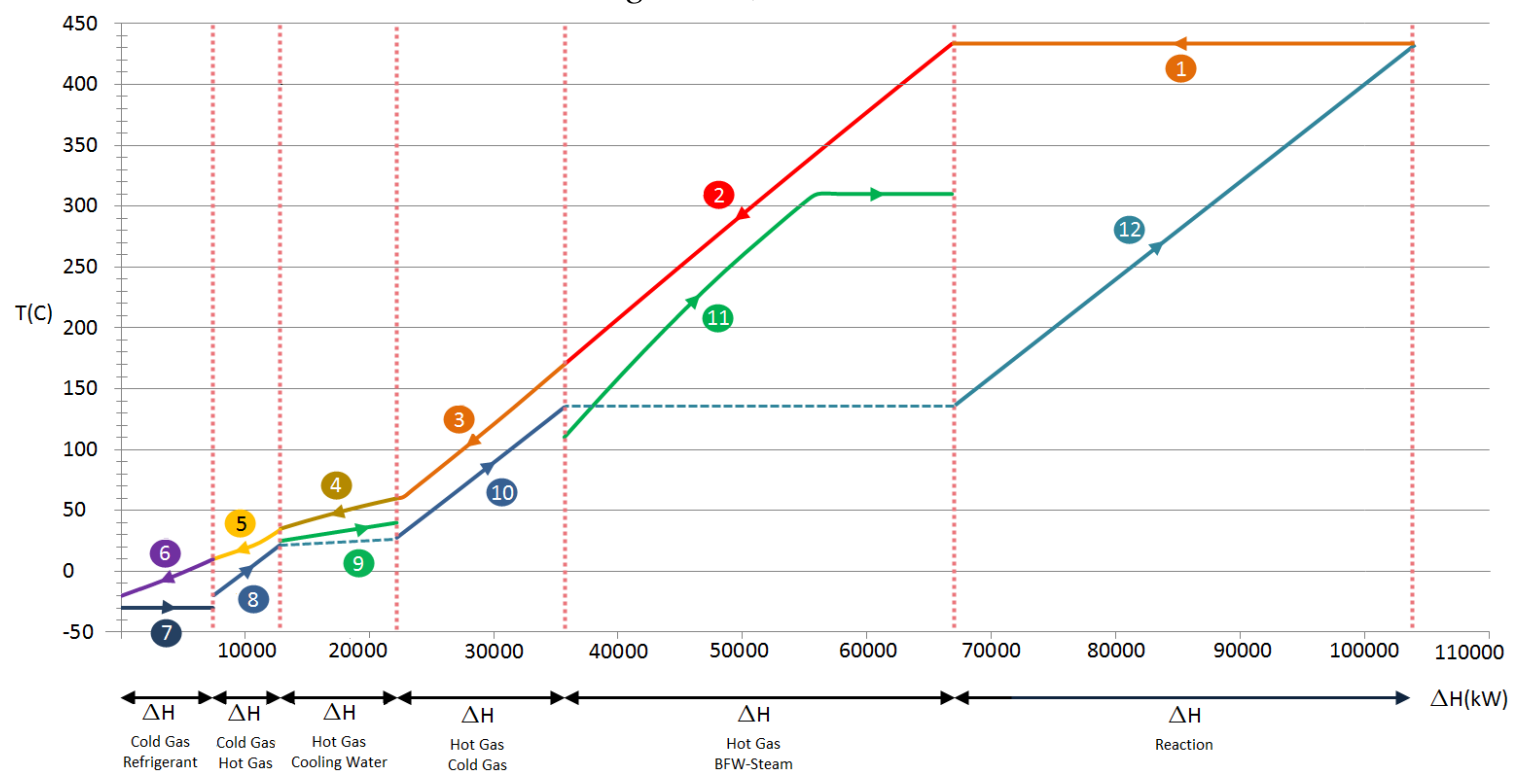

Source: Author.

As it can be seen, the hot reactor effluent (balloon 1 in Fig. 6.7) is initially cooled by heat exchange with the cold reactor feed (balloon 12) and then by steam raising in the waste heat steam boiler (balloon 11). Ammonia is the only component of the cold mixture (balloon 4) that can be partially condensed at non-cryogenic temperatures. Thus, a condensation system using cooling water (balloon 9) with a separator just above the ambient temperature can be used [41, 208]. However, at lower partial pressures (i.e. after the ammonia bulk is removed, balloon 6), the dew point is considerably reduced. For this reason, in order to achieve an ammonia content as low as $1.9 \% \mathrm{~mol}$ in the recycled synthesis gas, an additional refrigeration process $\left(-20^{\circ} \mathrm{C}\right)$ is required (balloon 7$)[19,41]$. Due to the large power and cooling requirement, the actual coefficient of performance (COP) of the refrigeration cycle achieves 2.43 (compared to the reference Carnot cycle with 5.42). Finally, the uncondensed, unreacted gas is recycled (balloons 8), then joins the fresh incoming syngas (balloon 10) and the mixture is preheated up to the reactor feed temperature [142]. 
Fig. 6.8. Carnot factor- $H$ diagram for the ammonia loop ( $T$ in $K$, and $T_{o}=298.15 K$ ). Note: If $T_{o}<25^{\circ} \mathrm{C}$, Carnot efficiency is calculated as $\left(T_{o} / T\right)-1$.

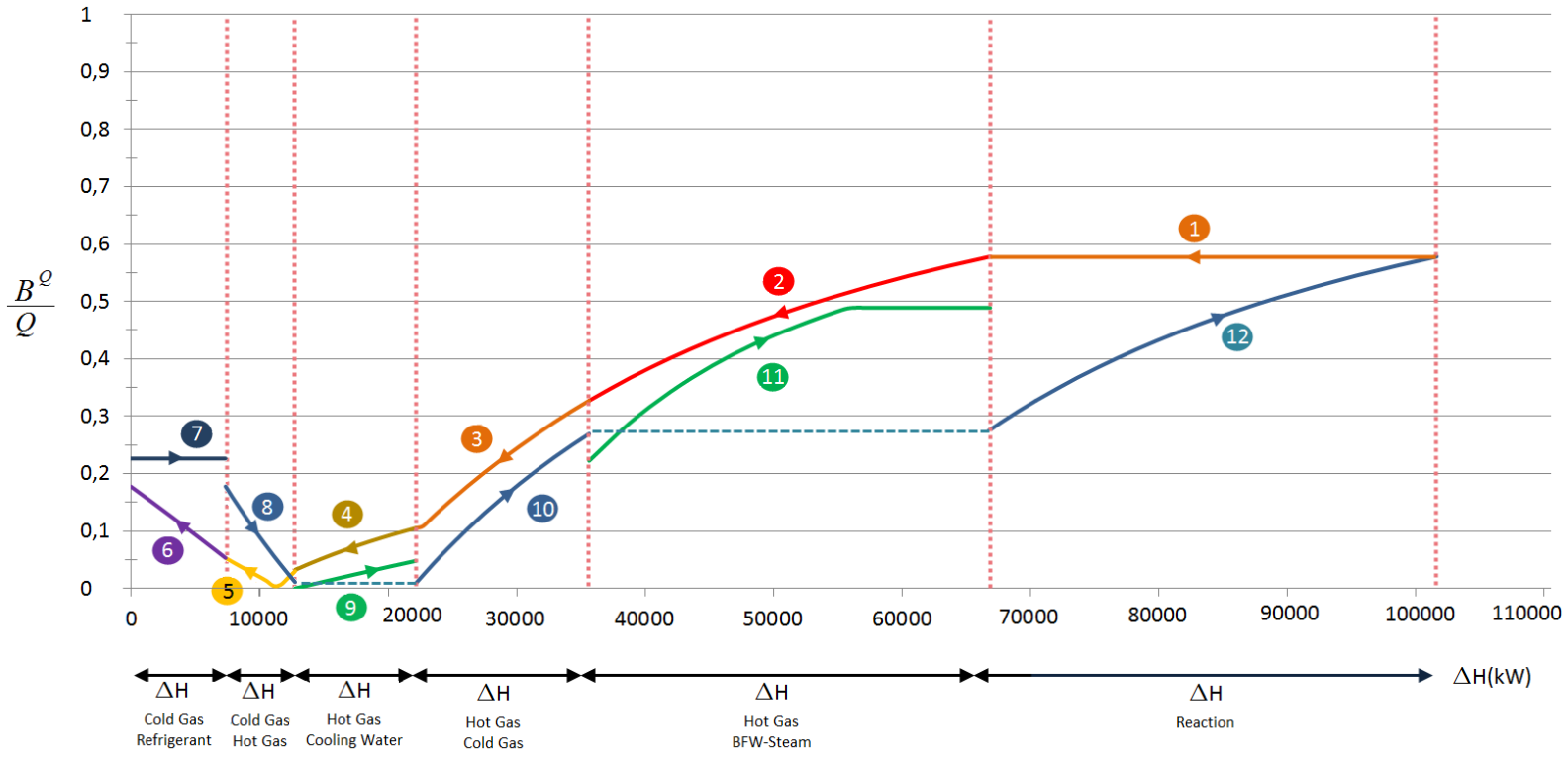

Source: Author.

Differently from Fig. 6.7, the corresponding Carnot factor- $H$ diagram (see Fig. 6.8) does evidence the remarkable change of behavior when the ammonia-rich stream is cooled below ambient temperature (balloons 5-8). Namely, the heat exergy associated with the cold streams (balloons 7,8) is actually transferred to the warmer ones (balloon 5,6). Consequently, the heat exchange at cross-ambient temperatures may require appropriate expressions to calculate exergy efficiency, as the consumed or produced exergy definitions differ from the aboveambient temperature counterparts [175].

Finally, in the cryogenic purge gas treatment section, an hydrogen recovery of $94.96 \%$ and a nitrogen recovery of $18.54 \%$ are achieved at conditions close to the ammonia loop pressure [97, 102]. The uncompressed fraction of the hydrogen-rich gas (71 bar) can be either recompressed externally and recycled to the ammonia loop [104] or used as fuel in the ammonia plant to reduce the feedstock consumption [103].

\subsection{Cumulative unit exergy cost and specific $\mathrm{CO}_{2}$ emissions}

The colocation of industrial ammonia plants together with facilities of urea, polymers or methanol production is an interesting way to use $\mathrm{CO}_{2}$ and other ammonia by-products (hot water, fuel gas, steam, etc.) in a marketable way. Thus, it would be desirable to evaluate the associated unit exergy cost and to quantify the related environmental impact of those substances. The cumulative exergy cost is an indicator of the deviation from thermodynamic perfection that allows performing comparisons that encompass the stages from the extraction of the natural resources up to the end-use of the final products.

Thus, based on the methodology proposed in Chapter 5, and by considering the cumulative unit exergy cost and $\mathrm{CO}_{2}$ emission cost data for the upstream processing stages of natural gas $\left(\mathrm{c}_{\mathrm{T}}=1.1780 \mathrm{~kJ} / \mathrm{kJ}_{\mathrm{NG}}, \mathrm{c}_{\mathrm{NR}}=1.1312 \mathrm{~kJ} / \mathrm{kJ}_{\mathrm{NG}}\right.$, and $\left.\mathrm{c}_{\mathrm{CO} 2}=0.0071 \mathrm{~kg}_{\mathrm{CO} 2} / \mathrm{MJ}_{\mathrm{NG}}\right)$ [189], the total 
and non-renewable unit exergy costs and $\mathrm{CO}_{2}$ emissions of the products of the ammonia plant can be calculated. Fig. 6.9 summarizes the unit exergy costs and specific $\mathrm{CO}_{2}$ emissions associated to the most important streams of the ammonia plant.

Fig. 6.9. Unit exergy costs and $\mathrm{CO}_{2}$ emissions of representative input and output streams of the integrated syngas and ammonia production plant.

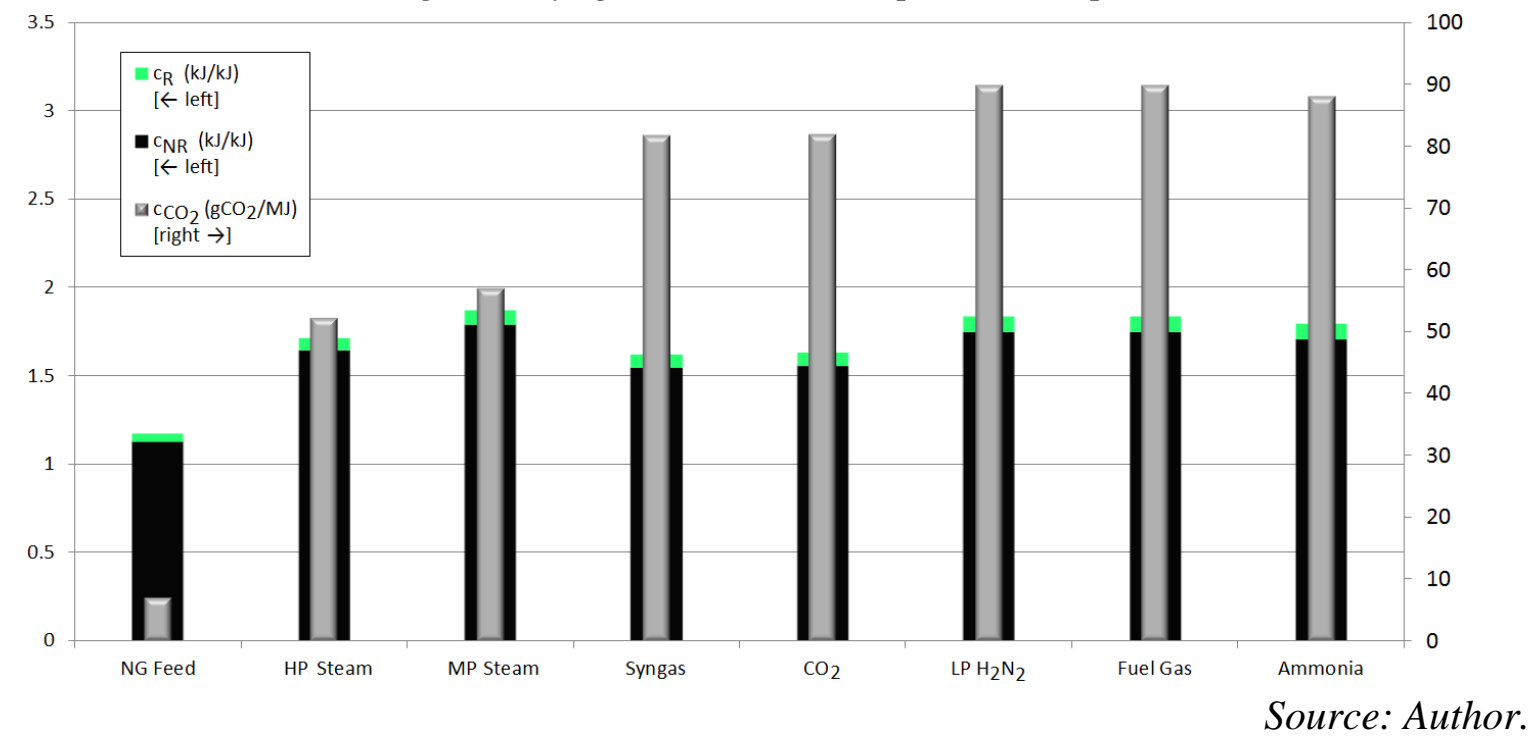

According to Fig. 6.9, the ammonia production requires an exergy consumption of $1.7950 \mathrm{~kJ}$ per $\mathrm{kJ}$ of ammonia produced, of which $95.32 \%$ corresponds to non-renewable sources. This is equivalent to an rational exergy efficiency of ammonia production of $55.71 \%$. Meanwhile, the specific $\mathrm{CO}_{2}$ emissions per unit of exergy of ammonia produced reaches $0.0881 \mathrm{~kg}_{\mathrm{CO} 2} / \mathrm{MJ}_{\mathrm{NH} 3}$. This is equivalent to $1.69 \mathrm{t}_{\mathrm{CO} 2} / \mathrm{t}_{\mathrm{NH} 3}$, close to $1.76-1.87 \mathrm{t}_{\mathrm{CO} 2} / \mathrm{t}_{\mathrm{NH} 3}$ estimated before. The difference between these two values lies in the use of exergy for the allocation of the specific $\mathrm{CO}_{2}$ emissions among all the products of the plant, instead of allocating all the emissions only to the ammonia produced. Meanwhile, the production of marketable $\mathrm{CO}_{2}$ in the plant requires $1.6370 \mathrm{~kJ}$ per $\mathrm{kJ}$ of $\mathrm{CO}_{2}$ (95.29\% coming from non-renewable sources) and entails specific $\mathrm{CO}_{2}$ emissions of $0.0821 \mathrm{~kg}_{\mathrm{CO} 2} / \mathrm{MJ}_{\mathrm{CO} 2}$.

As pointed out by Szargut et al. [151], unreasonable results could be obtained if the apportioning of the exergy consumption over the useful products in the complex chemical processes is performed on a mass basis. In fact, this approach could be only acceptable if the products are similar (e.g. hydrocarbons distilled from crude oil). The authors also argue that, in the case that the production method is the same for all products, the apportionment of exergy consumption could be based on the exergy values of the useful products. However, since ammonia, fuel gas, $\mathrm{CO}_{2}$, hot water, etc. are produced throughout a series of chemical processes, the calculation of the exergy costs and the allocation of the $\mathrm{CO}_{2}$ emissions requires of a more detailed study. In this way, the proposed exergy costing allows to analyze more rationally the production of the ammonia plant byproducts in terms of their specific exergy consumption and environmental impact. For the sake of comparison, other surveys dealing with the allocation of the unit exergy and energy costs and the specific $\mathrm{CO}_{2}$ emissions are briefly presented next. 
According to Szargut et al. [151] the Cumulative Exergy Consumption (CExC) and the Cumulative Degree of Perfection (CDP) of the ammonia production via the steam reforming of natural gas are $30.9 \mathrm{GJ} / \mathrm{t}_{\mathrm{NH} 3}$ and $64.2 \%$, respectively. Thus, by considering the exergy of the ammonia (337.9 MJ/kmol), the extended exergy consumption $\left(1.5574 \mathrm{~kJ} / \mathrm{kJ}_{\mathrm{NH} 3}\right)$ and the $\mathrm{CO}_{2}$ emissions $\left(0.0879 \mathrm{~kg}_{\mathrm{CO} 2} / \mathrm{MJ}_{\mathrm{NH} 3}\right)$ related to the ammonia production can be calculated. Moreover, if an additional electricity consumption of $0.108 \mathrm{kWh}$ per $\mathrm{kg}_{\mathrm{NH} 3}$ is assumed, the CDP would be strikingly reduced to $44.7 \%$. The authors do not report any apportionment of the exergy costs or the $\mathrm{CO}_{2}$ emissions among the various byproducts of the plant.

Kool et al. [209] reported a world's average energy consumption of the production of ammonia via steam reforming process as high as $37.5 \mathrm{GJ} / \mathrm{t}_{\mathrm{NH} 3}$ (or $1.9250 \mathrm{~kJ} / \mathrm{kJ}_{\mathrm{NH} 3}$ ) whereas the amount of $\mathrm{CO}_{2}$ emissions allocated only to the ammonia produced is about 0.1102 $\mathrm{kgCO} 2 / \mathrm{MJ}_{\mathrm{NH} 3}$. According to the authors, higher values are still possible for coal and oil-fueled plants $\left(>60 \mathrm{GJ} / \mathrm{t}_{\mathrm{NH} 3}\right)$. It is important to notice that these figures are not rationally calculated through a thermoeconomy analysis involving all the plant products, since all the irreversibility of the processes is charged to ammonia.

In fact, various surveys deal differently with the scenario of co-production of ammonia and its byproducts. For instance, Davis and Haglund [210] considered the benefits of the surplus steam production as a way to replace the combustion of fossil fuels elsewhere in the life cycle of the ammonia production, whereas Ahlgren et al. [211] applied an economic allocation methodology for the main products of the process (i.e. the nitrogen fertilizer and surplus electricity). From a world survey, William and Al-Ansari (2007) calculated an energy-based average specific emission of about $0.1063 \mathrm{~kg} \mathrm{CO}_{2} / \mathrm{MJ}_{\mathrm{NH} 3}$, with minimum values as low as $0.0831 \mathrm{~kg}_{\mathrm{CO} 2} / \mathrm{MJ}_{\mathrm{NH} 3}$ [212]. Rafiqul et al. [5] reported an energy-based consumption of $1.7915 \mathrm{~kJ} / \mathrm{kJ}_{\mathrm{NH} 3}$ and specific emissions of ammonia production via SMR of about $0.0975 \mathrm{~kg} / \mathrm{MJ}_{\mathrm{NH} 3}$ in plants operating back to 1995 . Meanwhile, the theoretical minimum methane consumption for the chemical process of ammonia production is estimated as 22.21 $\mathrm{GJ} / \mathrm{t}_{\mathrm{NH} 3}$ (energy-based) or equivalently as $1.1195 \mathrm{~kJ} / \mathrm{kJ}_{\mathrm{NH} 3}$, whereas the corresponding $\mathrm{CO}_{2}$ emissions are reported as $0.0625 \mathrm{~kg}_{\mathrm{CO} 2} / \mathrm{MJ}_{\mathrm{NH} 3}$ [9]. In spite of their relevance, none of those studies has allocated the unit exergy costs and $\mathrm{CO}_{2}$ emissions among the various products and byproducts by using a rational methodology, misleading the purchasers of the ammonia plant co-products to believe they are relying on low energy consumption process with reduced emissions.

Table 6.2 summarizes the thermodynamic properties and exergy costs calculated for selected streams in the baseline scenario for the syngas and ammonia production plant of this thesis. 
Table 6.2. Selected process data calculated for the baseline scenario for the integrated syngas and ammonia production plant $\left(1000 t_{\mathrm{NH} 3} / d\right)$, cf. Fig. 6.1 for stream number.

\begin{tabular}{|c|c|c|c|c|c|c|c|c|}
\hline $\mathbf{N}^{\circ}$ & Stream & $\begin{array}{c}\mathrm{n} \\
(\mathrm{kmol} / \mathrm{h})\end{array}$ & $\begin{array}{c}\mathbf{T} \\
\left({ }^{\circ} \mathbf{C}\right)\end{array}$ & $\begin{array}{c}\mathbf{P} \\
\text { (bar) }\end{array}$ & $\begin{array}{c}\text { B } \\
(k W)\end{array}$ & $\begin{array}{c}\mathbf{c}_{\mathbf{T}} \\
(\mathrm{kJ} / \mathbf{k J})\end{array}$ & $\begin{array}{c}\mathbf{c}_{\mathrm{NR}} \\
(\mathbf{k J} / \mathbf{k J})\end{array}$ & $\begin{array}{c}\mathbf{c}_{\mathrm{CO} 2} \\
\left(\mathrm{~kg}_{\mathrm{CO} 2} / \mathrm{MJ}\right)\end{array}$ \\
\hline 1 & Cold Process Air & 1,690 & 25 & 1 & 0 & 1.0000 & 0 & 0.0000 \\
\hline $2,4,7$ & $\mathrm{~W}_{\text {in }}$ Air Compression & -- & -- & -- & 6677 & 2.153 & 2.062 & 0.0657 \\
\hline 22 & NG fuel & 516 & 25 & 1 & 119,203 & 1.1780 & 1.1310 & 0.0071 \\
\hline 11 & NG feed & 1,200 & 25 & 35 & 4054 & 1.1780 & 1.1310 & 0.0071 \\
\hline 12 & MP Steam & 3,600 & 350 & 35 & 21,250 & 1.8700 & 1.7910 & 0.0571 \\
\hline 25 & HP Steam & 10,391 & 460 & 100 & 75,505 & 1.7170 & 1.6450 & 0.0524 \\
\hline 30 & Boiler Steam & 7,151 & 310 & 100 & 41,599 & 1.4850 & 1.4250 & 0.0452 \\
\hline 33 & BFW utilities & 6,395 & 297 & 100 & 14,477 & 1.4940 & 1.4230 & 0.0777 \\
\hline 36 & Cold BFW & 6,395 & 30 & 100 & 1,977 & 1.5590 & 1.0570 & 0.0337 \\
\hline 41 & Shift Product & 6,512 & 35 & 35 & 285,811 & 1.4940 & 1.4230 & 0.0777 \\
\hline 52 & DEA makeup water & 864 & 25 & 1.3 & 216 & 1 & 0 & 0.0000 \\
\hline 42 & Rich DEA & 30,224 & 93 & 2 & $1,883,324$ & 1.6260 & 1.5490 & 0.0819 \\
\hline 43 & Lean DEA & 28,187 & 111 & 1.37 & $1,883,394$ & 1.6370 & 1.5600 & 0.0821 \\
\hline 56 & $\mathrm{~W}_{\text {in }}$ DEA pump & -- & -- & -- & 896 & 2.1530 & 2.0620 & 0.0657 \\
\hline 60 & Feed gas to Compr. & 5,279 & 35 & 34.65 & 277,204 & 1.6260 & 1.5490 & 0.0819 \\
\hline $62 / 65 / 68 / 71$ & $\mathrm{~W}_{\text {in }}$ Syngas Compr. & -- & -- & -- & 9,883 & 1.8700 & 1.7910 & 0.0571 \\
\hline 105 & LP Steam & 3,997 & 191 & 7 & 16,966 & 2.1530 & 2.0620 & 0.0657 \\
\hline 49 & $\mathrm{CO}_{2}$ to Compressor & 1,223 & 35 & 1.20 & 6,859 & 1.6370 & 1.5600 & 0.0821 \\
\hline 88 & LP Condensate & 3,997 & 109 & 7 & 1,882 & 2.1530 & 2.0620 & 0.0657 \\
\hline 83 & Ammonia Product 1 & 1,565 & 35 & 190 & 146,627 & 1.8090 & 1.7240 & 0.0888 \\
\hline 74 & Fresh Syngas & 5,271 & 35 & 200 & 284,003 & 1.6530 & 1.5740 & 0.0819 \\
\hline 75 & Recycle gas to mixer & 10,157 & 35 & 190 & 678,809 & 1.8090 & 1.7240 & 0.0888 \\
\hline 93 & Feed to circulator & 14,478 & 22 & 190 & 874,011 & 1.7710 & 1.6870 & 0.0870 \\
\hline 94 & Purge gas & 268 & 35 & 190 & 17,883 & 1.8090 & 1.7240 & 0.0888 \\
\hline 91 & BFW HRCT & 1,646 & 310 & 100 & 4,123 & 1.8090 & 1.7240 & 0.0888 \\
\hline 90 & BFW NH $\mathrm{NH}_{3}$ Loop & 756 & 310 & 100 & 1,893 & 1.8090 & 1.7240 & 0.0888 \\
\hline 89 & $\mathrm{NH}_{3}$ Loop Steam & 1,594 & 310 & 100 & 9,275 & 1.8090 & 1.7240 & 0.0888 \\
\hline 82 & Ammonia Product 2 & 950 & -20 & 190 & 88,469 & 1.7710 & 1.6870 & 0.0870 \\
\hline 95 & Aqua ammonia & 131 & 101 & 190 & 2,923 & 1.8370 & 1.7480 & 0.0900 \\
\hline 99 & HP $\mathrm{H}_{2}-\mathrm{N}_{2}$ mixture & 98.5 & 67 & 189.6 & 6,313 & 1.8370 & 1.7480 & 0.0900 \\
\hline 97 & $\mathrm{LP} \mathrm{H}_{2}-\mathrm{N}_{2}$ mixture & 51.9 & 33 & 71 & 3,285 & 1.8370 & 1.7480 & 0.0900 \\
\hline 98 & Fuel Gas & 85.4 & 12 & 3 & 5,115 & 1.8370 & 1.7480 & 0.0900 \\
\hline 92 & Steam HRCT & 1,646 & 310 & 100 & 9,577 & 1.8550 & 1.7740 & 0.0446 \\
\hline 101 & Makeup water & 3,600 & 25 & 1 & 900 & 1.0000 & $\mathbf{0}$ & 0.0000 \\
\hline 107 & $\mathrm{~W}_{\text {out }}$ HP turbine & -- & -- & -- & 12,189 & 1.8700 & 1.7910 & 0.0571 \\
\hline 104 & $\mathrm{~W}_{\text {out }}$ MP turbine & -- & -- & -- & 11,414 & 2.153 & 2.062 & 0.0657 \\
\hline 86 & $\mathrm{~W}_{\text {in }}$ circulator & -- & -- & -- & 759 & 1.8700 & 1.7910 & 0.0571 \\
\hline 109 & Ammonia & 2,516 & 15 & 190 & 235,014 & 1.7950 & 1.7110 & 0.0881 \\
\hline-- & Makeup CW tower & 11,631 & 25 & 1 & 2,908 & 1.0000 & 0 & 0 \\
\hline
\end{tabular}

As it can be seen from Table 6.2, due to the large amount of power consumed in the process air compression, its related exergy costs and $\mathrm{CO}_{2}$ emissions are among the highest exergy and environmental costs in the ammonia plant. A similar exergy-intensive stream is the low pressure steam used to supply the duty of the stripper reboiler. Meanwhile, the high and medium pressure steam generation present exergy costs of about $1.8000 \mathrm{~kJ} / \mathrm{kJ}$ steam. In other words, HP and MP steam are produced with a rational exergy efficiency of about $55 \%$. Furthermore, steam generation (HP, MP and LP steam) also presents the highest ratio of nonrenewable to renewable exergy cost, since the largest exergy reduction of the flue gases occur 
in the first module of the HRCT. This exergy reduction also explains a higher allocation of $\mathrm{CO}_{2}$ emissions to the steam generation process. Clearly, this approach is more rational than simply allocating the emissions on the products of the last module of the HRCT. It should be also noted that higher exergy costs $(15 \%)$ are obtained for the power produced in the MP steam turbine compared to that produced in the HP turbine. It can be explained by a higher contribution of the MP steam turbine condensate (57\%) to the heat rejected in the condenser.

At a first glance, the splitting into renewable an non-renewable costs in a practically fossilbased process may seem pointless. However, reported data on exergy costs and $\mathrm{CO}_{2}$ emission cost of various fuels [186-190] have shown that a small part of the cumulative unit exergy costs of natural gas and petroleum derivatives corresponds to renewable sources. Thus, it must be accounted for if a fair level playing field for comparative assessments with other syngas and ammonia production technologies is intended. For instance, other scenarios, including the syngas production by using the steam reforming of ethanol, have earned more attention in Brazil, mainly due to its well-established sugar cane ethanol economy [213-217]. Thus, apart from the fossil-based ammonia production process, more recent works on biomass-based ammonia production may offer a more interesting opportunity to highlight the importance of the allocation of the renewable unit exergy costs and $\mathrm{CO}_{2}$ emissions. Finally, it is emphasized that, even if more stringent environmental policies may demand an additional amount of external exergy consumption (e.g. for abatement of emissions), the presented methodology would remain applicable, so that the costs of other externalities can also be included. However, post-combustion $\mathrm{CO}_{2}$ capture from the primary reformer stack will remain as the greater challenge due to the higher specific stripping exergy compared to pre-combustion $\mathrm{CO}_{2}$ capture in ammonia plants $[33,84]$.

\subsection{Exergy efficiency and exergy destruction}

Figure 6.10 shows the exergy efficiency for some representative components and units of the syngas and ammonia production plant. As expected, the input-output efficiencies present a reduced sensitivity to the process parameters. For instance, in the chemical absorption system of the syngas purification unit, the large mass chemical exergy of the solvent overwhelms the influence of other process inputs, such as steam and power, in the calculation of the efficiency. This shortcoming is also evident when calculating the exergy efficiency of the ammonia converter. For this reason, the causes of the reduced exergy performance as well as the suitable alternatives for the processes improvement are discussed next, but at the light of the consumed - produced efficiency. 
Fig. 6.10. Exergy efficiency for representative components and units.

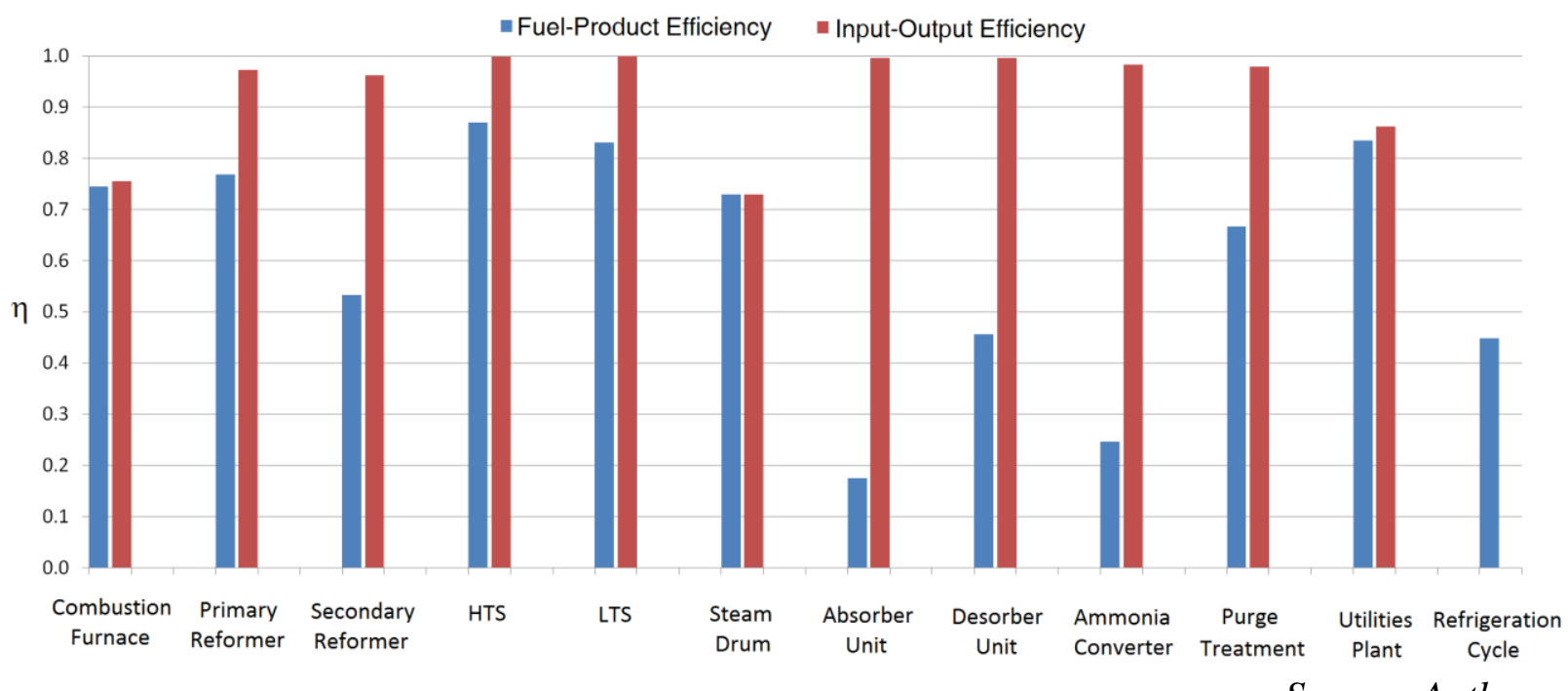

Source: Author.

\section{- Combustion Furnace and Utility System}

According to Fig. 6.10, the exergy efficiency of the combustion furnace is slightly higher than that of the boiler; maybe due to the higher mean thermodynamic temperature within the former compared with the steam drum. The principal irreversible phenomena that occur in radiant furnaces are the combustion reactions that lead to the diffusion of the species and the uncontrolled conversion/dissipation of the chemical exergy into physical exergy.

Despite the fact that most of the irreversibility owed to chemical conversion is inevitable, some exergy losses can still be reduced by preheating the reactants [151]. In the steam boilers, the typical large temperature differences between the hot and the cold streams, as well as the pressure losses, are among the most important sources of irreversibility. The exergy losses in the steam system can be reduced (i) by preheating the boiler feedwater using low-grade heat exergy sources (e.g. shift reactors effluents); (ii) by increasing the mean thermodynamic temperature of the steam and (iii) by superheating the steam in colder sections of the HRCT.

Some authors [9] have reported lower exergy efficiencies of the steam generation in a standalone combined heat and power production (CHP) plant when compared to the energy integration of an steam network to the chemical plant. Actually, the fuel consumption used to generate steam in the energy-integrated plant is reported as $10 \%$ lower than if steam is produced in isolation at the CHP plant. Alternatively, a gas turbine coupled to an autothermal reformer (ATR) could be used to provide the heat duty of the reforming process, as well as to produce electricity and process steam in a cogeneration system $[79,80]$. In this configuration, the exergy loss due to the excess steam generation and condensation could be avoided [42]. 


\section{- Primary and Secondary Reformers}

Since the volume of the reacting mixture increases in the primary reforming $\left(n_{P}>n_{R}\right)$, the exergy efficiency, contrary to the methane conversion, increases with higher reformer pressures [69]. Notwithstanding, as the pressure increases, the yield of syngas decreases because an increase of the methane slip [25]. To overcome this problem, some authors [69] have suggested to perform an incomplete methane conversion, favoring the recycle of the unreacted feedstock. However, it entails the utilization of further methods for separating the non-reacted gases (e.g. pressure swing absorption) [218]. Other alternatives include the increase of the steam to carbon ratio and the reactor temperature, but it implies the increase of steam and fuel consumption, and the use of costly high temperature resistant materials.

As discussed earlier, the use of a pre-reformer upstream of the primary reformer may help to cut down the reformer fuel consumption about 5-10\%, as well as to widen the alternatives of feedstock consumed [69]. Further developments such as the Unmixed Reforming (UMR) process [219], the intensified SMR based on the micro-channels reactor technology [26], and the chemical looping (CL) reactor producing discrete and pure streams of $\mathrm{H}_{2}$ and $\mathrm{N}_{2}$ [220] have been also reported as alternatives for increasing the steam reforming efficiency by means of the reduction of the large driving forces involved (conversion, heat transfer and mixing).

\section{- Syngas Purification}

The throttling process of the high pressure rich amine and the irreversible heat transfer from lean DEA to cooling water play an important role in the low exergy performance of the absorber unit. Actually, if the effect of DEA cooling and throttling is not included in the efficiency calculation, this value could be as high as $62 \%$ instead of $18 \%$, as shown in Fig 6.10. On the other hand, due to the highly irreversible heat exchange processes in the reboiler and the reflux condenser, the large amount of low pressure steam consumed drops down the exergy efficiency of the desorber unit. According to Leites et al. [69], the irreversibility in the absorption process can be minimized by reducing the absorbent circulation rate. Furthermore, the feed of two separate lean solvent streams with different optimal $\mathrm{CO}_{2}$ concentrations to the absorber column would be preferable over a single lean stream, as it allows an increase in the absorptivity of the solution, reducing the heat consumption of conventional flowsheets [69].

\section{- Ammonia Converter}

The exergy associated with the unreacted feed or inerts typically constitutes transiting exergy in the ammonia reactor [177]. In this way, the large recycle rate and low conversion make its exergy efficiency much lower than that of other exothermic reactors. Higher conversions can be achieved by using multiple reactor beds with optimized intercooling systems along with more active catalysts and increased pressures. This leads to the minimization of the recycle rates and thus to both power and cooling savings.

However, if the converter pressure is increased, the amount of power consumed in the circulator and the syngas compressor is also increased, along with the equipment cost. Furthermore, the reactor pressure affects the thermodynamics of the ammonia synthesis, since 
the volume of the reaction decreases as proceeds to equilibrium $\left(n_{P}<n_{R}\right)$. As a result, the reduction of the exergy loss (by reducing the driving force) enters automatically in conflict with any attempt to increase the process yield. In fact, by assuming that the heat is continuously removed so that ammonia is isothermally produced [25], it can be shown that the exergy efficiency increases as the pressure reduces below $\mathrm{P}_{\mathrm{o}}$, Eq. (6.1) [69]:

$$
\eta_{\text {reactor }}=\Delta B^{P H} / \Delta B^{C H}=\left(n_{P}-n_{R}\right) R T_{o} \ln \left(P / P_{o}\right) / \Delta B^{C H} \quad n_{P}<n_{R}
$$

This fact is evidently in opposition to Le Châtelier principle, which suggests increasing the pressure of a decreasing-volume reaction, whereas decreasing the temperature of an exothermic one. To conciliate this dilemma, the classical single bed ammonia converters have been superseded by multiple intercooled catalytic beds. Moreover, novel dual pressure ammonia loops (e.g. Udhe process) [221], that start the operation at a lower pressures and end at higher ones, have been also introduced. Those improvements aim to increase the exergy efficiency while maintaining high production rates.

More recently, the so-called heat and mass exchange technology partially (or completely) substitutes the conventional gas-gas heat exchanger at the reactor outlet [142]. Therein, the amount of unseparated ammonia recycled to the converter is reduced at the expense of the ammonia enrichment of the reactor effluent. Other authors studied the ammonia adsorption in a co-current gas-flowing/solids-fixed bed reactor (GFSFBR) [222]. In this process, the instantaneous removal of the ammonia produced shifts the equilibrium towards more ammonia production.

\section{- Cryogenic Purge Gas Treatment}

Despite the absence of chemical reactions in the cryogenic purge gas recovery unit, other dissipative components lead to an exergy efficiency much lower than expected by the simpler input-output definition. Among the most irreversible process in the system are the throttling process of the liquid effluent from the cold-box separator [94] and the temperature difference between the hot and cold streams in the cryogenic heat exchangers (ranging from $40^{\circ} \mathrm{C}$ to $\left.-191^{\circ} \mathrm{C}\right)$.

Despite the fact that the SNF plants contain many exergy conversion systems, there always exist some major components that dominate the overall exergy performance, e.g. reformers, steam boilers, ammonia converter, reboiler, refrigeration cycle or compression train. These components typically present the largest exergy consumption and exergy destruction rates [147], as shown in Fig. 6.11. In addition, Fig. 6.12 summarizes the exergy destruction in selected components. The total amount of exergy destruction achieves $136.48 \mathrm{MW}$, whereas global exergy consumption is calculated as $405.70 \mathrm{MW}$. This results in an overall exergy conversion efficiency of $66.36 \%$, and in close agreement with an extended exergy analysis for ammonia production reported by Szargut et al. [151]. According to Fig. 6.11, almost 59\% of the exergy destroyed corresponds to the reforming section, which also includes the highly irreversible fuel combustion in the reformer furnace. 
Moreover, $\mathrm{CO}_{2}$ desorption process is one of the main sources of exergy destruction in the ammonia plant (13.22\%). Lower steam consumption rates could be achieved by using better available techniques in gas purification systems, such as less exergy-intensive chemical (MDEA) or physical (Selexol, Rectisol) absorption solvents.

Fig. 6.11. Breakdown of the exergy destruction in the syngas and ammonia production plant. Total exergy destruction: $136.48 \mathrm{MW}$ for a $1000 t_{\mathrm{NH}} /$ day plant.

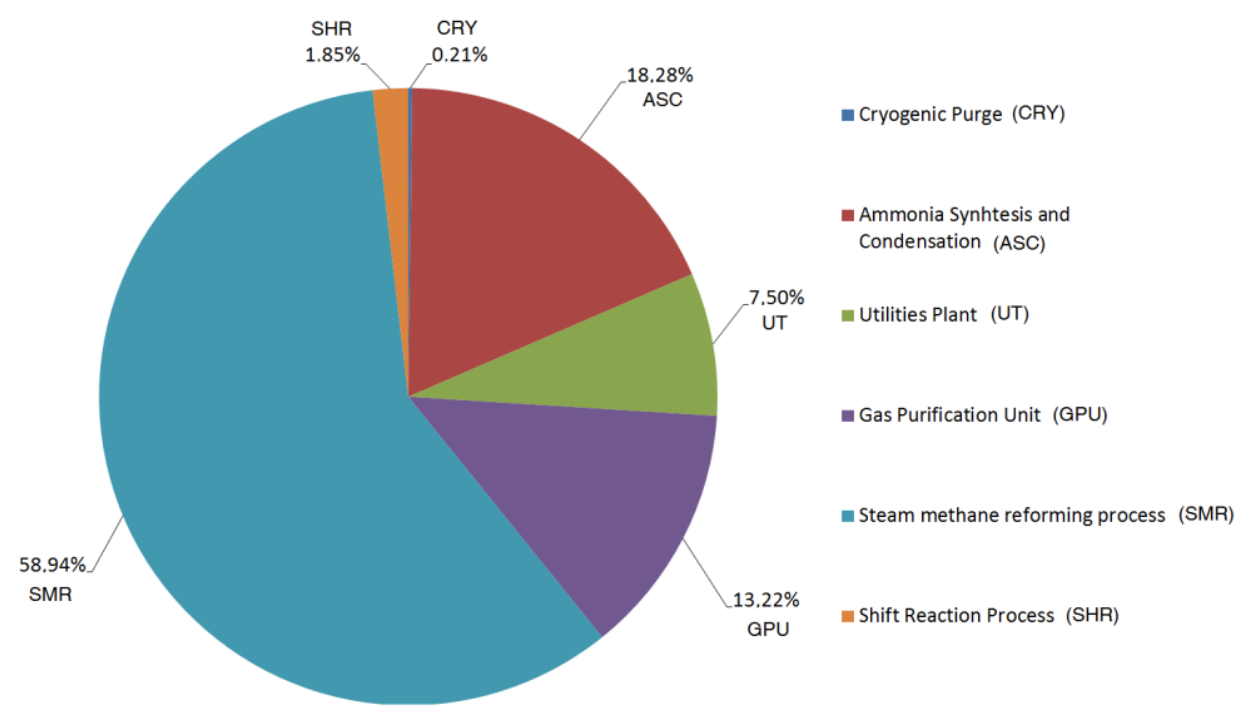

Source: Author.

Regarding the ammonia loop, the highly exothermic reaction of ammonia synthesis along with the exergy-intensive syngas compression and ammonia refrigeration processes are responsible for almost one fifth of the overall exergy destroyed.

Fig. 6.12. Exergy destruction rates for selected operation units of a $1000 t_{N H 3} /$ day plant.

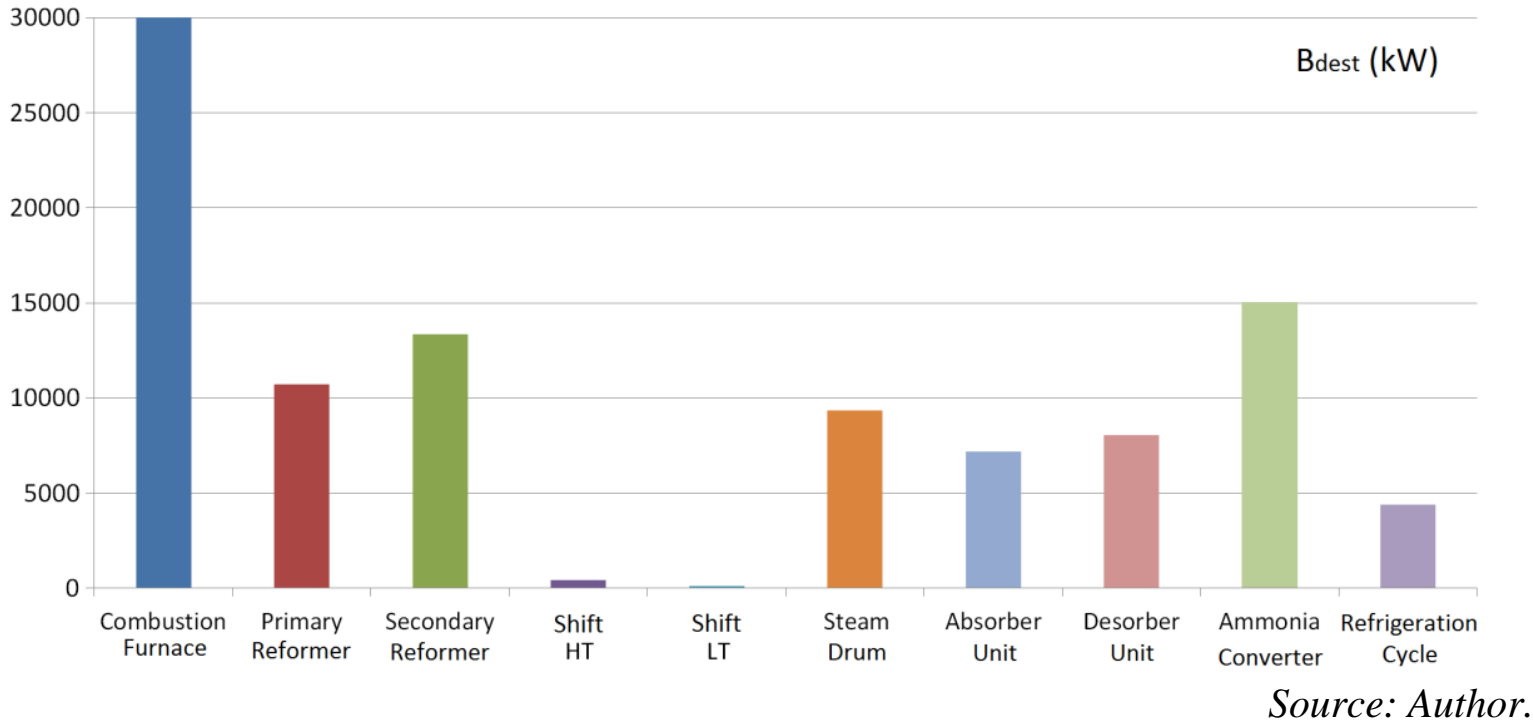

Better reactor catalysts (such as ruthenium-based catalyst operating at lower pressures) and waste heat recovery systems, alongside better ammonia refrigeration processes may reduce the power consumption, and consequently, exergy destruction in the ammonia loop. Yet, due to the dominant effect of the frontend syngas production process, some authors [223] have 
reported that, even though the irreversibility in the ammonia reactor unit decreases by more than $60 \%$, the overall exergy destruction would be reduced by only $6 \%$.

\subsection{Final considerations}

In this chapter a suitable methodology has been used to address the rational distribution of the exergy costs and $\mathrm{CO}_{2}$ emissions among the all streams of the chemical plant, with special attention given to the convection section of the primary reformer. In this way, neither the product of the last component of the HRCT is overcharged with the total irreversibility, nor any unit exergy cost has been left unallocated. The rational production efficiency of ammonia is calculated as $55.71 \%$, whereas the specific $\mathrm{CO}_{2}$ emissions of the ammonia produced reach $0.0881 \mathrm{~kg}_{\mathrm{CO} 2}$ per $\mathrm{MJ}_{\mathrm{NH} 3}$ (or $1.69 \mathrm{t}_{\mathrm{CO} 2} / \mathrm{t}_{\mathrm{NH} 3}$ ). For the $\mathrm{CO}_{2}$ byproduct, those figures are $61.08 \%$ and $0.0821 \mathrm{~kg}_{\mathrm{CO} 2} / \mathrm{MJ}_{\mathrm{CO} 2}$, respectively. The methodology proposed could be also applied to analyze the production of other byproducts of common chemical plants, such as sulfur compounds in refineries, $\mathrm{CO}_{2}$ produced by fermentation processes in sugar cane mills or alternatively, the $\mathrm{CO}_{2}$ reinjection in enhanced oil recovery applications [64, 224]. It is worthy to notice that this base-case configuration presented deals with a basically fossil-fueled ammonia production process. Thus, the renewable fraction of the unit exergy costs, coming from the indirect utilization of the electricity mix, might not have been evidenced, were it not for an extended exergy costing.

It is also found that any increase of the driving forces implies higher exergy destruction rates. Thus, the minimization of the irreversibility in the industrial ammonia plants is expected to be a trade-off between lower exergy destruction rates and higher yield rates. Hence, any choice between the conflicting targets will depend ultimately on the availability and price of feedstock and project specific conditions. Moreover, more detailed analyses to determine enhanced alternatives to mitigate the environmental impact are still required, showing that the decarbonization of this sector is still open to improvement. Certainly, the previous results allow identifying the most inefficient, energy intensive and environmentally unfriendly processes. Thus, they could be regarded as the starting point of the decision-making for the thermodynamic and economic optimization of the conventional and alternative production routes presented in the next sections at both component, sub-system and plantwide levels.

Hereunder, Chapter 7 deals with the assessment of the most suitable components for the utility systems, with the syngas purification unit playing an special role in the energy integration process. The target is the minimization of the operating costs of the chemical plant while reducing the overall energy requirements. A careful analysis of the energy integration curves along with the use of the exergy method provide the means to select the best alternatives among the available energy technologies and find out their optimal operating conditions. 
CHAPTER 7

\section{THE ROLE OF THE SYNGAS PURIFICATION TECHNOLOGY IN THE OVERALL ENERGY INTEGRATION}

Modern ammonia production plants are equipped with efficient energy integration networks able to recover an important fraction of the enthalpy of reaction released by the exothermic chemical systems. However, to fully supply the exergy demands of the highly endothermic reforming reactions and the syngas purification and compression systems, an additional energy consumption, typically provided by means of costly non-renewable resources, is still required. Consequently, an optimal energy integration of the reactive components to the remaining systems of the plant might allow not only reducing the amount of fuel consumed, but also minimizing the process irreversibility by pursuing enhanced heat recovery and power generation. Furthermore, the valorization of the plant byproducts (e.g. $\left.\mathrm{CO}_{2}\right)$ may also increase the overall efficiency of the process, whereas the reduction of wastes ensures a minimum degradation of valuable feedstock.

On the other hand, the choice of a carbon capture unit based on either physical or chemical absorption drastically affects the way in which the waste heat recovery (e.g. combustion air preheating) must be performed, and whether one or more energy technologies should or not be integrated (e.g. heat pump). Furthermore, the selection among various energy resources, such as the import of electricity over the autonomous combined heat and power production (CHP), strongly depends on the ratio between the prices of electricity and fuels consumed, as well as on the extent of the energy integration. Thus, evidently, a simple trial and error approach falls short in efficiently determining the most suitable energy technologies and the operating conditions that enable the chemical plant to operate under minimum cost.

Accordingly, in this chapter, a systematic methodology is used to identify the most suitable utility systems (steam network, refrigeration, cogeneration) that satisfy the minimum energy requirement (MER) with the lowest energy consumption and operating cost. In addition, the exergy analysis is used to determine the potential improvements that may remain hidden to the conventional energy integration analysis, regarding the minimization of the avoidable exergy losses and the integration of reactive, CHP and syngas purification systems. By applying this methodology, the best operating conditions of such systems are identified, as well as the opportunities for producing surplus electricity in complex ammonia production plants, typically associated to urea and nitric acid production facilities in SNF complexes.

\subsection{Superstructure adopted for the chemical process and the utility system.}

Figure 7.1 shows the simplified layout of an integrated syngas and ammonia production plant based on the steam reforming of natural gas. As described in previous chapters, the chemical plant is composed of the syngas production unit (feedstock saturator, prereformer, primary and secondary reformers and water gas shift reactors), the syngas purification process $\left(\mathrm{CO}_{2}\right.$ 
removal by either physical or chemical absorption systems and methanator) and the ammonia synthesis (multiple reactor beds with intercooling) and separation processes (condensation and refrigeration systems). Since these elements are strongly interrelated to each other, it is expected the operating conditions of a set of components to be simultaneously affected by the performance of the remaining sections of the plant concept, especially the heat and power balance of the whole plant.

Fig. 7.1. Superstructure of the chemical processes, utility systems and resources network in the ammonia production plant.

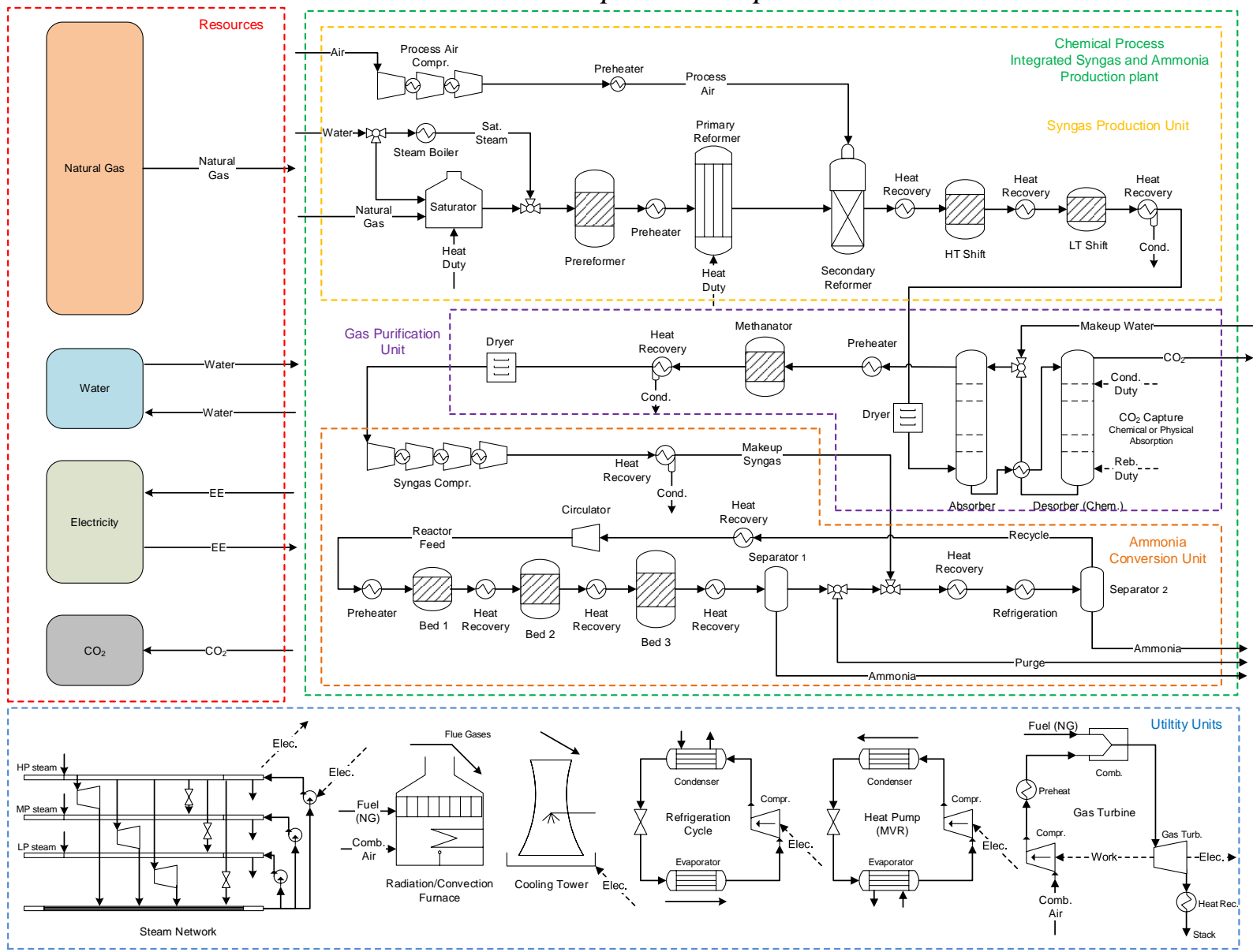

Source: Author.

According to Fig. 7.1, natural gas is consumed as both feedstock in the reformer and fuel to produce a relatively expensive, high temperature hot utility stream in a natural gas-fired furnace with combustion air preheating. This utility must be able to supply the heat duty to the externally-fired reformer, whereas the residual exergy of the flue gas can be utilized for raising and superheating steam, as well as to preheat other process streams.

It is worthy to notice that, since the flue gas must be cooled down to the stack temperature, some of its exergy heat may be available below the pinch temperature, representing so an increase in the cold utility consumption and, thus, an avoidable loss. For this reason, two different scenarios should be considered. The first one consists of a direct competition of the combustion gas with the steam network for satisfying the hot utility streams required at lower temperatures. Alternatively, the hot fumes could be used to preheat the combustion air by 
using the excess heat exergy available below the pinch point. In this way, the air preheating defines a cold stream whose target temperature is equal to the utility pinch point. The effect of air preheating is an increase of the adiabatic combustion temperature, which translates into an increase of the heat exergy available at high temperature [225]. Actually, the reactants preheating could be interpreted as a chemical heat pump [226].

Meanwhile, a mechanical draft cooling tower and a vapor compression refrigeration system together with a mechanical vapor recompression (MVR) process supply the cooling and heat pump requirements of the whole plant. Cooling water inlet and outlet temperatures are set as $40^{\circ} \mathrm{C}$ and $15^{\circ} \mathrm{C}$, respectively, and a ratio of power consumed-to-cooling duty of $0.021 \mathrm{~kW}_{\mathrm{el}} / \mathrm{kW}_{\text {th }}$ is assumed for the cooling tower [159]. The refrigeration and heat pump systems are defined in terms of their representative exergy efficiency (50\%) as shown in Eqs.(7.1-7.6):

$$
\begin{gathered}
C O P_{\text {refrig, actual }}=\eta_{e x} \cdot C O P_{\text {refrig, Carnot }} \\
=\eta_{e x} \cdot T_{\text {evap }} /\left(T_{\text {cond }}-T_{\text {evap }}\right) \\
W_{\text {refrig }}=\frac{\dot{Q}_{\text {evap }}}{C O P_{\text {refrig, actual }}} \\
\dot{Q}_{\text {cond }}=\frac{\dot{Q}_{\text {evap }} \cdot\left(C O P_{\text {refrig,actual }}+1\right)}{C O P_{\text {refrig, actual }}} \\
C O P_{\text {heatpump, actual }}=\eta_{\text {ex }} \cdot C O P_{\text {heatpump, Carnot }} \\
=\eta_{\text {ex }} \cdot T_{\text {cond }} /\left(T_{\text {cond }}-T_{\text {evap }}\right) \\
W_{\text {heatpump }}=\frac{\dot{Q}_{\text {cond }}}{C O P_{\text {heatpump, actual }}^{H P}} \\
\dot{Q}_{\text {evap }}=\frac{\dot{Q}_{\text {cond }} \cdot\left(C O P_{\text {heatpump, actual }}^{H P}-1\right)}{C O P_{\text {heatpump, actual }}^{H P}}
\end{gathered}
$$

On the other hand, the superstructure of the steam network is composed of a set of superheated steam headers and draw-off levels of steam (Fig.7.1) that allows for the recovery and distribution of the waste heat along the chemical plant. The choice of the optimal levels of steam is performed by inspecting the profile of the grand composite curve of the chemical process [227]. Thus, the power can be generated by optimally profiting the thermodynamic potential of the waste heat exergy via backpressure and extraction-condensation steam turbines. Moreover, as long as electricity can be imported from the grid, the trade-off between the additional fuel consumption and the electricity purchase to supply the power demand of the whole plant will be strongly influenced by the performance of the cogeneration and waste heat recovery systems, as well as by the ratio between the electricity 
and natural gas costs. This reasoning also applies to the suitability of the integration of a gas turbine system with regeneration, aiming to increase the cogeneration efficiency [228].

Accordingly, a methodology based on the combination of the exergy method [151] and the energy integration method [157] is used to assess the performance of the various components of the integrated syngas and ammonia production plant operating with different carbon capture configurations. Exergy indicators are used for estimating the performance of each configuration and some graphical representations allow performing systematic comparisons between the different designed setups. Table 7.1 compares the rational exergy efficiency, Eq. (7.7), with other exergy efficiency definition, Eqs.(7.8), proposed for evaluating the overall exergy performance [206]. It must be noticed that, the rational exergy efficiency is higher than the relative one as it accounts for the outlet exergy of other byproducts $\left(\mathrm{CO}_{2}\right.$, purge gas).

Table 7.1. Plantwide exergy efficiency definitions of the ammonia production plant.

\begin{tabular}{ccc}
\hline Definition & Formula & Equation \\
\hline Rational & $\eta_{\text {Rational }}=\frac{B_{\text {useful,output }}}{B_{\text {input }}}=1-\frac{B_{\text {Dest }}}{B_{\text {input }}}=1-\frac{B_{\text {Dest }}}{B_{C H_{4}}+B_{B F W}+W_{\text {Net }}^{\text {Import }}}$ \\
\hline Relative & $\eta_{\text {Relative }}=\frac{B_{\text {consumed } \text {, ideal }}}{B_{\text {consumed, actual }}}=\frac{B_{\text {Ammonia }}}{B_{C H_{4}}+B_{B F W}+W_{\text {Net }}^{\text {import }}}$ \\
\hline
\end{tabular}

$B=$ exergy rate or flow rate $(\mathrm{kW}), B F W=$ boiler feedwater, Dest $=$ destroyed.

\subsection{Optimization problem definition}

As it has been exposed hitherto, ammonia production plants are designed in complex formats where processes streams are integrated through recycle loops and an extensive heat recovery network. Accordingly, the selection among a set of proposed utility units (Fig. 7.1) of the most suitable alternatives that minimize the operating cost and the energy requirements is better addressed through the solution of a mixed integer linear programming (MILP) problem. This framework enables the reshaping of the composite curve of the chemical process, so that the operating cost of the ammonia production and the process irreversibility can be reduced.

\subsubsection{Minimum Energy Requirement}

As discussed in Chapter 5, in order to calculate the minimum energy requirements (MER), the contribution of each hot and cold streams to the overall heat balance is combined into the respective hot and cold composite curves [229]. These composite curves are shifted away from each other through a physical constraint, namely the minimum temperature approach $\Delta \mathrm{T}_{\min }$, so that reasonable heat transfer rates can be ensured. Clearly $\Delta \mathrm{T}_{\min }$ will depend on the nature of each stream [157]. Equation (7.9-7.11) shows the optimization problem set to find the MER:

$\min _{R_{r}} R_{N_{r}+1}$ 
Subject to

Heat balance of each interval of temperature $r \sum_{i=1}^{N} Q_{i, r}+R_{r+1}-R_{r}=0 \quad \forall r=1 . . N$

Feasibility of the solution

$$
\mathrm{R}_{r} \geq 0
$$

where $N$ is the number of temperature intervals defined, considering the supply and target temperatures of the entire set of streams; $Q$ is the heat exchanged between the process streams $\left(Q_{\mathrm{i}, \mathrm{r}}>0\right.$ hot stream, $<0$ cold stream) and $R$ is the heat cascaded from higher $(r+1)$ and to lower $(r)$ temperature intervals $(\mathrm{kW})$.

\subsubsection{Minimum Operating Cost}

In order to calculate the minimum operating cost of the ammonia plant, the modeling of the process flowsheet is separated from the heat integration problem, so that the calculation of the mass and energy balances and the complex energy conversions can be handled by the process modeler Aspen ${ }^{\circledR}$ Plus. Meanwhile, the determination of the minimum energy requirements (MER) and the solution of the energy integration problem is performed by using the OSMOSE Lua platform developed by the IPESE group at the École Polytechnique Fédérale de Lausanne, Switzerland [121].

Thus, the first step consists of the identification of a list of all the suitable utility systems [ $\omega=$ steam network, furnace, refrigeration system, heat pump, cogeneration system, etc.] based on the analysis of the shape of the grand composite curve (GCC) [227]. Then, the computational framework manages the data transfer with the ASPEN Plus ${ }^{\circledR}$ software and builds the mixed integer linear programming (MILP) problem described in the Eqs.(7.127.16). This optimization problem minimizes the resources consumption (water, natural gas) and, thus, the operating cost of the chemical plant, while satisfying the constraints of the MER problem [229]. In this way, the synthesis problem consists of finding the integer variables, $y_{w}$, associated to the existence or absence and the corresponding continuous load, $f_{w}$, of the utility system $\omega$ that minimizes the objective function given by Eq.(7.12):

$$
\min _{\substack{f_{\omega}, y_{\omega} \\ R_{r}, W}} \sum_{\omega=1}^{N_{\omega}} f_{\omega}\left[(B \cdot c)_{f e e d s t o c k}+(B \cdot c)_{\text {fuel }}+(V \cdot c)_{\mathrm{H}_{2} \mathrm{O}}+(W \cdot c)_{\text {power }}^{\text {net imp }}-(B \cdot c)_{\mathrm{NH}_{3}}-(\dot{m} \cdot c)_{C O_{2}}\right] \cdot t_{O P}(7
$$

Subject to:

Heat balance at the temperature interval $r \quad \sum_{\omega=1}^{N_{\omega}} f_{\omega} q_{\omega, r}+\sum_{i=1}^{N} Q_{i, r}+R_{r+1}-R_{r}=0 \quad \forall r=1 . . N$

Balance of generated/consumed power

$$
\sum_{\omega=1}^{N_{\omega}} f_{\omega} W_{\omega}+\sum_{\substack{\text { chemical } \\ \text { units }}} W_{\text {net }}+W_{\text {imp }}-W_{\text {exp }}=0
$$

Existence and load of the utility unit $w$

$$
f_{\text {min, }, \mathrm{y}_{\omega}} \leq f_{\omega} \leq f_{\text {max }, \omega} \mathrm{y}_{\omega} \quad \forall \omega=1 . . N_{\omega}
$$

Feasibility of the solution (MER)

$$
R_{1}=0, R_{N_{r}+1}=0, \mathrm{R}_{r} \geq 0 ; W_{\text {prod }} \geq 0, W_{\exp } \geq 0
$$


where $N_{w}$ is the number of units in the set of utility systems; $B$ is the exergy flow rate $(\mathrm{kW})$ of the resources going in and out of the plant; $c$ stands for the purchasing cost or the selling price (euro per $\mathrm{kWh}, \mathrm{m}^{3}$ or $\mathrm{kg}$ ) of the feedstock and electricity consumed or the marketable ammonia and $\mathrm{CO}_{2}$ produced; $V$ is the flowrate of water consumed $\left(\mathrm{m}^{3} / \mathrm{h}\right) ; q$ is the heating/cooling rates supplied by the utility systems $(\mathrm{kW}) ; t_{\mathrm{OP}}$ is the operation time $(\mathrm{h})$; and $W$ is the power produced by either the utility systems, the chemical operation units or imported from/exported to the grid $(\mathrm{kW})$.

It is important to emphasize that the process modeling and simulation of the chemical plant alone, including its mass and energy balances, is performed by using Aspen ${ }^{\circledR}$ Plus software. Meanwhile, the utility units shown in Fig. 7.1 are modeled via equation oriented subroutines written in the Lua programming language. Therefore, the additional equations required for the mass and energy balances of those units rely on the concept of layer (water, natural gas, ammonia, power, carbon dioxide, etc.), as shown in Fig. 7.2.

For instance, according to Eq. 7.14, the overall power generated by the utility systems (steam or gas power cycles) should be able to supply the demands of the chemical plant and other utility units (refrigeration, heat pump, cooling tower). Otherwise, the balance of the respective layer would include the possibility of importing electricity from the grid. Moreover, if a surplus of power could be produced at expense of the waste heat exergy available throughout the plant, the excess electricity could be sold to the grid, provided that the electricity export is economically attractive. Analogously, in the layer of natural gas (or other resource), the amount of energy supplied by the vendors is balanced with the fuel or feedstock consumption by the chemical plant and utility systems (gas turbine, furnace). In this way, not only the balances of the resources consumed (power, natural gas, biomass, water, etc.) and the products and byproducts produced (ammonia, biomethane, syngas, hydrogen, $\mathrm{CO}_{2}$, etc.), but as well as of the waste heat recovered, can be performed (Fig. 7.2).

To this end, representative market cost for the water $\left(3.69 \mathrm{euro} / \mathrm{m}^{3}\right)$ and natural gas consumed $\left(0.032\right.$ euro/kWh), as well as the selling prices of ammonia $(0.098$ euro/kWh $)$ and $\mathrm{CO}_{2}$ $(0.0084$ euro/kWh) produced are taken from literature [66, 230, 231]. 
Fig. 7.2. Definition of the layer concept used in the optimization of the components of the utility systems.

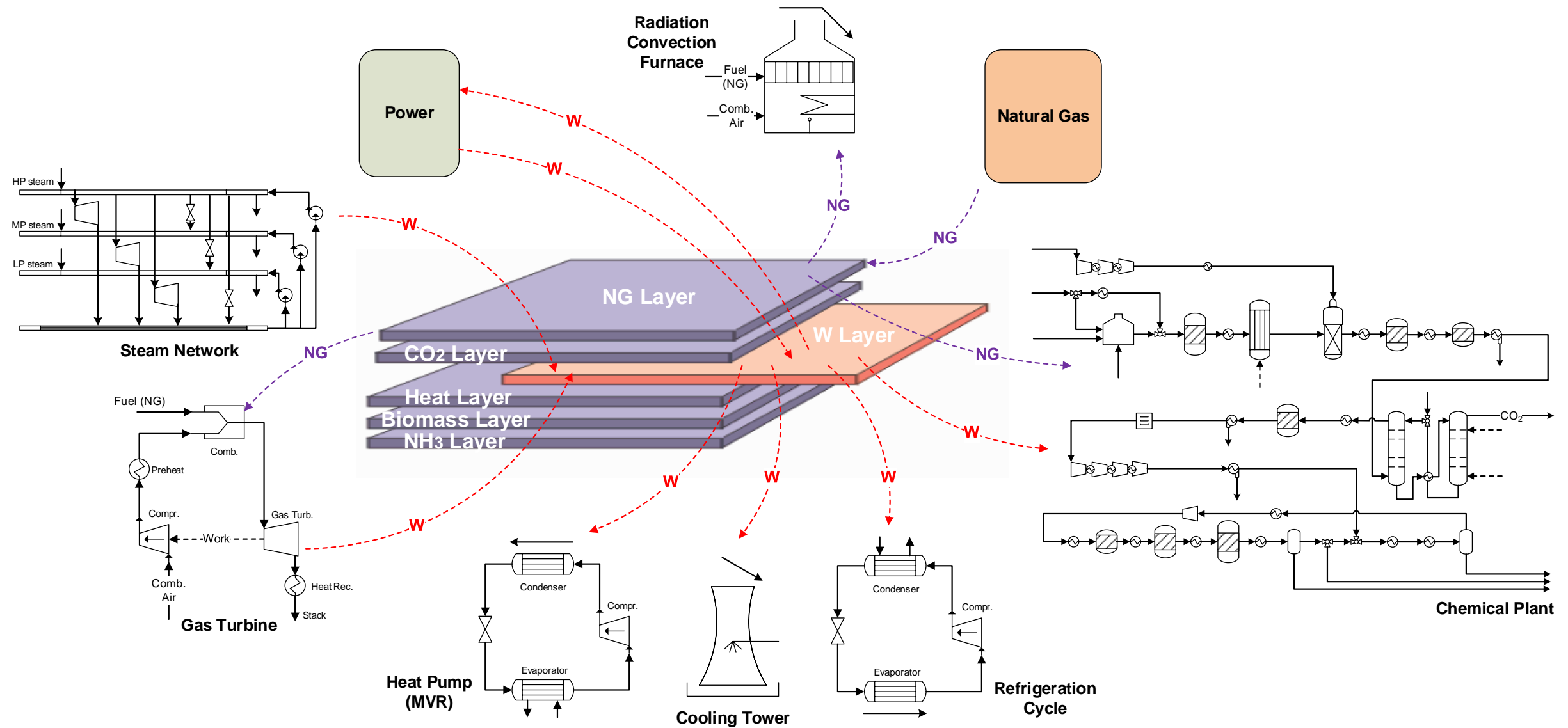

Source: Author. 


\subsection{Optimal operating conditions of the utility systems}

In this section, some graphical representations, proven to be fundamental tools in the optimal choice of the most suitable energy technologies and resources consumed, are examined. Afterwards, the solution of the optimization problems for the minimum energy requirement and operating cost are discussed. Finally, the summary of the main exergy consumption remarks is presented.

\subsubsection{Minimum Energy Requirement}

Figures 7.3a-c show the composite (CC) and grand composite (GCC) curves of the chemical process of syngas and ammonia production, operating under three different carbon capture technologies, namely MDEA, DEA and DEPG absorption systems. The minimum heating and cooling requirements, calculated by solving the MER problem, Eqs.(7.9-7.11), are also indicated.

A first inspection of the GCC curves evidences a marked shift of the pinch point temperature from about $120^{\circ} \mathrm{C}$ in the chemical absorption based (MDEA, DEA) configurations to about $500^{\circ} \mathrm{C}$ in the case of DEPG-based absorption system. The shape of the curves shown in Fig. $7.3 \mathrm{c}$ is inherent to the absence of the reboiler duty in a physical absorption-based syngas purification unit. This characteristic entails particular opportunities for the integration of the utility systems and demands an appropriate approach in order to fully exploit the thermodynamic potential of the excess heat available throughout the chemical plant. Among the most interesting opportunities are the reduction of the furnace fuel consumption by preheating the combustion air along with the enhancement of the cogeneration potential. Additionally, as the process steam generation occurs below the pinch temperature in Fig.7.3c, the use of heat recovery steam generators (HRSG) is preferable to the utilization of auxiliary fired boilers. Otherwise, the consumption of a high temperature utility source below the pinch temperature would lead to an avoidable increase of the cooling requirement and the fuel consumed. Accordingly, reduced cooling (18-23\%) and heating (40-51\%) requirements are expected when physical solvents are used for carbon capture purposes compared to chemical absorption-based configurations (see Fig. 7.3).

In the cases of ammonia plants with amine-based purification units (Figs. 7.3a-b), the reboiler and condenser streams in the desorption column generate a plateau-like pinch point at low temperatures. The desorption process demands an appreciable amount of hot utility, approx. 3.17 and 3.69 MJ/kg $/ \mathrm{CO}$ for MDEA and DEA technology, respectively. For this reason, the installation of a mechanical vapor recompression (MVR) unit, that transfers heat across the pinch temperature by using the power generated by the cogeneration system, seems to be a suitable energy integration approach that might help reducing the demand of the high temperature utility. According to Figs.7.3a-c, the target would consist of exploiting the opportunity of reactants preheating while avoiding excessive firing in the reformer furnace by maximizing the waste heat recovery. It is also recommendable to adjust the levels of pressure 
of the steam generation depending on the self-sufficient zones depicted in the GCC, and to reduce the surplus steam generation whenever it is possible.

Fig. 7.3. Composite (CC, left) and grand composite (GCC, right) curves for the simulated ammonia plants with (a) MDEA-based, (b) DEA-based and (c) DEPG-based carbon capture
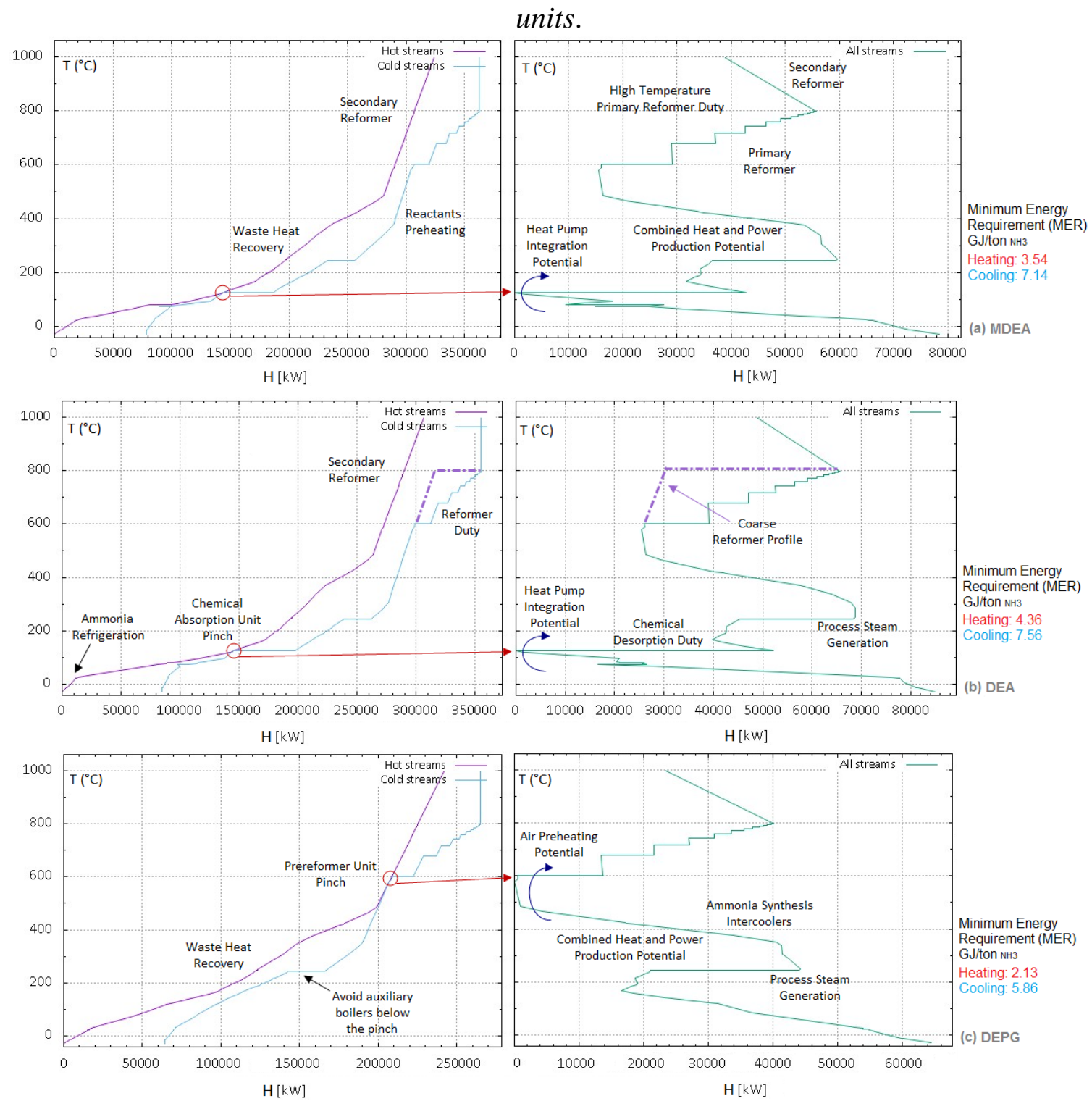

Source: Author.

The GCCs shown in Fig. 7.3 are not only helpful in devising the most appropriate energy technologies of the utility system that better exploit the waste heat recovery of the chemical process, but also for envisaging breakthrough approaches in the chemical plant. Figure 7.4 compares the integration profile of a feedstock saturator with that of a steam boiler. By profiting the partial pressure vaporization effect, up to $10-30 \%$ of the reforming steam can be generated through the saturator unit [166]. The saturator transforms the plateau-like profile of the vaporization process into a smoother one, thus facilitating the integration of low-grade waste heat available elsewhere in the chemical process [95]. Furthermore, by using a prereformer, the endothermic reforming reaction can be carried out at lower temperatures, reducing the firing in the reformer duty. 
In addition, by promoting the process-to-process heat exchange in the self-sufficient zones at higher temperatures, instead of raising low pressure steam, the energy degradation arisen with the large heat transfer driving forces can be minimized. For instance, according to Fig. 7.4, the use of a gas heated reformer in combination with an enriched air blown autothermal reformer cold may reduce the exergy destroyed at the frontend syngas production unit, while increasing the rate of carbon captured per unit of hydrogen produced [231].

Fig. 7.4. T-H profile modifications by means of the incorporation of other energy technologies in the chemical system.

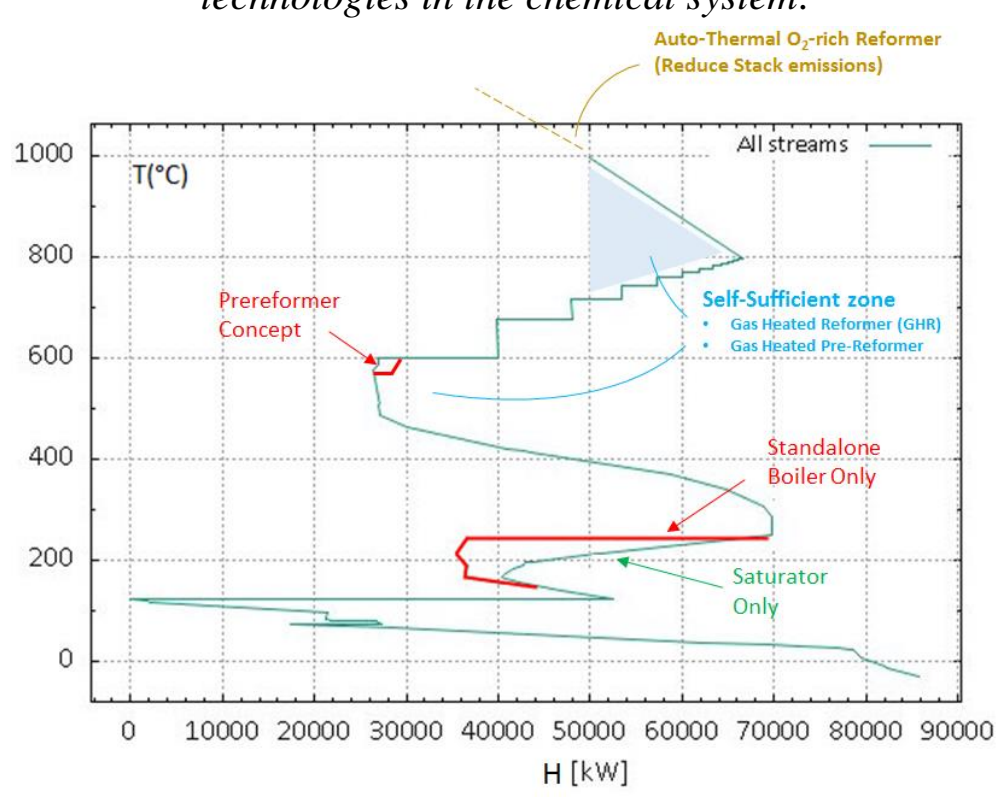

Source: Author.

\subsubsection{Minimum operating cost}

After performing the inspection of the GCCs presented in Figs. 7.3a-c, twelve optimal ammonia plant configurations are proposed and compared in terms of their exergy demands and the potential for their energy integration, when using three carbon capture technologies (i.e. MDEA, DEA and DEPG solvent, see Table 7.2). They also operate under different scenarios of resources consumption and cogeneration modes (i.e. grid, mixed, and autonomous plant powered by either Rankine or Combined cycles). The most suitable utility units as well as the operating conditions thereof are determined for each configuration, and represented in Figs.7.5a-i, in the so-called Integrated Curves.

Figures 7.5a-c show the integrated curves for the scenario in which electricity import is favored over the autonomous electricity generation in the cogeneration system (GRID mode). Since the steam superheating is hindered, the integration of a heat pump (MVR) is enabled to balance the heat demand of the chemical absorption carbon capture units. In other scenarios, Figs.7.5d-f, denominated MIXED mode, the steam network (0.12, 3, 40, 110 bar, superheating $200^{\circ} \mathrm{C}$ ) supplies a larger share of the power consumed, but the utility system still imports an important amount of electricity from the grid in order to balance the plantwide power requirements. Differently from the GRID mode, in the MIXED mode and especially for DEPG-based ammonia plants, there is a strong incentive for recovering as 
much as possible the excess heat exergy available below the pinch $\left(500^{\circ} \mathrm{C}\right)$ in order to increase the exergy available above it. It is also noteworthy that, in the MIXED mode, the heat pump integration is neglected by prioritizing the use of the surplus steam generated over the import of the costly electricity from the grid.

In a third scenario (AUTO mode, Figs. 7.5g-i), the total power consumption is entirely generated by the steam network by maximizing the waste heat recovery along the chemical plant and also consuming an important amount of natural gas. Even though this operation mode reproduces the typical conditions of modern ammonia plants [206], it is also responsible for the large irreversibility associated to the cogeneration system. This problem can be thermodynamically overcome by means of the integration of a combined cycle that provides the power required by the self-sufficient ammonia plant (AUTO GT, Figs. 7.5j-1). Table 7.2 summarizes the optimal process variables calculated for the various configurations of the ammonia plant shown in Fig. 7.5a-1. 
Fig. 7.5. Integrated Composite curves: (a-c) electricity grid import with limited steam superheating (Grid Mode), (d-f) electricity grid import with significant steam superheating (Mixed Mode), ( $g$-i) no electricity grid import with Rankine Cycle cogeneration (Auto Mode), (j-l) no electricity grid import with Combined Cycle cogeneration (Auto GT Mode), cf. Table 7.2. EE: electricity, Superh: superheating, Preh: preheating. Source: Author

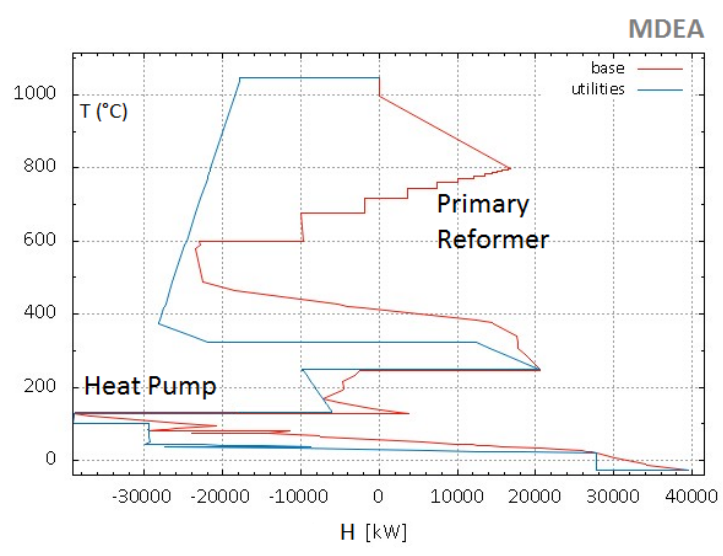

Conditions: EE grid, Steam superh. $50^{\circ} \mathrm{C}$, No air preh.

(a)

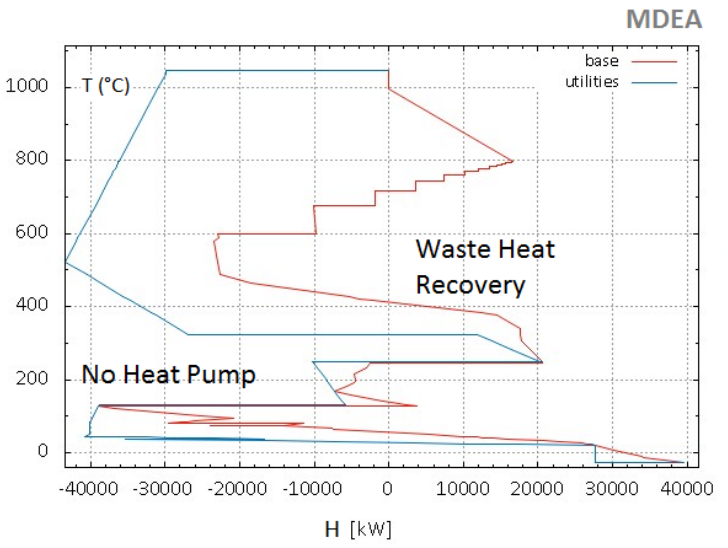

Conditions: EE grid, Steam superh. $200^{\circ} \mathrm{C}$, No air preh.

(d)

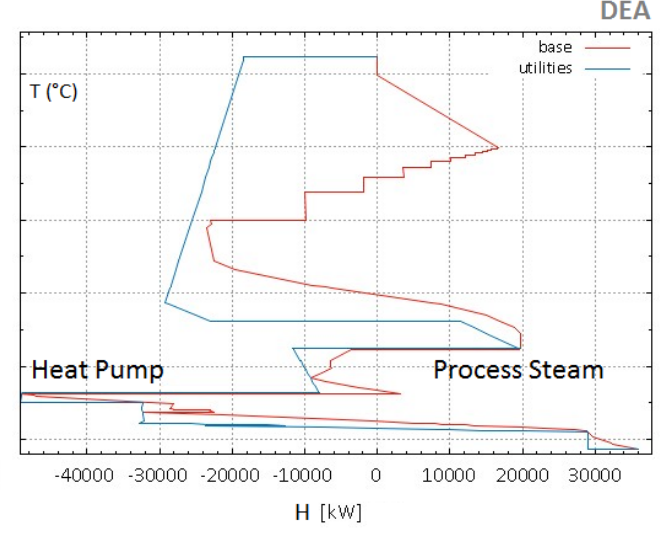

Conditions: EE grid, Steam superh. $50^{\circ} \mathrm{C}$, No air preh.

(b)

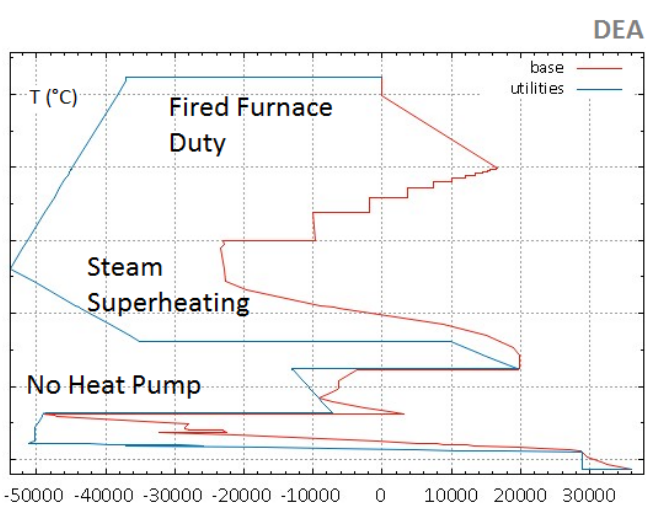

$\mathrm{H}[\mathrm{kW}]$

Conditions: EE grid, Steam superh. $200^{\circ} \mathrm{C}$, No air preh.

(e)

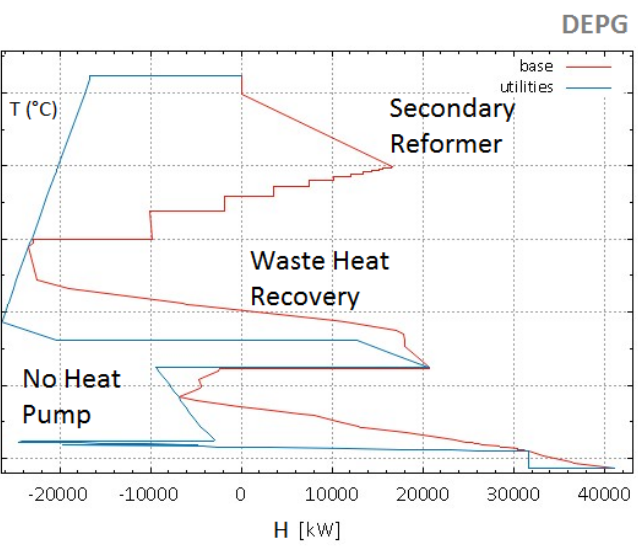

Conditions: EE grid, Steam superh. $50^{\circ} \mathrm{C}$, No air preh.

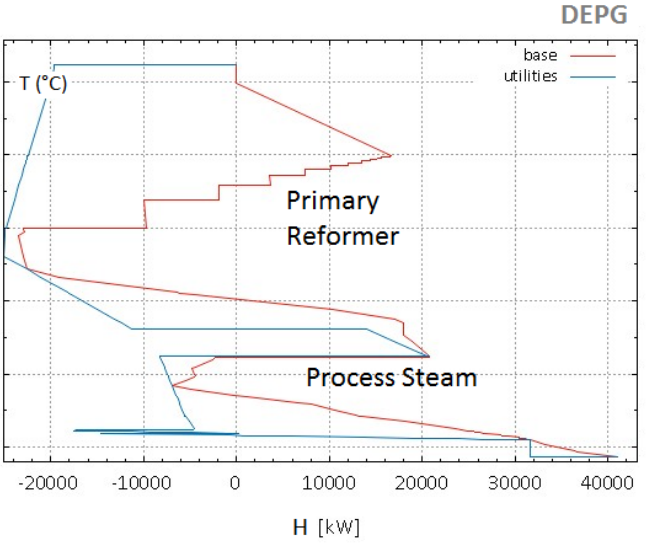

Conditions: EE grid, Steam superh. $200^{\circ} \mathrm{C}$, Air preh. $580^{\circ} \mathrm{C}$ 
Fig. 7.5. Integrated Composite curves: (a-c) electricity grid import with limited steam superheating (Grid Mode), (d-f) electricity grid import with significant steam superheating (Mixed Mode), ( $g$-i) no electricity grid import with Rankine Cycle cogeneration (Auto Mode), (j-l) no electricity grid import with Combined Cycle cogeneration (Auto GT Mode), cf. Table 7.2. EE: electricity, Superh: superheating, Preh: preheating. (cont'd)
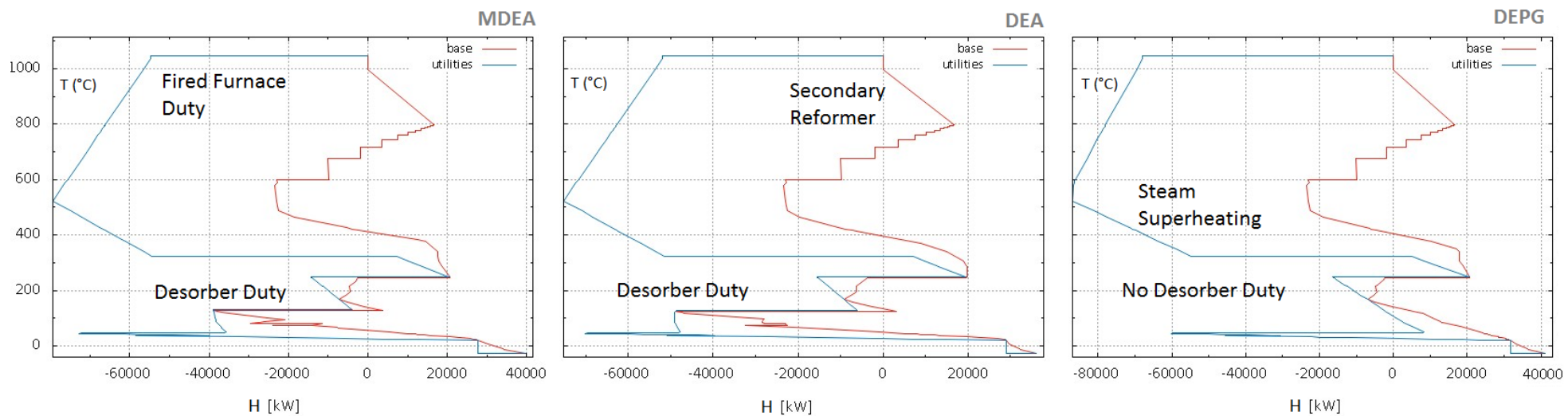

Conditions: No EE grid, Steam superh. $200^{\circ} \mathrm{C}$, No air preh. Conditions: No EE grid, Steam superh. $200^{\circ} \mathrm{C}$, No air preh. Conditions: No EE grid, Steam superh. $200^{\circ} \mathrm{C}$, Air preh. $580^{\circ} \mathrm{C}$

(g)

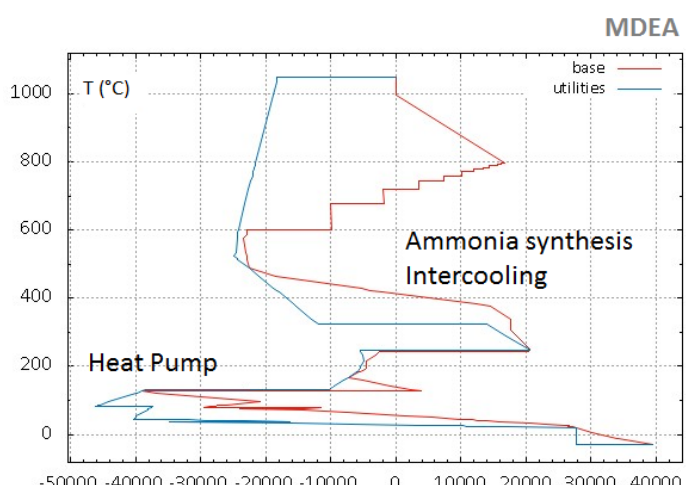

$H[k w]$

Conditions: No EE grid, Steam superh. $200^{\circ} \mathrm{C}$, No furnace air preh., Gas turbine regeneration $500^{\circ} \mathrm{C}$ (h)

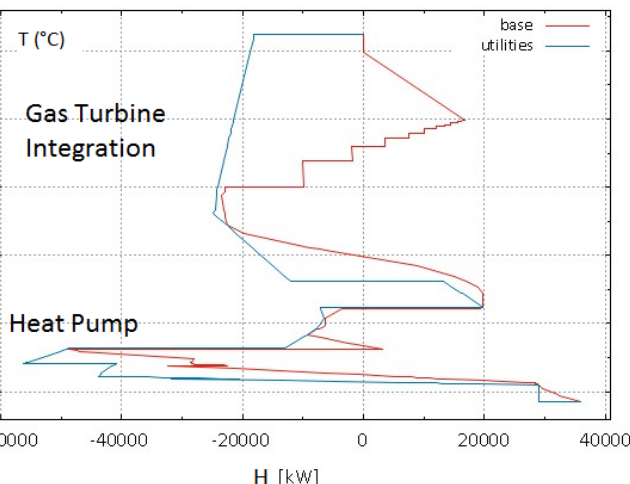

Conditions: No EE grid, Steam superh. $200^{\circ} \mathrm{C}$, No furnace air preh., Gas turbine regeneration $500^{\circ} \mathrm{C}$

(k) (i)

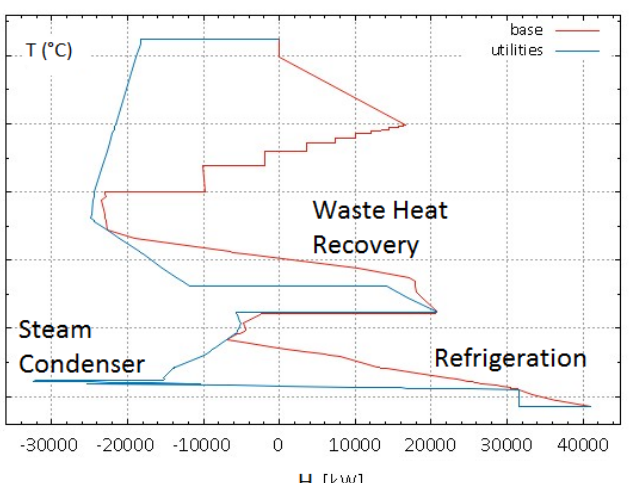

Conditions: No EE grid, Steam superh. $200^{\circ} \mathrm{C}$, furnace air preh. $580^{\circ} \mathrm{C}$, Gas turbine regeneration $500^{\circ} \mathrm{C}$ 
Table 7.2. Optimal process variables of the studied ammonia production facilities.

\begin{tabular}{|c|c|c|c|c|c|c|c|c|c|c|c|c|}
\hline Process parameter & $\begin{array}{l}\text { MDEA } \\
\text { Grid }\end{array}$ & $\begin{array}{l}\text { MDEA } \\
\text { Mixed }\end{array}$ & $\begin{array}{c}\text { MDEA } \\
\text { Auto RC }\end{array}$ & $\begin{array}{c}\text { MDEA } \\
\text { Auto GT }\end{array}$ & $\begin{array}{l}\text { DEA } \\
\text { Grid }\end{array}$ & $\begin{array}{l}\text { DEA } \\
\text { Mixed }\end{array}$ & $\begin{array}{c}\text { DEA } \\
\text { Auto RC }\end{array}$ & $\begin{array}{c}\text { DEA } \\
\text { Auto GT }\end{array}$ & $\begin{array}{l}\text { DEPG } \\
\text { Grid }\end{array}$ & $\begin{array}{l}\text { DEPG } \\
\text { Mixed }\end{array}$ & $\begin{array}{l}\text { DEPG } \\
\text { Auto RC }\end{array}$ & $\begin{array}{c}\text { DEPG } \\
\text { Auto GT }\end{array}$ \\
\hline Cogeneration system & Rankine & Rankine & Rankine & Combined & Rankine & Rankine & Rankine & Combined & Rankine & Rankine & Rankine & Combined \\
\hline Electricity import & Yes & Yes & No & No & Yes & Yes & No & No & Yes & Yes & No & No \\
\hline Feedstock consumption $\left(\mathrm{GJ} / \mathrm{t}_{\mathrm{NH} 3}\right)$ & 23.52 & 23.52 & 23.52 & 23.52 & 23.03 & 23.03 & 23.03 & 23.03 & 23.51 & 23.51 & 23.51 & 23.51 \\
\hline Utility fuel consumption $\left(\mathrm{GJ} / \mathrm{t}_{\mathrm{NH} 3}\right)$ & 3.28 & 5.50 & 10.06 & 7.02 & 3.32 & 6.68 & 9.34 & 6.99 & 3.07 & 2.55 & 8.83 & 6.21 \\
\hline $\begin{array}{l}\text { Utility elect. grid consumption } \\
\left(\mathrm{GJ} / \mathrm{t}_{\mathrm{NH} 3}\right)\end{array}$ & 2.15 & 1.15 & 0.00 & 0.00 & 2.04 & 0.67 & 0.00 & 0.00 & 1.90 & 1.71 & 0.00 & 0.00 \\
\hline Overall plant consumption $\left(\mathrm{GJ} / \mathrm{t}_{\mathrm{NH} 3}\right)$ & 28.94 & 30.17 & 33.58 & 30.53 & 28.39 & 30.38 & 32.37 & 30.02 & 28.47 & 27.76 & 32.34 & 29.72 \\
\hline $\begin{array}{l}\text { Extended plant consumption } \\
\left(\mathrm{GJ} / \mathrm{t}_{\mathrm{NH} 3}\right)^{1}\end{array}$ & 33.28 & 33.93 & 36.86 & 33.52 & 32.59 & 33.82 & 35.53 & 32.96 & 32.58 & 31.67 & 35.49 & 32.62 \\
\hline $\begin{array}{l}\text { Rankine cycle power generation } \\
\left(\mathrm{GJ} / \mathrm{t}_{\mathrm{NH} 3}\right)^{2}\end{array}$ & 0.44 & 1.25 & 2.46 & 0.72 & 0.43 & 1.47 & 2.18 & 0.66 & 0.53 & 0.70 & 2.49 & 0.80 \\
\hline $\begin{array}{l}\text { Brayton cycle power generation } \\
\left(\mathrm{GJ} / \mathrm{t}_{\mathrm{NH} 3}\right)^{2}\end{array}$ & 0.00 & 0.00 & 0.00 & 2.01 & 0.00 & 0.00 & 0.00 & 2.02 & 0.00 & 0.00 & 0.00 & 1.63 \\
\hline $\begin{array}{l}\text { Chemical process power demand } \\
\left(\mathrm{GJ} / \mathrm{t}_{\mathrm{NH} 3}\right)^{3}\end{array}$ & 1.61 & 1.61 & 1.61 & 1.61 & 1.61 & 1.61 & 1.61 & 1.61 & 1.81 & 1.81 & 1.81 & 1.81 \\
\hline Ancillary power demand $\left(\mathrm{GJ} / \mathrm{t}_{\mathrm{NH} 3}\right)^{4}$ & 0.98 & 0.79 & 0.85 & 1.12 & 0.86 & 0.53 & 0.57 & 1.07 & 0.62 & 0.60 & 0.68 & 0.62 \\
\hline Cooling requirement $\left(\mathrm{GJ} / \mathrm{t}_{\mathrm{NH} 3}\right)^{5}$ & 7.14 & 7.14 & 7.14 & 7.14 & 7.56 & 7.56 & 7.56 & 7.56 & 5.86 & 5.86 & 5.86 & 5.86 \\
\hline Heating requirement $\left(\mathrm{GJ} / \mathrm{t}_{\mathrm{NH} 3}\right)^{5}$ & 3.54 & 3.54 & 3.54 & 3.54 & 4.36 & 4.36 & 4.36 & 4.36 & 2.13 & 2.13 & 2.13 & 2.13 \\
\hline $\mathrm{CO}_{2}$ emissions avoided $\left(\mathrm{t}_{\mathrm{CO} 2} / \mathrm{t}_{\mathrm{NH} 3}\right)$ & 1.228 & 1.228 & 1.228 & 1.228 & 1.257 & 1.257 & 1.257 & 1.257 & 1.237 & 1.237 & 1.237 & 1.237 \\
\hline $\mathrm{CO}_{2}$ emitted -direct $\left(\mathrm{t}_{\mathrm{CO} 2} / \mathrm{t}_{\mathrm{NH} 3}\right)$ & 0.174 & 0.293 & 0.536 & 0.374 & 0.177 & 0.356 & 0.497 & 0.372 & 0.164 & 0.136 & 0.470 & 0.331 \\
\hline $\mathrm{CO}_{2}$ emitted - indirect $\left(\mathrm{t}_{\mathrm{CO} 2} / \mathrm{t}_{\mathrm{NH} 3}\right)^{6}$ & 0.053 & 0.047 & 0.049 & 0.034 & 0.051 & 0.044 & 0.046 & 0.035 & 0.048 & 0.042 & 0.043 & 0.031 \\
\hline $\mathrm{CO}_{2}$ emitted indirect $-\mathrm{EE}(\%)$ & 69.80 & 42.42 & 0.00 & 0.00 & 68.4 & 26.19 & 0.00 & 0.00 & 68.5 & 70.28 & 0.00 & 0.00 \\
\hline Total fossil $\mathrm{CO}_{2}$ emitted $\left(\mathrm{t}_{\mathrm{CO} 2} / \mathrm{t}_{\mathrm{NH} 3}\right)$ & 0.228 & 0.340 & 0.585 & 0.408 & 0.228 & 0.400 & 0.543 & 0.407 & 0.212 & 0.178 & 0.513 & 0.361 \\
\hline Operating Incomes $^{7}$ (euro/t $\left.\mathrm{t}_{\mathrm{NH} 3}\right)$ & 516.65 & 516.65 & 516.65 & 516.65 & 516.90 & 516.90 & 516.90 & 516.90 & 516.72 & 516.72 & 516.72 & 516.72 \\
\hline Operating $\operatorname{Costs}^{7}\left(\right.$ euro/t $\left.\mathrm{t}_{\mathrm{NH} 3}\right)$ & 297.85 & 287.10 & 291.74 & 265.57 & 290.85 & 278.75 & 281.47 & 261.26 & 288.29 & 278.29 & 281.05 & 258.51 \\
\hline Operating Revenues ${ }^{7}\left(\right.$ euro/t $\left.\mathrm{t}_{\mathrm{NH} 3}\right)$ & 218.79 & 229.55 & 224.91 & 251.07 & 226.05 & 238.15 & 235.43 & 255.63 & 228.43 & 238.43 & 235.67 & 258.21 \\
\hline Ammonia production (t/day) & 950.36 & 950.36 & 950.36 & 950.36 & 970.45 & 970.45 & 970.45 & 970.45 & 950.84 & 950.84 & 950.84 & 950.84 \\
\hline
\end{tabular}

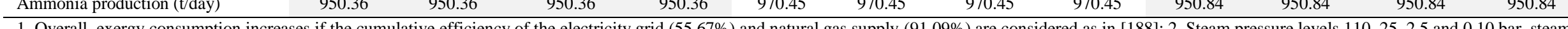
superh. $200^{\circ} \mathrm{C}$, Brayton cycle with regeneration, pressure ratio $20: 1 ; 3$. Power consumed by the chemical plant alone; 4 . Cooling tower, heat pump and vapor compression refrigeration systems; 5 . Heating requirements of the chemical processes (energy basis) determined from the composite curves; 6 . It considers the indirect emissions due to the upstream supply chains of natural gas $\left(0.0049 \mathrm{~g}_{\mathrm{CO} 2} / \mathrm{kJ}_{\mathrm{CH} 4}\right)$ and electricity $\left(62.09 \mathrm{~g}_{\mathrm{CO} 2} / \mathrm{kWh}\right)$ [188, 232]; 7. Operating revenues (only) calculated as the difference between the gross operating incomes minus the operating cost. See Appendix 2 for a detailed discussion on the influence of the share of the capital investment in the overall plant revenues. 
According to Fig. 7.6, the chemical absorption-based ammonia plant configurations present the highest overall exergy consumption figures, compared to the DEPG-based plants. Meanwhile, the lowest exergy consumption corresponds to the MIXED operating mode (partial electricity import and Rankine cycle-powered cogeneration system) by using a DEPG absorption unit $\left(27.76 \mathrm{GJ} / \mathrm{t}_{\mathrm{NH} 3}\right.$, see Table 7.2$)$. In practice, ammonia plants operate in an autonomous way in which the waste heat recovered along the plant is maximized to simultaneously produce steam and power, thus reducing the external fuel consumption. However, the AUTO (autonomous, Rankine cycle-powered) operation modes present the worst performance among all the optimized configurations due to the reduced cogeneration efficiency, attaining total power consumptions of about $32.27-33.5 \mathrm{GJ} / \mathrm{t}_{\mathrm{NH} 3}$, or $21 \%$ higher than the best overall exergy consumption calculated for MIXED modes. The integration of a combined cycle (AUTO GT) partly helps to overcome this problem by increasing the cogeneration efficiency, which clearly impacts the amount of fuel consumed in the AUTO operating modes (i.e. 25-30\% lower). It is also worthy to notice that, when the GRID mode is adopted (i.e. minimum steam superheating), the natural gas fuel consumed remains almost invariable regardless of the $\mathrm{CO}_{2}$ capture technology used, but a larger amount of electricity is consumed instead. As already explained, this follows the integration of a mechanical vapor recompression system, restricting the fuel consumption to the supply of the high temperature reformer duty. As a conclusion, both electricity import and fuel consumption can be reduced by preheating the combustion air and operating in a MIXED mode.

Fig. 7.6. Plantwide and extended exergy consumption and $\mathrm{CO}_{2}$ emission figures for the various configurations studied.

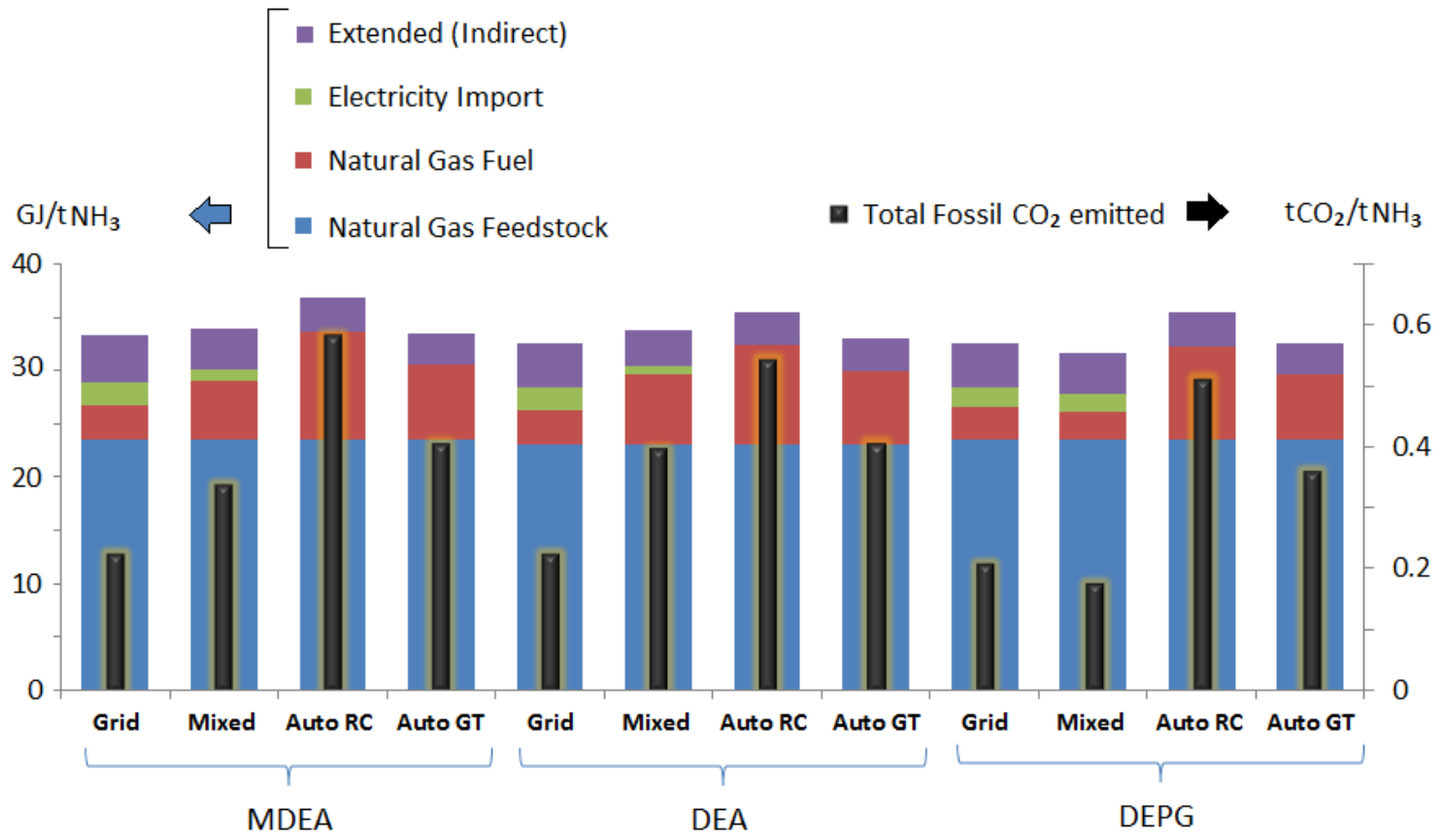

Source: Author

Additionally, Fig. 7.6 shows the Extended Exergy Consumption that takes into account the exergy efficiency of the electricity generation in the grid $(55.68 \%)$ as well as of the natural gas $(91.09 \%)$ supply chain [188]. Certainly, by including the upstream inefficiencies of the 
feedstock supply chains into the originally standalone ammonia plant analysis, the panorama is worsened as the cumulative irreversibility further impairs the global performance of the ammonia production. Actually, the extended exergy consumption can be $14 \%$ higher in the electricity import cases (larger cumulative losses) and 9.7\% higher in the case of the autonomous configurations (cf. Table 7.2). Although these figures may not be immediately interesting for ammonia producers when evaluating the performance of the plant itself, they certainly prove to be useful either for issuing public and environmental policies or carrying out decision-makings that aim to holistically compare the impact of the fertilizers sector with other industrial sectors in a fair level playing field.

Table 7.2 also shows the indirect and direct $\mathrm{CO}_{2}$ emissions associated to the ammonia production process. The former accounts for the emissions owed to the upstream supply chains of natural gas $\left(0.0049 \mathrm{~g}_{\mathrm{CO} 2} / \mathrm{kJ}_{\mathrm{CH} 4}\right)$ and electricity $\left(62.09 \mathrm{~g}_{\mathrm{CO} 2} / \mathrm{kWh}\right)[188,232]$. In turn, the direct $\mathrm{CO}_{2}$ emissions originate in the furnace of the externally fired primary reformer. On the other hand, the avoided $\mathrm{CO}_{2}$ emissions are those produced along the process unit reactions and captured by either the physical or chemical absorption system, and commercialized after its purification. As it can be observed, the contribution of the electricity generation to the indirect emissions can be as high as $70 \%$, indicating that the impact of this resource should not be lightly neglected if a broader comparison with other alternative hydrogen and ammonia production routes (biomass, solar, nuclear and wind energy) is aimed. In spite of this, the electricity import operating modes (GRID and MIXED) are still the ones with the lowest associated overall $\mathrm{CO}_{2}$ emissions $\left(0.217 \mathrm{t}_{\mathrm{CO} 2} / \mathrm{t}_{\mathrm{NH} 3}\right)$. This is not surprising due to the low $\mathrm{CO}_{2}$ footprint associated to the Brazilian electricity mix [58]. Thus, the environmental impact of the ammonia production process is a strongly location-dependent industrial activity, as it has been also demonstrated by using alternative ammonia production routes from biomass gasification [30]. Anyway, the greater challenge will remain in the $\mathrm{CO}_{2}$ capture from the fumes of the primary reformer furnace, as the desorption energy per ton of $\mathrm{CO}_{2}$ is generally increased in post-combustion applications $[33,84]$.

According to Fig. 7.7, the lowest revenue (i.e. highest operating cost) corresponds to the MDEA absorption-based ammonia plant operating under the GRID mode. It can be partly explained by an intensive import of expensive electricity. However, it is also shown that, even if the AUTO mode with Rankine cycle effectively reduces (2-3\%) the operating cost compared to GRID mode, the same cost is actually higher (1-2\%) than in the MIXED mode. Thus, although the costly electricity import may actually impair the operating cost of the utility system, the overall effect can be eventually compensated by an appropriate integration of the cogeneration system as in the MIXED mode. It is also noteworthy that, regardless of the absorption solvent used for carbon capture purposes, when the ammonia plant is powered by a combined cycle (steam network plus gas turbine system), the operating cost can be reduced from 1 up to $13 \%$ compared with other configurations, either autonomous or griddependent. For the sake of a more complete comparison, the trade-off between the capital cost increase and the operating cost reduction (i.e. the marginal purchasing cost of an additional gas turbine system) has been roughly estimated by assuming a specific open cycle gas turbine (OGCT) cost of $900 \mathrm{USD} / \mathrm{kW}$ incl. IDC [233] or 3.6-4.5 euro/ $\mathrm{t}_{\mathrm{NH} 3}$. This value is 
less than one fourth of the total reduction (19.7-39.3 euro/ $\left.\mathrm{t}_{\mathrm{NH} 3}\right)$ of the operating costs when a combined cycle system is integrated. Then, as a preliminary estimation, there is a net gain in the revenues even at expense of the introduction of an OGCT system, because the reduction in the respective operating cost offsets its increased capital cost. In this way, the best performance in terms of total revenues still corresponds to the ammonia plant powered by a combined cycle and using DEPG as the carbon capture solvent.

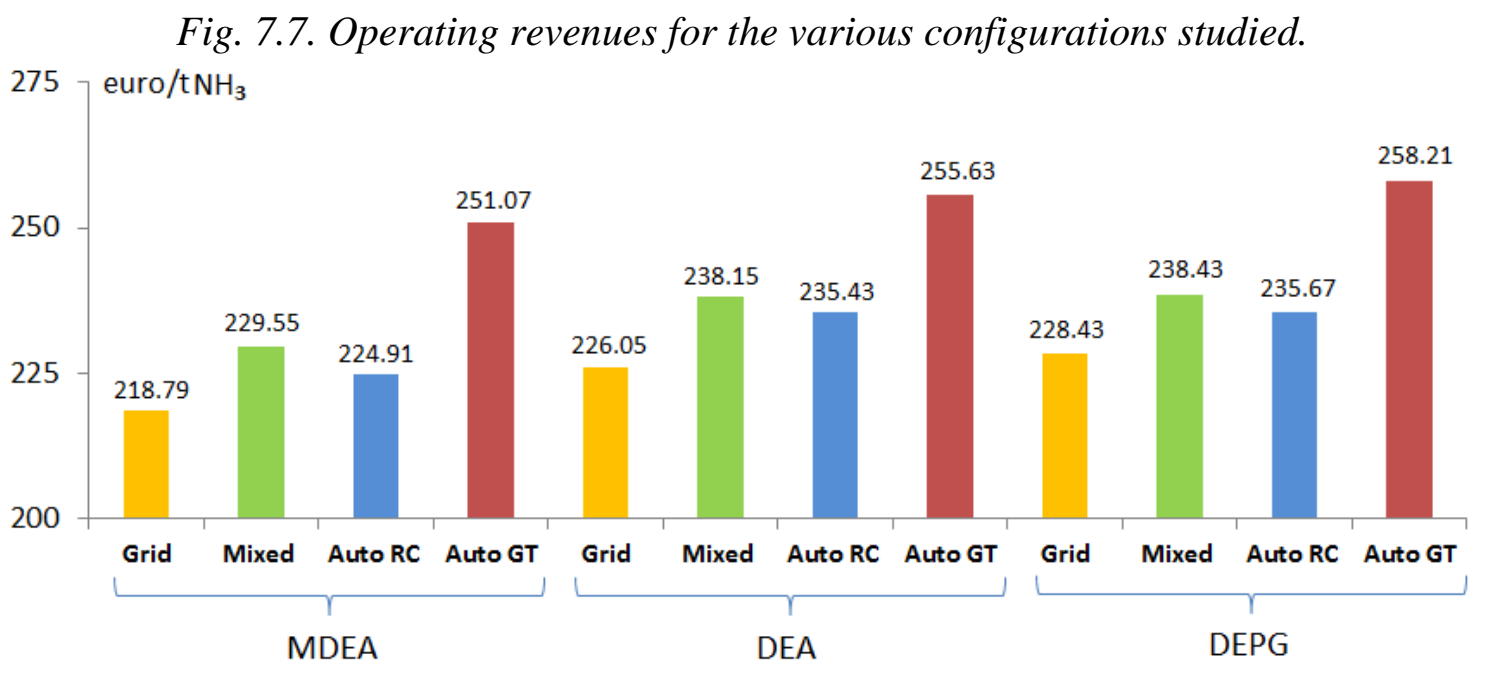

Source: Author

Fig. 7.8 shows the breakdown of the power generation and consumption amongst the main power consumers of the chemical plant as well as the ancillary utility systems (heat pump, refrigeration, cooling tower). As expected, the two largest power demands correspond to the process air and syngas compression systems, required to operate the ammonia plant at elevated pressures. The ammonia refrigeration-separation system is also responsible for about one fourth of the overall power consumption. Due to the economy of scale, higher pressures allow reducing the size of the plant components per unit of throughput of ammonia produced, as well as favors the syngas conversion and ammonia separation. However, the future trend points towards the utilization of lower reaction pressures as better catalyst with higher activities at milder operation conditions are constantly developed [41]. It must be also noticed the effect of the integration of a mechanical vapor recompression unit (heat pump) on the overall power consumption of the utility systems. Namely, a considerable amount of costly electricity import and an additional natural gas consumption are required when operating in either the GRID mode or the combined cycle-powered AUTO GT mode, respectively. In fact, the heat pump consumption is comparable to the total power produced by the Rankine cycle alone.

Finally, the highest power consumption related to the DPEG pumping must be compared to that of the amine-based systems. This is a well-known issue related to the DEPG absorption systems, i.e. the reduced heating requirement comes at expense of a higher amount of power consumed in the circulation pump [130]. Certainly, higher capture rates are desirable to avoid the consumption of the valuable hydrogen in the downstream methanator unit, also because slipped methane acts an inert in the ammonia loop, increasing the need of a larger purge. However, larger capture efficiencies with DEPG requires higher solvent circulation rates, 
since the solvent capacity is compromised as the temperature increases [234, 235]. In fact, for a $90 \% \mathrm{CO}_{2}$ capture efficiency, the solvent $/ \mathrm{CO}_{2}$ loading ratio can be as high as 5:1, i.e. threefold the value when more stringent capture efficiencies (> 95\%) are imposed [236]. Meanwhile, DEA recirculation rate is set as $716 \mathrm{~m}^{3} / \mathrm{h}$, with maximum and minimum $\mathrm{CO}_{2}$ loadings of 0.47 and $0.025 \mathrm{kmolCO}_{2} / \mathrm{kmol}_{\mathrm{DEA}}$, respectively. For the case of MDEA, those figures are set as $495 \mathrm{~m}^{3} / \mathrm{h}, 0.51$ and $0.013 \mathrm{kmolCO}_{2} / \mathrm{kmol}_{\mathrm{MDEA}}$, respectively. 
Fig. 7.8. Breakdown of power generation and consumption in each plant configuration shown in Table 7.2. Source: Author

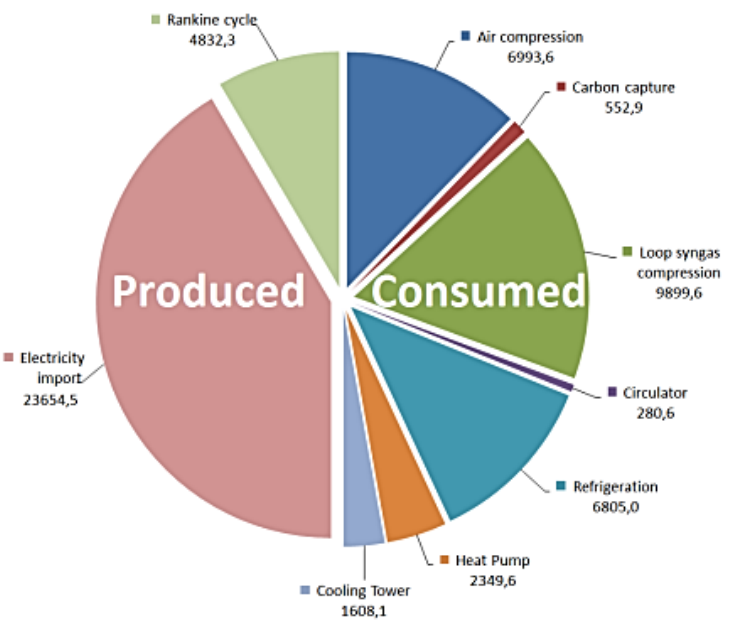

(a) MDEA Grid

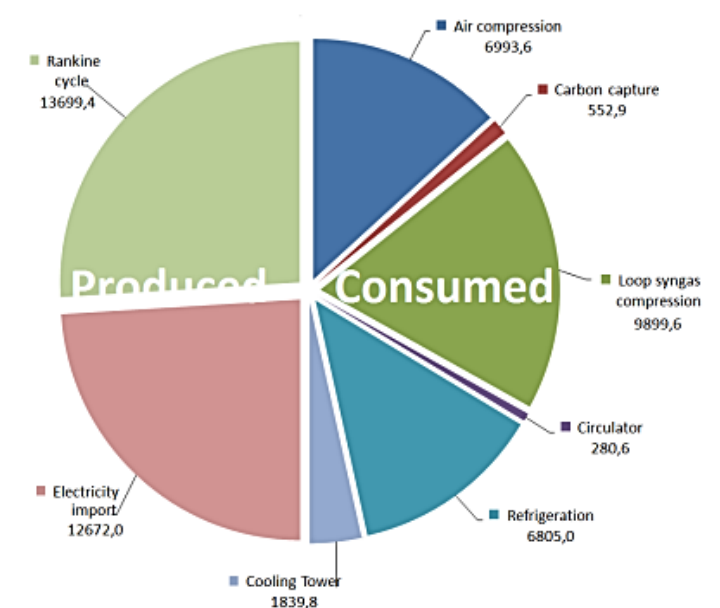

(d) MDEA Mixed

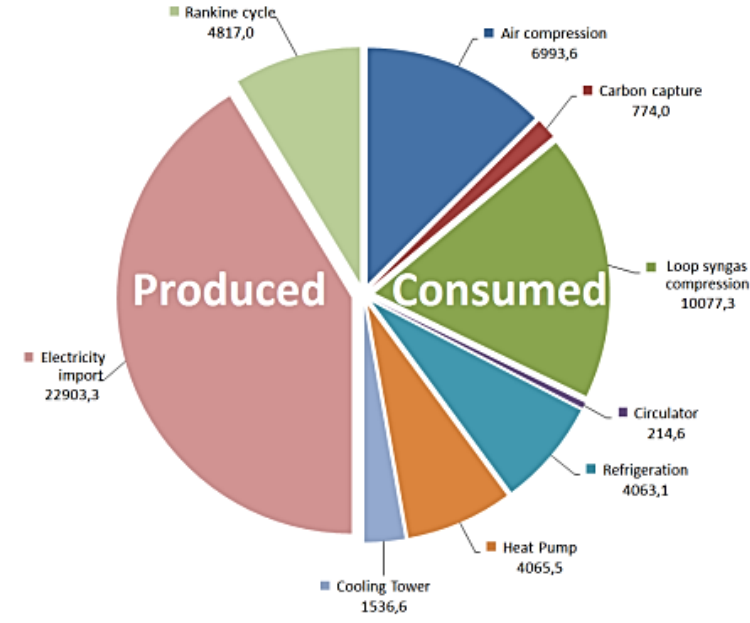

(b) DEA Grid

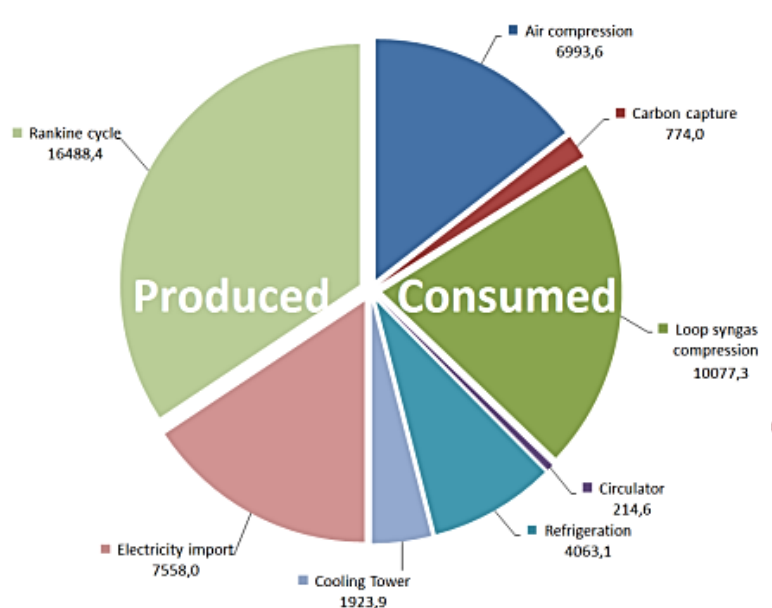

(e) DEA Mixed

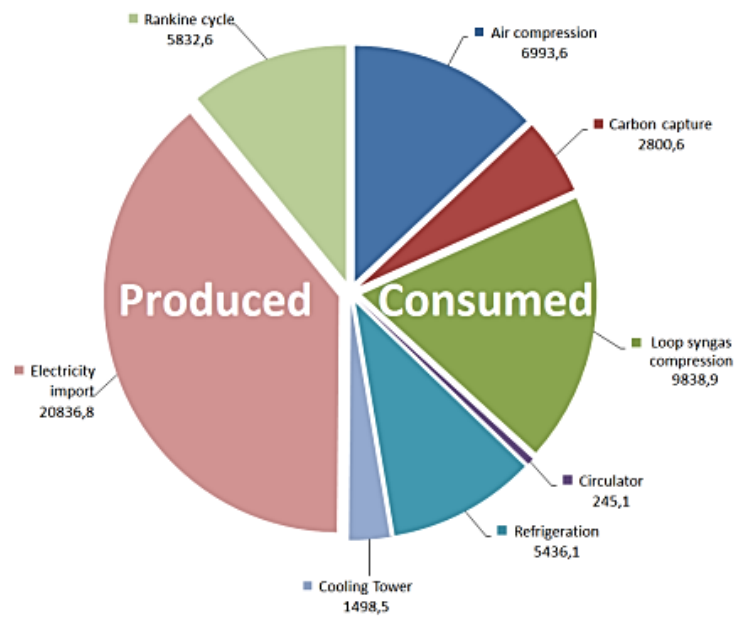

(c) DEPG Grid

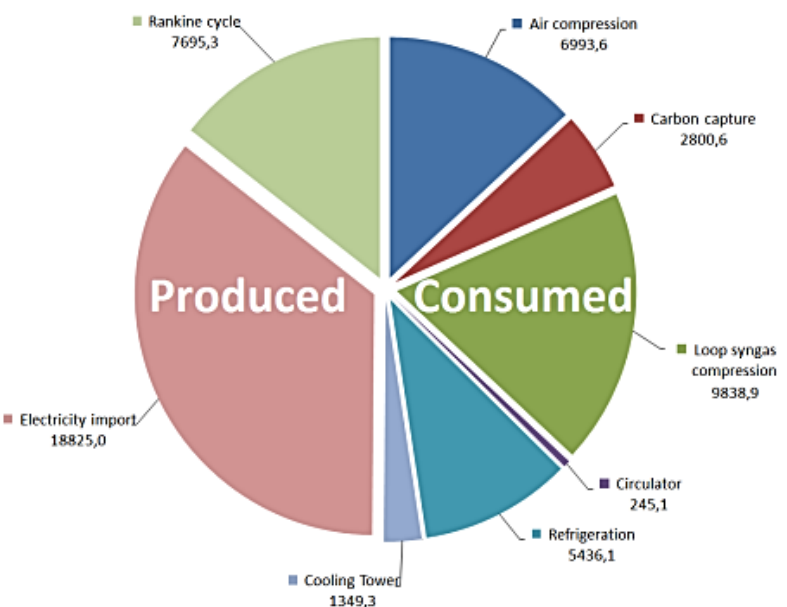

(f) DEPG Mixed 
Fig. 7.8. Breakdown of power generation and consumption in each plant configuration shown in Table 7.2. (cont'd). Source: Author

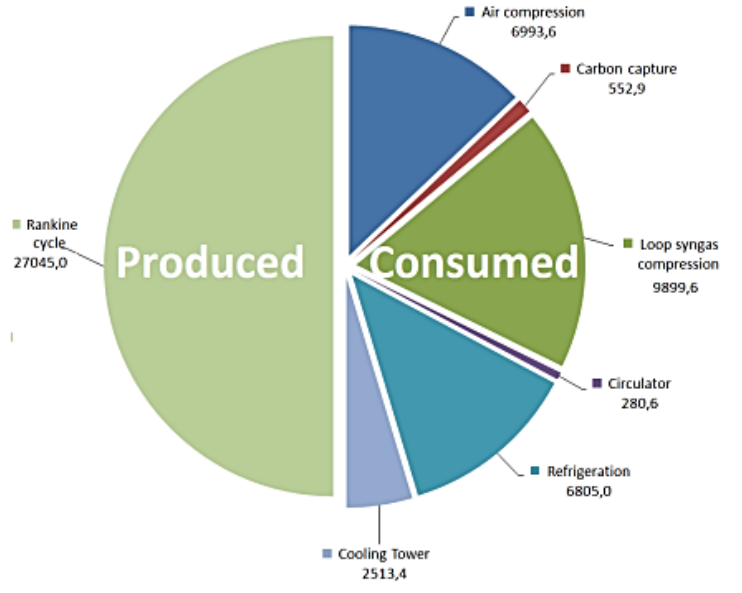

(g) MDEA Auto

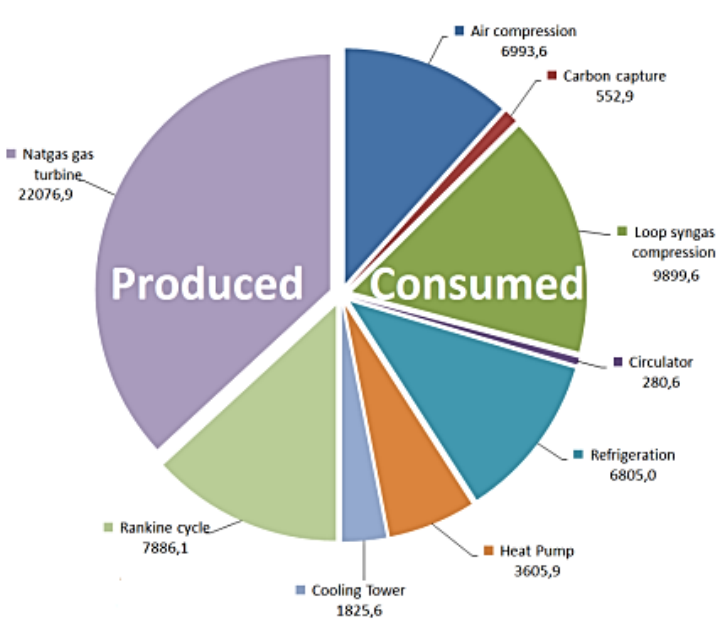

(j) MDEA Auto GT

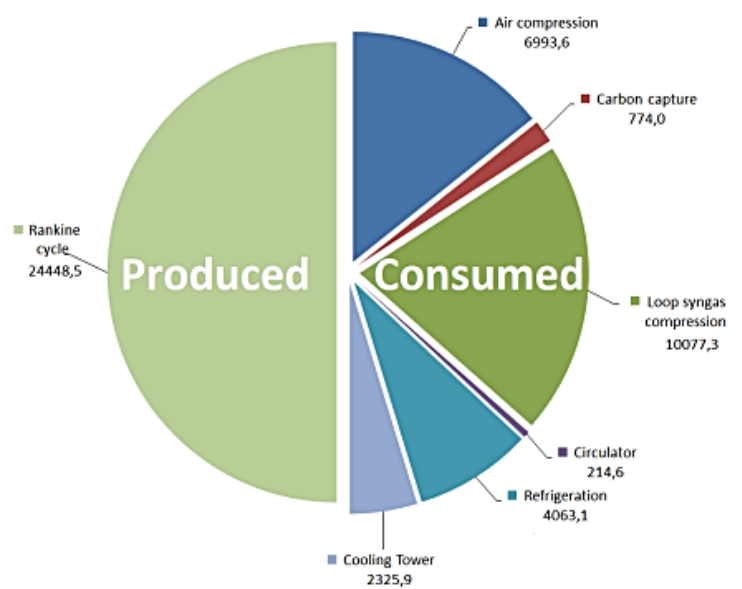

(h) DEA Auto

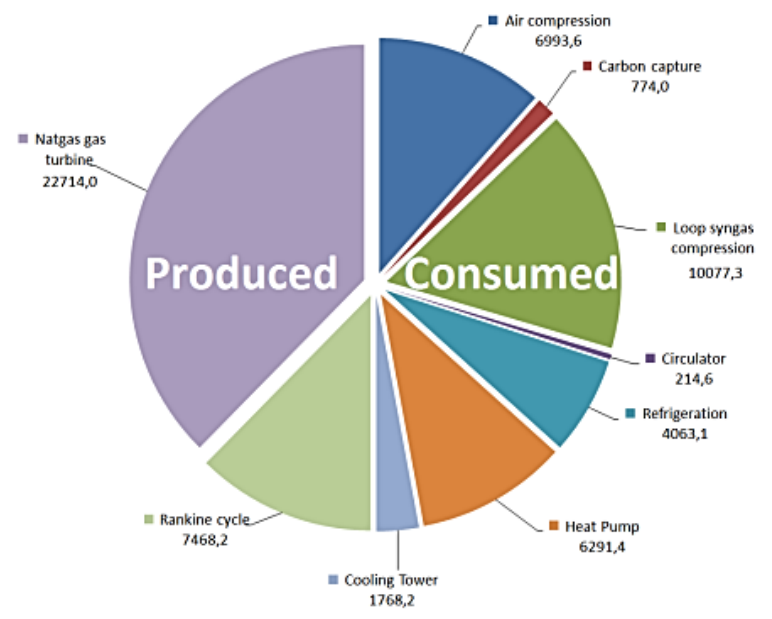

(k) DEA Auto GT

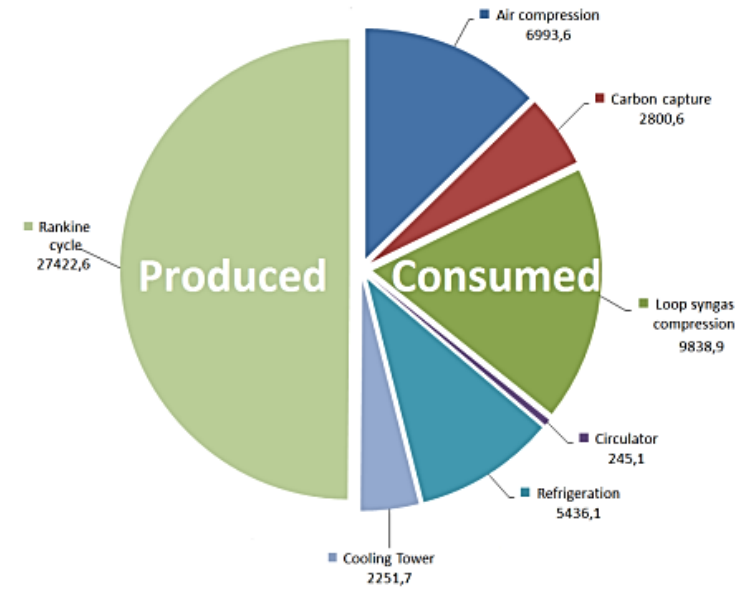

(i) DEPG Auto

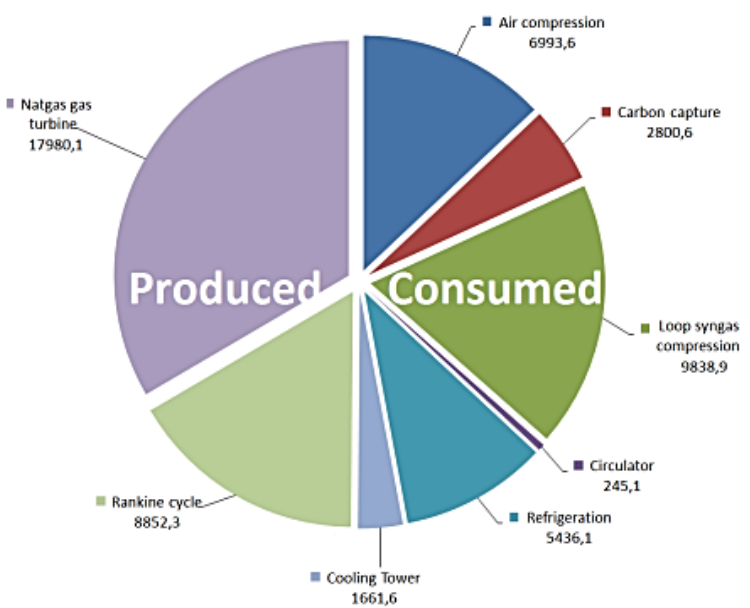

(I) DEPG Auto GT 


\subsection{Exergy analysis of the integrated ammonia production plants}

Figure 7.9 compares the relative and extended relative exergy efficiency of the various ammonia plant configurations and operation modes studied. As expected, the integration of a combined cycle into a traditionally Rankine cycle-powered ammonia plant increases the relative exergy efficiency about $8.8 \%$. Nontheless, the highest relative exergy efficiency still corresponds to MIXED operation mode with physical absorption-based syngas purification. This behavior is closely followed by the GRID operation modes, regardless of the syngas purification system utilized. It is also worthy to notice the appreciable reduction (>10\%) of the relative exergy efficiency when a broader scope, i.e. an extended framework that accounts for the irreversibilities in the natural gas and electricity upstream supply chains, is considered.

Fig. 7.9. Plantwide and extended exergy consumption figures for the various configurations studied.

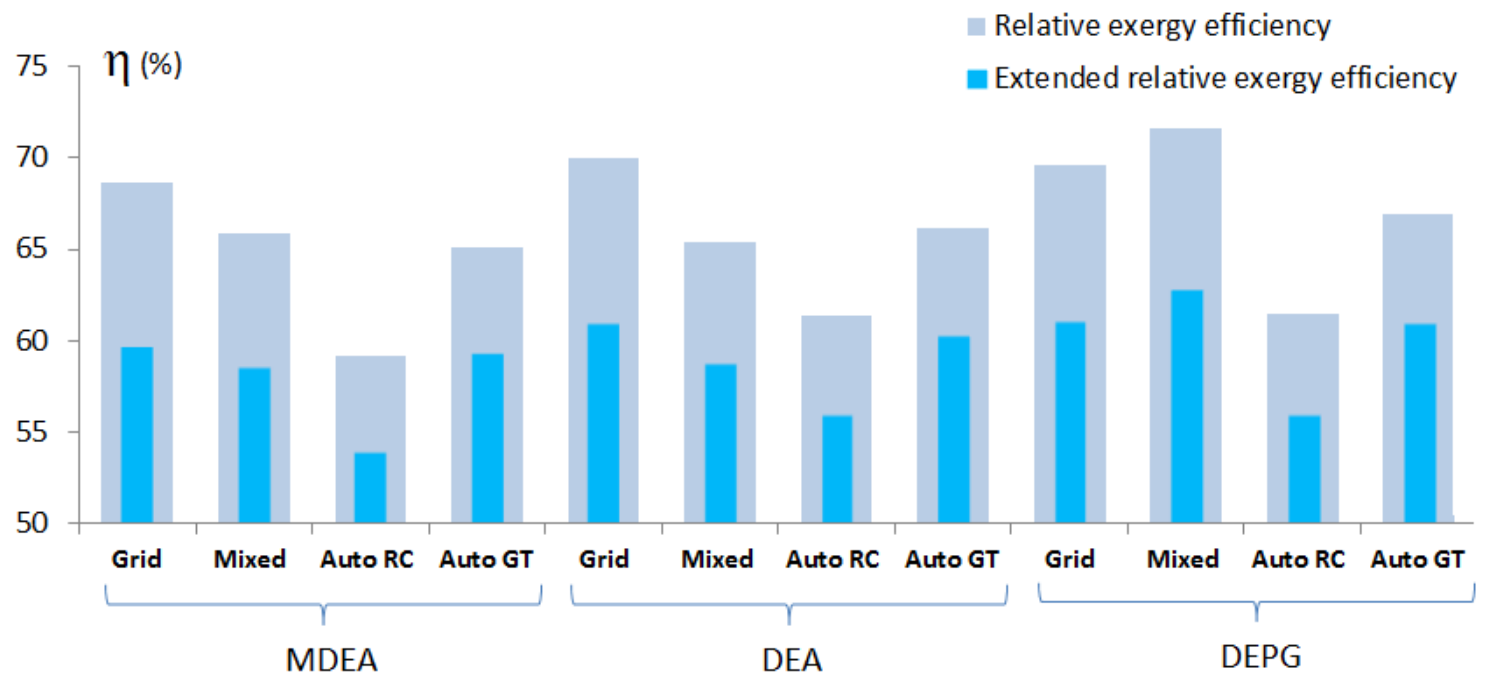

Source: Author

Table 7.3 summarizes the exergy efficiency and the specific exergy destruction, used as indicators to rank the performance of the analyzed ammonia plant configurations. It is interesting to point out the striking reduction of the overall exergy efficiency when going from its pure rational definition up to the extended relative one. In fact, the efficiency drop can be as high as 19.3-23.8\%, since this broader scope of reduction accounts for both the exergy destroyed along the upstream feedstock production stages as well as for the disregard of the potential utilization of the ammonia byproducts $\left(\mathrm{CO}_{2}\right.$, purge $)$.

Within the same scope, the increase in the exergy destruction may achieve $31 \%$ in the AUTO and $39 \%$ in the AUTO GT operating modes, or as much as $43-77 \%$ for the GRID and MIXED operation modes. At a first glance, the higher increase in the extended exergy destruction could be wrongly attributed to the intensive utilization of the less efficient electricity mix (55.67\%), if compared to the more efficient natural gas supply chain (91.09\%). However, the electricity utilization still remains to be the most efficient way of driving the ammonia plant configurations studied. In other words, although the electricity import entails the consumption of a relatively inefficiently produced input (i.e. grid electricity), the high-grade and readily 
available nature of the electricity consumed actually lead to an improved performance of the ammonia production process, only comparable to the utilization of a combined cycle (AUTO GT) in an autonomous operation mode (Tables 7.3, last column). This fact once again emphasizes the importance of the characteristics of the electricity mix in which the chemical process is embedded. In the hypothesis that much less efficient electricity mixes were considered, the AUTO and AUTO GT operation modes may become more attractive alternatives for electricity generation via CHP systems, than importing costly, less efficiently and less environmentally friendly electricity from 'dirtier' grids.

Table 7.3. Exergy destruction and exergy efficiencies for the chemical and physical absorption-based ammonia production plant configurations.

\begin{tabular}{lcccc}
\hline Process parameter & MDEA Grid & MDEA Mixed & MDEA Auto RC & MDEA Auto GT \\
\hline Rational exergy efficiency $(\%)$ & 78.40 & 75.21 & 67.59 & 74.32 \\
\hline Extended rational exergy efficiency $(\%)$ & 68.20 & 66.88 & 61.57 & 67.70 \\
\hline Relative exergy efficiency $(\%)$ & 68.67 & 65.87 & 59.20 & 65.09 \\
\hline Extended relative exergy efficiency $(\%)$ & 59.73 & 58.58 & 53.93 & 59.29 \\
\hline Exergy destruction $\left(\mathrm{GJ} / \mathrm{t}_{\mathrm{NH} 3}\right)$ & 6.25 & 7.48 & 10.88 & 7.84 \\
\hline Extended exergy destruction $\left(\mathrm{GJ} / \mathrm{t}_{\mathrm{NH} 3}\right)$ & 10.58 & 11.24 & 14.16 & 10.83 \\
\hline
\end{tabular}

1.The exergy efficiency and the specific exergy destruction change if the cumulative exergy efficiency of the electricity grid $(55.67 \%)$ and of the natural gas supply chain (91.09\%) are considered as in [188].

Table 7.3. Exergy destruction and exergy efficiencies for the chemical and physical absorption-based ammonia production plant configurations (cont'd).

\begin{tabular}{lcccc}
\hline Process parameter & DEA Grid & DEA Mixed & DEA Auto RC & DEA Auto GT \\
\hline Rational exergy efficiency $(\%)$ & 79.05 & 73.88 & 69.34 & 74.76 \\
\hline Extended rational exergy efficiency $(\%)$ & 68.86 & 66.36 & 63.17 & 68.10 \\
\hline Relative exergy efficiency $(\%)$ & 70.00 & 65.43 & 61.41 & 66.21 \\
\hline Extended relative exergy efficiency $(\%)$ & 60.98 & 58.77 & 55.94 & 60.31 \\
\hline Exergy destruction $\left(\mathrm{GJ} / \mathrm{t}_{\mathrm{NH} 3}\right)$ & 5.95 & 7.94 & 9.92 & 7.58 \\
\hline Extended exergy destruction $\left(\mathrm{GJ} / \mathrm{t}_{\mathrm{NH} 3}\right)$ & 10.15 & 11.38 & 13.09 & 10.51 \\
\hline $\begin{array}{l}\text { 1.The exergy efficiency and the specific exergy destruction change if the cumulative exergy efficiency of the electricity grid } \\
\text { (55.67\%) and of the natural gas supply chain }(91.09 \%) \text { are considered as in }[188] .\end{array}$
\end{tabular}

Table 7.3. Exergy destruction and exergy efficiencies for the chemical and physical absorption-based ammonia production plant configurations (cont'd).

\begin{tabular}{lcccc}
\hline Process parameter & DEPG Grid & DEPG Mixed & DEPG Auto RC & DEPG Auto GT \\
\hline Rational exergy efficiency $(\%)$ & 79.69 & 81.72 & 70.17 & 76.35 \\
\hline Extended rational exergy efficiency $(\%)^{1}$ & 69.81 & 71.63 & 63.92 & 69.55 \\
\hline Relative exergy efficiency $(\%)$ & 69.64 & 71.59 & 61.48 & 66.89 \\
\hline Extended relative exergy efficiency $(\%)^{1}$ & 61.01 & 62.76 & 56.00 & 60.93 \\
\hline Exergy destruction $\left(\mathrm{GJ} / \mathrm{t}_{\mathrm{NH} 3}\right)$ & 5.78 & 5.08 & 9.64 & 7.03 \\
\hline Extended exergy destruction $\left(\mathrm{GJ} / \mathrm{t}_{\mathrm{NH} 3}\right)^{1}$ & 9.89 & 8.99 & 12.81 & 9.93
\end{tabular}

1.The exergy efficiency and the specific exergy destruction change if the cumulative exergy efficiency of the electricity grid $(55.67 \%)$ and of the natural gas supply chain $(91.09 \%)$ are considered as in [188].

Figure 7.10 shows the exergy destruction breakdown among the most representative components of the ammonia plant configurations studied. As it can be seen, the primary and secondary reformers together are responsible for nearly $26-54 \%$ of the total exergy destroyed. As expected, the highest exergy destruction share of the utility systems (others) is attained 
through an intensive utilization of both the steam network and the cogeneration systems (27$52 \%$ ), e.g. in both AUTO and AUTO GT operating modes. The ammonia synthesis reactor has a contribution to the exergy destruction that may oscillates between 7.8-17.3\%, which depends on the amount of inerts, the recycling ratio and conversion in the ammonia synthesis loop [237]. A marked difference exists between the shares of the exergy destruction presented by the carbon capture units analyzed. The amine-absorption carbon capture units either by using DEA or MDEA solvent is responsible for one tenth to one fifth of the total exergy destruction. Those figures are smaller when physical absorption systems are used $(<4.6 \%)$. Accordingly, the use of carbon capture systems that spare an energy intensive desorption process can be effectively used to reduce the contribution of the syngas purification to the total amount of irreversibility in the ammonia plant.

Fig. 7.10. Exergy destruction breakdown for each plant configuration shown in Table 7.2.

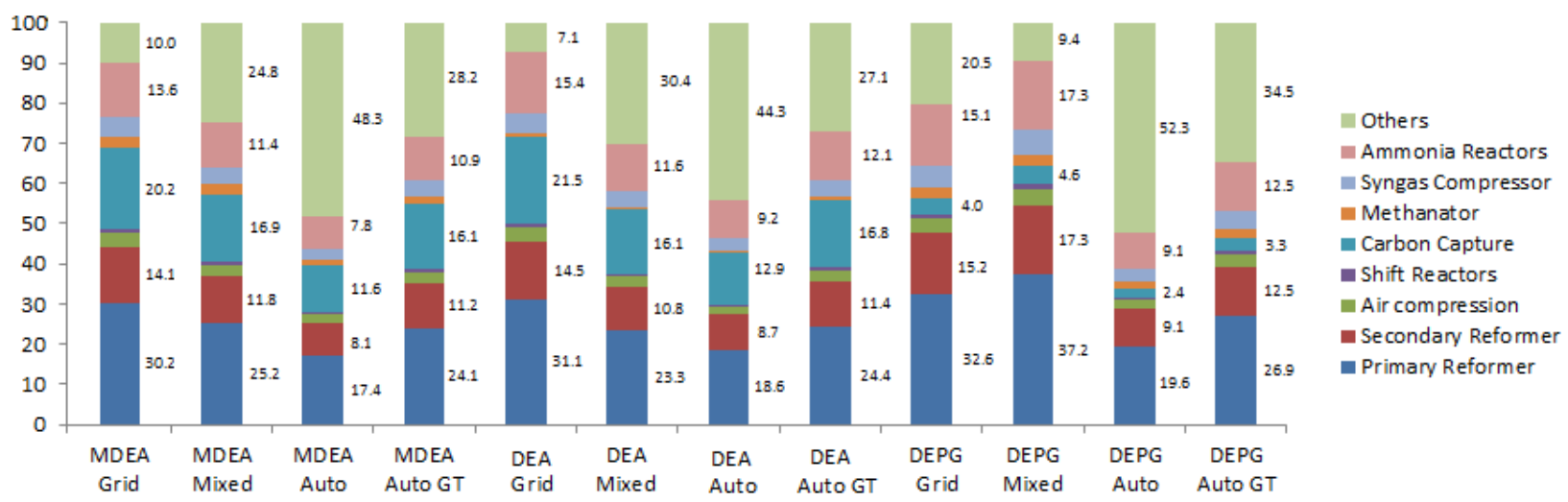

Source: Author.

The irreversibility associated to the heat exchange network (HEN) of the ammonia production plants operating in the AUTO and AUTO GT modes can be graphically represented by the Carnot Integrated Curves shown in Fig. 7.11. In this plot, the area enclosed by the curves of the utility systems and the chemical plant can be interpreted as the exergy destroyed. Thus, the closer the two curves, the lesser the irreversibility in the HEN. As expected, due to an upgraded utilization of the exergy heat of the high-grade utility produced by the fired furnace, the exergy destruction rate drastically drops when a Combined cycle is integrated to the ammonia production unit. The recovery of the exergy embodied in the high temperature flue gases is maximized by using the combustion gases to drive the gas turbine system, and only thereafter for gas turbine cycle regeneration purposes, process streams preheating or steam generation, respectively, instead of using the combustion gases for directly producing steam [80, 110].

Certainly, this modification perturbs the whole power and heat balance, particularly within the self-sufficient zones where the cogeneration has benefited from. In other words, since part of the excess heat exergy has been used to produce power, additional fuel consumption will be required to ensure the balance of the heating demands of the chemical process. In spite of this, the integration of a waste heat recovery steam network generally reduces the large driving forces related to the process-to-process heat exchange, even at the expense of supplementary fuel consumption. For instance, as shown in Figs.7.8a-b, it is preferable to 
generate a surplus of electricity for driving the heat pump (MVR) system by extracting most of the thermodynamic potential in the self-sufficient zones, than directly using waste heat from the process to supply the desorption reboiler duty [238].

Thus, roughly speaking, due to the technical and metallurgical limitations of the energy technologies considered, the use of a gas turbine system becomes advantageous when aiming to better exploit the thermodynamic potential at higher temperatures (above $500^{\circ} \mathrm{C}$ ) whereas the steam network represents the most suitable alternative for recovering the waste heat exergy available at lower ones $\left(300-500^{\circ} \mathrm{C}\right)$. Supercritical Rankine cycles or Graz-related power cycles may represent also interesting alternatives for utility systems with reduced exergy destruction rates, provided that industrial ammonia plants can afford the large capital investments. 
Fig. 7.11. Carnot integrated curves for the AUTO and AUTO GT scenarios shown in Figs. 7.5g-l: (a)MDEA-based, (b)DEA-based, (c)DEPGbased ammonia plants. $\Theta$ is the Carnot factor calculated as $1-(T O / T)$ where To $=298 \mathrm{~K}$

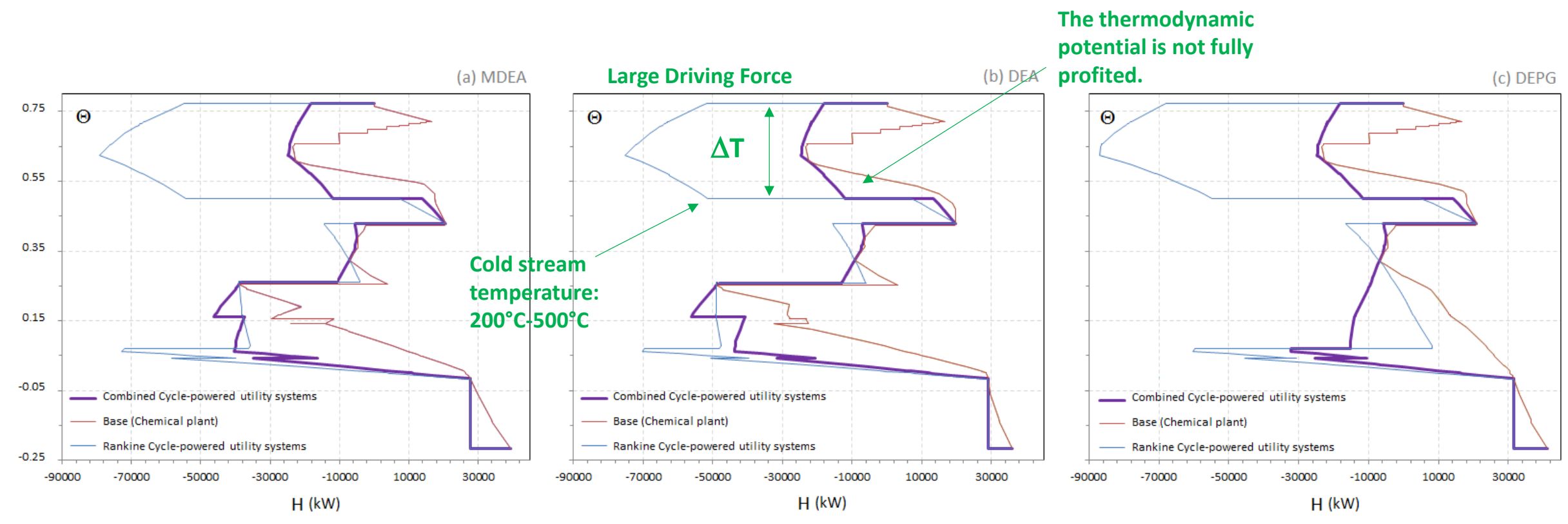

Source: Author. 


\subsection{Final considerations}

A systematic methodology based on a mixed integer linear programming (MILP) and a modular chemical process simulation approach allows determining the most suitable utility systems that satisfy the minimum energy requirement (MER) with the lowest resources consumption and cost in a highly integrated syngas and ammonia production plant. Various operating scenarios based on the utilization of three types of syngas purification systems, as well as several energy resources and cogeneration technologies, have been compared in terms of exergy consumption and efficiency as well as optimal operating revenues.

By using the energy integration method, the most appropriate utility systems and operation conditions that maximize the recovery of the thermodynamic potential of the excess heat produced along the chemical plant have been identified. This has been possible through the development a suitable representation of the energy integration profile for the reactive systems, acknowledged as the key components of the energy intensive chemical plant. Moreover, an extended exergy analysis has been performed so that a broader insight into the major sources of irreversibility can be hierarchized and the exergy destruction in the ammonia production can be reduced by proposing the utilization of better energy technologies for cogeneration.

Direct and indirect $\mathrm{CO}_{2}$ emissions generated in the ammonia production process are also calculated, allowing for better comparisons with other industrial and chemical process in terms of their environmental impact. On the other hand, the valorization of some plant byproducts (e.g. captured $\mathrm{CO}_{2}$ ) simultaneously increased the chemical plant efficiency while alleviating its atmospheric burden. As a result, by operating either in a mixed mode (i.e. partial electricity import with an improved waste heat recovery and cogeneration systems) or in a totally autonomous mode with combined cycle cogeneration systems, along with the use of physical absorption syngas purification units, the process irreversibility and operation cost can be appreciably reduced, whereas the conventional plant efficiency is increased (ca. 8$10 \%)$.

Thus, by considering that typical ammonia plants actually operate in autonomous modes with no other energy input that natural gas, the gas turbine system is the most rational alternative for increasing the exergy efficiency and the reduction of the operating costs in the SNF facilities. Accordingly, in the next chapter, a novel approach based on the chemically recuperated gas turbine concept is proposed for the production of syngas intended to be fed to an industrial ammonia loop. This setup aims to improve the energy integration capabilities at higher temperatures and reduce the amount of atmospheric emissions, by intensifying the rates of pre-combustion carbon capture. To the author's knowledge, similar approaches has been proposed for expanding the exothermic reactor effluents [239, 240], but none of them integrate the chemically recuperated gas turbine concept to the chemical plant in a same physical structure, as it has been firstly introduced in this thesis. 
CHAPTER 8

\section{IMPROVED SYNGAS PRODUCTION AND CARBON CAPTURE BY USING A NOVEL CRGT APPROACH}

In spite of the large degree of energy integration in the modern syngas production units, the highly endothermic reactions of steam methane reforming and the combined steam and power generation still require a huge amount of energy that is typically supplied by an expensive natural gas-fired furnace at very high temperatures. Since normally only half of the energy supplied by the furnace is used to carry out the reforming reactions, the remaining heat recovery must be performed in a separate convection train (HRCT).

Additionally, the high temperature effluent of the secondary reformer is generally cooled by producing low temperature steam, increasing the process irreversibility and the losses associated to the excessive condensate. Thus, in this chapter, the advantages of introducing a chemically recuperated gas turbine (CRGT) concept to simultaneously carry out the endothermic chemical reactions and recover the exergy available from the autothermal reformer effluent are discussed. In this way, higher temperatures and higher conversions can be attained with lower driving forces. The power required by the air compression as well as other ancillary systems (e.g. air separation unit, carbon capture system, boiler feedwater pumps and recompressor) can also be supplied, while the exergy of the exhaust gases from the turbine is used more efficiently than in typical steam generation systems. Thus, more compact and integrated syngas production plants can be envisaged [241]. Moreover, by introducing more advanced cogeneration features such as air enrichment, process gas reheating and incremental levels of pressures in the reaction-driven components, slight energy savings together with drastically reduced atmospheric $\mathrm{CO}_{2}$ emissions and irreversibility can be achieved in the frontend syngas production section.

\subsection{An overview on the conventional and novel approaches for syngas production.}

The worldwide demand for hydrogen faces an increasing trend, primarily driven by hydroprocessing in refineries and the chemical industry [232]. Moreover, its potential use in automotive applications will also likely push the production capacity even further [242]. Despite the fact that some efforts to produce hydrogen from renewable sources such as solar and biomass energy have been considered [243], the cheapest route remains to be the steam reforming of natural gas. However, conventional reforming still presents many disadvantages regarding the way in which the waste heat recovery is achieved [244]. The customary practice consists of the use of a natural gas-fired furnace to supply the energy to the catalytic tubes of the primary reformer. Nevertheless, since barely $50 \%$ of the exergy in the combustion gases can be effectively recovered in the reformer, the conventional reforming process requires the installation of an additional convection train (HRCT) for heat recovery purposes [34]. 
Therein, the waste heat is generally used to raise steam at lower temperatures $\left(<300{ }^{\circ} \mathrm{C}\right)$ than those found elsewhere in the conversion process, entailing an avoidable irreversibility [245].

As part of the efforts to improve these shortcomings, this chapter investigates the integration of a novel application for the Chemically Recuperated Gas Turbine (CRGT) concept to a syngas production plant, intended to supply a typical ammonia synthesis loop $\left(\mathrm{H}_{2} / \mathrm{N}_{2} 3: 1\right)$. The convenience of a CRGT system lies in the simultaneous existence of both endothermic and exothermic chemical conversion technologies in the syngas production units, which could be appropriately coupled via gas heated reformers (GHR) and waste heat recovery systems. Hence, the readily available exergy of the autothermal reformer effluent could be better exploited to produce power, replacing the conventional role of the ATR effluent as a source for steam generation.

The conventional CRGT concept has been previously studied by different authors looking for improvements in applications of electricity generation [246-250]. Harvey and Kane [246] performed a second law analysis based on an ABB GT26 gas turbine with reheat and chemical recuperation via methane steam reforming, achieving a methane conversion close to 27\%. As expected, an improved performance has been obtained at higher temperatures and lower pressures in the reformer. Moreover, the exergy destroyed in the heat recovery steam generator (HRSG) is reported as threefold that in the reformer, corroborating the fact that the generation of steam implies substantially higher exergy destruction rates due to the lower temperature involved. Finally, the authors concluded that further parametrical and process flowsheet modifications may improve the overall efficiency, e.g. by using dual pressure chemically recuperated gas turbine systems with different pressures and steam-to-carbon ratios that align the desired levels of pressure in each reformer. Bearing that in mind, Carapellucci and Milazzo [247] investigated the differences between one single and two dual pressure configurations with an ABB GT24 gas turbine. The dual pressure configurations differed in the steam-to-carbon ratio supplied to the reformer, one with the same steam-tocarbon ratio $(\mathrm{S} / \mathrm{C})$ in both reformers, and the other configuration with separately optimized $\mathrm{S} / \mathrm{C}$ ratios. As a results, when the $\mathrm{S} / \mathrm{C}$ ratios aligned with their optimal levels of pressure, the methane conversion, the heat recovery and plant efficiency are enhanced at the same time that steam production and power generation are increased. The result is a higher efficiency (53.8\%) and a power output comparable to that of a combined cycle, which reinforces the position of the CRGT cycle as a competitive thermoelectric alternative.

Kesser et al. [249] built, upon a basic CRGT cycle (i.e. no reheat), two reference cases with different compression ratios and compared them with a combined cycle (CC), a steam injected gas turbine cycle (STIG) and an open gas turbine system (OCGT). It is found that the cycle with chemical recuperation follows closely the performance of the combined cycle, presenting the second highest efficiency, whereas the STIG cycle showed the second highest specific power generation, rendering it a more attractive, simpler and less costly option than an advanced CRGT cycle. Adelman et al. [250] revised various conceptual designs for the steam methane reformer in terms of the performance and size of the CRGT system. The proposed designs evolved by either adding a preheater, changing the diameter of the catalytic 
tubes, adding heat transfer intensification methods such as roughness and fins to the tubes, or even utilizing different catalyst reactivity, all motivated by the goal to find near-minimum catalyst volumes. The results pointed towards higher reforming temperatures as a direct way to increase conversion of methane and reduce the complexity and volume of the system.

Although most of the efforts have been devoted to medium-to-large scale power generation systems, decentralized approaches have gained renovated interest. More recently, Nakagaki et al. [248] studied the feasibility of a chemical recuperation system applied to microturbines $(30-300 \mathrm{~kW})$. The study revealed the practicality of the integration of CRGT systems to existing power generation systems, either microturbine or MW-class turbines. The $2 \% \mathrm{Ru}$ content catalyst is reported to present the best performance in terms of reaction rates at temperatures as low as $350{ }^{\circ} \mathrm{C}$, whereas conversions as high as $51 \%$ have been claimed.

Differently from the research works reviewed hitherto, based on power generation concepts with dedicated combustion chambers, other opportunities for profiting the readily available exergy embodied in the exothermic reactor effluents have been thoroughly discussed (e.g. partial oxidation combustors, autothermal reformers, methanol and ammonia converters, etc.) $[25,240]$. Some examples include the implementation of an expansion device that directly produces shaft work from the exergy of the ammonia reactor effluent in lieu of using it for raising steam [239, 251]. Despite the fact that this approach does not actually render the ammonia production unit self-sufficient in terms of power supply, it is reported a reduction of the specific power consumed (44-75\%) when compared to the utilization of a heat recovery steam generator. Furthermore, as this approach represents a more efficient way of profiting the thermo-mechanical exergy of the process stream, it also reduces the degradation of the thermodynamic potential of the chemical system [252].

Similarly, Agee et al. [253] described the application of a sub-stoichiometric, simultaneous combustion and reforming process of natural gas in order to produce syngas in a methanol manufacturing plant. By using the autothermal reformer also as an enriched air combustor, the highly exothermal reaction supplies the reforming duty in a single pressurized vessel, whereas the expansion of the reactor effluent is able to provide some of the power required for air compression and separation purposes. In more advanced technologies, such as partial oxidation gas turbines [254], a mixture of methane and steam at high pressure is partially oxidized in a catalytic adiabatic reactor that replaces the conventional combustor chamber of a OCGT. In this way, the low molecular weight fuel gas produced at high temperature, mainly composed of hydrogen and carbon oxides, is expanded to generate mechanical power. Henceforth, the produced gas is completely burned in a bottoming cycle so that the reaction enthalpy released can be supplied to a downstream chemical process. The specific mechanical power produced is reportedly about 6-9\% of the LHV of the fuel, which renders the partial combustion gas turbine system also a 'fuel producer' technology [254]. It must be observed that these approaches substantially differ from that one patented more recently by Keller et al. [228] in which the cogeneration of hydrogen and power is achieved through the integration of two physically separate hydrogen and power generation units. The hydrogen production unit comprises a gas heated reformer, followed by a water gas shift reactor and a hydrogen 
separation unit. These units produce a purified hydrogen stream and a byproduct consisting of a hydrocarbon-rich fuel stream. The byproduct stream is burned in a conventional combustion chamber of a gas turbine along with an oxygen-rich stream, and eventually used to provide heat to the gas heated reformer. This approach has been also reported by Alves and Nebra [80, 255] who developed the exergoeconomy analysis of the hydrogen production from natural gas by using an autothermal reformer and a separate gas turbine cogeneration system.

In accordance with this brief review, the suitability of the integration of an autothermal reformer (ATR) to a chemically recuperated gas turbine (CRGT) concept, in order to exploit the exposed advantages of the simultaneous syngas and power production, has not been proposed so far. Moreover, it has neither been suggested before as a part of the frontend of an ammonia production plant. Thus, in this chapter, exergy is used to minimize the exergy destruction rates along the various components of the syngas production units and reduce the consumption of the non-renewable methane feedstock, based on four novel proposed configurations. As a result, the physical reconfiguration of the chemical plant layout is shown to greatly reduce the driving forces and, thus, the irreversibility arisen from the combined heat and power production (CHP) system, but more importantly, the net atmospheric emissions.

\subsection{Syngas Production with Thermo-Chemically Recuperated Gas Turbine Systems}

Figures 8.1-8.5 compare the five configurations of syngas production plants studied. The conventional system (Fig.8.1) is based on the most common arrangement for hydrogen production via SMR [206]. The processes comprises a primary reformer $\left(700-800^{\circ} \mathrm{C}\right)$ in series with an autothermal secondary reformer $\left(\sim 1000^{\circ} \mathrm{C}\right)$, where a fraction of the reformed gas is burnt with the aid of enriched air (30-40\%) to provide the heat required to further convert the methane feed. The air separation unit (ASU) consists of a dual pressure column equipped with a condenser-reboiler heat exchanger, whereas the cooling effect is produced by throttling a compressed stream of air that exchanges heat with its separate cryogenic fractions $\left(-190^{\circ} \mathrm{C}\right)$.

For the sake of an enhanced hydrogen production, two sequential reactors are used to perform the water gas shift reaction at lower temperatures $\left(200-350^{\circ} \mathrm{C}\right)$ [25]. Furthermore, in order to remove the carbon oxide components present in the syngas, a purification section composed of a chemical absorption and a methanation system is considered. In this configuration, the waste heat released throughout the chemical process is recovered by using an integrated steam network, so that the additional fuel consumption required to supply both the power and heat demands of the plant (feed preheating, endothermic reactions, etc.) can be reduced [231]. According to Fig. 8.1, the valuable waste heat available at the outlet stream of the secondary reformer is indeed used to produce steam, which in turn needs to be further superheated in the separate HRCT. The superheated steam is eventually expanded in a steam turbine, supplying both the power and steam demands. 
Fig.8.1. Conventional configuration. Steam network-based utility system.

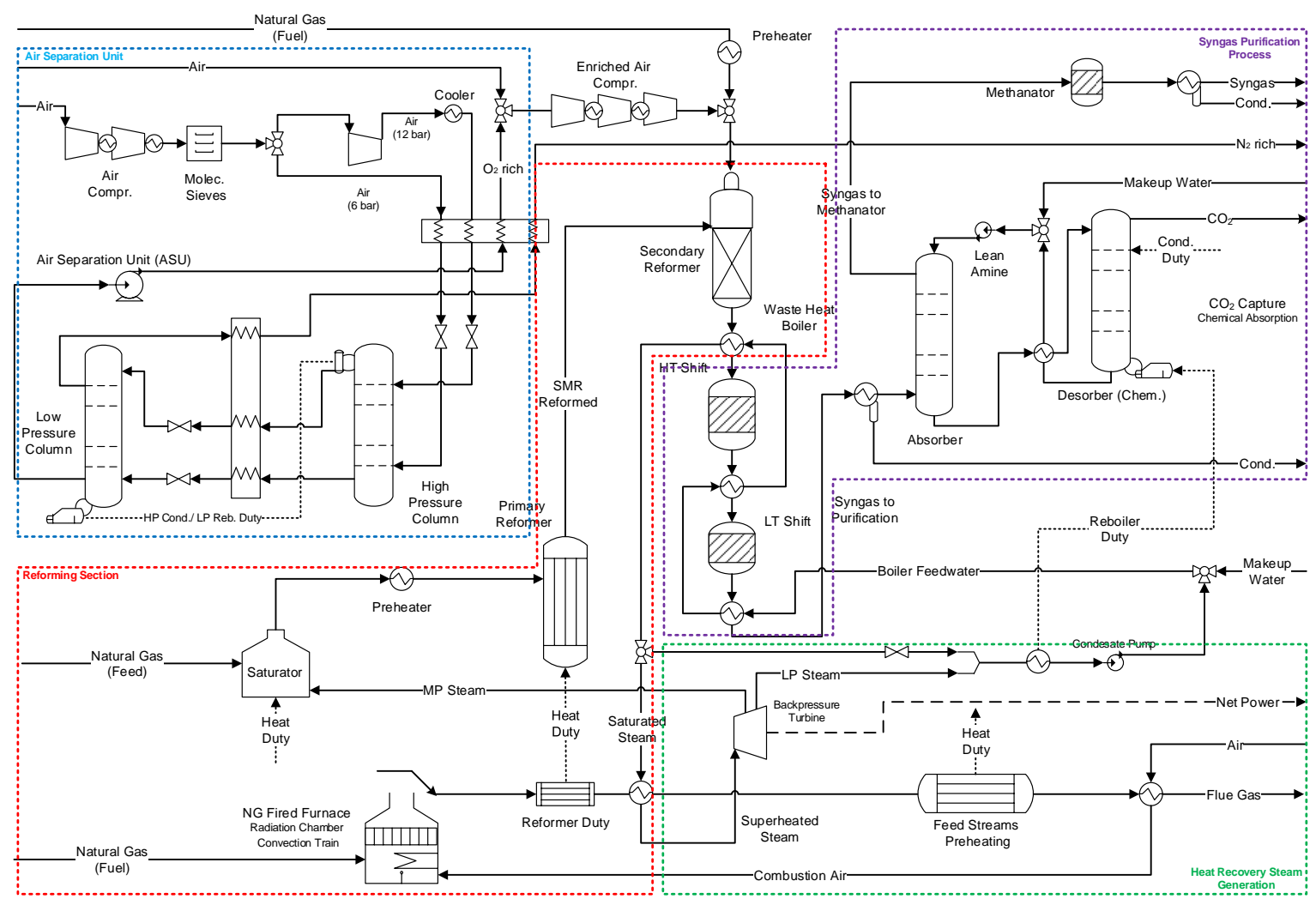

Source: Author.

The four novel approaches proposed for the syngas production, based on the chemically recuperated gas turbine (CRGT) concept, are illustrated in Figs. 8.2-8.5. Differently from the conventional layout (Fig. 8.1), the novel approaches aim to maximize the use of the readily available exergy of the high temperature, pressurized effluent of the secondary reformer. This is accomplished by chemically recuperating the physical exergy of the expander outlet stream to reform a saturated natural gas mixture and simultaneously supplying the total power demand of the chemical plant. Due to the characteristics of the chemical recuperation process, compared to either the simple combustion air preheating or the steam injection, a higher amount of exergy can be embodied in the chemical exergy of the reformed mixture, which further increases the efficiency of the waste heat recovery process [246].

Actually, these four novel approaches (Fig.8.2-8.5) have been conceived by realizing the opportunity to exploit the exposed advantages of the CRGT concept, which may lead to more compact syngas production systems. Furthermore, even if it could not be immediately evident, as the combustion progresses in the same autothermal reactor where the endothermic reaction occurs, the large amount of water produced in the combustion process at high temperature directly offers an increased amount of steam in the catalytic section, which in turn increases the equilibrium conversion of the reformer as the concentration of the reactants increases. This is threefold advantageous: (i) The losses associated to the release and condensation of the flue gases produced in a separate fired furnace can be reduced [34], as the exergy embodied in the water content of the combustion gases can be maximally exploited at higher temperatures, rather than producing low-grade superheated steam in the heat recovery 
convection train (HRCT) of the primary reformer; (ii) As the combustion gases become also process gas, the excessive dilution of the carbon dioxide with nitrogen is overcome, thus allowing for an easier capture process at the expense of an additional consumption in the syngas purification system; (iii) The water produced together with the other reaction products allows for a higher power throughput per unit of mass of gas produced, which enables the utilization of the expander exhaust gases as the heating medium at the downstream chemically recuperated, gas heated methane reformer.

Fig. 8.2. Single Pressure, Chemically Recuperated Gas Turbine-based configuration (Single Pressure GT).

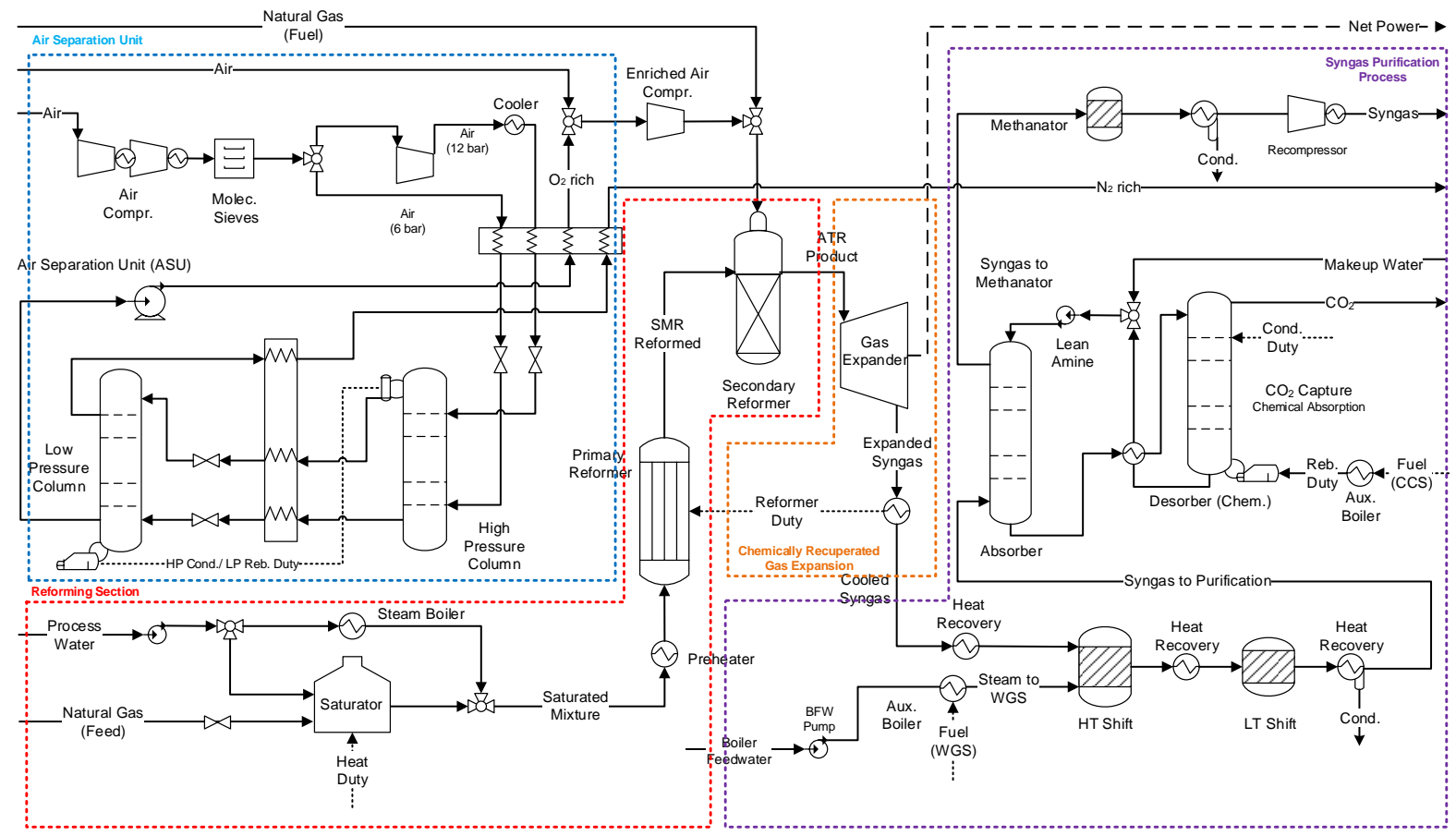

Source: Author.

Meanwhile, the relationship between the endothermic and exothermic nature of the reactions and the variation of the temperature and the molar volume of the reactants can be further examined in light of the Le Châtelier and the Counteraction principles, which rule the rates of hydrogen production and the extent of process irreversibility, respectively [69, 226]. For instance, the reforming process is recognized as an increasing-volume, endothermic reaction that proceeds faster at higher temperatures, but whose equilibrium is favored at lower pressures. Thus, it is interesting to inquire about the effect of the variation of some selected operating parameters (e.g. pressure, temperature and streams composition) as well as of the arrangement of the unit components on the overall plant performance. According to this, a dual pressure syngas production process, based on the CRGT concept (Fig. 8.3), is performed at two decremental levels of pressure, following the overall increase of the molar volume as the reaction progresses to equilibrium. It must be noticed that this process layout resembles the gas reheat process, already used in conventional gas turbine power systems as it is reported to increase the power throughput without increasing the inlet temperature of the turbine [246, 247]. 
Fig. 8.3. Dual Pressure, Chemically Recuperated Gas Turbine-based configuration (Dual Pressure GT).

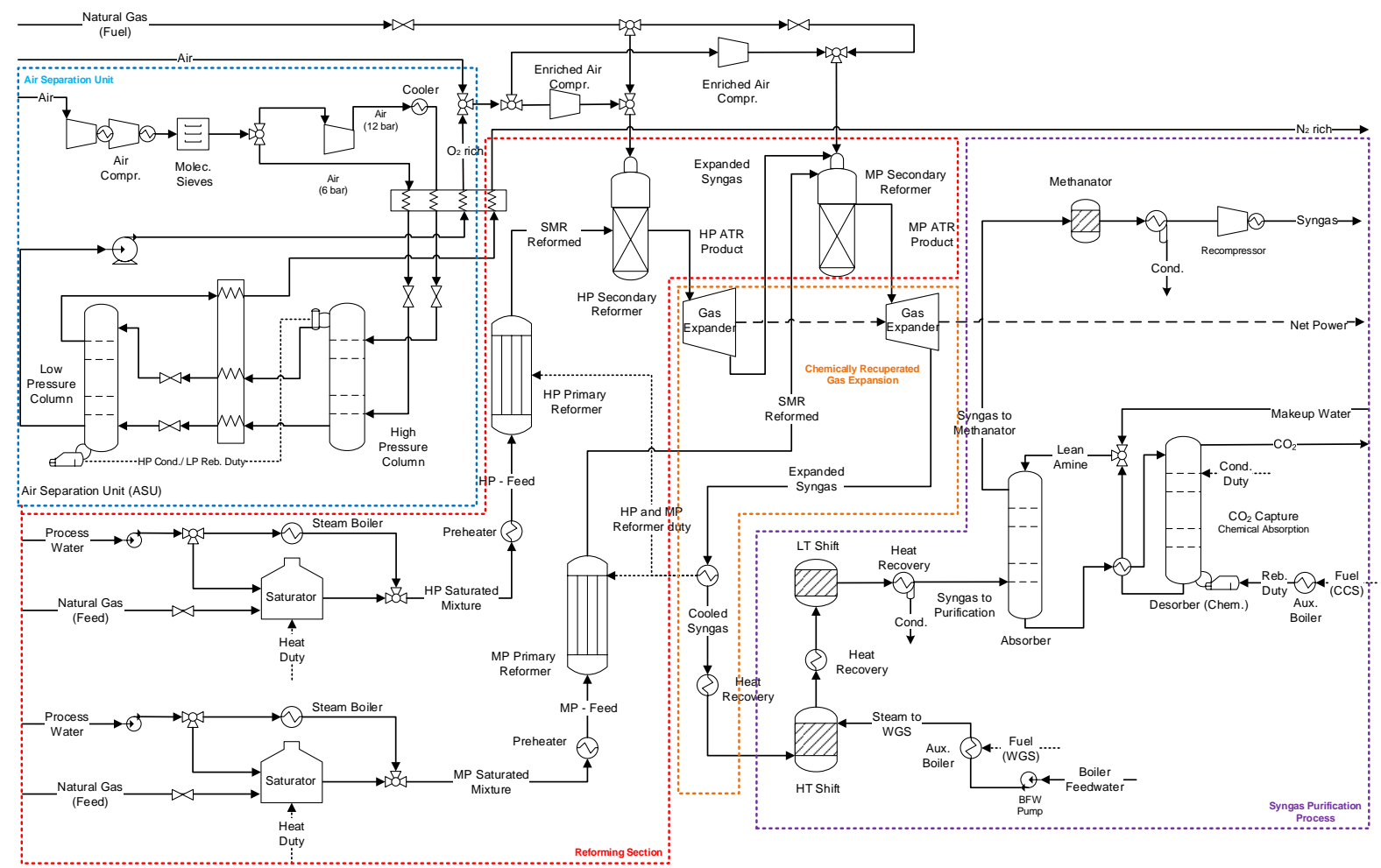

Source: Author.

Finally, motivated by a suitable combination of the previous energy conversion approaches and aiming to improve the energy integration potential of the single and dual pressure CRGTbased syngas production plants, two additional configurations have been proposed (Fig. 8.48.5). In those approaches, a comprehensive steam network recovers the waste heat along the chemical process in order to generate part of the power supply as well as the process steam consumed in the reforming, shift reactions and syngas purification. This improvement allows for a slight reduction of the fuel consumed that otherwise would be burnt in auxiliary boilers. Moreover, it also entails a substantial reduction of the associated greenhouse gas emissions and the process irreversibility. 
Fig. 8.4. Single Pressure, Chemically Recuperated Gas Turbine-based configuration with Steam Network (Single CC).

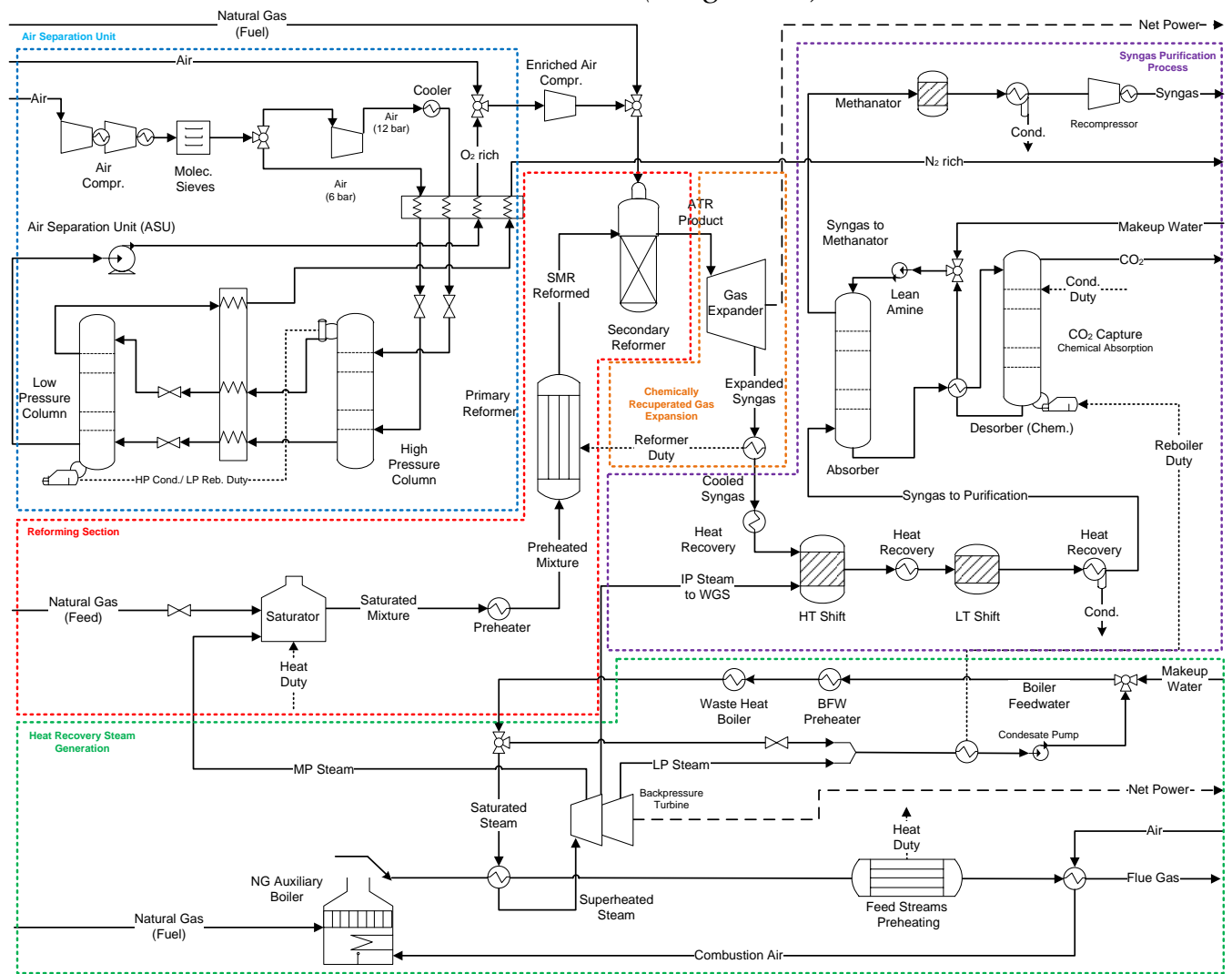

Fig. 8.5. Dual Pressure, Chemically Recuperated Gas Turbine-based configuration with Steam Network (Dual CC).

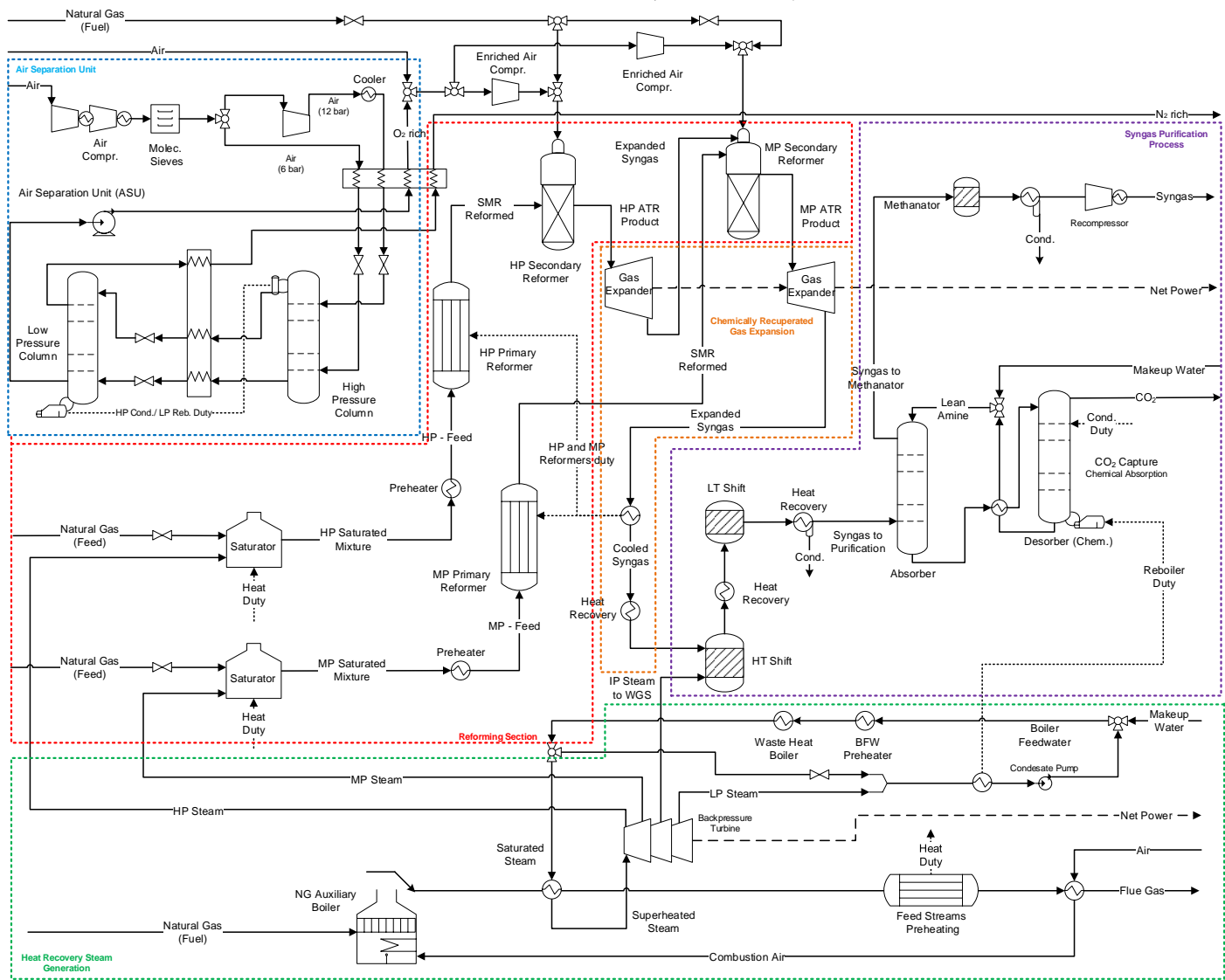

Source: Author. 
Table 8.1 compares two exergy efficiency definitions, Eqs.(8.1-8.2), proposed for better evaluating the overall performance of syngas production plants [206]. It must be noticed that, since the exergy of the input and output material flows are much larger than any other exergy flow rates, the relative exergy efficiency, Eq.(8.2) may lead to higher values. According to this, aiming to differentiate the exergy effectively transformed by the system from the untransformed exergy, the consumed-produced exergy efficiency is also calculated by using the exergy change of selected streams in the conversion processes. In other words, Eq. (8.1) aims to evaluate the extent at which the chemical exergy consumed in the process can be recovered in the form of an increased physical exergy of the products, while minimizing the system irreversibility. Evidently, the lower the surplus power produced and the higher the exergy recovered in the form of reformed mixture, the better the performance of syngas production. The relative exergy efficiency, in turn, determines the ratio between the minimum exergy required to materialize the syngas to the actual exergy consumption of the syngas production unit.

Table 8.1. Plantwide exergy efficiency definitions of the syngas production systems.

\begin{tabular}{lc}
\hline Definition & Formula \\
\hline $\begin{array}{c}\text { ConsProd } \\
(8.1)\end{array}$ & $\eta_{\text {ConsProd }}=\frac{B_{\text {Produced }}}{B_{\text {Consumed }}}=\frac{\left(B_{\text {outlet }}^{P H}-B_{\text {inlet }}^{\text {PH }}\right)+W_{\text {Net }}^{\text {imported }}}{\left(B_{\text {inlet }}^{C H}-B_{\text {oulet }}^{C H}\right)}$ \\
\hline $\begin{array}{l}\text { Relative } \\
(8.2)\end{array}$ & $\eta_{\text {Relative }}=\frac{B_{\text {Consumed, ideal }}}{B_{\text {Consumed, actual }}}=\frac{B_{\text {Syngas }}}{B_{C H_{4}}+B_{B F W}+W_{\text {Net }}^{\text {import }}}$ \\
\hline $\begin{array}{l}\text { B = exergy rate or flow rate (kW), PH: physical exergy, CH: chemical exergy, ConsProd: Consumed/Produced, BFW: boiler } \\
\text { feedwater, Dest: destroyed. }\end{array}$
\end{tabular}

\subsection{Optimization problem definition}

Syngas production units are designed in sophisticated ways in which the processes streams are interrelated through recycle operations and an intrincate waste heat recovery network. Consequently, the numerous design variables involved not only make the search space large in magnitude, but their interdependency renders the search for the optimality a complicated non-linear problem [146].

Fortunately, by separating the process flowsheet modeling from the heat integration problem, the calculation of the mass and energy balances and the simulation of the complex energy conversions can be handled by the modular simulation Aspen ${ }^{\circledR}$ Hysys, whereas the determination of the minimum energy requirements (MER) and the solution of the energy integration problem is performed by an additional Excel ${ }^{\circledR}$ platform. A systematic SQP approach is used to find the optimal solution subject to the technical, thermodynamic and operational constraints presented in Table 8.2. 
Table 8.2. Optimization problem definition.

Objective functions: Minimum Specific Exergy Destruction ( Minimum Specific Methane Consumption)

\section{Subject to the constraints:}

- No net power import. Preferably no net power export.

- Maximum ATR catalyst temperature, $1,100^{\circ} \mathrm{C}$ for safe hydrogen operation, due to metallurgical limitations at high pressure and to avoid adverse effects on catalyst activity and lifespan.

- Minimum and maximum primary reformer temperature $330^{\circ} \mathrm{C}-800^{\circ} \mathrm{C}$ for reasonable catalyst activity and methane conversion.

- Minimum energy requirements satisfied by the waste heat recovery or by the utility system.

- Maximum operating pressure of reformers, 35 bar.

- Minimum steam to carbon ratio, $\mathrm{S} / \mathrm{C}=2.5$ to avoid carbon deposition.

- No carbon oxide components in the purified syngas (ppm levels).

- Hydrogen production: $6,100-6,500 \mathrm{kmol} / \mathrm{h}$

- Fresh syngas molar composition suitable for ammonia production, $\mathrm{H}_{2} / \mathrm{N}_{2}$ ratio about $>2.999$, inerts content $\left(\mathrm{CH}_{4}+\mathrm{Ar}\right)<10 \%$.

\section{Design Variables:}

- Steam to Carbon ratio, 2.5-6

- Primary reformer outlet temperature, $\mathrm{T}_{\mathrm{SMR}}, 330-800^{\circ} \mathrm{C}$.

- $\mathrm{CH}_{4}$ fuel to feedstock ratio, $0.1-2$

- Oxygen enrichment, $0-40 \% \mathrm{O}_{2}$ in air

- Highest Reforming Pressure, 30-35 bar in both conventional and CRGT based configurations.

- Lowest Reforming Pressure (Expander outlet pressure for CRGT based configurations), 4 bar

- Split fractions (Enriched air, $\mathrm{CH}_{4}$ feed and $\mathrm{CH}_{4}$ fuel in dual pressure CRGT based configurations), $0.0-1.0$

- Steam split fractions in the steam network (to superheating coil after saturation), $0.0-1.0$

- Gas turbine bypass of the autothermal reactor effluent, $0.0-1.0$

- Primary reformer bypass of the preheated reactants, 0.0-1.0

- Steam superheating temperature in the steam network, $380^{\circ} \mathrm{C}-500^{\circ} \mathrm{C}$

- Steam to water gas shift reactors, $0-600 \mathrm{kmol} / \mathrm{h}$

- Normal air molar flow rate, 1,000-4,000 kmol/h

- $\mathrm{CH}_{4}$ feed molar flow rate, 1,000-2,000 kmol/h

\section{Constants:}

- Compression efficiency (85\%). Expander efficiency (90\%)

- Cooling water and boiler feedwater temperatures, $25^{\circ} \mathrm{C}$

- Minimum temperature approach in the $\operatorname{HEN}\left(20^{\circ} \mathrm{C}\right)$.

\subsection{Optimal operating conditions of the conventional and novel configurations}

Table 8.3 summarizes the optimal process variables calculated for the five configurations of the syngas production plant, according to the optimization problem given in Table 8.2. It is worthy to notice that, since the high-grade hot utility has been used more efficiently in the CRGT-based syngas production plants, compared to the conventional configuration, the 
specific consumption of natural gas of the proposed layouts is slightly reduced $(0.5-2.7 \%)$ whereas the cooling requirements also decrease (0.5-10.2\%).

On the other hand, an increased air enrichment is required in the CRGT-based configurations in order to provide the minimum exergy heat necessary to carry out the endothermic reactions and supply the power demand. Some authors reported no benefits in terms of methane conversion in the ATR when an air enrichment beyond $34 \% \mathrm{O}_{2}$ is used [256], although the recommended values range from 25 to $40 \% \mathrm{O}_{2}$ in air [257, 258]. In any case, the extent of air enrichment must consider the maximum outlet temperature of the ATR and, preferably, should not excessively produce $\mathrm{CO}_{2}$ gas, since it will consume the valuable hydrogen in the downstream methanator system. Thus, it is expected that, for a given steam to carbon ratio, there exists an optimal oxygen to fuel ratio that results in the highest production of hydrogen $[259,260]$, and also fulfills the overall heat and power requirements of the process.

Fig. 8.6. Comparison between the specific exergy consumption (left) and specific $\mathrm{CO}_{2}$ emissions (right) of the syngas production configurations.
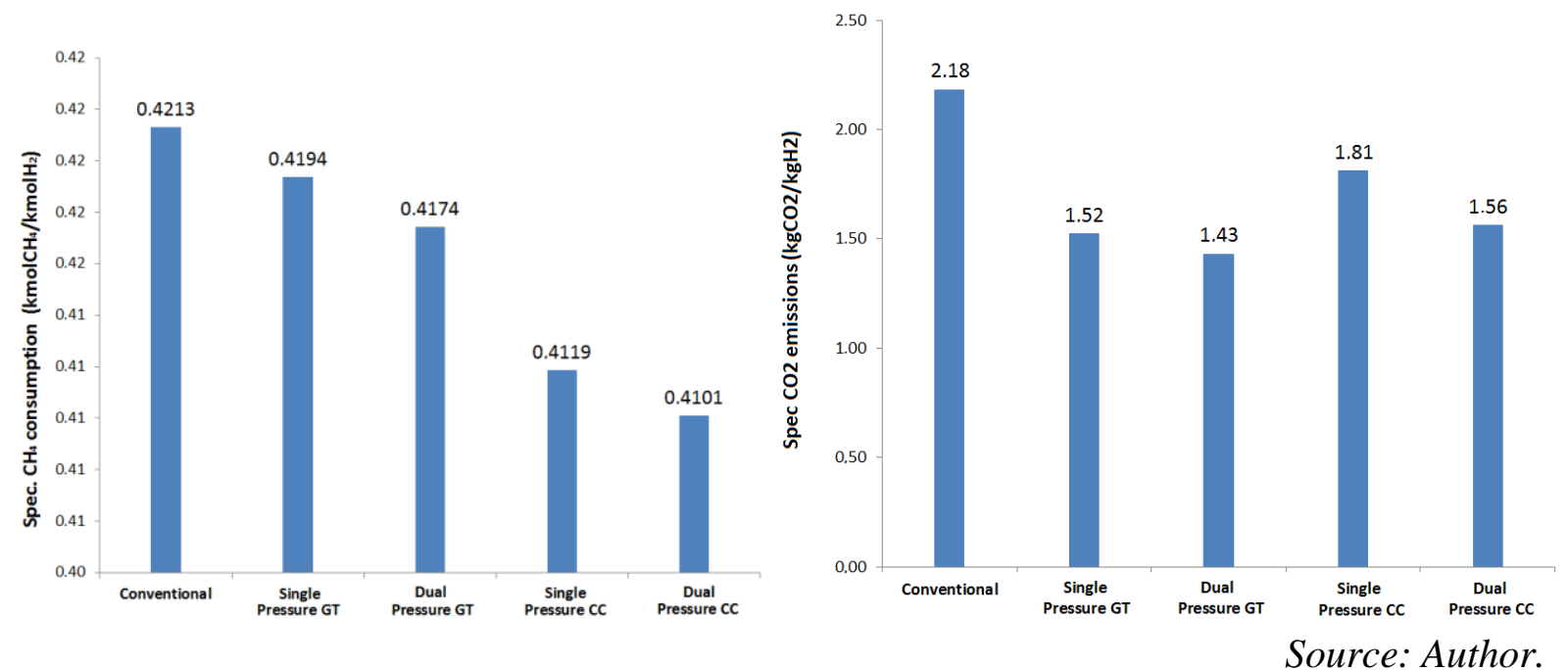

According to Fig. 8.6, the conventional configuration is not only responsible for the largest exergy consumption, but also for the highest specific $\mathrm{CO}_{2}$ emissions. Additionally, depending on the effectiveness and the amount of coils in the HRCT, the flue gases leaving the furnace stack might be rejected to the environment when a relatively large amount of exergy could be still profitable to preheat cold process streams [261, 262]. Moreover, the flue gases from the fired furnace are close to atmospheric pressure and, as such, they are not suitable for directly producing power. Instead, they are used for steam superheating as well as for preheating feed streams. Consequently, the reduction or the elimination of the firing in the primary reformer furnace, by means of a bypass of methane to the secondary reformer, may offset the flue stack losses and the emissions associated to that technology.

Meanwhile, in the CRGT-based configurations, the pressurized gases exiting from the adiabatic ATR reactor are readily expanded for producing power right before any heat exchange process may take place. Moreover, unlike the conventional design, the duty of the 
gas heated reformer is supplied by a gaseous mixture at higher pressures $[81,158]$. These features entail desirable operating conditions that partially enable to overcome the main disadvantages of the conventional primary reformer. Actually, in the conventional design, the maximum pressure differential and allowable heat flux across the reformer tubes are set by the mechanical and metallurgical restrictions. For this reason, the tube diameter cannot be arbitrarily enlarged, entailing also a restriction to the revamp of the conventional plant. In practice, the primary reformer currently poses the most critical bottlenecks that hinder the development of the so-called MEGAMMONIA® plants [29]. Fortunately, the proposed configurations shown in Figs. 8.2-8.5 ensure a good heat transfer performance and reduce the pressure differential across the catalytic tubes, thus, increasing the process reliability.

Table 8.3. Main process variables of the syngas production facilities studied.

\begin{tabular}{|c|c|c|c|c|c|}
\hline Process parameter & Conventional & $\begin{array}{c}\text { Single } \\
\text { Pressure } \\
\text { GT }\end{array}$ & $\begin{array}{c}\text { Dual } \\
\text { Pressure } \\
\text { GT }\end{array}$ & $\begin{array}{c}\text { Single } \\
\text { Pressure } \\
\text { CC }\end{array}$ & $\begin{array}{c}\text { Dual } \\
\text { Pressure } \\
\text { CC }\end{array}$ \\
\hline $\mathrm{CH}_{4}$ feed $(\mathrm{kmol} / \mathrm{h})$ & $1,800.0$ & $1,150.0$ & $1,085.0$ & $1,349.0$ & 851.9 \\
\hline $\mathrm{CH}_{4}$ bypass to ATR feed $(\mathrm{kmol} / \mathrm{h})$ & 274.7 & $1,202.0$ & $1,247.0$ & 893.0 & $1,364.0$ \\
\hline $\mathrm{CH}_{4}$ radiant fired furnace $(\mathrm{kmol} / \mathrm{h})$ & 612.4 & - & - & - & \\
\hline $\mathrm{CH}_{4}$ auxiliary boiler $(\mathrm{kmol} / \mathrm{h})$ & - & 450.0 & 403.4 & 535.1 & 439.4 \\
\hline $\mathrm{CH}_{4}$ slip $(\mathrm{kmol} / \mathrm{h})^{1}$ & 87.1 & 77.1 & 148.9 & 100.1 & 118.6 \\
\hline $\mathrm{H}_{2}$ production rate $(\mathrm{kmol} / \mathrm{h})$ & 6,171 & 6,498 & 6,196 & 6,499 & 6,185 \\
\hline Spec. $\mathrm{CH}_{4}$ cons. $\left(\mathrm{kmolCH}_{4} / \mathrm{kmolH}_{2}\right)$ & 0.4213 & 0.4194 & 0.4174 & 0.4119 & 0.4101 \\
\hline Air molar flow $(\mathrm{kmol} / \mathrm{h})$ & 2,524 & 2,610 & 2,483 & 2,671 & 2,448 \\
\hline Oxygen rich molar flow $(\mathrm{kmol} / \mathrm{h})$ & 440.0 & 919.9 & 911.9 & 578.4 & 718.3 \\
\hline Air enrichment (mol fraction) & 30.01 & 36.86 & 37.35 & 31.82 & 34.80 \\
\hline Nitrogen rich molar flow $(\mathrm{kmol} / \mathrm{h})^{2}$ & 1,440 & 2,942 & 2,984 & 1,892 & 2,349 \\
\hline Steam to carbon ratio $-\mathrm{HP}$ & 3.000 & 3.342 & 3.000 & 3.000 & 3.489 \\
\hline Steam to carbon ratio - MP & - & - & 3.000 & - & 4.154 \\
\hline System Pressure $-\mathrm{HP}(\mathrm{kPa})$ & 3,500 & 3,000 & 3,000 & 3,000 & 3,000 \\
\hline System Pressure - MP (kPa) & - & - & 1,341 & - & 1,400 \\
\hline Exhaust Pressure - LP $(\mathrm{kPa})$ & - & 1,095 & 600 & 1,400 & 900 \\
\hline Cooling Requirement $(\mathrm{kW})^{3}$ & 127,559 & 133,615 & 124,991 & 120,560 & 116,846 \\
\hline Spec. Cooling Req. $\left(\mathrm{kJ} / \mathrm{kmolH}_{2}\right)$ & 74,411 & 74,022 & 72,619 & 66,777 & 68,007 \\
\hline Methane conversion $(\%)$ & 96.76 & 99.58 & 96.68 & 96.39 & 98.67 \\
\hline Primary reformer outlet $\mathrm{T}\left({ }^{\circ} \mathrm{C}\right)$ - HP & 700 & 728 & 730 & 800 & 519 \\
\hline Secondary reformer outlet $\mathrm{T}\left({ }^{\circ} \mathrm{C}\right)$ - HP & 956 & 994 & 1089 & 1079 & 1052 \\
\hline Primary reformer outlet $\mathrm{T}\left({ }^{\circ} \mathrm{C}\right)$ - MP & - & - & 330 & - & 769 \\
\hline Secondary reformer outlet $\mathrm{T}\left({ }^{\circ} \mathrm{C}\right)$ - MP & - & - & 900 & - & 954 \\
\hline Enriched air split (\% to HP line) & - & - & 85.06 & - & 73.64 \\
\hline $\mathrm{CH}_{4}$ feed split (\% to HP line) & - & - & 94.87 & - & 4.89 \\
\hline $\mathrm{CH}_{4}$ fuel split (\% to HP line) & - & - & 77.19 & - & 93.03 \\
\hline $\mathrm{CO}_{2}$ captured flow rate $(\mathrm{kmol} / \mathrm{h})$ & 1,981 & 2,274 & 2,183 & 2,141 & 2,097 \\
\hline Spec. $\mathrm{CO}_{2}$ captured $\left(\mathrm{kmolCO}_{2} / \mathrm{kmolH}_{2}\right)$ & 0.321 & 0.350 & 0.352 & 0.329 & 0.339 \\
\hline Spec. $\mathrm{CO}_{2}$ captured $\left(\mathrm{t}_{\mathrm{CO} 2} / \mathrm{t}_{\mathrm{H} 2}\right)$ & 7.1 & 7.7 & 7.8 & 7.3 & 7.5 \\
\hline $\mathrm{CO}_{2}$ emitted flow rate $(\mathrm{kmol} / \mathrm{h})$ & 612.4 & 450.0 & 403.4 & 535.1 & 439.4 \\
\hline Spec. $\mathrm{CO}_{2}$ emitted $\left(\mathrm{kmolCO}_{2} / \mathrm{kmolH}_{2}\right)$ & 0.099 & 0.069 & 0.065 & 0.082 & 0.071 \\
\hline Spec. $\mathrm{CO}_{2}$ emitted $\left(\mathrm{t}_{\mathrm{CO} 2} / \mathrm{t}_{\mathrm{H} 2}\right)$ & 2.183 & 1.523 & 1.432 & 1.811 & 1.563 \\
\hline Water consumption $(\mathrm{m} 3 / \mathrm{h})$ & 97.48 & 69.40 & 69.13 & 73.07 & 65.90 \\
\hline Condensate prod $(\mathrm{m} 3 / \mathrm{h})$ & 57.93 & 40.86 & 34.65 & 32.47 & 29.02 \\
\hline
\end{tabular}




\begin{tabular}{lcccccc}
\hline \multicolumn{1}{c}{ Process parameter } & Conventional & Single & Dual & \multicolumn{2}{c}{ Single } & \multicolumn{2}{c}{ Dual } \\
& & Pressure & Pressure & Pressure & Pressure \\
& & GT & GT & CC & CC \\
\hline $\mathrm{CH}_{4}+\mathrm{Ar}$ content $(\%)^{4}$ & 1.54 & 1.56 & 2.44 & 1.67 & 2.02 \\
\hline $\mathrm{H}_{2} / \mathrm{N}_{2}$ ratio $^{4}$ molar & 3.036 & 2.999 & 2.999 & 3.000 & 3.075 \\
\hline
\end{tabular}

1. Methane slip in the product syngas can be recovered and recycled as fuel to the syngas production process; 2 . $97.75 \%$ Nitrogen at $27^{\circ} \mathrm{C}, 110 \mathrm{kPa} ; 3$. Cooling water $25-40^{\circ} \mathrm{C}, 60 \%$ relative humidity; 4 . Ammonia loop feed inerts content $\mathrm{CH}_{4}+\mathrm{Ar}$, and $\mathrm{H}_{2} / \mathrm{N}_{2}$ ratio.

As it can be also seen from Table 8.3, in the case of the dual pressure configuration with gas expansion only (i.e. Dual Pressure GT), the entire primary reforming process as well as most of the enriched air-blown partial combustion of the reformed mixture and the additional methane feed occur at higher pressures. Meanwhile, the medium pressure ATR is used to further convert the slipped methane from the HP reformers. The medium pressure ATR also reheats the expanded MP gas in order to increase the power generation, without exceeding the maximum allowable catalyst temperature in the ATR. However, when a steam network is integrated to the dual pressure configuration (i.e. Dual Pressure CC), the largest share of partial oxidation is still performed at the highest temperature and pressure, but most of the primary reforming is performed at medium pressure and lower temperature. This can be explained by a higher mechanical power required from the HP gas expander of the Dual Pressure GT configuration, due to the lack of an integrated waste heat recovery steam network (compare Figs. 8.7c and e). In other words, as long as an alternative technology of power generation relieves the load on the HP gas expansion system, the methane reforming is preferably addressed at a less equilibrium-limited, i.e. a lower pressure operating condition.

Although in the CRGT-based configurations an appreciable amount of fuel is still required by the auxiliary boiler, this consumption is about 12.1-34.6\% lower than the fuel consumed in the fired furnace of the tubular reformer, due to a higher extent of partial oxidation achieved in the exothermic ATR. Furthermore, since the $\mathrm{CO}_{2}$ produced by partial oxidation in the ATR is at a higher concentration, the capture process is facilitated compared to the challenging post-combustion carbon capture from the flue stack gases leaving the furnace of the externally fired primary reformer. As a result, the specific $\mathrm{CO}_{2}$ emissions to the atmosphere in the conventional scenario (approx. $2.2 \mathrm{t} \mathrm{CO}_{2} / \mathrm{t} \mathrm{H}_{2}$ ) are 21.5-52.4\% higher than those obtained for the CRGT-based configurations (see Fig. 8.6). Furthermore, regarding the reduced water demand in the CRGT-based systems, it can be explained by the reduced S/C ratios necessary in the ATR compared to the excess steam required in the conventional tubular reformer. Furthermore, it is important to highlight that in the CRGT-based configurations, an additional amount of water vapor is produced at high pressure in the ATR through partial oxidation. In this way, the surplus water produced at higher temperatures and pressures in the ATR serves not only as a means of temperature control, but also as additional process feedstock as well as working fluid, unlike the water vapor present in the stack gases.

Finally, Figs. 8.7a-e details the distribution of the power generated among the various consumers in the syngas production plants analyzed. Certainly, since no net export is aimed, the optimal solution determines the operating conditions that minimize the surplus power and maximize the hydrogen yield. It must be also born in mind that, differently from the 
conventional case, the CRGT concepts rely on the expansion of the pressurized ATR effluent. This features renders necessary the recompression of the syngas produced up to the original ATR outlet pressure after the excess water is removed. According to Fig. 8.7b-e, the share of power consumption of the post-compression can be as high as $40 \%$ of the overall power demand and, in some cases, it even surpasses the air compression duty, whereas the third largest consumption comes from the energy intensive air enrichment unit (20\%) and the amine circulation pump (10\%). 
Fig. 8.7. Distribution of the power supply and demand $(k W)$ for the $(a)$ conventional, $(b)$ single and (c) dual pressure GT, and (d) single and (e) dual pressure CC configurations.

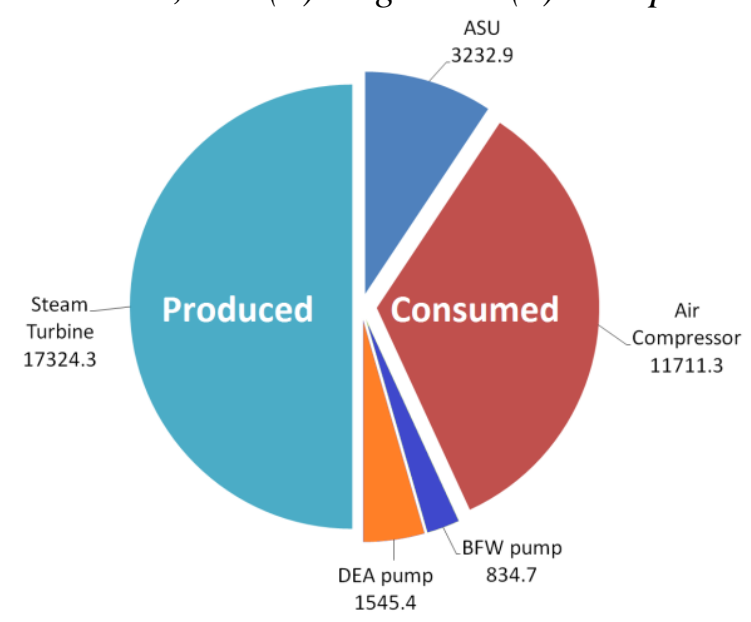

(a) Conventional

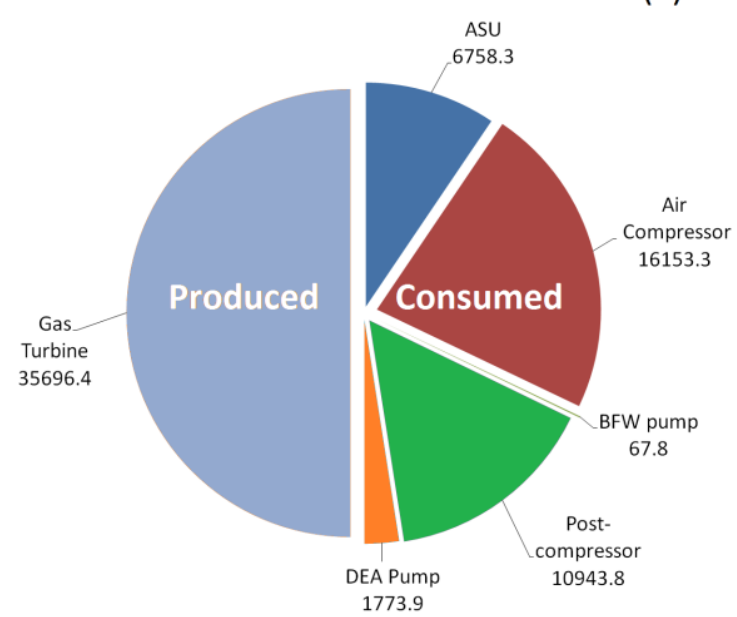

(b) Single Pressure GT

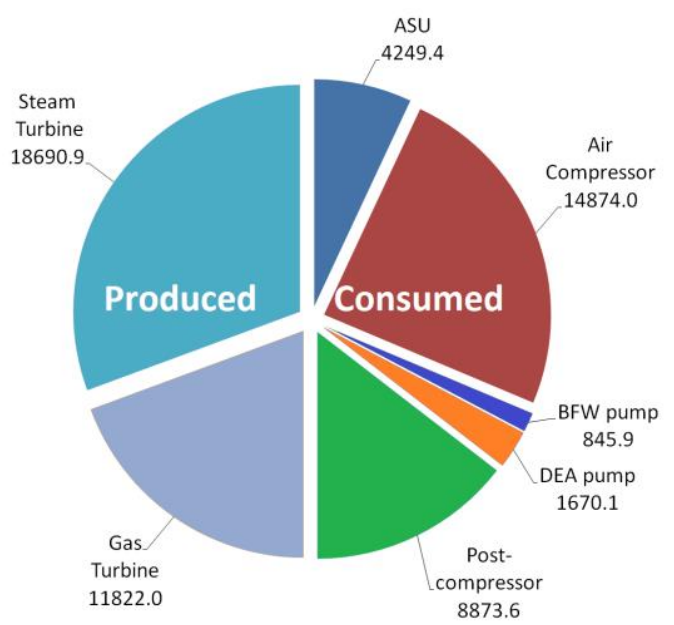

(d) Single Pressure CC

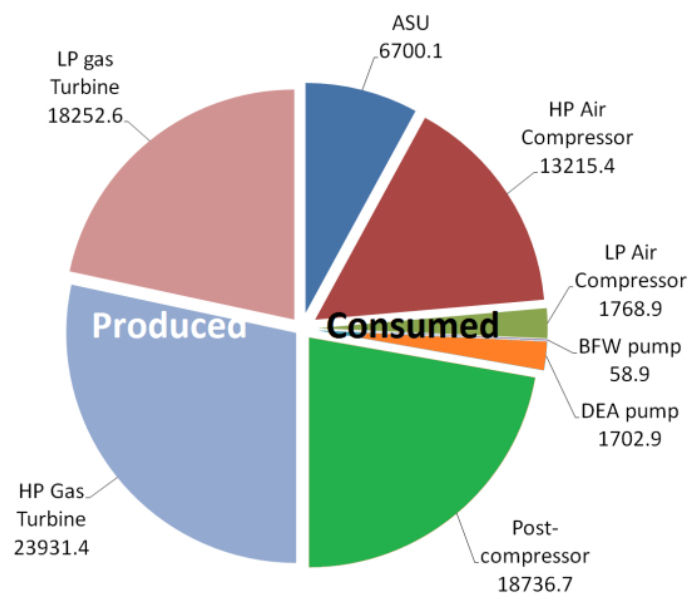

(c) Dual Pressure GT

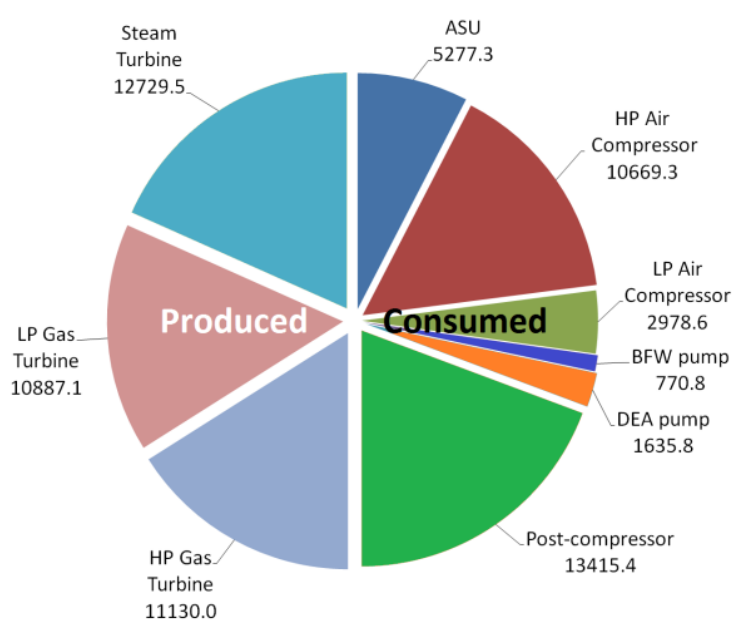

(e) Dual Pressure CC

Source: Author. 


\subsection{Energy Integration and Carnot Grand Composite Curves}

Figures 8.8 show the Carnot Grand Composite curves (CGCC) of the conventional and CRGT-based syngas production plants, reported in Table 8.3. As discussed in previous chapters, the area enclosed between the vertical axis and the CGCC represents the exergy destroyed in the heat exchanger network. According to Fig. 8.8, in the conventional configuration, a fraction of the exergy of the high-grade hot utility provided by the fired furnace is wastefully transferred to the environment. Actually, the flue stack gases leaving the convection train lead to an exergy loss equivalent to $6 \mathrm{MW}$, due to the practical limitations to fully recover this exergy. In contrast, in the CRGT-based configurations, the physical exergy of the excess reforming steam and the water produced by partial oxidation is mostly recovered up to its condensation [34].

According to Fig. 8.8, in all the configurations, an auxiliary boiler is still needed so that the total heating requirement for the steam generation can be balanced. However, regarding the CRGT-based configurations with gas expansion only (i.e. single and dual pressure-GT), the consumption of the chemical exergy of the fuel to merely produce steam suppose an avoidable exergy loss in the system. Effectively, the exergy destruction can be further reduced by integrating a heat recovery steam network, i.e. through the utilization of single and dual pressure-CC configurations. The resulting CGCCs (Fig. 8.8, right) resemble the combination of the conventional and the '-GT' profiles (Fig. 8.8, left). As a result, the plantwide and the cogeneration exergy efficiency increase compared to the conventional syngas production unit based only on heat recovery steam network [206].

Fig. 8.8. Carnot Grand Composite curves (CGCC) for the conventional, single and dual pressure GT configurations (left) and for single and dual pressure CC configurations (right).
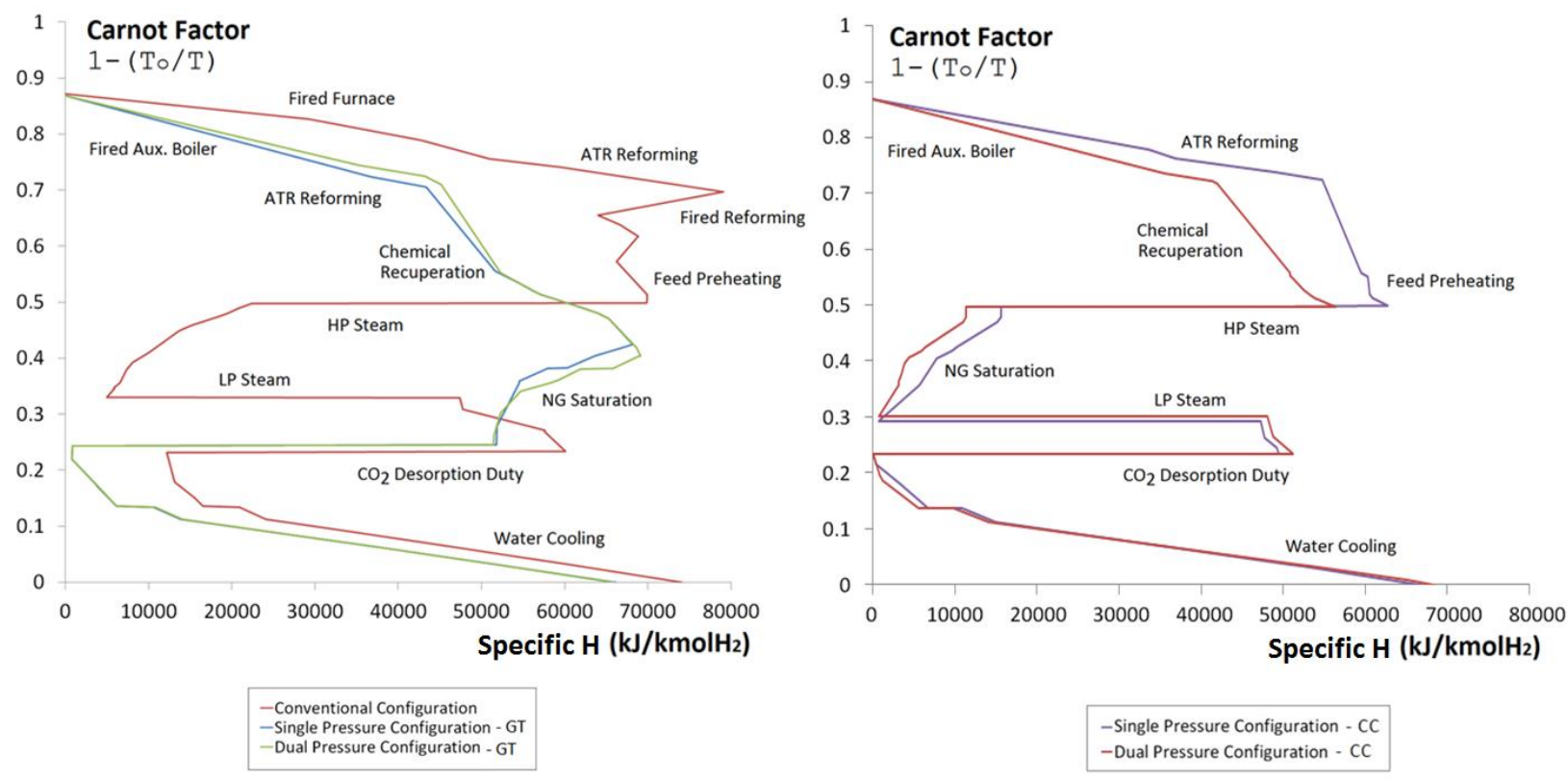

Source: Author.

Clearly, in the case of the CRGT-based configurations, the integration of an additional steam network entails a trade-off between the chemical recuperation and the combined steam and 
power generation. In fact, since the exergy available from the ATR effluent is used for power generation and heating the GHR, the plantwide steam and power balance is drastically affected [257]. Some authors suggested that this is actually a great opportunity to involve cheaper, more readily available resources such as coal, or even less pollutant resources, e.g. biomass, in order to supply the energy for the steam generation. The import of 'cleaner' electricity from the grid, may be also considered, obviously depending on the mix and the cost of the electricity [263]. In this way, further alternatives for reducing the large driving forces and the environmental impact triggered by the utilization of the auxiliary boiler can be endeavored. For example, the diversification of the supply chains of the syngas and hydrogen derivatives, traditionally dominated by non-renewable energy resources.

\subsection{Exergy analysis of the conventional and proposed syngas production units.}

Figure 8.9 shows the relative and consumed-produced exergy efficiencies calculated for the syngas production plants, as defined in Eqs. (8.1-8.2). Since the relative efficiency has been defined by using the minimum theoretical exergy consumption required to produce syngas from the elements of the environment, it accounts for the maximum potential of exergy savings. However, due to the large mass flow rates of the bulk chemical production process [55], the relative exergy efficiency is less sensitive than the consumed-produced counterpart.

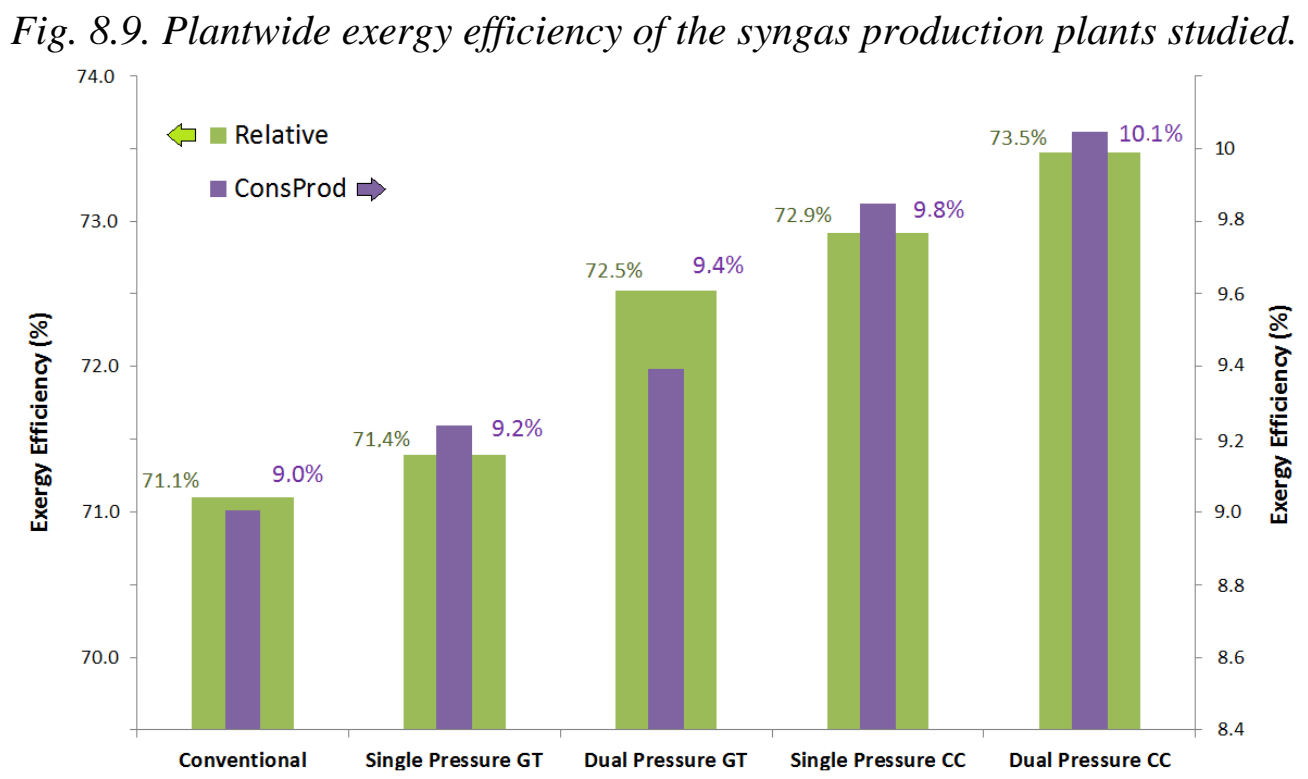

Source: Author.

As a result, the consumed-produced exergy efficiency (9-10\%) is a more suitable indicator for determining the overall plant performance, along with the cogeneration exergy efficiency (Fig. 8.10) and the specific exergy destruction (Fig. 8.11). As expected, the higher degree of energy integration between the utility system and the chemical processes in the CRGT-based configurations leads to an increased efficiency of the respective cogeneration systems. According to Fig. 8.10, the integration of a chemically recuperated gas turbine concept with reheat together with an extensive heat recovery steam network (i.e. Dual Pressure CC) achieves a cogeneration efficiency of $55 \%$, typical for a combined cycle. This value is $67 \%$ higher than that of the single pressure-GT configuration, analogous to a standalone open cycle 
gas turbine (OCGT). The capitalization on the other plant byproducts, such as the marketable $\mathrm{CO}_{2}$, potentially used in an associated urea or methanol, should be also included in the evaluation of the process efficiency.

Fig. 8.10. Exergy efficiency of the integrated cogeneration systems in the syngas production plants.

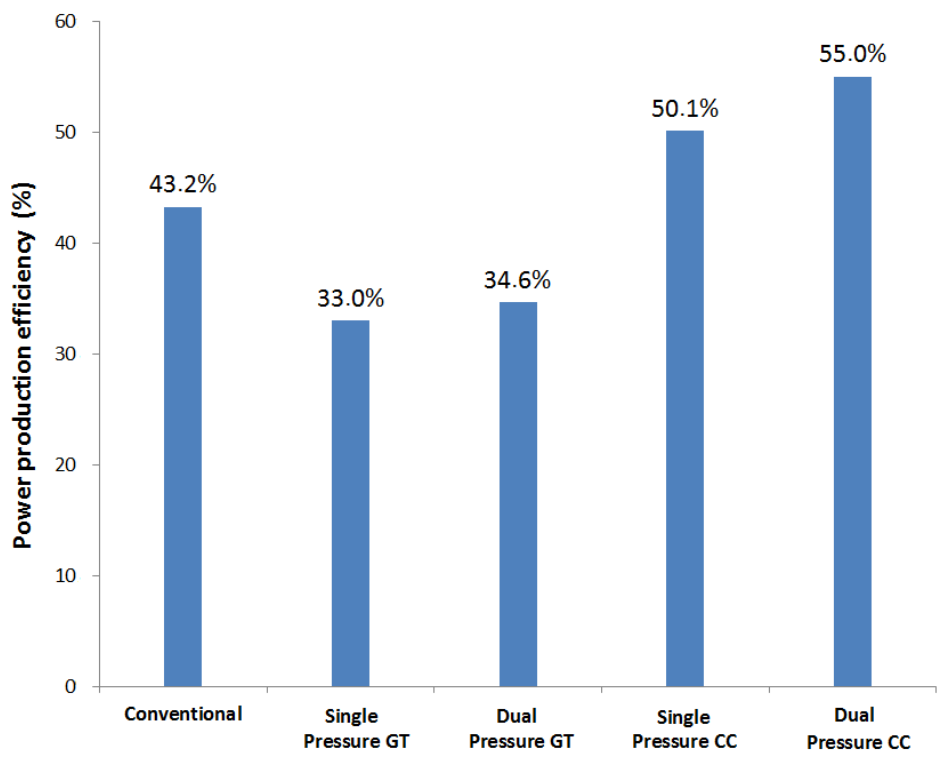

Source: Author.

Figure 8.11 compares the specific exergy destruction obtained for the studied configurations, which evidences that the higher the specific consumption, the higher the specific exergy destruction. Certainly, an increased fuel consumption often entails a worse utilization of the available resources to produce the same amount of product [34]. This explains an increase of $3-12 \%$ of the specific exergy destruction in the conventional system, compared to the CRGTbased configurations.

Fig. 8.11. Specific exergy destruction $\left(\mathrm{kJ} / \mathrm{kmol}_{\mathrm{H} 2}\right)$ for the studied syngas production plants.

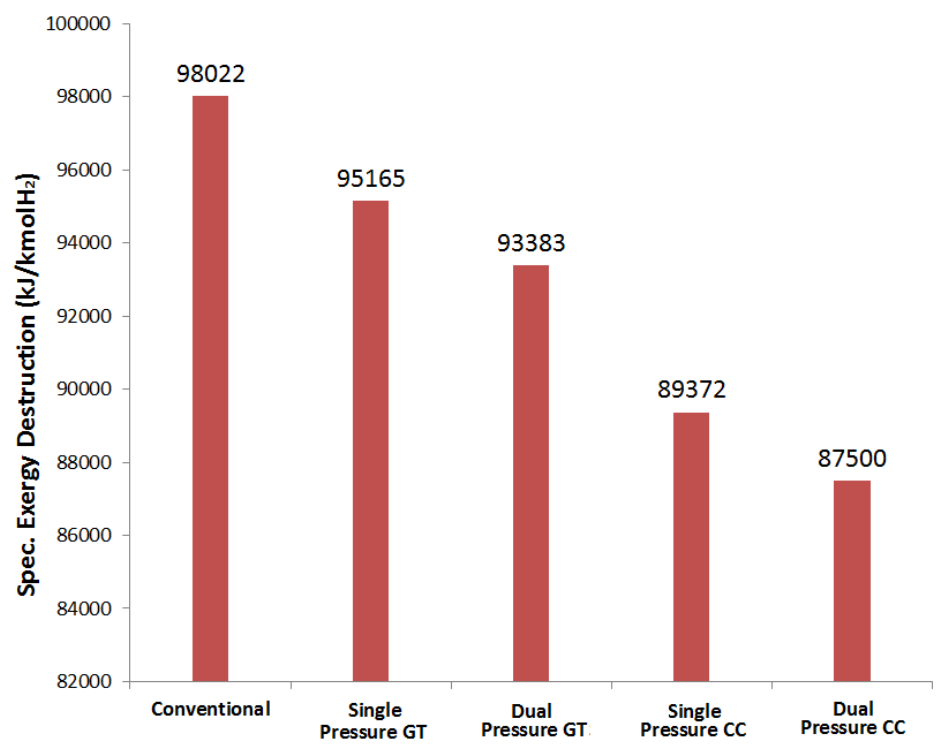

Source: Author. 
Finally, Fig. 8.12 shows the distribution of the overall exergy destruction among the components of the conventional syngas production process. As it can be seen, the largest shares of exergy destruction correspond to the waste heat recovery steam network and the HRCT of the primary reformer, together accounting for $60 \%$ of the exergy destroyed in the conventional system. If both primary and secondary were also included, the share of exergy destruction increases up to $80 \%$ of the total irreversibility.

Figure 8.12 also shows the exergy destruction breakdown of the most representative components of the syngas production plants based on the CRGT concept. It is worthy to notice that, in all the studied plants, the share of exergy destroyed in the reforming sections is relatively similar. Furthermore, the auxiliary boiler used in the CRGT-based configurations represents about $40 \%$ of the whole irreversibility. Also according to Fig. 8.12, the exergy destruction share associated to the reformers in the dual pressure CRGT-based configurations is effectively reduced (3-21\%) compared to the single pressure CRGT-based systems. This is owed to the application of the counteraction principle by operating the reforming sections at decremental levels of pressure [69]. Nevertheless, it is worthy to highlight that, in the optimal solutions, the lowest gas expansion pressure is not necessarily the atmospheric one, but rather an intermediate pressure. Indeed, higher operation pressures in the syngas production may lead to the reduction of the power consumption in the downstream syngas compressor train installed at the backend ammonia loop [257]. In practice, the natural gas supply is guaranteed at high levels of pressure, thus it seems to be preferable to maintain the operating conditions of the syngas production at higher pressures. Other benefits of an increased operating pressure in the overall plant include smaller reactors, better ammonia conversions, as well as improved refrigeration and separation conditions.

Fig. 8.12. Exergy destruction breakdown for representative components of the studied syngas production plants.

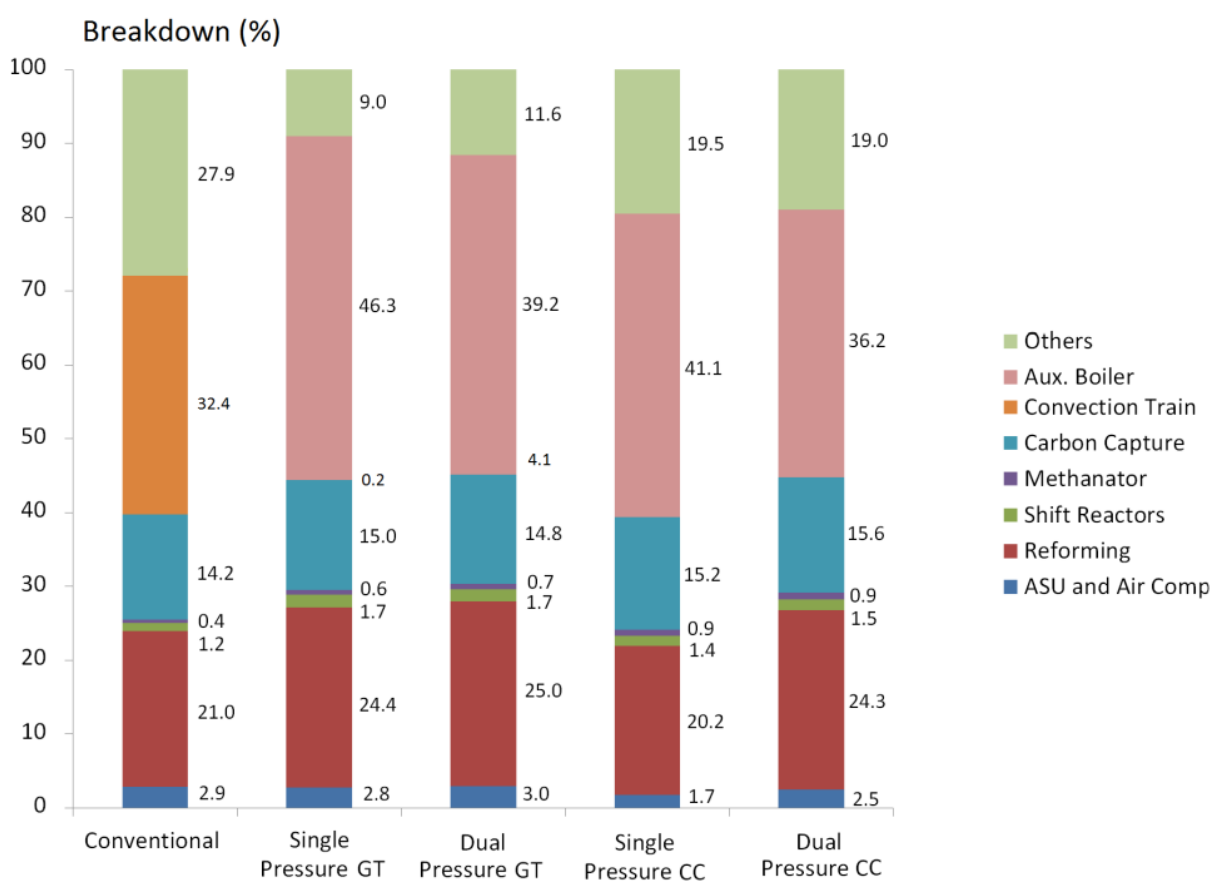

Source: Author. 
Some approaches found in the literature are in the same line of thinking of the subject presented in this chapter. As mentioned earlier, the partial oxidation gas turbine (POGT) simultaneously produces power and a secondary fuel that usually is a hydrogen-rich gas, which renders it well suited for the coproduction of power and chemicals. In this way, like the POGT systems, the configurations proposed here may also provide a secondary fuel that can be burned in a bottoming cycle (a cogeneration steam cycle, for instance) [264]. Moreover, according to some studies [265], the working fluid (fuel gas) provided by the partial oxidation reactor has a much higher specific heat than the complete combustion products. Thus, from an operational and thermodynamic point of view, more energy per unit mass of fluid can be extracted from the POGT expander compared to the typical gas turbine systems. As a result, a much smaller compressor is required, one that typically supplies less than half of the air flow required in a conventional gas turbine [264]. Other desirable features such as the ultra-low $\mathrm{NO}_{\mathrm{x}}$ emissions are also shared with the steam injected gas turbine systems.

Thus, as a preliminary conclusion, non-conventional approaches will be worthy to explore, considering the limited room for improvement in the reactive components. Actually, the energy-intensive nature of the current technical process is mainly due to the large amount of exergy consumed in the process air enrichment and compression, syngas recompression, the steam generation in auxiliary boilers and the carbon capture systems, from which the highest potential of exergy saving are expected [34]. Moreover, it has been also shown that the modification of the chemical process layout impacts the whole system irreversibility and allows for the minimization of the amount of exergy consumed in the syngas production unit. In this way, all the proposed novel configurations prove to be relevant in order to identify the opportunities of improvement in the syngas and hydrogen production systems. It is nevertheless important to point out that further economic evaluations should be performed in order to determine the marginal investment and risk cost involved [266]. However, exergy efficiency is not less important even at a cheaper natural gas cost scenario, because higher efficiencies are associated to smaller units and utility requirements, thus less operating costs, not to mention the striking reduction of the $\mathrm{CO}_{2}$ emissions achieved [34].

\subsection{Final considerations}

Novel syngas production plants represent interesting applications for improving the exergy efficiency and fuel consumption of systems traditionally based on non-renewable resources. However, the interrelation between the process parameters, the rearrangement of the new and the existing unit operations, as well as the perturbation of the combined steam and power production balances render the optimization of these systems a complex non-linear problem.

In this chapter, five different configurations have been compared, namely a conventional syngas production system and four proposed chemically recuperated gas turbine (CRGT)based concepts with single and dual levels of pressure. The minimization of the specific exergy destruction is considered as the objective function. More sensitive exergy efficiencies for both the overall plant and the cogeneration system have been also evaluated. As a result, even at optimal operating conditions, the proposed single and dual pressure configurations 
present a specific exergy destruction ranging from 87.5 to $95.2 \mathrm{MJ} / \mathrm{kmolH}_{2}$. However, those values are found to be $3-12 \%$ lower than the obtained for the conventional process.

Moreover, the exergy fuel consumption in the proposed CRGT configurations is estimated as $0.5-2.7 \%$ lower than in the conventional design. Meanwhile, the cooling requirement in the proposed setups is substantially reduced (up to $10 \%$ ) compared to the conventional plant. By using a dual pressure configuration, the reacting driving forces in the reforming sections are also reduced. Additionally, the integration of a waste heat recovery steam network actually leads to a reduction of the exergy losses associated to the consumption of high-grade utility streams (e.g. chemical exergy of the fuel) to merely produce steam in the auxiliary boilers.

Clearly, the minimization of the exergy destruction is not a priori an economic criterion, since this task is often achieved at the expense of additional capital investments. However, exergy efficiency is not less important, since higher efficiencies are associated to smaller units and utility requirements, not to mention the striking reduction of the $\mathrm{CO}_{2}$ emissions (useful in credit carbon economies). It is thus necessary to further discuss the relationship between the overall exergy destruction, its distribution throughout the chemical process and the economy thereof.

Analogously to the analysis that has been developed for the frontend syngas production process, in the next two chapters, the effect of the operating parameters and the process structure in the optimal configurations of the backend ammonia synthesis loop is thoroughly examined in light of the counteraction principle. 


\section{MODELING AND OPTIMIZATION OF AN INDUSTRIAL} AMMONIA SYNTHESIS LOOP

In this chapter, the modeling and optimization of an industrial ammonia synthesis loop that produces $1,000 \mathrm{t}_{\mathrm{NH} 3} /$ day is presented. The influence of some critical operation parameters on the exergy performance of the overall loop and the major components thereof is analyzed through a series of baseline and improved scenarios. It is worthy to notice that, the ammonia synthesis is a highly exothermic process, thus, higher per-pass conversions are only achieved by using various sequential catalyst beds. Thus, a near-optimum reactor conversion vs. temperature profile can be attained by regulating the inlet temperature of each bed. This is performed via indirect heat recovery, either by preheating the reactor feed gas or by using waste heat boilers.

Expectedly, by operating close to the line of optimum conversion for each reaction rate, lower catalyst volumes may be required. However, the optimal operating conditions of the catalytic beds alone do not seem to totally define the optimal performance of the overall ammonia loop. Indeed, it will be shown that the optimal design is rather a complex function of the standalone equipment performance and the interaction of all the synthesis loop components, even though largely influenced by the reactor performance.

In this chapter, exergy is used to quantify the efficiency and minimize the exergy destruction rate along the various components of the ammonia synthesis loop, as well as to optimize the process revenue as a function of the most critical operation parameters. Since the proposed objective function, namely the exergy destruction minimization, shows to be oversensitive to some specific process variables, some graphical maps based on extensive case studies have been prepared to illustrate the sharp variation of the objective function with the main process variables.

\subsection{Performance indicators proposed for the industrial ammonia synthesis loop.}

According to Fig. 9.1, the ammonia synthesis unit is composed of the makeup syngas compressor, the ammonia reactor, the waste heat recovery network, and the condensation and separation systems. Since the operating conditions of every component are interrelated to each other, the minor modification largely affects the different sections of the plant flowsheet.

Initiallt, the fresh syngas compressed above 150 bar is fed to the ammonia reactor, where a nitrogen conversion between $10-30 \%$ is achieved in presence of a magnetite catalyst [41]. In order to achieve a higher conversion per pass, the reactor is split into three sequential catalyst beds with intercooling, as discussed in previous chapters. The converter performance and, consequently, the loop efficiency, are affected by the pressure, temperature and composition of the reactor feed, as well as by the amount of inerts and ammonia recycled, the heat removal 
and the catalyst design. The build-up of inerts (i.e., argon and methane) is controlled by a continuous withdrawal of a portion of the hydrogen-rich recycle gas to keep inerts concentration down to an acceptable level [70]. This withdrawal is better performed after the ammonia bulk removal and before the fresh syngas addition, where the concentration of inerts is higher [94].

Fig. 9.1. Flowsheet of the industrial ammonia production unit.

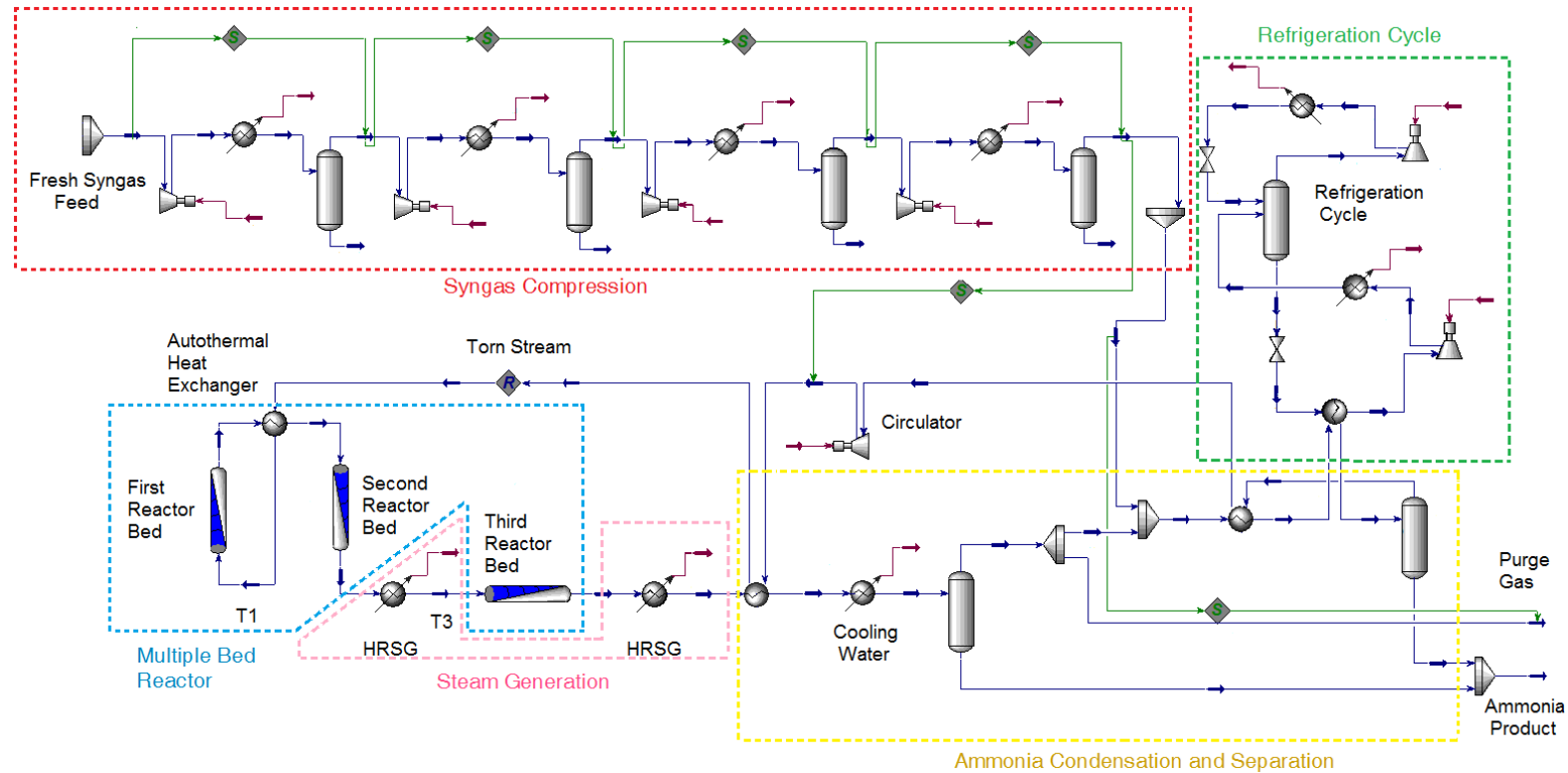

Source: [237].

Table 9.1 compares the rational exergy efficiency, Eq. (9.1), along with other exergy efficiency definitions, Eqs.(9.2-9.4), proposed for evaluating the overall performance of the ammonia loop [206]. It must be noticed that, since the exergy of the input and output material flows are much larger than the exergy flows (i.e., power), the rational exergy efficiency may untruthfully lead to high and similar values.

Table 9.1. Comparison between the exergy efficiency definitions of the overall ammonia synthesis unit.

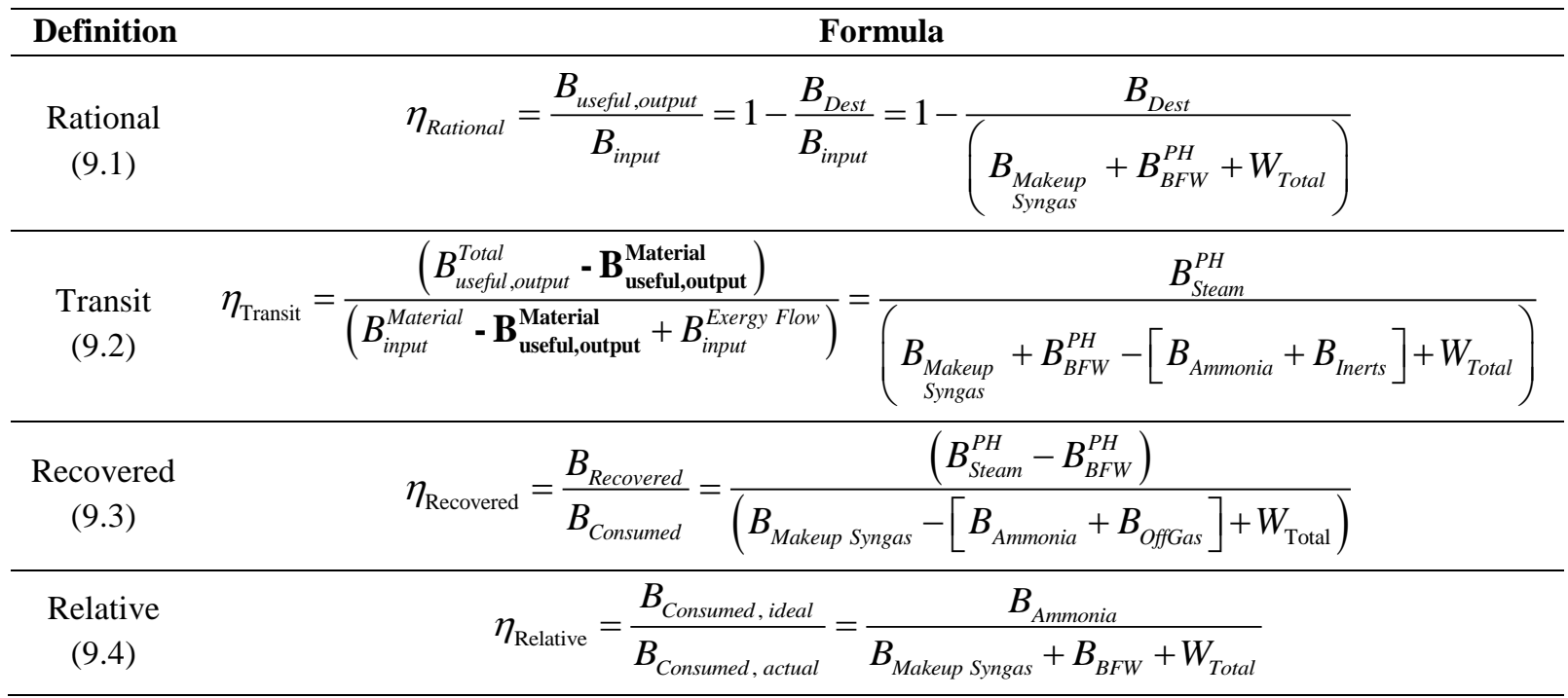


To overcome this problem, an alternative approach has been firstly suggested by KirovaYordanova [19]. The author proposal consists of considering the useful exergy of the material output (i.e. ammonia) as transit exergy, subtracting it from the numerator and denominator of Eq.(9.2), Table 9.1. However, Eq.(9.2) must be carefully used, since it considers that all the outlet material flow is exergy in transit, although a chemical reaction of the makeup syngas occurs in the ammonia loop [267]. Equation (9.2) also assumes that all the non-reacted nitrogen and hydrogen is recycled back to the ammonia converter and, thus, liquid ammonia, methane and argon are the only material exiting the system.

In an attempt to truly differentiate between the transiting exergy and the consumed exergy, the transiting exergy in the utilizable stream should be calculated as the part of the exergy entering a unit operation and going through it without undergoing any transformation [268]. However, according to Brodyansky et al. [176], apart from the inerts and the purged hydrogen-rich gas, the raw material fed to the ammonia synthesis loop has undergone chemical transformations. Accordingly, the transit exergy concept is useless in overcoming this problem.

Thus, in the light of the technical limitations of the previous exergy definitions and bearing in mind the net exothermic nature of the chemical process [245], an alternative way to calculate the loop performance is proposed as shown in Eq.(9.3), Table 9.1. By considering an ideal case in which all the exergy input that was neither embodied in the ammonia nor in the offgas products is recovered in the form of steam (i.e. the waste exergy recovery is maximized), the exergy balance could be written as in Eq.(9.5):

$$
B_{\text {Dest }}=0 \rightarrow B_{\text {Steam }}=B_{\text {Fresh Syngas }}-\left[B_{\text {Ammonia }}+B_{\text {Purge }}\right]+W_{\text {Compressor }}+\mathscr{W}_{\text {Circulator }}^{0}+W_{\text {Refrigerator }}+B_{B F W}
$$

Thus, Eq.(9.5) imposes a limit for the maximum exergy recovery in the form of steam, when pressure drop ( $W_{\text {Circulator }} \rightarrow 0$ ) and process irreversibility $\left(B_{\text {Dest }} \rightarrow 0\right.$ ) tends to zero. It is worthy to notice that, since the difference and not the absolute exergy flow rates are written in Eq.(9.3), the shortcomings of the exergy efficiency definition of systems with large recycle streams can be overcome. This exergy definition relies on the fact that the enthalpy of reaction of the ammonia synthesis represents about $8.8 \%\left(2.718 \mathrm{MJ} / \mathrm{t}_{\mathrm{NH} 3}\right.$, energy basis $)$ of the overall consumption of the ammonia production plant. For this reason, there is a strong incentive in recovering as much as possible this waste heat by maximizing the steam generation [41]. Furthermore, unlike the integration scenario in which the high-grade waste heat was available at a higher temperature (e.g. at the ATR outlet), the excess heat in the ammonia synthesis is available at temperatures closer to the operating conditions of the steam boiler. Consequently, the process irreversibility is reduced when steam is generated from this source. Thus, it is important to emphasize that the aim of the recovered exergy efficiency, Eq.(9.3), is not to merely account for the production of steam, but for the maximization of the exergy recovered.

On the other hand, it may be arguable that the objective of the chemical loop is the ammonia production, rather than expending the valuable exergy of the incoming syngas to produce low- 
grade steam. Therefore, a second exergy efficiency definition is proposed, Eq. (9.4), calculated as the ratio of the minimum exergy consumption in the ammonia production (i.e. the chemical exergy of ammonia, 327,000 kJ/kmol) to the actual exergy consumption of the loop. Despite of the physical meaning of Eq.(9.4), the denominator includes the absolute exergy values of the material flows, which patially compromises its sensitivity to the loop parameters. Anyhow, Eq.(9.4) gives a measure of the deviation from the scenario of minimum exergy demand when ammonia is built from the elements of the environment.

Table 9.2 contrasts the consumed-produced against the rational exergy efficiency definitions, proposed for representative components of the ammonia synthesis loop. In Eq.(9.7), the efficiency of the synthesis reactor is defined in terms of the increase of the physical exergy of the reactor effluent at the expense of a fraction of the chemical exergy of the reactants [69].

Table 9.2. Comparison of the exergy efficiency definitions for representative equipment.

\begin{tabular}{|c|c|c|}
\hline Unit (Eq.) & (a) Input-Output & (b) Consumed-Produced \\
\hline \multirow{2}{*}{$\begin{array}{c}\text { Syngas } \\
\text { Compression } \\
(9.6)\end{array}$} & $B_{\text {Dest }, \text { Comp }}$ & \multirow{2}{*}{ 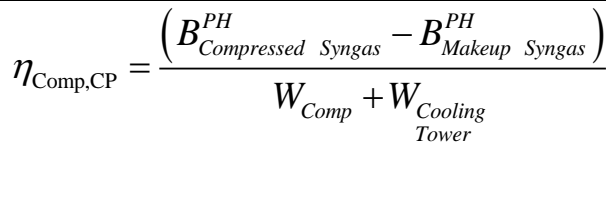 } \\
\hline & $\eta_{\text {Comp }, I O}=1-\frac{\left(B_{\text {Fresh Syngas }}^{T}+W_{\text {Comp }}+W_{\text {Cooling }}\right.}{\text { Tower }}$ & \\
\hline $\begin{array}{l}\text { Ammonia } \\
\text { Reactor } \\
(9.7) \\
\end{array}$ & $\eta_{\text {Reactor,IO }}=1-\frac{B_{\text {Dest }, \text { Reactor }}}{B_{\text {Reactor Feed }}^{T}}$ & $\eta_{\text {Reactor,CP }}=\frac{B_{\text {Reactor Product }}^{\text {PH }}-B_{\text {Reactor Feed }}^{\text {PH }}}{\left(B_{\text {Reactor Feed }}^{C H}-B_{\text {Reactor Product }}^{C H}\right)}$ \\
\hline \multirow{2}{*}{$\begin{array}{l}\text { Refrigeration } \\
\text { cycle } \\
\quad(9.8)\end{array}$} & \multirow{2}{*}{$\eta_{\text {Refrig,IO }}=1-\frac{B_{\text {Dest, Refrig }}}{W_{\text {Compressors }}+W_{\text {Cooling }}+B_{\text {Evap }}^{Q}}$} & $\eta_{\text {Refrig, CP }}=\frac{Q_{\text {Evap }}\left(T_{0} / T_{\text {Evap }}-1\right)}{W_{\text {Comp }}+W_{\text {Cooling }}}=\frac{C O P_{\text {actual }}}{C O P_{\text {Carnot }}}$ \\
\hline & & $\eta_{\text {Refrig, CP2 }}=\frac{B_{\text {Syngas, } \text {,in }}^{P H}-B_{\text {Syngas, out }}^{P H}}{W_{\text {Comp }}+W_{\text {Cooling }}}$ \\
\hline $\begin{array}{l}\text { Waste Heat } \\
\text { Boiler } \\
(9.9)\end{array}$ & $\eta_{\mathrm{HRSG}, \mathrm{IO}}=1-\frac{B_{\text {Dest }, \text { HRSG }}}{\left(B_{\text {Hot Gas }}^{T}+B_{B F W}^{T}\right)}$ & $\eta_{\mathrm{HRSG}, \mathrm{CP}}=\frac{\left(B_{\text {Steam }}^{P H}-B_{B F W}^{P H}\right)}{\left(B_{\text {Hot Gas }}^{P H}-B_{\text {Cold Gas }}^{P H}\right)}$ \\
\hline
\end{tabular}

Two approaches have been considered for calculating the exergy efficiency of the refrigeration cycle, Eq.(9.8). Differently from the first approach (identified as $C P$ ), the second definition (i.e. $C P 2$ ) includes the irreversibility arisen from the finite temperature difference in the evaporator. In this way, the term $B_{\text {ProcessGas }}^{P H}$ stands for both the inlet and outlet exergy flow rate of the refrigerated mixture.

\subsection{Optimization problem definition}

The equilibrium-limited ammonia synthesis is an interesting application for examining the difficulties faced in the optimization of chemical processes working under the reactionseparation-recycle-purge scheme. Notwithstanding, due to the interrelation between the feed temperatures and compositions, the reaction kinetics and the arrangement of the catalytic beds, the ammonia condensation, and the heat recovery network, finding out the operating parameters that optimize the overall performance of the ammonia loop is a formidable task. 
Considering only the catalytic beds, the kinetics already exhibit a strongly non-linear relationship with the reactor temperature. Away from the equilibrium, the kinetics are favored by a temperature increase, which also leads to an increase in the conversion [141]. However, the reaction rate slows down as approaches to the equilibrium, since the maximum conversion decreases at higher temperatures [23, 139]. Industrially, the most widespread strategy to increase the per-pass conversion consists of an intercooled, multiple bed ammonia reactor [25, 269]. Nevertheless, by continuously shifting the reaction away from equilibrium, not only the ammonia yield but also the irreversibility of the system are increased, thus, reducing the loop efficiency and increasing the utilities demand [69]. Accordingly, the intercooling introduces an additional but necessary source of irreversibility, since it simultaneously allows increasing the ammonia yield while integrates the chemical plant to the steam network [25, 41].

Meanwhile, some practitioners purposely increase the reactor space velocity (volumetric flow per unit of reactor volume) so that larger ammonia throughputs can be achieved, even at expense of a reduced per-pass conversion and reactor temperature [270]. The reasoning behind this deliberate increase in the circulation rate lies in the Le Châtelier Principle, which attempts to increase the ammonia production by increasing the reacting driving force [142]. As expected, the reduction of the per-pass conversion also leads to a reduced amount of perpass exergy destruction [69]. However, as it will be shown, it does not necessarily entails the reduction of the overall irreversibility in the loop. Moreover, by arbitrarily reducing the stoichiometric $\mathrm{H}_{2}$ to $\mathrm{N}_{2}$ ratio of the makeup syngas, whereas keeping the purge gas fraction as constant, the circulation rate can be willingly increased but only at the risk of the stability or blow-off of the exothermic reactor [270]. Additionally, a prohibitive pressure drop and an increase of the circulator power and refrigeration demands may be also triggered.

Regardless of the previous approaches adopted, according to the Counteraction Principle, the large driving forces $(-\Delta G)$ or, equivalently, the avoidable exergy losses occurring in the standalone chemical reactor may be reduced by applying a counteraction that increases the temperature of the exothermic reactor feed [69]. However, it should be also determined the effect of this perturbation on the overall ammonia synthesis loop. Table 9.3 details the optimization problem formulated for the minimization of the exergy destroyed in the whole loop per unit of exergy of useful product by considering that the capital costs are nearly constant for a given operating pressure $[169,271]$. The solution of this problem allows to determine the most suitable approach-to-equilibrium and the best temperature profile along the reactor beds of a $1,000 \mathrm{t}_{\mathrm{NH} 3} /$ day ammonia loop. Since the simulation of chemical processes is likely to pose convergence issues due to the snowball effect [142], a smart initial guess for the properties of the torn stream is necessary. Furthermore, advanced acceleration methods (dominant-eigenvalue) have been preferred over inefficient successive substitution methods. A sequential quadratic programming (SQP) tool in Aspen ${ }^{\circledR}$ Hysys is used to solve the optimization problem described in Table 9.3. 
Table 9.3. Optimization problem definition.

Objective function: Exergy destruction minimization.

Subject to the constraints:

- Minimum temperature approach at the gas-gas heat exchanger, $30^{\circ} \mathrm{C}$. The enthalpy of reaction of the first bed must be enough to raise the temperature of the incoming feed gas,

- Minimum temperature approach in the waste heat recovery exchangers, $30^{\circ} \mathrm{C}$. Avoid temperature cross between the process gas and steam,

- Convergence of the recycle tearing,

- Composition of inerts $(<15.4 \%)$ and ammonia $(<2.6 \%)$ in recycled gas are kept down to acceptable levels.

- Maximum catalyst temperatures, $550^{\circ} \mathrm{C}$. Metallurgical limitations at high pressure, adverse effects on catalyst activity and lifespan,

- Risk of poisoning by even low $\mathrm{O}_{2}$ concentrations sets a practical lower bound to the catalyst temperature, $290^{\circ} \mathrm{C}$.

\section{Design Variables:}

- Preheating temperature of the feed gas going to the first bed, $\mathrm{T}_{1}\left(290-500^{\circ} \mathrm{C}\right)$.

- Intercooling temperature of the feed gas going to the third bed, $\mathrm{T}_{3}\left(290-420^{\circ} \mathrm{C}\right)$.

- Makeup syngas molar composition (Fresh syngas $\mathrm{H}_{2} / \mathrm{N}_{2}$ ratio).

- Loop pressure (150 bar, 200 bar).

\section{Constants:}

- For both 150 and 200 bar, reactor volume $1^{\circ}$ bed: $29.4 \mathrm{~m}^{3} ; 2^{\circ}$ bed: $29.4 \mathrm{~m}^{3} ; 3^{\circ}$ bed: $39.3 \mathrm{~m}^{3}$.

- Reactor void fraction, 0.46 [79, 222]. Catalyst effective diameter, $3 \mathrm{~mm}$. Catalyst bulk density, $2300 \mathrm{~kg} / \mathrm{m}^{3}$,

- Circulator and refrigerator compression efficiency (85\%),

- Ammonia production rate (1000 metric t/day nominal),

- Cooling water and boiler feedwater temperatures, $25^{\circ} \mathrm{C}-40^{\circ} \mathrm{C}$,

- The diffusion and hydrodynamics effects are neglected.

\subsection{Base-case and optimal operation conditions}

Table 9.4 summarizes the process variables defined for the base-case and those calculated for the optimal operation conditions, according to the optimization problem given in Table 9.3. It is worthy to notice that, for both base-case operation pressures, a higher temperature $\mathrm{T}_{1}$ (cf. Fig. 9.1) has been purposely selected, promoting so a counteraction effect that aims to reduce the local irreversibility in the highly exothermic ammonia reactor. Consequently, a lower temperature $\mathrm{T}_{3}$ is chosen, so that, as the ammonia conversion progresses, the reacting driving force in the last reactor bed is reduced.

Yet, it is also evident that, despite the fact that this approach effectively allows for a reduction of the share of exergy destruction in the reactor, the overall performance of the ammonia loop is not proportionately improved. Indeed, when the base-case operation figures are compared with the optimal ones, the power consumption is considerably increased in the former cases. As it will be explained along this chapter, the observed behavior follows a more complex relationship between the different components of the ammonia unit.

For instance, the power consumption in the refrigeration system of the base-case design operating at 150 bar (SP150) is 69\% higher than in the optimal design, whereas the circulator power consumption is fivefold the power consumed in the optimal case. This is a direct consequence of an increased recycle rate, which reduces the reactor conversion in about $40 \%$. For the base-case operating at 200 bar (SP200), the refrigeration power consumption is 
threefold the power demand of the optimal case, whereas the circulator power consumption sharply increases as the reactor conversion is reduced in about $79 \%$. Thus, due to the lower nitrogen conversions and larger circulation rates, the worst refrigeration figures occur in the base-case operating conditions. On the other hand, the cooling water consumption is reduced by increasing the loop pressure and reducing the recirculation flow.

For the optimal setups of the SP150 and SP200 configurations, the syngas compression consumes almost $61 \%$ and $75 \%$ of the total power supply, respectively, followed by the ammonia refrigeration cycle (36\% and 24\%) and the circulator. Contrarily, in the base-case setups, the power consumption of the refrigeration and syngas compression systems are comparable. It is important to notice that, the overall power consumption is similar for both optimal cases (approx. $13 \mathrm{MW}$ ). Moreover, lower amounts of ammonia and inerts are recycled to the reactor when operating under the optimal case of the SP200 configuration, which reduces the recycle molar flow rate, and thus the refrigeration and the circulator power consumption. However, since the power required for makeup syngas compression purposes in the optimal case of the SP200 configuration is $21.5 \%$ higher than in the SP150 counterpart, the latter case remains competitive in terms of both power consumption (only $3.1 \%$ higher) and economic revenues (vide Table 9.4).

As a result, the highest exergy consumption corresponds to the base-case of the SP200 configuration (21.9 MW), whereas base-case scenario operating at 150 bar consumes approx. 18.9 MW. Given the compressor and circulator efficiencies and the pressure drop throughout the loop, some authors reported that a pressure-independent power consumption is obtained when operating between 140-315 bar, with a flat minimum between 155 [95] and 220 bar [56]. However, those claims should be interpreted with care since they are actually dependent on assumed conditions about the catalyst activity and the feed temperatures of each bed. Those studies also seem to ignore the relevance of the steam production and its impact on the plantwide energy balance [56]. Thus, in the subsequent sections it will be shown that the selection of a non-optimal reaction temperature, pressure and feed composition might rather entail an increased power consumption and a higher loop inefficiency.

Table 9.4. Main process variables of the base-case and calculated optimal operating conditions for a 1,000 $t_{N H 3} / d a y$ industrial ammonia unit.

\begin{tabular}{lcccc}
\hline \multicolumn{1}{c}{ Process parameter } & \multicolumn{2}{c}{ 150 bar (SP150) } & \multicolumn{2}{c}{ 200 bar (SP200) } \\
\hline First bed gas preheating temperature, $\mathrm{T}_{1}\left({ }^{\circ} \mathrm{C}\right)$ & Base-case & Optimal & Base-case & Optimal \\
\hline Third bed inlet gas temperature, $\mathrm{T}_{3}\left({ }^{\circ} \mathrm{C}\right)$ & 450 & 365 & 410 & 310 \\
\hline Fresh syngas $\mathrm{H}_{2} / \mathrm{N}_{2}$ ratio & 340 & 400 & 340 & 380 \\
\hline Fresh syngas inerts content $(\%)$ & 2.97 & 2.99 & 2.83 & 2.94 \\
\hline Recycle ammonia content $(\%)$ & 1.25 & 1.27 & 1.10 & 1.22 \\
\hline Recycle inerts content $(\%)$ & 2.38 & 2.23 & 2.58 & 1.90 \\
\hline Recycled reactor feed $\mathrm{H}_{2} / \mathrm{N}_{2}$ ratio & 13.55 & 11.40 & 15.40 & 8.43 \\
\hline Recycle molar flow rate $(\mathrm{kmol} / \mathrm{h})$ & 2.50 & 2.89 & 0.65 & 2.39 \\
\hline Fresh syngas compression power $(\mathrm{kW})$ & 34,642 & 20,889 & 37,998 & 15,118 \\
\hline Refrigeration power consumption $(\mathrm{kW})$ & 8,140 & 8,140 & 9,890 & 9,890 \\
\hline COP Carnot & 8,267 & 4,883 & 8,996 & 3,104 \\
\hline
\end{tabular}




\begin{tabular}{|c|c|c|c|c|}
\hline \multirow[b]{2}{*}{ Process parameter } & \multicolumn{2}{|c|}{150 bar (SP150) } & \multicolumn{2}{|c|}{200 bar (SP200) } \\
\hline & Base-case & Optimal & Base-case & Optimal \\
\hline COP actual & 2.43 & 2.43 & 2.43 & 2.43 \\
\hline Circulator power consumption $(\mathrm{kW})$ & 2,475 & 489 & 3,048 & 108 \\
\hline Purge gas fraction $(\%)^{2}$ & 7.0 & 7.0 & 7.0 & 7.0 \\
\hline First bed conversion $(\%)$ & 10.9 & 17.5 & 5.1 & 20.7 \\
\hline Second bed conversion $(\%)$ & 2.3 & 6.0 & 0.8 & 6.5 \\
\hline Third bed conversion $(\%)$ & 2.4 & 5.8 & 1.0 & 7.2 \\
\hline Reactor conversion $(\%)$ & 15.0 & 24.9 & 6.7 & 31.2 \\
\hline Waste heat recovery rate $(\mathrm{kW})^{3}$ & 22,870 & 27,826 & 20,686 & 28,622 \\
\hline Cooling water - gas condensation $(\mathrm{kW})^{4}$ & 9,010 & 9,122 & 10,880 & 11,190 \\
\hline Cooling water - refrigeration cycle $(\mathrm{kW})^{4}$ & 25,920 & 15,310 & 28,200 & 9,728 \\
\hline Incomes $\left(\$ / t_{\mathrm{NH} 3}\right)^{5}$ & 642.1 & 649.4 & 626.1 & 649.2 \\
\hline Costs $\left(\$ / t_{\mathrm{NH} 3}\right)^{6}$ & 472.1 & 417.3 & 440.4 & 416.6 \\
\hline Revenues $\left(\$ / t_{\mathrm{NH} 3}\right)$ & 169.9 & 232.1 & 185.6 & 232.6 \\
\hline Annualized Bare Module Cost $\left(\$ / t_{\mathrm{NH} 3}\right)^{7}$ & 73.2 & 24.1 & 51.0 & 26.9 \\
\hline $\begin{array}{l}\text { 1. Condenser pressure: } 13.6 \text { bar, evaporator } \\
\text { constant fraction of the total molar flow of th } \\
\text { maximum outlet temperature: } 35-40^{\circ} \mathrm{C} ; 5 \text {. Amr } \\
\text { cost included; } 7 \text {. Interest rate } 6 \% \text {, lifespan } 20 \mathrm{y}\end{array}$ & $\begin{array}{l}.2 \mathrm{kPa} . \mathrm{Min} \\
(\text { makeup); } 3 \\
32 / \mathrm{GJ} ; 6 . \mathrm{Nat} \\
550(2010)[1\end{array}$ & $\begin{array}{l}\text { temperat } \\
\text { urated stea } \\
\text { gas cost: } \$ \\
\text { 29]. }\end{array}$ & pproach: 5- & $\begin{array}{l}\text {; } 2 . \text { As a } \\
\text { ling water } \\
\text { re module }\end{array}$ \\
\hline
\end{tabular}

As expected, according to Table 9.4, if the circulation rate is increased at constant loop pressure and ammonia yield rate, a lower reactor conversion is obtained, with a subsequent depart from the equilibrium and an increase the reaction rate [25]. Higher recycle flow rates are reported as advantageous when an increase of ammonia plant capacity is aimed to [270]. Despite of this, due to an increased circulation rate in the base-case scenarios, a higher amount of ammonia is recycled to the reactor, eventually hindering the nitrogen conversion. In the latter case, lower separation temperatures would be necessary so that smaller amounts of recycled ammonia in the reactor feed stream are obtained [95]. On the other hand, since the level of temperature of the waste heat recovery also decreases with a higher recycle rate, larger heat exchanger areas and piping sections are required to account for increased flows, and also a higher power consumption is needed to overcome these pressure drops.

As a final remark, it must be observed that the higher the space velocity, the larger the separation vessels required for appropriate residence times, which increases the capital costs. Furthermore, due to the increased utilities consumption, lower revenues are achieved when operating under the base-case conditions. In conclusion, even if the base-case designs are in agreement with the counteraction principle applied to the reactor in isolation, the overall loop performance is not solely defined by the reactor performance.

\subsection{Reaction kinetics in the intercooled, multiple bed catalytic reactor}

Figures 9.2(a-d) graphically represent the relationship between the kinetics parameters (i.e., feed temperature, conversion and reaction rate) and the intercooling operating conditions. The kinetics properties correspond to the Montecatini catalyst (cf. Chapter 5). The contours of constant reaction rate, as well as the equilibrium and adiabatic operation lines have been determined for each one of the operating conditions reported in Table 9.4. 
It is important to notice that, even a slight deviation from the stoichiometric $\mathrm{H}_{2} / \mathrm{N}_{2}$ ratio in the makeup syngas fed to the ammonia loop may evolve into an appreciably different $\mathrm{H}_{2} / \mathrm{N}_{2}$ ratio in the converter feed stream (see Table 9.4) [65]. Such an extreme scenario is appreciated in the base-case case shown in Fig.9.2(c), in which the low reactor conversion and large recycle rate are responsible for a striking reduction of the calculated $\mathrm{H}_{2} / \mathrm{N}_{2}$ ratio of the recycled reactor feed $(\sim 1)$. Even though these extremely low $\mathrm{H}_{2} / \mathrm{N}_{2}$ operation ratios may not immediately represent practical applications, they are interesting for analyzing the effect of the variation of some loop parameters (particularly the reactor feed temperature) on the feasible window of the operating conditions. Some studies [25] reported a marked dependence of the reaction rate on the $\mathrm{H}_{2}$ to $\mathrm{N}_{2}$ ratio. For instance, the maximum reaction rates reportedly occur at lower $\mathrm{H}_{2}$ to $\mathrm{N}_{2}$ ratios when the variation with the temperature is found to be almost flat. However, at higher $\mathrm{H}_{2}$ to $\mathrm{N}_{2}$ ratios, e.g. close to $3: 1$, a sharp drop in the reaction rate is observed as the temperature falls. This is commonly attributed to a hindered condition of the hydrogen absorption at lower temperatures [25].

Furthermore, according to Fig. 9.2(a and c), as long as the departure from equilibrium and, thus, the reaction rate, are increased, the available enthalpy of reaction and, consequently, the temperature of the reactor effluent, are reduced. This drastically affects the heat recovery performance as well as the conversion in the second reactor bed. In this way, the base-case SP200 configuration represents a thermodynamically and kinetically feasible scenario, but also one in which the minimum ignition temperature of the autothermal reactor is barely achieved.

Some important conclusions drawn from the performance of the optimal setups point to the fact that the decision-making on the optimum reactor inlet and outlet temperatures is a tradeoff between the approach to and the departure from the equilibrium line [41]. Furthermore, even though the reacting driving force is readily modifiable by intercooling the reactor beds, the expected outcome of applying the Counteraction principle to the reactor in isolation is not necessarily equivalent to that in which the reactor is embedded in a broader, more complex thermal systems.

As it has been shown, the increase of the reacting driving forces in the ammonia converter does not always lead to an increased irreversibility of the whole system, since the global effect of the finite driving forces in that equipment may be compensated by the enhancement of the performance of the integrated chemical unit (enhanced power consumption, cooling duty, recycle composition, equipment size, etc.). Conversely, optimal operation conditions for the ammonia loop as a whole do not necessarily correspond to an optimal operation condition of the ammonia reactor alone [13, 41, 146, 147]. This is a characteristic of highly non-linear chemical systems where some changes that are individually detrimental may lead to improved performances when overlapped [272].

For instance, Sauar et al. [269] applied the principle of equipartition of forces that promots close to uniform driving forces along the reactor. The authors aimed to demonstrate the relevane of the evaluation of the isoforce operating lines for determining the trade-off between the augmentation of the ammonia throughput and the reduction of the entropy 
generation in the reactor. The procedure attempted to minimize the rate of entropy generation for a constant ammonia yield. The authors demonstrated that the optimum operation line (minimum entropy generation rate) lies between the line for the equilibrium and the line of maximum conversions for each reaction rate. They also suggested that, by operating the reactor at 0.95 times the maximum reaction rate, the entropy production rate could be reduced by $31 \%$. A smaller reaction rate means that, nevertheless, the reactor should be increased to maintain the ammonia yield.

Fig. 9.2. Conversion vs. Temperature. (a) Base-case 150 bar, (b) Optimal case 150 bar, (c) Base-case 200 bar, (d) Optimal case 200 bar. Reaction rates are given in kmol/ $\mathrm{m}^{3}-h$.
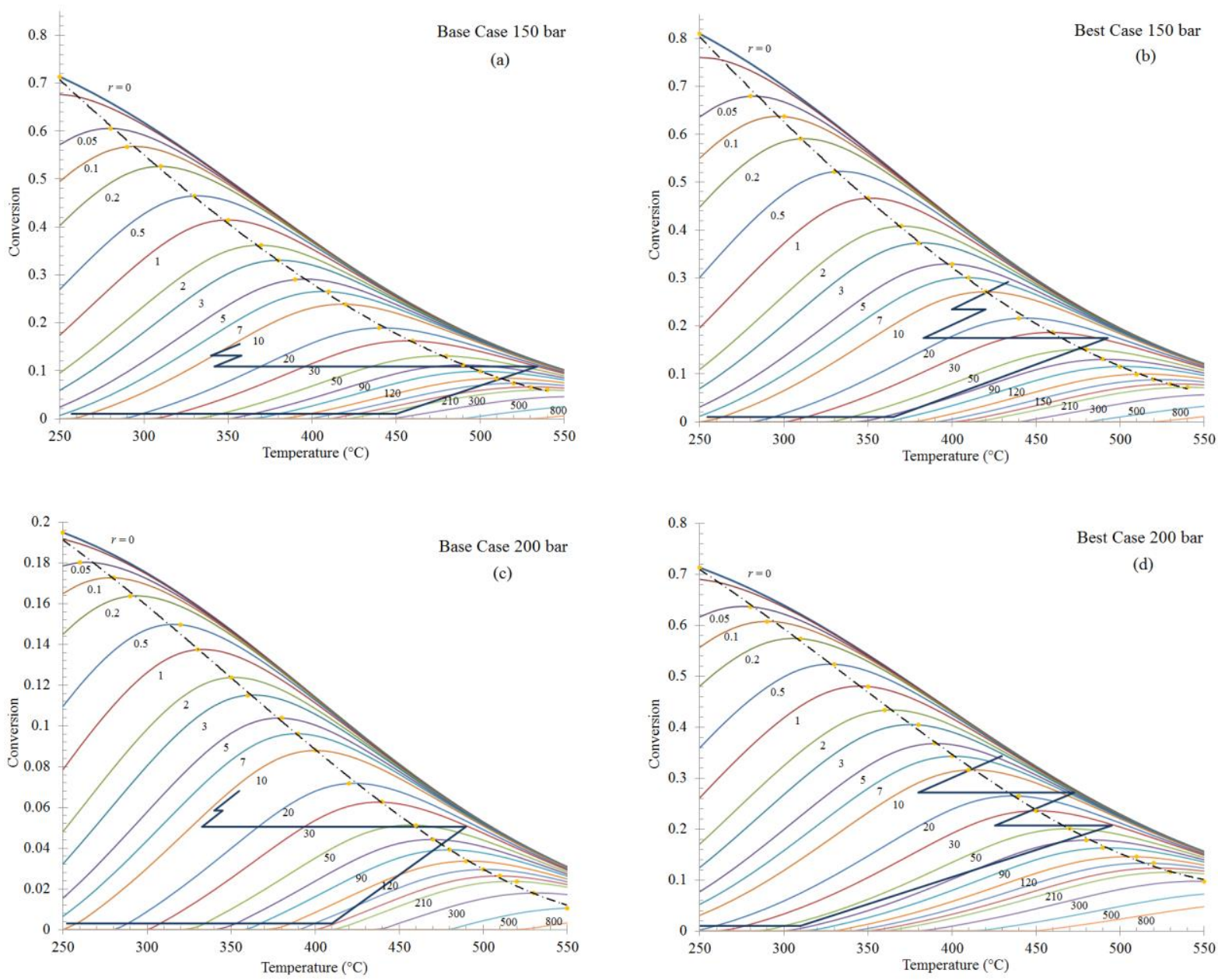

Source: Author.

9.5.Exergy analysis of the base-case and optimized ammonia loop configurations.

In this section, the exergy performance and some suitable alternatives for improving the exergy efficiency are discussed. Figures 9.3 and 9.4 show the exergy destruction rates and the exergy destruction breakdown, respectively, for the most representative components of the ammonia loop.

Even though the exergy associated to the unreacted feed or the inerts flows typically constitutes transit exergy in the reactor beds [177], the magnitude of those exergy flows 
fundamentally affects the reactor and, consequently, the performance of the entire loop. In fact, due to the large recycle rates and lower per-pass conversions, the exergy destruction in the ammonia reactor is appreciably higher than in other industrial exothermic reactors [55, 186]. Fortunately, although most of the exergy destruction in the chemical process is unavoidable, some exergy losses can still be reduced by reducing the internal irreversibility associated to the heat transfer (e.g. by preheating the reactants) [151] or by introducing configurations running on dual levels of pressure.

In fact, it is important to highlight that an increase of the reactor pressure entails a reduction in the power consumption of the circulator and the refrigeration systems, but only at expense of a higher exergy consumed in the makeup syngas compressor. Thus, aiming to reduce the syngas compression while maintaining the ammonia yield, dual pressure ammonia loop configurations (e.g. Udhe ${ }^{\circledR}$ dual process [221, 273]) have been lately introduced. This setups start at a lower operation pressure, only to proceed the conversion process at a subsequent elevated pressure. This case is analyzed in more detail in the next chapter. Certainly, nonconventional approaches will be worthy to explore considering the current limited room for improvement in the reactive components. In fact, the highest exergy savings are expected from the waste heat recovery and the syngas compression and refrigeration systems [274].

Fig. 9.3. Exergy destruction of representative components of the ammonia loop.

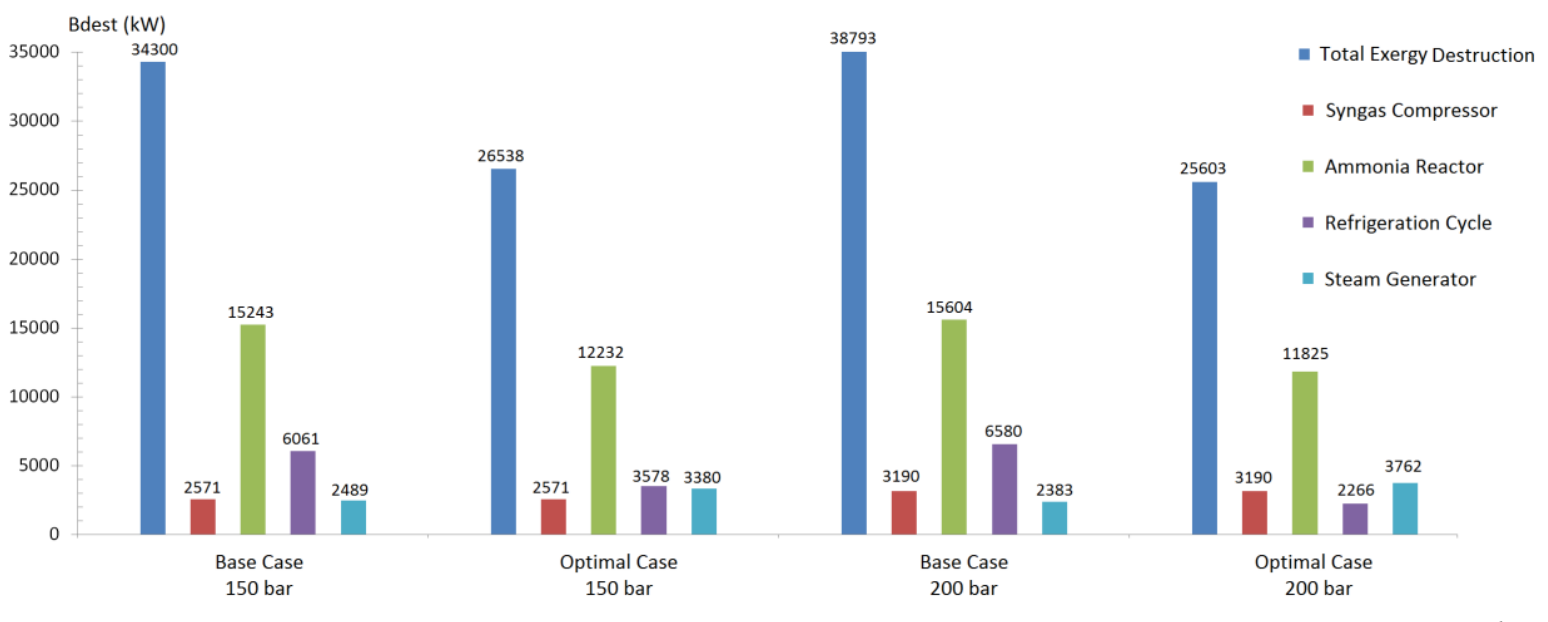

Source: Author.

According to Fig. 9.4, the exergy destruction share of the reactor is effectively reduced when a counteraction is applied in order to reduce the reactive driving force. However, a local reduction of the exergy destruction seems to be less important when the optimal operating conditions of the reactor are rather dictated by its interaction with the remaining energy systems. Meanwhile, Fig. 9.5 shows the overall exergy efficiency of the overall ammonia loop as defined by Eqs. (9.1-9.4). Higher rational exergy efficiencies are less sensitive to the variation of the process parameters. On the other hand, both transit and recovery definitions seem to better reflect the true nature of the energy intensive ammonia loop, inasmuch as they only take into account the variation of the loop parameters in the evaluation of the process performance. 
Fig. 9.4. Exergy destruction breakdown for representative components of the ammonia loop.

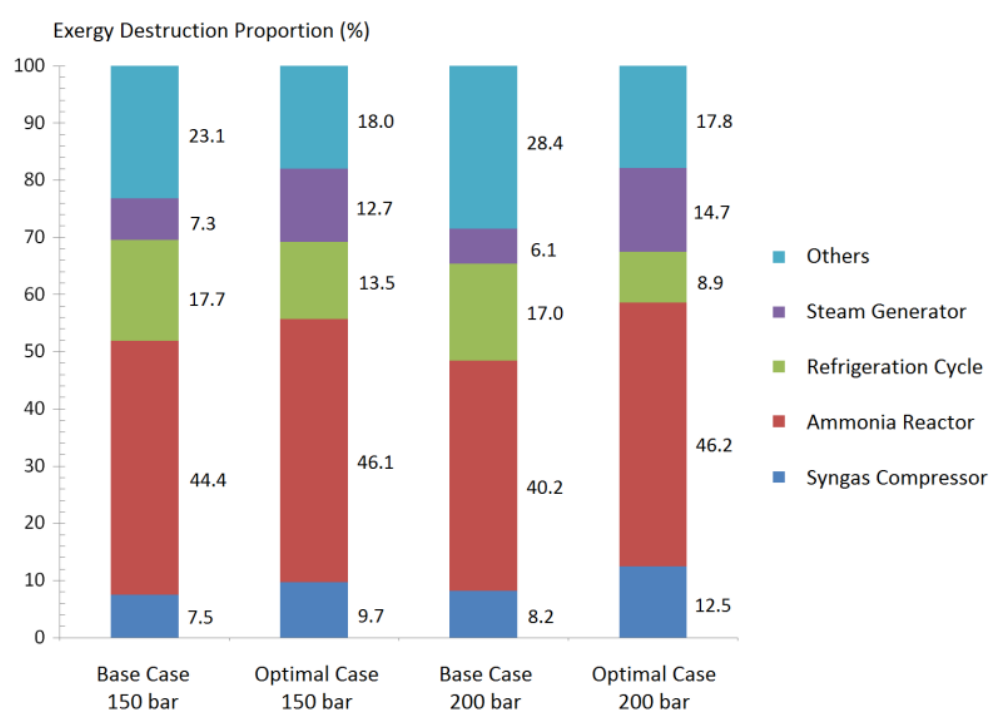

Source: Author.

Fig. 9.5. Plantwide exergy efficiencies of the base-case and optimal scenarios.

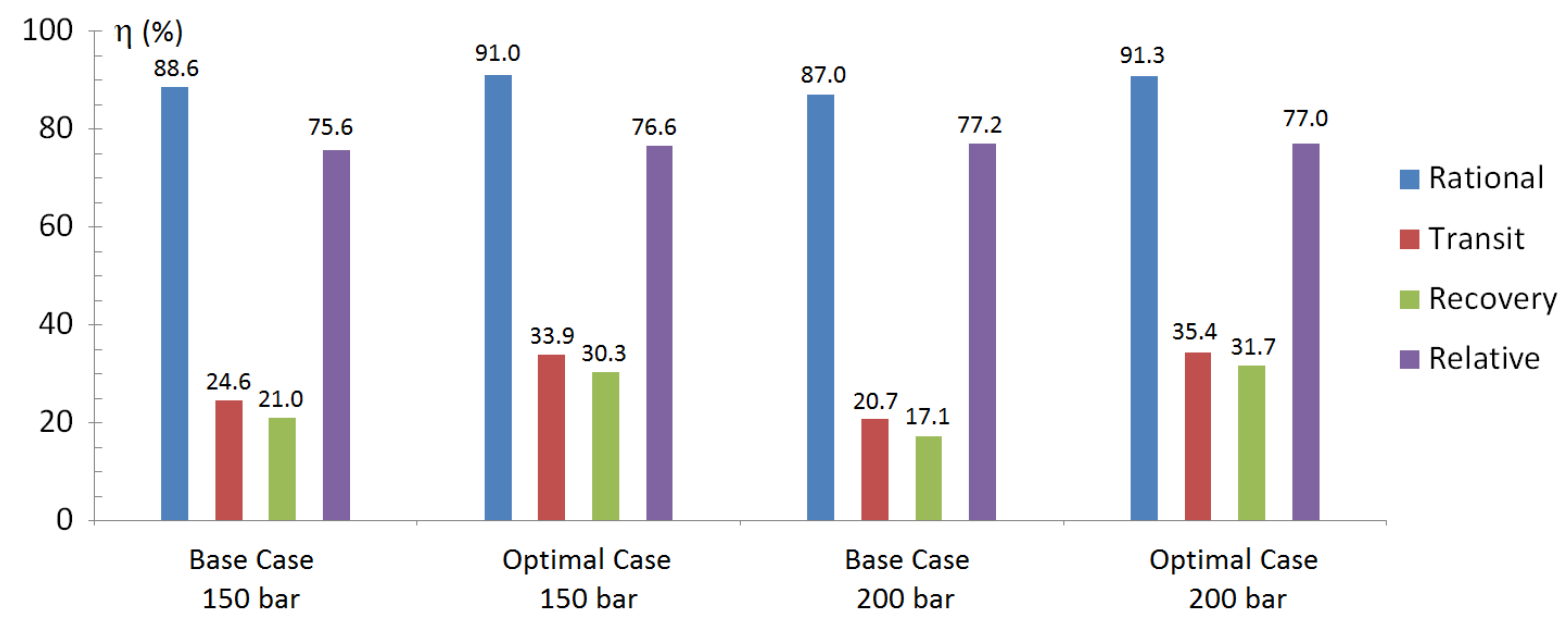

Source: Author.

The transit efficiency definition is nonetheless slightly higher than the recovery efficiency since the former assumes that all the non-reacted nitrogen and hydrogen is recycled back to the ammonia converter, and only liquid pure ammonia, methane and argon exit the loop. In other words, neither the purged offgas nor the low pressure hydrogen-rich stream are considered as products. Besides, the term $B_{B F W}^{P H}$ in Eq.(9.2) is considered as an exergy input, and not as transit exergy in the heat recovery steam generator. Actually, the numerator in Eq.(9.2) has been originally proposed in terms of the electricity generated in an additional energy conversion process, e.g. an associated Rankine cycle [19]. However, in the present work, the boundaries of the system studied are restricted to the steam generation process. The reason is that the recovery of the reaction enthalpy in the form of steam is actually linked to a more complicated combined steam and power generation system in the integrated syngas and ammonia production plant. Furthermore, fixed the available header pressure levels in the steam network system, there is not necessarily a direct relation between the power cycle 
performance and the ammonia loop parameters, since the steam system should be able to compensate the deficit in the steam network. Accordingly, the loop performance and the steam generation potential are suitably estimated by the recovery efficiency definition. This definition attempts to determine the opportunity to ideally recover all the dissipated heat in the form of a valuable ammonia unit byproduct, namely, high pressure steam.

On the other hand, the relative efficiency has been defined by using the minimum theoretical exergy consumption required to produce ammonia from the elements in the environment. As such, the relative efficiency accounts for the maximum potential of energy savings, including the upstream production processes of nitrogen and hydrogen. But, despite its broader scope and improved sensitivity compared to the abnormally higher rational efficiency, this indicator still copes with the reduced sensitiveness of other efficiency definitions used for evaluating the performance of the bulk chemicals production processes with large material flow rates. Finally, Figs.(9.6-9.7) depict the exergy efficiencies of representative components of the basecase and optimized ammonia loop setups for each pressure studied.

Fig. 9.6. Exergy efficiencies for representative components of the ammonia loop (150 bar).

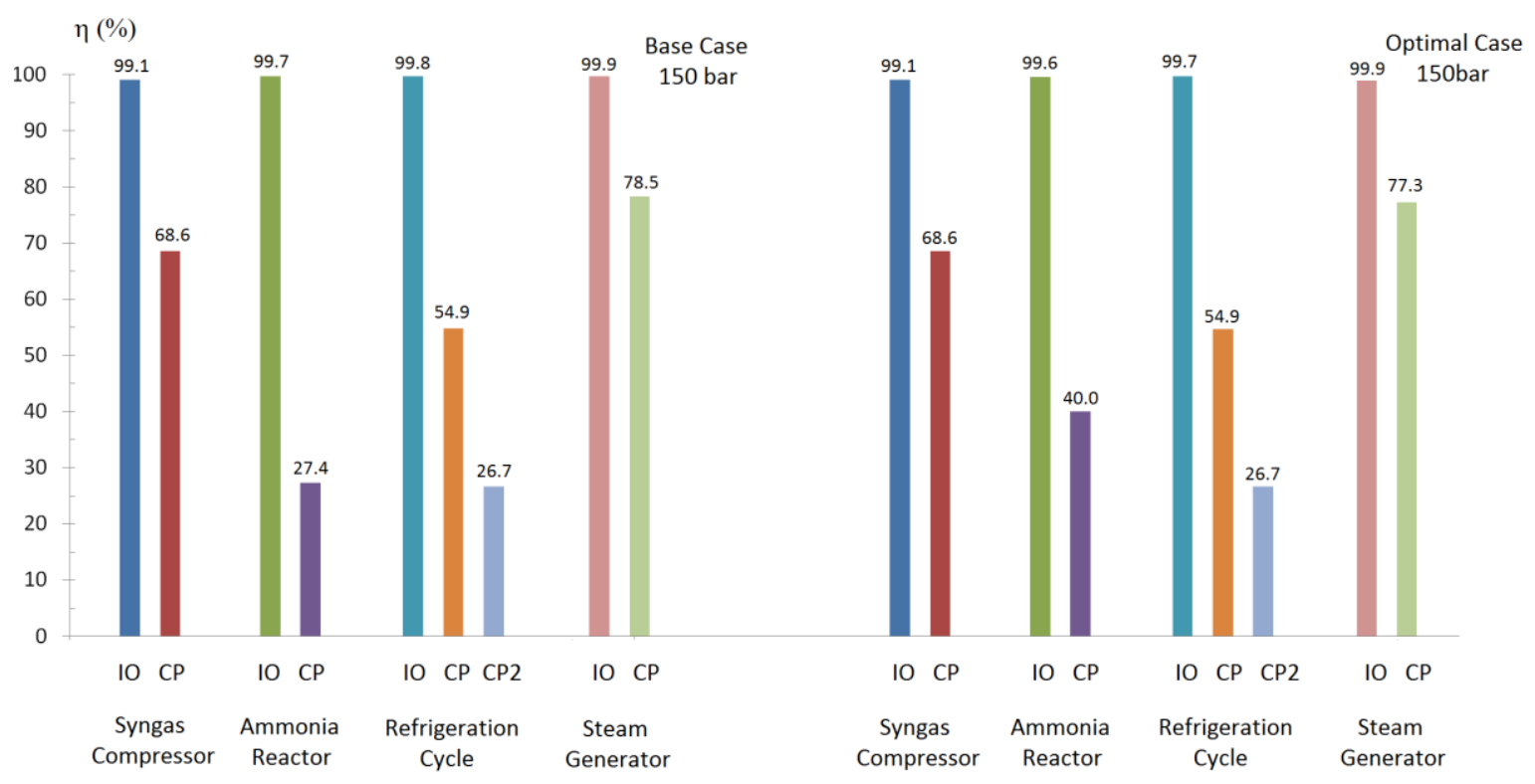

Source: Author.

It is interesting to remark the difference in the results obtained from the various exergy efficiency definitions for the refrigeration cycle. Since the consumed produced efficiency CP2 includes the exergy destroyed in the control volume of the evaporator, the exergy efficiency is appreciable lower than in the case in which only the exergy of the heat transferred at $-30^{\circ} \mathrm{C}$ is considered. Moreover, it is not surprising that the consumed-produced exergy efficiencies in the refrigeration cycle are similar in all the cases, considering that the isentropic efficiency of the refrigeration compressors is assumed equal, and the compression ratios were selected to reduce the refrigeration power consumption. Regarding the consumed-produced efficiency of the ammonia reactor for the base-case scenarios, these value are at least $40 \%$ lower compared to the optimized cases. 
Fig. 9.7. Exergy efficiencies for representative components of the ammonia loop (200 bar).

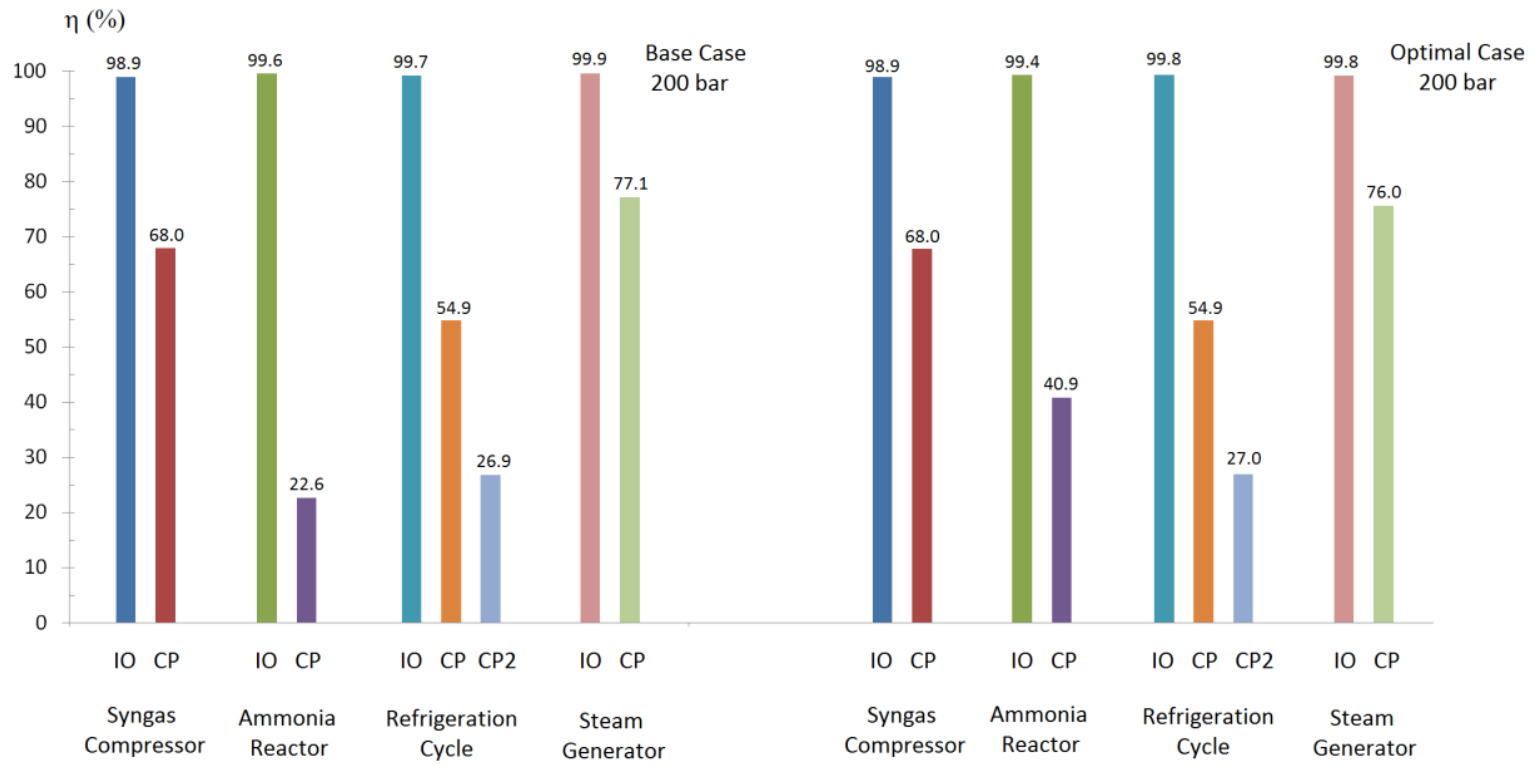

Source: Author.

Definitely, the experimental validation of the different configurations studied would be prohibitively expensive, and for this reason, some figures reported on the exergy efficiency and irreversibility are thoroughly discussed. Nielsen et al. [95] reported a significant influence of the variation of temperature of the cooling water on the power consumption of the makeup syngas compression system, but most critically of the ammonia refrigeration system. In this study, a typical 1,000 t/day ammonia synthesis unit operating at 140 bar, with a cooling water temperature of $30^{\circ} \mathrm{C}$ and ammonia product at $-33^{\circ} \mathrm{C}$ has been considered. For the simulated case, the maximum exergy loss in the ammonia loop attained 26.7-29.0 MW, of which 67.2\% are attributed to the ammonia synthesis and separation, and the remaining, to the compressor and other ancillary systems. Those values are in agreement with the irreversibility calculated in the present work (25-40 MW), which has been also found to be highly dependant on the utility consumption and the operation parameters, especially the loop pressure.

Sorin and Brodyansky [275] presented a thermodynamic optimization method that allows to target and reduce the transformed exergy input of the subsystems in the ammonia production unit. Those subsystems include the syngas compression, the chemical reactor, the separation of the loop products and the recycling of non-reactive components. The analysis also involves the combustion of the purge gas. Since the synthesis loop operates at 270 bar, the authors analyzed the advantages of reducing the pressure and the suitability of increasing the ammonia separation by providing a lower temperature cooling utility. It has been found that, by decreasing the system pressure from 270 bar to 150 bar, the efficiency of the original system $(74.3 \%)$ decreases only $2.3 \%$. The calculated efficiency is in close agreement with the relative definition of the exergy efficiency presented in this thesis.

Penkuhn et al. [274] compared the exergy efficiency of two ammonia loop configurations (1,600 t/day). The first configuration consists of a three stage adiabatic reactor (200 bar) with direct intercooling (quenching) and the second configuration consists of an intercooled reactor 
(140 bar) that uses Dowtherm A as coolant. The exergy efficiencies reported $(90.78 \%$ for the cold-shot cooled and $96.39 \%$ for the indirect cooled case) approach those calculated by the rational exergy efficiency, whereas the exergy destroyed is significantly different in both designs (39.85 MW for the cold-shot cooled and 15.18MW for the indirect cooled case). As it has been shown along this chapter, those results are seemingly a consequence of the careless calculation of the exergy efficiency in large-volume chemicals production systems. In other words, the imbalance between the material and exergy flow rates is likely to be ignored. The authors concluded that the quenching process results in a higher exergy destruction and a lower overall exergy efficiency. Moreover, the increase of the heat transfer coefficient reveals a slight improvement potential, in spite of the virtually unavoidable nature of the exergy destroyed in the reaction system.

A comparative analysis between a conventional ammonia unit (refrigeration only) and two alternative ammonia loops with additional recycled ammonia removal technologies have been studied by the Department of Energy of the United States [276]. One alternative includes a water stripping process whereas the other uses pressure swing adsorption (PSA). The total exergy losses for a 1,000 metric ton per day plant range from 39.2 MW (PSA-based) up to 41.0 MW (water scrubbing-based), with the conventional loop attaining 39.8 MW. According to the authors, the high solubility of ammonia in water renders the water stripping ammonia removal more energy intensive and less attractive, unless a fluid with less ammonia solubility is used. A more recent assessment [277] also uses water stripping for residual ammonia removal. The exergy destroyed in the ammonia loop (250 bar), calculated as $35.5 \mathrm{MW}$, represents almost one-third of the total exergy destroyed in the integrated syngas and ammonia production plant. The reduction of the inerts content and lower reactor outlet temperatures are envisaged as potential modifications that may drive losses down. Modified column designs could be helpful in reducing the amount of exergy destroyed (9.64 MW) in the residual ammonia removal system. Again, those figures are in close agreement with the irreversibility calculated in the present work (max. $40 \mathrm{MW}$ ). Furthermore, in the next chapter of this thesis, it will be shown how the integration of a cryogenic purge gas treatment unit may help increasing the ammonia loop efficiency by recycling a significant fraction of the hydrogen recovered at high pressure back to the synthesis loop.

Radgen and Lucas [278] performed a thermodynamic analysis of a fertilizer complex based on the combined pinch analysis and exergy analysis. According to the authors, although the pinch analysis is more straightforward as a methodology to predict the effect of the variation of a process parameters elsewhere in the ammonia loop, the exergy analysis allows for a more general insight of the problem, not only limiting the improvement potentials to the energy integration. Withal, the exergy analysis demands a heavier effort as it requires explicitly the entropy function of the streams. As a result, for a 1,000 t/day ammonia loop, the total exergy destruction rate is calculated as $30.9 \mathrm{MW}$ out of which $82.7 \%$ are only due to the syngas compression, the steam production and the ammonia synthesis reactor. Other irreversibility sources are attributed to the ammonia removal and refrigeration, and the purge gas purification systems. Other studies [19] analyzed the effect of the degree of conversion, the approach to equilibrium and the inert content $\left(\mathrm{mol} 7 \% \mathrm{CH}_{4}, 3 \% \mathrm{Ar}\right)$ on the irreversibility of an 
ammonia loop operating at much higher pressure (300 bar). The exergy destruction reported is as low as 18.06-18.95 MW for a loop with a single bed reactor, provided that no inerts are present in the reactor feed. A case study showed that the introduction of $10 \%$ of inerts on a 1,000 tons per day plant causes a marked increment $(45.5 \%)$ on the exergy destruction rate, which is worsened if a poor degree of conversion and relatively high circulation ratio $(5.7 \mathrm{~kg}$ gas/ $\mathrm{kg} \mathrm{NH}_{3}$ ) are considered. Certainly, the latter scenario is more likely to occur as an appreciable amount of inerts are normally recycled back to the converter together with the unseparated ammonia produced and the unreacted syngas. In that case, the exergy destruction figures achive values similar to those found in the previous analyses (>25 MW).

Accordingly, the mentioned research works prove to be relevant in order to estimate the opportunities of improvement in the ammonia loop, when compared with the results obtained in this work. It is nevertheless important to point out that, unlike the analysis performed in this chapter, most of these works do not consider the effect of the variation of other loop parameters such as bed intercooling, feed composition and loop pressure, and some of them were performed on the basis of energy data and assumed Carnot factors for specific temperature levels.

In summary, the ammonia unit performance is found to be fundamentally determined by the reactor parameters, followed by the purge and recycle rates and the refrigeration duty. For this reason, in the case that the operating conditions of those systems abruptly change, the other parameters are expected to change so that the feasible steady state is achieved again [95]. Thus, the chemistry and thermodynamics of the process define a window of possible operating conditions at which the ammonia synthesis is possible, and out of which no convergence of mass, energy and exergy balances could be obtained [95]. However, as the number of independent variables increases in that window, the computational effort for exploring the search space increases substantially, particularly for the reactor-separatorrecycle-purge schemes, because of their intricate non-linear characteristics [279].

Therefore, in order to determine a feasible dominion for the optimization problem, the effect of the most important parameters on the loop performance has been evaluated through a series of case studies. The most interesting of such case studies is the determination of the exergy destruction rate as a function of the inlet temperatures $T_{1}$ and $T_{3}$ of the catalytic bed for different fresh syngas $\mathrm{H}_{2} / \mathrm{N}_{2}$ ratios (Figs. 9.8-9.9). These plots have been used to explore the boundaries of the feasible region where the studied parameters satisfy the imposed restrictions, so they could be understood as feasible maps of the specific industrial ammonia production processes studied. It is important to point out that, differently from the SP150 case for which the maps exhibit a similar behavior, the case studies shown for SP200 are highly dependent on the $\mathrm{H}_{2} / \mathrm{N}_{2}$ ratio. Actually, a marked increase of the exergy destruction is observed for the cases in which the fresh syngas $\mathrm{H}_{2} / \mathrm{N}_{2}$ ratio is about $2.832\left(\mathrm{H}_{2} / \mathrm{N}_{2} \sim 1\right.$ in the reactor feed stream). Thus, despite the fact that the plots shown in Figs. 9.8-9.9 correspond to mathematically feasible solutions of the optimization problem, they must be interpreted with care. Those results are highly dependent on the model developed as well as on the correlations used for the thermophysical properties and vapor-liquid equilibrium (VLE) calculations, 
among other modeling limitations. At last, when dealing with exothermic autothermal reactors that present instability points (near blow off operation states), the convergence of the overall process simulation is hindered, which renders the process synthesis and optimization a challenging task. 
Fig. 9.8. Exergy destruction in the ammonia loop as a function of the inlet temperatures $T_{1}$ and $T_{3}$ of the catalytic bed (Loop pressure: 150 bar).
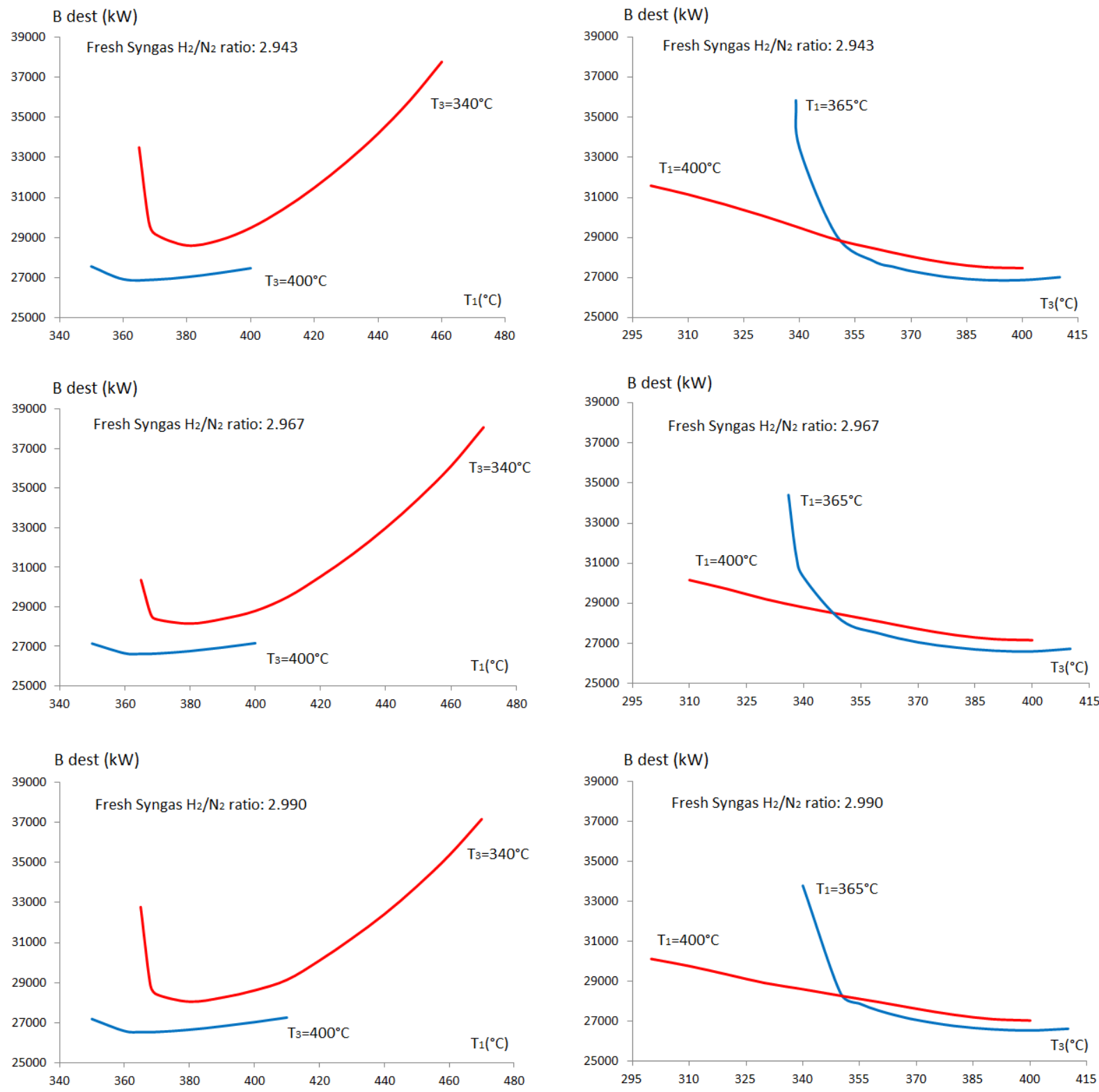

Source: Author. 
Fig. 9.9. Exergy destruction in the ammonia loop as a function of the inlet temperatures $T_{1}$ and $T_{3}$ of the catalytic bed (Loop pressure: 200 bar).
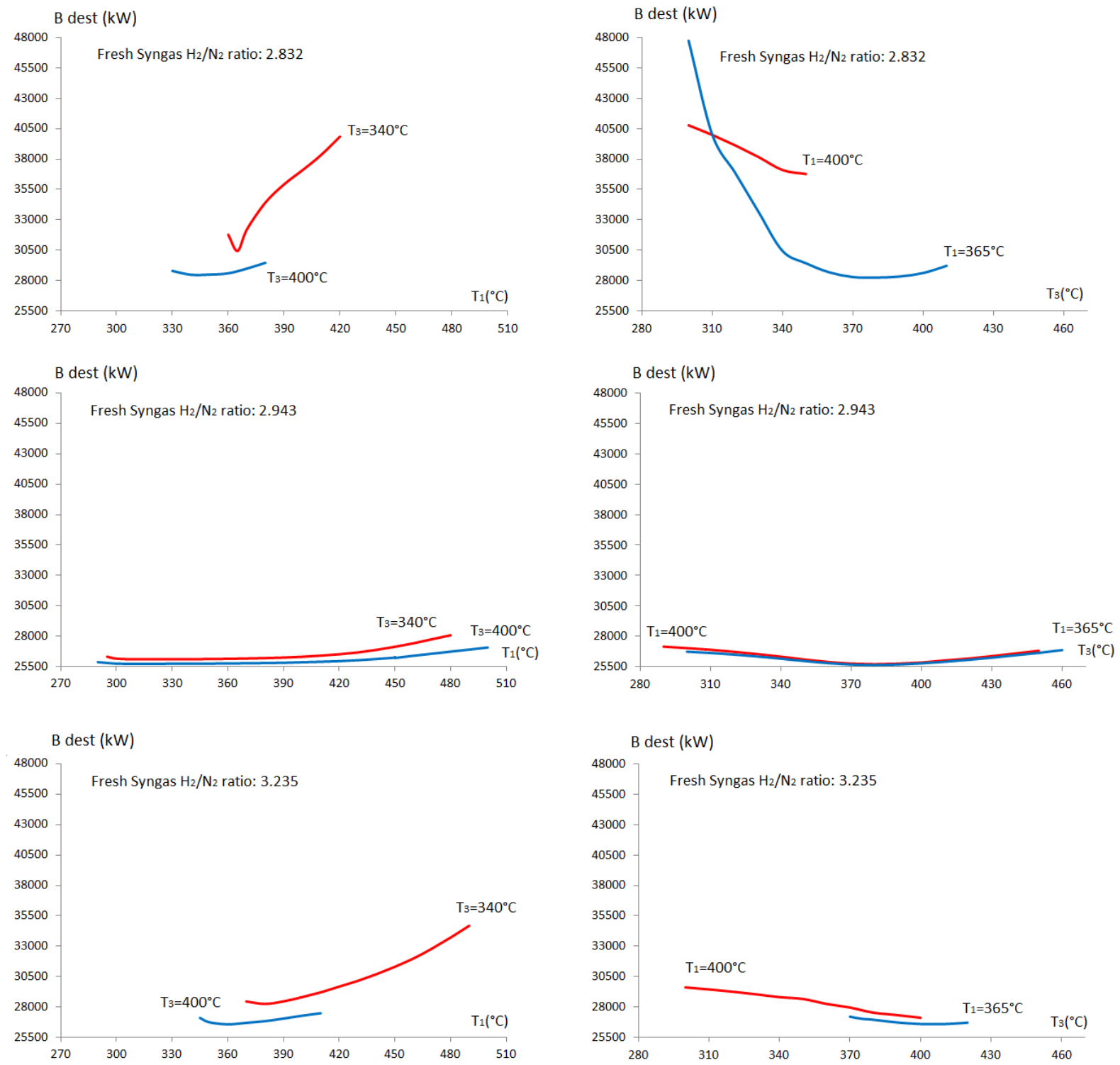

Source: Author. 


\subsection{Final considerations}

The interrelation between the kinetic, thermodynamic and technical operation parameters of the industrial ammonia synthesis unit has been examined by using both the Counteraction and the Le Châtelier Principle. Two different suitable approaches have been proposed in order to calculate the exergy efficiency of large volume chemicals production, as in the case of the ammonia production process. In fact, that kind of systems struggles to fit into an holistic and convenient exergy efficiency definition due to the dominance of the exergy of the material streams compared to the exergy associated to the power and heat consumption. Furthermore, as long as the chemical exergy is internally converted into other forms of exergy and exported in the form of steam, purge gas and ammonia product, the application of the transit exergy concept becomes inapplicable.

On the other hand, even at optimal operating conditions, the ammonia synthesis reactor, the syngas compression, the ammonia refrigeration and the waste heat recovery system are together responsible for about $26 \mathrm{MW}$ of exergy destruction, with the largest figures corresponding to the base case condition at 200 bar $(38.8 \mathrm{MW})$. The exergy destruction breakdown shows that the ammonia converter and the refrigeration system are together responsible for more than $71-82 \%$ of the overall irreversibility in the ammonia loop. Better catalysts with higher activities and higher reaction rates at lower pressures, and enhanced converter designs (dual pressure systems) along with an improved waste heat recovery system with more efficient refrigeration processes must be pursued if higher yield and lower exergy destruction rates are aimed.

Moreover, it has been found that, although an increase in the driving forces may imply higher exergy destruction rates according to the Counteraction Principle, by solely increasing the reacting driving forces in the ammonia converter, the whole system irreversibility is not necessarily increased. This is explained by the fact that the global effect of the finite driving forces in that equipment may be compensated by the enhancement of the performance of the integrated chemical unit. Thus, the minimization of the large amount of exergy consumed in the industrial ammonia units is rather a trade-off between lower exergy destruction rates and higher ammonia yields. Finally, a series of case studies, that prove to be useful to figure out the feasible dominion of the problem of the minimization of the exergy destruction, are also presented.

In the next chapter, the application of the Counteraction principle to the decreasing-volume ammonia synthesis reaction aims to reduce the overall irreversibility in the industrial ammonia production unit. To this end, the makeup syngas compression, the reactants conversion and the ammonia refrigeration and separation are performed at incremental levels of pressure, which in turn reduces the utilities consumption and enhance the hydrogen recovery from the purge gas. 


\section{SINGLE AND DUAL PRESSURE AMMONIA PRODUCTION UNITS: THE COUNTERACTION PRINCIPLE}

Higher per-pass conversions can be achieved by withdrawing the enthalpy of reaction of the ammonia synthesis before the reactants have attained the equilibrium. Notwithstanding, any increase of the reactive driving force $(-\Delta G)$ and, thus, the reactor conversion, also increases the irreversibility in that component. On the other hand, the reactor yield cannot be arbitrarily increased by solely increasing the reacting driving force, since the loop capacity may eventually come up against its practical limits. Accordingly, an alternative way of increasing the process capacity consists of producing an additional amount of ammonia outside the main synthesis loop [221, 273].

For instance, the installation of a pre-converter, i.e., an upstream, once-through ammonia reactor in series with the main ammonia loop, has been already proposed [95]. Analogously, a post-converter, i.e., a downstream purge gas converter, has been also suggested for revamping a 25 years old ammonia plant, but at the expense of a high capital cost [24]. However, since in both alternatives the sequential reaction stages operated at the same level of pressure, the potentials related to the reduction of the process irreversibility and the makeup syngas power consumption remained underexploited.

Meanwhile, in the Uhde Dual Pressure Process ${ }^{T M}$ [221, 273], the total ammonia production is split into an upstream, once-through conversion process at low pressure and other more compact, conventional synthesis loop at high pressure. According to some authors, the dual pressure system has been conceived to take advance of the economy of scale, since the potential reduction of the investment cost is about $20 \%$ by doubling the size of a single pressure train [41]. More recently, Lippman et al. [273] reported that, based on the same depreciation rate, feedstock and manufacturing costs, the specific ammonia production cost is $11 \%$ lower in a $3300 \mathrm{t}_{\mathrm{NH} 3}$ /day plant integrating the Dual Pressure System if compared with a conventional $2000 \mathrm{t}_{\mathrm{NH}_{3}}$ /day plant. Furthermore, by using a cryogenic purge gas treatment unit, a increase of $5 \%$ in the capacity of the ammonia unit has been reported (or the equivalent of feedstock economy) $[41,70]$.

Thus, in this chapter, the introduction of an additional catalytic once-through converter into an industrial ammonia synthesis unit is simulated. Exergy is used to quantify the efficiency and minimize the irreversibility generated in the various components of the ammonia synthesis loop, while looking for attractive economic revenues, as a function of the most critical operation parameters. Three unit configurations are analyzed: two are based on single pressure loops (SP150, SP200), whereas the other (DP) operates at two incremental levels of pressure (83/200bar). The dual pressure process aims to spotlight the relevance of the Counteraction Principle for driving the system irreversibility down and offset the unfavorable 
effects of the bed intercooling, considering the decreasing-volume equilibrium of the reactive mixture. Finally, the performance of an ammonia loop integrated to a cryogenic hydrogen recovery unit is compared with other unit that spares the purge gas treatment process.

\subsection{Description of the single and dual pressure ammonia synthesis units.}

Figures 10.1 and 10.2 show the flowsheets of the two types industrial ammonia unit analyzed. In the first configuration (Fig. 10.1), the ammonia synthesis is entirely achieved in a single pressure loop (cf. Ch. 9), where the unconverted mixture is recycled back to the reactor composed of three sequential catalytic beds. Meanwhile, in the dual pressure unit depicted in Fig. 10.2, the ammonia production occurs in two sequential conversion sections with incremental levels of pressure. In the first section, the fresh syngas is compressed up to 83 bar in an intercooled compressor. Next, the syngas is partially converted in a multiple bed oncethrough reactor, where about $18 \%$ of the overall amount of ammonia is produced. Then, $60 \%$ of the ammonia is condensed and separated out from the gas, whereas the remaining syngas is further compressed up to 200 bar and fed to the high pressure synthesis loop [41]. One benefit of the early removal of ammonia is that, after the first ammonia conversion stage, the volume of the gas handled is reduced and, consequently, the amount of circulated gas is also reduced. Besides, as it will be discussed in more detail later, by gradually increasing the pressure of a decreasing-volume reaction, the synthesis of ammonia can be performed in a more efficient way [69].

Fig. 10.1. Single pressure ammonia synthesis flowsheet, cf. Table 10.1 and 10.2 for stream properties.

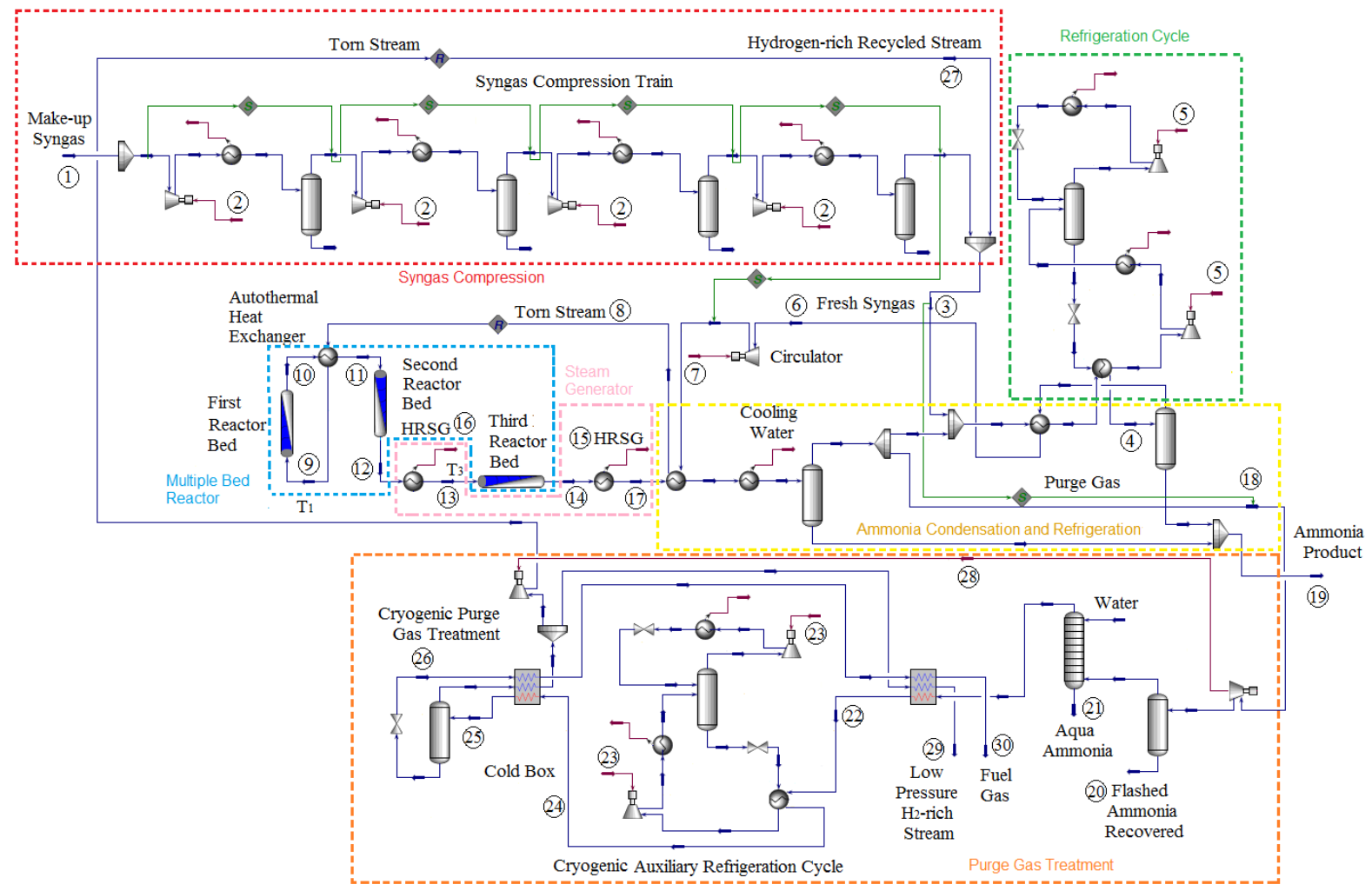

Source: [226]. 
Again, in both configurations, the loop efficiency is affected by the reactor pressure, the feed temperature and composition, the amount of inerts and recycled ammonia, as well as the heat removal and the catalyst design [280]. The build-up of inerts (argon, methane) in the loop is controlled by a continuous withdrawal of a portion of the hydrogen-rich recycle gas to keep the concentrations down to acceptable levels [70].

Fig. 10.2. Dual Pressure Ammonia Synthesis flowsheet, cf. Table 10.3 for stream properties.

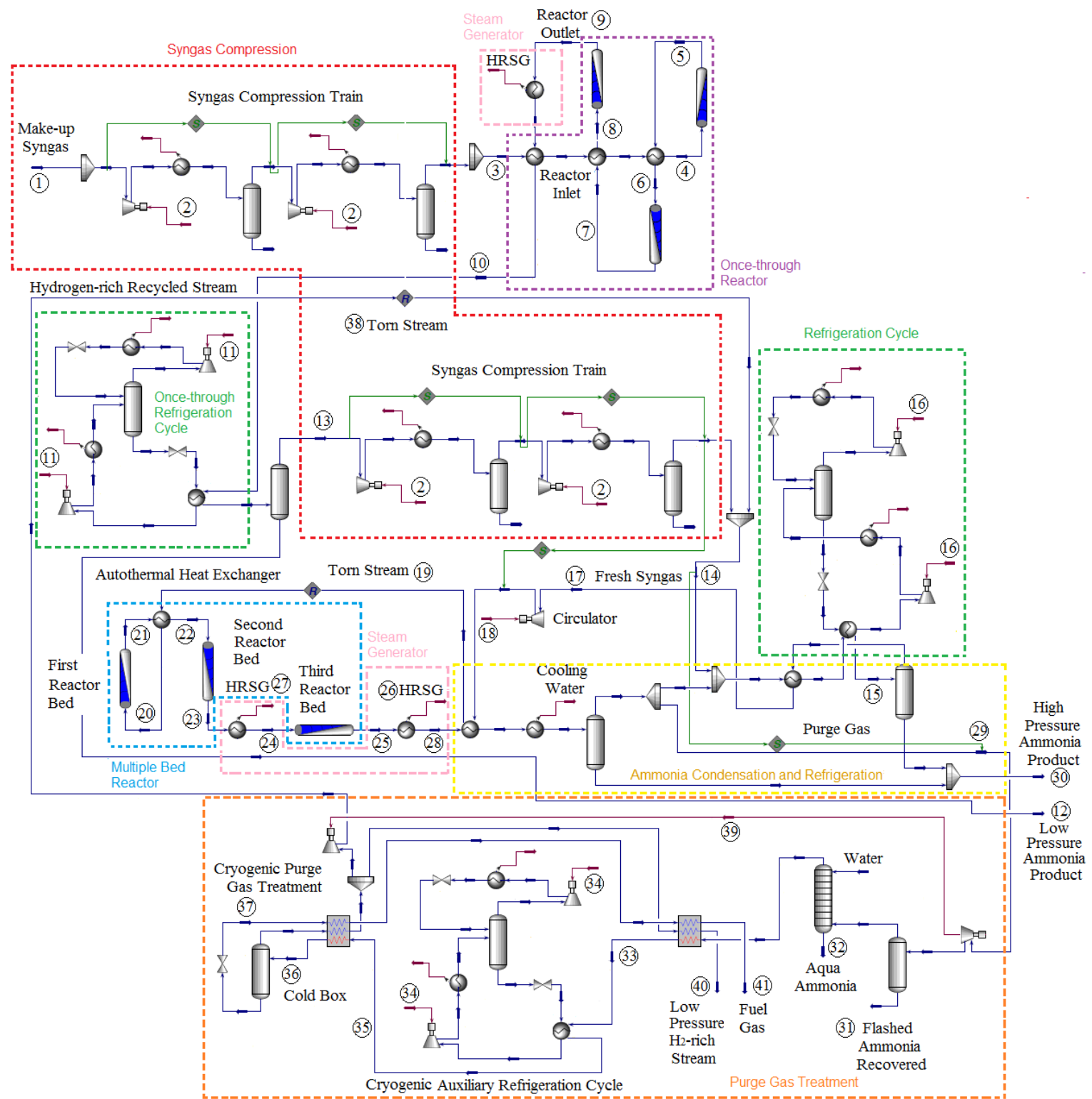

Source: [226].

In older ammonia plants, this purge gas has been typically used as supplemental fuel for the primary reformer [107]. However, since its valuable hydrogen content represents readily available feedstock for ammonia synthesis, and in view of the considerable amount of exergy that has been consumed to produce and compress the makeup mixture up to the loop pressure, in modern applications, hydrogen is preferably recovered and recycled to the synthesis loop [102]. Among various recovery processes, cryogenics can achieve a higher hydrogen recovery 
efficiency (99.5\%), while operating at higher pressures and larger flow rates [104]. Amongst the main advantages of the purge gas treatment process, shown in Figs. 10.1-10.2, are (i) the removal of the inerts from the ammonia loop with a reduced loss of valuable hydrogen, (ii) the enhancement of the reactivity of the system, which in turn reduces the size of the equipment, and (iii) the reduction of the power consumption in the syngas circulator and the refrigeration systems, since hydrogen and nitrogen are recovered at elevated pressures [94]. According to some studies, the hydrogen recovery could be used to increase the production about $4 \%$ or the equivalent energy savings on the original production capacity [281].

Accordingly, the purge gas is initially water scrubbed to remove ammonia, otherwise it would solidify in the cold box [102]. Ammonia can be distilled out of the aqueous mixture [22] or directly applied as a fertilizer [107]. Meanwhile, the moisture in the ammonia-free purge gas is removed by means of molecular sieves [22, 97]. In the cryogenic section, an auxiliary ammonia refrigeration system along with the heat recovery exchangers $[94,95]$ cool down the mixture to temperatures about $-190^{\circ} \mathrm{C}$, suitable to separate almost all the methane and argon contained in the purge gas stream [94]. Due to mechanical limitations on the brazed aluminum plate fin heat exchanger (PFHE), the recovery of the $\mathrm{H}_{2}-\mathrm{N}_{2}$ mixture from the purge gas is preferably carried out at pressures below 90 bar [97]. After the separation of the vapor (basically $\mathrm{N}_{2}$ and $\mathrm{H}_{2}$ ) and the liquid phases (mostly argon and methane), the vapor is reheated, and then the major part is recompressed at essentially the same pressure of the makeup syngas. The separated liquid phase is reheated and used in the plant fuel system. Meanwhile, the uncompressed fraction of the hydrogen-rich gas could be either externally recompressed and recycled to ammonia loop [104] or used as fuel in the ammonia plant [103].

Table 10.1-10.3 summarizes some thermodynamic properties of the process streams shown in the Figs. 10.1 and 10.2.

Table 10.1. Selected process data of the ammonia synthesis unit operating at 150 bar (1,000 $t_{N H 3} /$ day), cf. Figure 10.1 for stream number.

\begin{tabular}{|c|c|c|c|c|c|c|c|c|c|c|c|}
\hline $\mathbf{N}^{\circ}$ & Stream & $\begin{array}{c}\mathrm{n} \\
(\mathrm{kmol} / \mathrm{h})\end{array}$ & $\begin{array}{c}\mathbf{T} \\
\left({ }^{\circ} \mathbf{C}\right)\end{array}$ & $\begin{array}{c}\mathbf{P} \\
\text { (bar) }\end{array}$ & $\begin{array}{c}\mathbf{B}^{\mathrm{CH}} \\
(\mathbf{k W})\end{array}$ & $\begin{array}{c}\mathbf{B}^{\mathrm{PH}} \\
(\mathrm{kW})\end{array}$ & $\begin{array}{l}\mathrm{N}_{2} \\
(\%)\end{array}$ & $\begin{array}{c}\mathbf{H}_{2} \\
(\%)\end{array}$ & $\begin{array}{l}\mathrm{NH}_{3} \\
(\%)\end{array}$ & $\begin{array}{l}\mathrm{CH}_{4} \\
(\%)\end{array}$ & $\begin{array}{c}\text { Ar } \\
(\%)\end{array}$ \\
\hline 1 & Makeup syngas & $5,282.0$ & 35.1 & 34.7 & 265,469 & 12,927 & 25.0 & 73.6 & 0.0 & 1.0 & $0.4^{1}$ \\
\hline 2 & W compr. total & -- & -- & -- & -- & 8,193 & -- & -- & -- & -- & -- \\
\hline 3 & Fresh syngas & 5,362 & 35.9 & 150.8 & 270,836 & 18,825 & 24.8 & 74.0 & 0.0 & 1.0 & 0.2 \\
\hline 4 & To $2^{\circ}$ separator & 22,660 & -20.0 & 147.2 & $1,477,609$ & 80,025 & 20.9 & 59.6 & 9.4 & 7.9 & 2.2 \\
\hline 5 & W loop refrig. & -- & -- & -- & -- & 4,890 & -- & -- & -- & -- & -- \\
\hline 6 & Recycled syngas & 20,979 & 22.4 & 147.2 & $1,326,707$ & 73,057 & 22.5 & 64.4 & 2.2 & 8.5 & 2.4 \\
\hline 7 & W circulator & -- & -- & -- & -- & 498 & -- & -- & -- & -- & -- \\
\hline 8 & To ATHE & 20,979 & 254.2 & 150.8 & $1,326,707$ & 84,026 & 22.5 & 64.4 & 2.2 & 8.5 & 2.4 \\
\hline 9 & Bed 1 feed & 20,979 & 365.0 & 150.8 & $1,326,707$ & 94,013 & 22.5 & 64.4 & 2.2 & 8.5 & 2.4 \\
\hline 10 & Bed 1 outlet & 19,335 & 494.6 & 149.7 & $1,312,839$ & 100,885 & 20.2 & 57.1 & 10.9 & 9.2 & 2.6 \\
\hline 11 & Bed 2 feed & 19,335 & 384.7 & 149.7 & $1,312,839$ & 88,945 & 20.2 & 57.1 & 10.9 & 9.2 & 2.6 \\
\hline 12 & Bed 2 outlet & 18,861 & 422.9 & 148.6 & $1,309,138$ & 90,748 & 19.4 & 54.8 & 13.7 & 9.4 & 2.7 \\
\hline 13 & Bed 3 feed & 18,861 & 400.0 & 148.6 & $1,309,138$ & 88,367 & 19.4 & 54.8 & 13.7 & 9.4 & 2.7 \\
\hline 14 & Bed 3 outlet & 18,446 & 433.8 & 147.2 & $1,305,968$ & 89,963 & 18.8 & 52.7 & 16.2 & 9.6 & 2.7 \\
\hline 15 & BFW inlet & 3,997 & 110.1 & 100.0 & 999 & 1124 & -- & -- & -- & -- & -- \\
\hline 16 & Steam outlet ${ }^{2}$ & 3,997 & 310.0 & 100.0 & 999 & 12,909 & -- & -- & -- & - & -- \\
\hline
\end{tabular}




\begin{tabular}{|c|c|c|c|c|c|c|c|c|c|c|c|}
\hline $\mathbf{N}^{\mathbf{o}}$ & Stream & $\begin{array}{c}\text { n } \\
(\mathrm{kmol} / \mathrm{h})\end{array}$ & $\begin{array}{c}\mathbf{T} \\
\left({ }^{\circ} \mathbf{C}\right)\end{array}$ & $\begin{array}{c}\mathbf{P} \\
\text { (bar) }\end{array}$ & $\begin{array}{c}\mathrm{B}^{\mathrm{CH}} \\
(\mathrm{kW})\end{array}$ & $\begin{array}{c}\mathrm{B}^{\mathrm{PH}} \\
(\mathrm{kW})\end{array}$ & $\begin{array}{l}\mathrm{N}_{2} \\
(\%)\end{array}$ & $\begin{array}{c}\mathrm{H}_{2} \\
(\%)\end{array}$ & $\begin{array}{l}\mathrm{NH}_{3} \\
(\%)\end{array}$ & $\begin{array}{l}\mathrm{CH}_{4} \\
(\%)\end{array}$ & $\begin{array}{l}\text { Ar } \\
(\%)\end{array}$ \\
\hline 17 & To gas-gas HE & 18,446 & 300.0 & 147.2 & $1,305,968$ & 77,133 & 18.8 & 52.7 & 16.2 & 9.6 & 2.7 \\
\hline 18 & Purge gas & 284 & 30.0 & 147.2 & 19,838 & 982 & 19.6 & 55.2 & 12.3 & 10.0 & 2.9 \\
\hline 19 & $\mathrm{NH}_{3}$ Product & 2,544 & -2.6 & 147.2 & 233,816 & 4,456 & 0.2 & 0.4 & 98.3 & 1.1 & 0.0 \\
\hline 20 & Flashed $\mathrm{NH}_{3}$ & 8 & 8.6 & 80.0 & 756 & 14 & 0.2 & 0.2 & 98.8 & 0.8 & 0.0 \\
\hline 21 & Aquammonia $^{3}$ & 162 & 75.9 & 80.0 & 2,415 & 23 & -- & -- & 16.6 & -- & -- \\
\hline 22 & Purge to refrig. & 249 & 10.0 & 79.6 & 16,722 & 757 & 22.4 & 62.9 & 0.0 & 11.4 & 3.3 \\
\hline 23 & W cryo. refrig & -- & -- & -- & -- & 36 & -- & -- & -- & -- & -- \\
\hline 24 & To cold box & 249 & -30.0 & 79.6 & 16,722 & 769 & 22.4 & 62.9 & 0.0 & 11.4 & 3.3 \\
\hline 25 & To cryo. Sep. & 249 & -190.0 & 79.6 & 16,722 & 1,328 & 22.4 & 62.9 & 0.0 & 11.4 & 3.3 \\
\hline 26 & Expanded liquid & 89 & -197.1 & 1.0 & 6,899 & 478 & 52.0 & 7.7 & 0.0 & 31.8 & 8.5 \\
\hline 27 & $\mathrm{HP} \mathrm{H}_{2}-\mathrm{N}_{2}$ mixture & 87 & 87 & 232.0 & 5,344 & 338 & 6.1 & 93.3 & 0.0 & 0.2 & 0.4 \\
\hline 28 & W expander & -- & -- & -- & -- & 89 & -- & -- & -- & -- & -- \\
\hline 29 & $\mathrm{LP}_{2}-\mathrm{N}_{2}$ mixture & 74 & 30.0 & 80 & 5,344 & 225 & 6.1 & 93.3 & 0.0 & 0.2 & 0.4 \\
\hline 30 & Fuel Gas & 89 & -26.7 & 1.0 & 6,899 & 3 & 52.0 & 7.7 & 0.0 & 31.8 & 8.5 \\
\hline
\end{tabular}

1. Included $0.2 \%$ water; 2 . Vapor fraction $0.29 ; 3$. Aqueous solution $83.4 \%$ water.

Table 10.2. Selected process data of the ammonia synthesis unit operating at 200 bar, (1,000 $t_{N H 3} /$ day), cf. Figure 10.2 for stream number.

\begin{tabular}{|c|c|c|c|c|c|c|c|c|c|c|c|}
\hline $\mathbf{N}^{\mathbf{o}}$ & Stream & $\begin{array}{c}\mathrm{n} \\
(\mathrm{kmol} / \mathrm{h})\end{array}$ & $\begin{array}{c}\mathbf{T} \\
\left({ }^{\circ} \mathbf{C}\right)\end{array}$ & $\begin{array}{c}\mathbf{P} \\
\text { (bar) }\end{array}$ & $\begin{array}{c}\mathrm{B}^{\mathrm{CH}} \\
(\mathrm{kW})\end{array}$ & $\begin{array}{c}\mathrm{B}^{\mathrm{PH}} \\
(\mathrm{kW})\end{array}$ & $\begin{array}{l}\mathrm{N}_{2} \\
(\%)\end{array}$ & $\begin{array}{c}\mathbf{H}_{2} \\
(\%) \\
\end{array}$ & $\begin{array}{l}\mathrm{NH}_{3} \\
(\%) \\
\end{array}$ & $\begin{array}{l}\mathrm{CH}_{4} \\
(\%)\end{array}$ & $\begin{array}{l}\text { Ar } \\
(\%)\end{array}$ \\
\hline 1 & Makeup syngas & 5,282 & 35.1 & 34.7 & 264,574 & 12,927 & 25.2 & 73.4 & 0.0 & 1.0 & 0.41 \\
\hline 2 & W compr. Total & -- & -- & -- & -- & 9,954 & -- & -- & -- & -- & -- \\
\hline 3 & Fresh syngas & 5,421 & 36.2 & 200.0 & 273,712 & 20,206 & 24.7 & 74.1 & 0.0 & 1.0 & 0.2 \\
\hline 4 & To $2^{\circ}$ separator & 16,206 & -20 & 198.7 & 982,612 & 60,927 & 21.1 & 65.2 & 6.5 & 5.5 & 1.7 \\
\hline 5 & W loop refrig & -- & -- & -- & -- & 3,090 & -- & -- & -- & -- & -- \\
\hline 6 & Recycled syngas & 15,412 & 11.6 & 198.7 & 911,877 & 57,281 & 22.2 & 68.5 & 1.8 & 5.7 & 1.8 \\
\hline 7 & W circulator & -- & -- & -- & -- & 101 & -- & -- & -- & -- & -- \\
\hline 8 & To ATHE & 15,412 & 241.9 & 200.0 & 911,877 & 64,309 & 22.2 & 68.5 & 1.8 & 5.7 & 1.8 \\
\hline 9 & Bed 1 feed & 15,412 & 310.0 & 200.0 & 911,877 & 68,414 & 22.2 & 68.5 & 1.8 & 5.7 & 1.8 \\
\hline 10 & Bed 1 outlet & 13,731 & 496.9 & 199.6 & 897,816 & 74,705 & 18.8 & 58.6 & 14.3 & 6.3 & 2.0 \\
\hline 11 & Bed 2 feed & 13,731 & 429.3 & 199.6 & 897,816 & 69,333 & 18.8 & 58.6 & 14.3 & 6.3 & 2.0 \\
\hline 12 & Bed 2 outlet & 13,305 & 477.7 & 199.2 & 894,570 & 71,120 & 17.8 & 55.7 & 17.9 & 6.6 & 2.0 \\
\hline 13 & Bed 3 feed & 13,305 & 380.0 & 199.2 & 894,570 & 63,748 & 17.8 & 55.7 & 17.9 & 6.6 & 2.0 \\
\hline 14 & Bed 3 outlet & 12,873 & 430.0 & 198.7 & 891,367 & 65,364 & 16.8 & 52.4 & 21.9 & 6.8 & 2.0 \\
\hline 15 & BFW inlet & 3,997 & 110.1 & 100.0 & 999 & 1,124 & -- & -- & -- & -- & -- \\
\hline 16 & Steam outlet $^{2}$ & 3,997 & 310.0 & 100.0 & 999 & 13,466 & -- & -- & -- & -- & -- \\
\hline 17 & To gas-gas HE & 12,873 & 300.0 & 198.7 & 891,367 & 56,547 & 16.8 & 52.4 & 21.9 & 6.8 & 2.0 \\
\hline 18 & Purge gas & 312 & 30.0 & 198.7 & 20,527 & 1,151 & 19.4 & 60.7 & 9.8 & 7.7 & 2.4 \\
\hline 19 & NH3 Product & 2,570 & 15.0 & 198.7 & 235,915 & 4,637 & 0.4 & 0.7 & 97.7 & 1.2 & 0.0 \\
\hline 20 & Flashed NH3 & 11.5 & -2.6 & 80.0 & 1,057 & 19.2 & 0.1 & 0.2 & 99.1 & 0.6 & 0.0 \\
\hline 21 & Aquammonia3 & 118.9 & 52.5 & 80.0 & 1,739 & 10 & 0.0 & 0.0 & 16.2 & 0.0 & 0.0 \\
\hline 22 & Purge to refrig. & 281.5 & 10.0 & 79.6 & 17,786 & 855.6 & 21.5 & 67.3 & 0.0 & 8.5 & 2.7 \\
\hline 23 & W cryo. refrig & -- & -- & -- & -- & 40 & -- & -- & -- & -- & -- \\
\hline 24 & To cold box & 282 & -30.0 & 79.6 & 17,787 & 869 & 21.45 & 67.3 & 0.0 & 8.5 & 2.7 \\
\hline 25 & To cryo. Sep & 282 & -195.0 & 79.6 & 17,786 & 1,527 & 21.45 & 67.3 & 0.0 & 8.5 & 2.7 \\
\hline 26 & Expanded liquid & 89 & $\begin{array}{l}-199.1 \\
\end{array}$ & 1.0 & 5,867 & 500 & 57.6 & 7.7 & 0.0 & 26.6 & 8.1 \\
\hline 27 & $\mathrm{HP} \mathrm{H}_{2}-\mathrm{N}_{2}$ mixture & 146 & 83.2 & 225.1 & 9,121 & 564 & 4.8 & 94.9 & 0.0 & 0.1 & 0.2 \\
\hline 28 & $\mathrm{~W}$ expander & -- & -- & -- & -- & 144 & -- & -- & -- & -- & -- \\
\hline 29 & $\mathrm{LP} \mathrm{H}_{2}-\mathrm{N}_{2}$ mixture & 46 & 34.0 & 79.6 & 2,880 & 140 & 4.8 & 94.9 & 0.0 & 0.1 & 0.2 \\
\hline
\end{tabular}




\begin{tabular}{lccccccccccc}
\hline \multirow{2}{*}{$\mathbf{N}^{\mathbf{0}}$} & Stream & $\begin{array}{c}\mathbf{n} \\
(\mathbf{k m o l} / \mathbf{h})\end{array}$ & $\begin{array}{c}\mathbf{T} \\
\left({ }^{\circ} \mathbf{C}\right)\end{array}$ & $\begin{array}{c}\mathbf{P} \\
(\mathbf{b a r})\end{array}$ & $\begin{array}{c}\mathbf{B}^{\mathbf{C H}} \\
(\mathbf{k W})\end{array}$ & $\begin{array}{c}\mathbf{B}^{\mathbf{P H}} \\
(\mathbf{k W})\end{array}$ & $\begin{array}{c}\mathbf{N}_{\mathbf{2}} \\
(\boldsymbol{\%})\end{array}$ & $\begin{array}{c}\mathbf{H}_{\mathbf{2}} \\
(\boldsymbol{\%})\end{array}$ & $\begin{array}{c}\mathbf{N H}_{\mathbf{3}} \\
(\boldsymbol{\%})\end{array}$ & $\begin{array}{c}\mathbf{C H}_{\mathbf{4}} \\
(\boldsymbol{\%})\end{array}$ & $\begin{array}{c}\mathbf{A r} \\
(\boldsymbol{\%})\end{array}$ \\
\hline 30 & Fuel Gas & 89 & 12.5 & 1.0 & 5,867 & 0.1 & 57.6 & 7.7 & 0.0 & 26.6 & 8.1 \\
\hline \multicolumn{2}{l}{ 1. Included 0.2\% water; 2. Vapor fraction 0.34; 3. Aqueous solution $83.8 \%$ water. }
\end{tabular}

Table 10.3. Selected process data of the ammonia synthesis unit operating at dual pressure $83 / 200$ bar, $\left(1,000 t_{N H 3} /\right.$ day), cf. Figure 10.3 for stream number.

\begin{tabular}{|c|c|c|c|c|c|c|c|c|c|c|c|}
\hline $\mathbf{N}^{\mathbf{o}}$ & Stream & $\begin{array}{c}\mathrm{n} \\
(\mathrm{kmol} / \mathrm{h})\end{array}$ & $\begin{array}{c}\mathbf{T} \\
\left({ }^{\circ} \mathbf{C}\right)\end{array}$ & $\begin{array}{c}\mathbf{P} \\
\text { (bar) }\end{array}$ & $\begin{array}{c}\mathrm{B}^{\mathrm{CH}} \\
(\mathrm{kW})\end{array}$ & $\begin{array}{c}\mathbf{B}^{\mathrm{PH}} \\
(\mathbf{k W})\end{array}$ & $\begin{array}{l}\mathrm{N}_{2} \\
(\%)\end{array}$ & $\begin{array}{c}\mathbf{H}_{2} \\
(\%)\end{array}$ & $\begin{array}{l}\mathrm{NH}_{3} \\
(\%)\end{array}$ & $\begin{array}{l}\mathrm{CH}_{4} \\
(\%)\end{array}$ & $\begin{array}{l}\text { Ar } \\
(\%)\end{array}$ \\
\hline 1 & Makeup syngas & 5,282 & 35.1 & 34.7 & 264,574 & 12,927 & 25.2 & 73.4 & 0.0 & 1.0 & $0.4^{1}$ \\
\hline 2 & W compr. Total & -- & -- & -- & -- & 8,516 & -- & - & -- & -- & -- \\
\hline 3 & OTR feed preheat & 5,276 & 35.0 & 83.2 & 264,598 & 16,209 & 25.3 & 73.5 & 0.0 & 1.0 & 0.2 \\
\hline 4 & OTR Bed 1 feed & 5,276 & 290.0 & 83.2 & 264,598 & 19,455 & 25.3 & 73.5 & 0.0 & 1.0 & 0.2 \\
\hline 5 & OTR Bed 1 outlet & 4,751 & 466.9 & 83.1 & 259,918 & 21,638 & 22.5 & 65.0 & 11.1 & 1.2 & 0.2 \\
\hline 6 & OTR Bed 2 feed & 4,751 & 370.0 & 83.1 & 259,918 & 19,285 & 22.5 & 65.0 & 11.1 & 1.2 & 0.2 \\
\hline 7 & OTR Bed 2 outlet & 4,591 & 425.6 & 83.0 & 258,647 & 19,984 & 21.5 & 62.1 & 15.0 & 1.2 & 0.2 \\
\hline 8 & OTR Bed 3 feed & 4,591 & 370.0 & 83.0 & 258,647 & 18,687 & 21.5 & 62.1 & 15.0 & 1.2 & 0.2 \\
\hline 9 & OTR Bed 3 outlet & 4,497 & 402.9 & 82.9 & 257,923 & 19,084 & 20.9 & 60.2 & 17.4 & 1.3 & 0.2 \\
\hline 10 & To OTR refrig & 4,497 & 46.0 & 82.9 & 257,923 & 13,708 & 20.9 & 60.2 & 17.4 & 1.3 & 0.2 \\
\hline 11 & W refrig OTR & -- & -- & -- & -- & 827 & -- & -- & -- & -- & -- \\
\hline 12 & OTR $\mathrm{NH}_{3}$ product & 474 & 5.0 & 82.9 & 42,559 & 752 & 0.1 & 0.2 & 98.6 & 0.1 & 0.0 \\
\hline 13 & To loop Syngas & 4,023 & 5 & 82.9 & 216,033 & 12,338 & 23.4 & 67.3 & 7.8 & 1.3 & 0.2 \\
\hline 14 & Fresh syngas & 4,170 & 36.1 & 199.3 & 197,090 & 15,477 & 22.8 & 68.2 & 7.5 & 1.3 & 0.2 \\
\hline 15 & To $2^{\circ}$ separator & 8,467 & -20.0 & 199.3 & 562,287 & 31,737 & 19.9 & 59.1 & 10.0 & 8.3 & 2.7 \\
\hline 16 & W loop refrig & -- & -- & -- & -- & 2,022 & -- & -- & -- & -- & -- \\
\hline 17 & Recycled syngas & 7,763 & 19.2 & 199.3 & 498,763 & 28,819 & 21.7 & 64.5 & 1.9 & 9.0 & 2.9 \\
\hline 18 & W circulator & -- & -- & -- & -- & 42.9 & -- & -- & -- & -- & -- \\
\hline 19 & To ATHE & 11,933 & 257.2 & 200.0 & 723,500 & 50,466 & 22.1 & 65.7 & 3.9 & 6.3 & 2.0 \\
\hline 20 & Bed 1 feed & 11,933 & 350.0 & 200.0 & 723,500 & 55,145 & 22.1 & 65.7 & 3.9 & 6.3 & 2.0 \\
\hline 21 & Bed 1 outlet & 10,831 & 505.0 & 199.8 & 714,475 & 59,545 & 19.2 & 57.2 & 14.5 & 6.9 & 2.2 \\
\hline 22 & Bed 2 feed & 10,831 & 413.0 & 199.8 & 714,475 & 53,775 & 19.2 & 57.2 & 14.5 & 6.9 & 2.2 \\
\hline 23 & Bed 2 outlet & 10,444 & 468.6 & 199.6 & 711,544 & 55,363 & 18.1 & 53.8 & 18.7 & 7.2 & 2.2 \\
\hline 24 & Bed 3 feed & 10,444 & 400 & 199.6 & 711,544 & 51,227 & 18.1 & 53.8 & 18.7 & 7.2 & 2.2 \\
\hline 25 & Bed 3 outlet & 10,162 & 441.1 & 199.3 & 709,467 & 52,329 & 17.2 & 51.1 & 22.0 & 7.4 & 2.3 \\
\hline 26 & BFW inlet & 3,997 & 110.1 & 100.0 & 999 & 1,124 & -- & -- & -- & -- & -- \\
\hline 27 & Steam outlet $^{2}$ & 3,997 & 310.0 & 100.0 & 999 & 14,426 & -- & -- & -- & -- & -- \\
\hline 28 & To gas-gas HE & 10,162 & 300.0 & 199.3 & 709,467 & 44,668 & 17.2 & 51.1 & 22.0 & 7.4 & 2.3 \\
\hline 29 & Purge gas & 292 & 30.0 & 199.3 & 19,384 & 1,076 & 19.9 & 59.1 & 10.0 & 8.3 & 2.7 \\
\hline 30 & $\mathrm{NH}_{3}$ Product & 2,107 & 13.8 & 199.3 & 194,283 & 3,831 & 0.3 & 0.7 & 97.5 & 1.4 & 0.1 \\
\hline 31 & Flashed $\mathrm{NH}_{3}$ & 11 & -2.6 & 80.0 & 1,010 & 18 & 0.1 & 0.2 & 99.1 & 0.7 & 0.0 \\
\hline 32 & Aquammonia $^{3}$ & 118 & 53.2 & 80.0 & 1,638 & 10 & 0.0 & 0.0 & 15.4 & 0.0 & 0.0 \\
\hline 33 & Purge to refrig. & 263 & 10.0 & 79.5 & 16,789 & 799 & 22.1 & 65.6 & 0.0 & 9.3 & 3.0 \\
\hline 34 & W cryo. refrig & -- & -- & -- & -- & 40 & -- & -- & -- & -- & -- \\
\hline 35 & To cold box & 263 & -30 & 79.5 & 16,789 & 811 & 22.1 & 65.6 & 0.0 & 9.3 & 3.0 \\
\hline 36 & To cryo. sep. & 263 & -190 & 79.5 & 16,789 & 1,384 & 22.1 & 65.6 & 0.0 & 9.3 & 3.0 \\
\hline 37 & Expanded liquid & 85 & -198 & 1.0 & 5,953 & 457 & 55.1 & 8.2 & 0.0 & 28.3 & 8.4 \\
\hline 38 & $\mathrm{HP} \mathrm{H}_{2}-\mathrm{N}_{2}$ mixture & 146 & 73.2 & 215.0 & 8,954 & 556 & 6.3 & 93.1 & 0.0 & 0.2 & 0.4 \\
\hline
\end{tabular}




\begin{tabular}{llcccccccccc}
\hline $\mathbf{N}^{\mathbf{0}}$ & \multicolumn{1}{c}{ Stream } & $\begin{array}{c}\mathbf{n} \\
(\mathbf{k m o l} / \mathbf{h})\end{array}$ & $\begin{array}{c}\mathbf{T} \\
\left({ }^{\circ} \mathbf{C}\right)\end{array}$ & $\begin{array}{c}\mathbf{P} \\
(\mathbf{b a r})\end{array}$ & $\begin{array}{c}\mathbf{B}^{\mathbf{C H}} \\
(\mathbf{k W})\end{array}$ & $\begin{array}{c}\mathbf{B}^{\mathrm{PH}} \\
(\mathbf{k W})\end{array}$ & $\begin{array}{c}\mathbf{N}_{\mathbf{2}} \\
(\boldsymbol{\%})\end{array}$ & $\begin{array}{c}\mathbf{H}_{\mathbf{2}} \\
(\boldsymbol{\%})\end{array}$ & $\begin{array}{c}\mathbf{N H}_{\mathbf{3}} \\
(\boldsymbol{\%})\end{array}$ & $\begin{array}{c}\mathbf{C H}_{\mathbf{4}} \\
(\boldsymbol{\%})\end{array}$ & $\begin{array}{c}\mathbf{A r} \\
(\boldsymbol{\%})\end{array}$ \\
\hline 39 & W expander & -- & -- & -- & -- & & -- & -- & -- & -- & -- \\
\hline 40 & LP H $_{2}-\mathrm{N}_{2}$ mixture & 32.0 & 33.0 & 79.5 & 1,965 & 97.3 & 6.3 & 93.1 & 0.0 & 0.2 & 0.4 \\
\hline 41 & Fuel Gas & 85 & 19.4 & 1.0 & 5,953 & 0.1 & 55.1 & 8.2 & 0.0 & 28.3 & 8.4 \\
\hline 1. Included 0.2\% water; 2. Vapor fraction 0.41; 3. Aqueous solution 84.6\% water.
\end{tabular}

\subsection{Exergy efficiency definition of the industrial ammonia units.}

The same exergy efficiency definitions presented in Table 9.1 of the Chapter 9 of this thesis are used to evaluate the overall performance of the ammonia production units operating under the single and dual pressure configurations. In the same way, the proposed exergy efficiency definitions for representative components of the industrial ammonia units are equivalent to those presented in Table 9.2 of the Chapter 9, with exemption of an additional exergy efficiency, defined exclusively for the cryogenic purge gas treatment unit, Eq. (10.1):

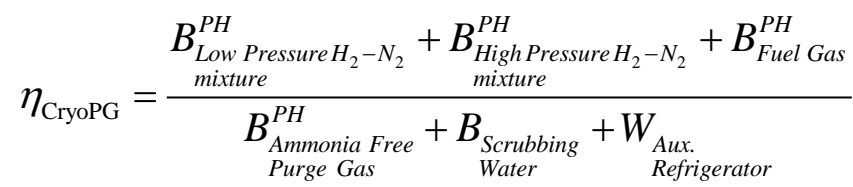

Since no chemical reactions occur in the treatment unit, the efficiency is calculated as the ratio of the physical exergy recovered in the high and low pressure hydrogen and methanerich streams to the total exergy consumed, including the power used in the auxiliary refrigeration system.

\subsection{Counteraction principle and the application to the ammonia synthesis}

As stated earlier, the variation of the loop pressure strongly affects the thermodynamics and economics of the ammonia synthesis. For example, higher loop pressures increase the ammonia equilibrium conversion and, thus, the waste heat recovery available for steam generation. Higher pressures also allow reducing the refrigeration power and improve the ammonia separation, reducing so the equipment size. Notwithstanding, higher operating pressures also demand an increased syngas compression power [69, 134].

On the other hand, if ammonia synthesis is carried out at lower pressures (and consequently, lower temperatures), the enthalpy of reaction is not anymore available for high pressure steam generation and, as such, the utility of this low-grade waste heat is doubtful [74]. However, by reducing the loop pressure, the overall irreversibility in the reactor is also reduced, in accordance with the Counteraction Principle. Clearly, the decision-making leads to diametrically opposite objectives [69], namely the reduction of the exergy losses by reducing the driving forces of the process and the increase of the process yield by increasing them. This can be better illustrated if all the reaction enthalpy is considered as isothermally removed at $\mathrm{T}_{0}$ during ammonia production. In fact, the exergy efficiency of an exothermic isothermal reactor, in which the number of moles is reduced along the conversion process, can be written as in Eq. (10.2):

$$
\eta_{p}=\Delta B^{P H} / \Delta B^{C H}=\frac{\left(n_{P}-n_{R}\right) R T_{o} \ln \left(P_{P} / P_{R}\right)}{\Delta B^{C H}}, \text { for } n_{P}<n_{R}
$$


Accordingly, the exergy efficiency increases as the pressure is reduced, since the exergy of the products exceeds the exergy of the reactants [69]. To tackle this dilemma, different solutions can be envisaged, including (i) the reduction of the exergy losses by keeping the reactions from running into completion [69], shifting the multiple bed reactor away from equilibrium by producing high pressure steam, while (ii) introducing a novel dual pressure ammonia loop (e.g. Udhe Dual Process ${ }^{T M}$ ), that operates by starting at lower pressures and proceeding at higher ones. For this reason, the Counteraction principle proves to be useful to drive the process irreversibility down by using incremental levels of pressure in the ammonia production processes. Meanwhile, the reduction of the recycle rates allows cutting down the energy required in the circulation and refrigeration systems.

\subsection{Operation conditions of the single and dual pressure ammonia synthesis units.}

Table 10.4 compares the processes parameters of the single and dual pressure configurations of the ammonia synthesis units studied. For the single pressure configuration at 150 bar (SP150), the total power consumed in refrigeration is about $69 \%$ higher than that of the dual pressure system (DP). The circulation power consumption is also considerably higher, as a direct consequence of a larger recycle flow rate and a higher loop pressure drop. The reactor conversion in the SP150 setup is also reduced in about 20.2\% compared with the DP system, producing thus a drop in the amount of steam generated. Yet, in spite of the lower inerts content of the recycled stream in the loop of the DP system, the higher amount of ammonia recycled, compared with the SP200 configuration, slightly reduces the reaction conversion.

Anyway, since an important percentage of the inerts and the ammonia converted in the low pressure section of the DP configuration is withdrawn ahead of reaching the loop, the compression power consumed in the fresh syngas compressor is significantly reduced, along with the recycle rate and the cooling water requirement. It is also worthy to notice that, even though an additional refrigeration stage is required downstream the once-through reaction section, the addition of a low pressure ammonia separation system in the DP unit reduces the refrigeration duty required in the loop by $58.6 \%$ and $34.6 \%$ compared with the SP150 and SP200 configurations, respectively. Thus, the advantage of the DP system over the SP150 and SP200 configurations does not only rely on the economic point of view (an improved investment and feedstock economy and higher revenue), but also on the increased efficiency and reduced environmental impact.

Furthermore, despite the fact that, for any scenario, similar amounts of fuel gas are produced (26.5\%-31.7\% $\mathrm{CH}_{4}, 52 \%-57.5 \% \mathrm{~N}_{2}$ and $8.0 \%-8.5 \% \mathrm{Ar}$ ), the highest hydrogen recovery rates do occur for the DP configuration. For instance, in the DP case, the proportion of hydrogenrich stream recovered at basically the same loop pressure is $80.2 \%$, while for the SP150 and SP200 setups, those figures are appreciable lower (53.9\% and 75.9\%, respectively). In this way, the use of a cryogenic purge gas treatment unit in combination with a dual pressure reaction system helps improving the utilization of the exergy of the natural gas consumed in the ammonia production process. Alternatively, in order to increase the overall efficiency of the system, argon, nitrogen and methane may be further separated by any suitable method. 
Table 10.4. Main process parameters of the various configurations studied.

\begin{tabular}{|c|c|c|c|}
\hline \multirow[b]{2}{*}{ Process parameter } & \multicolumn{2}{|c|}{ Single Pressure (SP) } & \multirow{2}{*}{$\begin{array}{c}\text { Dual Pressure (DP) } \\
\text { 83-200bar }\end{array}$} \\
\hline & 150 bar & 200 bar & \\
\hline Once-through reactor inlet temperature $\mathrm{T}_{\mathrm{OT}}\left({ }^{\circ} \mathrm{C}\right)$ & -- & -- & 290 \\
\hline First bed gas preheating temperature, $\mathrm{T}_{1}\left({ }^{\circ} \mathrm{C}\right)$ & 365 & 310 & 350 \\
\hline Third bed inlet gas temperature, $\mathrm{T}_{3}\left({ }^{\circ} \mathrm{C}\right)$ & 400 & 380 & 400 \\
\hline Once-through reactor pressure drop (bar) & -- & -- & 0.32 \\
\hline Loop reactor pressure drop (bar) & 3.6 & 1.3 & 0.70 \\
\hline Makeup syngas $\mathrm{H}_{2} / \mathrm{N}_{2}$ ratio & 2.94 & 2.91 & 2.91 \\
\hline Fresh syngas $\mathrm{H}_{2} / \mathrm{N}_{2}$ ratio & 2.99 & 3.00 & 2.99 \\
\hline Inerts mole fraction $(\%)$ & 1.40 & 1.40 & 1.40 \\
\hline Makeup syngas water molar fraction (\%) & 0.2 & 0.2 & 0.2 \\
\hline Recycle ammonia composition (\%) & 2.22 & 1.81 & 3.88 \\
\hline Recycle inerts composition (\%) & 10.86 & 7.43 & 8.29 \\
\hline Recycled reactor feed $\mathrm{H}_{2} / \mathrm{N}_{2}$ ratio & 2.86 & 3.08 & 2.98 \\
\hline Recycle molar flow rate $(\mathrm{kmol} / \mathrm{h})$ & 20,979 & 15,412 & $\mathbf{1 1 , 9 3 3}$ \\
\hline Fresh syngas compression power $(\mathrm{kW})$ & 8,193 & 9,954 & 8,516 \\
\hline Once-through refrigeration power $(\mathrm{kW})^{1}$ & -- & -- & 827 \\
\hline Loop refrigeration power $(\mathrm{kW})^{2}$ & 4,890 & 3,090 & 2,022 \\
\hline Cryo. Auxiliary refrigeration power $(\mathrm{kW})$ & 35.6 & 39.7 & 39.4 \\
\hline COP Carnot refrigeration & 4.42 & 4.42 & $4.42^{3}$ \\
\hline COP actual refrigeration & 2.43 & 2.43 & $2.43^{3}$ \\
\hline Circulator power consumption $(\mathrm{kW})$ & 498 & 101 & 42,9 \\
\hline Purge gas fraction $(\%)$ & 7.0 & 7.0 & 7.0 \\
\hline Once-through reactor conversion $(\%)^{4}$ & -- & -- & 29.3 \\
\hline Loop reactor first bed conversion $(\%)^{4}$ & 17.4 & 24.5 & 20.9 \\
\hline Loop reactor second bed conversion $(\%)^{4}$ & 6.1 & 8.2 & 9.3 \\
\hline Loop reactor third bed conversion $(\%)^{4}$ & 5.7 & 9.1 & 7.5 \\
\hline Reactor conversion $(\%)^{4}$ & 26.8 & 37.1 & 33.6 \\
\hline Waste heat recovery rate $(\mathrm{kW})^{5}$ & 28,379 & 29,518 & 31,482 \\
\hline Cooling water - gas condensation $(\mathrm{kmol} / \mathrm{h})^{6}$ & 9,373 & 11,280 & 8,928 \\
\hline Cooling water - refrigeration cycle $(\mathrm{kmol} / \mathrm{h})^{6}$ & 16,636 & 10,569 & $11,968^{7}$ \\
\hline Scrubbing water consumption $(\mathrm{kmol} / \mathrm{h})^{8}$ & 135 & 100 & 100 \\
\hline Aqua ammonia production $\left(\mathrm{m}^{3} / \mathrm{h}\right)^{9}$ & 2.25 & 2.28 & 2.25 \\
\hline High $\mathrm{P}, \mathrm{H}_{2}$-rich recovered stream $(\mathrm{kmol} / \mathrm{h})^{10}$ & 86.8 & 146.2 & 145.9 \\
\hline Low $\mathrm{P}, \mathrm{H}_{2}$-rich recovered stream $(\mathrm{kmol} / \mathrm{h})$ & 74.0 & 46.2 & 32.0 \\
\hline Fuel gas production $(\mathrm{kmol} / \mathrm{h})^{11}$ & 88.64 & 89.2 & 85.1 \\
\hline Incomes $\left(\$ / t_{\mathrm{NH} 3}\right)^{12}$ & 637.2 & 644.5 & 643.4 \\
\hline Costs $\left(\$ / t_{\mathrm{NH} 3}\right)^{13}$ & 403.1 & 402.7 & 397.8 \\
\hline Revenues $\left(\$ / t_{\mathrm{NH} 3}\right)$ & 234.1 & 241.8 & 250.8 \\
\hline Annualized Bare Module Cost $\left(\$ / t_{\mathrm{NH} 3}\right)^{14}$ & 23.8 & 26.3 & 25.8 \\
\hline
\end{tabular}

1. Condenser pressure: 13.6 bar, evaporator pressure: $424.4 \mathrm{kPa}$. Minimum temperature approach: $5^{\circ} \mathrm{C} .2$. Condenser pressure: 13.6 bar, evaporator pressure: $115.2 \mathrm{kPa}$. Minimum temperature approach: $5-10^{\circ} \mathrm{C} ; 3$. Except for the once-through section refrigeration, whose values are $\mathrm{COP}_{\text {actual }}=5.2$ and $\mathrm{COP}_{\text {Carnot }}=10.9 ; 4$. Reactor conversion $\xi$ as defined in Ch. 5; 5. Saturated steam as 100 bar; 6 . Cooling water maximum outlet temperature: $35-40^{\circ} \mathrm{C}$; 7 . Condenser water cooling duty: $57 \%$ loop refrigeration, $42 \%$ once-through refrigeration, $1 \%$ cryogenic unit auxiliary refrigeration; 8 . Water at $30^{\circ} \mathrm{C}$ and 79.5 bar; 9. Ref. [107]; 10. Hydrogen recovery efficiency > $93.11 \%$ - 94.92\%; 11. Methane $26.5 \%-31.7 \%$, Nitrogen $52 \%-57.5 \%$; 
Argon 8.0\%-8.5\%; 12. Ammonia price: \$32/GJ; 13. Natural gas cost: \$9.7/GJ. Annualized bare module cost included; 14 . Interest rate $6 \%$, lifespan 20 years. CEPCI: $550(2010)$ [115, 129].

According to Table 10.4, in the SP150 and SP200 configurations, the makeup syngas compression consumes almost $56.7 \%$ and $75.5 \%$ of the power supply, respectively, followed by the refrigeration cycles $(39.8 \%$ and $23.7 \%$, respectively) and the circulator $(<3.4 \%)$. It is also important to notice that the total power consumption (compression, refrigeration and circulation) in the DP configuration reaches only $10.6 \mathrm{MW}$, i.e. $24.1 \%$ and $35.9 \%$ lower than in SP150 and SP200 configurations, respectively. It is also striking that, even if an important amount of the makeup syngas must still be compressed to levels of pressure comparable to those of SP200 system, the total exergy consumption required in the syngas compression train of the DP design is almost the same that the consumed in the SP150 system. In this way, the more favorable kinetic and equilibrium conditions and ammonia separation characteristics at higher pressures can be exploited, whereas the losses associated to the compression and the safety and control issues related to the high operating pressures can be minimized.

Some authors claim that given the compressor and circulator efficiencies and the pressure drop in the loop, a pressure-independent power consumption exists between 140 and 315 bar, with a flat minimum at 155 - 220 bar $[56,95]$. Those claims are partially in agreement with the results shown in Table 10.4 for the SP scenarios (9\% difference). However, the overall power consumption, including the makeup syngas and refrigeration compressors and the circulator, is rather 19\%-25\% higher in the SP units compared to the DP setup.

\subsection{Reaction kinetics in the intercooled, multiple bed catalytic reactor}

The plots of conversion vs. temperature shown in Fig. 10.3 graphically represent the relationship between the kinetics parameters (temperature, pressure, conversion and reaction rate) and the intercooling system of the industrial ammonia production unit. These figures are based on the characteristics of the Montecatini catalyst, reported in Chapter 5. The contours of the constant reaction rates, the approach to the equilibrium and the adiabatic reactor lines have been determined for each one of the configurations studied (Table 10.4). Due to safety issues and the metallurgical limitations associated to the hydrogen-rich operation conditions at high pressure, the maximum reaction temperature is limited to $550^{\circ} \mathrm{C}$. Moreover, due to the risk of poisoning by even a small $\mathrm{O}_{2}$ content, the practical lower bound of the catalyst temperature is set about $290^{\circ} \mathrm{C}[95]$.

Although the avoidable exergy destruction in the reactor is generally small, the importance of a combined study of the reactor kinetics and its exergy efficiency lies rather on the fact that the exergy destroyed in the remaining components (compressor, exchangers, separators, etc.) strongly depends on the reactor performance $[163,274]$. In fact, fixed the production capacity, the specific exergy consumption in the whole loop depends on the reactor space velocity and the conversion rate which, in turn, depends on the catalyst activity, the reactor size and the recycled stream composition. Thus, the lower the space velocity, the closer the equilibrium may be attained, entailing a lower conversion rate. On the other hand, by increasing the circulation rate, a higher yield of ammonia could be achieved but only at expense of a lower conversion per pass, reducing the reactor irreversibility [221]. However, the circulation rate 
cannot be increased indefinitely, since the ignition temperature of the autothermal converter could not be ensured, not to mention the increased exergy losses due to an excessive pressure drop. Lower per-pass conversion also needs larger equipment and, thus, higher capital costs.

Accordingly, Figs. 10.3(a-d) can be used to analyze the temperature profile that allows the various setups to attain not only a higher conversion rate but also a reduced irreversibility associated to the reacting driving force. Thus, even though the adiabatic reaction lines in Fig. 10.3 closely follow the locus of maximum conversion rates, the irreversibility arisen from the higher conversions is compensated by an enhanced performance of the integrated chemical plant.

Fig. 10.3. Conversion vs. Temperature plots. The contours of constant reaction rates are given in kmol m${ }^{-3} h^{-1}$; (a) Single pressure 150 bar; (b) Single pressure 200 bar; Dual pressure: (c) once-through 83 bar; (d) loop 200 bar.
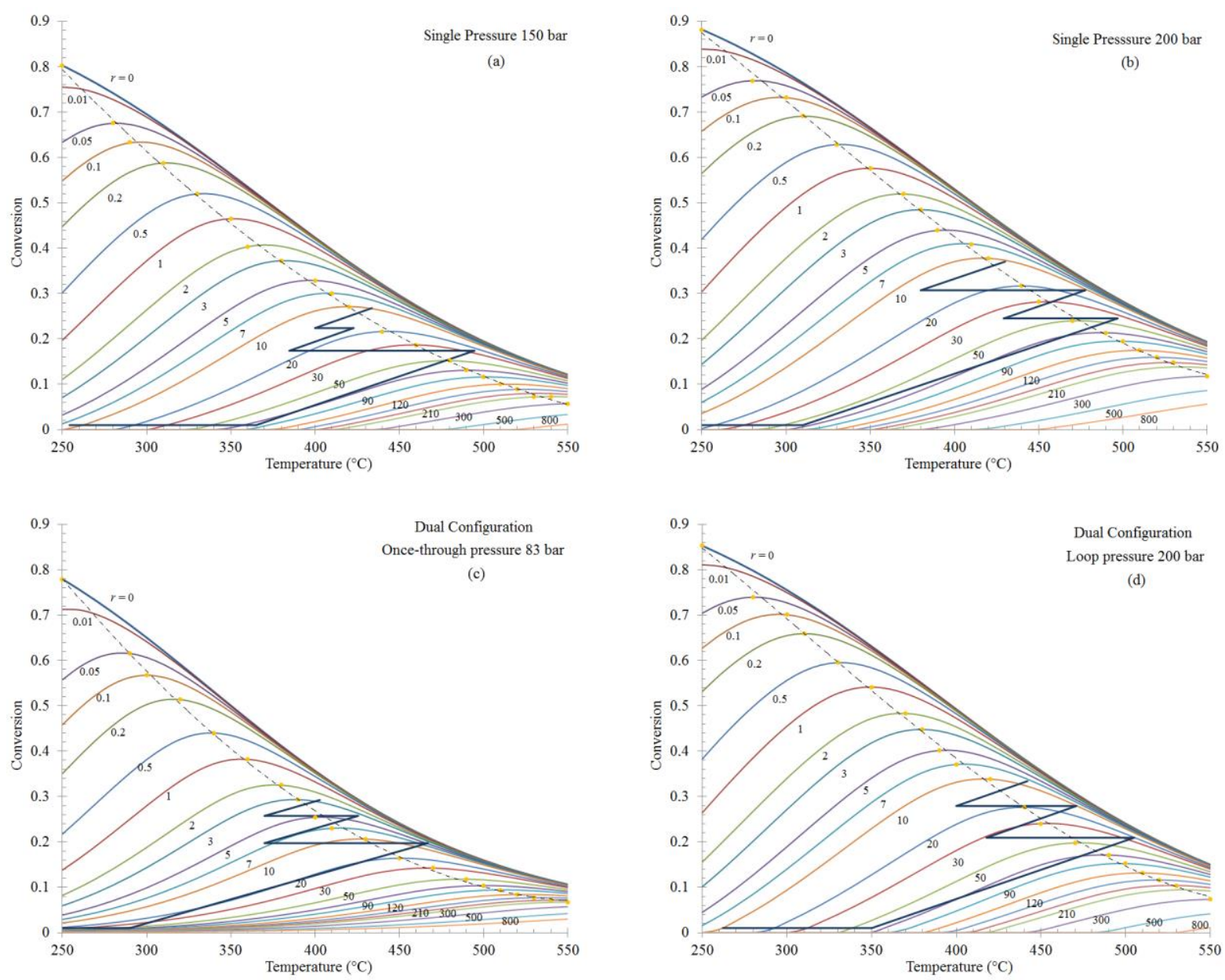

Source: Author.

It is also important to emphasize that, although a once-through ammonia converter working at a higher pressure may allow reducing the required catalyst volume, the concentration of the reagents would be so high, or equivalently, the amount of inerts entering such system would be so low (compared to the $8 \%$ inert $+2.5 \%$ ammonia content in the SP reactor feed), that an auxiliary ammonia injection system would be required to moderate the exit temperatures, due 
to extremely high conversion rates $[221,273]$. However, this system would introduce another source of irreversibility, not only because ammonia would be mixed to the reacting system, but also because it inevitably would trigger some hindering effects in the reactor conversion and the ammonia separation performance. Fortunately, the low pressure once-through reactor is thermodynamically limited to an acceptable temperature, avoiding the need of the ammonia injection.

Finally, it is worthy to notice that, differently from other reactor schemes that use direct cold shot [136] or autothermal reactors [282], the indirect cooling system depicted in Figs. 10.110.2 has gained preference as it allows producing high pressure steam. Other works discussed the advantage of a Dowtherm cooled-reactor system [274, 283], but according to Johannessen et al. [284], a cooled-reactor would result more difficult to control and increase the capital cost. Theoretically, the optimal configuration would consist of a separated adiabatic reactor operating in reaction-mode followed by a diathermic reactor operating in heat-exchangemode [285]. Yet, in practice, the near-optimal solution comprises one or more adiabatic reactor stages with intercooling, as adopted in this thesis [285]. In this way, more complex control problems can be avoided, and the search of an improved design can be simplified.

\subsection{Exergy analysis of the single and dual pressure ammonia synthesis units.}

Figure 10.4 shows the overall exergy efficiency of the SP and DP pressure configurations, as defined in Table 9.1, Chapter 9.

Fig. 10.4. Plantwide exergy efficiencies for the various ammonia production configurations.

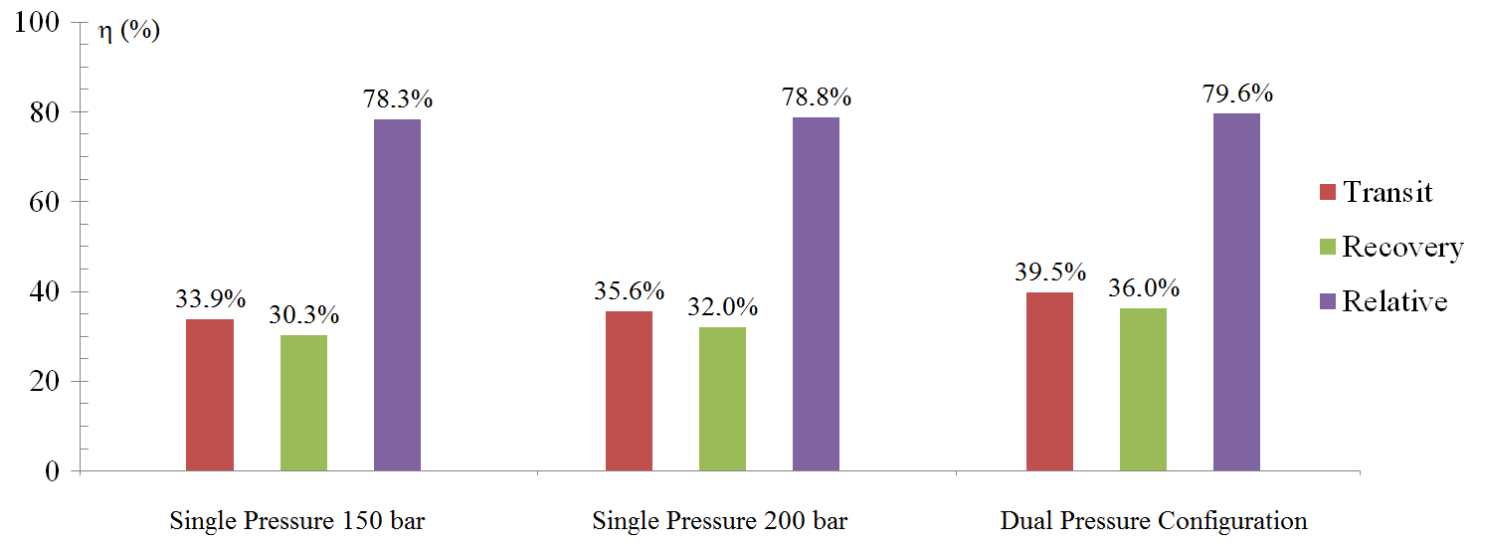

Source: Author.

The rational efficiency (>91\%) is found to be less sensitive to the variation of the process parameters and, thus, it is not shown in Fig. 10.4. On the contrary, both transit and recovery definitions reflect better the effect of the variation of the loop parameters on the performance of the chemical process. However, since the transit exergy efficiency definition inaccurately considers the ammonia product as if it were transit exergy, the loop performance and steam generation potential are more suitably estimated by using the recovery efficiency.

As expected, the DP configuration based on a stepwise, increased reactor conversion and a lower recycle rate outperforms the SP setups, wheres a high pressure steam generation is also achieved. On the other hand, the relative efficiency, even though lower than the abnormally 
high rational definition, still copes with the issues related to the exergy definition of largevolume chemical production processes.

Meanwhile, Fig. 10.5, shows the exergy efficiency calculated for representative components of the various analyzed layouts. The loop reactor efficiency remains almost invariable for all the configurations, but with the highest figures corresponding to the DP scheme. The main irreversible phenomena that occur in the ammonia converter are related to the highly irreversible reactions and the uncontrolled conversion of the chemical exergy of the reactants into the physical exergy of the products. Thus, very high circulation rates and low per-pass conversions in the ammonia reactor compromise the exergy efficiency. Despite the fact that most of the irreversibility due to chemical conversion is inevitable, performing the chemical reaction at lower pressures without attaining the completion may help increasing the reactor efficiency.

It also remarkable the different results obtained from the two exergy efficiency definitions for the refrigeration system. In fact, since the consumed-produced efficiency CP2 includes the exergy destroyed in the control volume of the evaporator, the exergy efficiency is appreciable lower. Regarding the syngas compressor, the consumed-produced exergy efficiency is quite similar for any configuration. Moreover, it is not surprising that the exergy efficiencies in the refrigeration cycle are similar in all the cases, considering that the isentropic efficiency of the refrigeration compressors is equal and the compression ratios are selected to reduce the power consumption. Finally, even though no chemical reactions are present in the cryogenic purge gas recovery unit, other dissipative components lead to a lower exergy efficiency.The throttling process of the cold box liquid effluent (whereby it partially vaporizes generating the refrigeration effect), the large temperature differences between the feed and exit streams in the cryogenic heat exchangers (ranging from $40^{\circ} \mathrm{C}$ to $-191{ }^{\circ} \mathrm{C}$ ), and the auxiliary refrigeration system [94] are examples of those components.

Accordingly, more sensitive exergy performance calculations can be achieved if the targets of the system and the role of the loop parameters are unequivocally defined, especially in the case of systems with large mass flow recycle streams. In the same way, a better understanding of the system irreversibility can be achieved by identifying the main components tasks, with the reactor performance playing the most important role in the ammonia loop. Figure 10.6 depicts a mechanical heat pump analogy of the exothermic adiabatic reactor, where the mechanical work substitutes the chemical work. This representation helps to visualize the extent in which the chemical exergy in the system can be totally or partially transformed in order to increase the physical exergy of the reactants [140, 169]. 
Fig. 10.5. Consumed-produced exergy efficiency of representative components of the ammonia production units: (a) single pressure 150 bar, (b) single pressure 200 bar, (c) dual pressure configuration.

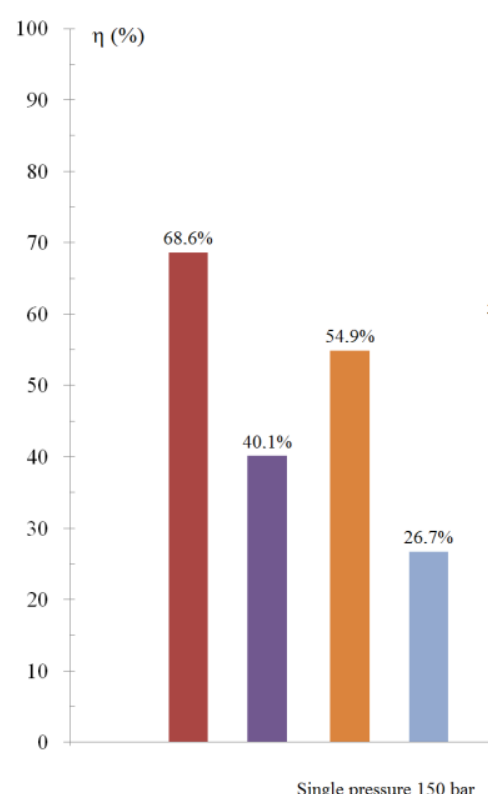

(a)
- Syngas Compressor

- Loop Reactor

= Loop Refrigeration

= Loop Refrigeration (includes evaporator)

= Cryogenic Purge Gas Treatment

Wteam Generator

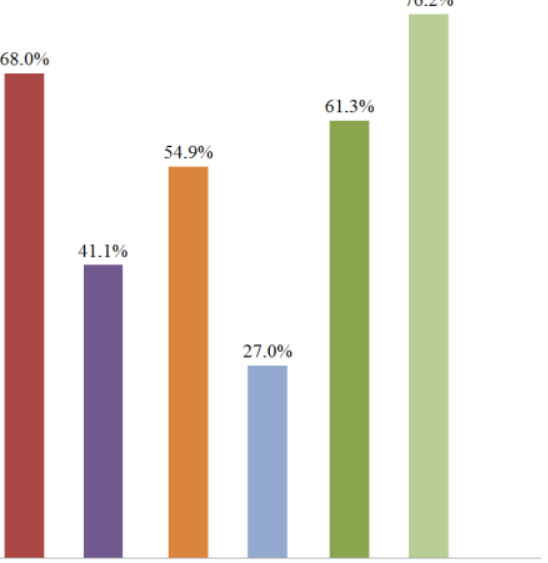

Single Pressure 200 bar

(b)

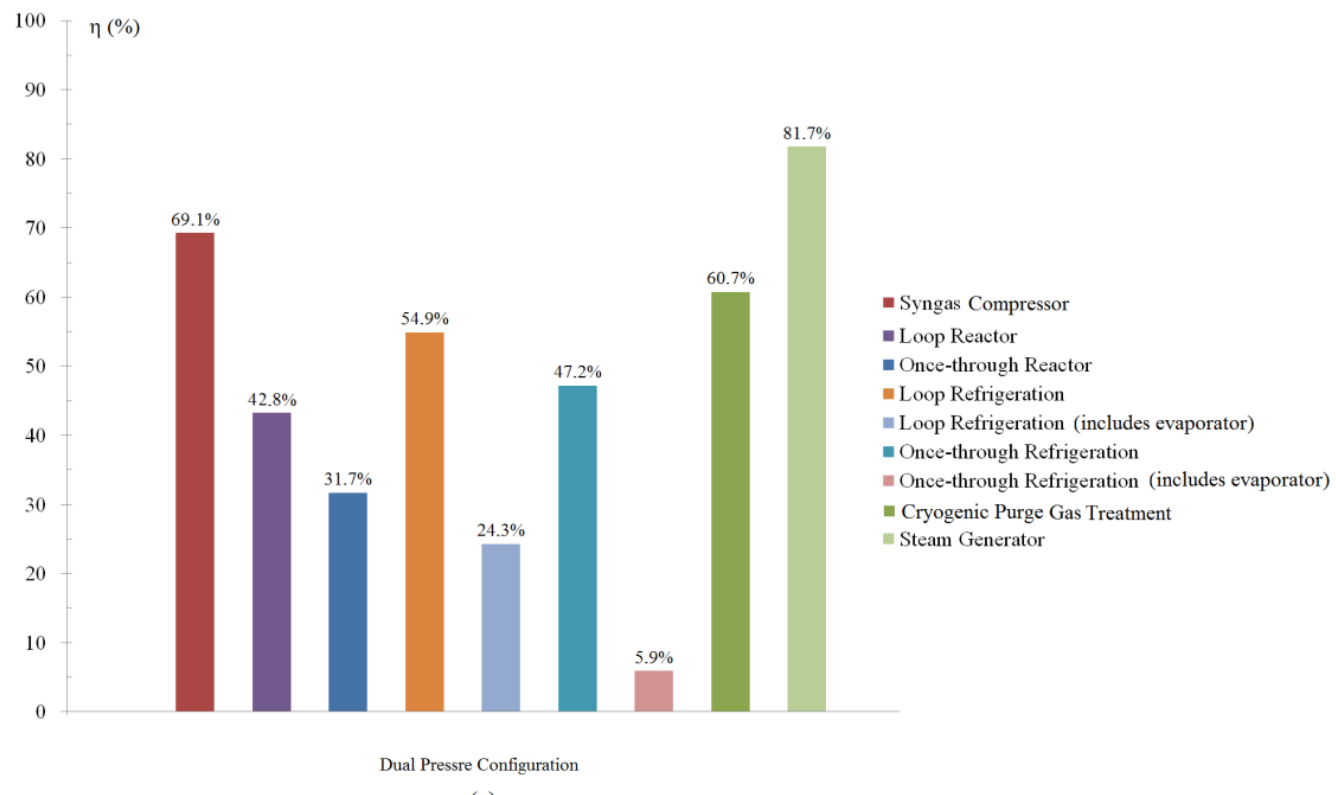

(c)

Source: Author.

According to Fig. 10.6, the recovery of chemical exergy of the reactants can be maximized by using a set of van't Hoff equilibrium boxes devised to carry out the reaction reversibly. Therein, the chemical species interact only with the ambient, exchanging heat and generating a net amount of work [178]. The maximum exergy recovery entails the full exploitation of the energy of the chemical reaction (Gibbs free energy) and the energy of mixing in a lesser extent [286]. Thus, if the reactor operation is performed reversibly, the variation of the chemical exergy equals the variation of the physical exergy between the reactants and products. To this end, a set of reversible heat pumps, operating at infinitesimal temperature 
differences between the 'evaporator' and 'condenser', $\mathrm{T}_{\mathrm{H}}$ and $\mathrm{T}_{\mathrm{L}}$, ensures the reversible conditions of the whole system. Equivalently, provide that no irreversibility occurs, the reactor exergy efficiency equals the unity.

Fig. 10.6. Exothermic reactor represented as a chemical exergy-driven heat pump. $Q$ ', $Q$ '” and $Q$ "' represent the reversible heat rates transferred to the ambient at the reference temperature for calculating the maximum net chemical work done by the species ( $W^{\prime}, W^{\prime}$ ', and $\left.W^{\prime \prime \prime}\right)$ in a set of van't Hoff equilibrium boxes.

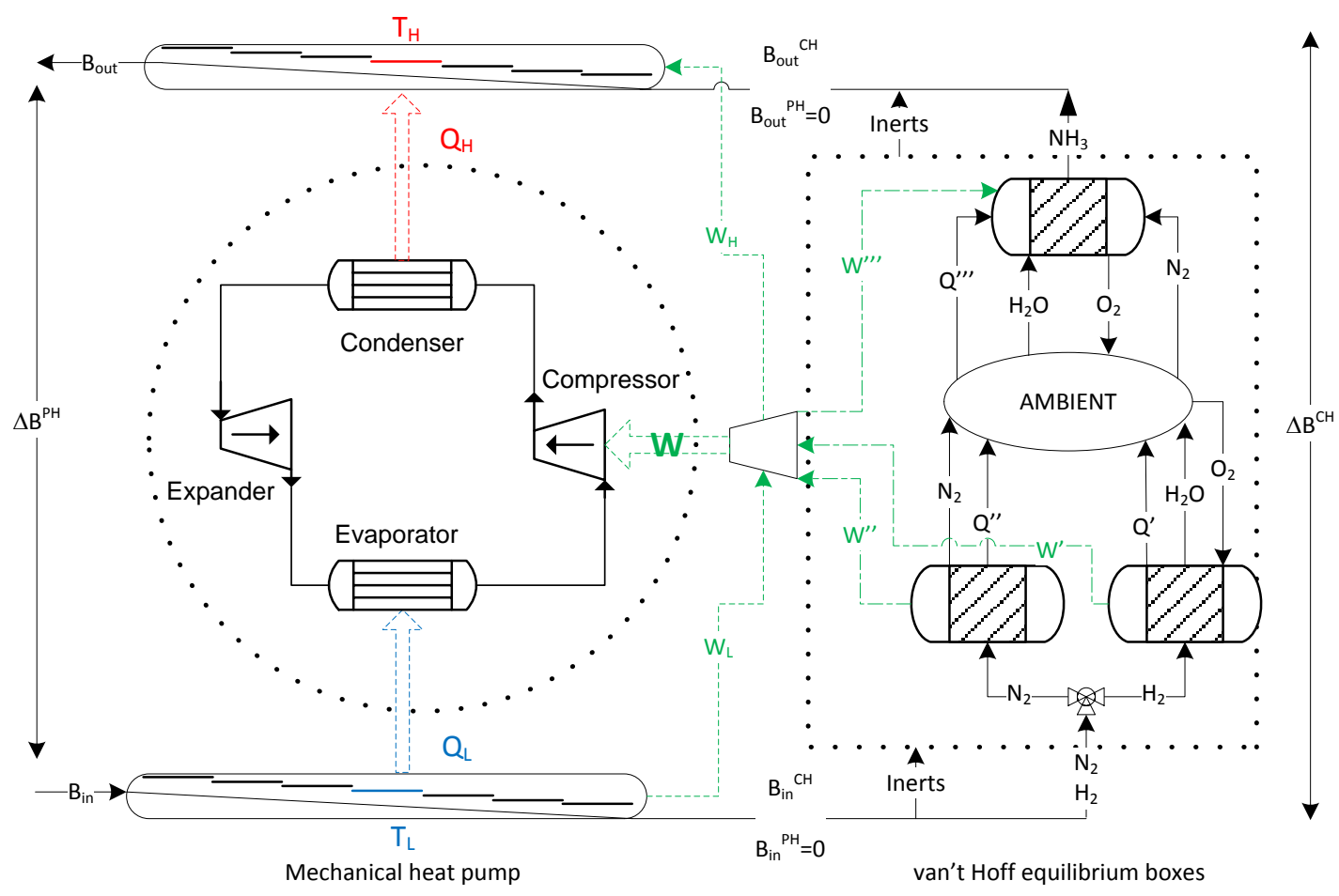

Source: Author.

However, in actual reactors, the chemical exergy consumed in the system is not entirely transformed into an equivalent form of work (i.e. variation of physical exergy) due to the spontaneous change in composition, unrestrained diffusion, pressure drop and heat transfer between species. In fact, the exergy destroyed in the reactor shown in Fig. 10.6 is accounted for the continuous release of the enthalpy of reaction in the uncontrolled form of internal energy, followed by the heat transfer from the hot products to the fresh reactants [140]. A mechanical analogy of such disordered transfer modes, in opposition to a reversible heat pump, would be an electric heater or a mechanical stirrer used to partially convert the chemical exergy of the reactants into exergy of heat available at high temperature $T_{H}$. The increase of the physical exergy by using the latter methods cannot be used to reverse the chemical reaction in the van't Hoff box, differently from the case of the reversible heat pump, because the energy quality has been degraded. Bringing back the system to the initial state would require a footprint in the ambient, or equivalently, more fuel is required to reverse the chemical process. Certainly, this represents a deeper insight of the exergy analysis when compared with the First law analysis.

Thus, on the one hand, the contribution of the reaction process to the exergy destruction relies, on the equilibrium approach: the more complete the reaction, the higher the exergy 
destruction [69]. On the other hand, the irreversibility associated to the heat exchange and the pressure drop depends the total conversion per pass: the higher the conversion, the lower the recycle flow rate of the unreacted syngas and the inert build-up. Moreover, since a large portion of the exergy entering the reactor is neither transformed nor destroyed throughout its volume, this transit also modifies the reactor performance. Consequently, the amount of exergy consumed in the condensation, refrigeration and circulation systems is also affected, not to mention the effect on the increased pressure drop, higher equipment sizes and space velocities. Lower conversions also lead to increased hydrogen content in the purge stream, and thus to a higher exergy destruction rate in the purge gas treatment. All in all, to an improper feedstock utilization.

According to some authors, the exergy destruction owed to the highly exothermic chemical reaction processes can be calculated by using the Eq.(10.3) [245, 285]:

$$
B_{\text {dest } \text {,react }}=T_{0} \frac{d S_{\text {gen }}}{d t}=T_{0}\left(n_{\text {out }} S_{\text {out }}-n_{\text {in }} S_{\text {in }}\right)=T_{0} \int_{V C} \sigma d z=T_{0} \int_{V C} r \frac{-\Delta G}{T} \mathrm{~A} d z
$$

where $-\Delta \mathrm{G} / \mathrm{T}$ is the reactive driving force. Eq. (10.3) integrates the contributions of the local entropy generation rates along the control volume, assuming local equilibrium conditions [284]. Denbigh [245] and Hinderink et al. [169] estimated the reactor irreversibility for the ammonia oxidation and hydrogen production processes, respectively, in a chemical reactor operating at constant pressure and temperature by using Eq.(10.4):

$$
B_{\text {dest }}=T_{o} S_{\text {gen }}=-T_{o} \frac{\Delta G_{\text {reaction }}}{T_{\text {reaction }}}
$$

Equation (10.4) is called 'unavoidable lost work' or 'dissipated energy' and can be used only for a constant reaction temperature $\mathrm{T}_{\text {reaction. }}$. However, in the adiabatic reactor studied in this chapter, both pressure drop and temperature variations occur. Therefore, the maximum work given by the Gibbs energy function is not equivalent to the maximum potential work attainable when the system achieves the equilibrium with the ambient, namely, its exergy.

Thus, a further understanding about the relation between the reactor parameters and the exergy destroyed on it can be achieved by considering the combined energy and entropy balance of a set of partially reversible fuel cell and heat pump. It can be demonstrated that the total irreversibility in the system is given by Eq.(10.5):

$$
B_{\text {dest }}=T_{o} S_{\text {gen }}=n_{N_{2}, \text { inlet }} \xi\left(T_{o} \Delta s_{\text {reaction }}-\Delta h_{\text {reaction }}\right)-\left[Q_{H}\left(1-\frac{T_{o}}{T_{H}}\right)-Q_{L}\left(1-\frac{T_{o}}{T_{L}}\right)\right]
$$

where the term in brackets represents the exergy recovered as work after accounting for the irreversibility present in the fuel cell and the actual heat pump. Thus, the combined efficiency of the mechanical analogy of the chemical reactor shown in Fig. 10.6 is calculated as:

$$
\eta_{\text {reactor }}=\eta_{\text {fuel cell }} \eta_{\text {heat pump }}=\frac{W}{n_{N_{2}, \text { inlet }} \xi\left(T_{o} \Delta s_{\text {reaction }}-\Delta h_{\text {reaction }}\right)} \cdot \frac{Q_{H}\left(1-\frac{T_{o}}{T_{H}}\right)-Q_{L}\left(1-\frac{T_{o}}{T_{L}}\right)}{W}
$$


where the reactor conversion $\xi$ reduces as the temperature increases [133] and its relation with the equilibrium constant $K_{P}$ is given by Eq.(10.7):

$$
K_{P}=\frac{a_{N_{3}}}{a_{H_{2}}^{1.5} \cdot a_{N_{2}}^{0.5}}=\frac{\Theta_{N H_{3}}+2 \xi}{(1-\xi)^{0.5}\left(\Theta_{H_{2}}-3 \xi\right)^{1.5}}\left(\frac{1}{y_{N_{2}}^{\text {inlet }}}-2 \xi\right) \frac{\gamma_{N H_{3}}}{\gamma_{N_{2}}^{0.5} \cdot \gamma_{H_{2}}^{1.5}} \frac{1}{P}=f(\mathrm{~T})
$$

where $a_{i}$ is the activity of the species $i$ (cf. Ch. 5).

Some conclusions can be withdrawn from Eqs. (10.5-10.7). First and foremost, it is important to notice that, for the adiabatic reactor, the temperatures $T_{H}$ and $T_{L}$ in Eq. (10.5) cannot vary independently since, as long as the inlet temperature $T_{L}$ increases, the outlet temperature $T_{H}$ also increases, but at expense of a decrease of the reactor conversion $\xi$, and thus, of the available enthalpy of reaction. Additionally, as the inlet temperature $\mathrm{T}_{\mathrm{L}}$ is reduced to the lowest allowable limit, the first term in the right hand side of Eq. (10.5) increases because the conversion of the adiabatic reaction increases, Eq. (10.7). However, the second term inside the brackets of Eq. (10.5) also is also reduced, which makes the total exergy destroyed dependent on the highest temperature $\mathrm{T}_{\mathrm{H}}$ attained. This temperature may vary depending on the recycle rate of inerts, ammonia and reactants, as well as on the approach to equilibrium. If the mixture is shifted away from equilibrium, i.e. if the reactor outlet temperature $T_{H}$ is reduced after the first conversion bed, the reactor conversion can be further increased elsewhere in a second bed. Then, the chemical exergy available can be further exploited, but not without triggering more exergy destruction.

On the other hand, if the reactor inlet temperature $\mathrm{T}_{\mathrm{L}}$ is increased (e.g. by preheating the reactor feed), not only the second term inside the brackets of Eq. (10.5) increases, but also the conversion-related term reduces, and the exergy destroyed is also reduced. Thus, were it not for the reactant preheating, the exergy recovered at high temperature would have a lower exergy value due to the reduced chemical conversion. This idea is the basis of the Counteraction principle applied to an exothermic reactor. Accordingly, there should be an intermediate optimal temperature that keeps the exergy destruction to the minimum, while guaranteeing the required reaction conversion [69]. Finally, as the reactor inlet temperature eventually approaches the reaction outlet temperature in the last reactor beds, the reactor conversion vanishes and the catalytic bed becomes a letdown system (i.e. pressure drop only). In this way, the pressure-related share of the physical exergy is degraded. Anyway, differently from Eq. (10.4), the pressure drop related irreversibility can still be accounted for by using the proposed Eq. (10.5). It is also noteworthy that, if the reactor pressure is increased, the activity coefficient of ammonia reduces while the respective coefficients of nitrogen and hydrogen increases, thus shifting the per-pass conversion to the product equilibrium. Pressure drop losses are generally around 5\% of total reactor losses [19] and have been reduced so far by using different radial-axial gas flow reactor configurations [95].

Figure 10.7 compares the chemical exergy consumed $\left(\Delta B^{C H}\right)$ and the exergy efficiency at which this exergy is converted into physical exergy $\left(\Delta B^{P H}\right)$ in the different beds of the 
various industrial ammonia units analyzed. The reactor bed conversion and the autothermal heat exchanger (ATHE) performance are also represented.

Fig. 10.7. Consumed-produced (CP) efficiency of the reactor beds, and its relation with the individual bed and overall reactor conversion and the autothermal heat exchanger (ATHE) performance.

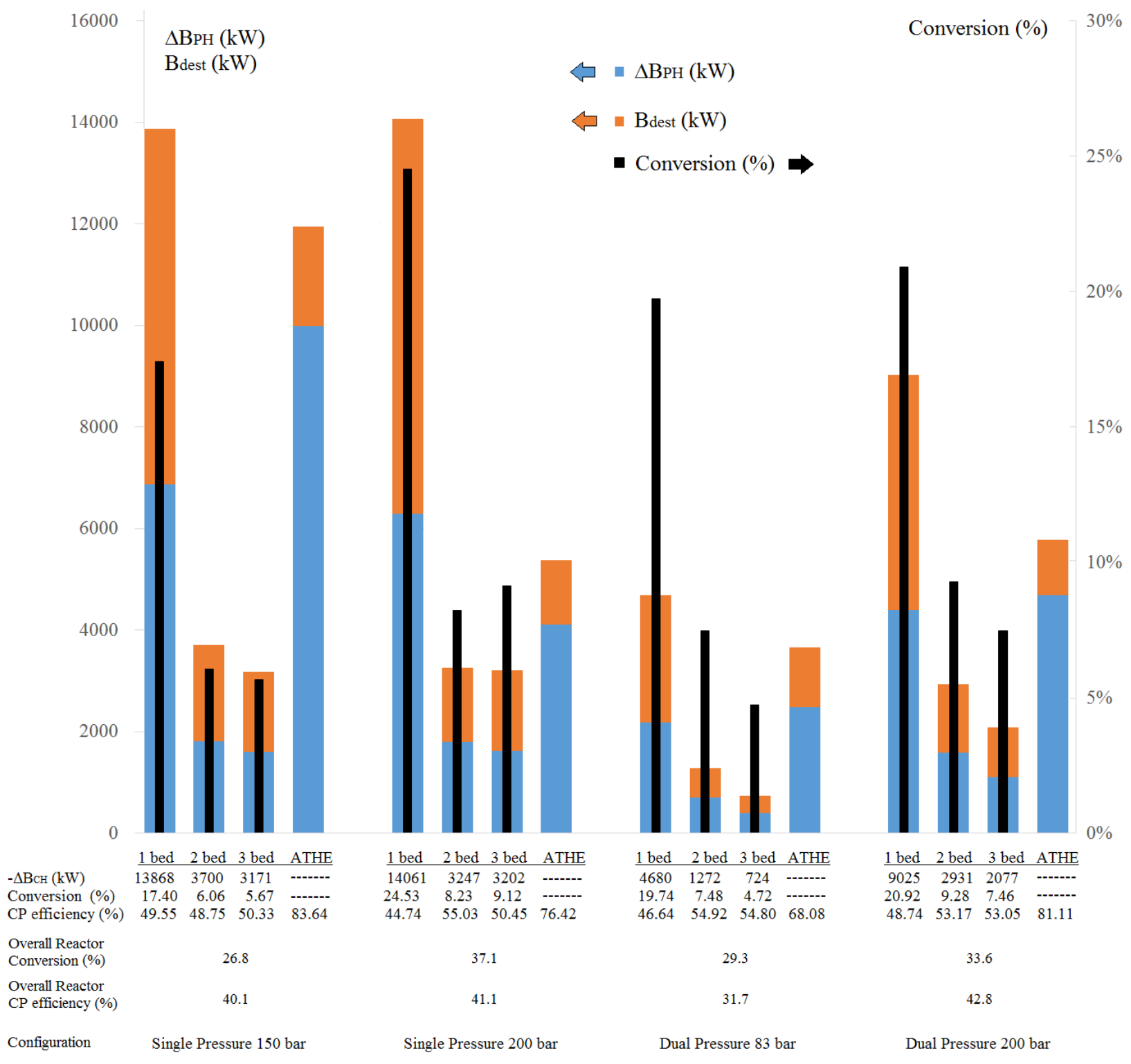

Source: Author.

Regarding the first reactor bed, the conversion in the SP200 setup is $29 \%$ higher than that of the SP150 scenario, whereas the exergy consumption is slightly higher and the irreversibility is somehow lower. Higher reaction conversions are also attained for the subsequent catalyst beds of SP200 at slightly higher exergy efficiency. The most remarkable difference comes from the ATHE responsible for preheating the inlet stream of the first reactor bed. For SP150 configuration, the exergy destroyed in the ATHE is almost twofold due to an increased molar flow in the reactor feed (27\%). The lower conversion and increased recycle rates of SP150 are indeed responsible for the largest heat transfer irreversibility associated. In the case of the 
DP configuration, both the reactor conversion and the chemical exergy consumption are reduced if compared with the SP200 case. As a result, the large driving forces are reduced while improving the exergy efficiency of the exothermic beds.

Figure 10.8 and 10.9 show the breakdown of the percentage and detailed exergy destruction rate for representative components of the industrial ammonia production units. According to Fig. 10.8, the introduction of an additional once-through reactor in the DP system increases the share of the reaction-based irreversibility about 16\%, if compared with SP150 and SP200 configurations.

Fig. 10.8. Exergy destruction breakdown for representative components of the ammonia production units: (a) single pressure 150 bar, (b) single pressure 200 bar, (c) dual pressure

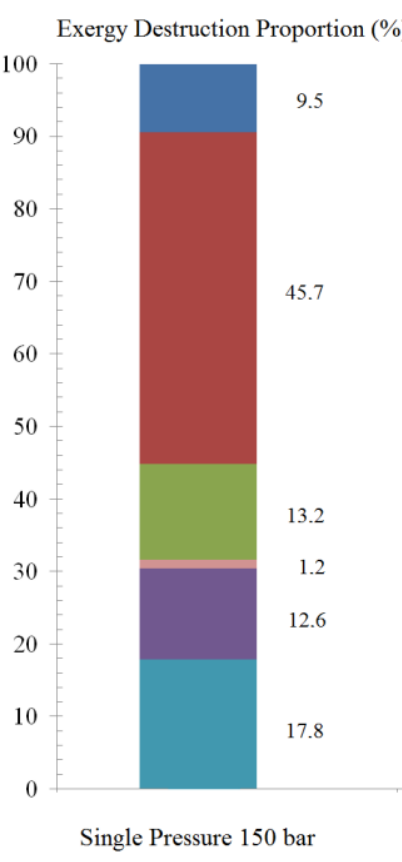

(a) configuration.

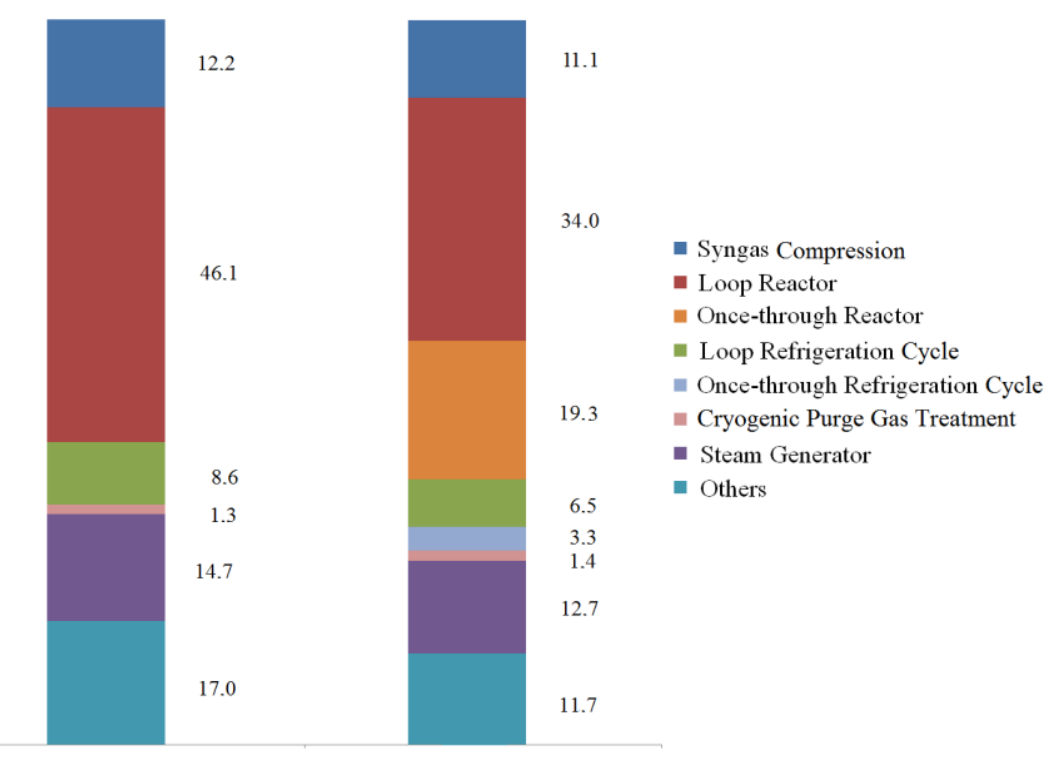

Single Pressure 200 bar

(b)
Dual Pressure Configuration

(c)

Source: Author.

However, according to Fig. 10.9, despite the increment of the share of irreversibility due to the introduction of an separate once-through reaction section, the overall exergy destruction rate in the DP system is much lower (3.7-15\%) than in the SP configurations. Consequently, the effect of locally increasing the exergy destruction rate in the reactive components of the DP configuration is offset by a more uniform distribution of the irreversibility within the whole unit. This is a consequence of the application of the Counteraction principle to a chemical conversion process of a mixture that reduces its volume. Moreover, the incremental pressures reduce the power consumption by lowering the makeup syngas compression and the refrigeration duty (e.g., avoidable losses), as more efficient conversions are carried out at lower pressures. 
Fig. 10.9. Exergy destruction of representative components of the ammonia production units: (a) single pressure 150 bar, (b) single pressure 200 bar, (c) dual pressure configuration.

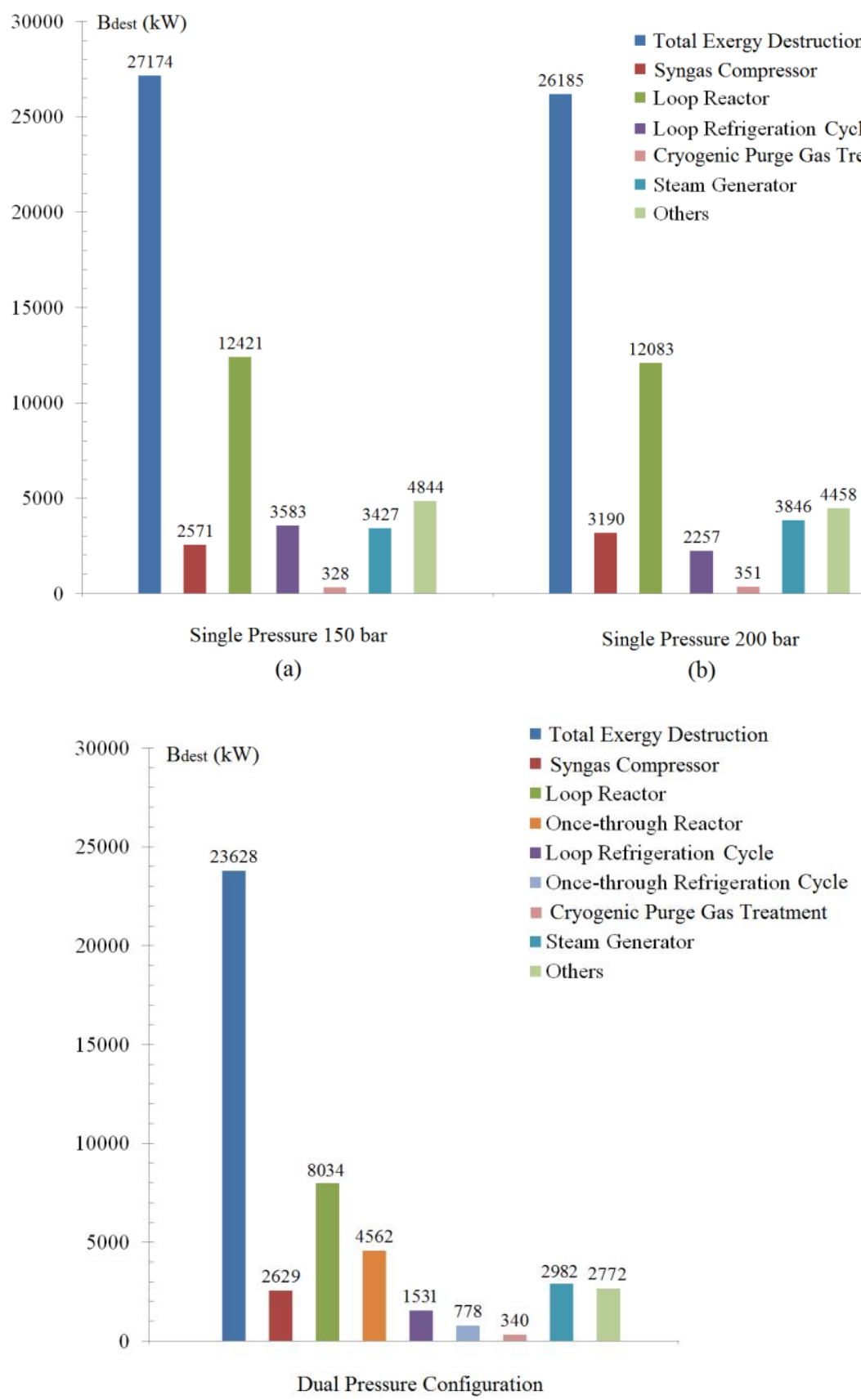

(c)

Source: Author.

Alternatively, better conversions at lower pressures and higher overall reactor efficiencies could be obtained if a set of low temperature catalysts were developed and suitably distributed along the reactor beds. In this way, improved conversion rates at different bed temperatures and compositions could be obtained [5, 22, 41]. Notably, it would be also desirable to perform the reaction under resisted conditions [245], so that the chemical exergy can be maximally exploited. Unfortunately, this is a practical shortcoming in large-scale industrial applications, 
because it would require slow enough, quasi-reversible conditions [169] or a strict coupling between thermal and chemical driving forces, which is often impracticable [286].

\subsection{Combined exergy and energy integration analysis}

Since the saturated steam is generated at a temperature of only $310^{\circ} \mathrm{C}$, lower than that of the ammonia reactor effluent $\left(>430^{\circ} \mathrm{C}\right)$, the heat recovery steam generator (HRSG) contributes to the degradation of the chemical exergy of the reactants. This condition is better appreciated in the Carnot composite curves (CCC, left) and Carnot grand composite curves (CGCC, right) shown in Figs. 10.10a-c, for the heat exchanger network (HEN) of the industrial ammonia production units analyzed.

Firstly, a wider span of the enthalpy flow rate is observed in the CCC of the SP150 setup, indicating larger heat transfer rates between the process streams and, thus, increased exergy destruction rates in the HEN, compared to the SP200 and DP arrangements. Furthermore, according to Figs. 10.10a-b, the plateaus, corresponding to the level of temperature of the evaporator and the condenser in the refrigeration cycle, point out a source of irreversibility in the ammonia condensation and separation processes. The latter can be tackled by carrying out the refrigeration process at different pressures, as in the case of the DP ammonia loop configuration (Fig. 10.10c). On the other hand, a large thermodynamic potential can be envisaged in all the cases, regarding the exploitation of the self-sufficient zones represented in the CGCCs (Figs.10.10a-c, right).

Actually, in order to profit from this potential, some authors proposed the expansion of the ammonia reactor effluent in gas expanders, in lieu of the typical utilization of steam boilers [239]. As a result, the authors reported that, although this modification does not necessarily render the ammonia production unit self-sufficient in terms of power demand, it is claimed that expansion process would profit in more efficient way the thermo-mechanical exergy embodied in the reactor effluent. Since the exergy of the heat is transformed directly into work, the exergy losses related to the heat transfer driving forces and associated to the condensation of the steam turbine could be avoided [239]. As a conclusion, non-conventional approaches are reportedly worthy to be explored, considering the current limited room for improvement in the reactive components.

In fact, the highest exergy saving potentials are expected from the reevaluation of the high temperature gas-gas heat exchangers, responsible for 1500 to $2800 \mathrm{~kW}$ of exergy destroyed, followed by the improvement of the syngas compression train [274] and a reduction of the costly refrigeration. Consequently, the current energy-intensive operationg of the technical process is mainly due to the large exergy consumption of the makeup syngas compression, refrigeration and circulation systems together (10.6-14.4 MW). A benefit of the DP system is that the makeup gas is incidentally refrigerated in the way to the high pressure loop. Since the compressor operates at lower temperatures than in the SP200 case, less exergy is consumed and destroyed, even compensating the pressure drop throughout the additional once-through converter [221]. 
Fig. 10.10. Carnot composite curves (CCC; left) and Carnot grand composite curves (CGCC; right) for the ammonia production units. The Carnot factor is calculated as $\left(T_{o} / T\right)-1$ if $T<T_{o}$.
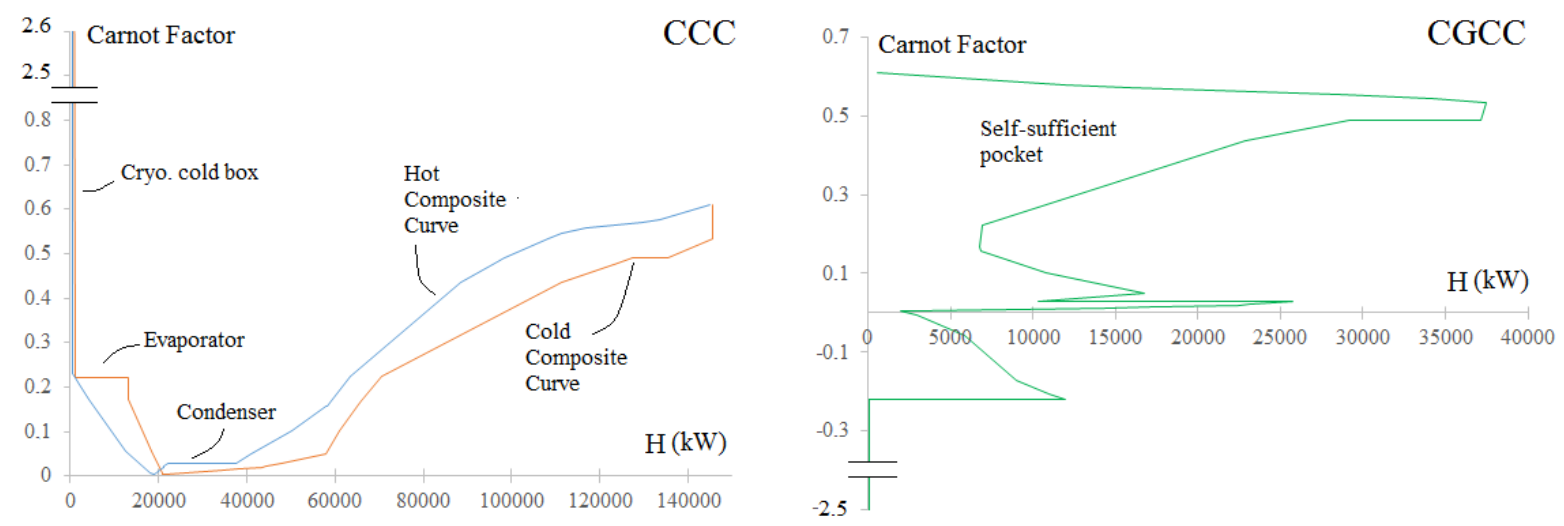

(a) Single pressure 150 bar
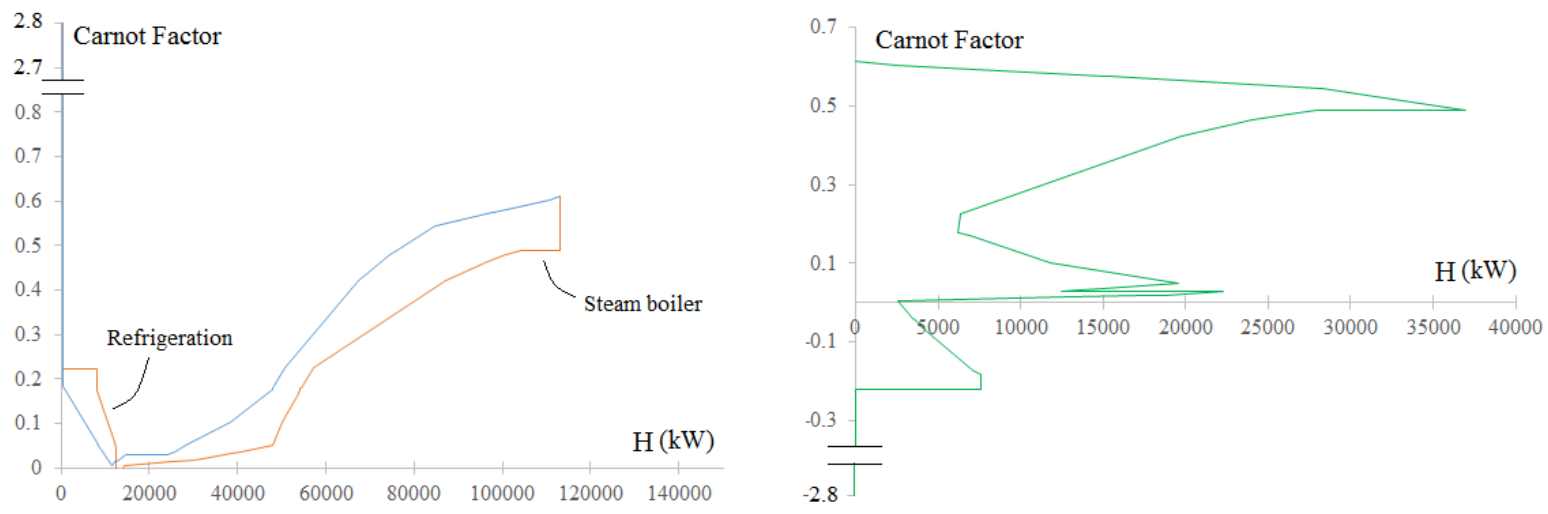

(b) Single pressure $200 \mathrm{bar}$
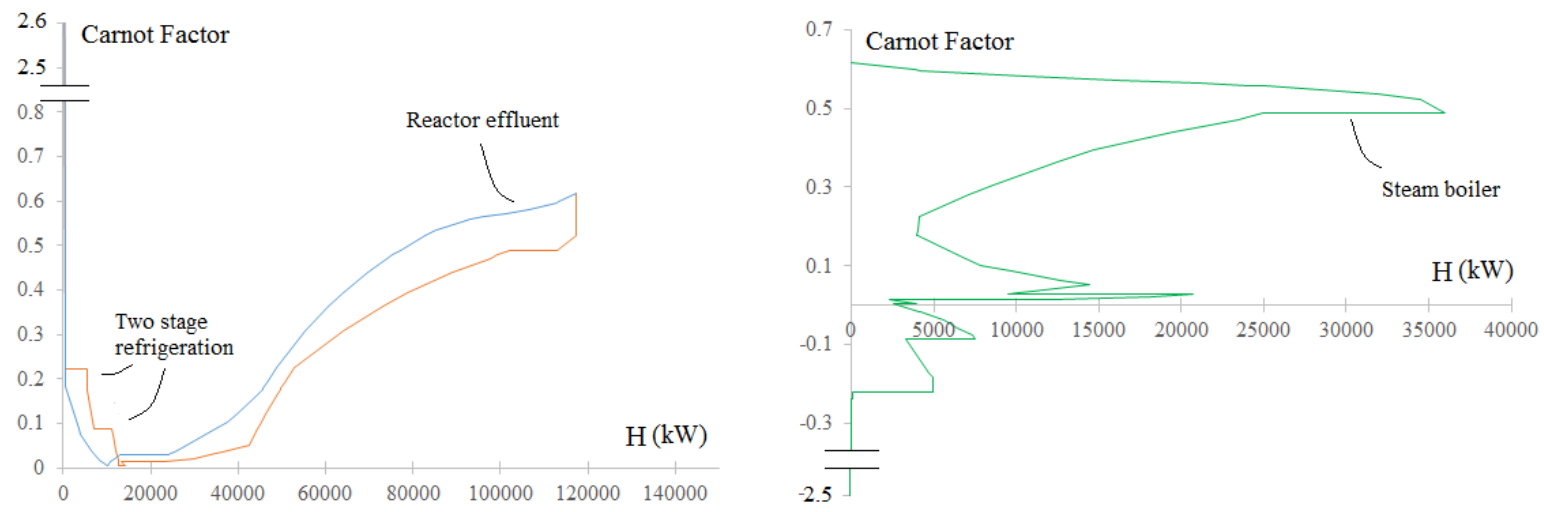

(c) Dual pressure configuration

Source: Author.

Finally, it should be noticed that a large amount of the power consumed eventually ends up in the cooling water and dissipated to the environment. However, both thermodynamic and economic restrictions often render any attempt to recover this low-grade heat exergy $(<360$ $\mathrm{K})$ impracticable, as summarized in Table 10.5: 
Table 10.5. Cooling duty and total exergy associated at selected heat transfer temperatures.

\begin{tabular}{lccc}
\cline { 2 - 4 } & SP150 & SP200 & DP \\
\hline Cooling duty $(\mathrm{kW})$ & 34,120 & 31,639 & 28,373 \\
\hline Exergy associated at 360 K (kW) & 1,902 & 2,114 & 1,720 \\
\hline Exergy associated at 300 K (kW) & 210 & 195 & 175 \\
\hline Exergy dissipation share & & & \\
\hline Compressor intercooling (\%) & 42.0 & 51.2 & 46.0 \\
\hline Refrigeration condenser (\%) & 27.4 & 15.7 & 21.8 \\
\hline CW for first separator $(\%)$ & 30.6 & 33.1 & 32.2 \\
\hline
\end{tabular}

Thus, since these losses are not anymore recovered, they are considered as exergy destroyed. As a final remark, it is pointed out that the intercooling system of the multistage syngas compressor accounts for $790-1082 \mathrm{~kW}$ out of $1700-2100 \mathrm{~kW}$ of the exergy destroyed through the cooling utility, as shown in Table 10.5 (i.e. 42-51\%). This loss could only be eliminated if ammonia loops were redesigned to operate at lower pressure and temperatures. Moreover, refrigeration is expensive and its use should be minimized favoring the use of cooling water when applicable.

\subsection{The role of the purge gas treatment unit}

For the sake of comparison, the results of this chapter are compared against the overall exergy destruction found for the best case scenarios of the SP150 and SP200 setups, reported in Chapter 9 for which the valuable hydrogen contained in the purge gas was neither recovered nor recycled to the ammonia loop.

As expected, by extending the boundaries of the thermodynamic system to encompass a cryogenic purge gas treatment unit, the overall irreversibility of the ammonia synthesis unit increases about 2.2 and $2.4 \%$ for the SP150 and SP200 configuration, respectively. However, by considering the total exergy destruction rate in the DP configuration $(23,628 \mathrm{~kW})$, this value is found to be considerably lower than those reported for those best case scenarios without purge gas recovery, or $13.0 \%$ and $9.8 \%$ lower, respectively. This highlights the potential benefits of using a cryogenic purge gas treatment unit in terms of exergy destruction minimization. It must be pointed out that the nitrogen recovery is less attractive than hydrogen, since hydrogen recovery along with syngas production with ratios of $\mathrm{H} / \mathrm{N}<3.0$ allow transferring some of the primary reformer duty to the secondary reformer [33]. Consequently, the current bottlenecks of the large capacity reformers, used for hydrogen production in MEGAMMONIA @ plants, can be partly overcome [35].

\subsection{Final considerations}

Exergy analysis is used to demonstrate the application of the Counteraction principle for reducing the irreversibility arisen from the lower conversions in the ammonia synthesis. By introducing an additional once-through reactor operating at lower pressure, the exergy losses have been driven down, whereas the amount of waste heat recovered has been increased. As a result, the introduction of a dual pressure process increases the overall exergy performance of 
the ammonia unit in more than $10.1 \%$. Moreover, the circulation rates are also reduced, which in turn cuts down the exergy consumption in the condensation and refrigeration systems and impacts the equippment sizing and the overall production cost. The syngas compressor, ammonia converter, the waste heat recovery and ammonia refrigeration systems are found to be responsible for about $80-86 \%$ of total irreversibility in the ammonia loop, which varies from 23.8MW for DP design up to 27.2MW for SP150 design.

Other non-conventional approaches are still worthy to be explored, considering the current limited room for improvement in the reactive components. Among the largest energy saving potentials are the reevaluation of the high temperature gas-gas heat exchangers, and the compression train and refrigeration systems. The challenge is thus to keep the rate of reaction at acceptable levels, whereas the effective driving forces are lowered and the net power input is reduced.

Regarding the use of non-conventional approaches, in the last chapters of this thesis, a combined exergy analysis and energy integration study attempts to determine the best operating parameters and the operating modes that minimize the reduction of the syngas and ammonia production costs, by using biomass as the energy source. The effect of the partial and total substitution of the natural gas by these renewable resources, in terms of both efficiency and environmental impact, are discussed. In the last chapter, the upgrade of the organic residues of the sugar cane biorefineries is briefly evaluated to determine the best options of upgraded products, either synthetic natural gas or hydrogen, intended to be used as feedstock in a separate ammonia production unit. 
CHAPTER 11

\section{SYNGAS AND AMMONIA PRODUCTION FROM BIOMASS}

The pursuit of alternative energy sources for the synthetic fertilizers sector has recently earned renewed interest due to increasing concerns regarding the marked dependence of the world economy on non-renewable energy resources and also motivated by the more stringent environmental regulations. The decarbonization of this productive sector, responsible for about $2 \%$ of the global energy consumption [42], might help not only improving the carbon footprint of these fundamental commodities, but also reducing their dependence on the international market prices, traditionally dominated by the main natural gas producers.

Accordingly, in this chapter, the use of biomass gasification for partially or totally replacing the use of methane in the integrated syngas and ammonia production plant is compared with the performance of the conventional route. However, by undertaking novel designs, additional or totally different demands can be created. Consequently, the optimal integration approach between the new chemical plant and the alternative utility systems must be updated, so that the power and steam requirements remain satisfied. To this end, a systematic framework that allows selecting the most suitable utility systems (refrigeration, waste heat recovery and cogeneration) that satisfy the minimum energy requirements with the lower operating cost, is adopted. Moreover, exergy analysis is used to identify the potential improvements that may remain hidden to the energy integration analysis.

\subsection{Biomass as a source of hydrogen for ammonia production}

The global supply of nitrogen fertilizers faces an increasing trend estimated in 176.5 million of tons in 2018 [45]. However, in the same year, the production of these inorganic chemicals, of which ammonia is an staple intermediate feedstock, involved an intensive generation of atmospheric emissions of about 353 million $\mathrm{t}_{\mathrm{CO} 2}$. In order to palliate the environmental impacts that nitrogen fertilizers production is responsible for, several efforts have been addressed to incentivize the partial or total decarbonization of the ammonia supply chain. Alternative pathways of hydrogen production, such as solar or wind energy, used for electrolyzing the water and for separating the nitrogen from air (cryogenic distillation, selective membranes, etc.), have been widely proposed [243]. However, as concerns the most promising alternative energy resources for hydrogen production, the thermochemical conversion routes of biomass have shown to be the most interesting opportunity to capitalize on the underexploited biomass potential in tropical countries [30].

The earliest example of pressurized steam/oxygen-blown, fluidized-bed biomass gasification technology (140 MW) coupled to an ammonia synthesis unit used to run on peat and saw dust (originally designed for heavy oil gasification) in the Kemira Oy ammonia plant in Oulu, Finland, during the late eighties [287]. Eventually, due to its limited competitiveness in a volatile ammonia price market, the plant had to be shut down [288]. Hitherto, the main 
drawbacks of the biomass-based ammonia synthesis remain related to the high investment risk, the biomass availability and the scale-up of the gasification concept [289], as it may not fully compete with current fossil-based commercial production capacities well above $1000 \mathrm{t}_{\mathrm{NH} 3} /$ day. For the sake of comparison, 'handier' coal feedstock generally allows for gasification capacities from ten to one hundred times larger $\left(>2000 \mathrm{MW}_{\text {th }}\right)$ than those of biomass gasification plants $\left(<170 \mathrm{MW}_{\text {th }}\right)$.

In spite of this limitations, it is expected that larger pressurized biomass gasifiers may help increasing the performance of biomass conversion processes [289]. Furthermore, it must be acknowledged that large scale biomass conversion systems are not without precedent, especially in Brazil, where the estimated national production capacity of bagasse in sugarcane mills has already surpassed 190 million tons per year [230]. In fact, the largest sugarcane mills have throughputs between 0.5 to 1 million tons per year of bagasse, equivalent to the biomass required to operate a $1000 \mathrm{t} /$ day ammonia plant $\left(1.8-2.7 \mathrm{t}_{\text {Biomass }} / \mathrm{t}_{\mathrm{NH} 3}\right)$ [30, 289, 290], even when the productive season covers typically less than half of a year [130, 230].

Additionally, unlike the well-established infrastructure and the mature technology of the conventional routes of ammonia production based on fossil fuels, better solutions for the supply management, energy conversion processes, as well as enhanced reliability and profitability of the renewable resources are still necessary to guarantee their competitiveness [289]. Fortunately, the technology is revisited when the access to fossil resources such as natural gas and coal is limited, mainly due to scarcity or unstable international prices [291]. Moreover, more stringent environmental regulations along with increasing concerns about the marked dependence on imported fertilizers, which renders the nation vulnerable to volatile international markets, have led the government and research institutions to seek for the utilization of the readily available bagasse feedstock in the production of hydrogen and other commodities traditionally based on fossil fuels [292-294]. Certainly, there are well-known applications for the residual bagasse, but gasification represents a more efficient and environmentally friendly alternative compared to current direct bagasse combustion. The economic aspects are also relevant, as the average price of bagasse oscillates around 15-20 USD per ton of wet biomass, $40 \%$ lower than the cost of the amount of available residue in the United States [295]. It makes bagasse one of the cheapest lignocellulosic agricultural byproduct [230, 296], not to mention that the transportation costs represent also a competitive advantage, as the bagasse is concentrated and available on site [295].

Some authors performed the energy and environmental analysis of the ammonia production via biomass gasification [115, 290], either considering its integration to other chemical facilities (e.g. Kraft pulp mill) or conditioning its operability to an external electricity supply. However, a more interesting scenario in the middle term should rather consider the energy, economic and environmental benefits of the use of the fairly available and affordable sugarcane bagasse to partially or totally replace the natural gas in the nitrogen fertilizers production facilities (FAFEN). This study is motivated by a broader assessment that projects to enable an integration of the Sao Paulo state natural gas distribution system to the State's sugar cane mills, as the former strategically goes through the area where the mills settle. 
Accordingly, in the following sections, by applying a systematic approach, an exergy and energy integration assessment of the most suitable technologies and parameters that minimize the utility systems operating costs of a conventional and various alternative syngas and ammonia production plants is discussed.

\subsection{Natural gas and Biomass-based Ammonia Production Plants}

Figure 11.1 shows the simplified layout of the conventional configuration of the ammonia production plant analyzed in previous chapters. Therein, a mixture of saturated natural gas (steam-to-carbon ratio, S/C 3:1) is firstly fed to an adiabatic prereformer, where heavier hydrocarbons are partially decomposed into hydrogen and carbon oxides at relatively lower temperatures $\left(<600^{\circ} \mathrm{C}\right)[69]$. Next, an endothermic primary reformer (SMR, 700-800 $\left.{ }^{\circ} \mathrm{C}\right)$ in series with an autothermal reformer (ATR, $1000^{\circ} \mathrm{C}$ ) is used to convert most of the methane feed [231]. The primary reforming is by far the most exergy-intensive processes, needing to outsource the energy required from a radiant furnace that sustains the reactions occurring in the catalytic tubes [72]. Meanwhile, in the secondary reformer, a portion of the reformed mixture is burnt with air in order to provide the energy to the endothermic reactions as well as to attain a $\mathrm{H}_{2} / \mathrm{N}_{2}$ ratio $3: 1$, suitable for ammonia production [25]. Downstream of the reforming sections, a set of high temperature $\left(350^{\circ} \mathrm{C}\right)$ and low temperature $\left(200^{\circ} \mathrm{C}\right)$ water gas shift reactors are used to further increase the hydrogen production by using the water and $\mathrm{CO}$ content in the reformed gas [25].

Fig.11.1. Conventional natural gas-fueled ammonia production plant.

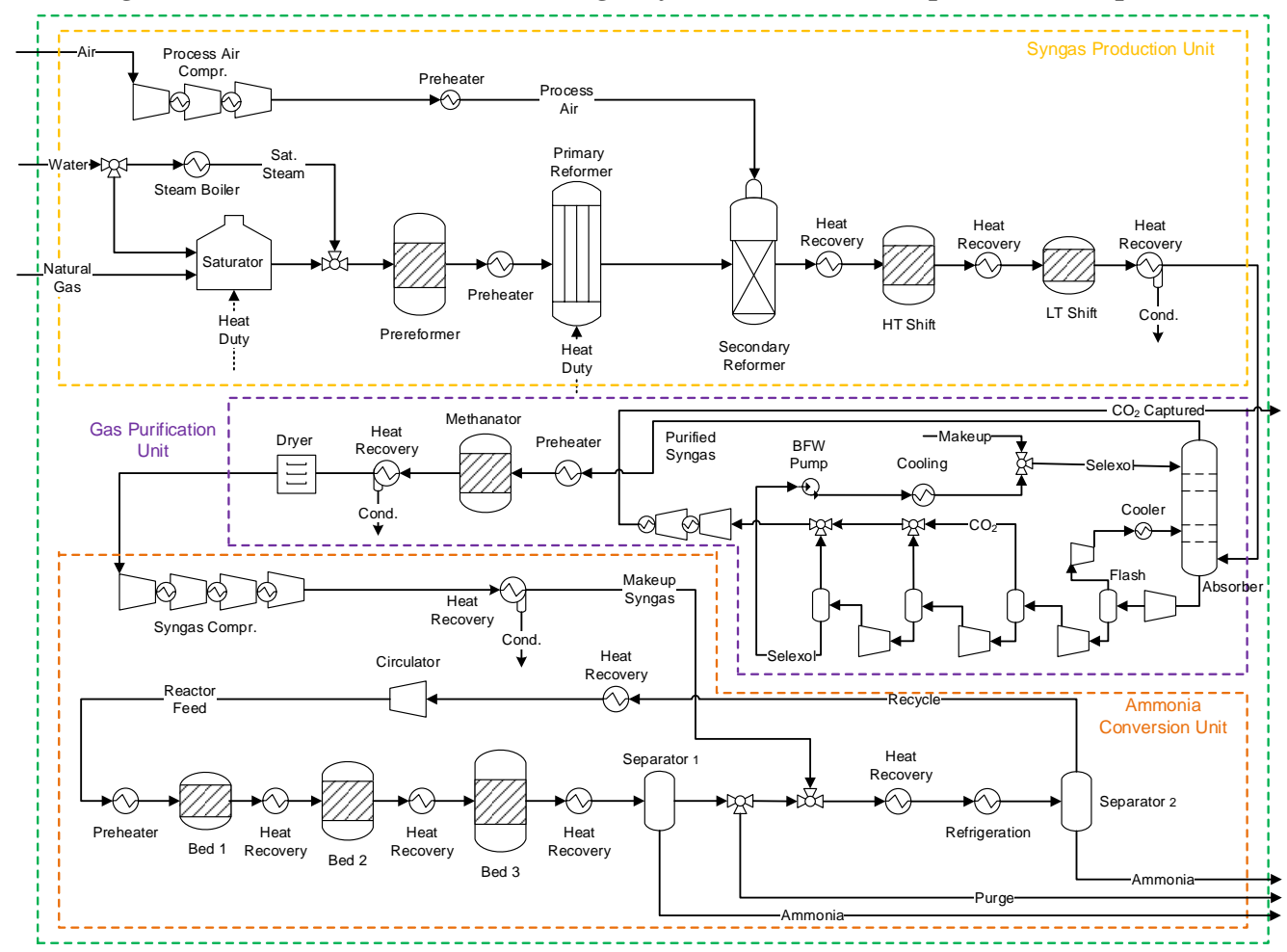

Source: [231].

Henceforth, a purification section, composed of a physical absorption unit (DEPG) and a methanation system, is used to remove the carbon oxide components present in the syngas 
produced [206]. The purified syngas is then compressed up to 200 bar and fed to a synthesis loop, where the $\mathrm{H}_{2} / \mathrm{N}_{2}$ mixture is partially converted into ammonia through a series of catalytic beds indirectly cooled, in order to shift the equilibrium conversion towards higher ammonia yield [226]. The reactor performance and, consequently, the loop efficiency are affected not only by the reactor operating conditions (feed pressure, temperature and composition, heat removal and catalysts design), but also by the amount of inerts (i.e., argon and methane) and ammonia recycled. Accordingly, most of the produced ammonia must be separated by using cooling water $\left(25-40^{\circ} \mathrm{C}\right)$ and a vapor compression refrigeration system ($30^{\circ} \mathrm{C}$ ). Moreover, in order to prevent the built up of inerts in the loop, a portion of the hydrogen-rich gas is continuously purged, whereas the rest of the unreacted mixture is recycled to the converter beds.

Meanwhile, Fig. 11.2 shows the process superstructure used to determine the performance of the ammonia production by using the gasification of sugar cane bagasse. The bagasse ultimate composition (mass) is set as $46.70 \% \mathrm{C}, 6.02 \% \mathrm{H}, 44.95 \% \mathrm{O}, 0.17 \% \mathrm{~N}, 0.02 \% \mathrm{~S}$ and $2.14 \%$ Ash, whereas proximate analysis (mass) is considered as $50 \%$ moisture (as-received), $14.32 \%$ fixed carbon, $83.54 \%$ volatiles, and ash in balance [294]. The large moisture content of bagasse is reduced to about $10 \%$ in a rotary dryer that consumes the power and heat supplied by the utility systems, as well as the heat recovered from the gasifier effluents [297]. Bagasse must be also chipped by means of an energy intensive process that may require between 1 to $3 \%$ of the total energy embodied (LHV basis) in the biomass consumed [290].

In the gasification step, the carbonaceous materials in the bagasse are converted into a gaseous mixture called syngas, rich in $\mathrm{CO}, \mathrm{H}_{2}, \mathrm{CO}_{2}$ and $\mathrm{CH}_{4}$, among other components [297]. This gas can be used as process feedstock or can even provide the combined heat and power required by the chemical processes, more efficiently than biomass combustion. It is thus not surprising that gasification has gained renewed interest worldwide mainly for the production of chemicals including fertilizers, liquid fuels as well as power and gaseous fuels [291]. However, the variable biomass composition and its relatively high moisture content, along with the complex gasification operation conditions strongly influence the process yield, the energy requirement and, consequently, the efficiency of the chemical process [298, 299]. 
Fig. 11.2. Superstructure used in the process synthesis and optimization of the utility systems of the biomass-based ammonia production plant.

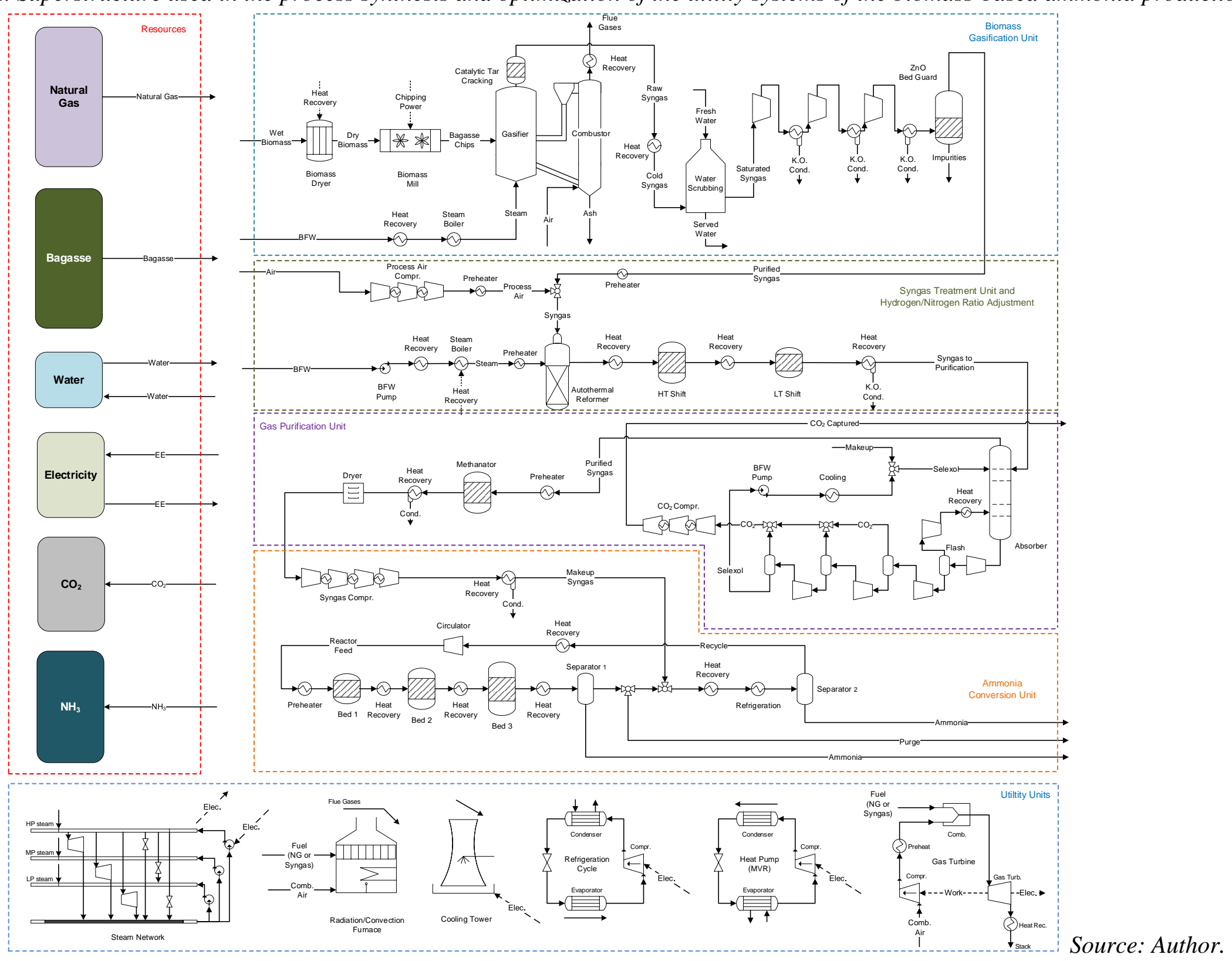


The Battelle Columbus Laboratory (BCL) indirect gasifier operating at atmospheric pressure, shown in Fig. 11.2, avoids the dilution with nitrogen of the syngas produced, as the combustion and gasification processes occur in a separate double column system [300]. Steam is used as the gasification medium (steam-to-biomass ratio 0.75), whereas the combustion with air of a fraction of the char produced in the bagasse pyrolysis step supplies the heat required by the endothermic drying, pyrolysis and reduction reactions. After the syngas produced leaves the gasifier, a thermal catalytic cracking of the tar produced is performed [32]. Then, the syngas is cooled down to $400^{\circ} \mathrm{C}$ and scrubbed with water, in order to remove the impurities that may affect the downstream equipment, and then compressed to 35 bar. As syngas still contains methane and carbon monoxide, an autothermal reforming and a water gas shift reaction process, analogous to that described in the conventional process of ammonia production, help increasing the hydrogen content, simultaneously producing more $\mathrm{CO}_{2}$. Henceforth, the hydrogen-rich syngas can be either sent to purification for ammonia production, or used in the utility system to generate electromechanical power or to supply the heat exergy to the chemical process.

Also according to Fig. 11.2, the various alternatives of utility systems available for supplying the power and heat demands of the chemical plant include syngas or natural gas-fired furnaces and gas turbine systems, a cooling tower, a vapor-compression refrigeration system, as well as the resources consumed (e.g. natural gas, biomass, water, electricity). The cooling water inlet and outlet temperatures are set as $40^{\circ} \mathrm{C}$ and $25^{\circ} \mathrm{C}$, respectively, and a cooling tower power-to-cooling duty ratio of $0.021 \mathrm{~kW}_{\mathrm{el}} / \mathrm{kW}_{\text {th }}$ is assumed [159]. The refrigeration system is, in turn, defined in terms of its exergy efficiency $(50 \%)$ and the evaporator and condenser temperatures [231]. Additionally, the waste heat available throughout the chemical processes is recovered by using an integrated steam network, so that the amount of fuel and cooling water, necessary to balance the power and steam demands of the chemical plant (feed preheating, endothermic reactions), can be reduced [231]. The steam network superstructure is composed of a set of superheated steam headers and draw-off levels of steam. The choice of the optimal levels of steam generation is performed by examining the profile of the Grand Composite Curve (GCC) of the chemical process [227]. In this way, more power can be generated by optimally profiting the thermodynamic potential of the waste heat exergy via backpressure and condensation steam turbines.

In order to perform the material and energy balances of the bagasse pretreatment processes (drying and chipping), a FORTRAN subroutine is implemented in Aspen ${ }^{\circledR}$ Plus. The amount of moisture removed in the rotary dryer $\mathrm{m}_{\mathrm{H}_{2} \mathrm{O} \text { removed }}(\mathrm{kg} / \mathrm{h})$ is calculated in terms of the initial bagasse moisture $\psi_{\mathrm{H}_{2} \mathrm{O} \text {, As-received }}(\%)$, the desired bagasse moisture at the inlet of the gasifier $\psi_{\mathrm{H}_{2} \mathrm{O} \text {, Dried bagasse }}(\%)$ and the feed mass rate of the wet bagasse, $\mathrm{m}_{\text {Wet bagass }}(\mathrm{kg} / \mathrm{h})$, according to Eq.(11.1):

$$
\mathrm{m}_{\mathrm{H}_{2} \mathrm{O} \text { removed }}=\left(\psi_{\mathrm{H}_{2} \mathrm{O} \text {, As-received }}-\frac{1-\psi_{\mathrm{H}_{2} \mathrm{O} \text {, As-received }}}{1-\psi_{\mathrm{H}_{2} \mathrm{O} \text {, Dried bagasse }}} \times \psi_{\mathrm{H}_{2} \mathrm{O} \text {, Dried bagase }}\right) \times \mathrm{m}_{\text {Wet bagasse }}
$$


On the other hand, gasification is modeled and simulated as a series of interrelated drying, pyrolysis, reduction and combustion processes, as shown in Fig.11.3.

Fig. 11.3. Modeling and simulation steps of the twin circulated bed BCL bagasse gasifier.

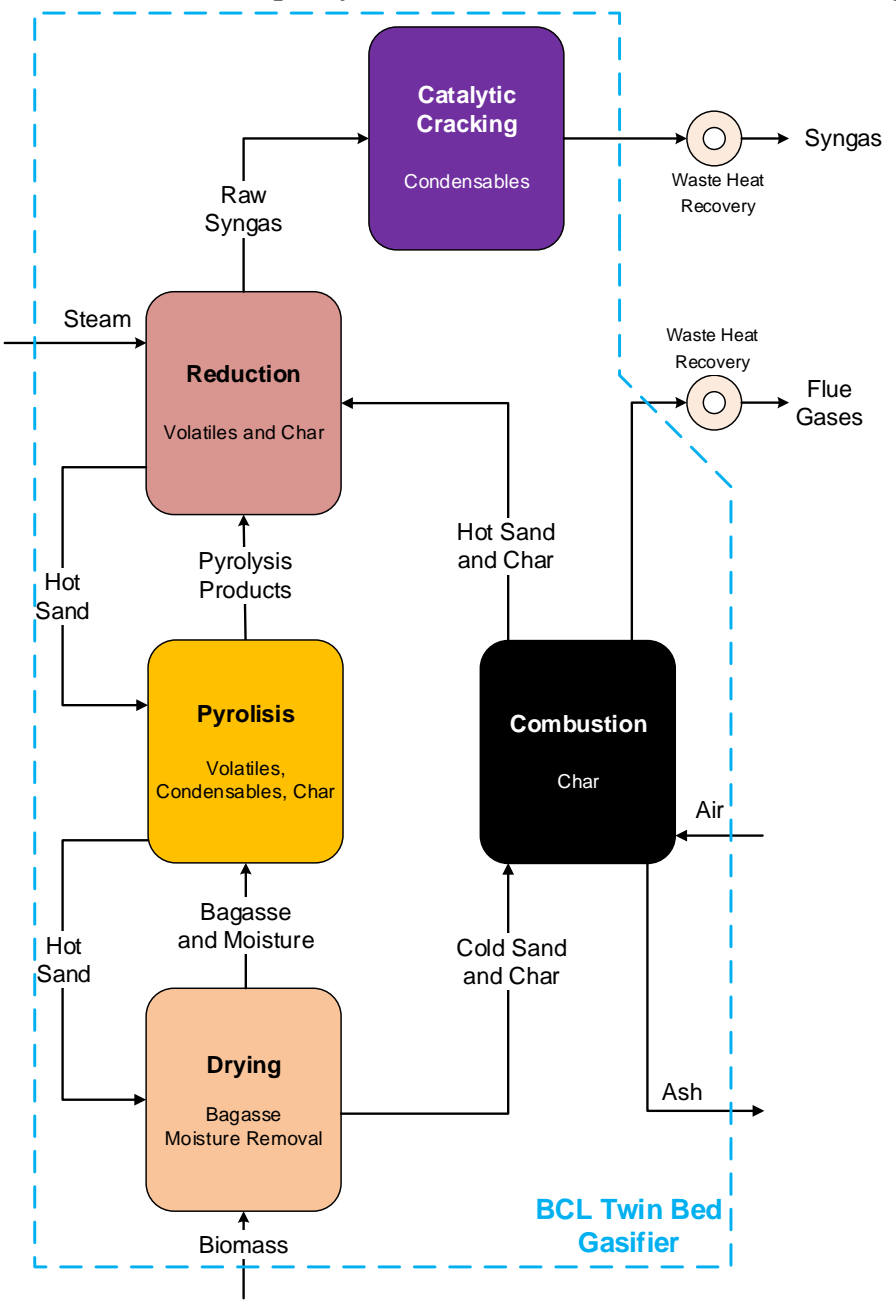

Source: Author.

The actual mass yields $\left(\mathrm{y}_{j}\right)$ of volatiles, condensables, and solids in the pyrolysis reaction step, as well as the gaseous volumetric fractions $v_{i}$ of the hydrogen, carbon monoxide, carbon dioxide and methane produced are calculated by means of a set of empirical correlations reported in the literature as a function of the reaction temperature $T$, Eqs.(11.2-11.8) [301, 302]. To this end, an Aspen-embedded Excel ${ }^{\circledR}$ spreadsheet calculator has been used to perform the atomic balance of species $(\mathrm{C}, \mathrm{H}, \mathrm{O}, \mathrm{N}$, and $\mathrm{S}$ ) comprised in the volatiles, condensables, char and ash produced in the pyrolysis section:

$$
\begin{array}{ll}
\mathrm{y}_{\text {Gas }}=311.10-351.45\left(\frac{T}{500}\right)+121.43\left(\frac{T}{500}\right)^{2} & \text { Gases } \quad \text { (\%wt. of dry biomass) } \\
\mathrm{y}_{\text {Char }}=-15.03+50.58\left(\frac{T}{500}\right)-18.09\left(\frac{T}{500}\right)^{2} & \text { Char (\%wt. of dry biomass) } \\
\mathrm{y}_{\text {Tar }}=-196.07+300.86\left(\frac{T}{500}\right)-103.34\left(\frac{T}{500}\right)^{2} & \text { Tar (\%wt. of dry biomass) }
\end{array}
$$




$$
\begin{array}{lll}
\mathrm{v}_{C O}=240.53-225.12\left(\frac{T}{500}\right)+67.50\left(\frac{T}{500}\right)^{2} & \mathrm{CO} \quad \text { (\%vol. of gas) } \\
\mathrm{v}_{\mathrm{CO}_{2}}=-206.86+267.66\left(\frac{T}{500}\right)-77.50\left(\frac{T}{500}\right)^{2} & \mathrm{CO}_{2} \quad \text { (\%vol. of gas) } \\
\mathrm{v}_{C H_{4}}=-168.64+214.47\left(\frac{T}{500}\right)-62.51\left(\frac{T}{500}\right)^{2} & \mathrm{CH}_{4} \quad \text { (\%vol. of gas) } \\
\mathrm{v}_{H_{2}}=234.97-257.01\left(\frac{T}{500}\right)+72.50\left(\frac{T}{500}\right)^{2} & \mathrm{H}_{2} \quad \text { (\%vol. of gas) }
\end{array}
$$

Additionally, aiming to correct the underestimation of the tar and methane produced (a wellknown shortcoming of the non-stoichiometric equilibrium methods [297]), the approach-toequilibrium temperatures for the char gasification and water gas shift reactions are adjusted to reflect the actual composition of the syngas produced in the BCL gasifier [303, 304]. Meanwhile, the ratio of specific chemical exergy to the lower heating value is calculated by means of the correlation proposed by Szargut et al [151], for solid fuels with the specified mass ratios, Eq.(11.9):

$$
\beta=\frac{b^{C H}}{L H V}=\frac{1.0438+0.1882 \cdot \frac{y_{H}}{y_{C}}-0.2509\left(1+0.7256 \cdot \frac{y_{H}}{y_{C}}\right)}{1-0.3035 \cdot \frac{y_{O}}{y_{C}}}
$$

where the bagasse lower heating value ( $\mathrm{LHV}, \mathrm{kJ} / \mathrm{kg}$ ) is estimated based on the correlations reported by Channiwala et al. Eq.(11.10) [172]:

$$
L H V=349.1 y_{C}+1178.3 y_{H}+100.5 y_{S}-103.4 y_{O}-15.1 y_{N}-21.1 y_{\text {Ashes }}-0.0894 \cdot h l v \cdot y_{H}
$$

and $y_{i}$ are the mass fractions of carbon $(\mathrm{C})$, hydrogen $(\mathrm{H})$, sulfur $(\mathrm{S})$, oxygen $(\mathrm{O})$, nitrogen $(\mathrm{N})$ and ashes (A) in the dry biomass, and $h_{l v}$ is the enthalpy of evaporation of water at standard conditions $(2442.3 \mathrm{~kJ} / \mathrm{kg})$. The so-calculated lower heating value and chemical exergy of dry bagasse are equal to 17.3 and $19.5 \mathrm{MJ} / \mathrm{kg}$, respectively.

Some performance indicators for each ammonia plant configuration are proposed to allow for systematic comparisons between the different designed setups, based on totally and partially natural gas-fueled, and totally biomass fueled ammonia plants with and without electricity import. Table 11.1 compares the overall rational exergy efficiency, Eq. (11.11), with other exergy efficiency definition, Eqs.(11.12), proposed for evaluating the overall performance of chemical production plants [206]. It must be noticed that, the rational exergy efficiency is higher than the relative one as it accounts for the outlet exergy of other byproducts $\left(\mathrm{CO}_{2}\right.$, purge gas). 
Table 11.1. Plantwide exergy efficiency definitions of the ammonia production plants.

\begin{tabular}{lcl}
\hline Definition & Formula & Eq. \\
\hline Rational & $\eta_{\text {Rational }}=\frac{B_{\text {useful,output }}}{B_{\text {input }}}=1-\frac{B_{\text {Dest }}}{B_{\text {input }}}=1-\frac{B_{\text {Dest }}}{B_{C H_{4}}+B_{\text {Bagasse }}+B_{B F W}+W_{\text {Net }}^{\text {Import }}}$
\end{tabular}

$B=$ exergy rate or flow rate $(k W), B F W=$ boiler feedwater, Dest $=$ destroyed

\subsection{Optimization problem definition}

As it has been shown hitherto, ammonia production plants are designed in complex ways in which the chemical units and the processes streams are interrelated through recycle loops and an extensive waste heat recovery network. Moreover, as long as electricity can be imported from the grid, the trade-off between an additional fuel consumption in the cogeneration system and the electricity purchase, both used to supply the power demand of the whole plant, will be strongly influenced by the performance of the cogeneration and waste heat recovery systems [228], as well as by the ratio between the cost of the electricity and fuels consumed [168]. Furthermore, since the conventional process flowsheet is drastically modified when alternative energy resources are considered, additional or totally different demands may be created. This requires a complete redesign of the energy integration approach between the new chemical units and the redefined utility systems, so that the power and steam requirements remain satisfied.

For instance, biomass can be chosen to either totally or partially replace the natural gas as feedstock, as fuel or as both, opening an opportunity to the diversification of the input of these chemical systems, depending on the availability and cost of the energy resources. In this way, cheaper energy resources such as bagasse may be favored over more expensive energy demands [305]. Moreover, by importing electricity in lieu of generating it in the utility systems, the energy, economic and environmental impacts are transferred to the outside of the battery limits. Thus, depending on the natur of the electricity mix, it may bring more energy and environmental benefits than using natural gas [188]. All these new features render the determination of the optimality a cumbersome task. It must be also noticed that the energy integration method alone falls short to put on evidence the exergy destruction in the heat exchanger network and reveal the potential for reducing the inherent driving forces by rationally performing the waste heat recovery and power generation. Fortunately, the selection of the most suitable alternatives of a set of proposed energy technologies for the utility systems allows reshaping the integrated curves of the chemical process aiming to minimize the exergy destruction. This procedure relies on an efficient mathematical programming approach in which all the potential energy technologies, resources and production routes are included in a comprehensive superstructure.

By separating the chemical process simulation from the energy integration problem, the calculation of the mass and energy balances and the simulation of the complex energy conversion systems can be handled by using the Aspen ${ }^{\circledR}$ Plus modeler [119]. Meanwhile, the 
determination of the minimum energy requirements (MER) and the solution of the energy integration problem is performed by using the OSMOSE Lua platform developed by the IPESE group at the École Polytechnique Fédérale de Lausanne - EPFL, Switzerland [121].

In order to calculate the minimum energy requirement (MER), the contribution of each hot and cold streams to the overall heat balance is combined into the respective hot and cold composite curves [229]. These composite curves are shifted away from each other through a physical constraint, namely the minimum temperature approach $\Delta \mathrm{T}_{\min }$, so that reasonable heat transfer rates can be ensured. $\Delta \mathrm{T}_{\min }$ will depend on the nature of each stream [157]. Equations (11.13-11.15) shows the optimization problem set to find the MER:

$\min _{R_{r}} R_{N_{r}+1}$

Subject to

Heat balance of each interval of temperature $r \sum_{i=1}^{N} Q_{i, r}+R_{r+1}-R_{r}=0 \quad \forall r=1 . . N$

Feasibility of the solution

$$
\mathrm{R}_{r} \geq 0
$$

where $N$ is the number of temperature intervals defined by considering the supply and the target temperatures of the entire set of streams; $Q$ is the heat exchanged between the process streams $\left(Q_{\mathrm{i}, \mathrm{r}}>0\right.$ hot stream, $<0$ cold stream) and $R$ is the heat cascaded from higher $(r+1)$ and to lower $(r)$ temperature intervals $(\mathrm{kW})$.

This framework allows to determine the most suitable utility systems (steam network, refrigeration system, heat pump, cogeneration system) and their operating conditions, that satisfy the minimum energy requirement (MER) with the lowest resources consumption (water, natural gas and bagasse) and optimal operating cost [229]. The computational framework manages the data transfer with the ASPEN Plus® software and builds the mixed integer linear programming (MILP) problem described in the Eqs.(11.16-11.20) that minimizes the operating cost of the chemical plant. In other words, the optimization problem consist of finding the integer variables, $y_{w}$, associated to the existence or absence of a given utility unit, $\omega$, and its corresponding continuous load, $f_{w}$, that minimizes the objective function given by Eq.(11.16):

$$
\min _{\substack{f_{\omega}, y_{\omega} \\ R_{r}, W}} \sum_{\omega=1}^{N_{\omega}} f_{\omega}\left[(B \cdot c)_{C_{4}}+(B \cdot c)_{\text {Biomass }}+(V \cdot c)_{\text {Water }}+(W \cdot c)_{\text {Power }}^{\text {import }}-(B \cdot c)_{N_{3}}-(\dot{m} \cdot c)_{C O_{2}}\right] \cdot t_{O P}
$$

Subject to:

Heat balance of temperature interval $r \quad \sum_{\omega=1}^{N_{\omega}} f_{\omega} q_{\omega, r}+\sum_{i=1}^{N} Q_{i, r}+R_{r+1}-R_{r}=0 \quad \forall r=1 . . N$

Balance of produced/consumed power $\quad \sum_{\omega=1}^{N_{\omega}} f_{\omega} W_{\omega}+\sum_{\text {chemical }} W_{\text {net }}+W_{\text {imp }}-W_{\exp }=0$

Existence and size of the utility unit $w$

$$
f_{\min , \omega} \mathrm{y}_{\omega} \leq f_{\omega} \leq f_{\max , \omega} \mathrm{y}_{\omega} \quad \forall \omega=1 . . N_{\omega}
$$

Feasibility of the solution (MER) $\quad R_{1}=0, R_{N_{r}+1}=0, \mathrm{R}_{r} \geq 0$ and $W_{\text {prod }} \geq 0, W_{\text {exp }} \geq 0$ 
where $N_{w}$ is the number of units in the set of utility systems; $B$ is the exergy flow rate $(\mathrm{kW})$ of the resources going in and out of the plant; $c$ stands for the purchasing cost (euro per $\mathrm{kWh}, \mathrm{m}^{3}$ or $\mathrm{kg} / \mathrm{h}$ ) of the feedstock and electricity consumed or the selling price of the marketable ammonia and $\mathrm{CO}_{2}$ produced; $V$ is the flowrate of the water consumed $\left(\mathrm{m}^{3} / \mathrm{h}\right) ; q$ is the heating/cooling rates supplied by the utility systems $(\mathrm{kW}) ; t_{\mathrm{OP}}$ is the operation time $(\mathrm{h}) ; W$ is the power produced by either the utility systems, the same chemical process or imported from/exported to the grid $(\mathrm{kW})$. It is important to emphasize that the process modeling and simulation of the chemical plant alone, including its mass and energy balances, is performed by using Aspen ® Plus software. Meanwhile, the utility units shown in Fig. 11.2 are modeled via equation oriented subroutines written in the Lua programming language. Therefore, the additional equations required for the mass and energy balances of those units rely on the concept of layer (water, natural gas, biomass, syngas, ammonia, power, carbon dioxide, etc.) as shown in Fig. 11.4.

Thus, according to Eq.(11.18), the overall power generated by the utility systems (steam or gas power cycles) should be able to supply the demands of the chemical plant and other utility units (refrigeration, heat pump, cooling tower). Otherwise, the balance of the respective layer considers the possibility of importing electricity from the grid. Moreover, if a surplus power could be produced at expense of the waste heat exergy available throughout the plant, the excess electricity could be sold to the grid provided that its export is economically attractive. Analogously, in the layer of natural gas (or other resource), the amount of energy supplied by the vendors is balanced with the fuel or feedstock consumption by the chemical plant and the utility systems (gas turbine, furnace). In this way, not only the balances of the resources consumed (power, natural gas, biomass, water, etc.) and the products and byproducts produced (ammonia, syngas, hydrogen, $\mathrm{CO}_{2}$, etc.), but as well as of the waste heat recovered, can be performed (cf. Fig.11.4). To this end, representative market cost for the water (3.69 euro $\left./ \mathrm{m}^{3}\right)$, bagasse $(0.0056$ euro/kWh) and natural gas consumed $(0.032 \mathrm{euro} / \mathrm{kWh})$, as well as the selling prices of ammonia $(0.098$ euro/kWh $)$ and $\mathrm{CO}_{2}(0.0084$ euro/kWh $)$ produced are taken from sorted literature $[66,230,231]$. 
Fig. 11.4. Concept of layer used in the optimization of the utility systems. W: power, NG: Natural Gas, BIOM: Biomass, SG: Syngas.

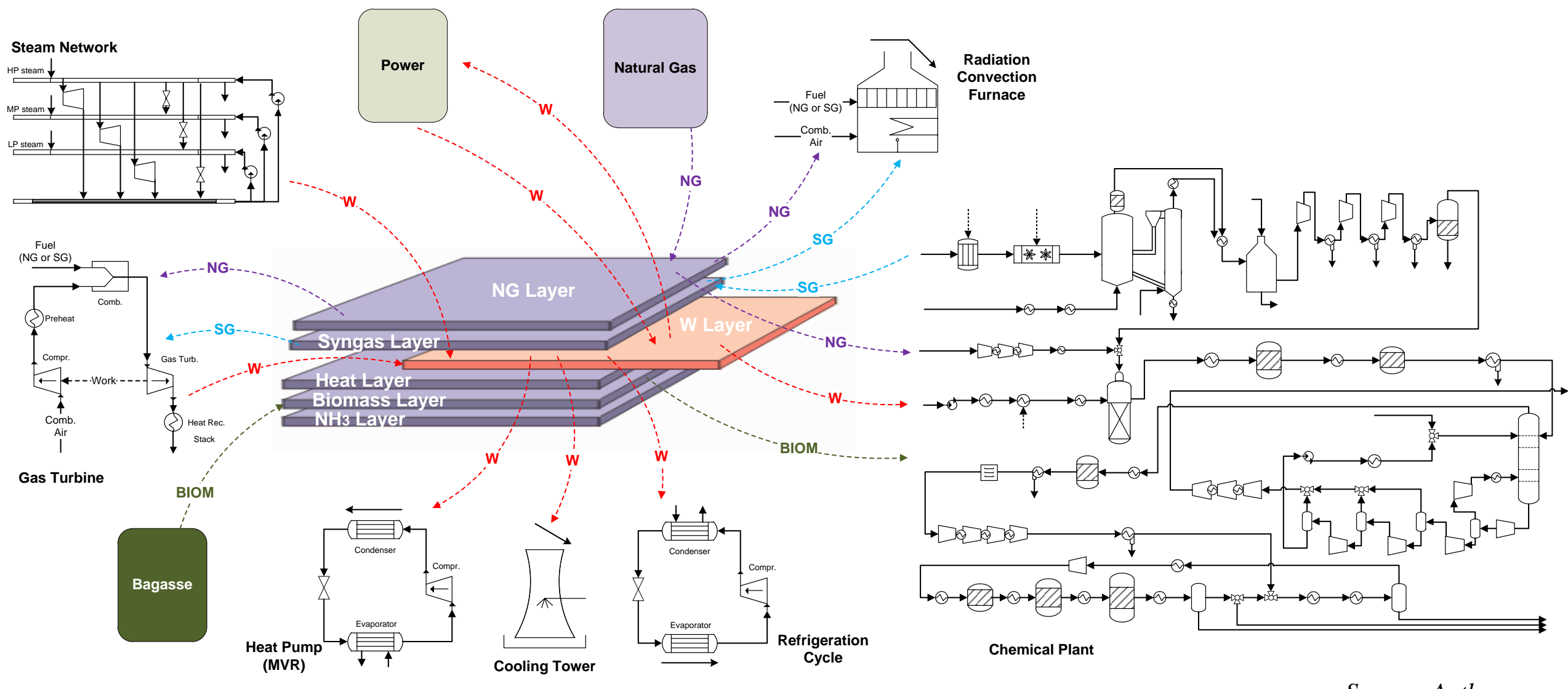




\subsection{Optimal senarios of the conventional and alternative routes of ammonia production.}

In this section, the energy consumption remarks and the performance of the optimized ammonia production setups are presented. First, it is important to notice that, unlike the conventional route that only consumes methane, in the alternative configurations studied, the biomass-derived syngas is used as the main process feedstock for hydrogen production. Meanwhile, natural gas can be still consumed in the fired furnace for balancing the heating requirements in the alternative ammonia production facilities. Natural gas can be also fed to a gas turbine system in order to supply the power required to drive the compressors and pumps, as well as the cooling tower and the vapor compression refrigeration systems.

As concerns the cases of total substitution of natural gas, a fraction of the biomass-derived syngas is consumed instead of importing natural gas, which directly affects the ammonia yield and, most importantly, the overall balance of the combined heat and power production of the plant. The decision making is therefore not a trivial problem, as it involves the iterative adjustment of the energy integration results [238]. Thus, by using a systematic optimization framework, the waste heat available for cogeneration purposes can be thoroughly exploited, whereas minimizing the operating cost of the plant and maximizing the total amount of ammonia produced.

Accordingly, apart from the conventional case using only natural gas as both feedstock and fuel (see Fig. 11.1), thirteen additional scenarios have been tested in which the biomassderived syngas is used as the main feedstock for ammonia production, but the input to the utility systems varies according to economic and environmental targets. These scenarios combine the utilization of the various energy resources in the utility systems (i.e. imported natural gas, electricity from the grid, as well as produced syngas) along with the integration of either a Rankine or a Combined cycle. However, only six out of all the combinations considered are found to be independent optimal solutions, namely:

Conventional case: the natural gas imported is used as both feedstock and fuel, without electricity import;

WF-RC-EE case: No gaseous fuels are consumed in the utility systems, thus the imported electricity along with the optimized steam network and Rankine cycle are responsible for the combined heat and power production;

NG-RC-no EE case: Only imported natural gas is consumed as fuel in the utility systems, whereas the optimized steam network and a Rankine cycle provide the required heat and power demands, without any electricity import required;

NG-CC-no EE case: Analogously to the previous case, except for the consumption of the natural gas imported in a more efficiency Combined cycle that supply the required heat and power demands of the chemical plant;

SG-RC-no EE case: Differently from the previous cases, here a fraction of the gasification syngas is consumed as fuel whereas an optimal steam network and a Rankine cycle provide the required heat and power demands, without the need of electricity import; 
SG-CC-no EE case: Similar to the previous case, except for the integration of a Combined cycle.

In other words, by enabling the natural gas turbine system (e.g. combined cycle) while the electricity import is not allowed (see case NG-CC-noEE), the optimization solution is found to be equivalent to that of another scenario in which, even if the electricity consumption is now enabled, the optimizer solution still favors the natural gas consumption in a combined cycle over the import of costly electricity from the grid (i.e. NG-CC-EE). Table 11.2 summarizes the results of the optimal process variables for the considered scenarios of ammonia production.

According to Fig. 11.5 (see also Table 11.2), the non-renewable exergy consumption in the conventional case achieves $32.34 \mathrm{GJ} / \mathrm{t}_{\mathrm{NH} 3}$, with combined $\mathrm{CO}_{2}$ emissions (i.e. in the fumes and the raw syngas) of $1.75 \mathrm{t}_{\mathrm{CO} 2} / \mathrm{t}_{\mathrm{NH} 3}$, out of which $29.3 \%$ are not captured and, thus, emitted to atmosphere (cf. Fig. 11.6). The source of these emissions is related to the use of natural gas in the fired furnace of the primary reformed. Nevertheless, the conventional case presents the lowest exergy consumption among all the analyzed configurations, due to the higher operating pressures of the primary reformer when compared to the atmospheric gasification processes.

Fig. 11.5. Plantwide and extended exergy consumption figures for the various configurations studied.

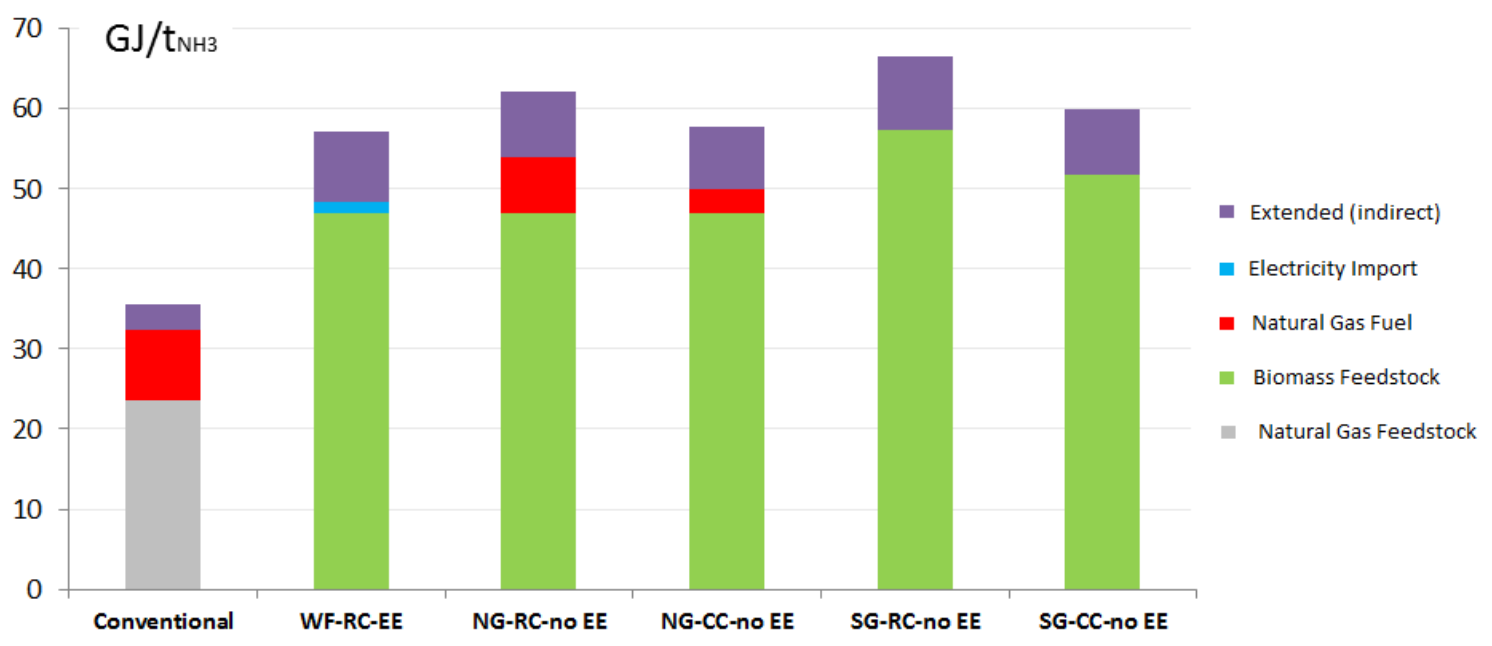

Source: Author

In fact, in the biomass-based setups, the water-scrubbed syngas must be further compressed, not to mention the large amoung of exergy consumed in the bagasse treatment process, which renders the biomass-based route more power intensive (see Fig. 11.7). The conventional case also presents the lowest overall power consumption $\left(2.49 \mathrm{GJ} / \mathrm{t}_{\mathrm{NH} 3}\right), 60 \%$ lower than the highest power consumption figure $\left(6.4 \mathrm{GJ} / \mathrm{t}_{\mathrm{NH} 3}\right)$ corresponding to the case in which only syngas is used as the fuel source for the combined heat and power generation (SG-RC-no $\mathrm{EE})$.Thus, it is not surprising that the SG-RC-noEE case also accounts for the highest overall exergy input $\left(57.32 \mathrm{GJ} / \mathrm{t}_{\mathrm{NH} 3}\right)$. 
From Table 11.2, it is also evidenced that even for a higher specific consumption of feedstock, cheaper biomass input allows for a lower operating cost and, thus, higher operating revenues, than if the utility system were fueled with costly natural gas. It explains why the lowest amount of operating revenues corresponds to the conventional case, in which natural gas is used as both feedstock and fuel. As a conclusion, the use of cheaper energy resources as well as the diversification of the energy inputs to the ammonia plant may serve not only for reducing the amount of emissions produced but also increasing the revenues obtained, even at expense of lower efficiencies [305].

Fig. 11.6. Overall and detailed (biogenic and fossil, directly and indirectly emitted, and avoided) $\mathrm{CO}_{2}$ emissions for the various configurations studied.

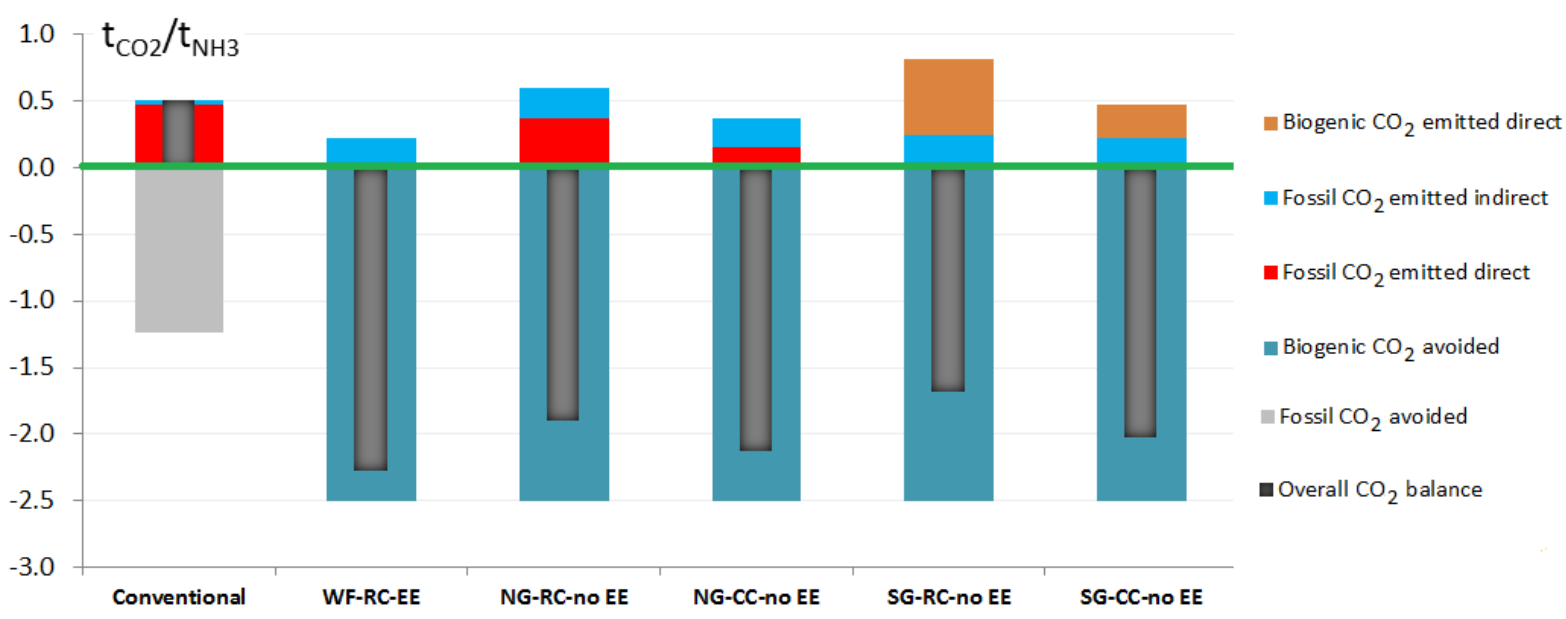

Source: Author

Additionally, Table 11.2 shows the Extended Exergy Plant Consumption that takes into account the exergy efficiency of the electricity generation (55.68\%), as well as of the natural gas $(91.09 \%)$ and bagasse $(86.13 \%)$ supply chains [188]. Certainly, by adding the upstream inefficiencies in the feedstock supply chains into the originally standalone ammonia plant analysis, the panorama is worsened as the exergy destroyed in the feedstock acquisition further impairs the global performance of the production process. This increase in the overall exergy consumption is not negligible, varying from $15.3-17.9 \%$ in the case of the biomassbased routes, but as low as $9.7 \%$ in the case of the conventional route. Although these figures may not be immediately interesting for ammonia producers when evaluating the performance of the plant itself, those figures certainly prove to be useful to public policy and decisionmakers in both environmental and benchmarking frameworks, since they allow to holistically compare the impact of the fertilizers sector with other industrial sectors from a fair level playing field. 
Table 11.2. Optimal process variables of the studied ammonia production facilities. WF: no fuel, NG: Natural Gas fuel, SG: Syngas fuel, EE: Electricity consumption, RC: Rankine cycle, CC: Combined cycle, no-EE: No electricity import.

\begin{tabular}{|c|c|c|c|c|c|c|}
\hline Process parameter & Conventional & WF-RC-EE & NG-RC-no EE & NG-CC-no EE & SG-RC-no EE & SG-CC-no EE \\
\hline Feedstock input & Natural Gas & Biomass & Biomass & Biomass & Biomass & Biomass \\
\hline Utility system input & Natural Gas & Electricity & Natural Gas & Natural Gas & Syngas & Syngas \\
\hline Cogeneration system & Rankine & Rankine & Rankine & Combined & Rankine & Combined \\
\hline Feedstock consumption $\left(\mathrm{GJ} / \mathrm{t}_{\mathrm{NH} 3}\right)$ & 23.51 & 47.04 & 47.04 & 47.04 & 57.32 & 51.66 \\
\hline Utility fuel consumption $\left(\mathrm{GJ} / \mathrm{t}_{\mathrm{NH} 3}\right)$ & 8.83 & 0.00 & 6.90 & 2.91 & $6.79^{10}$ & $3.05^{10}$ \\
\hline Utility electricity consumption $\left(\mathrm{GJ} / \mathrm{t}_{\mathrm{NH} 3}\right)$ & 0.00 & 1.41 & 0.00 & 0.00 & 0.00 & 0.00 \\
\hline Overall plant consumption $\left(\mathrm{GJ} / \mathrm{t}_{\mathrm{NH} 3}\right)$ & 32.34 & 48.45 & 53.95 & 49.95 & $\mathbf{5 7 . 3 2}^{10}$ & $51.66^{10}$ \\
\hline Extended plant consumption $\left(\mathrm{GJ} / \mathrm{t}_{\mathrm{NH} 3}\right)^{1}$ & 35.49 & 57.15 & 62.19 & 57.81 & 66.55 & 59.98 \\
\hline Rankine cycle power generation $\left(\mathrm{GJ} / \mathrm{t}_{\mathrm{NH} 3}\right)^{2}$ & 2.49 & 4.22 & 5.73 & 4.32 & 6.40 & 4.53 \\
\hline Brayton cycle power generation $\left(\mathrm{GJ} / \mathrm{t}_{\mathrm{NH}}\right)^{2}$ & 0.00 & 0.00 & 0.00 & 1.33 & 0.00 & 1.43 \\
\hline Chemical process power demand $\left(\mathrm{GJ} / \mathrm{t}_{\mathrm{NH} 3}\right)^{3}$ & 1.81 & 4.88 & 4.88 & 4.88 & 5.56 & 5.19 \\
\hline Ancillary power demand $\left(\mathrm{GJ} / \mathrm{t}_{\mathrm{NH} 3}\right)^{4}$ & 0.68 & 0.75 & 0.85 & 0.77 & 0.84 & 0.77 \\
\hline Cooling requirement $\left(\mathrm{GJ} / \mathrm{t}_{\mathrm{NH} 3}\right)^{5}$ & 5.86 & 10.92 & 10.91 & 10.92 & 10.95 & 10.93 \\
\hline Heating requirement $\left(\mathrm{GJ} / \mathrm{t}_{\mathrm{NH} 3}\right)^{5}$ & 2.13 & 0.00 & 0.00 & 0.00 & 0.00 & 0.00 \\
\hline Fossil $\mathrm{CO}_{2}$ emissions avoided $\left(\mathrm{t}_{\mathrm{CO} 2} / \mathrm{t}_{\mathrm{NH} 3}\right)^{6}$ & 1.237 & 0.000 & 0.000 & 0.000 & 0.000 & 0.000 \\
\hline Fossil $\mathrm{CO}_{2}$ emitted -direct $\left(\mathrm{t}_{\mathrm{CO} 2} / \mathrm{t}_{\mathrm{NH} 3}\right)$ & 0.470 & 0 & 0.368 & 0.155 & 0.000 & 0.000 \\
\hline Fossil $\mathrm{CO}_{2}$ emitted - indirect $\left(\mathrm{t}_{\mathrm{CO} 2} / \mathrm{t}_{\mathrm{NH} 3}\right)^{7}$ & 0.043 & 0.227 & 0.236 & 0.217 & 0.246 & 0.222 \\
\hline $\mathrm{CO}_{2}$ emitted indirect $-\mathrm{EE}$ grid $(\%)$ & 0.00 & 10.74 & 0.00 & 0.00 & 0.00 & 0.00 \\
\hline $\mathrm{CO}_{2}$ emitted indirect - Nat. Gas (\%) & 100.00 & 0.00 & 14.33 & 6.58 & 0.00 & 0.00 \\
\hline $\mathrm{CO}_{2}$ emitted indirect - Bagasse $(\%)$ & 0.00 & 89.26 & 85.67 & 93.42 & 100.00 & 100.00 \\
\hline Total fossil $\mathrm{CO}_{2}$ emitted $\left(\mathrm{t}_{\mathrm{CO} 2} / \mathrm{t}_{\mathrm{NH} 3}\right)$ & 0.513 & 0.227 & 0.604 & 0.372 & 0.246 & 0.222 \\
\hline Biogenic $\mathrm{CO}_{2}$ emissions avoided $\left(\mathrm{t}_{\mathrm{CO} 2} / \mathrm{t}_{\mathrm{NH} 3}\right)^{6}$ & 0.000 & 2.503 & 2.503 & 2.503 & 2.503 & 2.503 \\
\hline Biogenic $\mathrm{CO}_{2}$ emitted - direct $\left(\mathrm{t}_{\mathrm{CO} 2} / \mathrm{t}_{\mathrm{NH} 3}\right)$ & 0.000 & 0.000 & 0.000 & 0.000 & 0.569 & 0.256 \\
\hline Total atmospheric emissions $\left(\mathrm{t}_{\mathrm{CO} 2} / \mathrm{t}_{\mathrm{NH} 3}\right)$ & 0.513 & 0.227 & 0.604 & 0.371 & 0.816 & 0.478 \\
\hline${\mathrm{Overall} \mathrm{CO}_{2} \text { emissions balance }}^{8}$ & 0.513 & -2.276 & -1.899 & -2.131 & -1.687 & -2.025 \\
\hline Biomass consumption $\left(\mathrm{t}_{\text {Bagasse }} / \mathrm{t}_{\mathrm{NH} 3}\right)$ & -- & 2.41 & 2.41 & 2.41 & 2.94 & 2.65 \\
\hline Gasifier syngas production $\left(\mathrm{GJ} / \mathrm{t}_{\mathrm{NH} 3}\right)$ & -- & 31.06 & 31.06 & 31.06 & $37.85^{10}$ & $34.11^{10}$ \\
\hline Operating Incomes ${ }^{9}\left(\right.$ euro/t $\left.\mathrm{t}_{\mathrm{NH} 3}\right)$ & 516.72 & 529.34 & 529.34 & 529.34 & 529.98 & 529.63 \\
\hline Operating $\operatorname{Costs}^{9}\left(\right.$ euro/ $\left./ \mathrm{t}_{\mathrm{NH} 3}\right)$ & 281.05 & 107.59 & 124.73 & 90.31 & 79.53 & 71.67 \\
\hline Operating Revenues $^{9}\left(\right.$ euro/ $\left.\mathrm{t}_{\mathrm{NH} 3}\right)$ & 235.67 & 421.75 & 404.62 & 439.04 & 450.45 & 457.96 \\
\hline Ammonia production ( $\mathrm{t} /$ day) & 950.84 & 1119.22 & 1119.22 & 1119.22 & $918.45^{10}$ & $1019.14^{10}$ \\
\hline
\end{tabular}

1. Overall exergy consumption increases if the extended efficiency of the electricity grid (55.67\%), natural gas (91.09\%) and bagasse (86.13\%) supply are considered as in [188]; 2. Steam pressure levels $110,25,2.5$ and 0.10 bar, steam superh. $200^{\circ} \mathrm{C}$, Brayton cycle with regeneration, pressure ratio $20: 1 ; 3$. Power consumed by the chemical plant alone; 4. Cooling tower and vapor compression refrigeration systems; 5 . Heating requirements of the chemical processes (energy basis) determined from the composite curves; 6. $\mathrm{CO}_{2}$ emissions captured through the physical absorption system; 7. It considers the indirect emissions due to the upstream supply chains of natural gas $\left(0.0049 \mathrm{~g}_{\mathrm{CO} 2} / \mathrm{kJ}_{\mathrm{CH}}\right)$, electricity $\left(62.09 \mathrm{~g}_{\mathrm{CO} 2} / \mathrm{kWh}\right)$ and residual bagasse $\left(0.0043 \mathrm{~g}_{\mathrm{CO} 2} / \mathrm{kJ}_{\mathrm{Bagasse}}\right)[188,232] ; 8$. Consider overall $\mathrm{CO}_{2}$ emitted (either fossil or biogenic) minus biogenic $\mathrm{CO}_{2}$ captured; 9 . Operating revenues (only) calculated as the difference between the gross operating incomes minus the operating cost. See Appendix 2 for a detailed discussion on the influence of the share of the capital investment in the overall plant revenues; 10. It considers bagasse as the only energy input of the ammonia plant, thus the utility fuel consumption is a fraction of the syngas produced. It also reduces the amount of ammonia produced. 
Furthermore, the indirect emissions related to the supply chains of the electricity, natural gas and bagasse (production, distribution, etc.) have been included in the calculation of the actual $\mathrm{CO}_{2}$ fossil emitted in Table 11.2 [188, 232]. Such indirect contribution is not negligible and reveals environmental issues that otherwise may remain hidden if electricity imported is considered as an emissions-free input. For instance, the indirect emissions of the bagasse supply are the largest share of indirect emissions (85-100\%) in the biomass-based route $(0.23$ $\left.\mathrm{t}_{\mathrm{CO} 2} / \mathrm{t}_{\mathrm{NH} 3}\right)$. This value is on average fivefold the indirect emissions associated to the conventional route $\left(0.043 \mathrm{t}_{\mathrm{CO} 2} / \mathrm{t}_{\mathrm{NH} 3}\right)$ and can be explained by the large amount of bagasse required, which not only takes a toll to the efficiency of the overall plant, but proportionally increases the indirect emissions produced too.

On the other hand, the direct emissions are derived from the combustion of natural gas or raw syngas (with an important biogenic $\mathrm{CO}_{2}$ content), used to supply the combined heat and power production. The avoided emissions are related to the carbon capture system in the syngas purification section. Since direct biomass-derived emissions are considered as neutral emissions [191], the difference between the biogenic $\mathrm{CO}_{2}$ captured and the overall $\mathrm{CO}_{2}$ emitted is considered as the balance of $\mathrm{CO}_{2}$ emissions (see Table 11.2). The negative value indicates an overall positive impact in the depletion of $\mathrm{CO}_{2}$ present in the atmosphere, meaning that for each ton of ammonia produced, between 1.7 to 2.3 tons of $\mathrm{CO}_{2}$ are withdrawn from the environment. This is in close agreement with reported literature [115]. As a result, the indirect emissions from the bagasse utilization are not only offset by the captured biogenic emissions, but also the scenario of a 'greener' Brazilian electricity import (WF-RC-EE) leads to the best results in terms of overall exergy consumption and $\mathrm{CO}_{2}$ emissions among the partially and totally renewable routes analyzed in Table 11.2. 
Fig. 11.7. Power consumption breakdown of the selected scenarios, EE: electricity, superh.: steam superheating, (a) Conventional, (b) WF-RC-EE, (c) NG-RC-no E, (d) NG-CC-no EE,(e) SG-RC-no EE, (f) SGCC-no EE, see Table 11.2. Source: Author.

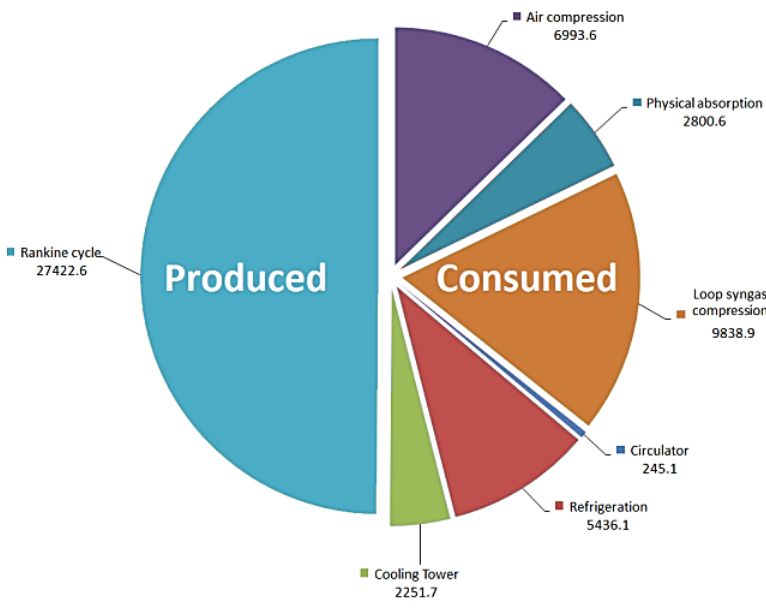

Rankine cycle steam pressure levels $110,40,3,0.12$ bar, steam superh. $200^{\circ} \mathrm{C}$, no EE import, natural gas fuel

(a)

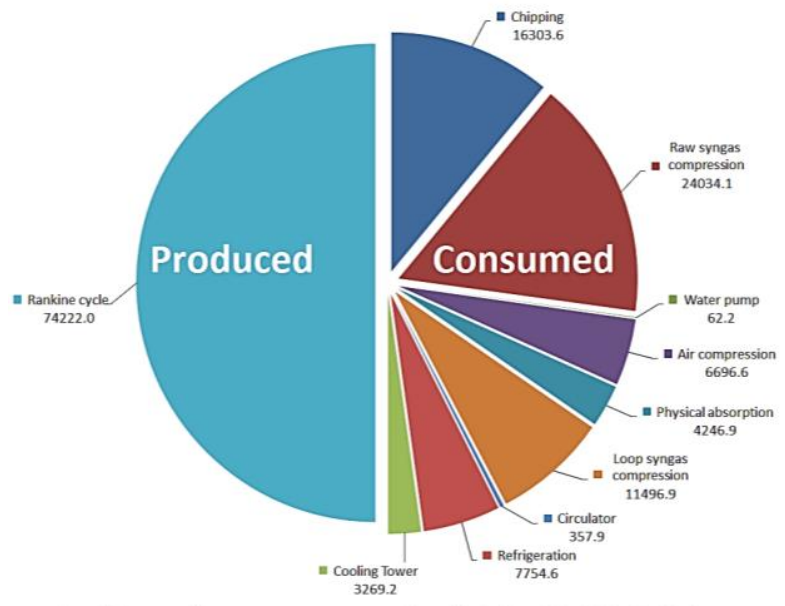

Rankine cycle steam pressure levels $110,25,2.5,0.10$ bar, steam superh. $200^{\circ} \mathrm{C}$, no EE import, natural gas fuel

(c)

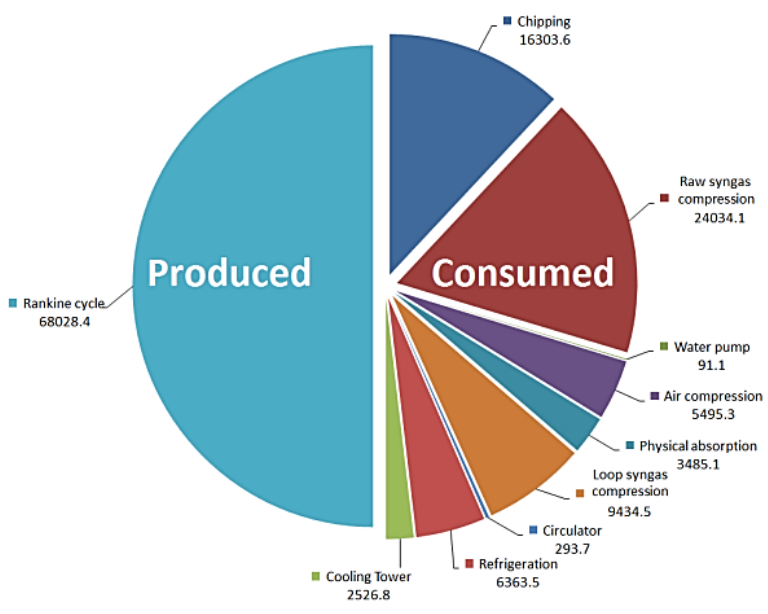

Rankine cycle steam pressure levels 110, 25, 2.5, 0.10 bar, steam superh. $200^{\circ} \mathrm{C}$, no EE import, syngas fuel

(e)

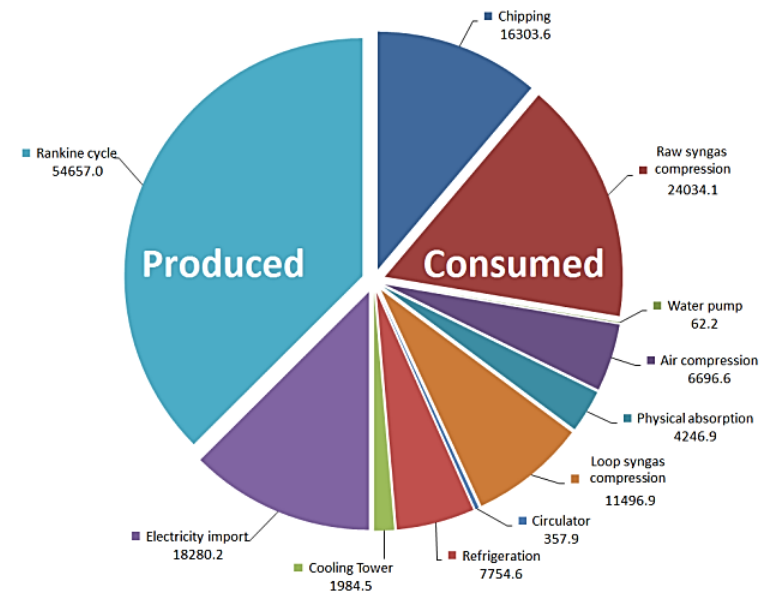

Rankine cycle steam pressure levels $110,25,2.5,0.10 \mathrm{bar}$, steam superh. $200^{\circ} \mathrm{C}$, EE import, no fuels

(b)

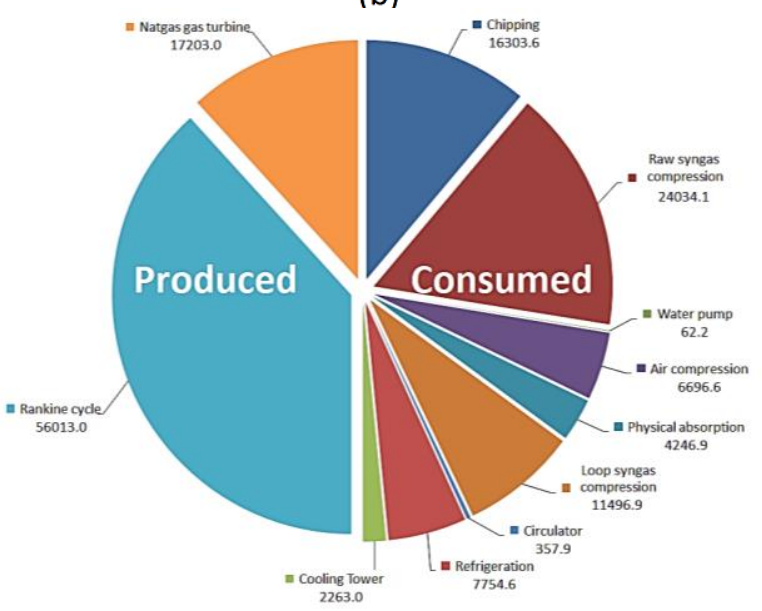

Combined cycle steam pressure levels 110, 25, 2.5, 0.10 bar, steam superh. $200^{\circ} \mathrm{C}$, no EE import, natural gas fuel, gas turbine 20:1 bar

(d)

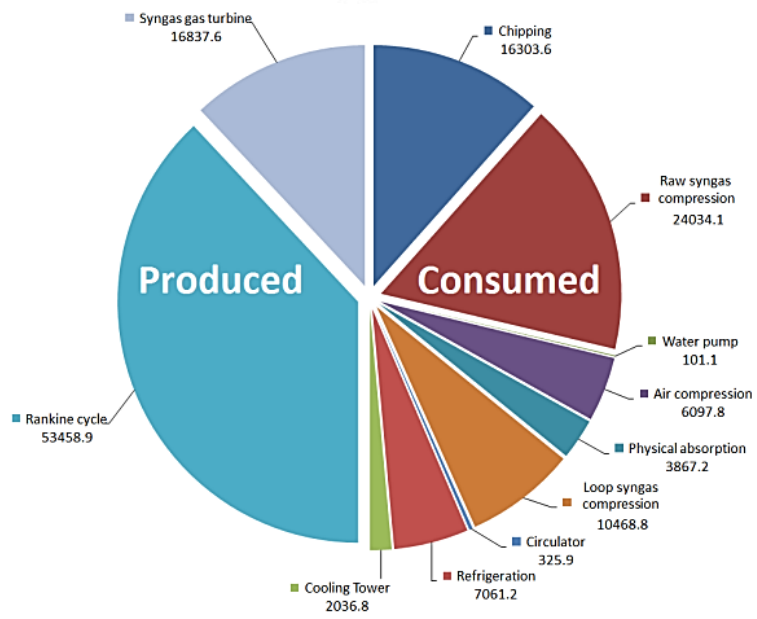

Combined cycle steam pressure levels 110, 25, 2.5, 0.10 bar, steam superh. $200^{\circ} \mathrm{C}$, no EE import, syngas fuel, gas turbine 20:1 bar 


\subsection{Combined exergy and energy integration analysis}

Figure 11.8a-f show the integrated curves corresponding to the simulated scenarios described in Table 11.2.

Fig. 11.8. Integrated composite curves. EE: electricity, superh.: steam superheating, (a) Conventional, (b) WF-RC-EE, (c) NG-RC-no E, (d) NG-CC-no EE,(e) SG-RC-no EE, (f) SG-CC-no EE, cf. Table 11.2

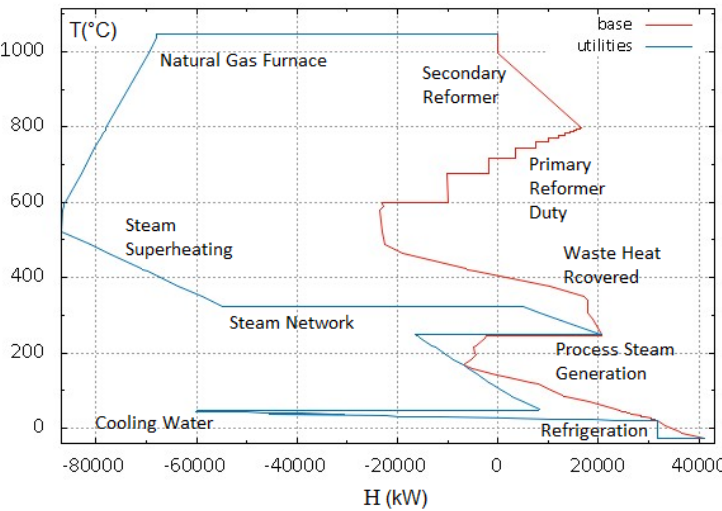

Natural gas feedstock, Natural gas fueled utility system, Steam pressure levels $110,40,3,0.12$ bar, Rankine cycle, steam superh. $200^{\circ} \mathrm{C}$, no EE import

(a)

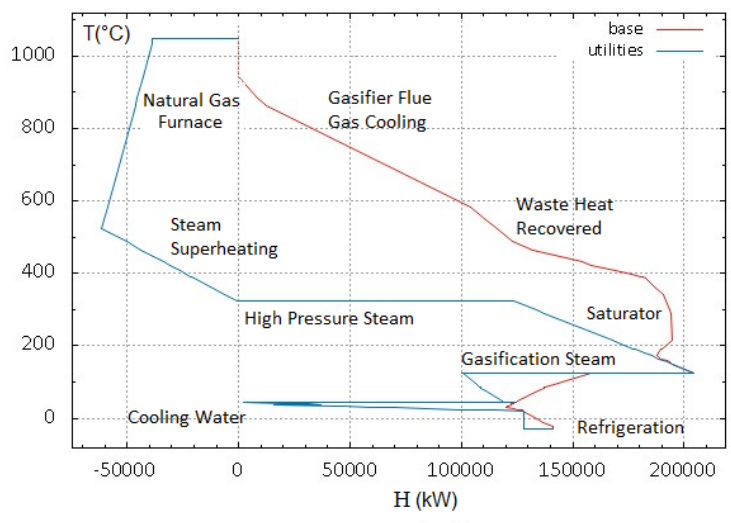

Biomass feedstock, Natural gas fueled utility system, Steam pressure levels $110,2.5,0.10$ bar, Rankine cycle, steam superh. $200^{\circ} \mathrm{C}, \mathrm{NG}$ furnace, no $\mathrm{EE}$ import

(c)

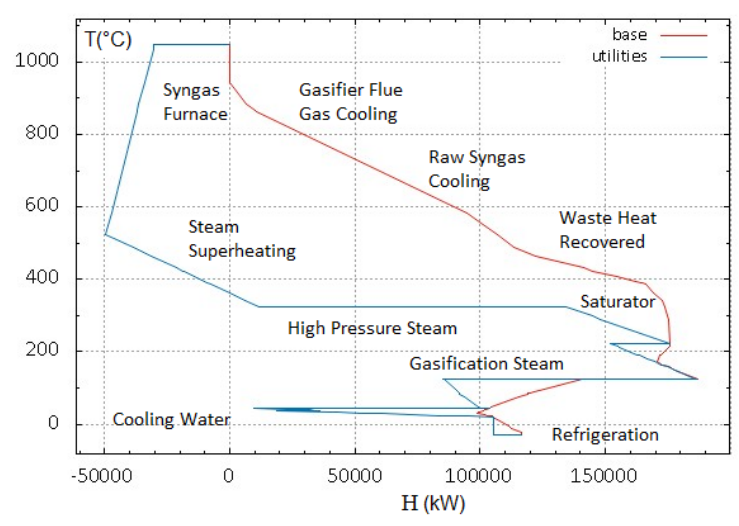

Biomass feedstock, Syngas fueled utility system, Steam pressure levels $110,25,2.5,0.10$ bar, Rankine cycle, steam superh. $200^{\circ} \mathrm{C}$, Syngas furnace, no EE import

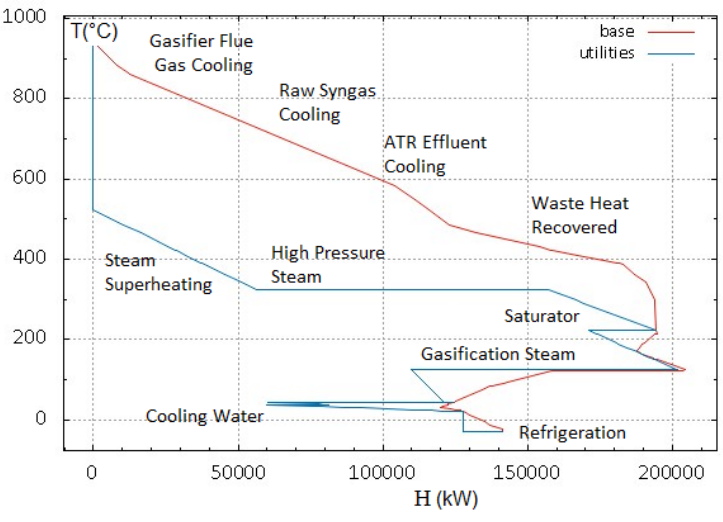

Biomass feedstock, No fuel used in utility system. Steam pressure levels 11 $25,25,0.10$ bar, Rankine cycle, steam superh. $200^{\circ} \mathrm{C}$, EE import

(b)

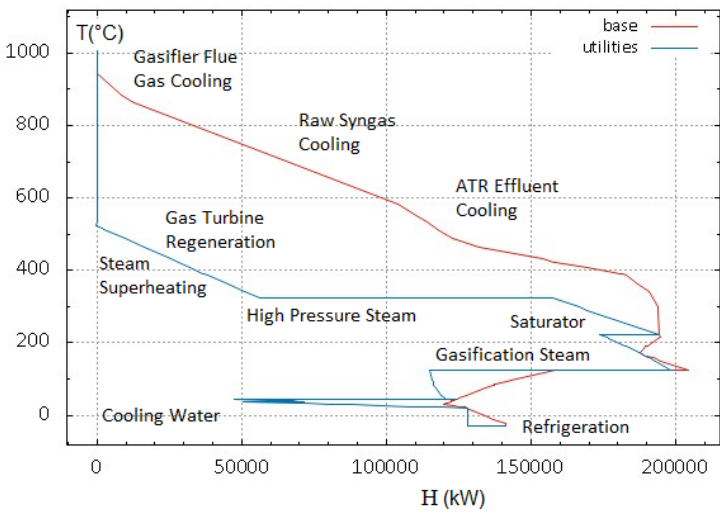

Biomass feedstock, Natural gas fueled utility system, Steam pressure level $110,25,2.5,0.10$ bar, Combined cycle, steam superh. $200^{\circ} \mathrm{C}$, Gas turbine 20:1 bar, no EE import

(d)

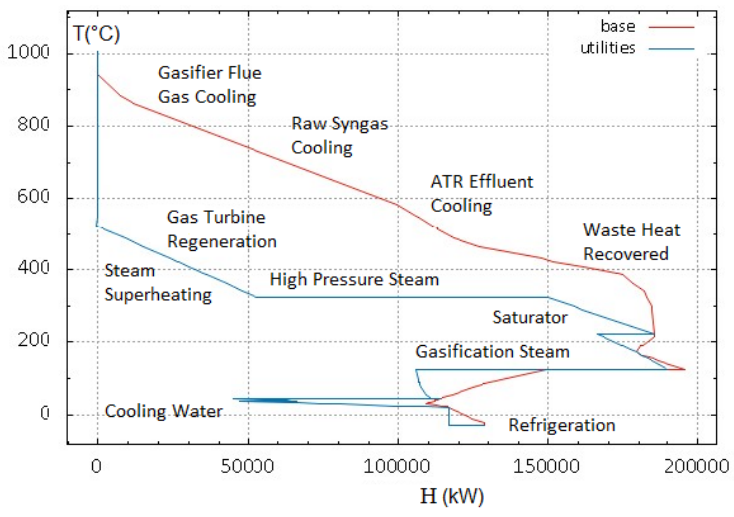

Biomass feedstock, Syngas fueled utility system, Steam pressure levels $110,25,25,0,10$ bar, Combined cycle, steam superh. $200^{\circ} \mathrm{C}$, Gas turbine 20.1 bar, no EE import

(e) 
As it can be observed, the bagasse-based ammonia production designs, Figs. 11.8 (b-c) present a higher potential of heat recovery due to the excess heat exergy available throughout the whole chemical plant. This is profited by integrating a steam network that recovers and then supplies the heat required, either by preheating process streams or by raising steam (used as both process reactant and power fluid).

On the other hand, the reduced excess heat exergy available in the conventional case (Fig. 11.8a) must be compensated by an increased consumption of fuel in the utility system, about 21 to $84 \%$ higher than in the cases (b-c). In spite of this fact, the share of exergy destruction in the natural gas-fired furnace and the primary reformer together $\left(6.7 \mathrm{GJ} / \mathrm{t}_{\mathrm{NH} 3}\right)$ is still much lower than the sum of the irreversibility comprised in the bagasse treatment (chipping, drying, scrubbing), gasification and syngas compression processes together, according to Fig. 11.9 and Table 11.3. Actually, the latter irreversibility accounts for approx. $16.2 \mathrm{GJ} / \mathrm{t}_{\mathrm{NH} 3}$ or $60-80 \%$ of the exergy destroyed in the bagasse-based ammonia production setups. The gasifier itself is responsible for half of the exergy destruction in the plant and, as the char combustion process is inevitable, there is a small room for reducing its contribution to the total process irreversibility. However, as concerns the drying, chipping and cold syngas cleaning (water scrubbing), and compression processes, better technologies for the removal of the bagasse moisture, hot catalytic cleaning of the syngas and increased gasifier pressures may help reducing the amount of avoidable exergy destroyed in the frontend of biomassbased ammonia production plants [32].

Fig. 11.9. Exergy destruction breakdown for the selected scenarios, EE: electricity, superh.: steam superheating, (a) Conventional, (b) WF-RC-EE, (c) NG-RC-no E, (d) NG-CC-no EE,(e) SG-RC-no EE, (f) SG-CC-no EE, see Table 11.2.

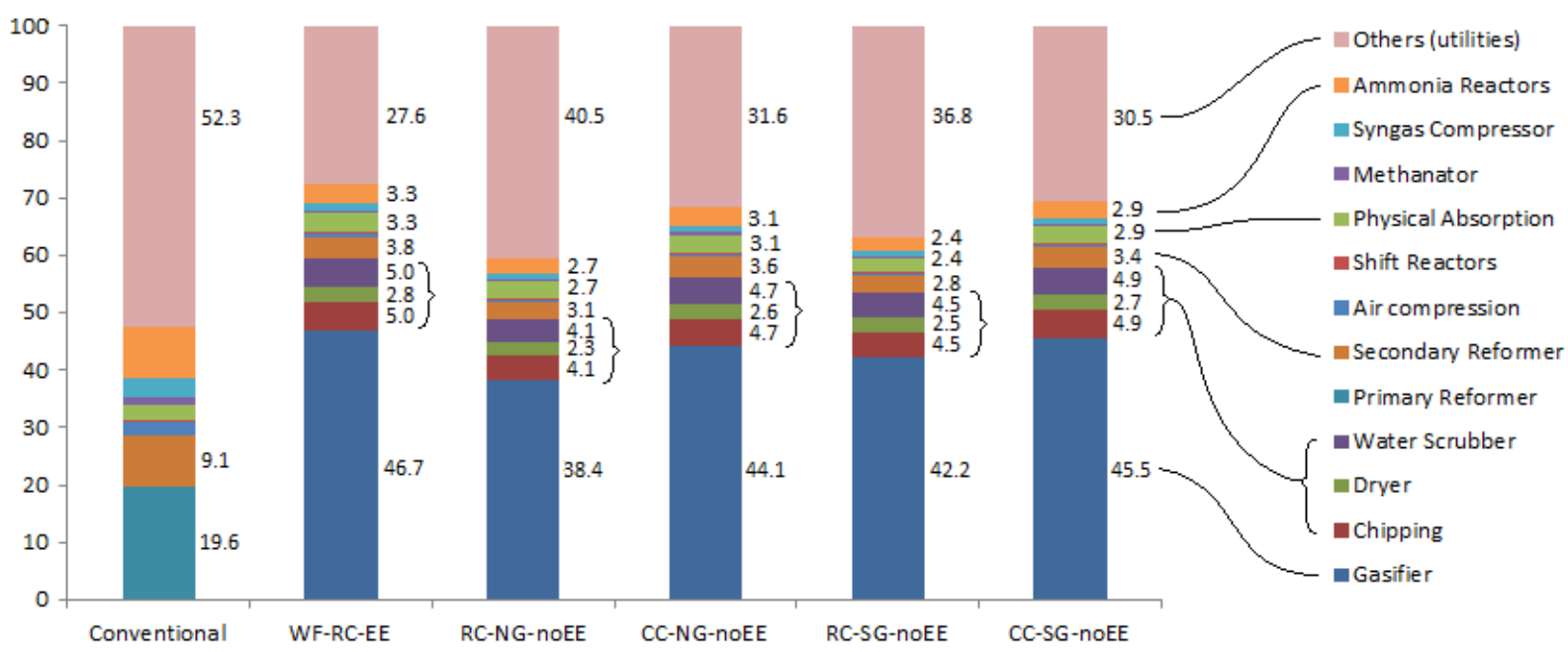

Source: Author.

The calculated plantwide rational efficiencies shown in Table 11.3 are in close agreement with those reported for the thermo-environonomic optimization of two biomass and natural gas-based ammonia production plants with electricity import [115]. 
Table 11.3. Exergy destruction and exergy efficiencies for the studied configurations

\begin{tabular}{lcccccc}
\hline Process parameter & Conventional & $\begin{array}{c}\text { WF-RC } \\
\text {-EE }\end{array}$ & $\begin{array}{c}\text { NG-RC } \\
\text {-no EE }\end{array}$ & $\begin{array}{c}\text { NG-CC } \\
\text {-no EE }\end{array}$ & $\begin{array}{c}\text { SG-RC } \\
\text {-no EE }\end{array}$ & $\begin{array}{c}\text { SG-CC } \\
\text {-no EE }\end{array}$ \\
\hline Rational exergy efficiency $(\%)$ & 70.17 & 47.87 & 42.99 & 46.43 & 40.46 & 44.89 \\
\hline $\begin{array}{l}\text { Extended rational exergy } \\
\text { efficiency }(\%)\end{array}$ & 63.92 & 40.58 & 37.29 & 40.12 & 34.85 & 38.67 \\
\hline Relative exergy efficiency $(\%)$ & $\mathbf{6 1 . 4 7}$ & 41.02 & 36.85 & 39.79 & $\mathbf{3 4 . 6 7}$ & 38.47 \\
\hline $\begin{array}{l}\text { Extended relative exergy } \\
\text { efficiency }(\%)\end{array}$ & $\mathbf{5 6 . 0 0}$ & 34.78 & 31.96 & 34.38 & $\mathbf{2 9 . 8 6}$ & 33.14 \\
\hline Exergy destruction $\left(\mathrm{GJ} / \mathrm{t}_{\mathrm{NH} 3}\right)$ & $\mathbf{9 . 6 4}$ & 25.26 & 30.75 & 26.75 & $\mathbf{3 4 . 1 3}$ & 28.47 \\
\hline $\begin{array}{l}\text { Extended exergy destruction } \\
\left(\mathrm{GJ} / \mathrm{t}_{\mathrm{NH} 3}\right)^{1}\end{array}$ & $\mathbf{1 2 . 8 1}$ & 33.96 & 39.00 & 34.61 & $\mathbf{4 3 . 3 6}$ & 36.79 \\
\hline
\end{tabular}

1.Overall exergy consumption increases if the cumulative efficiency of the electricity grid (55.67\%), natural gas $(91.09 \%)$ and bagasse $(86.13 \%)$ supply are considered as in [188].

It is also worthy to notice that, according to the calculated extended exergy efficiency (see Fig. 11.10), the standalone ammonia plant efficiency (i.e. not extended) are appreciably reduced (8.9-15.2\%) due to the incorporation of the irreversibility present in the upstream feedstock supply chains. These figures are of course dependent on other process externalities such as the transportation and distribution infrastructure, the production efficiency of the energy resources as well as the composition of the electricity mix concerned. It is important to mention other scenarios which recently have earned attention in Brazil to mitigate the fossil fuel consumption in the ammonia production process, which consider the syngas production by using the steam reforming of ethanol, encouraged by a well-established sugarcane ethanol economy [213-217].

Fig. 11.10. Comparison between the relative and extended relative exergy efficiency of the various conventional and alternative ammonia production plant configurations.

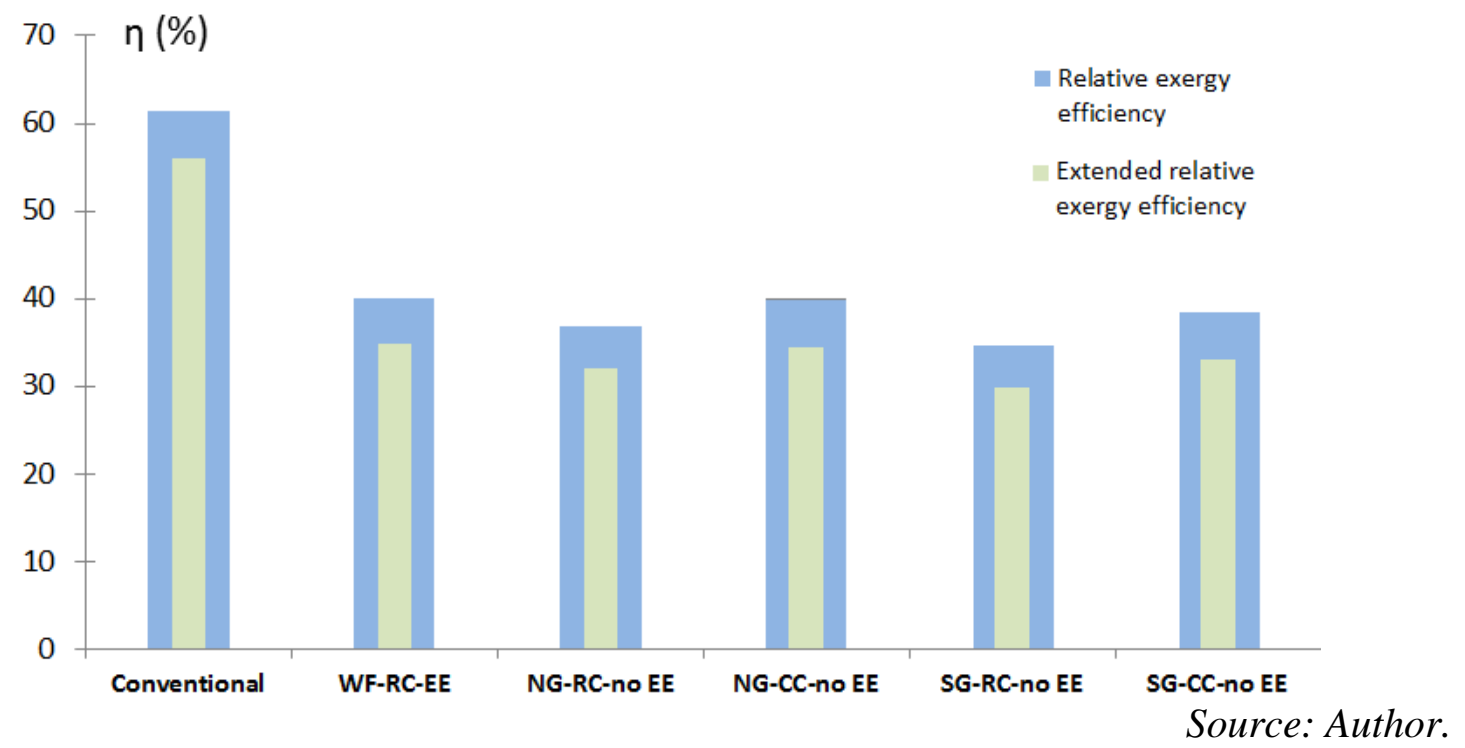

As a final remark, from a sustainability point of view, lower electricity selling prices promote the integration of improved energy conversion systems, since in this way the fuel consumption in the 'internal' cogeneration systems attempts to compensate the improved 'external' standalone cogeneration system (i.e. waste heat is upgraded to useful input). 
Paradoxically, higher purchasing electricity and natural gas prices are also necessary to encourage the rational transformation of the waste heat exergy into mechanical power by using cogeneration (since imported electricity cost becomes prohibitively high). At the end, the relative prices of electricity and fuels (natural gas and bagasse) play an important role when determining the pathway that must follow the economic process optimization. That explains why better solutions in terms of exergy efficiency or reduced environmental impact are not necessarily meant to be as well the most profitable ones [168], as it has been found from the calculated operating revenues.

\subsection{Final considerations}

A conventional natural gas-based ammonia production plant is compared with a series of alternative biomass-based ammonia production facilities, aiming to reduce the amount of nonrenewable exergy consumed, whereas increasing the operating revenues. The combined energy integration and exergy analyses performed allowed spotting the best alternatives of utility systems that increase the revenues, while maximize the recovery of the available waste heat exergy.

As a result, the exergy efficiencies of the natural and biomass-based ammonia production plants averaged $65.8 \%$ and $41.3 \%$, whereas the overall emission balance varies from 0.5 to $-2.3 \mathrm{t}_{\mathrm{CO} 2} / \mathrm{t}_{\mathrm{NH} 3}$, respectively. The negative values point towards the environmental benefits brought about the production of chemicals through the use of alternative energy sources such as biomass. However, in the short to medium terms, the global production of these important commodities are foreseen to remain dominated by the use of the non-renewable natural gas resources, especially coal and natural gas. Yet, further efforts on research and development of more efficient conversion technologies of renewable energy sources must look towards the introduction of alternative ammonia production routes at larger scales in the SNF industry, in spite of the current high investment risk and less mature energy conversion technologies of biomass.

Moreover, electricity import, whether available, may help reducing the irreversibility in the biomass-based ammonia production, as well as reducing the overall $\mathrm{CO}_{2}$ emissions. However, higher operating revenues are rather achieved by totally replacing the natural gas input and avoiding the electricity import, favoring the consumption of the syngas produced in a combined power cycle for supplying the heat and power demands. Finally, it must be noticed that by defining an extended plant consumption and efficiency concepts, the whole effect of the production process, including the inefficiencies of upstream feedstock supply chain can be evaluated. The results show to be strongly dependent on the indirect emissions, the energy resources used (natural gas, electricity or bagasse) and the ratio of the price of electricity to natural gas adopted.

In the next chapter, the upgrade of the organic residues of the sugar cane biorefineries is further evaluated to determine the best options of upgraded products, either synthetic natural gas or hydrogen, intended to be used as feedstock in a separate ammonia production unit, provided that the nitrogen byproduct of a nearby oxygen separation plant is available. 
CHAPTER 12

\section{UPGRADING BIOMASS RESIDUES TO HYDROGEN AND SNG USED AS FEEDSTOCK FOR SNF PRODUCTION}

As it has been shown in Chapter 11, the decarbonization of the synthetic nitrogen fertilizers (SNF) sector fundamentally relies on the availability of a large amount of inexpensive biomass resources [289]. In that regard, the demand might be suitably satisfied by means of the readily concentrated, residual organic material derived from sugar cane biorefineries. This approach is twofold advantageous, since those residues might be upgraded to feedstock and fuels used to supply the utility requirements in the SNF plants. Additionally, the capitalization on these waste effluents may reduce the energy consumption and the cost related to the treatment thereof. Hence, the global processes efficiency can be increased, while economical revenues from wastes, that otherwise would be disposed, can be obtained.

The main residues of the sugarcane industry are bagasse and vinasse. A fraction of the former is commonly used to provide the combined heat and power requirements of the sugarcane mill, whereas the excess bagasse is discarded [306]. Meanwhile, the vinasse is typically used in fertigation of the sugar cane crops [307]. The average quantity of residues generated by an sugarcane ethanol distillery is estimated as $474 \mathrm{~m}^{3} / \mathrm{h}$ of vinasse and $7.4 \mathrm{~kg} / \mathrm{s}$ of excess bagasse [232, 306, 308]. In light of this, enhanced conversion routes for these substances may bring about economic and environmental benefits, especially in the fertilizers sector, as long as the syngas, methane and hydrogen produced from alternative resources could replace the costly non-renewable natural gas used in the conventional route. Two biological and thermal conversion processes, namely anaerobic digestion and biomass gasification have gained recent interest due to their suitability to transform organic residues into value-added products.

Thus, in this chapter, the performance of different scenarios of the production of alternative feedstock intended to be used in SNF plants is evaluated. The process flowsheet integrates the anaerobic digestion and gasification with both Rankine and combined cycle cogeneration systems that provide the utility requirements of the chemical plant. As a result, the highest exergy efficiencies were obtained for the synthetic natural gas (SNG) production, whereas the highest irreversibility is due to the vinasse disposal, since anaerobic digestion cannot fully convert organic wastes. The bagasse gasification and the utility systems are also responsible for an important amount of exergy destroyed.

\subsection{Various SNF feedstock production from the upgrade of biomass residues}

Figure 12.1 shows the detailed superstructure used for each one of the feedstock production routes, including the energy resources, the integrated utility systems and the chemical plants for hydrogen, SNG and syngas production. The studied configurations were designed to be self-sufficient in terms of power and heat supply. This has been possible by using purified 
syngas or SNG as fuels (without electricity import) and integrating two different cogeneration systems based on either a waste heat recovery steam network or a combined cycle. The superstructure shown in Fig. 12.1 also comprises syngas and SNG-fired furnaces, a cooling tower and a vapor compression refrigeration system.

Fig. 12.1. Detailed superstructure of the various production routes of upgraded feedstock.

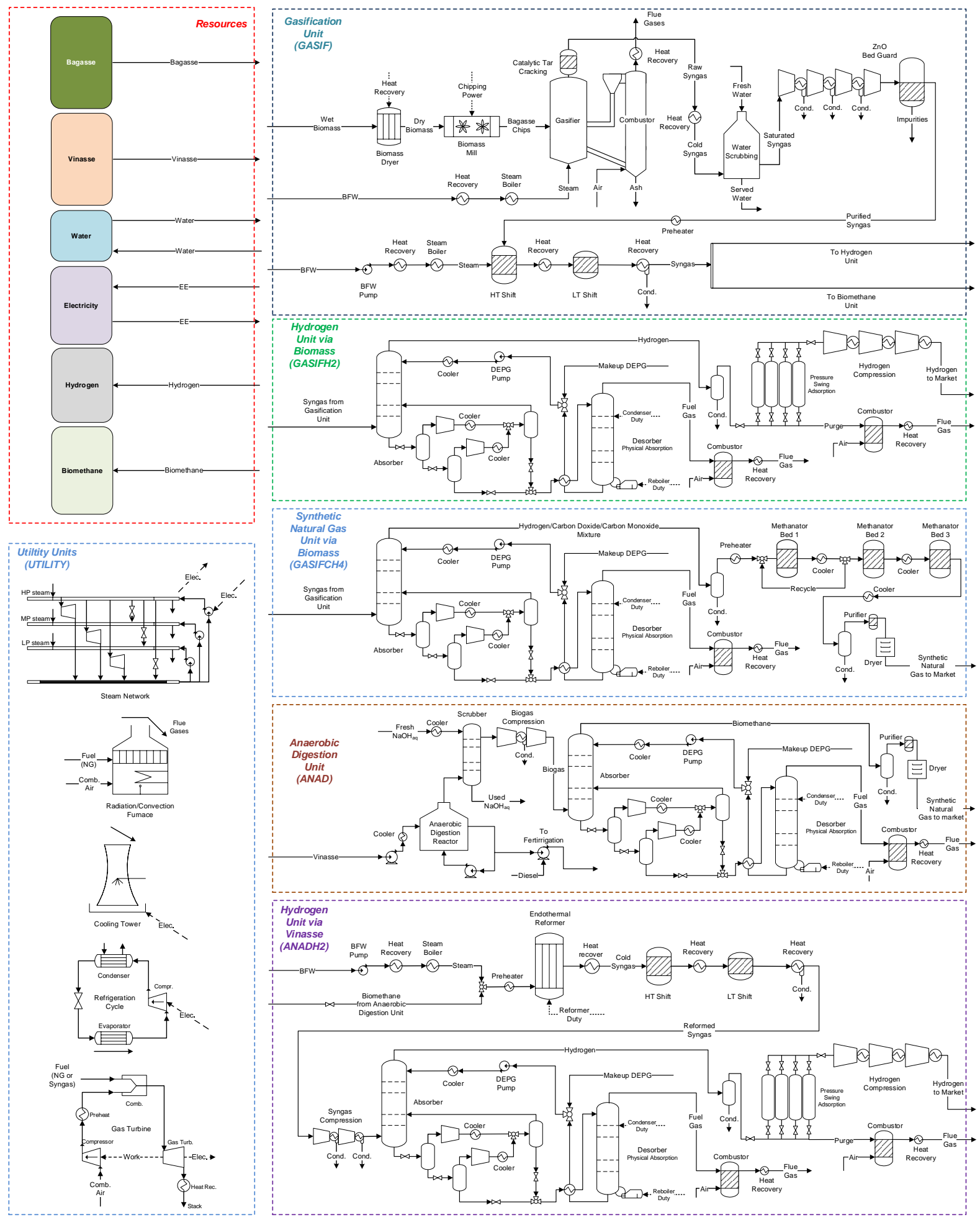

Source: Author. 
The bagasse gasification process (GASIF, Figs. 12.1-12.2) is similar to that described in the previous chapter, where a twin fast circulating bed gasifier that uses steam and air as gasifying and combustion agents, respectively, is utilized to produce a raw $\mathrm{H}_{2} / \mathrm{CO}$-rich stream. Next, a syngas purification system, comprising a downstream tar catalytic cracker and a water scrubbing column, is used to remove the impurities that may affect the downstream equipment. The syngas is then compressed to $30 \mathrm{bar}$ and a water gas shift conversion process is used to produce a further amount of hydrogen at the expense of the $\mathrm{CO}$ present in the gas. At this point, the hydrogen-rich syngas can be converted to either biomethane, hydrogen or used in the utility system to produce power. The production of pure hydrogen by using biomass-derived syngas (GASIFH2, Fig. 12.1-12.2) continues through a series of physical $\mathrm{CO}_{2}$ absorption (with an auxiliary reboiled column) and pressure swing adsorption units that deliver a pressurized hydrogen with $99 \%$ purity, suitable to be transported and used as feedstock in a separate SNF plant. On the other hand, if SNG production from biomass gasification is intended (GASIFCH4, Fig. 12.1-12.2), the hydrogen, $\mathrm{CO}$ and $\mathrm{CO}_{2}$ present in the syngas at the required stoichiometric molar fractions are converted into methane in three sequential methanator reactor beds, which operate between $250^{\circ} \mathrm{C}$ to $700^{\circ} \mathrm{C}$. The methanation reactions are exothermic and, thus, an intercooler system is required to avoid the fast catalyst deterioration. SNG may be henceforth fed to a downstream associated SNF plant.

Fig. 12.2. SNG and hydrogen production routes.

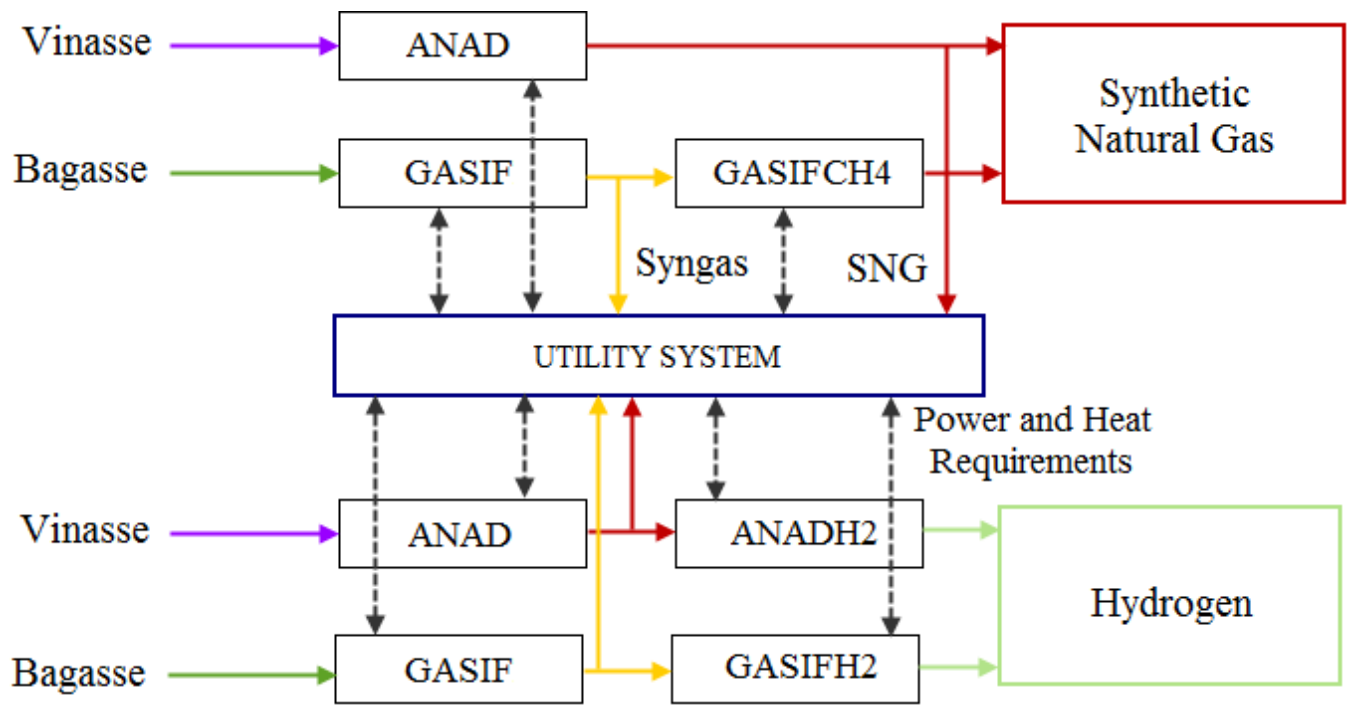

Source: Author.

In the following, a brief description of the vinasse treatment process is presented. A detailed assessment can be found in the works of Nakashima and Oliveira Junior [307, 309]. Since the vinasse separated in the ethanol distillation column is at relatively high temperatures $\left(60^{\circ} \mathrm{C}\right)$ and presents a low $\mathrm{pH}$ (3.75-5) [308], it must be mixed with a recycled digestor effluent for $\mathrm{pH}$ correction and cooled down to $35^{\circ} \mathrm{C}$ [310]. This temperature is suitable for biogas production via anaerobic digestion (ANAD, 12.1-12.2) [311]. In order to produce SNG, the biogas is desulfurized using a cold sodium hydroxide solution [309], whereas the remaining biogas impurities, mainly $\mathrm{CO}_{2}$, are removed by using a physical absorption process with an auxiliary reboiled column, similar to that used in the gasification route. Since the overhead 
gas of the auxiliary desorption column still contains methane, it can used as fuel gas to provide the utility requirements of the overall system. In the hydrogen production via vinasse anaerobic digestion (ANADH2, Fig. 12.1-12.2), the SNG produced is further reformed by using steam (S/C ratio 3.0) similarly to the SMR process in the conventional natural gasfueled route. Two sequential high and a low temperature water gas shift reactors are used to increase hydrogen yield. The additional $\mathrm{CO}_{2}$ produced is removed again by using physical absorption and pressure swing adsorption systems. At last, the purified hydrogen is compressed (200 bar) for being transported and fed to a separate SNF plant.

Equations 12.1-12.2 are used to calculate the plantwide exergy efficiency as well as the exergy efficiency of the utility system for the various waste upgrading routes studied:

$$
\begin{gathered}
\eta_{\text {utility }}=\frac{W_{\text {net }}+B_{\text {delivered }}^{Q}}{B_{\text {recovered }}^{Q}+B_{\text {fuel gas }}} \\
\eta_{\text {overall }}=\frac{B_{\text {high-grade feedstock }}}{B_{\text {biomass residues }}}
\end{gathered}
$$

where $B_{\text {biomass residues, }}, B_{\text {fuel gas }}$, and $B_{\text {high-grade feedstock }}$ correspond to the exergy of the biomass waste effluent from the biorefinery, the intermediate fuel consumed in the utility systems, and the upgraded gas that can be used as feedstock in an associate SNF plant. Meanwhile, $B_{\text {recovered, delivered }}^{Q}$ stands for the heat exergy recovered by the utility system and distributed throughout the chemical plant. The net power produced $W_{\text {net }}$ by the utility system is used to drive the syngas compressors and other ancillary components, without electricity import.

\subsection{Optimization problem definition}

The following optimization problem is formulated to assess the most suitable utility systems that satisfy the minimum energy requirement (MER) with the lowest resources consumption and optimal operating revenues. The computational framework OSMOSE Lua ${ }^{\circledR}$ manages the data transfer with Aspen $®$ Plus software and builds the mixed integer linear programming (MILP) problem described in the Eqs.(12.3-12.6) that maximizes the operating revenues of the chemical plant, while satisfying the constraints of the MER problem. The solution consists thus of the size factors, $f_{w}$, and the integer variables, $y_{w}$, that determines the existence of each utility unit $w$ that minimizes the objective function given by Eq.(12.1):

$$
\min _{\substack{f_{\omega}, y_{\omega} \\
R_{r}}} \sum_{\omega=1}^{N_{\omega}} f_{\omega}\left[\sum_{\substack{\text { Bagasse, } \\
\text { Vinasse }}}\left(c \cdot B^{C H}\right)_{\text {residue }}+(\dot{V} \cdot c)_{\text {water }}-\left(c \cdot B^{C H}\right)_{\begin{array}{c}
\text { high-grade } \\
\text { feedstock }
\end{array}}\right] \cdot t_{O P}
$$

Subject to:

Heat balance of temperature interval $r \sum_{\omega=1}^{N_{\omega}} f_{\omega} q_{\omega, r}+\sum_{i=1}^{N} Q_{i, r}+R_{r+1}-R_{r}=0 \quad \forall r=1 . . N$

Existence and size of the utility unit $w$

$$
f_{\min , \omega} \mathrm{y}_{\omega} \leq f_{\omega} \leq f_{\max , \omega} \mathrm{y}_{\omega} \quad \forall \omega=1 . . N_{\omega}
$$

Feasibility of the solution (MER)

$$
R_{1}=0, \quad R_{N_{r}+1}=0, \quad \mathrm{R}_{r} \geq 0
$$


where $N_{w}$ is the number of units in the set of utility systems; $B$ and $c$ stand, respectively, for the exergy flow rate $(\mathrm{kW})$ and costs (euro per $\mathrm{kWh}, \mathrm{m}^{3}$ or $\mathrm{kg} / \mathrm{h}$ ) of the residues consumed (or selling prices of the $\mathrm{H}_{2}$ and SNG produced); $V$ is the flowrate of water consumed $\left(\mathrm{m}^{3} / \mathrm{h}\right) ; Q$ and $q$ are the heat exchanged between the process streams $\left(Q_{\mathrm{i}, \mathrm{r}}>0\right.$ hot stream, $<0$ cold stream), and the heating/cooling supplied by the utility systems $(\mathrm{kW})$, respectively; $t_{\mathrm{OP}}$ is the operation time (h); and $R$ is the heat cascaded from higher $(r+1)$ and to lower $(r)$ temperature intervals $(\mathrm{kW})$.

Additional equations for the mass and energy balances on each stream layer (water, biomass, upgraded products), along with the equations for modeling each one of the different energy technologies of the utility systems, are included in the optimization problem, analogously to the procedure explained in Chapter 7, Fig. 7.2. Representative market prices for the water $\left(3.03 \mathrm{euro} / \mathrm{m}^{3}\right)$, bagasse $(0.0056 \mathrm{euro} / \mathrm{kWh})$, vinasse $(0.0006$ euro $/ \mathrm{kWh})$ as well as the selling prices of the upgraded SNG (0.032 euro/kWh) and hydrogen $(0.072$ euro/kWh), are taken from sorted literature [66, 230, 231].

\subsection{Optimal operating conditions of the upgrading processes of the biorefinery residues.}

The main exergy consumption and production remarks for each analyzed case are summarized in Table 12.1 and briefly discussed next. According to those results, the biomass route produces higher amounts of upgraded hydrogen and SNG, partly explained by an incomplete conversion of the vinasse in the anaerobic digestion compared with the bagasse gasification. This difference is nonetheless offset when SNG is the desired product, since the anaerobic digestion readily produces a valuable methane-rich stream, whereas the gasification process relies on further purification and methanation processes.

Meanwhile, the use of a more efficient Combined cycle reduces the fuel consumption of the utility systems, which slightly increases the amount of exported SNG product (6\%). However, a thorough evaluation of the gas turbine integration may show that the marginal investment cost may eventually outweigh this thermodynamic advantage. For this reason, an effectively integrated steam network could be more favorable for supplying the total electricity demand by using the waste heat exergy recovered throughout the chemical plant together with an additional consumption of syngas or SNG.

On the other hand, the hydrogen production scenario seems to be more favorable in terms of higher operating revenues, even for utility fuel consumptions comparable to those of the SNG production routes. Ammonia can be then produced in a downstream synthesis loop (See Chapter 9 and 10) provided that the nitrogen byproduct of a nearby oxygen separation plant is available [81], so that the hydrogen logistics can be simplified. Thus, the choice of the most favorable upgraded feedstock will be necessarily subject to the available infrastructure for storage and transportation, as well as to other economic criteria [31]. 
Table 12.1. Exergy consumption and production remarks for each production route shown in Figs. 12.1-12.2

\begin{tabular}{|c|c|c|c|c|}
\hline & \\
\hline & \multicolumn{4}{|c|}{ Upgraded product } \\
\hline & $\mathrm{CH}_{4}$ & $\mathrm{CH}_{4}$ & $\mathbf{H}_{2}$ & $\mathbf{H}_{2}$ \\
\hline Cogeneration cycle & $\begin{array}{l}\text { Combined } \\
\text { cycle }\end{array}$ & $\begin{array}{l}\text { Rankine } \\
\text { cycle }\end{array}$ & $\begin{array}{l}\text { Combined } \\
\text { cycle }\end{array}$ & $\begin{array}{l}\text { Rankine } \\
\text { cycle }\end{array}$ \\
\hline \multicolumn{5}{|l|}{ Overall biomass consumption } \\
\hline As vinasse $(\mathrm{MW})$ & \multicolumn{4}{|c|}{69.1} \\
\hline As bagasse (MW) & \multirow{2}{*}{\multicolumn{4}{|c|}{72.3}} \\
\hline Overall fuel consumption & & & & \\
\hline Gaseous fuel fed to utility system & $\begin{array}{l}\text { Purified } \\
\text { syngas }\end{array}$ & $\begin{array}{l}\text { Purified } \\
\text { syngas }\end{array}$ & $\begin{array}{l}\text { Purified } \\
\text { syngas }\end{array}$ & $\begin{array}{l}\text { Vinasse- } \\
\text { derived SNG }\end{array}$ \\
\hline LHV (MJ/kg) & 14.3 & 14.3 & 14.3 & 48.1 \\
\hline Molar flow rate $(\mathrm{kmol} / \mathrm{h})$ & 110 & 188 & 116 & 46 \\
\hline$[$ Exergy $(\mathrm{MW})]$ & [6.9] & {$[11.8]$} & [7.3] & [10.7] \\
\hline $\begin{array}{l}\text { Fraction of the overall syngas or SNG } \\
\text { produced }(\%)\end{array}$ & 14.45 & 24.77 & 15.26 & 37.49 \\
\hline \multicolumn{5}{|l|}{ Exported product } \\
\hline Biomass route contribution (\%) & 0.54 & 0.51 & 0.58 & 0.72 \\
\hline Vinasse route contribution ( \%) & 0.46 & 0.49 & 0.42 & 0.28 \\
\hline Total exported product $(\mathrm{kmol} / \mathrm{h})$ & 266 & 249 & 695 & 670 \\
\hline [Exergy (MW)] & [62.3] & [58.2] & [48.2] & [45.7] \\
\hline Operating Revenues (euro/h) & 1,569 & 1,438 & 2,990 & 2,806 \\
\hline
\end{tabular}

It is worthy to notice that, the scenarios presented in Table 12.1 mostly rely on the use of the purified syngas as the fuel consumed in the utility system over the SNG utilization. This circumstance can be explained by the fewer conversion steps that lead to a lower exergy cost of the upgrading process of vinasse to $\mathrm{SNG}$, in comparison to the more intricate gasification process combined with methanation. In other words, the best solutions for SNG production prioritize the utilization of the vinasse anaerobic digestion, whereas a fraction (14.5-24.8\%) of the intermediate syngas produced in the gasification process is used as the only fuel input to the utility system.

This trend drastically changes in the case of the hydrogen production route. Indeed, the choice of the fuel consumed seemingly depends on the type of the cogeneration plant that is integrated. For instance, by introducing a more efficient combined cycle in the $\mathrm{H}_{2}$ production route, the optimum solution only consumes $15.3 \%$ of the syngas produced. In contrast, when a cogeneration system based solely on a steam network is operated, the fraction of vinassederived SNG used as fuel in the utility system can be as much as $37.5 \%$ of the total SNG produced. This situation is related to the higher energy requirements (compression, reforming, purification, etc.) of the hydrogen production route via vinasse-derived SNG, which leads the optimal solutions to prioritize the gasification route. In this way, provided that more efficient cogeneration systems are integrated, the energy intensive conversion of the vinasse-derived SNG to $\mathrm{H}_{2}$ is preferred. However, as soon as less efficient cogeneration systems are considered, the utilization of SNG as the fuel of the utility system can be justified. Clearly, this leads to a penalty on the efficiency of the utility system due to an increased consumption of fuel, and consequently to the lowest overall exergy efficiency among the upgrading routes studied (see Fig. 12.3). 
Fig. 12.3. Overall and utility system exergy efficiency in the various upgraded SNG and hydrogen production routes

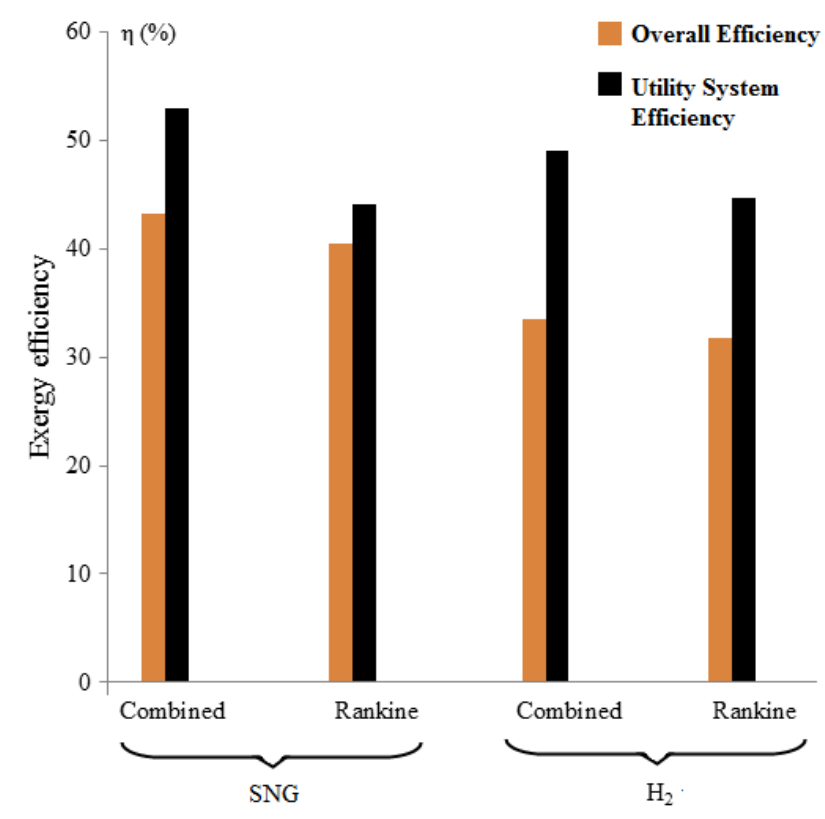

Source: Author.

Figure 12.4 evidences a major concentration of exergy destruction in the anaerobic digestion, the gasification and the utility systems. Since the SNG production by using a combined cycle has the lowest exergy requirements in terms of both heat and power, the irreversibility in the utility system and, consequently, in the overall plant is the lowest. On the other hand, regardless of the cogeneration process used, the more numerous hydrogen production steps introduce further sources of irreversibility, more precisely about $9.3 \%$ of the overall exergy destroyed in the $\mathrm{H}_{2}$ production routes (see GASIFH2 and ANADH2, Fig. 12.4).

Accordingly, the exergy analysis points toward the syngas and biomethane production processes as the ones facing the most important challenges for upgraded syngas, hydrogen and SNG production, intended to be used in a downstream industrial ammonia loop. According to Nakashima and Oliveira Junior [307], the treated vinasse effluent has a high concentration of inert material that is discarded in the sugarcane crops. Thus, the exploitation of vinasse organic inerts (e.g. concentration and combustion) could significantly reduce the exergy destruction in the routes analyzed. As concerns the gasification process, a significant part of the energy of the biomass (3\% of the LHV) [290] must be still internally consumed in the syngas production, which significantly reduces the yield of syngas and the value-added products. In this regard, higher gasification pressures may lead to reduced power consumption in the syngas compression and, consequently, lower exergy destruction in the utility systems [32]. 
Fig. 12.4. Exergy destruction breakdown in the various $S N G$ and hydrogen production routes

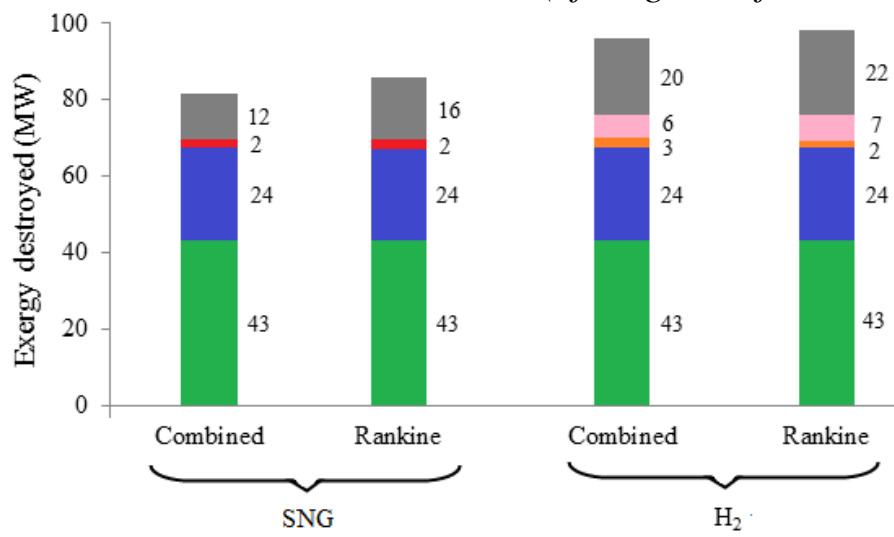

Utility Systems

(GASIFH2)

- Syngas-derived hydrogen production

Biomethane-derived hydrogen production (ANADH2)

Syngas-derived biomethane production (GASIFCH4)

Biomass-derived syngas production

(GASIF)

Vinasse-derived biomethane production

(ANAD)

Source: Author.

The corresponding integrated curves indicating the location of the various utility and chemical energy conversion systems of the studied SNG and hydrogen production routes are illustrated in Figs. 12.5 a-d.

Fig. 12.5. Integrated curves of the various production routes for $S N G(a)$ without and $(b)$ with the integration of a combined cycle. The corresponding figures of hydrogen production are shown in (c) and (d).

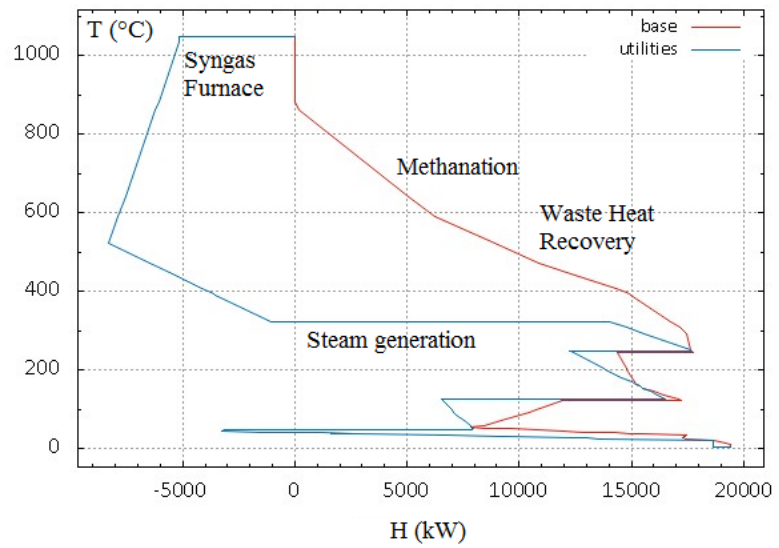

(a)

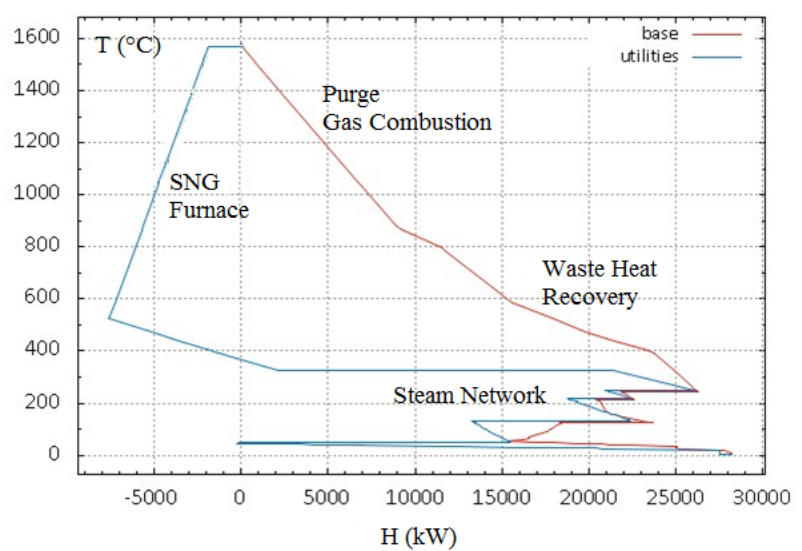

(c)

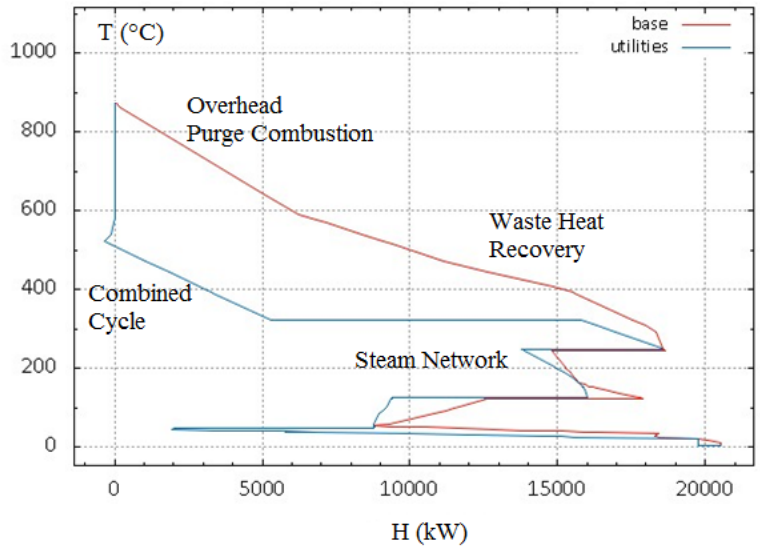

(b)

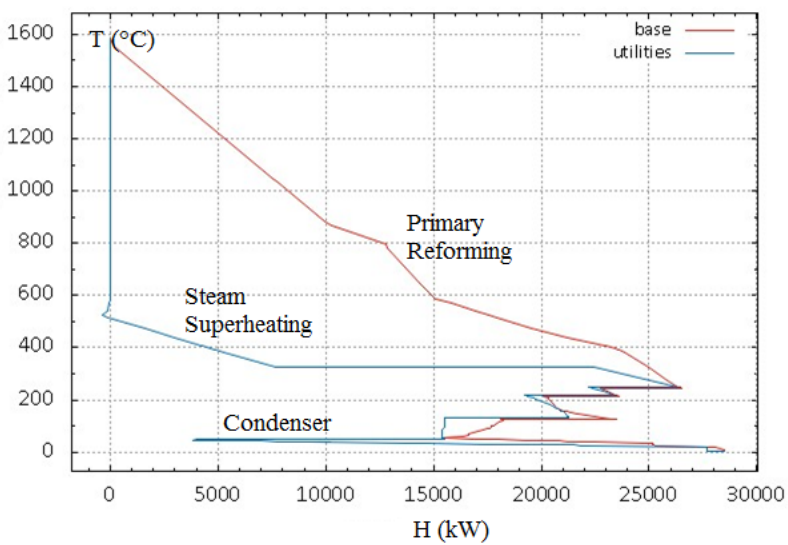

(d)

Source: Author. 


\subsection{Final considerations}

Various scenarios for upgrading the main sugarcane residues into value-added products, namely synthetic natural gas and hydrogen, have been examined in terms of operating revenues, fuel consumption, exergy efficiency and exergy destruction. To this end, an exergy analysis and energy integration study is performed in order to determine the best combination of chemical processes and utility systems that allow reducing the process irreversibility. Among the scenarios studied, SNG production presented the highest exergy efficiency due to the lower exergy consumption and simpler conversion route. Nevertheless, higher revenues can be obtained by producing hydrogen at comparatively similar exergy efficiencies. The integration of a combined cycle leads to slight efficiency gains, which may eventually offset by increased marginal investment costs, which must be further analyzed in future works.

Technically and thermodynamically enhanced chemical conversion processes for biomass pretreatment, gasification and syngas purification, along with improved vinasse treatments are key issues for the efficient and economic production of alternative, high-grade feedstock useable in associate synthetic nitrogen fertilizers plants. Meanwhile, the pursuit of the decarbonization of the SNF sector will remain an active and open field of research. 
CHAPTER 13

\section{CONCLUSIONS AND SUGGESTIONS FOR FUTURE WORKS}

In this thesis, a systematic methodology is proposed and applied for the process synthesis and optimization of the facilities of a highly integrated syngas and ammonia production plant as part of a synthetic nitrogen fertilizer (SNF) complex. The mathematical modeling, simulation and optimization framework considers the actual operational constraints and the limitations of industrial ammonia production plants, but also attempts to propose novel approaches in light of the limited room for improvement in the old-fashioned, existing facilities. Nevertheless, the limited knowledge of the most advanced commercially available technologies and operating conditions (know-how) is admittedly recognized as the chief barrier in the development of this thesis.

For this reason, initially, the most recent developments on the energy savings and energy integration processes in the chemical industry of SNF have been reviewed. Next, a baseline flowsheet including the most representative processes existing in the SNF plants is adopted. Then, a set of potential improvements that entails a reduction of the production costs and an increase of the exergy efficiency of the conventional unit is outlined. The developments and modifications suggested for the improvement of the different sections of the baseline design are ranked through the conception and proposition of indicators for the exergy, economic and environmental performance. For instance, an appropriated methodology has been proposed to address the rational distribution of the exergy costs and $\mathrm{CO}_{2}$ emissions among the streams of the chemical plant, with special attention given to the convection section of the primary reformer. The exergy efficiency of ammonia is calculated as $55.71 \%$, whereas the specific $\mathrm{CO}_{2}$ emissions of the ammonia produced reach $0.0881 \mathrm{~kg}_{\mathrm{CO} 2}$ per $\mathrm{MJ}_{\mathrm{NH} 3}$ (or $1.69 \mathrm{t}_{\mathrm{CO} 2} / \mathrm{t}_{\mathrm{NH} 3}$ ). For the $\mathrm{CO}_{2}$ byproduct, those figures are found to be $61.08 \%$ and $0.0821 \mathrm{~kg}_{\mathrm{CO} 2} / \mathrm{MJ}_{\mathrm{CO} 2}$, respectively.

Furthermore, from the analysis of the baseline flowsheet, a directly proportional correlation between the increased exergy destruction rate and larger driving forces in the chemical process has been evidenced. Thus, since the irreversibility in real ammonia production facilities cannot be totally eliminated (because reasonable product yields and reduced equipment sizes are desirable), any improvement in the performance of an industrial ammonia production plant must be always considered a trade-off between lower exergy destruction rates and higher yield rates. For this reason, different process synthesis and optimization techniques, including heuristic, thermodynamic and algorithmic methods have been used to find out the best process flowsheet configurations that operate under various pathways of energy and material inputs and satisfy the specified targets.

For instance, the study of the intricate relationship between the chemical plant and the utility systems has been better evaluated by using a systematic methodology based on a mixed 
integer linear programming (MILP) and a modular chemical process simulation approach. This procedure allowed for the determination of the most suitable utility systems that satisfy the minimum energy requirement (MER) with the lowest resources consumptions and operating cost. This has been possible thanks to a thorough characterization of the energy integration curves of the chemical process, but especially of the reactive systems. Three different types of syngas purification units, namely chemical and physical absorption systems, have been also employed. As a result, by operating under various scenarios of energy inputs (electricity, natural gas, biomass) and cogeneration systems (steam network, combined cycle), the most appropriate utility systems and operation conditions, that maximize the recovery of the thermodynamic potential associated to waste heat exergy produced along the chemical plant, have been identified.

Furthermore, the calculation of the direct and indirect $\mathrm{CO}_{2}$ emissions generated in the optimal configurations of ammonia production allowed for better comparisons with other industrial and chemical process in terms of the environmental impact. As a result, by operating either in a mixed mode (i.e. by means of a partial electricity import along with an improved waste heat recovery and cogeneration system) or in a totally autonomous mode with a combined cyclebased cogeneration system, together with the use of physical absorption-based syngas purification unit, the process irreversibility and the operation cost can be reduced, whereas the efficiency of the conventional plant is increased (ca. 8-10\%). Thus, by considering the current autonomous operation mode (i.e. no electricity import) of most of the moderns ammonia production plants, the integration of a combined cycle is the most rational alternative for increasing the exergy efficiency and maximizing the process revenues in the SNF facilities.

By considering (i) the highly irreversible nature of complete combustion and (ii) the associate stack losses in both the fired furnace of the primary reformer and in the combustion chamber of the gas turbine, along with (iii) the exergy losses associated to the excess steam generation and its subsequent condensation; four novel approaches, based on the chemically recuperated gas turbine concept (CRGT), have been proposed for the production of syngas, intended to be fed to a backend industrial ammonia loop. These novel setups were aimed to improve the energy integration capabilities at higher temperatures and reduce the amount of atmospheric emissions, by intensifying the rates of pre-combustion carbon capture. Five different configurations have been compared, namely a conventional syngas production system and four proposed configurations based on the chemically recuperated gas turbine (CRGT) concept, with single and dual levels of pressure. As a conclusion, even at optimal operating conditions, the proposed single and dual pressure configurations presented a specific exergy destruction ranging from 87.5 to $95.2 \mathrm{MJ} / \mathrm{kmolH}_{2}$, although 3-12\% lower than those found for the conventional process. Moreover, the exergy fuel consumption in the proposed CRGT configurations has been estimated as $0.5-2.7 \%$ lower than in the conventional design. Meanwhile, the cooling requirement in the proposed setups is substantially reduced (up to $10 \%$ ) compared to the conventional plant. It is thus verified that by using a dual pressure configuration that aims to generate a counteraction in the reactive components, the reacting driving forces can be effectively reduced. However, the most striking characteristic of these proposed setups has been their capability of cutting down up to $25 \%$ the specific atmospheric 
$\mathrm{CO}_{2}$ emissions, compared to the conventional case. To the author's knowledge, similar approaches has been proposed for expanding the exothermic reactor effluents, but none of them have integrated the CRGT concept to the chemical and cogeneration plant in a same physical structure.

Proceeding from these reasonings, the interrelation between the kinetic, thermodynamic and technical operation parameters of the backend industrial ammonia synthesis unit has been also examined by using both the Counteraction principle and the Le Châtelier Principle. Two new different approaches have been proposed to calculate the exergy efficiency of industrial processes that cope with relatively large material exergy flow rates, compared to other exergy flow rates (e.g. power). As a result, even at optimal operating conditions, the ammonia synthesis reactor, the syngas compression, the ammonia refrigeration and the waste heat recovery network were together responsible for about $26 \mathrm{MW}$ of exergy destruction in backend ammonia production unit (1,000 $\mathrm{t}_{\mathrm{NH} 3} /$ day), with the largest figures related to the base case operating condition at 200 bar (38.8 MW). Furthermore, the exergy destruction breakdown shows that the ammonia converter and the refrigeration system were together responsible for more than $71-82 \%$ of the overall irreversibility in the ammonia loop. More importantly, it has been found that, despite the fact that increased driving forces may imply higher exergy destruction rates in the reactor beds, by solely increasing the reacting driving forces in the ammonia converter, the whole system irreversibility has not been necessarily increased. Indeed, the global effect of the finite driving forces in that particular equipment was seemingly compensated by the enhancement of the performance of the integrated ammonia loop (namely, lower circulation rates, higher reactor conversions and reduced power consumption for recycling and refrigeration purposes).

On the other hand, by introducing an additional once-through conversion section operating at a lower pressure ( 83 bar) in series with the main ammonia loop, the exergy losses have been driven down by $13 \%$, whereas the extent of recovery of the waste heat from the enthalpy of synthesis reaction has been also increased. As a result, the implementation of a dual pressure configuration increased the overall exergy efficiency of the industrial ammonia unit up to $30 \%$, by significantly reducing the compression demand. In spite of these advantages, the syngas compressor, the ammonia converter, the waste heat recovery and the ammonia refrigeration systems were still responsible for about $80-86 \%$ of total irreversibility in the backend industrial ammonia unit, which varies from $23.8 \mathrm{MW}$ for the dual pressure up to $27.2 \mathrm{MW}$ for the single pressure (150 bar) configuration, respectively. Consequently, it could be expected that the development of better catalyst that operate at lower or gradual levels of pressure bring about a reduction of the power consumption in the ammonia loop. However, since a decrease of the loop pressure entails a reduction of the available waste heat from the synthesis reactor, the overall steam and power generation balance may be drastically modified. Accordingly, the design and integration of advanced catalytic reactors into the conventional production facilities should be thoroughly evaluated including the secondary effects. 
In the last chapters of this thesis, the minimization of the operating costs in the ammonia production processes that use an inexpensive, readily concentrated source of biomass from sugar cane mills has been addressed. Actually, this analysis has been motivated by the underexploited biomass potential in the tropical countries as well as due to the more stringent environmental regulations in the SNF industry. A detailed evaluation of the economic and environmental impacts of the partial or total substitution of the natural gas in conventional ammonia production plants permited the calculation of the exergy efficiencies of natural and biomass-based ammonia production routes, averaging $65.8 \%$ and $41.3 \%$, respectively. Meanwhile, the overall $\mathrm{CO}_{2}$ emission balance attained 0.5 to $-2.3 \mathrm{t}_{\mathrm{CO} 2} / \mathrm{t}_{\mathrm{NH} 3}$, for the fossil and alternative production routes, respectively. Yet, the current high investment risk and less mature renewable energy conversion technology compared with the mature infrastructure of the coal and petrochemical industry hinder the integration of alternative ammonia production routes at larger scales in the near-medium term. In the meantime, electricity import, whether available, may help reducing the irreversibility in the biomass-based ammonia production plants, as well as reducing the overall $\mathrm{CO}_{2}$ emissions, provided that the domestic electricity generation carries reduced indirect $\mathrm{CO}_{2}$ emissions and higher generation efficiencies. Notwithstanding, the highest operating revenues can be rather achieved by totally replacing the natural gas input and avoiding the electricity import, favoring the consumption of the gasification syngas produced in a combined power cycle for supplying the heat and power demands of the chemical plant and the utility systems.

All in all, as concerns the natural gas substitution by using biomass and 'greener' electricity sources, the results showed to be strongly dependent on the indirect emissions, the energy resources used (natural gas, electricity or bagasse) and the ratio of the price of electricity to natural gas. Furthermore, technically and thermodynamically enhanced chemical conversion processes for biomass pretreatment, gasification and syngas purification, along with improved vinasse treatments are found to be key issues for the efficient and economic production of alternative, high-grade feedstock useable in associate synthetic nitrogen fertilizers plants. Meanwhile, the pursuit of the decarbonization of the SNF sector will remain an active and open field of research.

Some general recommendations can be issued from the main results obtained in this thesis:

- Advanced energy integration approaches that allow for increased waste heat recovery and improved cogeneration are unfortunately not costumary practices in the conservative chemical and industrial sector. However, due to the reduced room for the energy consumption cutdown and, thus, the overall efficiency increase, the exploration of breakthrough approaches for energy conversion technologies, arrangements and energy resources will become necessary, at least for elucidating the truly thermodynamic and technological potentials available in modern ammonia production facilities.

- The combined application of the exergy method and other physical principles based on the Second Law of the Thermodynamics along with economic and extended environmental 
analyses prove to be useful when comparing the utilization of various energy resources and operation modes in complex chemical production plants. This approach also reflects the true impact of the chemical production facilities on the market and the natural environment in which they operate (and viceversa).

- Despite of lower exergy efficiencies and, consequently, increased exergy consumption, alternative energy resources may help reducing the fossil fuel dependence and intensive $\mathrm{CO}_{2}$ emissions of the SNF industry, as well as increasing the operating revenues given the large biomass potential in tropical countries. However, in the near term, the most likely scenario might rather consists of the combined use of the available renewable and fossil energy resources to achieve a more competitive position in the volatile international market.

$* * *$

Certainly, the various aspects presented in this thesis can be questioned, improved, extended or used to derive other subjects of study in the Laboratory of Environmental and Thermal Engineering (LETE) research group and in the scientific community in general. Indeed, the variety of processes, operating conditions, arrangements and uncertainties associated mantain this field of research under constant development. Some additional suggestions for futures research work can be summarized but not limited to:

- Energy integration and economic analyses of the integration of the syngas and ammonia production facilities and associated urea plants. Urea plants generally consume the carbon dioxide captured from the reforming, partial oxidation and shift reactions. However, since in the conventional ammonia production process, the $\mathrm{CO}_{2}$ captured falls short to completely supply the total amount of $\mathrm{CO}_{2}$ required as feedstock, the integration of the novel approaches with enhanced energy consumption and $\mathrm{CO}_{2}$ absorption rates may help increasing the availability of this input in the urea unit.

- Detailed economic evaluation and equipment design of the novel approaches proposed for the production of syngas and ammonia by using either non-renewable and alternative energy resources. Although a detailed quantification of the investment costs and financial risk is not mandatory in order to assess the thermodynamic and environmental potential, they may certainly contribute to a more insightful decision-making on the commercial feasibility of the breakthrough proposed configurations.

- Preliminary assessment of the potential of decarbonization of the chemical and industrial sector through the upgrade of alternative energy resources, such as the residues of sugar cane biorefineries, and the integration of the existing infrastructure of fossil fuels distribution. The mapping of the localization of the Sao Paulo's natural gas pipelines superposed to the analysis of the distribution of the mains sugar cane mills can be used to project the building of the future ammonia production plants aiming to partially (or totally) replace the natural gas utilization in the manufacturing of synthetic nitrogen fertilizers. 
- Evaluation of the production of alternative energy carriers/transportation fuels such as methanol, synthetic natural gas and ammonia by integrating the novel approaches proposed in this thesis and the Second Law Analysis, including the Counteraction principle.

- Sensitivity analysis of the uncertainty associated to the market prices of the resources consumed, since it may disclose radically different optimal configurations when the ratio between the electricity and the fuel prices becomes numerically comparable to the cogeneration efficiencies of the existing and revamped utility systems. 


\section{CITED REFERENCES}

1. Follett, R., Hatfield, J., Chapter 4. Utilization and Metabolism of Nitrogen by Humans, in Nitrogen in the Environment: Sources, Problems and Management. 2008, Elsevier USDA, Soil-Plant-Nutrient Research Unit. p. 720.

2. Erisman, J., Sutton, M., Galloway, J., Klimont, Z., Winiwarter, W., How a century of ammonia synthesis changed the world. Nature Geosci, 2008. 1(10): p. 636-639.

3. Alhammadi, H.R., J., Chapter B4 - Process design and operation: Incorporating environmental, profitability, heat integration and controllability considerations, in Computer Aided Chemical Engineering, S. Panos and C.G. Michael, Editors. 2004, Elsevier. p. 264-305.

4. Dias, V., Fernandes, E., Fertilizers: A synthetic global insight [In Portuguese]. BNDES Setorial Rio de Janeiro, set. 2006, 2006(24): p. 97-138.

5. Rafiqul, I.W., C., Lehmann, B., Voss, A., Energy efficiency improvements in ammonia production—perspectives and uncertainties. Energy, 2005. 30(13): p. 2487-2504.

6. Hajjaji, N., Pons, M., Houas, A., Renaudin, V., Exergy analysis: An efficient tool for understanding and improving hydrogen production via the steam methane reforming process. Energy Policy, 2012. 42(0): p. 392-399.

7. Simpson, A., Lutz, A., Exergy analysis of hydrogen production via steam methane reforming. International Journal of Hydrogen Energy, 2007. 32(18): p. 4811-4820.

8. Kirova-Yordanova, Z., Application of the exergy method to the environmental impact estimation: The nitric acid production as a case study. Energy, 2011. 36(6): p. 3733-3744.

9. Kirova-Yordanova, Z., Thermodynamic Evaluation of Energy Integration and Cogeneration in Ammonium Nitrate Production Complexes. International Journal of Thermodynamics (IJoT), 2013. 16(4): p. 163-171.

10. Panjeshahi, M.H., Ghasemian Langeroudi, E., Tahouni, N., Retrofit of ammonia plant for improving energy efficiency. Energy, 2008. 33(1): p. 46-64.

11. Papoulias, S., Grossmann, I., A structural optimization approach in process synthesisII: Heat recovery networks. Computers \& Chemical Engineering, 1983. 7(6): p. 707-721.

12. Papoulias, S., Grossmann, I., A structural optimization approach in process synthesis-I: Utility systems. Computers \& Chemical Engineering, 1983. 7(6): p. 695-706. 
13. Papoulias, S., Grossmann, I., A structural optimization approach in process synthesisIII: Total processing systems. Computers \& Chemical Engineering, 1983. 7(6): p. 723-734.

14. Yee, T.F., Grossmann, I. E., Kravanja, Z., Simultaneous optimization models for heat integration-I. Area and energy targeting and modeling of multi-stream exchangers. Computers \& Chemical Engineering, 1990. 14(10): p. 1151-1164.

15. Yee, T.F., Grossmann, I. E., Kravanja, Z., Simultaneous optimization models for heat integration-III. Process and heat exchanger network optimization. Computers \& Chemical Engineering, 1990. 14(11): p. 1185-1200.

16. Yee, T.F., Grossmann, I. E., Simultaneous optimization models for heat integration-II. Heat exchanger network synthesis. Computers \& Chemical Engineering, 1990. 14(10): p. 1165-1184.

17. Worrell, E., Blok, K., Energy savings in the nitrogen fertilizer industry in the Netherlands. Energy, 1994. 19(2): p. 195-209.

18. Rosen, M.A., Comparative assessment of thermodynamic efficiencies and losses for natural gas-based production processes for hydrogen, ammonia and methanol. Energy Conversion and Management, 1996. 37(3): p. 359-367.

19. Kirova-Yordanova, Z., Exergy analysis of industrial ammonia synthesis. Energy, 2004. 29(12-15): p. 2373-2384.

20. Norskov, J.K., Bligaard, T., Rossmeisl, J., Christensen, C. H., Towards the computational design of solid catalysts. Nat Chem, 2009. 1(1): p. 37-46.

21. Liu, H., Ammonia Synthesis Catalysts: Innovation and Practice. 2013, Beijing: Chemical Industry Press, ISBN 978-981-4355-77-3.

22. UNIDO, Fertilizer Manual: UN Industrial Development Organization. 3rd ed, ed. I.F.D. Center. 1998, Dordrecht, Netherlands: Kluwer Academic Publishers. 616.

23. Chorkendorff, I., Niemantsverdriet, J. W., Concepts of Modern Catalysis and Kinetics. 2nd ed. 2007: Wiley-VCH Verlag GmbH \& Co, ISBN: 978-3-527-31672-4.

24. Witt, J.J., Riezebos, A., Upgrading a 25-year-old ammonia plant resulting in lower energy consumption and higher production capacity. in AIChE Ammonia Safety Symposium. 1998. Charleston, South Carolina.

25. Appl, M., Ullmann's encyclopedia of industrial chemistry, Vol.11. Chapter 2. 2012, Wiley-VCH Verlag GmbH \& Co., Weinheim. 
26. Dopper, J.G., European Roadmap for Process Intensification. 2007, Ministry of Economic Affairs: Delft, The Netherlands. p. 53.

27. Canadian.Fertilizer.Institute, Benchmarking energy efficiency and carbon dioxide emissions. 2007, Canadian Industry Program for Energy Conservation.

28. Strait, R.B. New KBR Technologies available for revamping ammonia plants. in Nitrogen + Syngas International Conference and Exhibition. 2008. Moscow, 20-23 April.

29. Filippi, E., Davey, W., Thomas Wurzel, T. MEGAMMONIA- The Mega-Ammonia process for the new century. in AICHE Ammonia Safety Symposium. 2004.

30. Arora, P., Hoadley, A., Mahajani, S., Ganesh, A., Multi-objective optimization of biomass based ammonia production - Potential and perspective in different countries. Journal of Cleaner Production, 2017. 148: p. 363-374.

31. Ruth, M., Laffen, M., Timbario, T., Hydrogen Pathways: Cost, Well-to-Wheels Energy Use, and Emissions for the Current Technology Status of Seven Hydrogen Production, Delivery, and Distribution Scenarios Technical Report NREL/TP-6A1-46612. 2009, National Renewable Energy Laboratory - NREL.

32. Spath, P.L., Dayton, D.C. , Preliminary Screening - Technical and Economic Assessment of Synthesis Gas to Fuels and Chemicals with Emphasis on the Potential for Biomass-Derived Syngas. NREL/TP-510-34929. 2003, National Renewable Energy Laboratory: Golden, Colorado. p. 160.

33. Strait, R., Nagvekar, M., Carbon dioxide capture and storage in the nitrogen and syngas industries. Nitrogen+Syngas. January-February (303), 2010.

34. Rostrup-Nielsen, J., Natural Gas: Fuel or Feedstock?, in Sustainable Strategies for the Upgrading of Natural Gas: Fundamentals, Challenges, and Opportunities, E.G. Derouane, Parmon, V., Lemos, F., Ramôa-Ribeiro, F., Editor. 2005, Springer, Dordrecht. p. 1-22.

35. Bhat, M., English, B.,Turhollow, A., Nyangito, H., Energy in Synthetic Fertilizers and Pesticides: Revisited, 1994, Oak Ridge National Laboratory: Tennessee, USA.

36. Beaton, J., Efficient Fertilizer Use Manual: History of Fertilizer. 2006, Plymouth, Minnesota, USA: Mosaic-Back to Basics Publisher.

37. IFA. Statistics - Fertilizer Indicators - Details - Raw material reserves. 2002. Accessed 15/10/13; Available from: http://www.fertilizer.org/ifa/statistics/indicators/ind_reserves.asp.

38. YARA, Yara Fertilizer Industry Handbook, February 2014. 2014, Yara Internatioal: Oslo, Norway. 
39. Stewart, W.M., Dibb, D.W., Johnston, A.E., Smyth, T.J., The contribution of commercial fertilizer nutrients to food production. Agron. J., 2005. 97: p. 1-6.

40. Ayres, R., Ayres, L., Warr, B., Exergy, power and work in the US economy, 1900-1998. Energy, 2003. 28(3): p. 219-273.

41. Tamaru, K., The History of the Development of Ammonia Synthesis, in Catalytic Ammonia Synthesis, J.R. Jennings, Editor. 1991, Springer US. p. 1-18.

42. IPTS, Reference Document on Best Available Techniques for the Manufacture of Large Volume Inorganic Chemicals - Ammonia, Acids and Fertilisers Industries. 2007, Integrated Pollution Prevention and Control

43. Geman, H., Agricultural Finance: From Crops to Land, Water and Infrastructure. 2015: Wiley. 288 pages.

44. Glass, A., Nitrogen Use Efficiency of Crop Plants: Physiological Constraints upon Nitrogen Absorption. Critical Reviews in Plant Sciences, 2003. 22(5): p. 453-470.

45. FAO, World fertilizer trends and outlook to 2018. 2015: Rome.

46. POTASHCORP. Nitrogen - A Simplified Flow Diagram. 2014. Accessed 31.08.2014; Available from:

http://www.potashcorp.com/overview/nutrients/nitrogen/overview/simplified-flow-diagram.

47. CIESP. Fertilizers: Petrobras widens the participation, may 12, 2014 [In Portuguese]. 2014. Accessed 14-05-14; Available from:

http://www.ciesp.com.br/cubatao/noticias/fertilizantes-petrobras-amplia-atuacao.

48. Ribeiro, P.H., Contribution to the databank for supporting the evaluation of the life cycle of nitrogen fertilizers [In portuguese], MSc thesis in Polytechnic School, Department of Chemical Engineering. 2009, University of Sao Paulo: São Paulo.

49. PETROBRAS. Fact and data: understand why we invest in fertilizers [In Portuguese]. 2014. Accessed 14-12-2014; Available from: http://www.petrobras.com.br/fatos-edados/entenda-por-que-investimos-em-fertilizantes.htm.

50. FAO. Current world fertilizer trends and outlook to 2015. 2011. Accessed 15-10-14; Available from: ftp://ftp.fao.org/ag/agp/docs/cwfto15.pdf.

51. Hernandez, M., Torero, M., Fertilizer Market Situation: Market Structure, Consumption and Trade Patterns, and Pricing Behavior. IFPRI Discussion Paper 01058. January 2011, International Food Policy Research Institute. 
52. FAO, Brazil: Country fact sheet on food and agriculture policy trends. Food and Agriculture Policy Decision Analysis. 2014, Food and Agriculture Organization.

53. Portal.Brasil (2014) Dilma: "The Fertilizers production is strategic to Brazilian Economy" [In Portuguese] INFRAESTRUTURA, Nitrogenados, 03/05/2014. Portal Brasil.

54. CGEE, Sustainability of sugarcane bioenergy, in Centro de Gestão de Estudos Estratégicos. 2012: Brasilia, Brasil. p. 360.

55. Flórez-Orrego, D., Oliveira Jr, S. On the Allocation of the Exergy Costs and CO2 Emission Cost for an Integrated Syngas and Ammonia Production Plant. in 28th International Conference on Efficiency, Cost, Optimization, Simulation and Environmental Impact of Energy Systems, ECOS, 2015. Pau, France.

56. Appl, M., Ammonia: Principles and Industrial Practice. 1999, New York: Wiley-VCH Verlag.

57. Lee, S., Methane and Its Derivatives. 1997: CRC Press.

58. Flórez-Orrego, D., Silva, J. A. M., Oliveira Jr, S., Renewable and non-renewable exergy cost and specific CO2 emission of electricity generation: The Brazilian case. Energy Conversion and Management, 2014. 85: p. 619-629.

59. EFMA, Booklet No. 1 of 8: Production of Ammonia, in Best Available Techniques for Pollution Prevention and Control in the European Fertilizer Industry. 2000: Brussels, Belgium.

60. Crews, M.A., Shumake, B. G., Hydrogen Production and Supply: Meeting Refiners' Growing Needs, in Practical Advances in Petroleum Processing, C. Hsu, Robinson, P., Editor. 2006, Springer New York. p. 771-817.

61. Meyers, R.A., Handbook of Petroleum Refining Process. Third ed. 2004, New York: MacGraw-Hill.

62. Holladay, J.D., Hu, J., King, D. L., Wang, Y., An overview of hydrogen production technologies. Catalysis Today, 2009. 139(4): p. 244-260.

63. Fixen, P., World reserves of fertilizer nutrients [in Portuguese]. International Plant Nutrition Institute IPNI. Piracicaba: Informações Agronômicas, 2009. 126: p. 8-14.

64. Bartels, J.R., A feasibility study of implementing an Ammonia Economy, in Mechanical Engineering Dept. 2008, Iowa State University: Ames, Iowa. p. 111. 
65. Hocking, M.B., 11 - Ammonia, Nitric acid, and their derivatives, in Handbook of Chemical Technology and Pollution Control, M.B. Hocking, Editor. 1998, Academic Press: San Diego. p. 311-354.

66. Hotza, D., Diniz da Costa, J. C., Fuel cells development and hydrogen production from renewable resources in Brazil. International Journal of Hydrogen Energy, 2008. 33(19): p. 4915-4935.

67. Aasberg-Petersen, K., Dybkjær, I., Ovesen, C. V., Schjødt, N. C., Sehested, J., Thomsen, S. G., Natural gas to synthesis gas - Catalysts and catalytic processes. Journal of Natural Gas Science and Engineering, 2011. 3(2): p. 423-459.

68. Shumake, B.G., Abu Diab, T., Operation Hydrogen. Hydrocarbon Engineering, 2006. February: p. 43-48.

69. Leites, I.L., Sama, D. A., Lior, N., The theory and practice of energy saving in the chemical industry: some methods for reducing thermodynamic irreversibility in chemical technology processes. Energy, 2003. 28(1): p. 55-97.

70. Maxwell, G., Synthetic Nitrogen Products: A Practical Guide to the Products and Processes, 2004, New York: Springer US.

71. Henderson, M., Gandhi, M., Consider cryogenic methods to improve ammonia production. Hydrocarbon Processing, 2001. 80(10): p. 100-102.

72. Hou, K., Hughes, R., The kinetics of methane steam reforming over a Ni/-Al2O3 catalyst. Chemical Engineering Journal, 2001. 82: p. 311-328.

73. Van Beurden, P., On the catalytic aspects of steam-methane reforming. Energy Research Centre of the Netherlands (ECN), Technical Report I-04-003, 2004.

74. Mudahar, M., Hignett, T., Energy efficiency in nitrogen fertilizer production. Energy Agric, 1985. 4: p. 159-177.

75. Meerman, J., Hamborg, E., van Keulen, T., Ramírez, A., Turkenburg, W. C., Faaij, A. P. C., Techno-economic assessment of CO2 capture at steam methane reforming facilities using commercially available technology. International Journal of Greenhouse Gas Control, 2012. 9(0): p. 160-171.

76. Anderson, M., Osborne, S., Key lessons to optimise ammonia plant performance. Nitrogen+Syngas, Ammonia operations, 2013. 321(January - February): p. 1-6.

77. Rase, H., Chemical Reactor Design for Process Plants: Vol. 2, Case Studies. 1977, New York: Wiley. 
78. Edwards, J.H., Maitra, A. M., The chemistry of methane reforming with carbon dioxide and its current and potential applications. Fuel Processing Technology, 1995. 42(2-3): p. 269-289.

79. Knopf, C., Modeling, Analysis and Optimization of Process and Energy Systems, 1st Ed. 1st Ed. ed. 2011: Wiley, ISBN: 978-0-470-62421-0.

80. Alves, L., Nebra, S. Exergoeconomic analysis in hydrogen production by Autothermal reforming of natural gas with Cogeneration. in 22nd International Conference on Efficiency, Cost, Optimization, Simulation and Environmental Impact of Energy Systems, August 31 September 3. 2009. Foz do Iguaçu, Paraná, Brazil: ABCM.

81. Aasberg-Petersen, K., Christensen, T.S., Dybkjaer, I., Sehested, J., Ostberg, M., Coertzen, R.M., Keyser, M.J., Steynberg, A. P., Chapter 4 - Synthesis gas production for FT synthesis, in Studies in Surface Science and Catalysis, A. Steynberg, Dry, M., Editor. 2004, Elsevier B.V. p. 697.

82. Kohl, A.L., and Nielsen, R.B., Gas Purification. 5th ed. 1997: Gulf Publishing Company.

83. Movagharnejad, K., Akbari, M., Simulation of CO2 Capture Process. World Academy of Science, Engineering \& Technology, 2011(58): p. 192-196.

84. CHEMCAD. Power Plant Carbon Capture with CHEMCAD. rev. 031109. Technical Articles. Engineering advanced, 2009, Accessed 12-10-14; Available from:

http://www.chemstations.com/content/documents/Technical_Articles/Power_Plant_Carbon_C apture_with_CHEMCAD.pdf.

85. DOW, Gas Sweetening - The Dow Chemical Company. Form No. 170-01395. 1998, The Dow Chemical Company.

86. Addington, L., Ness. C., An Evaluation of General Rules of Thumb in Amine Sweetening Unit Design and Operation, in GPA Europe Sour Gas Processing Conference. 2009: Sitges, Spain.

87. Desideri, U., Paolucci, A., Performance modelling of a carbon dioxide removal system for power plants. Energy Conversion and Management, 1999. 40(18): p. 1899-1915.

88. Oi, L.E., Aspen HYSYS Simulation of CO2 Removal by Amine Absorption from a Gas Based Power Plant, in SIMS2007 Conference. 2007: Goteborg, Sweden

89. Polasek, J., Bullin, J. Selecting Amines for Sweetening Units, Process Considerations in Selecting Amine. in Proceedings GPA Regional Meeting. 1994. Tulsa, OK: BRE - Bryan Research and Engineering, Inc. - Technical Papers. 
90. Guo, W.F., F. Song, G. Xiao, J. Shen, L., Simulation and energy performance assessment of CO2 removal from crude synthetic natural gas via physical absorption process. Journal of Natural Gas Chemistry, 2012. 21(6): p. 633-638.

91. Dyment, J., Watanasiri, S., White Paper: Acid Gas Cleaning using DEPG Physical Solvents: Validation with Experimental and Plant Data, Aspen Technology Inc. 11-76771215. 2015.

92. Olsson, H., Advanced Process Integration Aspects of Tubular Reactors, in Department of Energy and Environment. 2013, Chalmers University of Technology: Goteborg, Sweden.

93. NETL, Cost and Performance Baseline for Fossil Energy Plants - Volume 2: Coal to Synthetic Natural Gas and Ammonia. DOE/NETL-2010/1402, NETL. 2011.

94. Ostuni, R., Filippi, E., Skinner, G.F., Hydrogen and Nitrogen Recovery from Ammonia Purge Gas. US20130039835 A1. 2013, Ammonia Casale Sa.

95. Nielsen, A., Ammonia: Catalysis and Manufacture. 1995, Springer, ISBN 9783540583356

96. Nevers, N., Physical and Chemical Equilibrium for Chemical Engineers. 2 ed. 2012, New York, USA: Wiley AIChE. 384.

97. Kent, J., Handbook of Industrial Chemistry and Biotechnology. 12th ed. Vol. 1. 2012.

98. Pearson, A., High Pressure Ammonia Systems - New Opportunities in International Refrigeration and Air Conditioning Conference, July 12-15, 2010. Paper 1111. Purdue Unviversity: Purdue, USA.

99. Smith, R., Chemical Process: Design and Integration. 2005, Manchester: Wiley and Sons.

100.Jekel T. B., R.D.T., Single-or two-stage compression. ASHRAE Journal, 2008. 50(8): p. 46-51.

101.Ludwig. P.E, Chapter 12 Compression equipment (Including Fans), in Applied Process Design for Chemical \& Petrochemical Plants, E.L. Ernest, Editor. 2001, Gulf Professional Publishing. p. xi-xii.

102.Isalski, W.H., Tomlinson, T.R., Expansion and compression using work generated in expansion. US4312851 A. 1982.

103.Mukhopadhyay, M., Fundamentals of Cryogenic Engineering. 2010, Delhi: PHI Learning Pvt. Ltd. 
104.Howell, J., The Membrane Alternative: Energy Implications for Industry: Watt Committee Report Number 21. 1990, New York, USA: CRC Press

105.Meindersma, G.W., Kuczynski, M., Implementing membrane technology in the process industry: problems and opportunities. Journal of Membrane Science, 1996. 113(2): p. 285292.

106.Mendivil, R., Fischer, U., Hirao, M., Hungerbühler, K., A New LCA Methodology of Technology Evolution (TE-LCA) and its Application to the Production of Ammonia (19502000). The International Journal of Life Cycle Assessment, 2006. 11(2): p. 98-105.

107.Hodge, C., Popovici, N., Pollution Control in Fertilizer Production (Environmental Science and Pollution Control Series Book 10). 1 ed. 1994: CRC Press.

108.Huchler, L., Cooling towers part 2: operating, monitoring and maintaining. Chemical Engineering Progress, 2009. October: p. 38-41.

109.Kirova-Yordanova, Z. Energy Integration and Cogeneration in Nitrogen Fertilizers Industry: Thermodynamic Estimation of the Efficiency, Potentials, Limitations and Environmental Impact. Part 1: Energy Integration in Ammonia Production Plants. in 25th International Conference on Efficiency, Cost, Optimization, Simulation and Environmental Impact of Energy Systems, ECOS, 2012. Perugia, Italy.

110.Weston, C.W., Papcun, J. R., Dery, M., Ammonium Compounds, in Kirk-Othmer Encyclopedia of Chemical Technology. 2000, John Wiley \& Sons, Inc.

111.Nishida, N., Stephanopoulos, G., Westerberg, A. W., A review of process synthesis. AIChE Journal, 1981. 27(3): p. 321-351.

112.Ravindran, A., Ragsdell, K. M., Reklaitis, G. V., Engineering Optimization: Methods and Applications. 2 ed. 2006: John Wiley and Sons.

113.Bradley, S., Hax, A., Magnanti. T., Applied Mathematical Programming, ed. A. Wesley. 1977: Addison-Wesley Publishing Company.

114.Dimian, M.C., Chapter 2 Introduction in process simulation, in Computer Aided Chemical Engineering, M.C. Dimian, Editor. 2003, Elsevier. p. 33-58.

115.Tock, L., Maréchal, F., Perrenoud, M., Thermo-environomic evaluation of the ammonia production. The Canadian Journal of Chemical Engineering, 2015. 93(2): p. 356-362.

116.Jaluria, Y., Design and Optimization of Thermal Systems, Second Edition. 2007: CRC Press. 
117.Anantharaman, R., Energy Efficiency in Process Plants with emphasis on Heat Exchanger Networks: Optimization, Thermodynamics and Insight, in Department of Energy and Process Engineering. 2011, Norwegian University of Science and Technology: Trondheim.

118.ASPENTECH, Aspen Hysys V8.8, Aspen technology Inc. 2015: Bedford, United States.

119.ASPENTECH, Aspen Plus V8.8, Aspen technology Inc. 2015: Bedford, United States.

120.Klein, S., Engineering Equation Solver - EES, F-Chart Software. 2018: Wisconsin, United States.

121.Yoo, M., Lessard, L, Kermani, M., Maréchal, F. OSMOSE Lua: A Unified Approach to Energy Systems Integration with Life Cycle Assessment. in 12th International conference PSE 2015 and 25th International conference ESCAPE 2015. Copenhagen, Denmark.

122.ASPENTECH, Aspen Physical Property System - Physical Property Methods V7.3 - User Guide. 2011.

123.NIST, Reference Fluid Thermodynamic and Transport Properties Database (REFPROP) - $V$ 10, National Institute of Standards and Technology Material Measurement Laboratory: Gaithersburg, United States.

124.Ben, I.H., Wronski, J., Quoilin, S., Lemort, V., Pure and Pseudo-pure Fluid Thermophysical Property Evaluation and the Open-Source Thermophysical Property Library - CoolProp. Industrial and Engineering Chemistry Research, 2014. 53(6): p. 2498-2508.

125.Abdollahi-Demneh, F., Moosavian, M., Omidkhah, M., Bahmanyar, H., Calculating exergy in flowsheeting simulators: A HYSYS implementation. Energy, 2011. 36(8): p. 53205327.

126.Ghannadzadeh, A., Thery-Hetreux, R., Baudouin, O., Baudet, P., Floquet, P., Joulia, X.,, General methodology for exergy balance in ProSimPlus ${ }^{\circledR}$ process simulator. Energy, 2012. 44(1): p. 38-59.

127.Gourmelon, S., Thery-Hetreux, R., Floquet, P., Baudouin, O., Baudet, P., Campagnolo, L., Exergy analysis in ProSimPlus ${ }^{\circledR}$ simulation software: A focus on exergy efficiency evaluation. Computers \& Chemical Engineering, 2015. 79: p. 91-112.

128.Querol, E., Gonzalez-Regueral, B., Ramos, A., Perez-Benedito, J. L., Novel application for exergy and thermoeconomic analysis of processes simulated with Aspen Plus ${ }^{\circledR}$. Energy, 2011. 36(2): p. 964-974. 
129.Turton, R.B., R. Whiting, W., Shaeiwitz, J., Analysis, Synhtesis and Design of Chemical Processes. 3rd ed. 2009, New York: Prentice Hall.

130.Hamelinck, C., Faaij, A.P.C., Future prospects for production of methanol and hydrogen from biomass. Journal of Power Sources, 2002. 111(1): p. 1-22.

131.Fogler, S.H., Elements of Chemical Reaction Engineering. 4th Edition ed. 1986: PrenticeHall.

132.Dyson, D.C., Simon, J. M., Kinetic Expression with Diffusion Correction for Ammonia Synthesis on Industrial Catalyst. Industrial \& Engineering Chemistry Fundamentals, 1968. 7(4): p. 605-610.

133.Gillespie, L., Beattie, J. A., The Thermodynamic Treatment of Chemical Equilibria in Systems Composed of Real Gases. I. An Approximate Equation for the Mass Action Function Applied to the Existing Data on the Haber Equilibrium. Physical Review, 1930. 36(4): p. 743753.

134.Denbigh, K.G., The Principles of Chemical Equilibrium: With Applications in Chemistry and Chemical Engineering, 1981: Cambridge University Press.

135.Seider, W., Seader, J., Lewin, D., Widagdo, S., Product and Process Design Principles: Synthesis, Analysis and Design. 3 ed. 2008: Wiley, ISBN: 978-0-470-04895-5.

136.Araújo, A., Skogestad, S., Control structure design for the ammonia synthesis process. Computers \& Chemical Engineering, 2008. 32(12): p. 2920-2932.

137.Singh, C.P., Saraf, D. N., Simulation of Ammonia Synthesis Reactors. Industrial \& Engineering Chemistry Process Design and Development, 1979. 18(3): p. 364-370.

138.Imperial Chemical Industries, Catalyst handbook: With special reference to unit processes in ammonia and hydrogen manufacture. 1970: Wolfe Publishers.

139.Kandiyoti, R., Fundamentals of Reaction Engineering. 2009: Ventus Publishing APS.

140.Glavič, P., Kravanja, Z., Homšak, M., Heat integration of reactors-I. Criteria for the placement of reactors into process flowsheet. Chemical Engineering Science, 1988. 43(3): p. 593-608.

141.Jacobsen, C., Dahl, S., Boisen, A., Clausen, B., Topsøe, H., Logadottir, A., Nørskov, J. K., Optimal Catalyst Curves: Connecting Density Functional Theory Calculations with Industrial Reactor Design and Catalyst Selection. Journal of Catalysis, 2002. 205(2): p. 382387. 
142.Seider, W., Seader, J., Lewin, D., Product Process and Desing Principles: Synthesis, Analysis and Evaluations. 2 ed. 2004: John Wiley and Sons. pp1122.

143.Sandler, S., Orbey, H., Modeling Vapor-Liquid Equilibria: Cubic Equations of State and Their Mixing Rules, 1998: Cambridge Series on Chemical Engineering.

144.Siddiq, S., Khushnood, S., Koreshi, Z.U., Shah, M.T., Process simulation of ammonia synthesis for increasing heat recovery in a thermal storage plant: A review. Agris - Food and Agriculture Organization of the United Nations, 2011. 16: p. 68-91.

145.Douglas, J., Conceptual Design of Chemical Processes. 1988: McGraw Hill, ISBN: 007017762-7.

146.Grossmann, I., Mixed-integer programming approach for the synthesis of integrated process flowsheets. Computers \& Chemical Engineering, 1985. 9(5): p. 463-482.

147.Tsatsaronis, G., Thermoeconomic analysis and optimization of energy systems. Progress in Energy and Combustion Science, 1993. 19(3): p. 227-257.

148.Lazzaretto, A., Tsatsaronis, G., SPECO: A systematic and general methodology for calculating efficiencies and costs in thermal systems. Energy, 2006. 31(8-9): p. 1257-1289.

149.Frangopoulos, C., Spakovsky, M., Sciubba, E., A Brief Review of Methods for the Design and Synthesis Optimization of Energy Systems. Int.J. Applied Thermodynamics, 2002. 5(4): p. 151-160.

150.Linnhoff, B., Flower, J., Synthesis of heat exchanger networks: I. Systematic generation of energy optimal networks. AIChE Journal, 1978. 24(4): p. 633-642.

151.Szargut, J., Morris, D., Steward, F., Exergy analysis of thermal, chemical, and metallurgical processes. 1988, New York: Hemisphere Publishing Corporation.

152.Le Chatelier, H.L., Sur un énoncé général des lois des équilibres chimiques. Comptes Rendus Académie de Sciences, 1884. 99: p. 786-789.

153.Leites, I.L., Calculation of exergy of nonideal systems and principle of counteraction for energy saving, in International Conference on Energy, Cost, Optimization and Simulation of Energy Systems, ECOS, 1996, Royal Institute of Technology: Stockholm, Sweden, June 2527. p. 25-28.

154.Sieniutycz, S., A synthesis of thermodynamic models unifying traditional and work-driven operations with heat and mass exchange. Open Systems \& Information Dyn, 2003. 10(31-49). 
155.Lazzaretto, A. and G. Tsatsaronis, SPECO: A systematic and general methodology for calculating efficiencies and costs in thermal systems. Energy, 2006. 31(8-9): p. 1257-1289.

156.Lozano, M.A. and A. Valero, Theory of the exergetic cost. Energy, 1993. 18(9): p. 939960.

157.Linnhoff, B., Hindmarsh, E., The pinch design method for heat exchanger networks. Chemical Engineering Science, 1983. 38(5): p. 745-763.

158.Marechal, F., Kalitventzeff, Boris, Computer-Aided Integration of Utility Systems, in Computer Aided Process and Product Engineering. 2006, Wiley-VCH Verlag GmbH. p. 327381.

159.Couper, J., Penney, W. R., Fair, J. R., Walas, S. M., Chemical Process Equipment (Third Edition). 2012, Boston: Butterworth-Heinemann.

160.Kemp, I.C., Pinch Analysis and Process Integration: A User Guide on Process Integration for the Efficient Use of Energy. 2 ed. 2006.

161.Rev, E., Process Engineering II. Process Synthesis. 2013, Budapest, Hungary: Budapest University of Technology and Economics

162.Linke, P., Kokossis, A., Alva-Argaez, A., Process Intensification, in Computer Aided Process and Product Engineering. 2006, Wiley-VCH Verlag GmbH. p. 297-326.

163.Flórez-Orrego, D., Oliveira Jr, S., Exergy Modeling and Optimization of an Ammonia Production Plant, in 29th International Conference on Efficiency, Cost, Optimization, Simulation and Environmental Impact of Energy Systems, ECOS, 2016. Portoroz, Slovenia.

164.Marechal, F., Palazzi, F., Godat, J., Favrat, D., Thermo-Economic Modelling and Optimisation of Fuel Cell Systems. Fuel Cells, 2005. 5(1): p. 5-24.

165.Canmet ENERGY, Nitrogen-Based Fertilizer Industry: Energy Recovery at an Ammonia Plant. Cat. No.: M154-60/4/2009E-PDF, in Pinch Analysis: For the Efficient Use of Energy, Water \& Hydrogen. 2012.

166.Godat, J., Marechal, F., Optimization of a fuel cell system using process integration techniques. Journal of Power Sources, 2003. 118(1-2): p. 411-423.

167.WBG, Nitrogenous Fertilizer Plants, in Pollution Prevention and Abatement Handbook. 1999, World Bank Group. p. 472. 
168.Kalitventzeff, B., Maréchal, F., Closon, H., Better solutions for process sustainability through better insight in process energy integration. Applied Thermal Engineering, 2001. 21(13-14): p. 1349-1368.

169.Hinderink, A.P., Kerkhof, F. P. J. M., Lie, A. B. K., De Swaan Arons, J., Van Der Kooi, H. J., Exergy analysis with a flowsheeting simulator-II. Application; synthesis gas production from natural gas. Chemical Engineering Science, 1996. 51(20): p. 4701-4715.

170.Perry, R.H., Green, D. W., Perry's chemical engineers' handbook. 2008, New York: McGraw-Hill.

171.Lior, N., Zhang, N., Energy, exergy, and Second Law performance criteria. Energy, 2007. 32(4): p. 281-296.

172.Channiwala, S., Parikh, P.,, A unified correlation for estimating HHV of solid, liquid and gaseous fuels. Fuel, 2002. 81(8): p. 1051-1063.

173.Cheremisinoff, N., Industrial Solvents Handbook, Revised And Expanded, 2003, New Yor, USA: CRC Press. 344. ISBN 9780824740337.

174.Song, G., Xiao, J., Zhao, H., Shen, L., A unified correlation for estimating specific chemical exergy of solid and liquid fuels. Energy, 2012. 40(1): p. 164-173.

175.Marmolejo-Correa, D., Gundersen, T., A comparison of exergy efficiency definitions with focus on low temperature processes. Energy, 2012. 44(1): p. 477-489.

176.Brodyansky, V.M., Sorin, M.V. and Le Goff, P., The Efficiency of Industrial Processes: Exergy Analysis and Optimization. 1994, Amsterdam: Elsevier.

177.Sorin, M., Lambert, J., Paris, J., Exergy Flows Analysis in Chemical Reactors. Chemical Engineering Research and Design, 1998. 76(3): p. 389-395.

178.Kotas, T., The exergy method of thermal planta analysis. 2 ed. 1995, Malabar, Florida: Krieger Publishing Company.

179.Ayres, R., Warr, B., Accounting for growth: the role of physical work. Structural Change and Economic Dynamics, 2005. 16(2): p. 181-209.

180.Flórez-Orrego, D., Henriques, I. B., Nguyen, T., Silva, J. A. M., Mady, C. E., Pellegrini, L., Gandolfi, R., Velasquez, H., Burbano, J., Lattouf, R., Oliveira Junior, S., The contributions of Prof. Jan Szargut to the exergy and environmental assessment of complex energy systems. Energy, 2018. 161: p. 482-492. 
181.Frangopoulos, C.A., Thermo-economic functional analysis and optimization. Energy, 1987. 12(7): p. 563-571.

182.Lozano, M., Valero, A., Theory of the exergetic cost. Energy, 1993. 18(9): p. 939-960.

183.Erlach, B., Serra, L., Valero, A., Structural theory as standard for thermoeconomics. Energy Conversion and Management, 1999. 40(15-16): p. 1627-1649.

184.Torres, C., Valero, A., Rangel, V., Zaleta, A., On the cost formation process of the residues. Energy, 2008. 33(2): p. 144-152.

185.Santos, J.C.S., Nascimento, M. A. R., Lora, E. E. S., Reyes, A. M. M, On the Negentropy Application in Thermoeconomics: A Fictitious or an Exergy Component Flow? International Journal of Thermodynamics, 2009. 12(4): p. 163-176.

186.Silva, J.A.M., Flórez-Orrego, D., Oliveira Jr, S., An exergy based approach to determine production cost and CO2 allocation for petroleum derived fuels. Energy, 2014. 67(0): p. 490495.

187.Silva, J.A.M., Oliveira Jr, S., An exergy-based approach to determine production cost and CO2 allocation in refineries. Energy, 2014. 67(0): p. 607-616.

188.Florez-Orrego, D., Silva, J.A.M., Oliveira Jr, S., Renewable and Non-Renewable Exergy Cost and Specific CO2 Emission of Electricity Generation: The Brazilian Case. Energy Conversion and Management, 2014.

189.Florez-Orrego, D., Silva, J.A.M., Velasquez, H., Oliveira, S., Renewable and NonRenewable Exergy Costs and CO2 Emissions of Fuels Production for Brazilian Transportation Sector, in 27th International Conference on Efficiency, Cost, Optimization, Simulation and Environmental Impact of Energy Systems, ECOS, 2014, Turku, Finland.

190.Flórez-Orrego, D., Thermodynamic and Environmental Comparison (CO2 emissions) of the route of the production and end use of vehicle fuels derived from Petroleum, Natural gas, Biofuels, Hydrogen and Electricity [In Portuguese], MSc Thesis in Department of Mechanical Engineering. 2014, University of São Paulo: São Paulo. p. 229.

191.Flórez-Orrego, D., Silva, J. A. M., Oliveira Jr, S., Exergy and environmental comparison of the end use of vehicle fuels: The Brazilian case. Energy Conversion and Management, 2015. 100: p. 220-231.

192.Flórez-Orrego, D., Silva, J.A.M, Oliveira Jr., S. Renewable and Non-Renewable Exergy Cost and Specific CO2 Emission of Electricity Generation: The Brazilian Case. in Efficiency, Cost, Optimization, Simulation and Environmental Impact of Energy Systems, ECOS, 2013. Guilin, China. 
193.Gomes, C., Thermodynamic, exergetic and thermoeconomic analysis of a thermoelectric powerplant with combined cycle and cogeneration [in Portuguese] PhD Thesis, in Mechanical Engineering Faculty. 2001, State University of Campinas: Campinas, Brazil.

194.Silva, M., Repowering in electricity generation systems in the siderurgic industry [in Portuguese], PhD Thesis in Mechanical Engineering Faculty. 2004, State University of Campinas: Campinas, Brazil.

195.Agudelo, A., Valero, A., Torres, C., Allocation of waste cost in thermoeconomic analysis. Energy, 2012. 45(1): p. 634-643.

196.Borelli, S., Oliveira Junior, S., Exergy-based method for analyzing the composition of the electricity cost generated in gas-fired combined cycle plants. Energy, 2008. 33(2): p. 153-162.

197.Pout, C., Hitchin, R., Apportioning carbon emissions from CHP systems. Energy Conversion and Management, 2005. 46(18-19): p. 2980-2995.

198.Rosen, M., Allocating carbon dioxide emissions from cogeneration systems: descriptions of selected output-based methods. Journal of Cleaner Production, 2008. 16(2): p. 171-177.

199.DEFRA, 2012 Guidelines to Defra / DECC's GHG Conversion Factors for Company Reporting, Departament of Energy and Climate Change 2012: London.

200.Agudelo, A., Valero, A., Usón, S., The fossil trace of CO2 emissions in multi-fuel energy systems. Energy, 2013. 58(0): p. 236-246.

201.Flórez-Orrego, D., Silva, J.A.M., Oliveira Jr, S., An Exergy and Environmental Comparison of End Use of Vehicle Fuels in Brazilian Transportation Sector., in 15th Brazilian Congress of Thermal Sciences and Engineering - ENCIT 2014, November 10-13. 2014: Belém, PA, Brazil.

202.Lozano, M.A., Valero, A., Thermoeconomic Analysis of Gas Turbine Cogeneration Systems. ASME Book H00874, 1993: p. 311-320.

203.Silva J.A.M, S., J, Oliveira Jr, S. The condenser product and residues allocation in thermoeconomics. in 23rd International Conference on Efficiency, Cost, Optimization, Simulation and Environmental Impact of Energy, ECOS, 2010. Lausanne, Switzerland.

204.Grossmann, I.E., Global Optimization in engineering design - Non convex optimization and its applications. 1996: Springer. 
205.Gong, M., Using Exergy and Optimization Models to Improve Industrial Energy Systems Towards Sustainability, PhD Dissertation No. 868, in Department of Mechanical Engineering, Energy Systems. 2004, Linköping University: Linköping.

206.Flórez-Orrego, D., Oliveira Junior, S., On the efficiency, exergy costs and CO2 emission cost allocation for an integrated syngas and ammonia production plant. Energy, 2016. 117, Part 2: p. 341-360.

207.GEA. Cooling Tower Calculator - GEA Heat Exchangers, GEA Aircooled Systems. 2008 Accessed 10-10-14; Available from:

http://www.gea-energytechnology.com/opencms/opencms/gas/en/calculators/.

208.Koyikkal, S., Chemical Process Technology and Simulation. 2013, Delhi: PHI Learning Pvt. Ltd.

209.Kool, A., Blonk, H., LCI data for the calculation tool Feedprint for greenhouse gas emissions of feed production and utilization: GHG Emissions of N, P and $K$ fertilizer production. 2012, Blonk Consultants: Netherlands.

210.Davis, J., Haglund, C., Life Cycle Inventory (LCI) of Fertiliser Production: Fertiliser Products Used in Sweden and Western Europe, SIK-report No. 654. 1999: Gothenburg, Sweden.

211.Ahlgren, S., Baky, A., Bernesson, S., Nordberg, A., Norén, O., Hansson, P.A., Ammonium nitrate fertiliser production based on biomass. Environmental effects from a life cycle perspective. Bioresource Technology, 2008. 99: p. 8034 - 8041.

212.Haas, M., Dijk, T., Inventarisatie klimaatvriendelijke kunstmest .Nutriënten management instituut (NMI). 2010: Wageningen.

213. Caetano de Souza, A., Luz-Silveira, J., Sosa, M., Physical-Chemical and Thermodynamic Analyses of Ethanol Steam Reforming for Hydrogen Production. Journal of Fuel Cell Science and Technology, 2006. 3(3): p. 346-350.

214.Silveira, J., Souza, A., Silva, M., Thermodynamic Analysis of Direct Steam Reforming of Ethanol in Molten Carbonate Fuel Cell. Journal of Fuel Cell Science and Technology, 2008. $\mathbf{5}(2)$.

215.BNDES, Bioethanol from sugar cane: Energy for the sustainable development [In Portuguese]. 2008, Rio de Janeiro: BNDES.

216.Rossi, C., Alonso, C., Antunes, O., Guirardello, R., Cardozo-Filho, L., Thermodynamic analysis of steam reforming of ethanol and glycerine for hydrogen production. International Journal of Hydrogen Energy, 2009. 34(1): p. 323-332. 
217.Lima da Silva, A., Malfatti, C., Müller, I., Thermodynamic analysis of ethanol steam reforming using Gibbs energy minimization method: A detailed study of the conditions of carbon deposition. International Journal of Hydrogen Energy, 2009. 34(10): p. 4321-4330.

218.Appl, M., Modern Amonia Technology. Nitrogen, 1992. 199 (September-October)(46).

219.Kumar, R., Lyon, R., Cole, J., Unmixed Reforming: A Novel Autothermal Cyclic Steam Reforming Process, in Advances in Hydrogen Energy C. Padró, Lau, F., Editor. 2002, Kluwer Academic/Plenum Publishers: New York. p. 31-45.

220.Edrisi, A., Mansoori, Z., Dabir, B., Using three chemical looping reactors in ammonia production process - A novel plant configuration for a green production. International Journal of Hydrogen Energy, 2014. 39(16): p. 8271-8282.

221.Lippmann, D., Larsen, J., The Uhde Dual Pressure Process - Reliability Issues and Scale Up Consideration, in The 47th Annual Safety in Ammonia Plants and Related Facilities Symposium,16 - 19 September 2002. San Diego, California, USA.

222.Nikačević, N., Jovanović, M., Petkovska, M., Enhanced ammonia synthesis in multifunctional reactor with in situ adsorption. Chemical Engineering Research and Design, 2011. 89(4): p. 398-404.

223.Maansson, B., Andresen, B., Optimal temperature profile for an ammonia reactor. Industrial \& Engineering Chemistry Process Design and Development, 1986. 25(1): p. 59-65.

224.Audus, H., Kaarstad, O., Kowal, M. Decarbonization of fossil fuels: hydrogen as an energy carrier. in Proceedings of the 11th World Hydrogen Energy Conference. 1996. Stuttgart, Germany.

225.Maréchal, F., Kalitventzeff, B., Effect modelling and optimization, a new methodology for combined energy and environment synthesis of industrial processes. Applied Thermal Engineering, 1997. 17(8): p. 981-992.

226.Flórez-Orrego, D., Oliveira Junior, S., Exergy assessment of single and dual pressure industrial ammonia synthesis units. Energy, 2017. 141: p. 2540-2558.

227.Maréchal, F., Kalitventzeff, B., Identification of the optimal pressure levels in steam networks using integrated combined heat and power method. Chemical Engineering Science, 1997. 52(17): p. 2977-2989.

228.Keller, A., Viswanathan, S., Integrated pressurized steam hydrocarbon reformer and combined cycle process, U.S.Patents Office. 2013, Phillips 66 Company, Houston, TX (US): United States. 
229.Maréchal, F., Kalitventzeff, B., Process integration: Selection of the optimal utility system. Computers \& Chemical Engineering, 1998. 22: p. S149-S156.

230.Santos, V.E.N., Ely, R. N., Szklo, A. S., Magrini, A., Chemicals, electricity and fuels from biorefineries processing Brazil's sugarcane bagasse: Production recipes and minimum selling prices. Renewable and Sustainable Energy Reviews, 2016. 53: p. 1443-1458.

231.Florez-Orrego, D., Sharma, S., Oliveira Jr, S., Marechal, F. Combined Exergy Analysis and Energy Integration for Design Optimization of Nitrogen Fertilizer Plants. in 30th International Conference on Efficiency, Cost, Optimization, Simulation and Environmental Impact of Energy Systems, ECOS, 2017, San Diego, United States of America: San Diego State University.

232.Flórez-Orrego, D., Silva, J.A.M., Velásquez, H., Oliveira Jr., S., Renewable and nonrenewable exergy costs and $\mathrm{CO} 2$ emissions in the production of fuels for Brazilian transportation sector. Energy, 2015.

233.Seebregts, A.J., Gas-Fired Power. 2010, Energy Technology Systems Analysis Programme - IEA ETSAP - Technology Brief E02- April.

234.Mokhatab, S., Poe, W., Mak, J. Y., Chapter 6 - Natural Gas Treating, in Handbook of Natural Gas Transmission and Processing (Third Edition). 2015, Gulf Professional Publishing: Boston. p. 181-222.

235.Chen, C., A Technical and Economic Assessment of CO2 Capture Technology for IGCC Power Plants. 2005, Carnegie Mellon University: Pittsburgh, Pennsylvania.

236.Adams-Ii, T., Salkuyeh, Y., Nease, J., Chapter 6 - Processes and simulations for solventbased CO2 capture and syngas cleanup A2 - Shi, Fan, in Reactor and Process Design in Sustainable Energy Technology. 2014, Elsevier: Amsterdam. p. 163-231.

237.Florez-Orrego, D., Oliveira Jr, S., Modeling and optimization of an industrial ammonia synthesis unit: An exergy approach. Energy (Oxford), 2017. 137(15): p. 234-250.

238.Gassner, M., Maréchal, F., Increasing Efficiency of Fuel Ethanol Production from Lignocellulosic Biomass by Process Integration. Energy \& Fuels, 2013. 27(4): p. 2107-2115.

239.Greeff, I.L., Visser, J. A., Ptasinski, K. J., Janssen, F. J. J. G., Integration of a turbine expander with an exothermic reactor loop-Flow sheet development and application to ammonia production. Energy, 2003. 28(14): p. 1495-1509. 
240.Greeff, I.L., Visser, J. A., Ptasinski, K. J., Janssen, F., Using turbine expanders to recover exothermic reaction heat-flow sheet development for typical chemical processes. Energy, 2004. 29(12): p. 2045-2060.

241.Florez-Orrego, D., Nascimento Silva, F., Oliveira Jr., S. Syngas Production with ThermoChemically Recuperated Gas Turbine Systems: An Exergy Analysis and Energy Integration Study. in 31th International Conference on Efficiency, Cost, Optimization, Simulation and Environmental Impact of Energy Systems - ECOS, 2018. Guimaraes, Portugal: University of Minho.

242.Rostrup-Nielsen, J., Rostrup-Nielsen, T. Large-scale Hydrogen Production. 6th World Congr. of Chemical Engineering, Melbourne, Australia 2001, Accessed 20.01.2018; Available from:

https://www.topsoe.com/sites/default/files/topsoe_large_scale_hydrogen_produc.pdf.

243.Frattini, D., Cinti, G., Bidini, G., Desideri, U., Cioffi, R., Jannelli, E., A system approach in energy evaluation of different renewable energies sources integration in ammonia production plants. Renewable Energy, 2016. 99: p. 472-482.

244.Liu, X., Zheng, H, Liu, Q., Research of Methane Reforming and Combustion Characteristics in Chemically Recuperated Gas Turbine. Industrial \& Engineering Chemistry Research, 2014. 53(5): p. 1940-1946.

245.Denbigh, K.G., The second-law efficiency of chemical processes. Chemical Engineering Science, 1956. 6(1): p. 1-9.

246.Harvey, S., Kane, N., Analysis of a reheat gas turbine cycle with chemical recuperation using ASPEN. Energy Conversion and Management, 1997. 38(15-17): p. 1671-1679.

247.Carapelluci, R., Milazzo, A., Thermodynamic optimization of a reheat chemically recuperated gas turbine. Energy Conversion and Management, 2005. 46: p. 2936-2953.

248.Nakagaki, T., Ogawa, T., Hirata, H., Kawamoto, K., Ohashi, Y., Tanaka, K., Development of Chemically Recuperated Micro Gas Turbine. Journal of Engineering for Gas Turbines and Power, 2003. 125: p. 391-397.

249.Kesser, K.F., Hoffman, M. A., Baughn, J. W. , Analysis of a Basic Chemically Recuperated Gas Turbine Power Plant. Journal of Engineering for Gas Turbines and Power, 1994. 116: p. 277-284.

250.Adelman, S.T., Hoffman, M. A., Baughn, J. W., A Methane-Steam Reformer for a Basic Chemically Recuperated Gas Turbine. Journal of Engineering for Gas Turbines and Power, 1995. 117: p. 16-23. 
251.Sahafzadeh, M., Ataei, A., Tahouni, N., Panjeshahi, M., Integration of a gas turbine with an ammonia process for improving energy efficiency. Applied Thermal Engineering, 2013. 58(1-2): p. 594-604.

252.Bie, Y., Hu, M.F., Zhang, X., Zhu, X. Q. , Chang, J.H, Mao, W. Y., Thermodynamic Analysis of an Ammonia Synthesis Process Based on Brayton Cycle. Advanced Materials Research, Switzerland, 2012 396-398 p. pp. 939-945.

253.Agee, M.A., Weick, L.J., Trepper, E.L., Agee, K.L. , Synthesis Gas Production System And Method, U.S.Patents Office, 2000, Syntroleum Corporation, Tulsa, Okla: United States.

254.Marechal, F., Kalitventzeff, B., Study of the insertion of partial oxidation gas turbine to satisfy high temperature requirements of industrial processes using energy integration techniques, in Computer Aided Chemical Engineering, S. Pierucci, Editor. 2000, Elsevier. p. 679-684.

255.Alves, L.G., Exergoeconomimic analysis and optimization of various hydrogen production routes from methane [In Portuguese], $\mathrm{PhD}$ Thesis in Department of Mechanical Engineering. 2007, UNICAMP: Campinas, Brazil.

256.Yu, Y.H., Simulation of Secondary Reformer in Industrial Ammonia Plant. Chemical Engineering \& Technology, 2002. 25(3): p. 307-314.

257.Cromarty, B.J., Hooper, C. W., Increasing the throughput of an existing hydrogen plant. International Journal of Hydrogen Energy, 1997. 22(1): p. 17-22.

258. Kirova-Yordanova, Z. Thermodynamic evaluation of the air separation units integration in nitrogen fertilizers production complexes. in 4th International Conference on Contemporary Problems of Thermal Engineering, CPOTE, 2016. Institute of Thermal Technology, Katowice, Poland.

259.Halabi, M.H., Croon, M. H. J. M., van der Schaaf, J., Cobden, P. D., Schouten, J. C., Modeling and analysis of autothermal reforming of methane to hydrogen in a fixed bed reformer. Chemical Engineering Journal, 2008. 137(3): p. 568-578.

260.Zahedi nezhad, M., Rowshanzamir, S. Eikani, M. H., Autothermal reforming of methane to synthesis gas: Modeling and simulation. International Journal of Hydrogen Energy, 2009. 34(3): p. 1292-1300.

261.Dybkjaer, I., Tubular reforming and autothermal reforming of natural gas - an overview of available processes. Fuel Processing Technology, 1995. 42(2): p. 85-107.

262.Rostrup-Nielsen, J., Aasbeerg-Petersen, K., Chapter 14 - Steam Reforming, ATR, partial oxidation: Catalysts and Reaction Engineering in Handbook of Fuel Cells - Fundamental 
Technology and Applications, W. Vielstich, Gasteiger, H., Lamm, A., Editor. 2003, John Wiley and Sons, Ltd.

263.Sandberg, P., White Paper - Optimal performance - integration of Haldor Topsoe Heat Exchange Reformer in ammonia plants. 2015, Asia Nitrogen and Syngas 2015 Conference, Haldor Topsoe A/S: Copenhagen, Denmark.

264.Rabovitser, J.K., Khinkis, M. J., Bannister, R. L., Miao, F. Q. , Evaluation of Thermochemical Recuperation and Partial Oxidation Concepts for Natural Gas-Fired Advanced Turbine Systems, in International Gas Turbine and Aeroengine Congress and Exhibition. 1996: Birmingham UK.

265.Rabovitser, J.K., Nester, S., White, D.J., 1.3.3. Partial Oxidation Gas Turbine (POGT) Cycles, in The Gas Turbine Handbook - National Energy Technology Laboratory. 2006, US Department Energy Office.

266.Cornelissen, R., Tober, E., Kok, J., van de Meer, T., Generation of synthesis gas by partial oxidation of natural gas in a gas turbine. Energy, 2006. 31(15): p. 3199-3207.

267.Szargut, J., Exergy in thermal systems analysis, in Thermodynamic optimization of complex energy systems, NATO Science Series 69, A. Bejan, Mamut, E., Editor. 1999, Springer.

268.Sorin, M., Paris, J., Integrated exergy load distribution method and pinch analysis. Computers \& Chemical Engineering, 1999. 23(4-5): p. 497-507.

269.Sauar, E., Nummedal, L., Kjelstrup, S., The principle of equipartition of forces in chemical reactor design: The ammonia synthesis. Computers \& Chemical Engineering, 1999. 23: p. S499-S502.

270.Froment, G., Bishoff, K. Wilde, J., Chemical Reactor Analysis and Design. 3ed ed. 2010, New York: John Wiley and Sons.

271.van der Ham, L.V., J. Gross, and S. Kjelstrup, Two performance indicators for the characterization of the entropy production in a process unit. Energy, 2011. 36(6): p. 37273732 .

272.Rangaiah, P., Stochastic Global Optimization: Techniques and Applications in Chemical Engineering. 2010: World Scientific.

273.Lippmann, D., Larsen, P., Uhde dual-pressure process for large-scale ammonia plants, in ThyssenKrupp techforum July 2004, Thyssenkrupp Uhde GmbH, Dortmund. 
274.Penkuhn, M., Tsatsaronis, G., Comparison of different ammonia synthesis loop configurations with the aid of advanced exergy analysis, in 29th International Conference on Efficiency, Cost, Optimization, Simulation and Environmental Impact of Energy Systems, ECOS, 2016. Portoroz, Slovenia.

275.Sorin, M.V., Brodyansky, V. M., A method for thermodynamic optimization-I. Theory and application to an ammonia-synthesis plant. Energy, 1992. 17(11): p. 1019-1031.

276.DOE, Evaluation of Alternative Technologies for Ethylene, Caustic-Chlorine, Ethylene Oxide, Ammonia, and Terephthalic Acid I. JVP International, Editor. 2007, Departamento fo Energy, Industrial Technologies Program: New York. p. 135.

277.Ghannadzadeh, A., Sadeqzadeh, M., Diagnosis of an alternative ammonia process technology to reduce exergy losses. Energy Conversion and Management, 2016. 109: p. 6370 .

278.Radgen, P., Lucas, K., Energy system analysis is fertilizer complex - pinch analysis vs. Exergy analysis. Chemical Engineering \& Technology, 1996. 19(2): p. 192-195.

279.Biegler, L.T., Grossmann, I.E., Westerberg, A.W., Systematic methods for chemical process design. 1997: Prentice Hall. 700pp.

280.IPCC, Reference Document on Best Available Techniques for the Manufacture of Large Volume Inorganic Chemicals - Ammonia, Acids and Fertilisers Industries. 2007, Integrated Pollution Prevention and Control

281.Paul, D., Yampol'skii, Y., Polymeric Gas Separation Membranes. 1993: CRC Press.

282.Murase, A., Roberts, H., Converse, A., Optimal Thermal Design of an Autothermal Ammonia Synthesis Reactor. Industrial \& Engineering Chemistry Process Design and Development, 1970. 9(4).

283.Luyben, W., Chapter 13 - Design and Control of a Cooled Ammonia Reactor, in Plantwide Control: Recent Developments and Applications, G. Rangaiah, ariwala, V., Editor. 2012, John Wiley \& Sons. p. 273-292.

284.Johannessen, E., Kjelstrup, S., Minimum entropy production rate in plug flow reactors: An optimal control problem solved for SO2 oxidation. Energy, 2004. 29(12-15): p. 24032423.

285.Kjelstrup, S., Bedeaux, D., Johannessen, E., Gross, J., Non-Equilibirum Thermodynamics for Engineers. 2010, Trondheim: World Scientific Publishing. 
286.Kjelstrup, S., Swaan A.,, Denbigh revisited: Reducing lost work in chemical processes. Chemical Engineering Science, 1995. 50(10): p. 1551-1560.

287.Hannula, I., Hydrogen production via thermal gasification of biomass in near-to-medium term. 2009, VTT Technical Research Centre of Finland.

288.Kurkela, E., Nieminen, M., Simell, P. Development and commercialization of biomass and waste gasification technologies from reliable and robust co-firing plants towards synthesis gas production and advanced power cycles. in 2nd World Conference and Technology Exhibition on Biomass for Energy, Industry and Climate Protection, ETA Florence, WIP - Munich. 2004. Florence, Italy.

289.Gilbert, P., Alexander, S., Thornley, P., Brammer, J., Assessing economically viable carbon reductions for the production of ammonia from biomass gasification. Journal of Cleaner Production, 2014. 64: p. 581-589.

290.Andersson, J., Lundgren, J., Techno-economic analysis of ammonia production via integrated biomass gasification. Applied Energy, 2014. 130: p. 484-490.

291.NETL. Gasification Background, Markets for Gasification, 2014, Accessed 29.10.2017; Available from:

https://www.netl.doe.gov/research/coal/energy-systems/gasification/gasifipedia/markets.

292.Ett, G., Landgraf, F., Derenzo, S., Yu, A., Reis, L., Mazzonetto, A., Antonoff, H., Souza, L., Brazilian Bio Fuels Production Scenario (Biogas, Biomethane and Biosyngas), in International Seminar on Biomass, Biogas and Energy Efficiency - Regional Leaders Summit. 2013: Sao Paulo, Brazil.

293.Ett, G., Landgraf, F., Sin Yu, A., Nunis, A., Poco, J., Silveira, J., Derenzo, S., Biosyngas Biomass entrained flow gasfication, in 5th International Conference on Engineering for Waste and Biomass Valorization. 2014: Rio de Janeiro, Brazil.

294.Camacho-Ardila, Y., Figueroa, J., Lunelli, B., Maciel Filho, R., Wolf Maciel, M. Syngas production from sugar cane bagasse in a circulating fluidized bed gasifier using Aspen PlusTM: Modelling and Simulation. in 2nd European Symposium on Computer Aided Process Engineering. 2012. London, UK: Elsevier.

295.Marques, F., Bagasse is the target. Revista Pesquisa FAPESP, 2009(Special issue May 2009/Dec 2010 ): p. 32-36.

296.Reddy, N., Yang, Y., Fibers from Sugarcane Bagasse, in Innovative Biofibers from Renewable Resources, N. Reddy and Y. Yang, Editors. 2015, Springer Berlin Heidelberg: Berlin, p. 29-30. 
297.Basu, P., Biomass Gasification and Pyrolysis: Practical Design. 2010: Academic Press.

298.Vitasari, C.R., Jurascik, M., Ptasinski, K., Exergy analysis of biomass-to-synthetic natural gas (SNG) process via indirect gasification of various biomass feedstock. Energy, 2011. 36(6): p. 3825-3837.

299.Juraščík, M., Sues, A., Ptasinski, K., Exergy analysis of synthetic natural gas production method from biomass. Energy, 2010. 35(2): p. 880-888.

300.Kinchin, C.M., Bain, R.L., Hydrogen Production from Biomass via Indirect Gasification: The Impact of NREL Process Development Unit Gasifier Correlations, Technical Report NREL/TP-510-44868, May 2009. 2009, National Renewable Energy Laboratory.

301.Puig-Arnavat, M., Bruno, J., Coronas, A., Modified Thermodynamic Equilibrium Model for Biomass Gasification: A Study of the Influence of Operating Conditions. Energy \& Fuels, 2012. 26(2): p. 1385-1394

302. Gomez-Barea, A., Nilsson, S., Vidal Barrero, F., Campoy, M., Devolatilization of wood and wastes in fluidized bed. Fuel Processing Technology, 2010. 91(11): p. 1624-1633

303.Duret, A., Friedli, C., Maréchal, F., Process design of Synthetic Natural Gas (SNG) production using wood gasification. Journal of Cleaner Production, 2005. 13(15): p. 14341446.

304.Doherty, W., Reynolds, A., Kennedy, D., The effect of air preheating in a biomass CFB gasifier using ASPEN Plus simulation. Biomass and Bioenergy, 2009. 33(9): p. 1158-1167.

305.Ingham, A., Reducing the Carbon Intensity of Methanol for Use as a Transport Fuel. Johnson Matthey Technology Review, 2017. 61(4): p. 297-307

306.Palacios-Bereche, R., Mosqueira-Salazar, K., Modesto, M., Ensinas, A., Nebra, S., Serra, L., Lozano, M.,, Exergetic analysis of the integrated first-and second-generation ethanol production from sugarcane. Energy, 2013. 62(0): p. 46-61.

307.Nakashima, R.N., Oliveira Junior, S. Exergy assessment of vinasse disposal alternatives : concentration, anaerobic digestion and fertirrigation. in 31st International Conference on Efficiency, Cost, Optimization, Simulation and Environmental Impact on Energy Systems, ECOS, 2018. Guimaraes, Portugal.

308. ANA, Handbook of conservation and reuse of water in the sugarcane energy industry [In Portuguese], in ANA Agência Nacional de Águas. 2009, UNICA: Brasilia. p. 290. 
309. Nakashima, R.N., Oliveira Junior, S. Exergy analysis of biogas production in ethanol distilleries. in 24th ABCM International Congress of Mechanical Engineering - COBEM, 2017. Curitiba, Brasil.

310.Barrera, E.L., Spanjers, H., Romero, O., Rosa, E., Dewulf, J., Characterization of the sulfate reduction process in the anaerobic digestion of a very high strength and sulfate rich vinasse. Chemical Engineering Journal, 2014. 248: p. 383-93.

311.Wilkie, A.C., Riedesel, K.J., Owens, J.M., Stillage characterization and anaerobic treatment of ethanol stillage from conventional and cellulosic feedstocks. Biomass and Bioenergy, 2000. 19(2): p. 63-102.

312.Haftka, R., Gurdal, Z., Elements of Structural Optimization. 3 ed. Vol. 11. 1991, Dordrecht: Kluder Academic Publishers.

313.Ozaki, Y., Yano, M., Onishi, M., Effective hyperparameter optimization using NelderMead method in deep learning. IPSJ Transactions on Computer Vision and Applications, 2017. 9(1): p. 20.

314.Suman, B., Study of simulated annealing based algorithms for multiobjective optimization of a constrained problem. Computers \& Chemical Engineering, 2004. 28(9): p. 1849-1871.

315.Edgar, F., Himmelblau, D., Lasdon, L., Optimization of chemical processes. 2 ed. 2001: McGraw-Hill, ISBN 0-07-039359-1.

316.Deb, K., Agrawal, S., Pratap, A., Meyarivan, T. A Fast Elitist Non-dominated Sorting Genetic Algorithm for Multi-objective Optimization: NSGA-II. in Parallel Problem Solving from Nature PPSN VI. 2000. Springer Berlin Heidelberg.

317.Sharma, S., Rangaiah, G. P., Cheah, K. S., Multi-objective optimization using MS Excel with an application to design of a falling-film evaporator system. Food and Bioproducts Processing, 2012. 90(2): p. 123-134.

318.Ponsich, A., Azzaro-Pantel, C., Domenech, S., Pibouleau, L., About the Relevance of Mathematical Programming and Stochastic Optimisation Methods : Application to Optimal Batch Plant Design Problems, in 15th European Symposium on Computer Aided Process Engineering (ESCAPE-15), May 29-June 1, L. Puigjaner, Espuña, A. (Editors), Editor. 2004, Elsevier: Barcelona, Spain.

319.Ayres, R.U., Ayres, L.W., Martináz, K.,, Exergy,Waste Accounting and Life-Cycle Analysis. Energy, 1998. 23: p. 255-363. 
320. Seddon, D., Gas Usage \& Value: The Technology and Economics of Natural Gas Use in the Process Industries. 2006, Tulsa, Oklahoma: PennWell Books, ISBN 1-59370-073-3.

321.Rutkowski, M. Current hydrogen from coal without CO2 capture and sequestration. 2008, Accessed 19.09.2017; Available from:

https://www.hydrogen.energy.gov/h2a_analysis.html.

322.Mann, M. Current hydrogen from biomass via gasification and catalytic steam reforming. 2005, Accessed 19.08.2017; Available from:

http://www.hydrogen.energy.gov/h2a_prod_studies.html.

323.Padro, C., Putsche, V., Survey of the economics of hydrogen technologies, NREL/TP-57027079. 1999.

324.Glatzmaier, G., Blake D., Showalter, S., Assessment of methods for hydrogen production using concentrated solar energy. NREL/TP-570-23629. 1998.

325.Levene, J., Kroposki, B., Sverdrup, G., Wind energy and production of hydrogen and electricity - opportunities for renewable hydrogen, NREL/CP-560-39534. 2006.

326.Maung, T., Ripplinger, D., McKee, G., Saxowsky, D., Economics of Using Flared vs. Conventional Natural Gas to Produce Nitrogen Fertilizer: A Feasibility Analysis. Agribusiness \& Applied Economics, Department of Agribusiness and Applied Economics, Fargo, United States, 2012. 699(September 2012): p. 38

327.Arora, P., Hoadley, A., Mahajani, S., Ganesh, A., Small-Scale Ammonia Production from Biomass: A Techno-Enviro-Economic Perspective. Industrial \& Engineering Chemistry Research, 2016. 55(22): p. 6422-6434.

328.Sarkar, S., Kumar, A., Sultana, A., Biofuels and biochemicals production from forest biomass in Western Canada. Energy, 2011. 36(10): p. 6251-6262 
APPENDIX 1

\section{ALGORITHMIC METHODS USED IN PROCESS SYNTHESIS AND OPTIMIZATION OF CHEMICAL PROCESSES}

The classification of the optimization problems is quite important from both computational and methodological points of view, as there are specific methods that provide more efficient solutions of a particular class of problems. Thus, in this section, an overview of the most common numerical methods used in optimization of chemical processes, broadly classified in deterministic and stochastic, is briefly presented [99].

The deterministic methods rely on pattern or lattice search procedures that may require direct evaluations of the objective function, calculation of gradients or even determination of second order derivatives [116]. These methods can be further classified according to the type of problems that can deal with, e.g. linear or non-linear; sequential approaches (i.e. successive solution of approximate subproblems); constrained or unconstrained; single or multivariable; involving either integer or continuous variables, or even a mixture of both.

Some methods like the Nealder Mead simplex (Fig. A1.1) and the univariate search are examples of direct search deterministic methods that proceed from an initial guess towards the optimum without requiring derivative calculations or other assumptions on the form of the objective function [312]. Since direct search methods allows handling discrete decision variables, topological changes in the structure of the process synthesis can be handled by using these methods [116].

Fig. A1.1. Graphical representation of the Nelder Mead method.

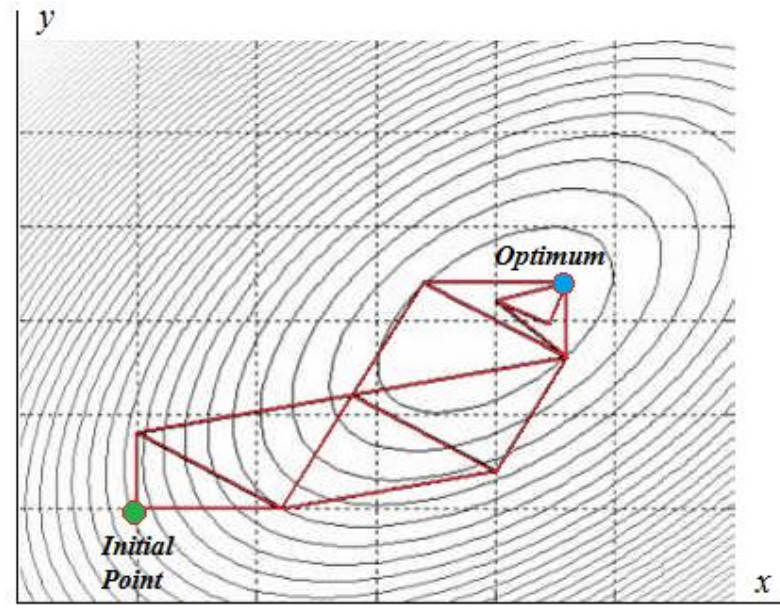

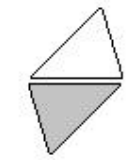

Reflection

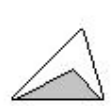

Contraction

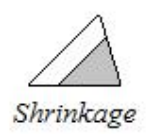

Adapted from source: [313].

Among the most traditional deterministic methods is the calculus method, based on the calculation of the Lagrange multipliers $\lambda$. Thus, the optimization problem can be addressed as 
the solution of a system of non-linear equations written in terms of the multipliers $\lambda$ and the independent variables $\mathbf{x}$. The system of non-linear equations results from the application of the Karush-Kuhn-Tucker (KKT) stationary conditions to the Lagrangian of the optimization problem, given in Eq.(A1.1), along with the complementary conditions for the multipliers and the constraints [312]. However, in order to determine whether the KKT conditions are sufficient, further analyses based on the positive definiteness of the Hessian matrix may be still required.

$$
\begin{aligned}
& L(\mathbf{x}, \lambda, \boldsymbol{\mu})=f(\mathbf{x})+\lambda^{T} h(\mathbf{x})+\boldsymbol{\mu}^{T} g(\mathbf{x}) \Rightarrow \nabla f(\mathbf{x})+\sum_{i} \lambda^{T} \nabla h_{i}(\mathbf{x})+\sum_{i} \mu^{T} \nabla g_{j}(\mathbf{x})=0 \\
& \mu^{T} g(\mathbf{x})=0 \\
& \mu \geq 0 \\
& h(\mathbf{x})=0 \\
& g(\mathbf{x}) \leq 0
\end{aligned}
$$

Some deterministic methods, applicable to unconstrained non-linear problems, are based on indirect search procedures and can be divided into zeroth, first (gradient-based) and second (hessian-based) order. The zeroth order methods rely on the calculation of the so-called Powell's conjugated directions, whereas the first and second order methods are based on the calculation of the first and second derivatives of the objective function [112]. The search starts from a suitable initial point and, based on a direction and step length criteria properly defined for each method, the search moves towards the point that presents the largest improvement of the objective function [312]. For instance, in the steepest descent method (first order), the direction of the next step is given by the gradient vector of the objective function calculated at the current point (Fig. A1.2). Moreover, the step length is determined by minimizing the local value of the objective function. Sometimes the gradient vector and hessian matrix might be obtained numerically rather than analytically, thus, multimodal functions can make these methods unstable [116].

Fig. A1.2. Steepest descent method applied to the function $F(x, y)=3 x^{2}+y^{2}-2 x y-2 x-2 y$

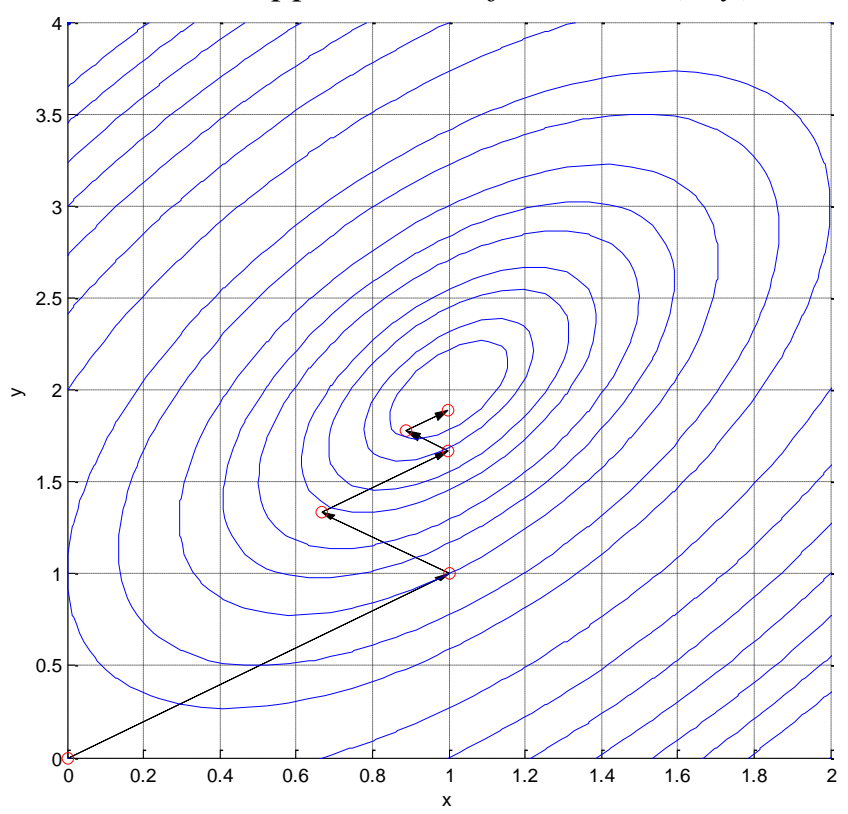


Among the methods used for solving constrained optimization problems, more specifically those in which the objective function as well as the constraints are linear functions of the independent variables (i.e. a linear programming problem), the simplex method is a widely used and efficient scheme. This method searches along the boundary of the feasible domain from one vertex (given by the constraints), to the next until the optimum is obtained [116].

In the case of nonlinear constrained optimization problems, the generalized reduced gradients and projected gradient methods, as well as the feasible direction methods can be used [312]. In these methods, the initial guess or starting point is in the feasible domain. Then, the search is moved to the constraint and obtain a point on the constraint. From this point, the search is moved tangentially to the constraint. Since by doing this the trial point violates the constraint, the next step is used to bring the point back to the feasible region [116]. Other methods, known as penalization methods and augmented lagrangian methods convert the constrained problem to an unconstrained one by modifying the search space (Fig. A1.3 right) [314]. This methods uses penalty parameters $r$, that modify a composite unrestricted function due to the violation of the constraints, Eq.(A1.2). The larger the violation, the higher the penalty. The composite function is then optimized using any of the techniques applicable for unconstrained problems [116].

$$
\begin{aligned}
& \underset{\mathbf{x}}{\operatorname{Min}} \phi(r, \mathbf{x})=f(\mathbf{x})+r \sum_{j=1}^{n_{g}=m}\left\langle-g_{j}\right\rangle^{2} \\
& \text { where } r=r_{1}, r_{2}, \ldots r_{i}, \ldots \rightarrow \infty \\
& \text { and }\langle a\rangle=\max (0, a)
\end{aligned}
$$

Fig. A1.3. Penalization method with $r=1$ (left) and $r=10$ (right) applied to the extended function $\phi(r, \mathbf{x})=f(\mathbf{x})+r \sum_{j=1}^{n_{g}=2}\left\langle-g_{j}\right\rangle^{2}=x_{1}^{2}+x_{2}^{2}+r\left(\left\langle-\left(x_{1}+x_{2}-1\right)\right\rangle^{2}+\left\langle-\left(x_{2}-x_{1}-1\right)\right\rangle^{2}\right)$
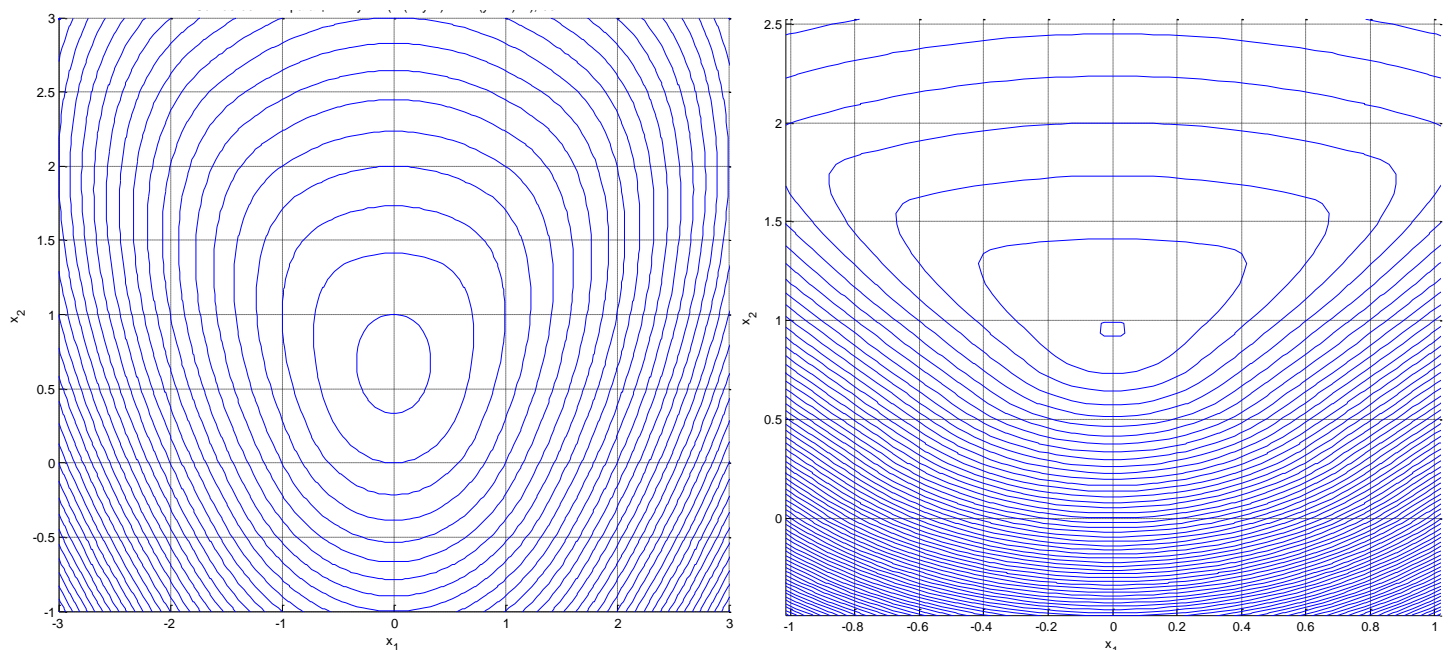

Source: Author.

Other numerical methods, used for the solution of constrained non-linear optimization problems, are based on sequential (linear or quadratic), local approximations of the original 
non-linear problem around the current iterate. Thus, either linear or quadratic programming can be used to solve the local optimization problem. For instance, in the sequential linear programming (SLP) method, the objective and constraints are linearized about the initial point, so that the local problem fits the linear programming (LP) format [112]. After an improved solution is locally obtained by using, e.g. the simplex method, the procedure is repeated until the fitness function is not further improved (Fig. A1.4).

Fig. A1.4. Sequential linear programming (SLP) method applied to the optimization of the objective function $f(\mathbf{x})=x_{1}^{2}+x_{2}^{2}+4 x_{1}+2 x_{2}$ subject to the constraints:

$$
g_{1}(\mathbf{x})=9 x_{1}-x_{1}^{2}-x_{2}^{2}-x_{2} \geq 0 \text { and } g_{2}(\mathbf{x})=x_{1}^{2}+x_{2}^{2}-7 x_{1}+2 x_{2} \geq 0 \text {. }
$$

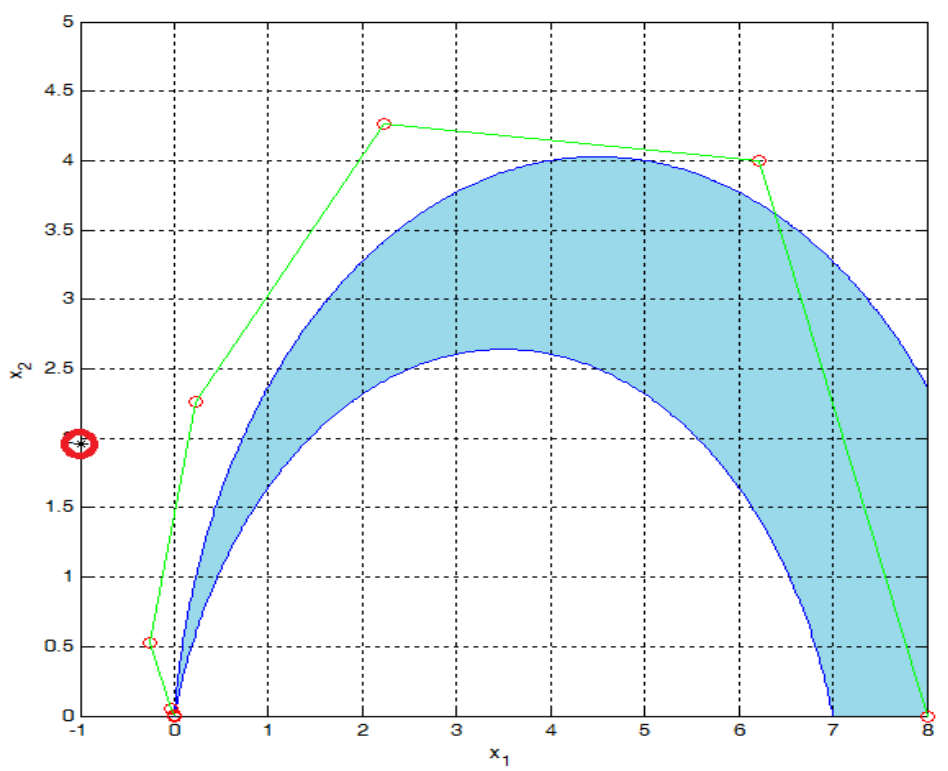

Source: Author.

Meanwhile, in the sequential quadratic programming (SQP) approach, the objective function is locally approximated as a quadratic function, whereas the restrictions are suitably linearized, according to Eq. (A1.3).

$$
\begin{gathered}
f(\mathbf{x}) \approx f\left(\mathbf{x}^{k}\right)+\nabla f\left(\mathbf{x}^{k}\right)^{T}\left(\mathbf{x}-\mathbf{x}^{k}\right)+\frac{1}{2}\left(\mathbf{x}-\mathbf{x}^{k}\right)^{T}\left[H_{f}\left(\mathbf{x}^{k}\right)\right]\left(\mathbf{x}-\mathbf{x}^{k}\right) \\
h(\mathbf{x}) \approx h\left(\mathbf{x}^{k}\right)+\nabla h\left(\mathbf{x}^{k}\right)\left(\mathbf{x}-\mathbf{x}^{k}\right) \\
g(\mathbf{x}) \approx g\left(\mathbf{x}^{k}\right)+\nabla g\left(\mathbf{x}^{k}\right)\left(\mathbf{x}-\mathbf{x}^{k}\right)
\end{gathered}
$$

In this way, the optimization problem takes the form of a quadratic programming problem, Eq.(A1.4), to be solved in each iteration by using, e.g. augmented lagrangian methods. Indeed, if it were not for the presence of constraints, the SQP approach would resemble the application of the Newton method for the numerical solution of nonlinear algebraic systems of equations [99]. 
$\underset{\mathbf{x}}{\operatorname{Min}} \nabla f\left(\mathbf{x}^{k}\right)\left(\mathbf{x}-\mathbf{x}^{k}\right)+\frac{1}{2}\left(\mathbf{x}-\mathbf{x}^{k}\right)^{T}\left[H_{f}\left(\mathbf{x}^{k}\right)\right]\left(\mathbf{x}-\mathbf{x}^{k}\right)$

subject to

$$
\begin{aligned}
& h\left(\mathbf{x}^{k}\right)+\nabla h\left(\mathbf{x}^{k}\right)\left(\mathbf{x}-\mathbf{x}^{k}\right)=0 \\
& g\left(\mathbf{x}^{k}\right)+\nabla g\left(\mathbf{x}^{k}\right)\left(\mathbf{x}-\mathbf{x}^{k}\right) \leq 0 \\
& \mathbf{x}-\mathbf{x}^{k} \in \mathbb{R}^{n}
\end{aligned}
$$

More recently, the structural process synthesis of complex chemical plants has taken advantage of robust optimization methods that can deal with mixed integer linear (MILP) and non-linear programing (MINLP). Branch and Bound and Outer Approximation approaches are examples of them [146]. In the former, the exhaustive evaluation of the integer variables is avoided by using a relaxed problem and using a decision tree that stands for all the potential solutions, either feasible or unfeasible (Fig. A1.5). This collection of nodes can be linked so that an unique path from any initial to final node represents the combination of the integer variables that enable or disable certain components and interconnections within the process flowsheet. Moreover, the main hypothesis consists of the idea that the relaxed model (point 1, Fig A1.5) holds the bound of the original mixed integer optimization problem. Thus, the other nodes are investigated either breadth first or depth first, until they are found to be inferior or they prove to be the optimal solution [99]. Since some infeasible nodes will not need to be further examined, a fathoming of the branch eliminates implicitly large groups of potential solutions without explicitly evaluating them [111].

Fig. A1.5. Breadth first (left) and depth first (right) searches used in the solution of MILP and MINLP problems with the Branch and Bound method
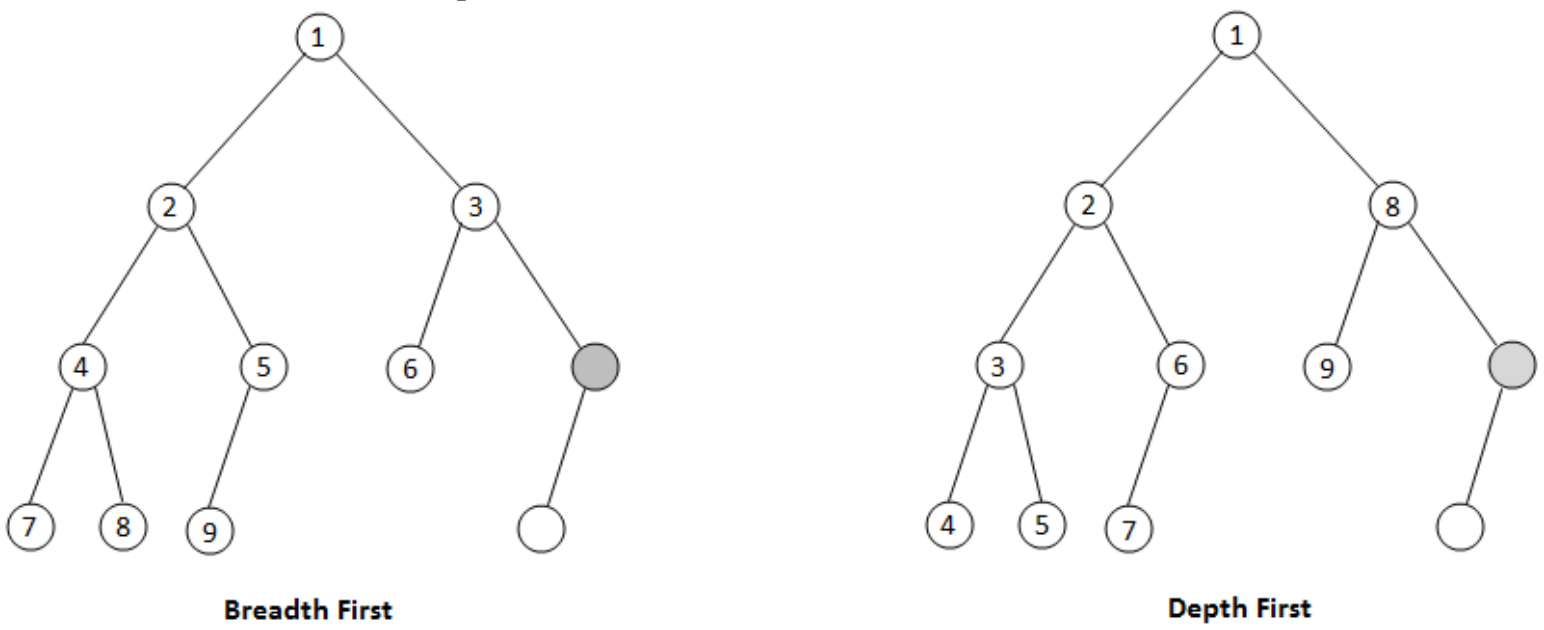

Source: Author.

Notwithstanding, the conventional deterministic methods often fail to find the optimal solution if the objective function is not continuous and smooth, or has many local extrema [315]. In fact, non-convex models with local optima and combinatorial explosion are the likeliest problems encountered in the optimization of complex chemical plant flowsheets involving integers variables [117]. Therefore, there has been a considerable development in optimization methods that include stochastic techniques, which use randomness to guide the 
search and allow intermediate deterioration of the objective function [99]. In this way, they are less prone to be stuck in a local optimum than deterministic methods. These methods can handle problems in which the derivatives would be cumbersome to calculate, causing the deterministic methods to fail [99].

For instance, in the genetic algorithm, populations of strings (the analogy of chromosomes) are created to represent a set of parameters (e.g. temperature, pressure, concentration, etc.), so that basic operations of natural genetics (i.e., reproduction, crossover and mutation) can be used in the search procedure [316]. Differently from deterministic approaches, genetic algorithms move from one set of points (population) to another set of points [317]. Other stochastic methods are often called global search metaheuristic methods due to the use of a set of heuristics combined with random search [318]. For instance, in the simulated annealing the probability that the objective function deteriorates is initially high, but as the temperature is gradually reduced, the probability of accepting new deteriorated solution is lower. At the end, when the temperature has been decreased enough, the rejection of non-optimal solutions is at its highest likelihood [314].

In summary, heuristics, thermodynamics and algorithmic approaches have been widely applied in process synthesis and optimization, but they are not exempt of some drawbacks. The first two do not have any guarantee that the optimum solutions are actually found, since the optimum configuration may be one that is currently unknown. Furthermore, they do not provide a global framework for synthetizing a variety of systems that are composed of different types of major components (chemical plants, heat recovery networks and utility plant) [146]. Meanwhile, the last one requires a significant computational effort and, inevitably, depends on and is restricted by the initial superstructure [111]. Thus, heuristics could be used in a preliminary screening to eliminate non-promising alternatives or generate good estimates; whereas thermodynamic approaches could be used to develop bounds or eliminate energy inefficient alternatives. In fact, the utilization of the heuristic and thermodynamic approaches for the improvement in isolation of the most important components represents rather a good starting value for a broader iterative optimization of the total plant $[146,148]$. In turn, the algorithmic approaches could be useful to automatically generate integrated flowsheets.

By considering the pervasive impact of the chemical production processes in the economic, energetic and environmental systems in which are contained, it is coherent to question whether the best solution in terms of economics simultaneously optimizes the other facets. Certainly, the economic performance represents an important criterion, but the chemical process must be also designed as a part of a sustainable industrial activity [99]. In other words, the chemical systems should not compromise the ability of the ecosystem to maintain the natural cycles that hold the species and provide society with the goods and services. Accordingly, the consumption of raw materials and the production of wastes should be minimized by performing the process as efficiently as it is economic and practicable.

However, when two or more optimization criteria are simultaneously imposed, different solutions can be acceptable, depending on the particular target that is assumed. For this 
reason, the multi-objective optimization appears as an attempt to reconcile the decision making perspectives by conveniently displaying a set of optimal solutions that are not dominated by the remaining solutions, known as Pareto optimal set. Therefore, the optimal solution may not be unique and is 'optimal' only in the strict mathematical sense. Thus, the expert human intervention is needed to weigh the alternatives and reach a final decision [149].

On the one hand, the thermodynamic criterion attempts to minimize the entropy generation (or exergy destruction) along the various components of the process. It can be achieved by means of the integration of the energy systems and the reduction of the avoidable losses derived from large driving forces. On the other hand, the thermoeconomy optimization use performance criteria also based on costs engineering and economy, looking towards the minimization of the costs of production. These costs may include initial investment costs, marginal revamping costs, operational costs, and so forth. Regarding the environmental optimization, some authors have proposed the exergy as an measure of the deviation from environmental conditions and for the renewability assessment [319]. Yet, exergy is not necessarily related to other undesirable environmental burdens, e.g. toxicity, greenhouse gas emissions, etc.

It is also important to notice that the industrial processes are often required to operate under variable feedstock supply and cost, catalyst and equipment performance, as well as variable environmental and commercial factors along the plant lifespan. Thus, a process configuration found to be optimum for certain conditions may not be reliable when operating under different specifications. Accordingly, sometimes it is recommended to accomplish an analysis of sensitivity of the optimum to determine the rate at which the objective function changes as one of the decision variables is changed. Such perturbations show the sensitiveness of the objective function, allowing to determine the control precision required or the penalty for failure to control the variable within the imposed restrictions.

Consequently, the selection of the adequate design variables, and the objective and constraint functions is fundamental for the success of the optimization process. In fact, as the number of independent variables increases, the computational effort for solving the optimization problem increases, particularly for thermodynamic systems, due to the complicated non-linear characteristics [279]. Therefore, it is desirable to focus in the dominant variables rather than considering all that might affect the solution. Additionally, the determination of more flexible constraints would be advantageous for reducing or increasing the value of the objective function. 
APPENDIX 2

\section{AN OVERVIEW OF THE INVESTMENT AND OVERALL COSTS OF THE AMMONIA PRODUCTION}

In the following, an overview on the investment and overall production costs in the integrated syngas and ammonia production plants is firstly introduced. Next, a sensitivity analysis on the effect of the share of the capital investment in the overall plant revenue for the conventional and alternative production routes, studied in Chapters 7 and 11, are dicussed.

\section{A2.1. Investment and operating cost of conventional and alternative production routes:} A literature review.

The reported investment costs present a marked dependence on the technology and the nature of the energy resources selected, as well as on the individual economic data assumed in each study (interest rate, year of analysis, plant lifespan, among others).

- Review on the investment costs of the conventional and alternative ammonia production plants.

In an early study of the World Bank [22], an economic assessment of a 1500 t/day ammonia plant included different scenarios of existing and new plants settled in developed, developing or remote locations. The estimated capital costs range from 170 to 350 million dollars (1998), which after updated and annualized (20 years, 6\% interest rate, CEPCI 550) are equivalent to overall ammonia plant costs between 38.28-78.8 US dollars/ $\mathrm{t}_{\mathrm{NH} 3}$, respectively. Those values certainly contrast to the investment cost reported by Seddon [320] equal to 1000 million dollars (2006) for a $2320 \mathrm{t}_{\mathrm{NH} 3}$ /day ammonia yield, or currently equivalent to about $100 \mathrm{US}$ dollars $/ \mathrm{t}_{\mathrm{NH} 3}$. In other study, Bartels [64] reports the investment cost of an ammonia synthesis plant based on the various hydrogen production scenarios presented by Rutkowski [321]. The study compared the capital cost of four ammonia production pathways via natural gas reforming (2004 $\mathrm{t}_{\mathrm{NH}_{3}} /$ day) and coal gasification (1563 $\mathrm{t}_{\mathrm{NH} 3} /$ day) with and without taking into account the capital cost of the carbon capture system (CCS). In the case of the SMR-based configurations, the updated investment costs range from 72.79 to $81.53 \mathrm{US}$ dollars $/ \mathrm{t}_{\mathrm{NH} 3}$, with the difference corresponding to the approximate cost of the CCS (8.74 US dollars/ $\left.\mathrm{t}_{\mathrm{NH} 3}\right)$. In the case of the ammonia production plant based on coal gasification, the respective figures for the capital costs can be as high as 146.61 - 175.50 US dollars/ $\mathrm{N}_{\mathrm{NH} 3}$. Accordingly, the CSS would represent between $11-16 \%$ of the overall investment cost of the integrated syngas and ammonia production facility [64].

Based on more recent data, Bartels [64] tabulated the variation of the total investment cost of an industrial ammonia plant based in the conventional SMR showing that the capital cost reduces from 100.78 US dollars/ $\mathrm{t}_{\mathrm{NH} 3}$ for $1000 \mathrm{t}_{\mathrm{NH} 3} /$ day yield rate up to $79.07 \mathrm{US}$ dollars/ $\mathrm{t}_{\mathrm{NH} 3}$ for a $2200 \mathrm{t}_{\mathrm{NH} 3}$ /day plant. These figures apply for an ammonia production facility that does 
not integrate either a gas turbine or an air separation unit; otherwise, they may increase up to 146.46-186.67 US dollars/ $\mathrm{t}_{\mathrm{NH} 3}$, with the lower figures corresponding to a $2200 \mathrm{t}_{\mathrm{NH} 3} / \mathrm{day}$ ammonia yield due to the economy of scale. The share of the investment cost of the ammonia synthesis loop alone and the air separation unit (ASU) are, in turn, estimated as being 20-30\% and $20 \%$ of the overall ammonia plant cost, respectively [64]. These results agree with the values estimated by Appl [218] after updated for an ammonia production plant (1800 $\mathrm{t}_{\mathrm{NH} 3} /$ day) based on natural gas steam reforming (85.58 US dollars/ $\mathrm{t}_{\mathrm{NH} 3}$ ) and coal gasification (171.16 US dollars/t $\left.\mathrm{t}_{\mathrm{NH} 3}\right)$.

Regarding the ammonia production via biomass gasification, the research works of Mann (736 $\mathrm{t}_{\mathrm{NH} 3} /$ day) [322], and Padró and Pusche (1022 $\mathrm{t}_{\mathrm{NH} 3} /$ day) [323] apud Bartels [64] can be used to estimate the investment cost of the entire ammonia production facility including a gas turbine and an auxiliary ASU. After updated from 2005 and 1992 US dollars, respectively, the reported figures vary between 194.67 - 241.98 US dollars $/ \mathrm{t}_{\mathrm{NH} 3}$. Furthermore, the share of the investment cost of the Haber-Bosch ammonia loop alone is reportedly to be at least $50-52 \%$ of the overall investment cost of the plant. Similarly, Arora et al [30] calculated the capital cost of a small scale $120 \mathrm{t}_{\mathrm{NH} 3}$ /day ammonia production plant, based on the gasification of wood, straw and bagasse, ranging between 127.66 - 237.09 US dollars/ $\mathrm{t}_{\mathrm{NH} 3}$. However, unlike the works of Mann and Padró and Pusche, in the Arora's research work, the gasifier cost and gas conditioning account for about $70-80 \%$ of the overall investment cost, whereas the ammonia loop barely contributes to $10 \%$ of the overall investment cost. Indeed, the share of investment cost of the ammonia loop alone is reportedly comparable to the respective shares of the ASU and the utility system [30]. This fact highlights the large uncertainty that the calculation of the capital cost of unconventional ammonia production plants reported in the literature cope with.

Meanwhile, despite of the expensive figures exhibited by the biomass-based industrial ammonia plants, the highest investment costs actually arise from the utilization of water electrolysis powered by alternative energy technologies such as wind farms and photovoltaic panels. For instance, based on the studies of hydrogen production reported by Glatzmaier et al [324], Bartels estimated a total capital cost of approximately 1438.17 US dollars $/ t_{\mathrm{NH} 3}$ for a 7 $\mathrm{t}_{\mathrm{NH} 3}$ /day ammonia production plant when an interest rate of $15 \%$ and 20 years lifespan is considered [64]. The ammonia synthesis loop alone represents again close to $48 \%$ of the overall plant cost. Certainly, the lower ammonia yield rate is responsible for the highest capital cost that the solar ammonia production is responsible for. Meanwhile, the research works of Levene et al [325] apud Bartels can be used to estimate the updated investment cost of an ammonia production plant based on the water electrolysis by using the electricity harvested from wind farms. Anyway, in spite of a slightly reduced investment cost of the wind-based ammonia production (970.23 US dollars/ $\mathrm{t}_{\mathrm{NH} 3}$ ) compared to the solar-driven designs, most of these alternative energy conversion systems remains to be uncompetitive when compared to either the natural gas, coal or even biomass-based ammonia production configurations. 
- Review on the overall production (operating plus investment) costs of the conventional and alternative ammonia production plants.

Several authors reported the overall cost of the ammonia production for both natural gas and biomass-based routes. Maung et al [326] estimated the overall costs of the ammonia production based on the use of both flared and conventional natural gas as US dollars 400$600 / t_{\mathrm{NH} 3}$. In other words, for a conventional ammonia plant configuration, the average investment cost (100 US dollars/ $/ \mathrm{t}_{\mathrm{NH} 3}$ ) corresponds from one fifth to one fourth of the overall ammonia production cost. These values are in close agreement with those obtained in Chapter 4, 9 and 10 of this thesis. On the other hand, the utilization of biomass brings about a marked increase of the overall ammonia production costs compared to an scenario of natural gas consumption. This can be explained by a strong dependency on the economy of scale and the cost of the biomass consumed as feedstock, which may sharply vary from US dollar 20 for bagasse up to 260 for wood per ton of dry biomass (tdb) [327].

For instance, Gilbert et al [289] evaluated the ammonia production based on the gasification of wood in a $1200 \mathrm{t}_{\mathrm{NH} 3}$ /day plant, obtaining a production cost of only $500 \mathrm{US}$ dollars/ $\mathrm{t}_{\mathrm{NH} 3}$ for a relatively low biomass feedstock cost of $60 \mathrm{USD} / \mathrm{tdb}$. Meanwhile, the work of Andersson and Lundgren [290] suggests an overall production cost as high as 750-1150 US dollars/ $\mathrm{t}_{\mathrm{NH} 3}$ for a biomass feedstock cost of 70-200 USD/tdb [30, 327]. Interestingly, Andersson and Lundgren's figures show not only of a twofold production cost but they also correspond to half of the ammonia throughput ( $700 \mathrm{t}_{\mathrm{NH}_{3}} /$ day) of the Gilbert's calculations (1200 $\left.\mathrm{t}_{\mathrm{NH} 3} / \mathrm{day}\right)$. As a conclusion, Gilbert's findings points towards the halving of the total production cost by barely doubling the ammonia yield rate. Additionaly, the production cost of Andersson and Lundgren are similar to those reported by Tock and Maréchal (970 US dollars/ $\mathrm{t}_{\mathrm{NH} 3}$ ), when gasified wood (70-200 USD/tdb) is used to produce about $1190 \mathrm{t}_{\mathrm{NH} 3} /$ day at a relatively low interest rate $(6 \%)$ for investment calculation purposes.

Sarkar et al [328] apud Arora [30] calculated the highest ammonia production cost for a 2000 $\mathrm{t}_{\mathrm{NH} 3}$ /day production plant based on gasification of wood chips as being equal to $2000 \mathrm{US}$ dollars $/ t_{\mathrm{NH} 3}$. More recently, Arora et al [327] estimated an overall production cost of about 1150 US dollars $/ \mathrm{t}_{\mathrm{NH} 3}$ for the small scale ammonia production from biomass when a relatively expensive (100 USD/tdb) and high interest rate is assumed (15\%). Later, Arora et al [30] calculated the total production cost of a small scale ammonia plant (120 t/day) based on the gasification of different types of biomass feedstock. The authors concluded that the investment cost contributes to $35 \%, 45 \%$, and $52 \%$ of the total ammonia production cost in the case of wood, straw, and bagasse feedstock, respectively. The remaining costs correspond to the sum of the operation and maintenance costs, as well as the cost of the electricity and biomass consumed, among other expenses.

\section{A2.2. Influence of the investment cost share on the overall production revenues in the conventional and alternative ammonia production routes.}

As it has been shown, the reported data about the overall and investment costs of modern ammonia production plants may present noticeable divergences between the studies. 
Nonetheless this information is still relevant for estimating the effect of the investment cost share on the overall ammonia production revenues, as shown in Fig. A2.1. In this figure, the 'net' revenue (REV) curves are calculated as the difference between the gross incomes (INCO) and the overall production expenditure (TOEX i.e. the sum of investment,CAPEX, and operating, OPEX, costs) as a function of the CAPEX-to-TOEX ratio (share \%):

$$
\mathrm{REV}=I N C O-\left(1+\frac{\text { share } \%}{1-\text { share } \%}\right) \text { OPEX }
$$

Thus, the intercepts of the revenue curves with the vertical axis (euro/ton $\mathrm{NH}_{\mathrm{NH}}$ ) stand for the case in which only the operating costs are considered, i.e. when the investment cost share is not under scrutiny. Those values have been reported as operating revenues in Table 7.2 and Table 11.2 and may be interpreted as upper bounds for the achievable revenues in retrofitted $n$-th plants (existing, operational and mature plants).

Fig. A2.1. Influence of the investment cost share on the overall production revenues for the

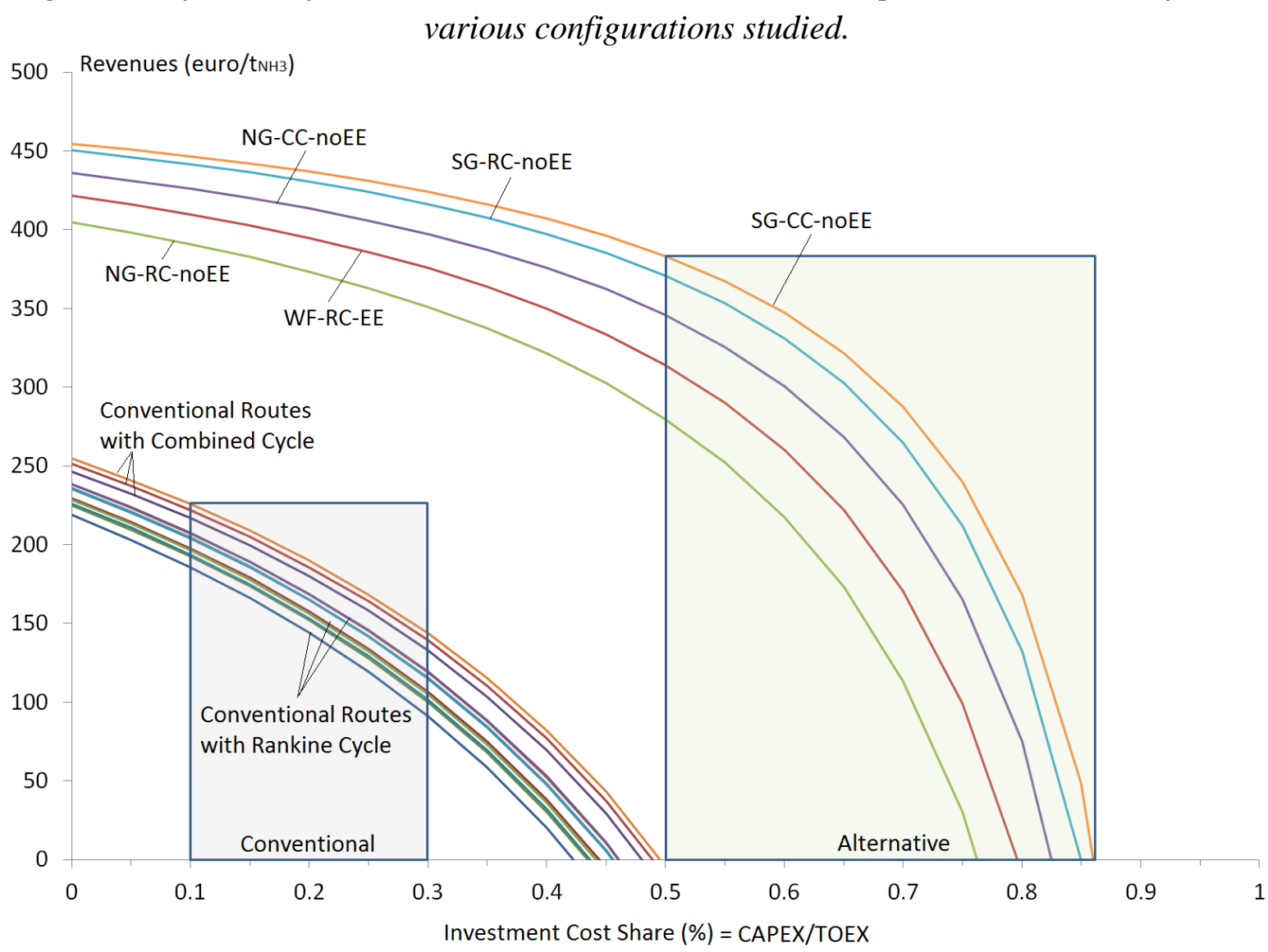

The rectangular demarcated zones in Fig. A2.1 enclose the representative intervals in which the share of the investment cost (CAPEX/TOEX) is assumed to vary, according to the reviewed literature. For the conventional case, an investment cost share between 10-30\% of the overall ammonia production cost is in strong agreement with the reported values of investment cost, ranging between 80-210 euro/ton $\mathrm{NH}_{3}$. As a result, if the investment costs of the grassroot ammonia production plants are considered, an important reduction of about $50 \%$ of the operating revenues, shown in Table 7.2, is expected. 
Meanwhile, the investment cost contribution of the alternative ammonia production plants is here assumed to be higher due to factors related with less mature, energy intensive biomass pretreatment and syngas purification technologies, economy of scale of the gasification of renewable energy resources and the investment risk, as discussed in Chapters 11 and 12. Thus, the investment cost share interval is assumed varying from a typical 50\% contribution up to the worst case scenario in which the overall revenues become null, in order to guarantee at least a positive value. In this way, the maximum investment costs is assumed to be comparable to the maximum operating revenues $\left(400-450\right.$ euro/ton $\left.\mathrm{NH}_{3}\right)$, which is close to some values reported in the available literature [30]. Thus, from these assumptions, if the investment cost share exceeds $75-85 \%$ of the overall cost, the alternative scenarios studied are not anymore profitable, due to the incapacity of the productive system to compensate the annualized investment cost. In any case, as concerns the alternative ammonia production configurations studied in Chapter 11, the utilization of more efficient cogeneration systems (e.g. combined cycles) that consume syngas derived from inexpensive bagasse for combined heat and power production is expected to increase the process revenues, as long as the costly natural gas and electricity consumption are spared.

However, according to Fig. A2.1, the advantage offered by a less expensive feedstock (bagasse) may be still offset by an increment of the investment cost associated to a more complex gasification and treatment processes, characteristic of the current biomass energy conversion systems. This fact reinforces the idea that, even though an abundant and cheap feedstock brings about benefits in terms of both operating costs and environmental impact, the investment and risk costs along with economy of scale still represent important challenges to be adressed for achieving the decarbonization of the SNF sector. 


\section{APPENDIX 3}

\section{PAPERS PUBLISHED AND CONFERENCES ATTENDED}

- Papers published in international journals.

- FLÓREZ-ORREGO, D.; OLIVEIRA JR, S. On the efficiency, exergy costs and CO2 emission cost allocation for an integrated syngas and ammonia production plant. Energy (Oxford), v. 117, Part 2, Pages 341-360, 2016.

- FLÓREZ-ORREGO, D.; OLIVEIRA JR, S. Modeling and optimization of an industrial ammonia synthesis unit: An exergy approach. Energy (Oxford), v. 137, 15, pp 234-250, 2017.

- FLÓREZ-ORREGO, D.; OLIVEIRA JR, S. Exergy assessment of single and dual pressure industrial ammonia synthesis units. Energy (Oxford), v. 141, pp 2540-2558. 2017.

- FlÓREZ-ORREGO, D. HENRIQUES, I. B., NGUYEN, T., MENDES DA SILVA, J., MADY, C.E., PELLEGRINI, L.F., GANDOLFI, R., VELÁSQUEZ, H., BURBANO, J.C., LATTOUF, R., DE OLIVEIRA JUNIOR, S. The contributions of Prof. Jan Szargut to the exergy and environmental assessment of complex energy systems, Energy (Oxford), v.161, pp. 482-492. 2018.

- SANTOS, R.G.; FARIA, P. R.; FLÓREZ-ORREGO, D.; SANTOS, J.J.C.S.; SILVA, J.A.M., Thermoeconomic Modeling for CO2 Allocation in Steam and Gas Turbine Cogeneration Systems, Energy (Oxford), v.117, Part 2, pp 590-603. 2016.

- Papers submitted to international journals.

- FLÓREZ-ORREGO, D., SHARMA, S., OLIVEIRA JR, S., MARECHAL, F., Combined Exergy Analysis and Energy Integration for Design Optimization of Nitrogen Fertilizer Plants. Frontiers in Energy Research.

FLÓREZ-ORREGO, D, NASCIMENTO SILVA, F., OLIVEIRA JUNIOR, S., Syngas Production with Thermo-Chemically Recuperated Gas Turbine Systems: An Exergy Analysis and Energy Integration Study. Energy (Oxford).

- OROZCO LOAIZA, C., MAYA LOPEZ, J., VELASQUEZ ARREDONDO, H.I. FLÓREZORREGO, D. Exergy analysis of the Colombian energy matrix: A view from its economic sectors and energy resources, International Journal Of Sustainable Energy Planning And Management. 
- NASCIMENTO SILVA, F., FLÓREZ-ORREGO, D, OLIVEIRA JUNIOR, S. Oxycombustion Gas Turbine for Increased Efficiency in Offshore Platform Applications, Energy Conversion and Management.

- FLÓREZ-ORREGO, D, OLIVEIRA JUNIOR, S., MARECHAL, F. Comparative Exergy and Technoeconomic Assessment of Fossil and Biomass-Based Routes for Hydrogen and Ammonia Production, Energy Conversion and Management.

- NAKASHIMA, R., FLÓREZ-ORREGO, D, OLIVEIRA JUNIOR, S. Integration of Anaerobic Digestion and Biomass Gasification for combined Hydrogen, Biomethane and Power Production, Journal Of Power Technology.

- SILVA ORTIZ, P., FLÓREZ-ORREGO, D., OLIVEIRA JUNIOR, S., MARÉCHAL, F., MACIEL FILHO, R. Exergetic, Environmental And Economic Assessment Of Sugarcane First-Generation Biorefineries, Journal Of Power Technology.

- Technical contributions to the proceedings of international conferences.

- FLÓREZ-ORREGO, D.; OLIVEIRA JR, S. On the Allocation of the Exergy Costs and CO2 Emission Cost for an Integrated Syngas and Ammonia Production Plant. 28th International Conference on Efficiency, Cost, Optimization, Simulation and Environmental Impact of Energy Systems, ECOS 2015, Pau, France, June 29th - July 3rd, 2015.

- FLÓREZ-ORREGO, D.; OLIVEIRA JR, S. Exergy Modeling and Optimization of an Ammonia Production Plant. 29th International Conference on Efficiency, Cost, Optimization, Simulation and Environmental Impact of Energy Systems, ECOS 2016, Portoroz, Slovenia, June 19th- 23rd, 2016. Best Conference Paper Award.

- FLÓREZ-ORREGO, D.; OLIVEIRA JR, S, Exergy Assessment of Single and Dual Pressure Ammonia Production Plants, International Conference on Contemporary Problems of Thermal Engineering - CPOTE 2016, Gliwice, Poland, September 14-16, 2016.

- FLÓREZ-ORREGO, D.; SHARMA S.; OLIVEIRA JR, S.; MARECHAL, F. Combined Exergy Analysis and Energy Integration for Design Optimization of Nitrogen Fertilizer Plants. 30th International Conference on Efficiency, Cost, Optimization, Simulation and Environmental Impact of Energy Systems, ECOS 2017, San Diego, United States of America, July 2nd - 6th, 2017.

- FLÓREZ-ORREGO, D, NASCIMENTO SILVA, F., OLIVEIRA JUNIOR, S., Syngas Production with Thermo-Chemically Recuperated Gas Turbine Systems: An Exergy Analysis and Energy Integration Study. 31th International Conference on Efficiency, Cost, Optimization, Simulation and Environmental Impact of Energy Systems, ECOS 2018, Guimaraes, Portugal, June 17th - 22nd , 2018.

- FlÓREZ-ORREGO, D., NGUYEN, T., SILVA, J.A.M., OLIVEIRA JR, S. The contributions of Prof. Jan Szargut to the exergy and environmental assessment of fuels and 
chemicals production, 31th International Conference on Efficiency, Cost, Optimization, Simulation and Environmental Impact of Energy Systems, ECOS 2018, Guimaraes, Portugal, June 17th - 22nd, 2018.

- OROZCO LOAIZA, C., MAYA LOPEZ, J., VELASQUEZ ARREDONDO, H.I. FLÓREZORREGO, D. Exergy analysis of the Colombian energy matrix: A view from its economic sectors and energy resources, 31th International Conference on Efficiency, Cost, Optimization, Simulation and Environmental Impact of Energy Systems, ECOS 2018, Guimaraes, Portugal, June 17th - 22nd, 2018.

- NASCIMENTO SILVA, F., FLÓREZ-ORREGO, D, OLIVEIRA JUNIOR, S. Oxycombustion Gas Turbine for Increased Efficiency in Offshore Platform Applications, International Conference on Contemporary Problems of Thermal Engineering - CPOTE 2018, Gliwice, Poland, 18th - 21st September 2018.

- FLÓREZ-ORREGO, D, OLIVEIRA JUNIOR, S., MARECHAL, F. Comparative Exergy and Technoeconomic Assessment of Fossil and Biomass-Based Routes for Hydrogen and Ammonia Production, International Conference on Contemporary Problems of Thermal Engineering - CPOTE 2018, Gliwice, Poland, 18th - 21st September 2018.

- NAKASHIMA, R., FLÓREZ-ORREGO, D, OLIVEIRA JUNIOR, S. Integration of Anaerobic Digestion and Biomass Gasification for combined Hydrogen, Biomethane and Power Production, International Conference on Contemporary Problems of Thermal Engineering - CPOTE 2018, Gliwice, Poland, 18th - 21st September 2018

- SILVA ORTIZ, P., FLÓREZ-ORREGO, D., OLIVEIRA JUNIOR, S., MARÉCHAL, F., MACIEL FILHO, R. Exergetic, Environmental And Economic Assessment Of Sugarcane First-Generation Biorefineries, International Conference on Contemporary Problems of Thermal Engineering - CPOTE 2018, Gliwice, Poland, 18th - 21st September 2018.

- Other conferences and seminars attended.

- 15th Brazilian Congress of Thermal Sciences and Engineering - ENCIT 2014, November 10-13, 2014, Belem, PA, Brazil. Oral Presentation.

- 27th International Conference on Efficiency, Cost, Optimization, Simulation and Environmental Impact of Energy Systems, ECOS 2014, Turku, Finland, June 15-19. Oral Presentation.

- FAPESP-NERC's Workshop on Sustainable Gas Futures, February 25, 2015, FAPESP, São Paulo. Attendee.

- Summer Workshop - Petroleum and Natural Gas Resources PRH19/PRH04, March 3-5, 2015, University of Sao Paulo. Attendee 
- Introduction to Optimization Technics, Webinar - Engineering Simulation and Scientific Software - ESSS, São Paulo, Brazil, March, 17 and 24,2015. Attendee

- 13th Science and Engineering Fair - FEBRACE, University of Sao Paulo - LSI-EPUSP, March, 2015. Referee

- 28th International Conference on Efficiency, Cost, Optimization, Simulation and Environmental Impact of Energy Systems, ECOS 2015, Pau, France, June 29th to July 3rd, 2015. Oral Presentation

- 14th Science and Engineering Fair - FEBRACE, University of Sao Paulo - LSI-EPUSP, March, 2016. Referee

- 29th International Conference on Efficiency, Cost, Optimization, Simulation and Environmental Impact of Energy Systems, ECOS 2016, Portoroz, Slovenia, June 19th- 23rd, 2016. Oral Presentation

- 30th International Conference on Efficiency, Cost, Optimization, Simulation and Environmental Impact of Energy Systems, ECOS 2017, San Diego, United States of America, July 2nd - 6th, 2017. Oral Presentation

- Conference on Contemporary Problems of Thermal Engineering - CPOTE 2016, Gliwice, Poland, September 14-16, 2016. Oral Presentation

- Graduate course on Fundamentals of Biofuels Production, June-December, 2016, PRH/ANP 44, Federal University of Sao Paulo, Brazil. Attendee

- Graduate course on Modeling, Optimization, Design and Analysis of Integrated Energy Systems (Dec. 13-16, 2016), École Polytechnique Fédérale de Lausanne, Switzerland. Attendee

- Seminar on the state of art of the energy storage and the introduction of renewable energy sources, energy and Environment Institute, University of São Paulo, March, 19-20, 2018, ANEEL PD-00061-0054/2016, Attendee

- 31th International Conference on Efficiency, Cost, Optimization, Simulation and Environmental Impact of Energy Systems, ECOS 2018, Guimaraes, Portugal, June 17th 22nd , 2018. Oral Presentation

- Sao Paulo School of Advanced Science on Renewable Energies, July 23rd - August 3, 2018. University of Sao Paulo, Brazil. Participant 
- Fellowships and Recognitions.

- Swiss Government Excellence Fellowship for Young Scientists and Artists, 2016-2017 PhD Research Scholarship (12 months)

- Outstanding Contribution in Reviewing, Energy (Oxford), Elsevier, 2016 Amsterdam, The Netherlands.

- Outstanding Contribution in Reviewing, Journal of Cleaner Production, 2017 Elsevier, Amsterdam, The Netherlands.

- Awards.

- Best Conference Paper Award - ECOS 2016 - 29 $9^{\text {th }}$ Conference on Energy, 2017 Cost, Optimization and Simulation of Energy Systems, Portoroz, Slovenia

- Peer Review Activities.

Journal of the Faculty of Sciences - National University of Colombia, International Journal of Hydrogen Energy, Applied Energy, Journal of Cleaner Production, Energy Journal, International Journal of Electrical Power and Energy Systems Engineering 


\section{COLCIENCIAS}

Colombian Department of Science, Technology and Innovation

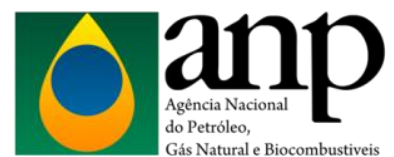

Brazilian National Agency of Petroleum, Natural Gas and Biofuels

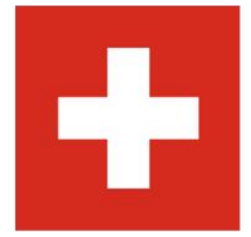

Swiss Federal Commission for Scholarships for Foreign Students 